COOPERATIVE RESEARCH IN COAL LIQUEFACTION

Final Report for the Period May 4, 1989-May 3, 1990

By

G. P. Huffman

Lyle V. A. Sendlein

May 28, 1991

Work Performed Under Contract No. FC22-89PC89851

For

U.S. Department of Energy

Pittsburgh Energy Technology Center

Pittsburgh, Pennsylvania

By

University of Kentucky

Lexington, Kentucky 


\section{DISCLAIMER}

This report was prepared as an account of work sponsored by an agency of the United States Government. Neither the United States Government nor any agency thereof, nor any of their employees, makes any warranty, express or implied, or assumes any legal liability or responsibility for the accuracy, completeness, or usefulness of any information, apparatus, product, or process disclosed. or represents that its use would not infringe privately owned rights. Reference herein to any specific commercial product, process, or service by trade name, trademark. manufacturer, or otherwise does not necessarily constiiute or imply its endorsement, recommendation, or favoring ty the United States Government or iny agency thereof. The views and opinions of authors ex. pressed herein do not necessarily state or reflect those of the United States Government or any agency thereof.

This report has been reproduced directly from the best available copy.

Available to DOE and DOE contractors from the Office of Scientific and Technical Information, P.O. Box 62, Oak Ridge, TN 37831; prices available from $(615) 576.8401$, FTS 626-8401.

Available to the public from the National Technical Information Service, U.S. Department of Commerce, 5285 Port Royal Rd., Springfield, VA 22161. 


\title{
Cooperative Research In Coal
}

\section{Liquefaction}

\author{
Final Report on DOE Contract \\ No. DE-FC22-89PC89851
}

Period: May 4, 1989 to May 3, 1990

Submitted to the

U.S. Department of Energy

Edited by:

G. P. Huffman, Director

Consortium for Fossil Fuel Liquefaction Science

233 Mining \& Mineral Resources Building

Lexington, KY 40506-0107

Phone: (606) 257-4027

Lyle V. A. Sendlein, Director

Institute for Mining \& Minerals Research

May 28, 1991

Submitted by the University of Kentucky

On Behalf of the

Consortium for Fossil Fuel Liquefaction Science

Auburn University

University of Kentucky

University of Pittsburgh

West Virginia University

University of Utah 
Table of Contents

Page

Overview of Research Activities $\ldots \ldots \ldots \ldots \ldots \ldots \ldots \ldots$

Task I - University of Kentucky . . . . . . . . . . . 17

Task II - University of Pittsburgh $\ldots \ldots \ldots \ldots \ldots \ldots \ldots$

Task III - West Virginia University $\ldots \ldots \ldots \ldots \ldots \ldots \ldots$

Task IV - Auburn University . . . . . . . . . . . 207

Task V - University of Utah . . . . . . . . . . . . 301

Task VI - Kentucky Center for Applied Energy Research .. 367 
.

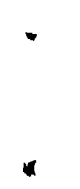

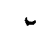


Overview of Research Conducted by the

Consortium for Fossil Fuel Liquefaction Science

on DOE Contract \#DE-FC22-89PC89851

Period: May 4, 1989 - May 3, 1990

G. P. Huffman, Director

Consortium for Fossil Fuel Liquefaction Science 


\section{SUMMARY}

Significant progress was made by the CFFLS in the May, 1990 to May, 1991 contract period in three primary coal liquefacation research areas: catalysis, structurereactivity studies, and novel liquefaction research areas: catalysis, structure-reactvity studies, and novel liquefaction processes. A brief summary of the accomplishmenis in the past year in each of these areas is given below.

\section{Catalysis}

An $\mathrm{Fe}(\mathrm{CO})_{5}$ precursor was effective at increasing the conversions of three types of coal tested in coprocessing at $425^{\circ} \mathrm{C}$. The use of mlinois No.6 high volatile bituminous and Wyodak subbituminous coals resulted in conversions of greater than $70 \%$ using $0.5 \mathrm{wt} \% \mathrm{Fe}$. The Upper Freeport medium volatile bituminous coal had a low conversion even with a catalyst, but there was still a significant increase over the negative conversions obtained without catalyst. Each of these conversions represented a large increase over the uncatalyzed reaction. The addition of small amour of Mo from soluble precursors increased conversions when used alone and with $\mathrm{Fe}(\mathrm{CO})_{5}$, but conversions greater than $90 \%$ were not obtained. The effect of the Mo catalyst was to increase conversions while also increasing the hydrogen content of the liquid products. The images of the Mo and Fe-Mo catalysts obtained using TEM show a highly dispersed catalyst, with particle sizes below $50 \mathrm{~nm}$.

Further studies were performed on the improvement and application of a recently-developed procedure for low-temperature coal depolymerization-liquefaction [35, 39-43]. The procedure includes a two step initial depolymerization stage in which different types of intercluster linkages are cleaved: (1) intercalation of the coal with catalytic amounts (3-15\%) of $\mathrm{FeCl}_{3}$ followed by mild hydrotreatment (HT) of the coal$\mathrm{FeCl}_{3}$ intercalate; and (2) base-catalyzed depolymerization (BCD) of the product from step 1. In the final stage of the procedure, the depolymerized coal product from the HT-BCD treatment, consisting primarily of monocluster compounds, is subjected to hydroprocessing (HPR) with sulfided catalysts to obtain light hydrocarbon fuels as final products.

Research activities this year included systematic studies of the lowtemperature depolymerization-liquefaction of three selected U.S. coals provided by the Argonne National Laboritory, i.e., Blind Canyon (UT), Beulah Zap (ND), and Pittsburgh \#8 (PA) coals; (2) preparation of larger amounts of depolymerized coals for the purpose of in-depth characterization of both prcducts and the iron catalyst used in the first step. The total conversion of these coals into depolymerized (THF-soluble product yielded ahout 68-71 wt\% of cyclohexane-solubles (oils) which predominantly consist of monocluster compounds containing a single ring system with molecular 
weights in the approximate range of 100-300. Hydroprocessing (HPR) of the depolymerized coals ( $365^{\circ} \mathrm{C}, 2700$ psig $\mathrm{H}_{2}$, RuMo or CoMo sulfided catalyst) yielded hydrocarbon fuels with a very high proportion (70.8-74.2\%) of low-boiling fractions (gasoline, kerosene, and gas oil; b.p. up to $325^{\circ} \mathrm{C} / 760$ torr). The high values for total distillables $\left(<538^{\circ} \mathrm{C}\right)$ are consistent with TG/MS analysis of the same products.

Significant progress has been made in characterizing both the structure and size distribution of iron catalysts. ${ }^{57} \mathrm{Fe}$ Mossbauer spectroscopy and X-ray absorption fine structure (XAFS) spectroscopy have been the primary techniques used in this work. The results indicate a remarkable degree of uniformity of catalyst structure for a variety of synthesis methods. The suite of iron catalysts that has been investigated following preparation and at different stages of hydrotreatment or liquefaction includes samples from the Shabtai $\mathrm{FeCl}_{3}$ impregnation process, $\mathrm{Fe}_{2} \mathrm{O}_{3} / \mathrm{SO}_{4}^{-2}$ specimens, samples prepared by treatment with iron carbonyl, and lignite samples prepared by cation exchange in both $\mathrm{FeCl}_{2}$ and $\mathrm{Fe}$ acetate. Both the Mossbauer spectra and the XAFS spectra show that initially the added iron is predominantly in the form of a highly dispersed ferric oxide that resembles a very fine particle iron oxyhydroxide or hematite. The temperature dependence of the Mössbauer spectra allow a measurement of the size distribution of these very fine, superparamagnetic particles by determining the percentage of iron contained in particles large enough to exhibit magnetic hyperfine spectra as a function of temperature. Typical size distributions derived for the iron added by the Shabtai method and the cation exchange method show most of the iron to be present in particles smaller than 20-70 $\dot{A}$ in diameter, while the $\mathrm{Fe}_{2} \mathrm{O}_{3} / \mathrm{SO}_{4}^{-2}$ particle sizes range from 30 to over $100 \AA$ in diameter.

Radial distribution functions (RDF) derived from XAFS spectra also exhibit the effects of particle size. Specifically, the amplitudes of third and fourth nearest neighbor iron shells in the RDFs are significantiy smaller than those of the bulk phases of hematite and goethite. This reflects the fact that the iron atoms in particles 20-100 $\AA$ in diameter have less third and fourth nearest neighbors, on average, than they do in bulk phases. During liquefaction, if sufficient sulfur is present, the added iron is converted to pyrrhotite, as indicated by both Mossbauer XAFS spectroscopy. If sulfur content of the coal and solvent is low, part or all of the iron may be unconverted during liquefaction and remain as a fine particle ferric oxide. It may be that the ferric iron-oxygen particles and molecular complexes act as acid catalysts, promoting bond cleavage reactions, while the pyrrhotite acts as a hydrogenation catalyst.

A catalytic system based on the combination of triflic acid and iodine was found to be effective for three types of coal: Illinois No.6, Wyodak, and Pittsburgh seam. Weaker acids such as p-toluenesulfonic acid and $98 \%$ sulfuric acid were less 
effective than triflic acid in this catalytic coal conversion. From the dependence of the product selectivity on the catalytic system and reaction conditions, and from the elemental analyses of liquids and residues and ${ }^{1} \mathrm{H}$ and ${ }^{13} \mathrm{C} N M R$ measurements, it is concluded that the main function of the acid is to enhance coal depolymerization to asphaltenes while the main role of iodine in the combined catalytic system is to hydrogenate and hydrocrack the asphaltenes to oils. Finally, the combined catalytic system removed more than $50 \%$ of the nitrogen and over $90 \%$ of the sulfur from the original coal. Triflic acid by itself was found to be a very efficient denitrogenation catalyst.

Thermogravimetry (TG) and in situ electron spin resonance (ESR) studies have demonstrated that zinc halides $\left(\mathrm{ZnCl}_{2} \mathrm{ZnBr}_{2}\right.$ and $\left.\mathrm{ZnI}_{2}\right)$ promote significant catalytic cracking of coal in flowing nitrogen gas at temperatures as low as $\mathbf{1 5 0}$ to $175^{\circ} \mathrm{C}$. Peaks in the free radical density as a function of temperature are shifted to significantly lower temperatures. Such peaks are markedly reduced in $f$ owing hydrogen, presumably because of hydrogen quenching of free radicals.

Sulfur isotope studies were carried out on presulfided $\mathrm{NiMo} / \mathrm{Al}_{2} \mathrm{O} 3$ catalysts from the first and second stage reactors at Wilsonville. Ordy about $1 / 3$ of the sulfur in the catalyst exchanges rapidly (with $\sim 1-2$ days). The $\delta^{34} \mathrm{~S}$ value of the catalyst then approaches that of the coal slurry very slowly.

New bimodal and unimodal catalysts with a suite of additional coals from the DOE Argonne Premium Coal Bank. Five catalysts, with different pore structures, containing nickel and molybdenum supported on alumina were investigated. A continuous-flow micro-reactor was used to study the deactivation of these catalysts with model compound hydrogenation/demetallation reactions. In addition pore diffusion experiments were performed to obtain effective diffusivities for these catalysts. Our results in these areas are summarized in the following. The results indicate that the presence of macropores decreases the rate of catalyst deactivation in coal liquefaction.

\section{Structure - Reactivity Research}

One of the most challenging tasks involved in characterizing the molecular properties of coal derived liquid (CDL) is to obtain a reliable estimate of the molecular weight distribution. Due to the relatively high MW, aromaticity and/or polarity of the major components, conventional MW determination techniques, e.g. based on colligative properties, tend to perform poorly with coal liquids. A novel $M W$ determination method has been developed based on MS measurtment of the relationship between vacuum distillation temperature and average molecular weight in combination with thermogravimetric (TG) measurement of the relationship between 
vacuum distillation temperature and relative mass loss. Combination of these two measurements then produces the relationship between molecular weight and mass fraction, in other words the molecular weight distribution of the sample. Obviously, this method can only be applied to vacuum distillable components. Within this limitation, however, the technique is relatively insensitive to mass losses during sample introduction and/or MW dependent differences in instrument response. Analysis of the oil fraction produced from a number of coals by the Shabtai hydrotreatment-base catalyzed depolymerization (HT-BCD) treatment by this TG/MS method yielded mean molecular weights of 235 to 270 .

A number of advanced NMR techniques are being applied in the investigation of the molecular structure of coal, coal pyrolysis products, and coal liquefaction products. These techniques include measurement and analysis of the static powder lineshape, analysis of the variable angle sample spinning lineshapes, and analysis of the intensity pattes $D$ obtained from spinning at the magic angle at a rotation rate less than the width of the anisotropy. Techniques that require more elaborate equipment and control over the orientation of the sample with respect to the magnetir field include the magic angle hopping experiment and the dynamic angle spinaing technique. In both of these two dimensional (2-D) experiments the orifntation of the sample is changed during each scan. Another technique involves spinning at the magic angle and using of pulses to regain the chemical shift anisotropy information that is averaged by spinning. In most of these experimental methods the aromatic region of the spectrum is analyzed. It is found that the aromatic carbons can be quantitatively grouped into four categories: protonated, substituted (having an alkyl carbon substituent), phenolic (having an oxygen substituent) and bridgehead or condensed. Such detailed structural information is important for assessing the efficacy of different liquefaction processes and catalysts in producing coal liquids with desirable characteristics.

Electron nuclear double resonance (ENDOR) has been used to identify three distinct types of free radicals in coal. The temperature dependence of the ENDOR signal has been measured at temperatures up to $150^{\circ} \mathrm{C}$. This has been made possible through the development of a new ENDOR probe that enhanced signal to noise ratios by a factor of 200 .

61 coal samples obtained from Ohio, Ilinois, Indiana and the Wilsonville pilot plant were characterized and subjected to thermal liquefaction tests at temperatures of 385 to $455^{\circ} \mathrm{C}$. The eight coals from the Argonne Premium Coal Sample Bank (APCSB) were also thermally liquefied at $385^{\circ} \mathrm{C}$. The largest range of conversion (30-95\%) is observed at $385^{\circ} \mathrm{C}$; as conversion increases, the asphaltene plus preasphaltene yield increases linearly, while the oil plus gas yield remains fairly constant. Secondary 
reactions (conversion of asphaltenes plus preasphaltenes to oil plus gas) becomes dominant as the temperature is increased.

The APCSB coals exhibited systematic liquefaction behavior with both chemical composition and structure, as determined from the NMR data of Pugmire and coworkers. With increasing carbon content, conversion increases to a peak at $77 \% \mathrm{C}$ maf) then decreases fairly rapidly, while oil plus gas $(0+g)$ yields also exhibit systematic behavior with coal structure as indicated by NMR. The $0+g$ yield increases with increasing fractions of both aliphatic carbon bonded to oxygen and aliphatic carbon present as $\mathrm{CH}$ or $\mathrm{CH}_{2}$, but decreases rapidly with increasing aromaticity.

The CFFLS has decided to concentrate much of its research effort on the Argonne Premium Coal Sample Bank (APCSB) coals, in order to facilitate comparison of results from different investigators at different CFFLS universities. Because mineral matter and sulfur forms can significantly affect liquefaction behavior, a detailed characterization of these coal properties was conducted using computercontrolled scanning electron microscopy (CCSEM), Mössbauer spectroscopy, and X-ray absorption fine structure (XAFS) spectroscopy. In order to obtain more meaningful correlations with liquefaction behavior, the data set was augmented by investigating eight Kentucky coals selected from many coals to exhibit a range of sulfur contents, rank, and liquefaction yields. Meaningful correlations were observed between liquefaction behavior and carbon content, pyrite surface area, and silicate minerals surface area.

X-ray photoelectron spectroscopy (XPS) has been used to study the surface characteristics of a suite of coal samples that included the eight APCSB coals and a number of coals of interest in the CFFLS program. The technique provides elemental identification and chemical information about the outermost 3-4 nm of the exposed surface of a solid. The surface composition of the coal samples was measured and compared with the results of bulk analysis. A good correlation with the bulk analysis was obtained for the total carbon and nitrogen using XPS quantitative analysis, although the accuracy of the XPS determinations seems to be lower, in view of uncertainties in determining sensitivity factors. The surface and bulk concentrations differ in the case of sulfur, oxygen, silicon, aluminum and iron. Silicon, aluminum and oxygen are enriched at the surface, for example, while iron is surface depleted.

Peak deconvolution was conducted to obtain information on the chemical environments of elements, such as sulfur, carbon and nitrogen. These results have been compared with model compound studies and good agreement was observed.

The ASED-MO (atomic superposition and electron delocalization molecular orbital) method was used to calculate bond dissociation energies in a compound studied 
recently as a model for coal fragments. The method has yielded results in qualitative agreement with experiment and appears to be promising for studying coal liquefaction processes. General trends appear to be reproduced, such as product distribution comparisons, but not absolute numbers. The study of the model compound, as well as a fragment of the Shinn model, clearly show the interplay of structure and reactivity, and underscore the importunce of such fundamental studies in the development of novel catalysts or processes. Further testing and improvements of the method are underway.

A group-contribution equation of state for coal liquids has been tested and found to be more flexible, more widely applicable, and more internally consistent than other available models. An adaptive algorithm for continuous-thermodynamics phaseequilibrium calculations has been tested and published. Five papers describing the details of the work have been published and two are under review for publication. Contacts have been developed with industry to try to obtain more data for representative systems.

Spectroscopic methods for the study of the pyrolysis processes of a variety of organic sulfur compounds have been developed. Thiophenoxy radicals have been detected directly by laser induced fluorescence in the pyrolysis products of phenyl disulfide. A pulsed pyrolysis jet source has been built, tested and proven to be a useful apparatus for producing pulses of pyrolysis products which are subsequently cooled by supersonic expansion. A new, precise wavelength calibration method has been developed for use with high resolution pulsed dye lasers in the $500-350 \mathrm{~nm}$ region.

The deuterium content of 1-methylnaphthalene, a non-donor solvent, increased substantially during liquefaction of a Western Kentucky No. 9 coal in deuterium gas. For a $30 \mathrm{~min}, 450^{\circ} \mathrm{C}$ experiment, deuterium NMR showed that $78 \%$ of the deuterium was at ring positions and $22 \%$ in methyl groups. At $300^{\circ} \mathrm{C}$ and one hour, essentially all of the deuterium is at ring positions.

\section{Novel Liquefaction Processes}

In continuing with the search to identify bacteria that will hydrogenate organic compounds and can eventually be applied to coal, experiments were performed using Rhodopseudomonas palustris, Desulfotomaculum nigrificans, Desulfovibrio desulfuricans and a fungus Poria placenta. The model compounds used were naphthalene, benzoic acid and diphenyl methane. Experiments were conducted using UV spectroscopy in the aqueous phase to see the action of bacteria on coal.

Sodium lactate and diphenyl methane proved to be good substrates for Rhodopseudomonas palustris. Benzoic acid enhanced its growth on yeast extract while 
naphthalene showed some inhibitory effect. Desulfotomaculum nigrificans acting on naphthalene increased the total organic carbon in the aqueous phase thus implying that it had broken down naphthalene into more soluble products. Desulfovibrio desulfuricans acting on coal resulted in increased absorbance and formation of more peaks in the UV spectra of the supernatant liquid phase. Coal was treated anaerobically with Sulfolobus brierleyi using appropriate media in an atmosphere of $15 \% \mathrm{CO}_{2}$ and $85 \% \mathrm{H}_{2}$. A selective removal of sulfur from the coal along with hydrogen sulfide production was observed. Additionally, a literature review was used to identify potential microorganisms that would be suitable for bioprocessing of coal.

A short study was made to investigate the possible advantages of the coliquefaction of coal and liguin. The main reasons for looking into this subject were: (a) lignin is the second most abundant polymeric organic product found in nature; (b) native lignin is a complex 3-dimensional polymer which contains about 0.3 wt\% sulfur and essentiaily nu nitrogen; addition of lignin (in significant amounts) in coal liquefaction would cut down unwanted emissions; and (c) lignin-coal coliquefaction may increase distillate yields. A problem immediately became apparent: not only does the word "lignin" mean different things to different people but the difficulty and expense of obtaining pure "native" lignin with low sulfur and nitrogen is too great for an investigation of coliquefaction to be worthwhile. A few experiments (without a catalyst) were run.

Extraction kinetics using the solvent $N$-methyl pyrollidone (NMP) were measured for three different bituminous coals which exhibited distinct extraction behavior and characteristics. These kinetic data were modeled by a newly-developed reversible kinetic model. The nnodel simulates not only the behavior of the extraction at short times, but can also be iased to predict the ultimate extraction yield of each coal at any desired temperature.

Removal of organic sulfur species was attempted using a metal : metal-salt redox couple. When applied to the raw coal, organic sulfur in the extract dropped from $238 \%$ down to $0.84 \%$. Model compound studies showed the method to be applicable to thiol type linkages but not effective in dibenzothiophenic bonds.

Hydrogenation experiments using NMP both as a solvent during hydrogenation and as a catalyst impregnation solvent in pretreatment showed some effects on the overall product yield and distribution. Shifts in the product slate towards the heavier asphaltols indicated that the iron chloride catalyst promoted condensation reactions rather than further ring cleavage and hydrogenation. However, such experiments may be useful in the future for determining the effects of iron-based catalytic hydrogenation since the coal extracts as prepared do not contain any iron species.

Thermal liquefaction experiments at 385,427 , and $445^{\circ} \mathrm{C}$ were carried out on 
demineralized Ohio No. 5 and Ohio No. 6 coals and on vitrinite maceral separates from those coals. Although the coals are very similar, the vitrinite from Ohio No. 5 exhibits a significantly different liquefaction pathway from the demineralized coals or the Ohio \#6 vitrinite.

Pyrolysis and solvent extraction of several Argonne Premium Coal Samples have been studied. For pyrolysis runs, three samples from each coal were prepared: undried raw coal, dried raw coal and pre-extracted coal. The reaction conditions were $350^{\circ} \mathrm{C}, 1$ atmosphere of nitrogen (initial pressure) and a reaction time of 3 hours. When pyrolyzed, dried coals (at $100^{\circ} \mathrm{C}$, in vacuo) gave significantly lower liquid yields compared to undried coals. On the other hand, pre-extraction with tetrahydrofuran (THF) showed no apparent effect on subsequent pyrolysis yields, as compared with yields obtained without pre-extraction. Hydrogen contents of the liquid products were significantly higher than those of the raw coals. The liquid products of Blind Canyon coal contained as much as $\mathbf{7 . 7 \%}$ hydrogen, presumably because of the high resinite content of this coal.

Thermal liquefaction reactions in nitrogen with added cyclic olefins (CLO) resulted in a substantial increase in coal conversion in comparison with their respective hydroaromatic analogues. More hydrogen was correspondingly released from the CLO's than from the hydroaromatic donors. Likewise, substantial differences were observed among the CLO's and their hydroaromatic analogues in hydrogen in an inert solvent, hexadecane. The CLO's converted substantially more coal and released more hydrogen than their hydroaromatic analogues.

Catalytic reactions with presulfided $\mathrm{NiMo} / \mathrm{Al}_{2} \mathrm{O}_{3}$, hydrogen, hexadecane as solvent, and added donors resulted in isotetralin (ISO) converting more coal than tetralin (TET) and hexahydro anthracene (HHA) more than octahydro anthracene (OHA); however, dihydroanth racene (DHA) converted more coal than either and appeared to incorporate molecular hydrogen from the coal reaction system. The major reaction product from DHA was OHA. The reason for high reactivity of DILA under these conditions has not yet been determined but may in part result from ste-ic or configural interactions between $\mathrm{DHA}$ and the $\mathrm{NiMo} / \mathrm{Al}_{2} \mathrm{O}_{3}$ catalyst. The other threering donor species formed the same reaction products but did not form DHA or show the high reactivity level of DHA.

Theoretical calculations using a molecular orbital model, modified neglect of differential overlap (MNDO), predict that ISO dehydrogenated to 1,4dehydronaphthalene (DHN) and subsequently to naphthalene (NAP) via a free radical mechanism. The calculated heats of formation for the required transitions were less than with either an anionic or a cationic mechanism. MNDO calculations with steric considerations also predicted that the ground state of ISO is less stable and more 
reactive than that of TET because ISO has a higher heat of formation.

Four model probe compounds were introduced to investigate the complex chemistry of coal-oil coprocessing reactions in this study. These four probe reactants served as monitors for evaluating the catalytic inhibitory effects of different hydrocarbonaceous substances. The four probe systems used were dibenzofuran for hydrodeoxygenation, dibenzothiophene for hydrodesulfurization, quinoline for hydrodenitrogenation, and anthracene for hydrogenation. The four model compounds were reacted individually with resid, coal, two different resid fractions and two prehydrogenated residua respectively, in order to discover how different compositional factors affected the reactivity of the individual probe reactions.

The results of these probe reactions indicate that when resid coal or a coinbination of coal plus resid was added to all probe reactions the HYD reaction as well as HDO, HDS and HDN were decreased. The resid participated in competitive catalytic reactions with the model reactions where the resid molecules compete for the active catalytic sites, perhaps even poisoning the active site thereby inhibiting the catalytic activity of the sites. A comparison of the heteroatom removal probe reactions showed that added untreated resid, coal, and coal plus resid had little effect on sulfur and nitrogen removal from dibenzothiophene and quinoline, but inhibited oxygen removal from dibenzofuran. All HYD reactions were affected; the most affected was the HYD of dibenzofuran, followed by benzothiophene, anthracene and quinoline in that order.

In the detailed reactions using hydrocarbon fractions, hexane soluble and prehydrogenated resid, prehydrogenated resid inhibited reactions more than did hexane soluble resid which in turn was more inhibitory than hydrocarbon fraction residua. The addition of untreated resid showed the greatest inhibitory effects on model compound reactions in comparison with the addition of different specified resids. The four model compounds had the same trend of reaction with the different added resids. The added resids and coals, in order of their inhibitory effect on the probe reactions, are: coal-resid mixture $>$ coal $>$ untreated resid $>$ thirty-minute prehydrogenated resid $>$ three-hour prehydrogenated resid $>$ hexane soluble resid $>$ hydrocarbon fraction resid.

Compared with reactions with untreated resid, any additional pretreatment of resid to remove heavy materials, such as prehydrogenation of some heavier components, contributed to some improvement of the four probe reactions. In a comparison of the four model compound reactions, quinoline consistently showed more HYD reaction than other model compounds and a high mole percent of HDN reaction while anthracene did not show much variation in HYD reactions with addition of the different resids. 
A slurry phase reactor system was developed during this past year that offers sufficient versatility in reaction rate controllability and the necessary mass transfer/heat transfer characteristics to prevent retrogressive reactions from occurring due to $\mathrm{H}_{2}$ starvation, while attaining a very high conversion of reactants. During the initial stages of liquefaction, free-radical reactions occur rapidly and reaction rates increase almost exponentially. As a result, no practical, commercial gas-liquid contactor was found that was capable of providing $\mathrm{H}_{2}$ at a sufficiently high enough rate to prevent $\mathrm{H}_{2}$ starvation, and thus retrogressive reactions, from occurring. Therefore, an alternative design approach was used. The reactor system was designed in order to control the rate of free-radical generation so that only practical, achievable $\mathrm{H}_{2}$ masstransfer rates would be required.

The approach taken in developing the reactor system was two-fold: 1) a reactant flow pattern was selected which would allow versatility in reaction rate controllability and 2) a gas-liquid contactor design was sought that provided the highest mass-transfer rates per unit volume without significantly affecting reaction rate controllability.

Basically, a bang-bang control strategy was implemented to regulate reaction rate. That is, a cyclic switching from high to low reaction temperatures was used to control reaction rate. The adjustable control parameter selected was the frequency of the cyclic switching in reaction temperature. Physically this was accomplished using a $r$ ecycle loop-type reactor in which the temperature in part of the loop was kept high (the reaction zone) and that in the remainder of the loop was kept low (the quenching zone). This reactor flow arrangement has been commonly used with jet-type reactors which have been utilized commercially when reaction rates are very high. The frequency of cyclic switching in reactant temperature can be varied simply by changing the reactant recycle flow rate.

A variety of designs for gas-liquid contactors were evaluated: gas-liquid jet injectors, motionless mixers, column packings, and high-shearing mechanical agitators. Of the contactors studied to date, each type was evaluated not only in terms of its relative effect on gas-liquid mass transfer but also on the feasibility of its use in the proposed reactor system. The effective mass transfer coefficients for the contactors in a low-temperature sodium sulfite oxidation system and in a slurry-phase catalytic naphthalene hydrogenation system were determined. Naphthalene hydrogenation was used as a model reaction to direct developmental efforts.

A mathematical model for the reactor system was developed for interpreting experimental observations and parameterizing the system. The $\mathrm{H}_{2}$ mass-transfer coefficient in the current system was determined to be only about 0.13 per second, which is about six times higher than that of a bubble column (0.02 per second). But 
even with this low order of improvement in the $\mathrm{H}_{2}$ mass-transfer rate, a significant hydrogen concentration appeared to be maintained throughout the reaction zone, and the overall reaction was observed to not be strongly mass-transfer limiter

A mechanical high-shearing gas-liquid contactor now under costruction will be used in future studies. Upon completion of limited developmental studies, coal liquefaction studies will then be performed to observe the responses in product distribution to well-controlled liquefaction under high $\mathrm{H}_{2}$ mass transfer rate conditions. 


\section{TASK I}

\section{UNIVERSITY OF KENTUCKY}




\section{Task I.1}

\section{BIOPROCESSING OF COAL}

PI's:
D. Bhattacharyya
M. I. H. Aleem
R. I. Kermode

\section{Report}

Coordinator:

D. Bhattacharyya

Phone:

Period:
(606) 257-2794
A. M. Khalid
H. Venkatachalam
F. Paryavi

\section{SUMIMARY}

In continuing with the search to identify bacteria that will hydrogenate organic compounds and can eventually be applied to coal, experiments were performed using Rhodopseudomonas palustris, Desulfotomaculum nigrificans, Desulfovibrio desulfuricans and a fungus Poria placenta. The model compounds used were naphthalene, benzoic acid and diphenyl methane. Experiments were done to see the action of bacteria on coal (KCERL \# 91182). The effect of the bacteria was observed by UV spectroscopy in the aqueous phase.

Sodium lactate and diphenyl methane proved to be good substrates for Rhodopseudomonas palustris. Benzoic acid enhanced its growth on yeast extract while naphthalene showed some inhibitory effect. Desulfotomaculum nigrificans acting on naphthalene increased the total organic carbon in the aqueous phase thus implying that it had broken down naphthalene into more soluble products. Desulfovibrio desulfuricans acting on coal resulted in increased absorbance and formation of more peaks in the UV spectra of the supernatant liquid phase. Coal was treated anaerobically with Sulfolobus brierleyi using appropriate media in an atmosphere of $15 \% \mathrm{CO}_{2}$ and $85 \% \mathrm{H}_{2}$. A selective removal of sulfur from the coal along with hydrogen sulfide production was observed. Also a literature review was used to identify potential microorganisms that would be suitable for bioprocessing of coal.

\section{Literature review}

The general area of biological conversion of substrate materials to fuels and chemicals presently comprises five basic areas of research. These include: wastewater treatment, 
biomass degradation and conversion, hydrogen production via microorganisms, bioconversion of coal, lignite, and peat to useful chemicals and energy and sulfur removal.

The research reported on wastewater, focused exclusively on treatment. It was concerned primarily with the biological destruction and elimination of water soluble compounds produced by conventional conversion and processing. Both experimental and design feasibility studies, for wastewater treatment, were reported. Several laboratory scale studies reported phenol degradation results including its toxicity to various microorganisms. One study reported the results of a one ton per day PDU which utilized H-coal wastewater. Although methane was produced, the focus of this research was destruction of water soluble compounds in H-coal wastewater, and not fuel production.

Current biomass conversion research is focussed almost exclusively on the anaerobic conversion of cellulosic materials to a mixture of $\mathrm{CH}_{4}$ and $\mathrm{CO}_{2}$. In general, these processes occurred in rather dilute suspensions of cellulosic substrate. Two abstracts reported efforts of producing chemicals such as acetone, butanols, ethanol, monocarboxylic acids, etc., from cellulose rather than combustible gases.

Three references $(1,2,3)$ reported experimental results on Rhodopseudomonas palustris and Rhodopseudomonas molischianum to convert paper mill and sugar refinery waste streams to hydrogen. Molecular hydrogen was produced for 30 days at a rate of 50 to $139 \mathrm{mu}$ liter per hour of $\mathrm{H}_{2}$ per mg of cells; depending on the microorganism and the substrate. Approximately $30 \%$ of the COD was converted to hydrogen. Other gases produced in the process were not reported. One study (2) involved the use of hydrogenases in purple bacteria, such as Thiocapsa oseopersicinia BBS and Ectothiorbodospira shaposbnikovii 1K, under anaerobic conditions. Finally, one abstract reported the cloning of the cellulose gene of $C$. cellobioparun using $E$. coli (4). The resulting material degraded carboxymethyl cellulose and p-nitrophenyl - beta - D - cellobioside. In no case was coal suggested as a substrate for a hydrogen producing process.

An overview of progress in the conversion of coal, lignite, and peat to other useful energy forms and chemicals, can be found in several review articles (5-10). These are, in general, rather broad in scope covering numerous aspects of bio-conversion. These usually reviewed progress - to - date and suggested potential general research areas using coal as a part of the substrate. Two studies $(11,12)$ focussed on the biological conversion of coal and lignite to methane. The first of these (11) proposed bench scale evaluation of a North Dakota lignite, Wyodak subbituminous, and an Ohio bituminous coal. The ultimate goal was to develop scale-up data for a larger system. The bacteria and fungi used in this project were to be supplied by Atlantic Research. Pretreatment via thermal softening for breaking 
the cross-linked matrix was also to be studied. The second study (12) had similar objectives; however, the focus was on converting model compounds anaerobically to methane. These included phenol, 2-naphthol, 1-naphthoic acid, and 1-phenanthrol. Both studies indicated that this type of process could economically convert lower ranked coals to a more useful fuel, such as, methane. Neither report was specific in terms of the bacteria or fungi to be used. One study (13) reported the potential for converting peat to methane.

Three investigations (14-16) examined the potential for converting a synthesis produced gas from coal to useful chemicals such as alcohols, acids and aldehydes. The reactions occurred anaerobically and at "reasonable rates". Again, potential bacteria and fungi were not identified.

The remaining papers in this area $(4,17-25)$ were concerned with solubilization and liquefaction of coal and lignite. Two of these $(18,19)$ utilized fungi isolated from lignite outcroppings. These were $P$. chrysosporium and $T$. versicolor. Both were concerned with producing water soluble products from lignite. Two abstracts $(20,22)$ reported biodegradation of model compounds. Included were 2-naphthol, 1-naphthoic acid, and 9-phenanthrol. Microorganisms used were isolated from sewage and anaerobic swamp sediment. Finally a Japanese patent (17) reported that Penicillium MT - 6001 converted coal to a liquid material with an analysis pattern similar to a naphthenic petroleum containing a lipid with polar radicals.

As indicated the original approach to biological coal liquefaction implied the aerobic production of water soluble species from lignite or low rank coals. An analysis of the water soluble material showed that in general it contained more oxygen than the original coal, thus decreasing its potential fuel value.

Recently two different approaches (23-25) have been proposed to overcome this. The first method attempts to duplicate the desirable characteristics of chemical liquefaction without the disadvantages of high temperature and pressure. That is, liquefy coal at room temperature and atmospheric pressure using microorganisms and enzymes under anaerobic conditions. Both elemental hydrogen and hydrogen donor solvent have served as the source of hydrogen. In addition to the benefits of very mild operating conditions, the anaerobic process is carried out in an organic solvent such as benzene. The resulting products are non-polar in nature and more similar to the products of chemical liquefaction.

The initial phase of this research (23) indicated that $20 \%$ of bituminous and subbituminous coal could be solubilized using a modified hydrogenase. The resulting products were aromatic in character. One of the encouraging aspects of this research is that hydrogenases from organisms such as P. vulgaris, Desulfotomaculum, and Desulforibrio, 
which can utilize molecular hydrogen, are relatively stable in solvents such as benzene. Both the stability and concentration were increased by attaching the enzyme to polyethylene glycol.

The second approach $(24,25)$ was to biologically depolymerize a water soluble lignite fraction. The resulting material was lower in molecular weight. Lignite was dissolved in sodium hydroxide and then reprecipitated at $\mathrm{pH} 7,5.5$, and 1.5 with dilute $\mathrm{HCl}$. The fraction at each $\mathrm{pH}$ was separated prior to lowering the $\mathrm{pH}$. The material collected at $\mathrm{pH}$ 1.5 , approximately $10 \%$ of the starting lignite, was then redissolved by raising the pH to 5.5 . This soluble "coal polymer" was used as substrate for $P$. capacia. Shake flask experiments were carried out for 60 days with molecular weight determinations made initially and at 30 and 60 days. After 60 days the average molecular weight had been reduced from 101,000 to 79,500 with shoulders at 96,000 and 39,000 . The complex nature of the materials precluded analysis at this stage of the research.

\section{Experiments with Rhodopseudomonas palustris.}

Rhodopseudomonas palustris is a phototrophic bacteria belonging to the class Rhodospirillales. The photosynthesis of the Rhodospirillales depends on the presence of oxidizable electron donors, such as reduced sulfur compounds, molecular hydrogen or organic carbon compounds. Rhodopseudomonas palustris has been known to reduce cyclic organic compounds (eg. benzoic acid) and since coal consists of cyclic organics, this made it an ideal choice for investigation regarding the hydrogenation of coal.

Rhodopseudomonas palustris (ATCC 17000) was grown phototropically on Van Niel's medium in the presence of $1 \%$ Naphthalene. The medium composition is $\mathrm{K}_{2} \mathrm{HPO}_{4}, 1 \mathrm{~g} \Lambda$; $\mathrm{MgSO}_{4}, 0.5 \mathrm{~g} /$; Naphthalene, 1.0\%; Tap water, 11 . The experiment was performed to determine the capabilities of this bacterium to carry out any reduction or hydrogenation of Naphthalene. The optical density of these cultures was determined daily at $410 \mathrm{~nm}$ to check for growth. After 6 days, when the cultures were at stationary phase, the cells were harvested. Naphthalene was recovered by extracting it with $\mathbf{n}$-hexane. The growth curves (at pH 6.5) shown in Figure 1, showed a slight inhilitory effect of naphthalene on the growth of Rhodopseudomonas palustris.

A second set of experiments was carried out with benzoic acid. Rhodopseudomonas palustris (ATCC 1700) was grown on Van Niel's medium with yeast extract as the substrate. The $\mathrm{pH}$ was adjusted to around 7 and Benzoic acid was added to two separate flasks. (0.5 g/ and $1 \mathrm{~g} / 1$ ). The $\mathrm{pH}$ was readjusted to 7 after the benzoic acid was added. Anaerobic conditions were maintained by bubbling nitrogen for 10 minutes through each flask initially 
and also each time the flasks were opened to withdraw a sample. The optical density (O.D) values at 420 and $520 \mathrm{~nm}$ were observed by UV spectroscopy. The characteristic absorption spectrum of benzoic acid at 272 and $280 \mathrm{~nm}$ was also recorded. Figure 2 shows that there is good growth of Rhodopseudomonas palustris, and that the presence of benzoic acid caused considerable enhancement of the bacterial growth.

Finally for the experiment on diphenyl methane, Rhodopseudomonas palustris (ATCC 17000) was grown phototropically on sodium lactate. The cells were centrifuged and stored in buffered solution $\mathrm{pH}=7.2$ under refrigeration. For the action on diphenyl methane, $2 \mathrm{ml}$ of this cell suspension was added to $100 \mathrm{ml}$ of media solution containing diphenyl methane $(0.5 \%)$ as the sole substrate. The composition of the medium is as follows. $\mathrm{MgSO}_{4}, 0.20 \mathrm{~g} /$; $\mathrm{CaCl}_{2} 0.05 \mathrm{~g} / ; \mathrm{KH}_{2} \mathrm{PO}_{4} 0.30 \mathrm{~g} / ; \mathrm{K}_{2} \mathrm{HPO}_{4} 0.7 \mathrm{~g} / ; \mathrm{NH}_{4} \mathrm{Cl} 1.0 \mathrm{~g} / ;$ Yeast extract $0.05 \mathrm{~g} /$; and diphenyl methane $0.5 \mathrm{ml} / 100 \mathrm{ml}$ medium solution.

The absorption spectra over the range 190 - $820 \mathrm{~nm}$ was observed. Figure 3 shows that there is good observed growth of R.palustris on diphenyl methane. Figure 4 shows the change in the spectra of the centrifuged samples. A change in absorbance values is seen at $256 \mathrm{~nm}$. When this change is plotted versus time (Figure 5), it is seen that there is a sharp rise in the absorbance within the first three days after which the absorbance drops and then begins to rise gradually. A similar trend is observed in the experiment with Desulfotomaculum nigrificans on naphthalene.

\section{Bacterial Action on Coal}

A lactate grown culture of Rhodopseudomonas palustris was used for the experiment with coal. The medium used was the same as that for growth on sodium lactate, the only change being that the substrate used was replaced by a $3 \%$ slurry of coal (KCERL \#91182). $3 \mathrm{ml}$ of bacterial suspension was used for $100 \mathrm{ml}$ of media solution. The experiment was run for 12 days. After 12 days the coal was filtered, washed with distilled water and dried in an oven at $105^{\circ} \mathrm{F}$ for an hour. The coal was sent to the Center For Applied Energy Research (UK), 3572 Iron Works Pike, Lexington, KY 40511 for chemical liquefaction studies to determine if the bacterial pretreatment had enhanced the liquefaction of coal. The supernatant liquid was centrifuged and the UV spectra was observed over the range from 190 to $820 \mathrm{~nm}$. A slight increase in absorbance was seen in the 200-300 $\mathrm{nm}$ range.

\section{Experiments with Desulfotomaculum Nigrificans.}

Desulfotomaculum nigrificans belong to the genus Desulfotomaculum. There are three species of bacteria under this genus. They are gram negative and their most common 
characteristic is their ability to reduce sulfate to sulfide. Of the three species only $D$. nigrificans is thermophilic. The reducing properties of this bacteria made it a choice for hydrogenation studies.

A culture of Desulfotomaculum nigrificans (ATCC 19858) was revived from a freeze dried culture and grown on modified Starkey's medium C (ATCC \#207). Its composition is as follows; $\mathrm{KH}_{2} \mathrm{PO}_{4}, 0.5 \mathrm{~g} / ; \mathrm{NH}_{4} \mathrm{Cl}, 1.0 \mathrm{~g} / ; \mathrm{Na}_{2} \mathrm{SO}_{4}, 1.0 \mathrm{~g} / \mathrm{CaCl}_{2} .2 \mathrm{H}_{2} \mathrm{O}, 0.1 \mathrm{~g} / ; \mathrm{MgSO}_{4} .7 \mathrm{H}_{2} \mathrm{O}$, $2.0 \mathrm{~g} /$; Sodium lactate $(60 \%), 3.5 \mathrm{~g} /$; Yeast extract $1.0 \mathrm{~g} /$.

The $\mathrm{pH}$ was adjusted to 7.6. The medium was autoclaved for 15 minutes at $15 \mathrm{psi}$. It was then cooled and the precipitate illtered off. The $\mathrm{pH}$ was readjusted to 7.4. The medium was then resterilized at 10 psi for 20 minutes. A $2 \mathrm{~N} \mathrm{NaOH}$ solution, filter sterilized 7.5\% cystine- $\mathrm{HCl}$, and filter sterilized $1 \%$ ferrous ammonium sulfate solutions were prepared separately. $1.0 \mathrm{ml}$ of the cystine solution and $5.0 \mathrm{ml}$ of the ferrous ammonium sulfate was added to $100 \mathrm{ml}$ of the basal medium. The $\mathrm{pH}$ was adjusted using the sterile $\mathrm{NaOH}$ to 7.5. The bacteria were grown under anaerobic conditions at eq 35-40 degree C. The revival time was two weeks. The growth time was one week.

For the test on naphthalene, $150 \mathrm{mi}$ of the medium solution was taken and the substrate was replaced with $\mathbf{5} \mathrm{g}$ of solid naphthalene. This solution was inoculated with 2.5 $\mathrm{ml}$ of the culture solution. The experiment was run under anaerobic conditions at .eq 35 degree C. The UV spectra near the characteristic absorption wavelength for naphthalene $(282 \mathrm{~nm}$ ) was observed. From Figure 6 it can be seen that the absorbance of naphthalene in the solution as a function of time drops a little initially, then rises and begins to drop back again. The sharp rise could be attributed to the bacteria acting on solid naphthalene and making it more soluble thus bringing it into solution. An analysis of the total organic carbon in the solvent phase gave the following result.

\begin{tabular}{|c|c|c|}
\hline SAMPLE & Avg ppm & Variance \\
\hline Blank & 110 & 1.39 \\
\hline \multicolumn{3}{|c|}{ (Medium+naphthalene) } \\
\hline Sample 1 (8 days) & 193 & 1.66 \\
\hline Sample 2 (10 days) & 154 & 1.71 \\
\hline
\end{tabular}

This increase in the total organic carbon further supports the previous view.

\section{Experiments with Desulfovibrio Desulfuricans.}

These bacteria belong to the genus Desulfovibrio. They are Chemoorganotrophs. They are anaerobic and urilize substrates like lactate, pyruvate and malate which they 
oxidize to acetate and carbon dioxide. They have the ability to reduce sulfate to $\mathrm{H}_{2} \mathrm{~S}$, and this property of theirs makes them a bacteria of interest in hydrogenation studies.

Desulfovibrio desulfuricans was grown anaerobically on Starkeys medium with sodium lactate as the substrate. For the experiment on coal the lactate was replaced by a 3 $\%$ slurry of KCERL \#91182 coal. The run time was 12 days. The coal was filtered, washed and dried in an ovisn at .eq 105 degree $F$ for an hour. The supernatant was centrifuged and its UV spectra was observed over the range 190 to $820 \mathrm{~nm}$. The UV spectra showed an increased number of peaks and an increased absorbance as seen in Figure 7. The dried coal will undergo chemical liquefaction studies at the Center for Applied Energy Research (UK), 3572 Iron Works Pike, Lexington, KY 40511. A change in liquefaction yield could indicate that the bacteria had had an effect on the coal.

\section{Action of Fungi on Coal}

Coal (\# 85089) was treated with Poria placenta, according to the outline shown in Figure 8, for 7 days at $\mathrm{pH}=7$. After treatment, the filtered culture broth was checked for solubilization products by determining it's absorption at $400 \mathrm{~nm}$. Since no absorption was detected, it was assumed that the fungus was unable to solubilize the bituminous coal. However the total sulfur contents of the pre-treated coals decreased from 5.9 to $4.2 \%$. This fungus treated coal was then desulfurized using thermophilic bacteria Sulfolobus brierleyi at eq 60 degree $\mathrm{C}$. The results showed that $50 \%$ of the total sulfur was removed by the bacteria from the treated coals as compared to $45 \%$ from the untreated coals in the same period of one week.

\section{Action of Sulfolobus Brierleyi on Coal Under Anaerobic Conditions}

This experiment was performed to study the selective removal of sulfur from coal and possible hydrogenation of coal organics. Sulfolobus brierleyi was grown on DSM-150 medium whose composition is as follows. $\left(\mathrm{NH}_{4}\right)_{2} \mathrm{SO}_{4}, 3.0 \mathrm{~g} / ; \mathrm{K}_{2} \mathrm{HPO}_{4} \cdot 3 \mathrm{H}_{2} \mathrm{O}, 0.5 \mathrm{~g} / ; \mathrm{MgSO}_{4} .7 \mathrm{H}_{2} \mathrm{O}, 0.5$

. $\mathrm{g} / \mathrm{KCl}, 0.1 \mathrm{~g} / ; \mathrm{Ca}\left(\mathrm{NO}_{3}\right)_{2}, 0.01 \mathrm{~g} / ;$ Yeast extract $0.2 \mathrm{~g} /$. A $1000 \mathrm{ml}$ fermenter was filled with $750 \mathrm{ml}$ of the above media solution and autoclaved for 20 minutes. $3 \mathrm{ml}$ of $5 \%$ yeast

- extract and $3 \mathrm{ml}$ of $5 \%$ casamino acids were added to the fermenter. The fermenter was connected to $\mathrm{CO}_{2}(15 \%)$ and $\mathrm{H}_{2}(85 \%)$ gas cylinders.

To collect the $\mathrm{H}_{2} \mathrm{~S}$ formed during the growth of the bacteria the following method was used. All the openings to the fermenter were bled into a flask containing $1 \mathrm{~L}$ of $6 \% \mathrm{NaOH}$ solution. A bismuth nitrate - acetic acid reagent was prepared by dissolving $4.28 \mathrm{~g}$.eq $\mathrm{Bi}\left(\mathrm{NO}_{3}\right) 3 . \mathrm{H}_{2} \mathrm{O}$ in $300 \mathrm{ml}$ acetic acid and diluted with $1.5 \mathrm{~L}$ distilled water. The $\mathrm{NaOH}$ was 
mixed with an equal volume of the bismuth nitrate-acetic acid reagent and the absorbance of the bismuth sulfide in solution was measured at $350 \mathrm{~nm}$. The fermenter was inoculated with $20 \mathrm{ml}$ of an active aerobically grown culture of Sulfoiobus brierleyi.

To increase the cell yield the culture was first grown aerobically for 3 days at .eq 61 degree C. The initial $\mathrm{pH}$ was 2.4. After this period $12 \mathrm{~g}$ of coal (KY \#85098) was added to the fermenter and the experiment was performed under anaerobic conditions. The experiment was run for 7 days. During this period, the absorbance of the bismuth sulfide solution and the $\mathrm{pH}$ was noted. Coal samples were also withdrawn at periodic intervals. Figure 9 shows the $\mathrm{pH}$ profile over the seven day period. The drop in $\mathrm{pH}$ is indicative of bacterial growth. It must be noted that the sharp rise in $\mathrm{pH}$ between days 4 and 5 is due to a $\mathrm{pH}$ adjustment that was done in the course of the experiment and not due to any bacterial action. Figure 10 shows an increase in the absorbance of the bismuth sulfide solution, indicating the formation of $\mathrm{H}_{2} \mathrm{~S}$ gas. Since no external sulfur was available from the medium, the bacteria had to have utilized the sulfur present in the coal. The coal samples taken out were dried and have been sent to the Center for Applied Energy Research (UK), 3572 Iron Works Pike, Lexington, KY 40511. An analysis for total carbon, hydrogen nitrogen and sulfur will be done on the coal to determine the effect of the bacteria on the coal. .pa

\section{REFERENCES}

1. Vincenzini, M.; Materassi, R.; Sili, C.; Florenzano, G., "Hydrogen production by immobilized cells. III-Prolonged and stable $\mathrm{H}_{2}$ photoevolution by Rhodopseudomonas palustris in light-dark cycles", Int. J. Hydrogen Energy (U.S.), v 11:10, 1986, p. 623-626.

2. Kondratieva, E.N.; Gogotov,I.N.; Crawford, R.L.; Hanson, R.S. (eds.), "Hydrogenase activity of purple bacteria under different growth conditions and properties of Rhodopseudomonas capsulata hydrogenase. Proceedings of the International Symposium on eq $C_{1}$ compounds, Minneapolis, MN, USA, Sep 1983, Report No. : CONF-8309357, 1984, p.59-61.

3. Vincenzin, M.; Materassi, R.; Tredici, M.R.; Florenzano, G., "Hydrogen production by immobilized cells. II. Hydrogen photoevolution and wastewater treatment by agar entrapped cells of Rhodopseudomonas palustris and Rhodospirillum molischianum", Int. J. Hydrogen Energy (U.K.), v 7:9, 1982, p. 725-728.

4. Fakoussa, R.M., "Production of water-soluble coal-substances by partial microbial liquefaction of untreated hard coal", Resour., Conserv. Recycling (Netherlands), v 1:3/4, Aug. 1988, p. 251-260. 
5. Narayan, R; "Objectives of coal Bioprocessing", First Annual Workshop on Biological Processing of Coal, Monterary, CA, USA. Electrical Power Research Institute., Pao Alto, CA, Report No. EPRI-ER-5709-SR; March 1988, p. 2.1-2.26.

6. Lee, D.D.; Scott, C.D., "Impact of Biotechnology on Coal Processing (list of substrates and products;', Oak Ridge National Lab, Tennessee, Report No. ORNL-6459 May 1988, p.71.

7. Couch, G.R., "Biotechnology and coal', IEA Coal Research, London, (UK),March 1987, $56 \mathrm{p}$.

8. "Biotechnology in hard coal mining", Colloquium on Biotechnology in Fiard Coal Mining, Essen,F.R.G, Steinkohlenbergbauverein,Essen, F. R. Germany, Report No.: CONF-8301117, 1983, p. 97.

9. Jones, W.J.,"Biological degradation of low-rank coal", Quarterly technical progress report, Jan. 1-Mar. 31, 1986, Report No.: DOE/FC/10625-T1, May 1986, p. 26.

10. Jones, W.J., "Biological degradation of low-rank coal", Quarterly technical progress report, Sept. 30-Dec. 31., 1985, Report No.: DOE/FC/10625-2029, Feb. 1986, p. 25.

11. Isbister, J.D.; Ghate, M.R.; Markel K.E.Jr.; Jarr, L.A.; Bossart, S.J., "Biological gasification", Proceedings of the Seventh Annual Gasification and Gas Stream Cleanup Systems Contractors Review Meeting, Electric Power Research Inst., Vol 2, Report No. : DOE/METC-87/6079, Aug. 1987, p. 530-536.

12. Jones, W.J.; Chian, E.S.K; Ghate, M.R.; Markel, K.E.Jr.; Jarr, L.A.; Bossert, S.J., "Biological degradation of low-rank coal", Proceedings of the Seventh Annual Gasification and Gas Stream Cleanup Systems Contractors Review Meeting, Electric Power Research Inst., Vol 2, Report No. : DOE/MEIC-87/6079, Aug. 1987, p. 382-392.

13. Kopstein, M.J.; Kube, W.R.; Sonreal, E.A.; White, D.M., "Peat conversion and utilization in the United States", Biennial Lignite Symposium, San Antonio, TX, USA, Report No.: GFETC/IC-82/1, 1981, p. 48-53.

14. "Biological production of fuels from coal derived gases: Quarterly repoĩt, Sept. 20 Dec. 20, 1986", Report No.: DOE/PC/80012-T6, 1986, p. 25.

15. "Biological production of fuels from coal derived gases: quarterly report, June. 20 Sept. 20, 1986", Report No.: DOE/PC/80012-T5, 1986, p. 11.

16. "Biological production of fuels from coal derived gases: a review of the literature", Report No.: DOE/PC/80012-T1, 1986, p. 33.

17. Takakuwa, Masayoshi, Gotaro Hashimoto, "New micro-organism - Patent No.: JP 62-208270", Kurushima Group Corp., Ehime, Japan, March 7, 1986.

18. Hasnain, S.; Overend, S.R.; Lowenstein, M.Z., (ed.), "Energy-related activities of the 
Canadian biomass research program", Proceedings of National Meeting on Biomass Research and Development for Energy Applications, Arlington, VA, Elsevier Applied Science Publishers, NY, 1985, p. 91-106.

19. Yen, Teh Fu, "Microbial screening test for lignite degradation: Quarterly progress report No. 9 for the period January-March 1987", Report No.: DOE/PC/70809-T-13, March 1987, p. 28.

20. Cooney, C.L., "Direct microbiological fermentation of lignocellulosic biomass", Biochemical Conversion Program Semi-Annual Review Meeting, Golden, CO, Solar Energy Research Inst., Report No.: SERICP-231-2726, p. 273-284.

21. Himmel, M.E.; Tucker, M.; Baker, J.; Mohagheghi, A; Grohmann, K., "Enzymatic hydrolysis research at SERI : an overview", Biochemical Conversion Program Semi-Annual Review Meeting, Golden, CO, Solar Energy Research Inst. Report No.: SERI/CP-231-2726, p. 201-242.

22. Jones, W.J., "Biological degradation of low-rank coal, Quarterly technical progress report: April 1-June 30, 1986," Report No.: DOE/FC/10625-2171, 1986, p. 25.

23. Scott, C.D.,C.A. Woodward, B.D. Faison, "Solubilization of Coal by Reducing Enzymes in Organic and Aqueous Media", First International Symposium on the Biological Processing of coal, Orlando, Florida, May 1990.

24. Crawford, D.L. ,R.K.Gupta, "Influence of Cultural Parameters on the Depolymerization of a Soluble Lignite Coal Polymer by Pseudomonas cepacia DLC-07, DOE Workshop on Bioprocessing of Fossil Fuels 1989.

25. Crawford, D.L. ,R.K. Gupta, L.A. Deobald, D.J. Foberts, "Biotransformations of Coal and Coal Substructure Model Compounds by Bacteria Under Aerobic and Anaerobic Conditions", First International Symposium on the Biological Processing of Coal, Orlando, Florida. May 1990. 
Figure 1 : Growth of Rhodopseudomonas palustris

In the Presence of Naphthalene.

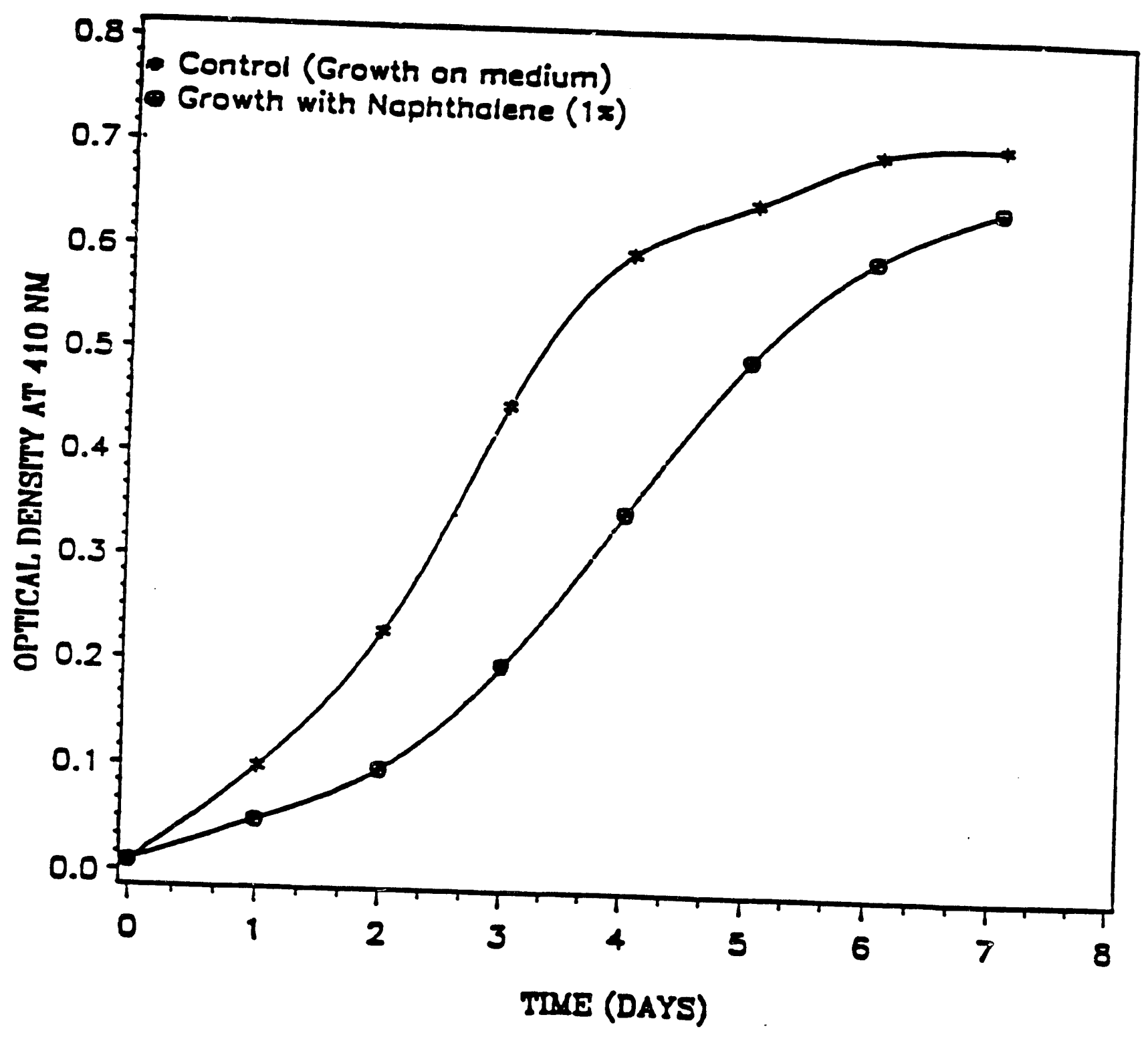


Figure 2 : Growth Curves of R.polustris on Benzoic Acid (0.5 x) at $420 \mathrm{~nm} \& 520 \mathrm{~nm}$

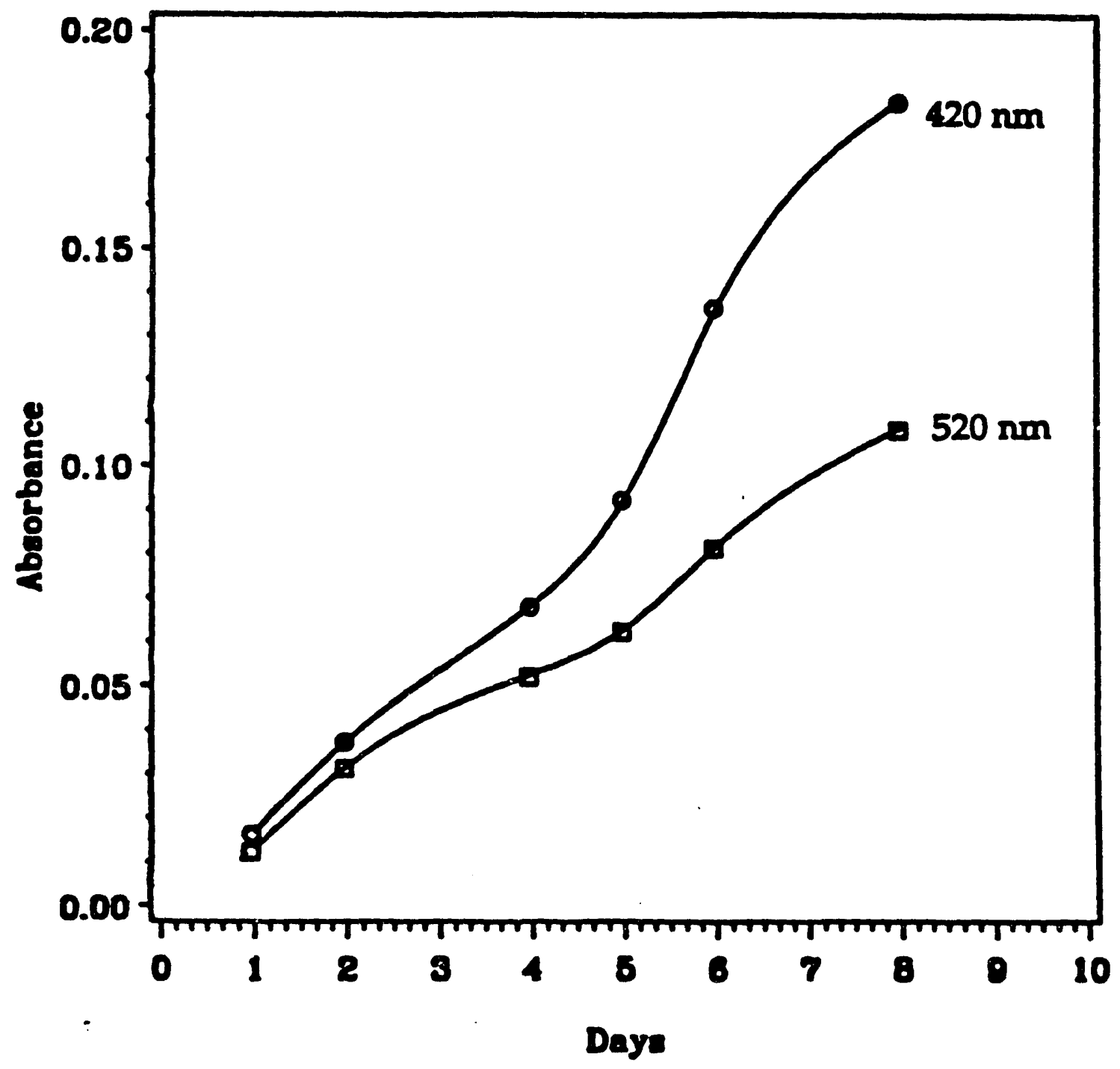


Figure 3 : Growth Curve of R.polustris on Diphenyl methone. Absorbonce of $420 \mathrm{~nm}$

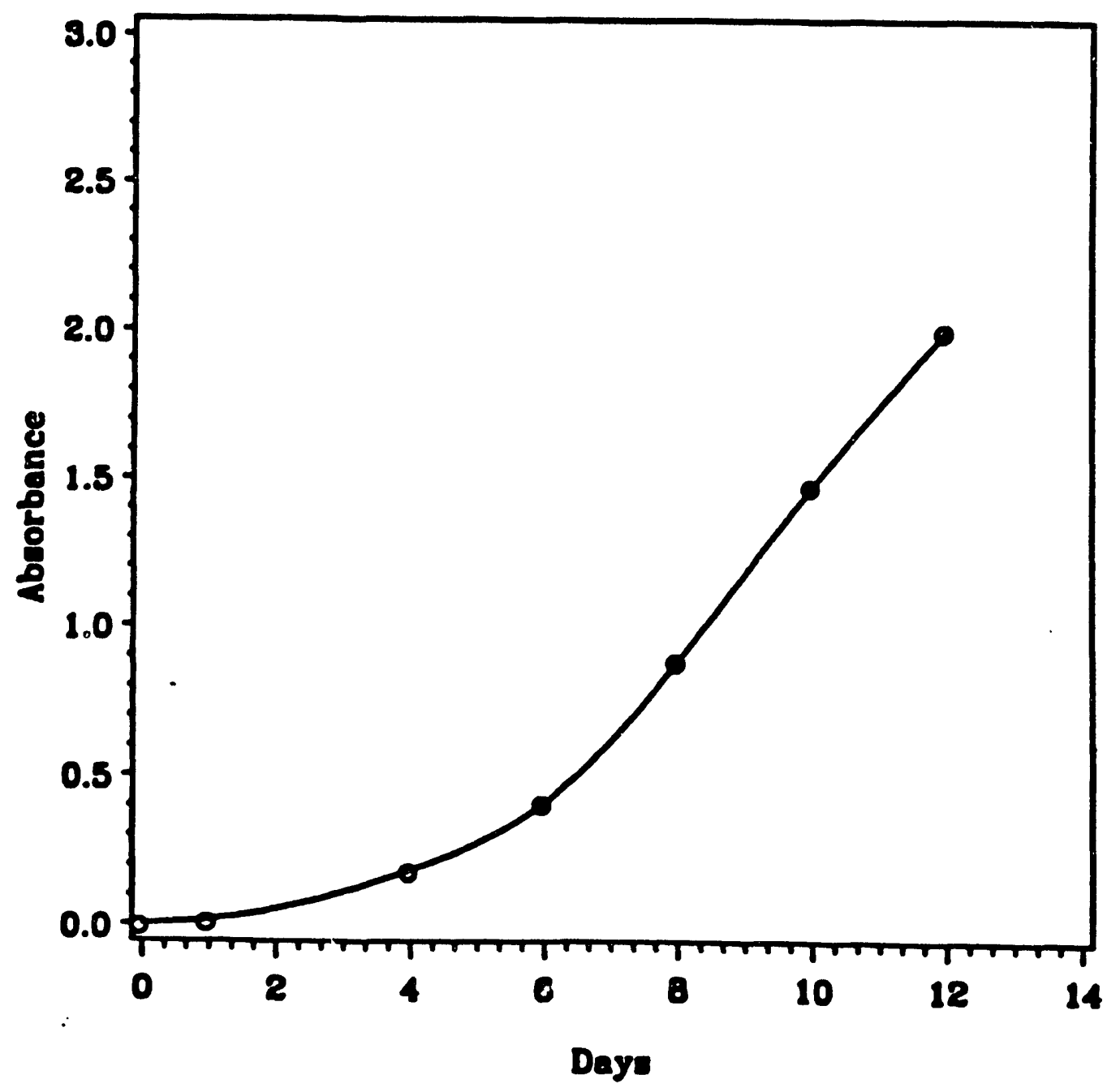


Figure 4 : UV Spectra of Supernatant From a Centrifuged Sample of Rhodopseudomonas palustris on Diphenyl methaine.

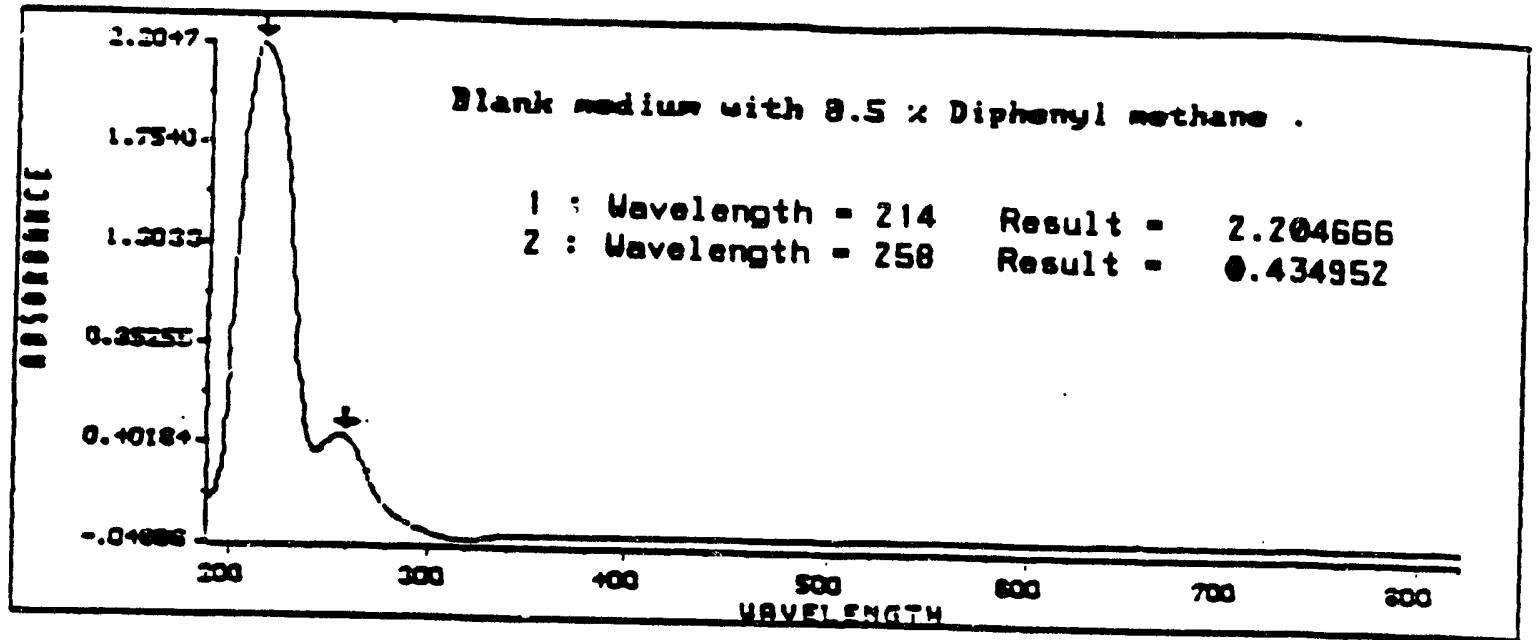

a) Absorbance of Blank Medium.

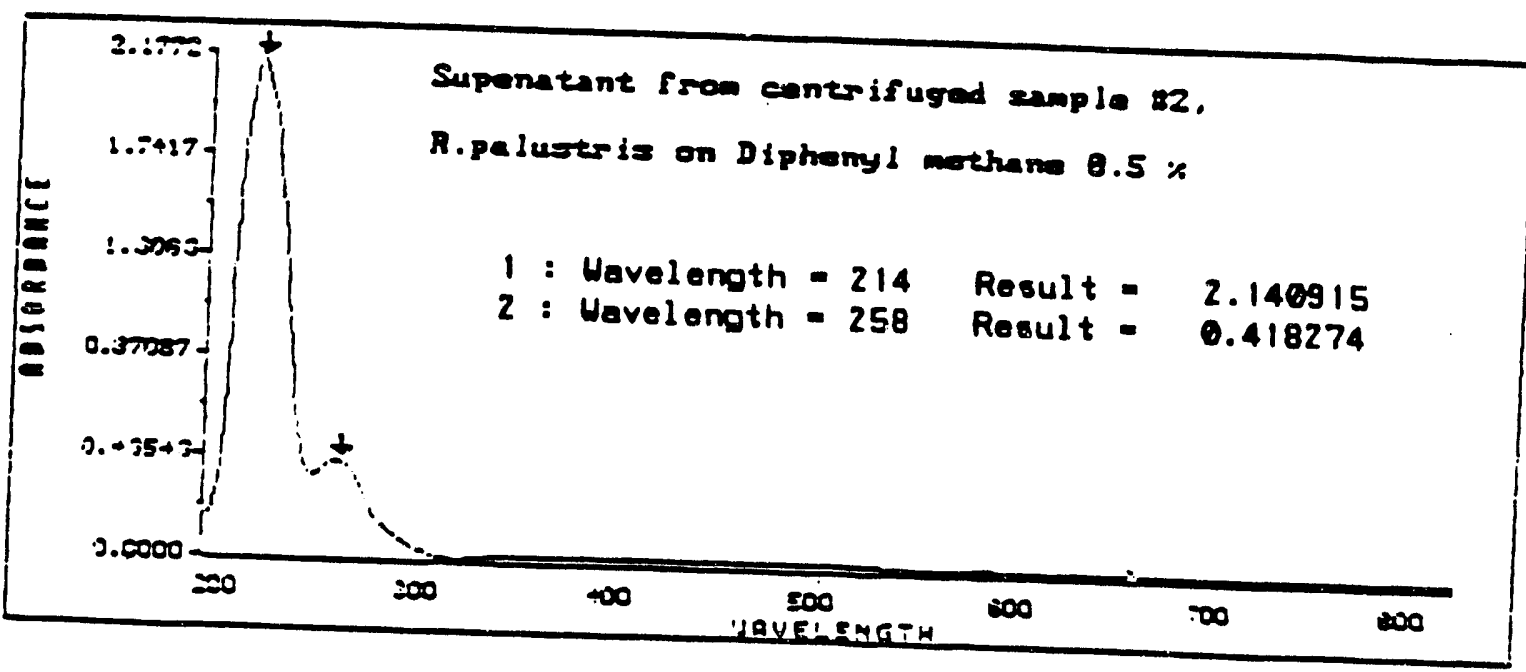

b) Absorbance after 1 day.

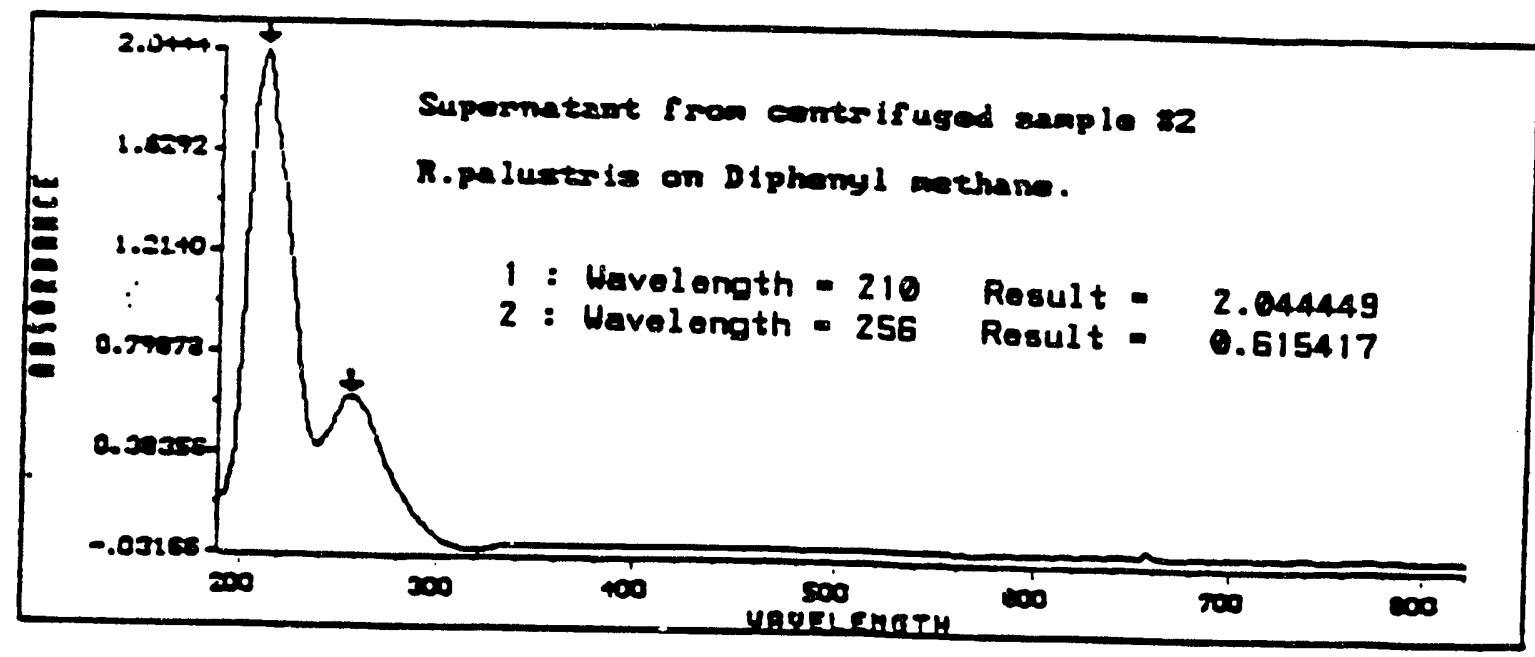

c) Absorbance after 3 days. 
Figure 5 : R.polustris on Diphenyl methone (0.5 x). Absorbance ot $256 \mathrm{~nm}$

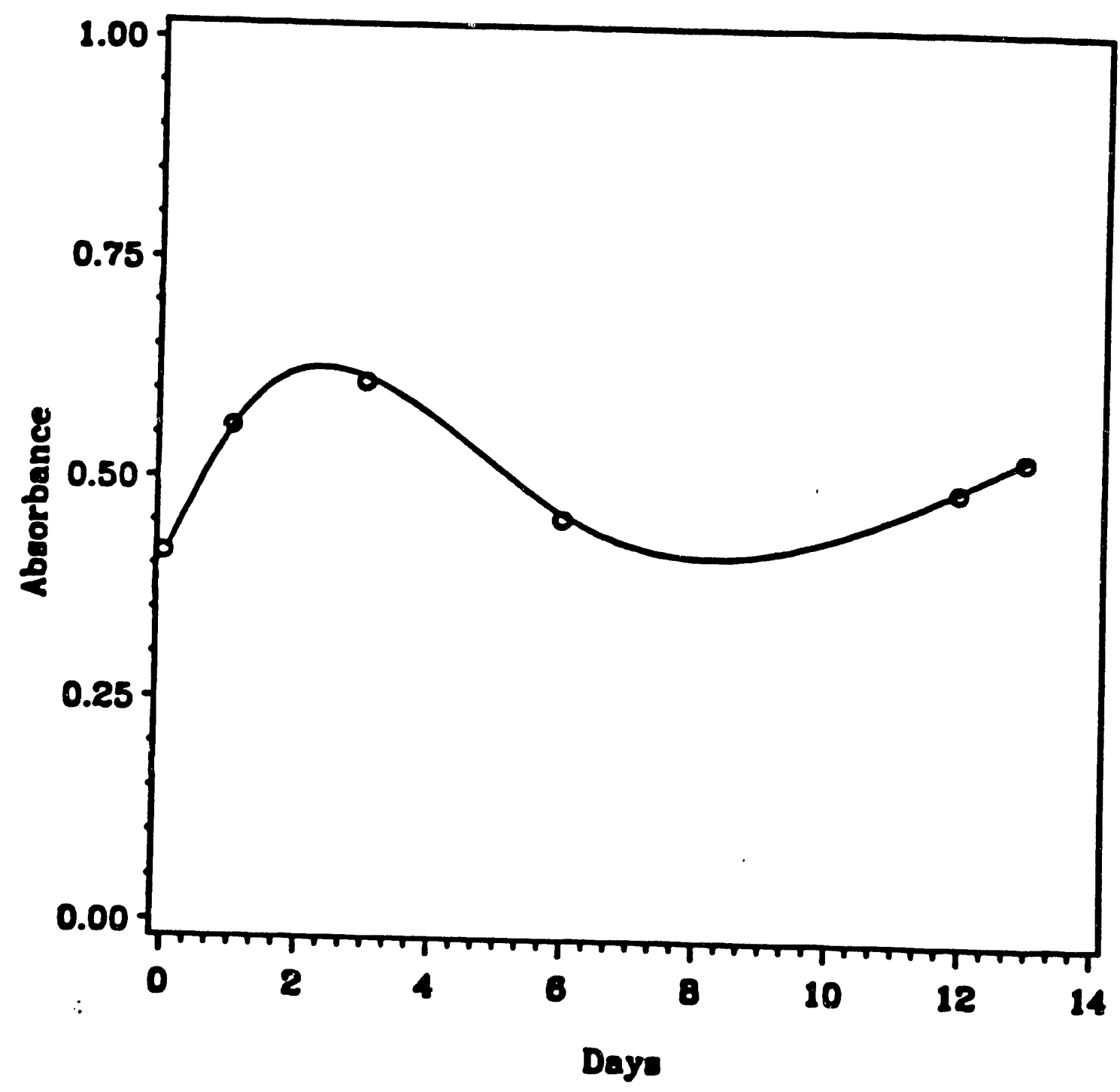


Figure 6 : Desulfotomoculum nigrificons on Nophtholene Absorbance Vs Time ot $282 \mathrm{~nm}$

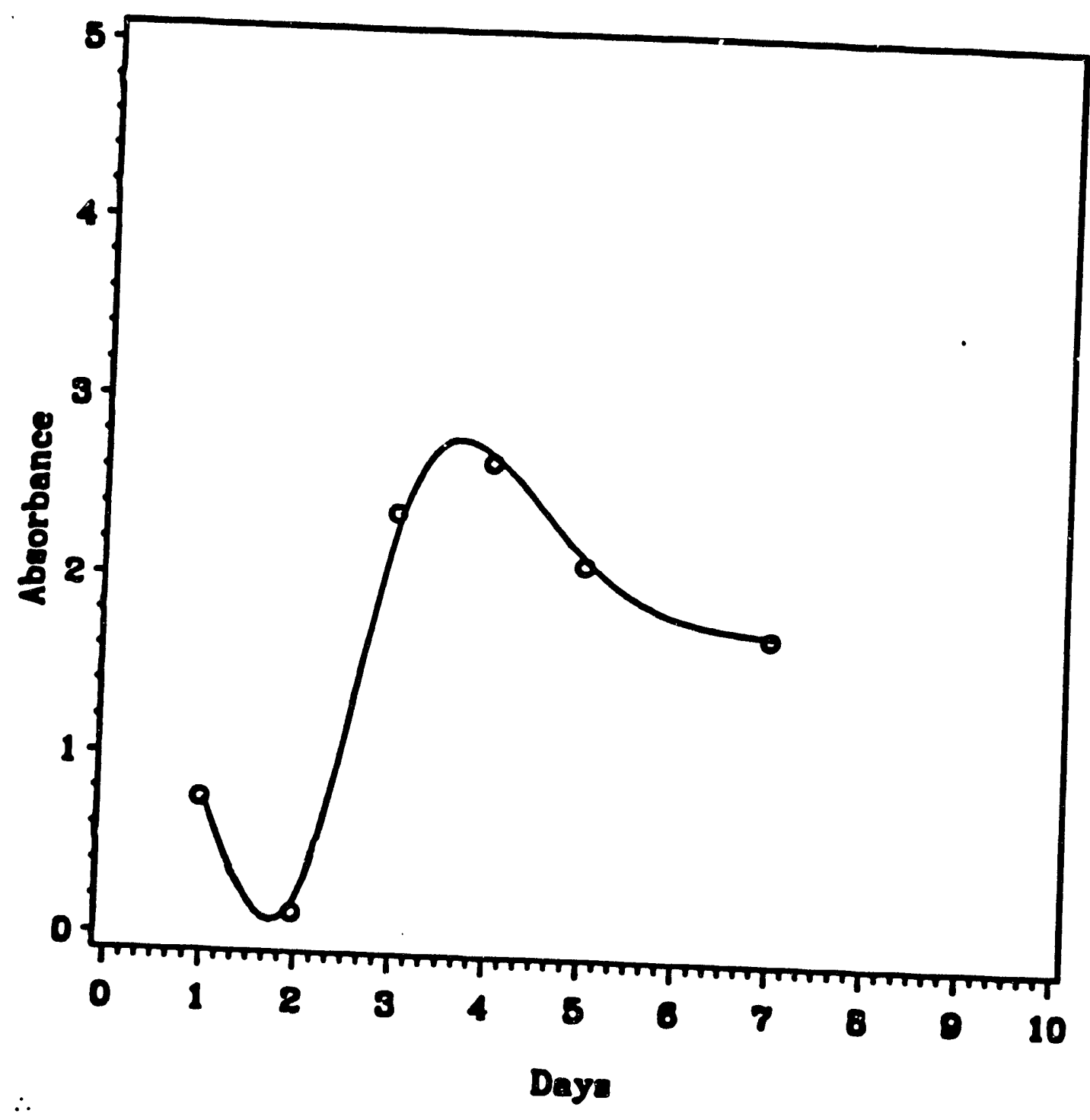


Figure 7 : UV Spectra of Supernatant From A Centrifuged Sample of

Desulfovibrio desulfuricans On Coal.

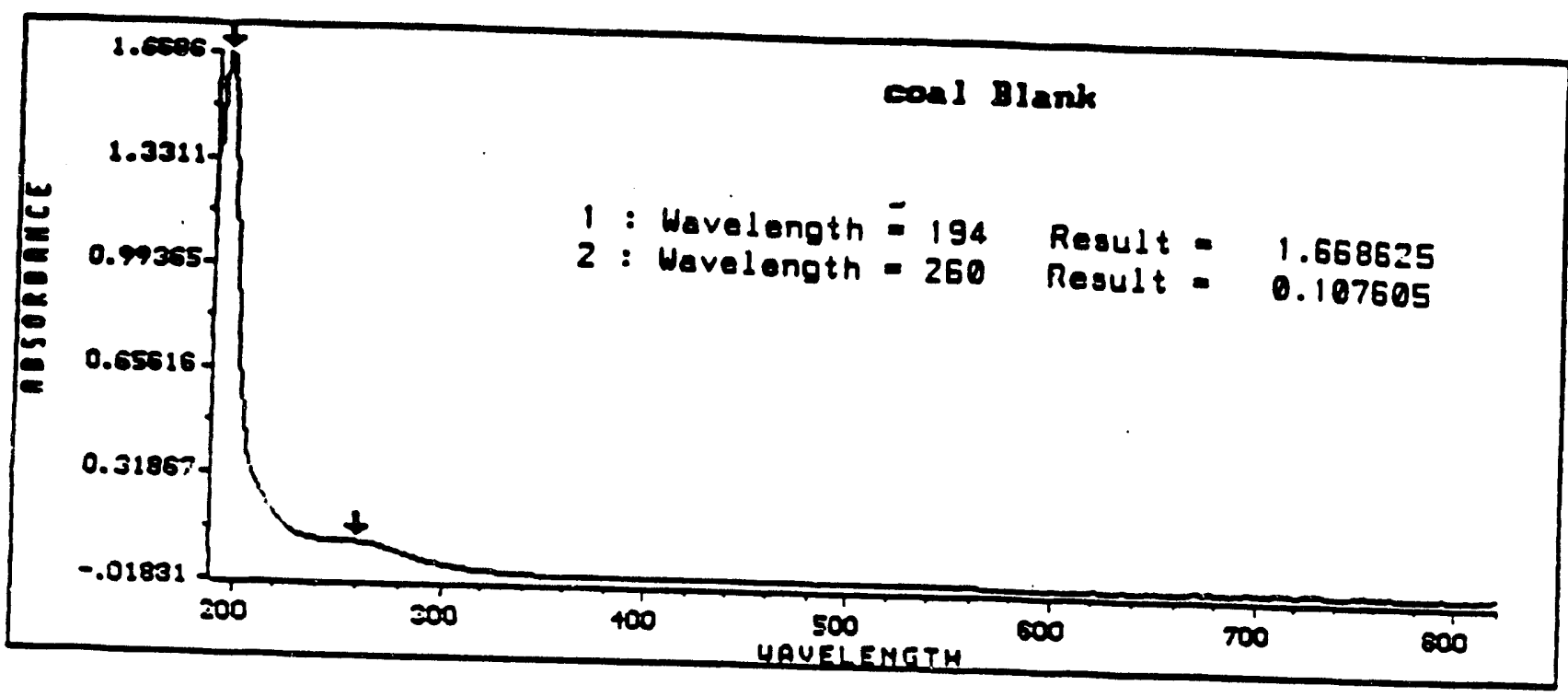

Absorbance of Blank Medium.

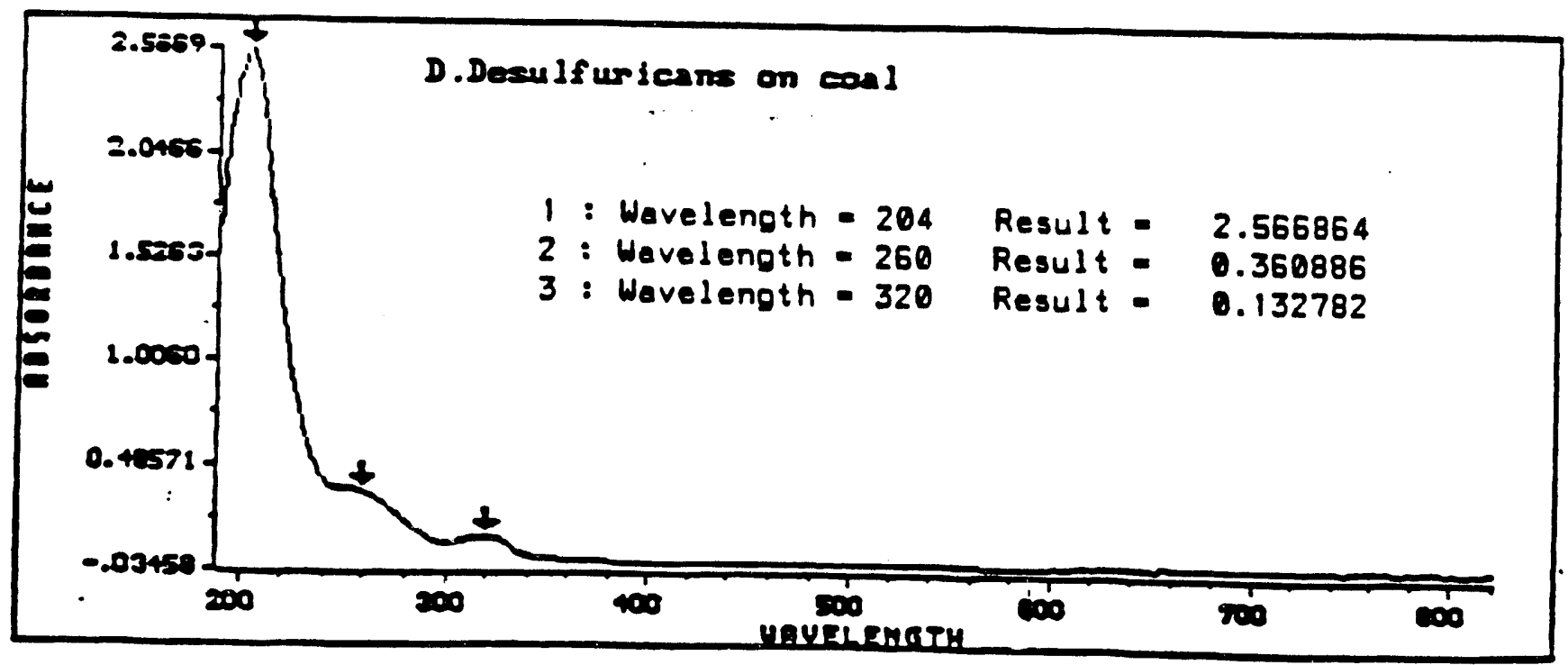

Absorbance after 12 days. 
Figure 8 : Procedure For Treating Coal With Fungus.

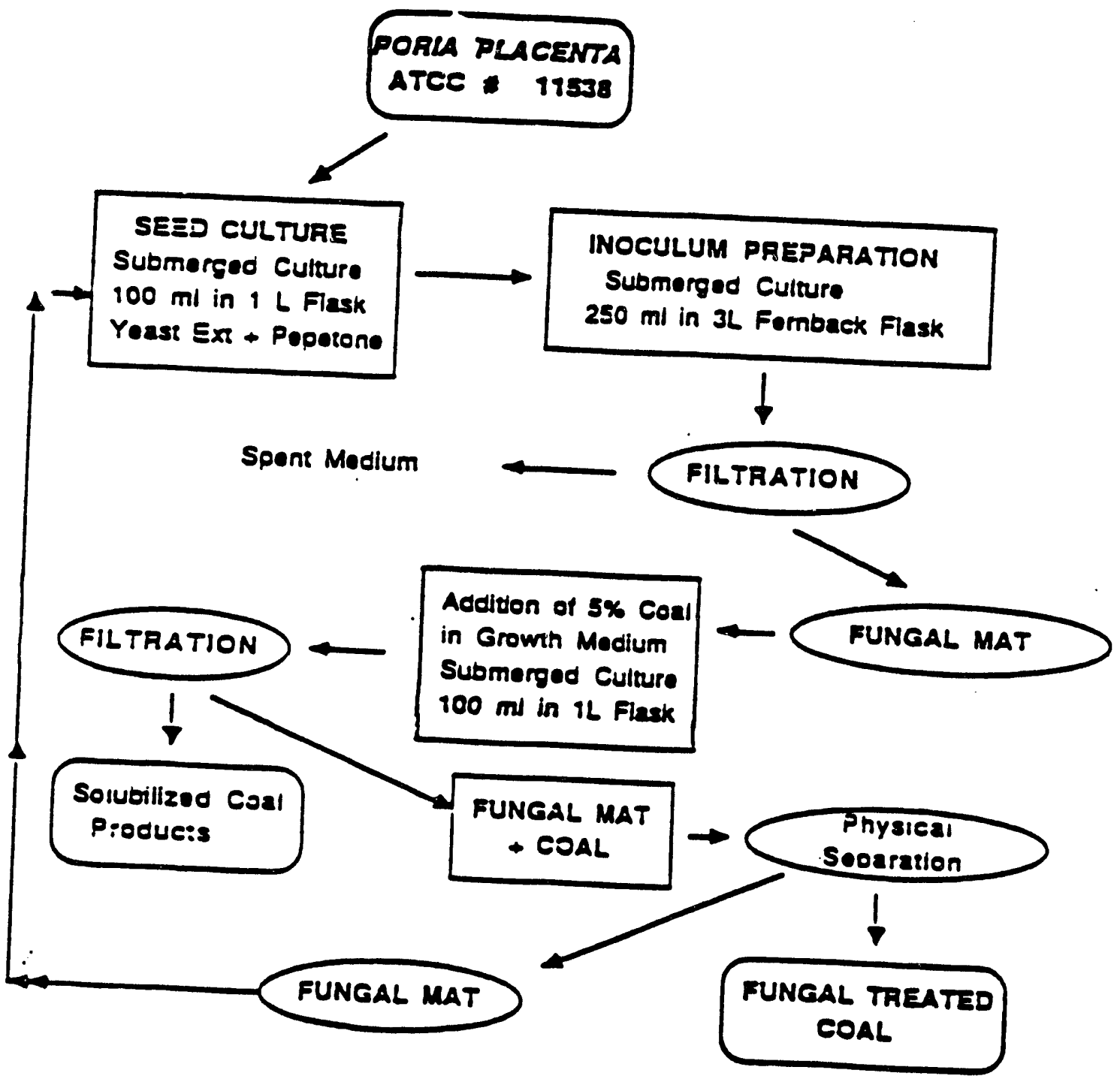


Figure 9 : pH Profile During Anaerobic Treatment Of Coal (KY \$85098) By Sulfolobus brierleyi.

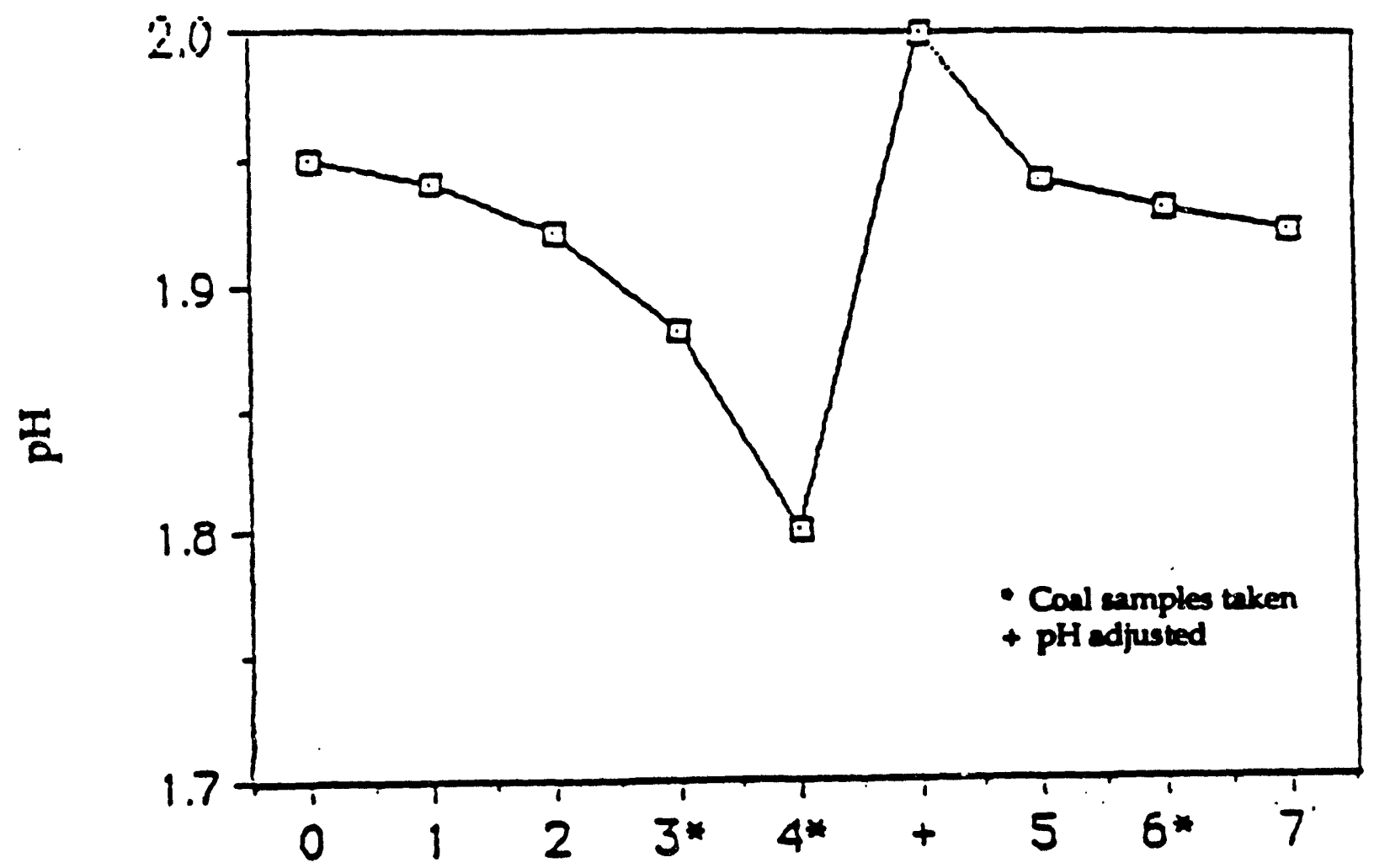

Duration (days) 
Figure 10 : HS Production During Araerobic Treatment Of Coal (KY \$85098) By Sulfolobus brierleyi.

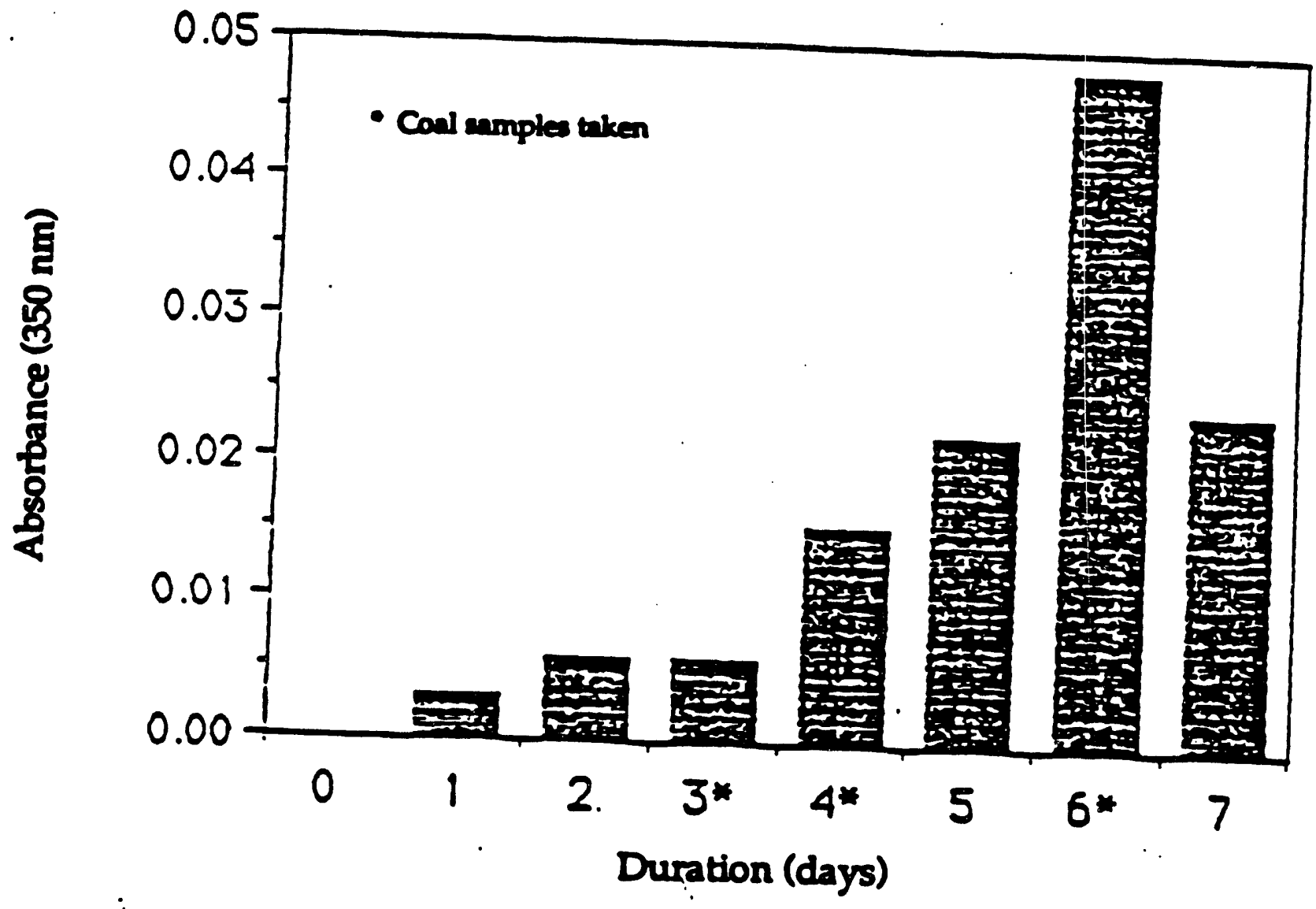




\title{
TASK I.2
}

\section{Materials Characterization Study of Coal Liquefaction Processes}
PI's:
G. P. Huffman
F. E. Huggins
P. J. Reucroft
K. Subbaswamy
Dennis Clouthier
Naresh Shah

Report

Coordinator: $\quad$ G. P. Huffman

Phone:

(606) $257-4027$

Period:

May 4, 1989 - May 3, 1990

\section{XAFS and Mössbauer Spectroscopy and CCSEM Studies of Coals of Interest in the CFFLS Program}

\author{
(N. Shah, F. E. Huggins and G. P. Huffman)
}

\section{Coal Characterization Studies}

The CFFLS has decided to concentrate much of its research effort on the Argonne Premium coal samples, in order to facilitate comparison of results from different investigators at different CFFLS universities. Because mineral matter and sulfur forms can significantly affect liquefaction behavior, we have carried out detailed characterization of these coal properties by computer-controlled scanning electron microscopy (CCSEM), Mössbauer spectroscopy, and X-ray absorption fine structure (XAFS) spectroscopy. In order to obtain more meaningful correlations with liquefaction behavior, the data set was augmented by investigating eight Kentucky coals selected from many coals to exhibit a range of sulfur contents, rank, and liquefaction yields. Table I lists the carbon and sulfur contents of all coals studied.

\section{Liquefaction Results}

In order to maximize any catalytic or retrograde effects of mineral matter, the liquefaction experiments were carried out at a relatively low temperature $\left(385^{\circ} \mathrm{C}\right)$ with tetralin as the solvent (1:1.5 coal/solvent ratio), without any added catalyst for a reaction time of 15 minutes. Microclaves (tubing bombs) were cold pressurized to 800 psi with hydrogen before heating to reaction conditions. Products of liquefaction were classified as: 
oils - pentane soluble; asphaltenes - pentane insoluble, benzene soluble; preasphaltenes benzene insoluble, pyridine soluble; insoluble organic matter (IOM) - pyridine insoluble. Total conversion is defined as 100 - IOM. A more detailed discussion of apparatus and procedures is given elsewhere. ${ }^{(1)}$ Figure 1 is a bar chart showing the liquefaction results for all coals.

\section{CCSEM}

Different mineral and inorganic species can act as catalysts or retardants during liquefaction, depending on reaction conditions. It is, therefore, important to know the types of minerals present and how they are distributed in coal. Computer controlled scanning electron microscopy (CCSEM) is an excellent tool to rapidly characterize the mineral matter in coal. As discussed elsewhere, ${ }^{(2,3,4)}$ CCSEM is capable of measuring the size and composition of 200-400 particles per hour. In the current study, 1200 mineral particles were measured for each sample.

Tables 2 and 3 list the mineralogical data obtained to date by CCSEM examination of Argonne and Kentucky coals. Column 1 lists the mineral categories observed in these coals, while remaining columns give the weight percentages of the total mineral matter accounted for by each category. With CCSEM, size distributions of various minerals present in the coal can also be determined. The results obtained for pyrite in several of the Argonne coals are shown in Figure 2. Because the Argonne coals are currently under investigation by many coal scientists, we have included the size distribution information for the major mineral phases in all of these coals in Table 4.

It may be noted the "mixed silicates" account for a fairly large percentage of the mineral matter in these raw coal samples. This phase primarily includes clay minerals and quartz in juxtaposition to each other, perhaps in partings. This is illustrated by the ternary diagrams in Figure 3. Each point in Figure 3a represents a mineral feature identified in CCSEM analysis that contains $\geq 90 \%$ of $(\mathrm{K}+\mathrm{Fe})+\mathrm{Al}+\mathrm{Si}$ on the basis of its energy dispersive X-ray (EDX) spectrum. The c mposition is normalized to these four elements and plotted in a ternary representation, as shown. As discussed in detail in earlier CCSEM pasers, ${ }^{(2-4)}$ aluminosilicate minerals are placed in one of several mineral categories depending on the approximate particle composition indicated by the EDX spectrum. The compositional ranges being used for quartz, kaolinite, illite and other silicate minerals are indicated in Figure $3 b$. It is evident that particles exhibit a range of compositions extending between the quartz, kaolinite, and illite composition areas. It is these intermediate compositions that the coal mineral analysis (CMA) program identifies as mixed silicates. 
it may also be noted that the Wyodak and Beulah Zap coals from the Argonne suite contain a significant amount of the phases identified as "miscellaneous sulfide," "miscellaneous mixed," and "mixed carbonates." These are, for the most part, not discrete mineral phases, but the contribution of $\mathrm{Ca}$ and $\mathrm{S}$ molecularly dispersed in the coal matrix. Normally, CCSEM analyses are run in a fashion that detects only discrete mineral matter and the measurements on these coals will be repeated on new samples in this manner.

\section{Mössbauer Spectroscopy}

${ }^{67} \mathrm{Fe}$ Mössbauer spectroscopy was used to identify and quantify iron bearing phases present in the coals. ${ }^{(5,6)}$ Iron predominantly exists as pyrite, siderite or Fe-containing clay in coals. Mössbauer spectroscopy is the most accurate technique for quantitative analysis of the iron-bearing phases in coal and provides a direct, non-destructive method of measuring the pyritic sulfur coisant of the coal. Table 5 lists the Mossbauer spectroscopy results for all coals. The pyritic sulfur determined by the Mössbauer measurement ${ }^{(5)}$ is given in column 2 , while columns 3-6 give the percentages of the total sample iron contained in the various iron-bearing phases observed. It is evident that the Argonne coals were in pristine condition and exhibited no pyrite oxidation. However, some of the Kentucky coals, mainly those with high pyrite contents have undergone minor oxidation that converts pyrite to ferrous sulfate form.

In order to examine the transformations of Fe-bearing minerals in coals as they undergo liquefaction and thereby assess the role of the inherent iron minerals as catalysts, the insoluble organic matter (IOM) of the Argonne Premium Sample coals were also investigated using Mössbauer spectroscopy. Figure 4 shows room temperature Mössbauer spectra of 1 linois \#6 coal before and after liquefaction treatment. In Illinois \#6 coal, almost all $\mathrm{Fe}$ is present in the form of pyrite; after the liquefaction test, $60 \%$ of the pyrite converts to pyrrhotite, while the remaining pyrite is unconverted. Under more severe conditions and/or longer liquefaction times all of the pyrite would convert to pyrrhotite. In the Pocahontas \#3 coal, iron is distributed among clays, siderite, and pyrite; however, in the Pocahontas \#3 IOM, Fe in clays and siderite remains unchanged whereas pyrite has been - converted to pyrrhotite. As summarized in the Table 5 for the Argonne Premium Samples, this observation appears quite general: the Fe-bearing minerals, other than pyrite, do not appear to undergo significant transformation during liquefaction, whereas some or all of the pyrite converts to pyrrhotite. According to Montano et al., ${ }^{(7-9)}$ pyrrhotite is the primary Fe containing catalytic species derived from coal minerals that is correlated with liquefaction. Iron in the form of clays or carbonates does not appear to be connected with liquefaction 
behavior.

Correlations Between Coal Properties and Thermal Liquefaction Behavior at Mild Conditions

Several correlations between coal structure and thermal liquefaction behavior under mild conditions $\left(385^{\circ} \mathrm{C}, 800 \mathrm{psig}_{2}\right.$ at room temperature, 15 minute reaction time) in tubing bomb reactors have been observed. Correlations with organic structure are shown in Figures 5 and 6. The decrease of gas production with increasing carbon content shown in Figure 5 is not surprising. The behavior of the total conversion percentage with carbon content (Figure 6) is more interesting, showing a bell-shaped maximum between approximately 77 and $83 \%$ carbon. This type of behavior has been observed previously. ${ }^{(1)}$

To explore correlations between mineral matter and liquefaction behavior, the total surface areas per gram of coal of the principal minerals were calculated. This calculation uses mean mineral diameters determined from the CCSEM measurement. For each mineral, the average particle diameter, $\left\langle\mathrm{d}_{n}\right\rangle$, for each of the seven size bins (see Figure 2 ) is calculated by adding the mean diameters of all the individual particles and dividing by the number of particles in that bin. If $w_{n}$ is the weight percentage pyrite in size bin $n$, it can be shown that the total pyrite surface area per gram of coal is given approximately by

$$
A_{\mathrm{tot}}^{\mathrm{pyx}}=\frac{6 \cdot W_{\mathrm{pyr}}^{\text {(Moss.) }}}{10^{4} \cdot p_{\mathrm{pyr}}} \sum_{n=1}^{7} \frac{w_{n}}{\left\langle d_{n}\right\rangle}
$$

where $W_{p y r}^{(\text {(com) }}$ is the weight percentage of pyrite determined from the Mossbauer measurement ${ }^{(5)}$ and $\rho_{p y r}$ is the density of pyrite $\left(5.0 \mathrm{~g} / \mathrm{cm}^{3}\right)$. For other minerals, we use the formula

$$
A_{\text {tot }}^{\min }=\frac{6 \cdot W_{\min }^{(\operatorname{ccs} x)} \cdot W_{a x h}}{10^{4} \cdot \rho_{\min }} \sum_{n=1}^{7} \frac{w_{n}}{\left\langle d_{n}\right\rangle}
$$

where $W_{\text {anh }}$ is the ASTM ash percentage and $W_{\min }^{(\text {(C)sBu) }}$ is the CCSEM determined weight percentage of the total mineral matter contained by the mineral in question. Equations (1) and (2) are rather rough approximations in an absolute sense. First, the mineral particles are, of course, not spherical and many are far from it. Second, the resolution of the SEM is only about $0.1 \mu \mathrm{m}$ and particles below that size are not measured. Our objective, however, is to identify meaningful trends between CCSEM mineral size and weight distributions and thermal liquefaction behavior. In that context, Equations (1) and (2) are reasonable first approximations. Moreover, these mineral surface areas are more meaningful parameters for 
catalytic activity than, say, the weight percentages of mineral phases.

In Figure 7, the oil production is plotted against the pyrite surface area per gram of coal determined from the CCSEM data as discussed above. The data show large scatter, but a generally increasing trend of oil production with pyrite surface area is observed. The large amount of scatter is not surprising, in view of the fact that coals covering a fairly large range of ranks and carbon content have been studied. Moreover, only one sample (Kentucky coal \#5416, Beaver Creek seam) has a pyritu surface area per gram of coal large enough (nearly $1 \mathrm{~m}^{2} / \mathrm{g}$ ) to be expected to give significant catalytic activity. It may be noted from Figures 5 and 6 that this sample exhibits significantly more total conversion and less gas production than would be expected on the basis of its carbon content alone. Presumably this is because of catalytic hydrogenation activity of the pyrrhotite derived from the comparatively high surface area pyrite in this coal.

Figure 8 shows the tota: conversion percentage plotted against the surface area per gram of all "silicate" minerals; that is, illite, kaolinite, quartz and mixed silicates (see Figure 3). Again, the scatter is large and no simple trends are easily discernible. If, however, we try to eliminate the superimposed effect of coal rank by ignoring coals of his and low carbon content (Beulah-Zap, Wyodak, Upper Freeport, Pocahontas, KY sample \#6398, and KY sample \#5416), there appears to be a general decrease of conversion percentage with increasing "silicate" surface area per gram of coal. A similar trend has been observed by $\mathrm{Stohl}^{(10)}$ for kaolinite, in a more controlled experiment. These results suggest that the surfaces of clay minerals and quartz may serve as sites for initiation of retrograde reactions.

\section{XAFS Studies of Sulfur in the Argonne Coals}

As part of another research contract, ${ }^{(11)}$ we have developed a method of least squares analysis of the X-ray absorption near edge structure (XANES) of sulfur XAFS spectra that allows us to make quantitative determinations of six different forms of sulfur in coal: pyrite, organic sulfide, thiophene, sulfoxide, sulfone and sulfate. This research is summarized in several recent ${ }^{(12-14)}$ and forthcoming papers. ${ }^{(16)}$ For the current report, we simply summarize in Table 6 the percentages of the total sample sulfur determined from the XANES to be in each identified sulfur form for the Argonne coals. Except for the Beulah-Zap sample, which contained a minor amount of gypsum, no oxidized sulfur was observed in these pristine coals. The reader is referred particularly to reference 15 for further detail.

\section{Summary}

A fairly complete database for the mineral matter, forms of iron, and forms of sulfur 
in the Argonne Premium Coal Samples has been generated by CCSEM, Mössbauer spectroscopy, and XAFS spectroscopy. Mineralogical and forms of iron data were also obtained for a number of Kentucky coals to broaden the database somewhat. Correlations of thermal liquefaction behavior with the surface areas per gram of pyrite and silicate minerals (clays and quartz) were examined. Oil production showed a general increase with total pyrite surface area, while total conversion exhibited a decreasing trend with increasing silicate surface area. These correlations were fairly weak, however, and it is likely that such mineral matter interactions play a secondary role for most coals in liquefaction processes where a catalyst is added.

\section{References}

1. R. A. Keogh and B. H. Davis, J. Coal Quality, 7(1), (1988), 27.

2. F. E. Huggins, D. A. Kosmack, G. P. Huffman, and R. J. Lee, Scanning Electron Microscopy/1980/I, 531-540, SEM Inc., AMF O’Hare, Chicago, IL 60666, USA.

3. F. E. Huggins, G. P. Huffman, and R. J. Lee, ACS Symposium, Series, 205, (1982), 239.

4. G. P. Huffman and F. E. Huggins, R. A. Shoenberger, J. S. Walker, R. B. Greegor, and F. W. Lytle, Fuel 65, 621-632 (1986).

5. G. P. Huffman and F. E. Huggins, Fuel, 57 (1978), 592.

6. F. E. Huggins, G. P. Huffman, R. J. Lee, ACS Symposium Series, 205, (1982), 239.

7. P. A. Montano and B. Granoff, Fuel $\underline{59}$ 214-216 (1980).

8. A.S. Bommannavar and P. A. Montano, Fuel 61, 523-528 (1982).

9. A.S. Bommannavar and P. A. Montano, Fuel 62, 932-935 (1983).

10. F. V. Stohl, ACS Div. of Fuel Chem. Preprints 34, No.3, 814-818 (1989).

11. G. P. Huffman and F. E. Huggins, "Comprehensive Investigation of Inorganic Constituents in Coal and Coal Derivatives," EPRI Contract No. RP-8803-20.

12. G. P. Huffman, F. E. Huggins, S. Mitra, N. Shah, R. J. Pugmire, B. Davis, F. W. Lytle, R. B. Greegor, Energy and Fuels, 3 , 200-205, (1989).

13. G. P. Huffman, F. E. Huggins, S. Mitra, and N. Shah, Proceedings of the $\underline{1989}$ International Conference on Coal Science, pp. 47-50, Int. Eng. Agency (1989).

14. G. P. Huffman, F. E. Huggins, H. E. Francis, S. Mitra and N. Shah, "Structural Characterization of Sulfur in Bioprocessed Coal," pp. 21-32, Processing and Utilization of High Sulfur Coals III Eds., R. Markuszewski and T. D. Wheelock, Elsevier, 1990. 
15. S. Mitra, F. E. Huggins, N. Shah, M. Taghiei, S. Vaidya, and G. P. Huffman, "Quantitative Analysis of All Major Functional Forms of Sulfur in Coal by Direct Analysis of XANES Spectra," in preparation. 
Table 1. Rank and total sulfur content of Kentucky and Argonne coals

\begin{tabular}{|c|l|l|c|c|}
\hline $\begin{array}{c}\text { COal \# } \\
\text { (UKCAER) }\end{array}$ & Seam & $\begin{array}{l}\text { County / } \\
\text { State }\end{array}$ & $\begin{array}{c}\% C \\
\text { DAF }\end{array}$ & $\begin{array}{c}\% S \\
\text { DAF }\end{array}$ \\
\hline 91864 & WKY \#9 & Union & 82.15 & 3.93 \\
6398 & Harlan & Harlan & 85.33 & 0.86 \\
2145 & Peach Orchard \#3 & Magoffin & 83.66 & 0.63 \\
71302 & WKY \#6 & Caldwell & 82.50 & 3.37 \\
3913 & Stockton & Martin & 82.34 & 0.75 \\
2167 & Cannel City & Magoffin & 80.97 & 1.62 \\
5416 & Beaver Creek & Pulaski & 74.45 & 13.92 \\
71464 & WKY \#9 & Henderson & 79.44 & 3.84 \\
71468 & WKY \#9 & Muhlenberg & 80.27 & 4.15 \\
\hline \multicolumn{7}{|c|}{ Pocahontas \#3 } & Virginia & 91.05 & 0.66 \\
& Upper Freeport & Pennsylvania & 85.5 & 2.32 \\
& Pittsburgh \#8 & Pennsylvania & 83.2 & 2.19 \\
& Lewiston-Stockton & West Virginia & 82.58 & 0.71 \\
& Blind Canyon & Utah & 80.69 & 0.62 \\
& Illinois \#6 & Illinois & 77.67 & 4.83 \\
& Wyodak-Anderson & Wyoming & 75.01 & 0.6 \\
& Beulah-Zap & North Dakota & 72.9 & 0.8 \\
\hline
\end{tabular}


Table 2. Mineral Composition of Argonne Coals

\begin{tabular}{|c|c|c|c|c|c|c|c|c|}
\hline \multirow[b]{2}{*}{ Mineral Species } & \multicolumn{8}{|c|}{ Wt\% of Mineral Matter } \\
\hline & $\begin{array}{l}\text { Up. } \\
\text { Fr. }\end{array}$ & $\begin{array}{l}\text { Wyo } \\
\text { dak }\end{array}$ & $\begin{array}{l}\text { I11 } \\
\# 6\end{array}$ & $\begin{array}{l}\text { Pitt } \\
\# 8\end{array}$ & $\begin{array}{l}\text { Poca } \\
\# 3\end{array}$ & $\begin{array}{l}\text { B1. } \\
\text { Cany }\end{array}$ & $\begin{array}{l}\text { Lew } \\
\text { Stoc }\end{array}$ & $\begin{array}{l}\text { Beu1 } \\
\text { Zap }\end{array}$ \\
\hline $\begin{array}{l}\text { Quartz } \\
\text { Kaolinite } \\
\text { Illite } \\
\text { Chlorite } \\
\text { Misc. Silicates }\end{array}$ & $\begin{array}{r}5 \\
4 \\
48 \\
\\
15\end{array}$ & $\begin{array}{r}8 \\
3 \\
1 \\
15\end{array}$ & $\begin{array}{r}9 \\
3 \\
18 \\
23\end{array}$ & $\begin{array}{r}12 \\
8 \\
20 \\
25\end{array}$ & $\begin{array}{r}4 \\
10 \\
8 \\
13 \\
37\end{array}$ & $\begin{array}{r}4 \\
9 \\
40 \\
37\end{array}$ & $\begin{array}{l}10 \\
15 \\
56 \\
16\end{array}$ & $\begin{array}{r}6 \\
5 \\
1 \\
12\end{array}$ \\
\hline $\begin{array}{l}\text { Pyrite } \\
\text { Misc. Sulf. }\end{array}$ & $\begin{array}{r}21 \\
1\end{array}$ & $\begin{array}{r}6 \\
28\end{array}$ & $\begin{array}{r}27 \\
1\end{array}$ & $\begin{array}{r}25 \\
1\end{array}$ & 4 & 3 & 1 & $\begin{array}{r}16 \\
2\end{array}$ \\
\hline $\begin{array}{l}\text { Fe-rich } \\
\text { Calcite } \\
\text { Mixed Carbonates } \\
\text { Sil-Sulfur }\end{array}$ & 4 & $\begin{array}{l}2 \\
2 \\
3\end{array}$ & $\begin{array}{l}8 \\
1 \\
3\end{array}$ & $\begin{array}{l}1 \\
2\end{array}$ & $\begin{array}{l}4 \\
7 \\
5\end{array}$ & $\begin{array}{l}2 \\
1\end{array}$ & & $\begin{array}{r}5 \\
27\end{array}$ \\
\hline $\begin{array}{l}\text { Other } \\
\text { Misc. Mixed }\end{array}$ & 1 & $\begin{array}{r}3 \\
29\end{array}$ & 3 & 4 & $\begin{array}{l}4 \\
4\end{array}$ & $\begin{array}{l}1 \\
3\end{array}$ & & 26 \\
\hline
\end{tabular}


Table 3. Mineral Composition of Kentucky Coals

\begin{tabular}{|l|r|r|r|r|r|r|r|r|r|}
\hline & \multicolumn{8}{|c|}{ Wt\% of Mineral Matter } \\
\cline { 2 - 10 } Mineral Species & 6398 & 71468 & 2145 & 71302 & 3913 & 91864 & 2167 & 5416 & 71464 \\
\hline Quartz & 3 & 8 & 11 & 7 & 10 & 13 & 6 & 1 & 8 \\
Kaol inite & 12 & 4 & 7 & 3 & 8 & 3 & 2 & & 7 \\
Illite & 7 & 18 & 45 & 22 & 29 & 16 & 21 & 1 & 13 \\
Misc. Silicates & 56 & 18 & 35 & 26 & 47 & 22 & 33 & 7 & 25 \\
\hline Pyrite & 2 & 31 & & 25 & & 26 & 6 & 55 & 26 \\
Misc. Sulf. & 1 & 1 & & 4 & 1 & 5 & 1 & 13 & 3 \\
Fe-rich & 1 & 11 & & & & & 14 & 1 & 4 \\
Calcite & 1 & 1 & & & & & 11 & & 1 \\
Mixed Carbonates & 3 & & & & & & & & \\
Ti rich & & & & 3 & & 1 & & 6 & \\
\hline Qtz-Pyrite & 5 & 3 & & 2 & 2 & 5 & & 2 & 5 \\
Sil-Sulfur & & & 2 & & 1 & & 7 & 1 \\
Sil-Pyrite & 9 & 3 & 1 & 5 & 2 & 5 & 5 & 6 & 5 \\
\hline Misc. Mixed & & & & & &
\end{tabular}


Table 4 Size distributions of the major mineral phases in the Argonne coals Size ranges of average particle diameter in microns for more than 7 wt 8
minerals.

Pyodak coal

\begin{tabular}{|c|c|c|c|c|c|c|c|c|}
\hline \multirow[b]{2}{*}{ MINERAL SPECIES } & \multirow[b]{2}{*}{ WT. 8} & \multirow[b]{2}{*}{$\begin{array}{l}0.0 \\
2.5 \\
\end{array}$} & \multirow[b]{2}{*}{$\begin{array}{l}2.5 \\
5.0 \\
\end{array}$} & \multirow{2}{*}{$\begin{array}{r}\text { Size } \\
5.0 \\
10.0 \\
\end{array}$} & \multirow{2}{*}{$\begin{array}{r}\text { Ranges } \\
10 \\
20 \\
\end{array}$} & \multirow[b]{2}{*}{$\begin{array}{l}20 \\
40 \\
\end{array}$} & \multirow[b]{2}{*}{$\begin{array}{l}40 \\
80\end{array}$} & \multirow[b]{2}{*}{$\begin{array}{r}80 \\
500 \\
\end{array}$} \\
\hline & & & & & & & & \\
\hline $\begin{array}{|ll|}\text { QUARTZ } \\
\text { MISC } & \text { SIIICATES } \\
\text { MISC } & \text { SULF } \\
\text { MISC } & \text { MIXED } \\
\text { MINOR } & \text { MINERAIS } \\
\end{array}$ & $\begin{array}{r}7.9 \\
14.8 \\
27.8 \\
28.6 \\
20.9 \\
\end{array}$ & $\begin{array}{r}5 \\
18 \\
22 \\
26 \\
15\end{array}$ & $\begin{array}{r}0 \\
29 \\
64 \\
50 \\
36 \\
\end{array}$ & $\begin{array}{r}17 \\
15 \\
8 \\
11 \\
14 \\
\end{array}$ & $\begin{array}{r}52 \\
29 \\
5 \\
8 \\
22\end{array}$ & $\begin{array}{r}17 \\
6 \\
0 \\
2 \\
6 \\
\end{array}$ & $\begin{array}{l}8 \\
3 \\
0 \\
2 \\
8\end{array}$ & $\begin{array}{l}0 \\
0 \\
0 \\
0 \\
0\end{array}$ \\
\hline GRAND TOTALS & 100.0 & 20 & 44 & 12 & 17 & 4 & 3 & 0 \\
\hline
\end{tabular}

Upper Freeport coal

\begin{tabular}{|c|c|c|c|c|c|c|c|c|}
\hline MINERAL SPECIES & WT. 8 & $\begin{array}{l}0.0 \\
2.5\end{array}$ & $\begin{array}{l}2.5 \\
5.0\end{array}$ & $\begin{array}{r}\text { Size } \\
5.0 \\
10.0\end{array}$ & $\begin{array}{l}\text { ges } \\
10 \\
20\end{array}$ & $\begin{array}{l}\text { rons } \\
20 \\
40\end{array}$ & $\begin{array}{l}40 \\
80\end{array}$ & $\begin{array}{r}80 \\
500\end{array}$ \\
\hline $\begin{array}{l}\text { ILIITE } \\
\text { MISC SILICATES } \\
\text { PYRITE } \\
\text { MINOR MINERALS }\end{array}$ & $\begin{array}{l}47.7 \\
14.5 \\
20.5 \\
17.3 \\
\end{array}$ & $\begin{array}{r}14 \\
18 \\
6 \\
8\end{array}$ & $\begin{array}{l}30 \\
27 \\
27 \\
24\end{array}$ & $\begin{array}{l}15 \\
20 \\
15 \\
21\end{array}$ & $\begin{array}{l}24 \\
17 \\
13 \\
22\end{array}$ & $\begin{array}{r}6 \\
6 \\
16 \\
8\end{array}$ & $\begin{array}{r}5 \\
5 \\
16 \\
10\end{array}$ & $\begin{array}{l}6 \\
7 \\
6 \\
7\end{array}$ \\
\hline GRAND TOTALS & 100.0 & 12 & 28 & 17 & 21 & 8 & 8 & 6 \\
\hline
\end{tabular}

Iewiston-stockton coal

\begin{tabular}{|c|c|c|c|c|c|c|c|c|}
\hline MINERAL SPECIES & WT. . & $\begin{array}{l}0.0 \\
2.5\end{array}$ & $\begin{array}{l}2.5 \\
5.0\end{array}$ & $\begin{array}{r}\text { Size } \\
5.0 \\
10.0\end{array}$ & $\begin{array}{l}\text { ges } \\
10 \\
20\end{array}$ & $\begin{array}{l}\text { rons } \\
20 \\
40\end{array}$ & $\begin{array}{l}40 \\
80\end{array}$ & $\begin{array}{r}80 \\
500\end{array}$ \\
\hline \begin{tabular}{l|} 
QUARTZ \\
KAOLINITE \\
IILITE \\
MISC SILICATES \\
MINOR MINERALS \\
\end{tabular} & $\begin{array}{r}10.2 \\
14.6 \\
56.4 \\
16.0 \\
2.8 \\
\end{array}$ & $\begin{array}{r}8 \\
13 \\
23 \\
15 \\
15\end{array}$ & $\begin{array}{l}44 \\
25 \\
49 \\
34 \\
22\end{array}$ & $\begin{array}{r}23 \\
32 \\
8 \\
14 \\
7\end{array}$ & $\begin{array}{l}18 \\
25 \\
13 \\
15 \\
43\end{array}$ & $\begin{array}{l}4 \\
4 \\
5 \\
6 \\
4\end{array}$ & $\begin{array}{r}2 \\
1 \\
2 \\
3 \\
10\end{array}$ & $\begin{array}{r}1 \\
0 \\
0 \\
12 \\
0\end{array}$ \\
\hline GRAND TOTALS & 100.0 & 19 & 42 & 14 & 17 & 5 & 2 & 2 \\
\hline
\end{tabular}


Table 4 (Continued) Size distributions of the major mineral phases in the Argonne coals Size ranges of average particle diameter in microns for more than 7 wt o minerals.

IIIIno18 \#6 cosl

\begin{tabular}{|c|c|c|c|c|c|c|c|c|}
\hline MINERAL SPECIES & WT. . & $\begin{array}{l}0.0 \\
2.5\end{array}$ & $\begin{array}{l}2.5 \\
5.0\end{array}$ & $\begin{array}{r}\text { Size } \\
5.0 \\
10.0\end{array}$ & $\begin{array}{l}\text { ges } \\
10 \\
20\end{array}$ & $\begin{array}{l}\text { cons } \\
20 \\
40\end{array}$ & $\begin{array}{l}40 \\
80\end{array}$ & $\begin{array}{r}80 \\
500\end{array}$ \\
\hline $\begin{array}{l}\text { QUARTZ } \\
\text { ILIITE } \\
\text { MISC SIIICATES } \\
\text { PYRITE } \\
\text { CALCITE } \\
\text { MINOR MINERALS }\end{array}$ & $\begin{array}{r}9.1 \\
18.3 \\
23.0 \\
27.1 \\
8.0 \\
14.5\end{array}$ & $\begin{array}{r}9 \\
18 \\
30 \\
6 \\
0 \\
41\end{array}$ & $\begin{array}{r}40 \\
71 \\
53 \\
3 \\
3 \\
34\end{array}$ & $\begin{array}{r}30 \\
6 \\
7 \\
13 \\
19 \\
11\end{array}$ & $\begin{array}{r}19 \\
3 \\
6 \\
23 \\
29 \\
6\end{array}$ & $\begin{array}{r}1 \\
1 \\
2 \\
19 \\
17 \\
2\end{array}$ & $\begin{array}{r}1 \\
1 \\
1 \\
28 \\
27 \\
4\end{array}$ & $\begin{array}{l}0 \\
0 \\
0 \\
8 \\
6 \\
3\end{array}$ \\
\hline GRAND TOTALS & 100.0 & 19 & 35 & 12 & 13 & 8 & 11 & 3 \\
\hline
\end{tabular}

Beulah-zap coal

\begin{tabular}{|c|c|c|c|c|c|c|c|c|}
\hline MINERAL SPECIES & WT. 8 & $\begin{array}{l}0.0 \\
2.5\end{array}$ & $\begin{array}{l}2.5 \\
5.0\end{array}$ & $\begin{array}{r}\text { Size } \\
5.0 \\
10.0\end{array}$ & $\begin{array}{r}\text { ges } \\
10 \\
20\end{array}$ & $\begin{array}{l}\text { cons } \\
20 \\
40\end{array}$ & $\begin{array}{l}40 \\
80 \\
\end{array}$ & $\begin{array}{r}80 \\
500 \\
\end{array}$ \\
\hline $\begin{array}{l}\text { MISC SILICATES } \\
\text { PYRITE } \\
\text { MIXED CARBONATE } \\
\text { MISC MIXED } \\
\text { MINOR MINERAIS }\end{array}$ & $\begin{array}{l}11.5 \\
15.9 \\
26.9 \\
26.0 \\
19.7\end{array}$ & $\begin{array}{r}17 \\
0 \\
19 \\
30 \\
5\end{array}$ & $\begin{array}{l}35 \\
69 \\
50 \\
13 \\
24\end{array}$ & $\begin{array}{r}9 \\
12 \\
12 \\
24 \\
14\end{array}$ & $\begin{array}{r}36 \\
4 \\
17 \\
30 \\
31\end{array}$ & $\begin{array}{r}4 \\
10 \\
1 \\
2 \\
11\end{array}$ & $\begin{array}{r}0 \\
3 \\
1 \\
1 \\
13\end{array}$ & $\begin{array}{l}0 \\
2 \\
0 \\
0 \\
1\end{array}$ \\
\hline GRAND TOTALS & 100.0 & 16 & 37 & 15 & 23 & 5 & 3 & 1 \\
\hline
\end{tabular}

Pittaburgh $\# 8$ coal

\begin{tabular}{|c|c|c|c|c|c|c|c|c|}
\hline MINERAI SPECIES & WT. \& & $\begin{array}{l}0.0 \\
2.5\end{array}$ & $\begin{array}{l}2.5 \\
5.0\end{array}$ & $\begin{array}{r}\text { Size } \\
5.0 \\
10.0\end{array}$ & $\begin{array}{l}\text { ges } \\
10 \\
20\end{array}$ & $\begin{array}{l}\text { rons } \\
20 \\
40\end{array}$ & $\begin{array}{l}40 \\
80\end{array}$ & $\begin{array}{r}80 \\
500\end{array}$ \\
\hline $\begin{array}{l}\text { QUARTZ } \\
\text { KAOIINITE } \\
\text { IILITE } \\
\text { MISC SILICATES } \\
\text { PYRITE } \\
\text { MINOR MINERALS }\end{array}$ & $\begin{array}{r}12.4 \\
8.2 \\
20.4 \\
24.6 \\
25.1 \\
9.3\end{array}$ & $\begin{array}{l}8 \\
13 \\
19 \\
31 \\
11 \\
42\end{array}$ & $\begin{array}{r}42 \\
32 \\
42 \\
29 \\
9 \\
21\end{array}$ & $\begin{array}{l}25 \\
28 \\
14 \\
12 \\
15 \\
14\end{array}$ & $\begin{array}{r}18 \\
15 \\
14 \\
8 \\
15 \\
11\end{array}$ & $\begin{array}{r}4 \\
10 \\
7 \\
7 \\
13 \\
8\end{array}$ & $\begin{array}{r}0 \\
2 \\
3 \\
7 \\
27 \\
4\end{array}$ & $\begin{array}{r}3 \\
0 \\
2 \\
5 \\
11 \\
0\end{array}$ \\
\hline GRAND TOTALS & 100.0 & 20 & 28 & 16 & 13 & 8 & 10 & 3 \\
\hline
\end{tabular}


Table 4 (Continued) Size distributions of the major mineral phases in the Argonne coals Size ranges of average particle diameter in microns for more than 7 wt of minerals.

\section{Blind Canyon coal}

\begin{tabular}{|c|c|c|c|c|c|c|c|c|}
\hline MINERAI SPECIES & WT. . & $\begin{array}{l}0.0 \\
2.5\end{array}$ & $\begin{array}{l}2.5 \\
5.0\end{array}$ & $\begin{array}{r}\text { Size } \\
5.0 \\
10.0\end{array}$ & $\begin{array}{l}\text { ges } \\
10 \\
20\end{array}$ & $\begin{array}{l}\text { rons } \\
20 \\
40\end{array}$ & $\begin{array}{l}40 \\
80\end{array}$ & $\begin{array}{r}80 \\
500\end{array}$ \\
\hline $\begin{array}{l}\text { KAOIINITE } \\
\text { ILLITE } \\
\text { MISC SILICATES } \\
\text { MINOR MINERALS }\end{array}$ & $\begin{array}{r}9.2 \\
39.6 \\
37.0 \\
14.2\end{array}$ & $\begin{array}{l}11 \\
25 \\
32 \\
14\end{array}$ & $\begin{array}{l}77 \\
73 \\
65 \\
38\end{array}$ & $\begin{array}{r}7 \\
2 \\
2 \\
13\end{array}$ & $\begin{array}{r}4 \\
0 \\
1 \\
15\end{array}$ & $\begin{array}{l}1 \\
0 \\
0 \\
8\end{array}$ & $\begin{array}{l}0 \\
0 \\
0 \\
9\end{array}$ & $\begin{array}{l}0 \\
0 \\
0 \\
2\end{array}$ \\
\hline GRAND TOTALS & 100.0 & 25 & 65 & 4 & 3 & 1 & 1 & 0 \\
\hline
\end{tabular}

Pocahontas \#3 coal

\begin{tabular}{|c|c|c|c|c|c|c|c|c|}
\hline MINERAL SPECIES & WT. 8 & $\begin{array}{l}0.0 \\
2.5\end{array}$ & $\begin{array}{l}2.5 \\
5.0\end{array}$ & $\begin{array}{r}\text { Size } \\
5.0 \\
10.0\end{array}$ & $\begin{array}{r}\text { Ranges } \\
10 \\
20\end{array}$ & $\begin{array}{l}\text { rons } \\
20 \\
40\end{array}$ & $\begin{array}{l}40 \\
80\end{array}$ & $\begin{array}{r}80 \\
500\end{array}$ \\
\hline $\begin{array}{l}\text { KAOLINITE } \\
\text { ILLITE } \\
\text { CHLORITE } \\
\text { MISC SILICATES } \\
\text { CALCITE } \\
\text { MINOR MINERALS }\end{array}$ & $\begin{array}{r}9.6 \\
8.2 \\
12.5 \\
36.8 \\
7.3 \\
25.6\end{array}$ & $\begin{array}{r}4 \\
24 \\
18 \\
24 \\
3 \\
13\end{array}$ & $\begin{array}{r}36 \\
62 \\
71 \\
48 \\
8 \\
29\end{array}$ & $\begin{array}{r}21 \\
7 \\
8 \\
11 \\
27 \\
13\end{array}$ & $\begin{array}{r}23 \\
2 \\
2 \\
11 \\
31 \\
21\end{array}$ & $\begin{array}{r}8 \\
2 \\
1 \\
2 \\
16 \\
12\end{array}$ & $\begin{array}{r}2 \\
2 \\
0 \\
2 \\
12 \\
8\end{array}$ & $\begin{array}{l}6 \\
0 \\
0 \\
1 \\
3 \\
4\end{array}$ \\
\hline GRAND TOTALS & 100.0 & 17 & 43 & 13 & 15 & 6 & 4 & 2 \\
\hline
\end{tabular}


Table 5. Mössbauer Data for Argonne Premium and Selected Kentucky Coals

\begin{tabular}{|c|c|c|c|c|c|}
\hline \multirow{2}{*}{$\begin{array}{l}\text { Argonne Premium } \\
\text { or } \\
\text { Kentucky coals }\end{array}$} & \multirow{2}{*}{$\begin{array}{l}\text { Wt\% } \\
\text { pyritic } \\
\text { sulfur }\end{array}$} & \multicolumn{4}{|c|}{ \%Fe in } \\
\hline & & Clay & Siderite & Pyrite & Sulfate \\
\hline $\begin{array}{l}\text { Upp. Freeport } \\
\text { Wyodak } \\
\text { Ill inois \#6 } \\
\text { Pittsburgh \#8 } \\
\text { Pocahontas \#3 } \\
\text { Blind Canyon } \\
\text { Lewis-Stockton } \\
\text { Beulah-Zap }\end{array}$ & $\begin{array}{l}1.60 \\
0.13 \\
2.14 \\
1.26 \\
0.11 \\
0.25 \\
0.20 \\
0.22\end{array}$ & $\begin{array}{r}6 \\
3 \\
1 \\
33 \\
32\end{array}$ & $\begin{array}{l}46 \\
28 \\
15\end{array}$ & $\begin{array}{r}94 \\
74 \\
97 \\
99 \\
20 \\
72 \\
53 \\
100\end{array}$ & \\
\hline $\begin{array}{r}91864 \\
6398 \\
2145 \\
71302 \\
3913 \\
2167 \\
5416 \\
71464 \\
71468\end{array}$ & $\begin{array}{r}2.04 \\
0.03 \\
0.15 \\
1.86 \\
0.17 \\
0.93 \\
11.50 \\
1.87 \\
2.14\end{array}$ & $\begin{array}{r}61 \\
57 \\
5 \\
34 \\
9\end{array}$ & 72 & $\begin{array}{l}95 \\
22 \\
43 \\
88 \\
66 \\
19 \\
92 \\
96 \\
95\end{array}$ & $\begin{array}{l}5 \\
7 \\
7 \\
8 \\
4 \\
5\end{array}$ \\
\hline
\end{tabular}

Mössbauer Data for Argonne Coal IOMs

\begin{tabular}{|l|c|c|c|c|}
\hline \multirow{2}{*}{$\begin{array}{c}\text { Argonne } \\
\text { Premium } \\
\text { Coal }\end{array}$} & \multicolumn{4}{|c|}{ \%Fe in } \\
\cline { 2 - 5 } & Clay & Siderite & Pyrite & Pyrrhotite \\
\hline Upper Freeport & & & & 100 \\
Wyodak & & 21 & 42 & 37 \\
IIlionis \#6 & & & 40 & 60 \\
Pittsburgh \#8 & & 50 & 33 & 67 \\
Pocahontas \#3 & 38 & 51 & & 12 \\
Blind Canyon \\
Lewis-Stockton & 63 & 31 & & 69 \\
Beulah-Zap & & & 31 & 69 \\
\hline
\end{tabular}


Table 6. Percentages of the total sulfur contained in different sulfur forms derived from analysis of XANES spectra.

\begin{tabular}{|l|c|c|c|c|c|c|c|}
\hline & \multirow{2}{*}{$\begin{array}{l}\text { Wt\% } \\
\text { Argonne Premium } \\
\text { Coal Sample }\end{array}$} & \multicolumn{6}{|c|}{ Sulfur Forms, \% } \\
\cline { 4 - 9 } & Sulfur & Pyrite & Sulfide & Thioph. & Sulfox. & Sulfone & Sulfate \\
\hline Pocahontas \#3, VA & 0.66 & 24 & 0 & 75 & 0 & 1 & 0 \\
\hline Upp. Freeport, PA & 2.32 & 62 & 6 & 33 & 0 & 1 & 0 \\
\hline Pittsburgh \#8, PA & 2.19 & 52 & 13 & 35 & 0 & 1 & 0 \\
\hline Lewis.-Stock., WV & 0.71 & 26 & 16 & 56 & 1 & 1 & 0 \\
\hline Blind Canyon, UT & 0.62 & 40 & 15 & 46 & 0 & 0 & 0 \\
\hline Illinois \#6, IL & 4.83 & 48 & 19 & 33 & 0 & 0 & 0 \\
\hline Wyodak-Anders., WY & 0.63 & 24 & 29 & 46 & 0 & 0 & 0 \\
\hline Beulah, ND (fresh) & 0.80 & 29 & 28 & 30 & 2 & 0 & 12 \\
\hline Beulah, ND (2nd.) & 0.80 & 37 & 24 & 30 & 2 & 0 & 7 \\
\hline
\end{tabular}




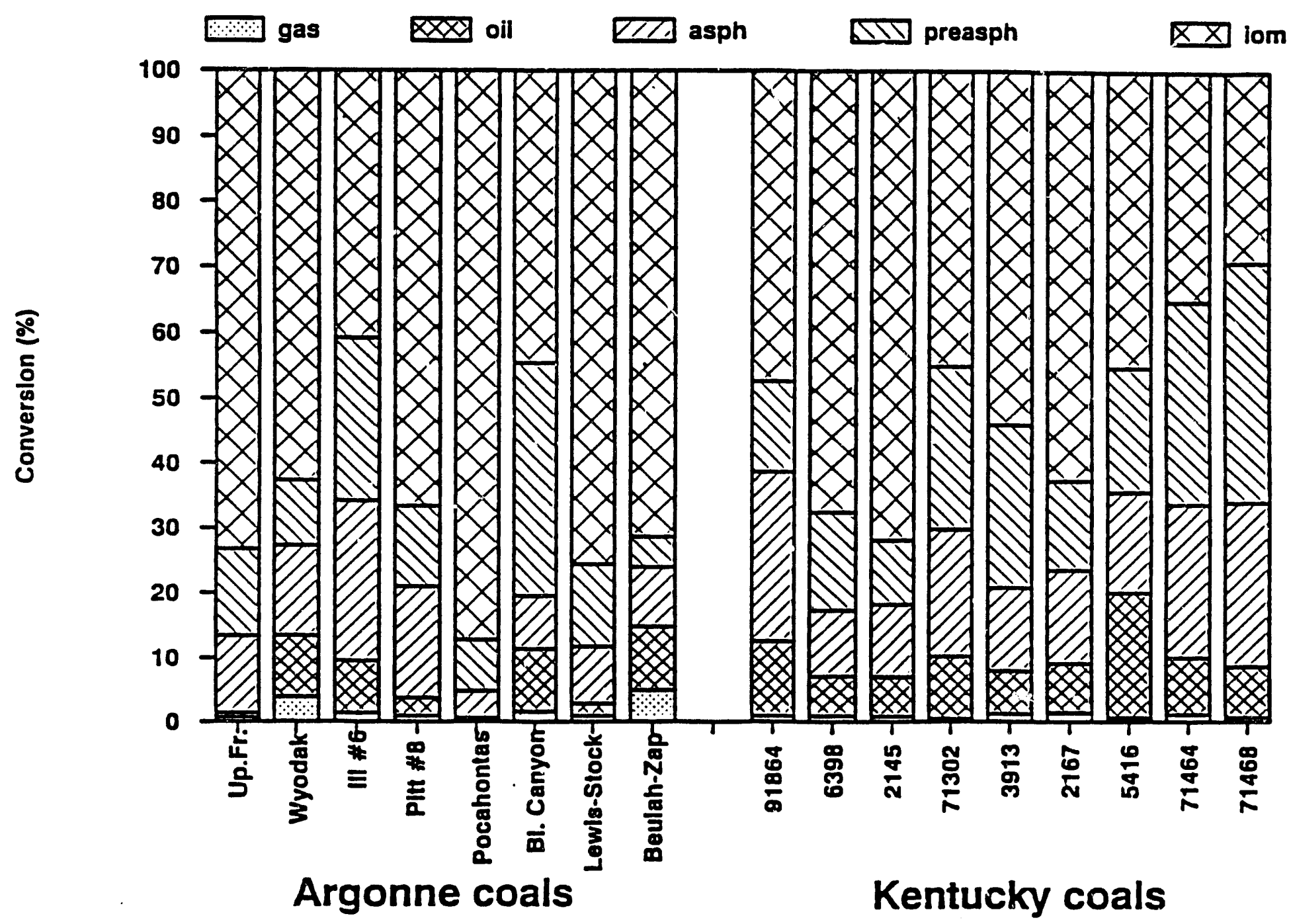

Figure 1 - Liquefaction yields for Argonne Premium coals and a suite of Kentucky coals. 


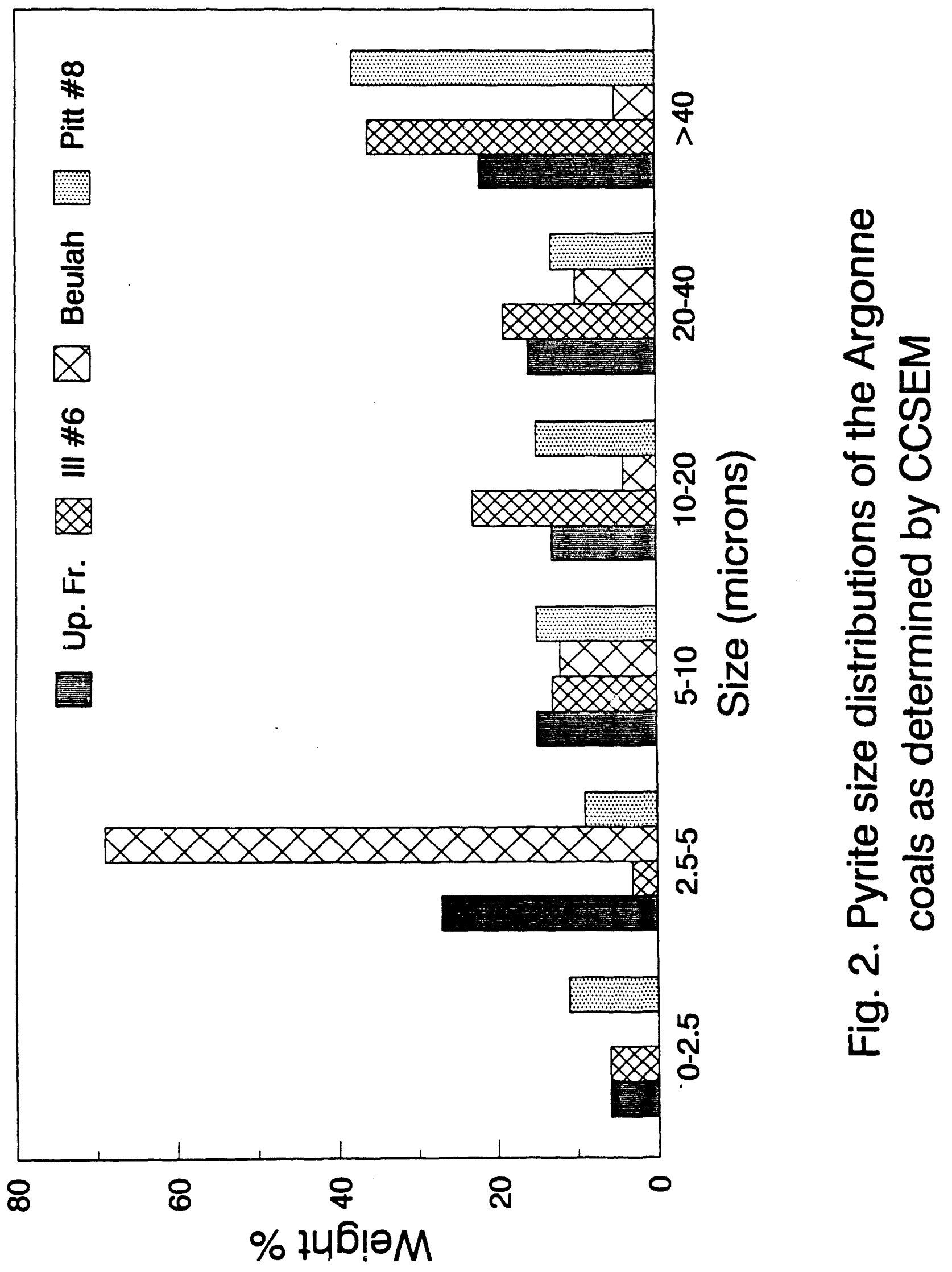




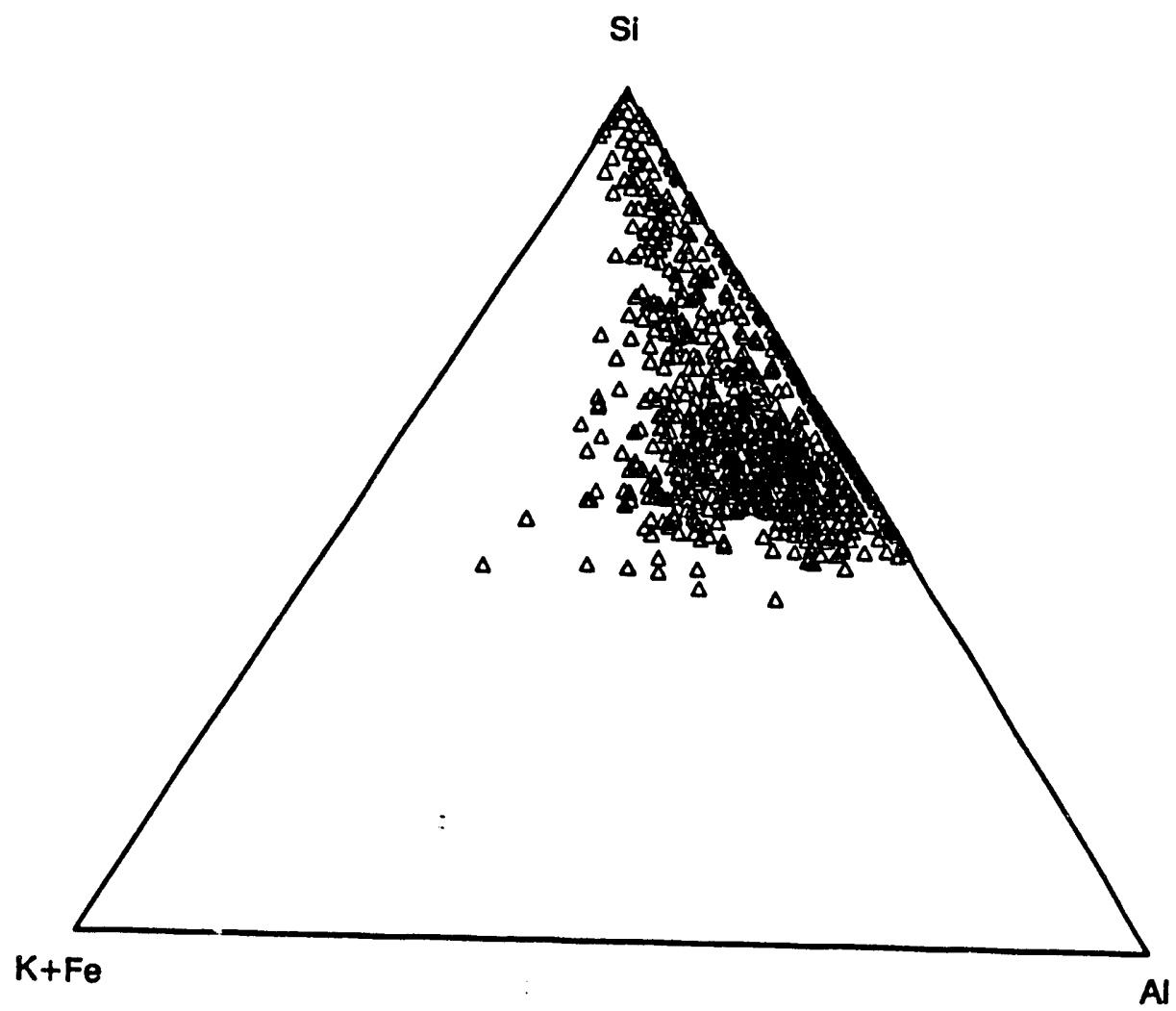
Figure 3a - Ternary representation of CCSEM compositional data for quartz and clay minerals in the Kentucky
Stockton coal, "3913

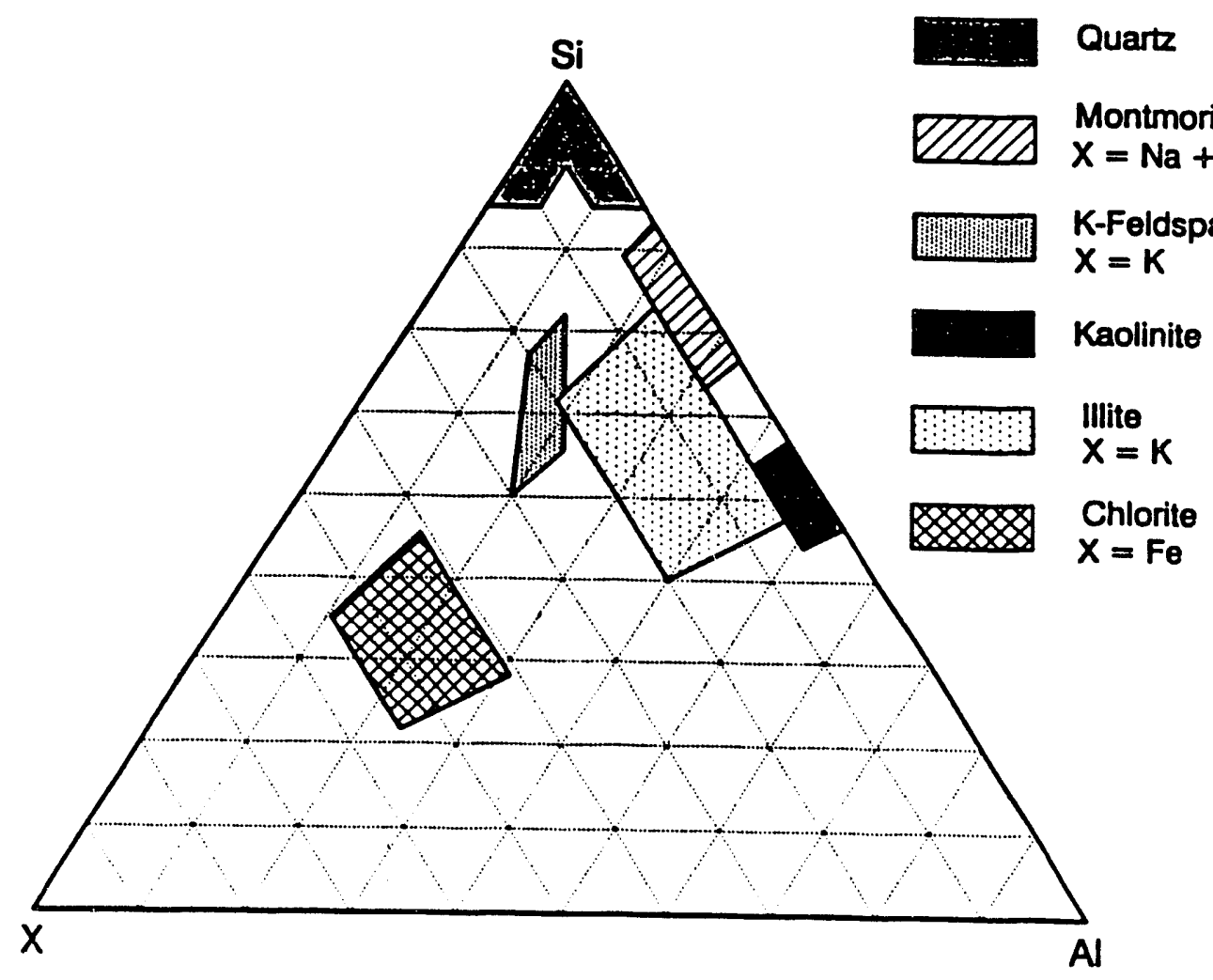

Figure 3b - Compositional ranges used to identify quartz, kaolinite, illite, and other silicate minerals. 

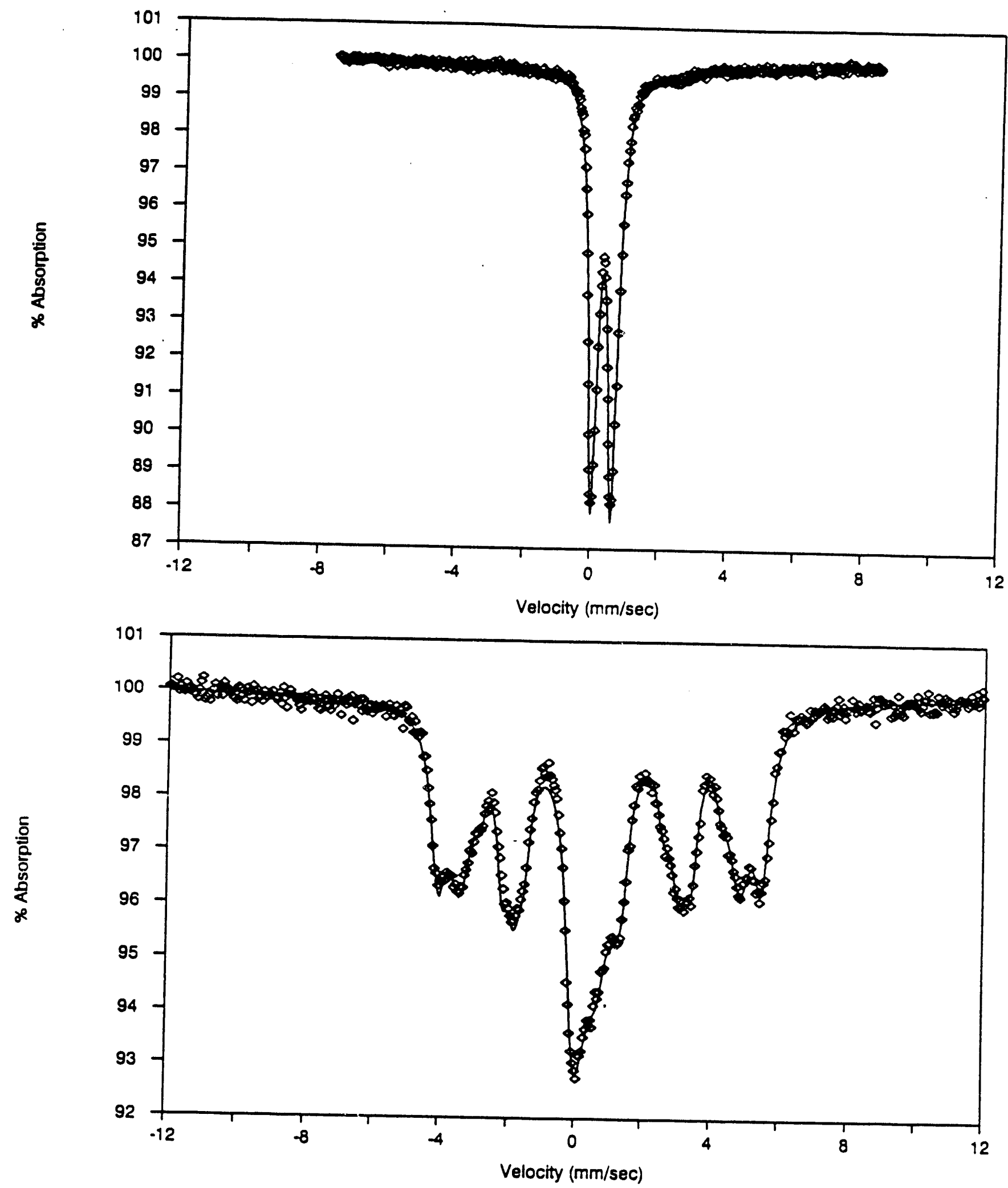

igure 4. Mössbauer spectra of Argonne Illinois \#6 raw coal (top) and IOM (bottom). 


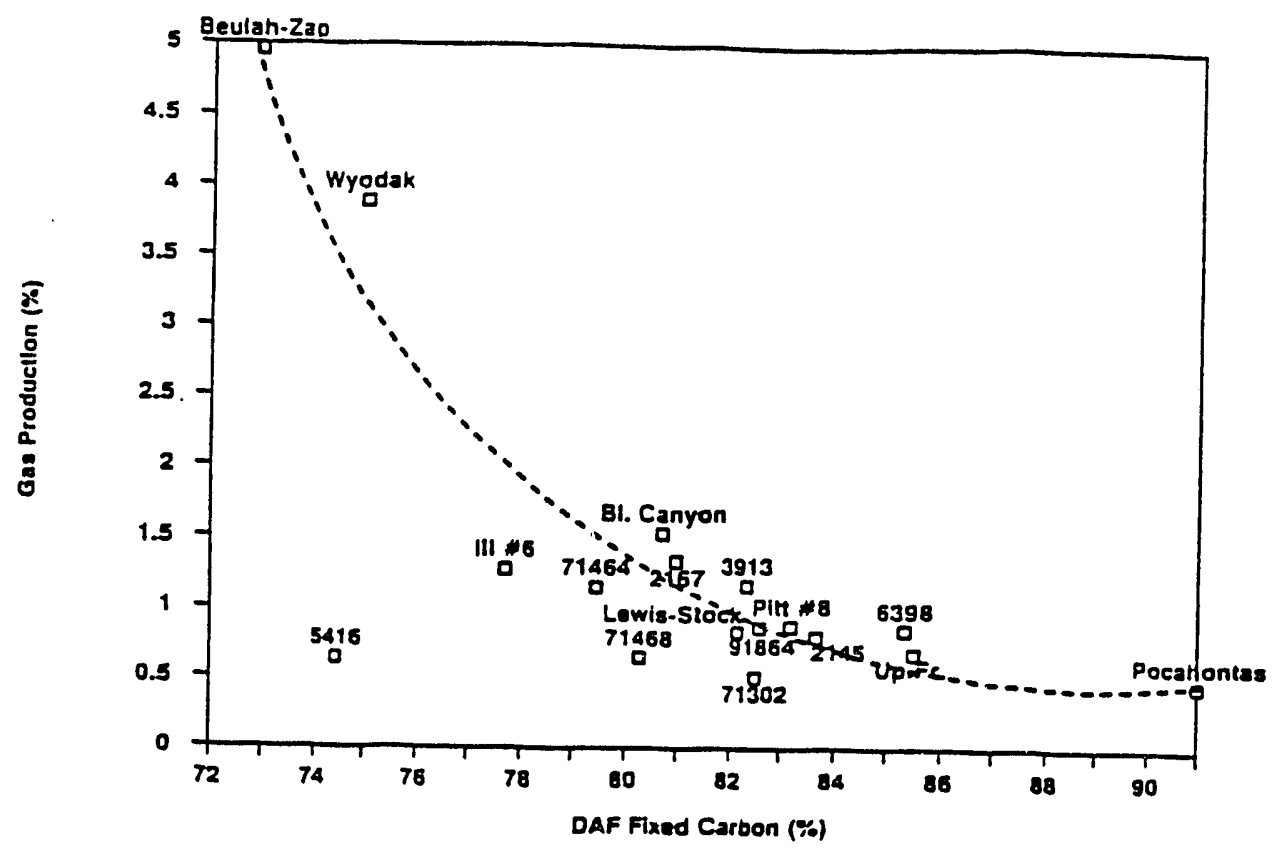

Figure 5. Gas production as a function of carbon content.

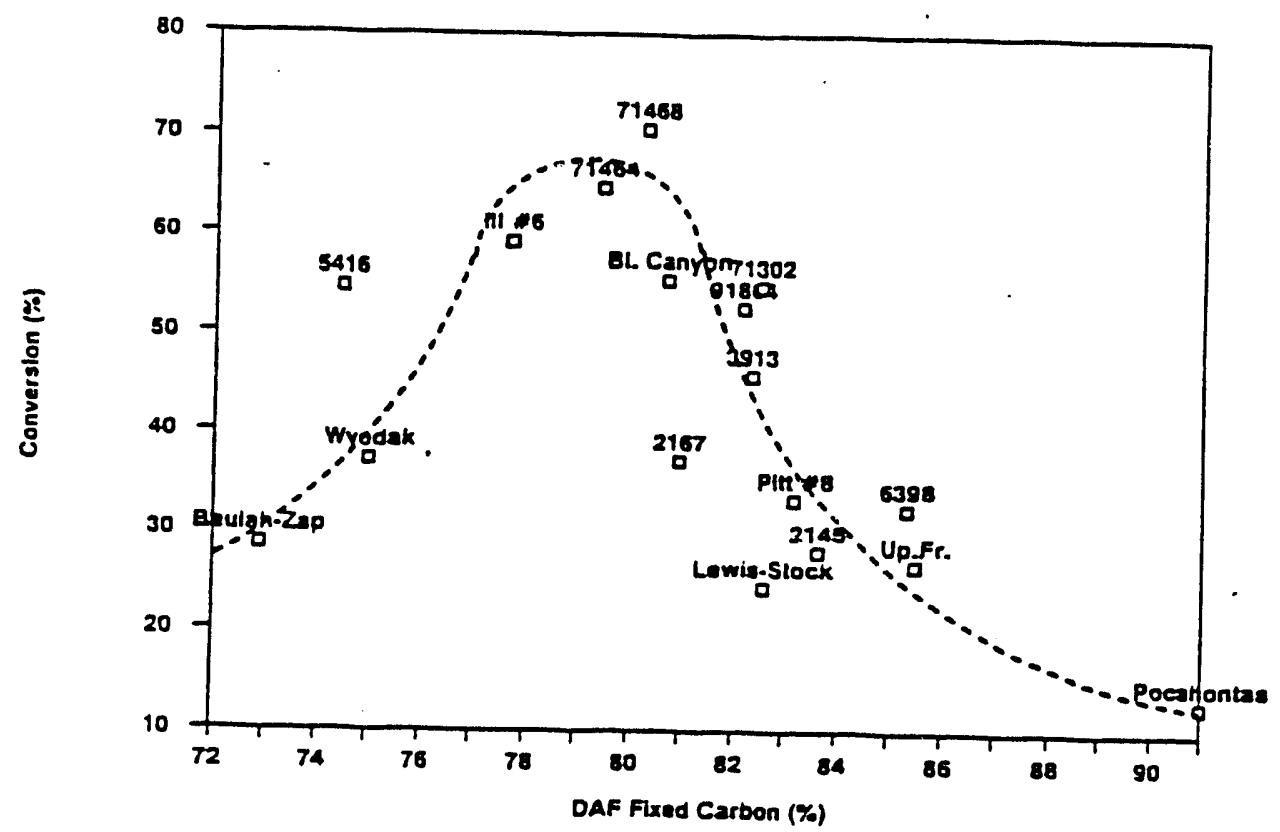

Figure 6. Total conversion percentage as a function of carbon content 


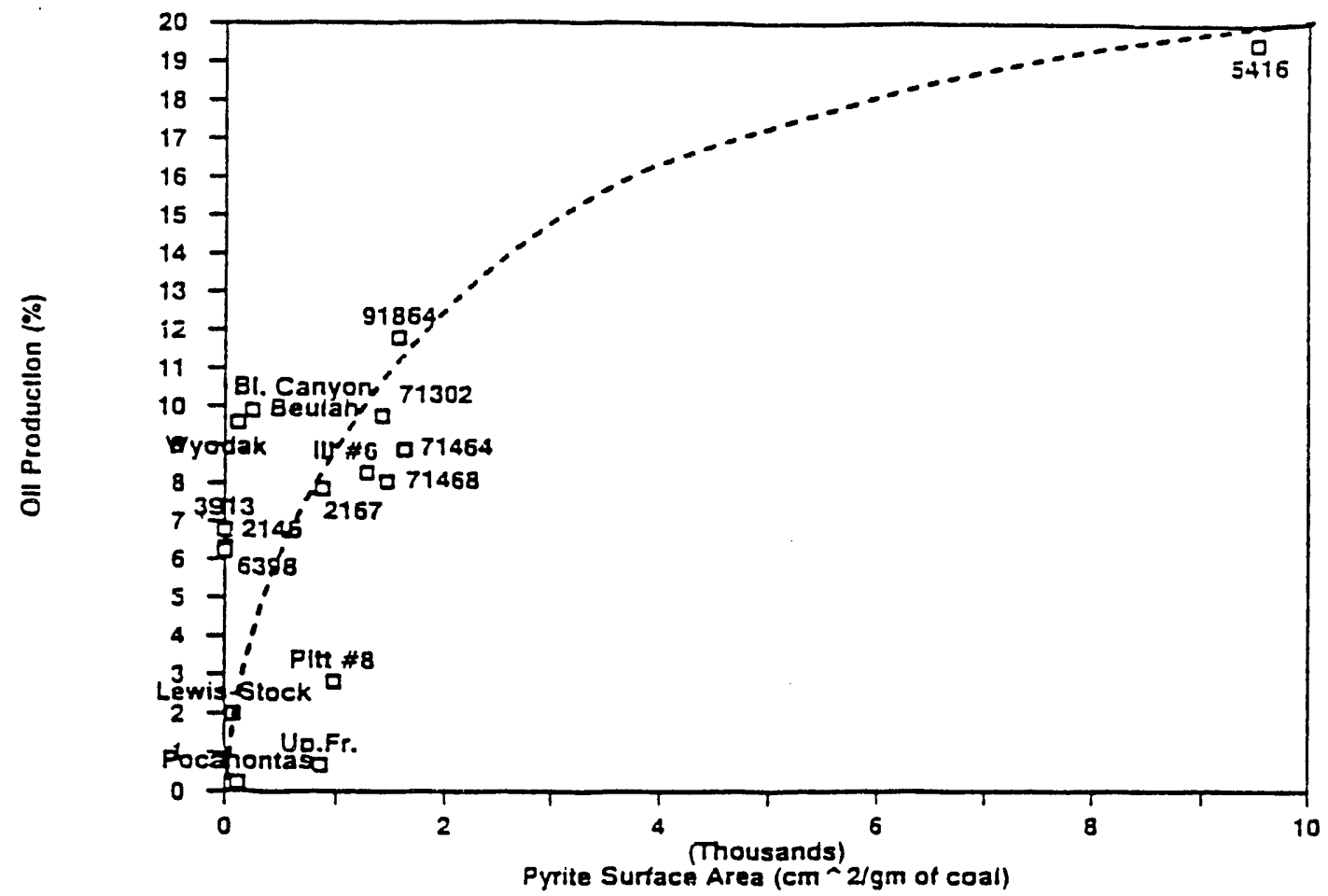

Figure 7. Oil production under mild thermal liquefaction conditions $\left(385^{\circ} \mathrm{C}, 100\right.$ psig $\mathrm{H}_{2} \mathrm{cold}, 15$ min.) versus CCSEM pyrite area per gram of coal.

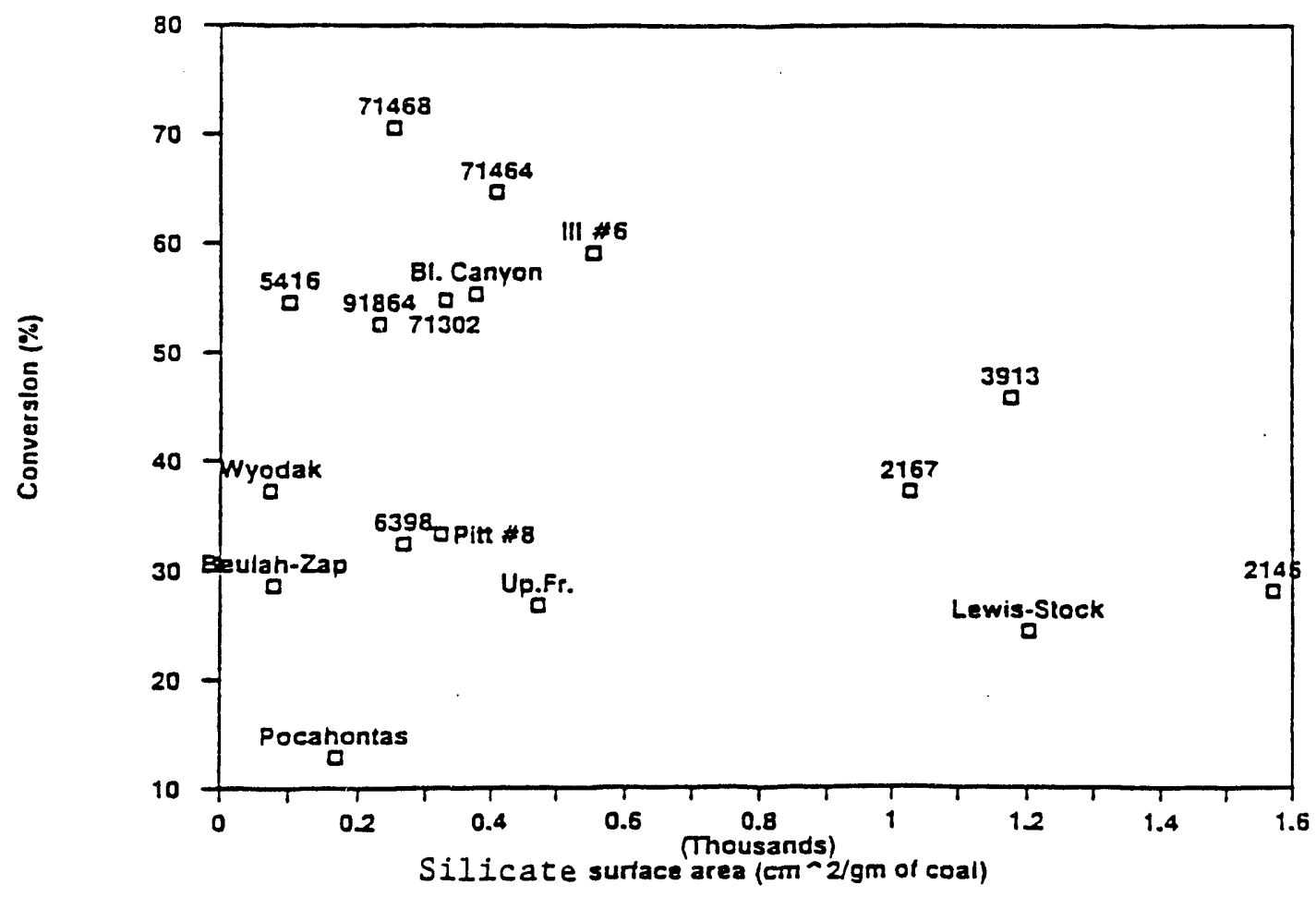

Figure 8. Total conversion under mild conditions versus CCSEM silicate surface area per gram of coal. 


\section{Structure and Size Distributions of Highly Dispersed Iron-Based Catalysts}

(G. P. Huffman, B. Ganguly, M. Taghiei, F. E. Huggins, and N. Shah)

\section{Introduction}

Iron-based catalysts for direct coal liquefaction has been identified as a principal research thrust area for the CFFLS. Much of our research in the past year has been directed at developing new methods for utilizing Mössbauer and X-ray absorption fine structure (XAFS) spectroscopy for determining the size and structure of such catalysts in their initial, as-dispersed state, and at various stages of the liquefaction process. The results are summarized below and in two recent papers. ${ }^{(2,2)}$

\section{Experimental Procedure}

A variety of samples from various CFFLS groups have been investigated. These samples included:

(1) Specimens of both Wyodak and Blind Canyon coal after impregnation with $\mathrm{FeCl}_{3}$ by Shabtai and coworkers. ${ }^{(3-6)}$

(2) Samples from (1) after a mild hydrotreatment $\left(290^{\circ} \mathrm{C}, 1500 \text { psig } \mathrm{H}_{2}, 2 \mathrm{hrs} .\right)^{(3-5)}$

(3) A highly dispersed, sulfated hematite catalyst precursor synthesized by a treatment described elsewhere. ${ }^{\left({ }^{()}\right.}$

(4) Iron added to lignite by cation exchange.

(5) Highly dispersed hematite on carbon black ${ }^{(7)}$

(6) Catalytic iron species derived from $\mathrm{Fe}(\mathrm{CO})_{6}{ }^{(1,8)}$ and from several of the catalyst precursors above under typical liquefaction conditions.

The synthesis and testing of most of these catalyst precursor forms have been described elsewhere. ${ }^{(1-8)}$ No previous description of the cation exchange process to generate a liquefaction catalyst has been given, however. The basic procedure was similar to that used by Walker, Jenkins and coworkers. ${ }^{(2-12)}$ The coal was first floated in a mixture of 1:10 by wt. of coal and carbon tetrachloride $(\rho=1.55)$ in a separating funnel which was shaken to a homogenous slurry then left undisturbed until two distinct layers were formed. The minerals in the sink fraction were drawn off and the process was repeated until no sink fraction formed. The floated coal was then filtered off and treated with 5N HCL. The coal was further washed with distilled water and air dried to constant weight. While this step is 
not essential, it was felt to be desirable in order to remove most of the pyrite from the coal, thereby eliminating its interference from Mössbauer and XAFS spectra.

In order to cation-exchange the iron into the coal, the following procedures were used. The sample of demineralized and air dried coal was mixed with freshly made iron chloride solution with a $2.5 \mathrm{pH}$ value. A mass ratio of 100 part $\mathrm{FeCl}_{2}$ solution to 1 part of dry coal was used. This mixture was stirred at $40^{\circ} \mathrm{C}$ for 24 hours. In a second treatment, an $\mathrm{Fe}$ acetate solution was used. Two samples were prepared, one using an iron acetate solution concentration of $0.026 \mathrm{M}$, the second using a concentration of $0.05 \mathrm{M}$ under a helium atmosphere. In order to remove any adhering salt from impregnated coal, the coal was repeatedly washed with distilled water until the $\mathrm{pH}$ value of filtrate for two consecutive washes was constant. The three samples prepared had total iron concentrations of $1.01 \%$, $8.49 \%$ and $10.4 \%$.

The prinicpal methods used for catalyst characterization have been ${ }^{67} \mathrm{Fe}$ Mössbauer spectroscopy and XAFS spectroscopy. As discussed below, these two techniques provide complementary information on both the structure and size of highly dispersed iron species. A recent paper summarizing the complementary investigation of iron dispersed in Australian brown coal by these techniques has been published by Yamashita et al. ${ }^{(13)}$

A standard constant acceleration Mössbauer spectrometer was used, ${ }^{(13,14)}$ employing either a $50 \mathrm{mCi}^{57} \mathrm{Co}$ in $\mathrm{Rh}$ source or $100 \mathrm{mCi}{ }^{57} \mathrm{Co}$ in $\mathrm{Pd}$ source. Sample cooling was accomplished using a Displex cryogenic system manufactured by Air Products. All isomer shifts are measured with respect to iron metal.

The XAFS experiments were carried out at the National Synchrotron Light Source (NSLS) at Brookhaven National Laboratory and at the Stanford Synchrotron Radiation Laboratory. Si(III) double crystal monochromators were used for all measurements, most of which were carried out in the $x$-ray fluorescent mode using a Stearn-Heald detector described elsewhere. ${ }^{(16)}$ The zero of energy for all spectra is taken as the first inflection point of the spectrum of iron metal.

\section{Results and Discussion}

For all of the as-synthesized samples examined, Mössbauer spectroscopy showed the iron to be in the form of a highly-dispersed, superparamagnetic, ferric oxide. This is illustrated by Figures 1-3. Figure 1 compares the room temperature and $12 \mathrm{~K}$ spectrum of a Wyodak coal impregnated with $\mathrm{Fe}$ from an $\mathrm{FeCl}_{3}$ solution by Shabtai and coworkers. ${ }^{(3-5)}$ In Figure 2, the $12 \mathrm{~K}$ spectrum of a lignite (Hagel seam, PSOC 1482) subjected to a cation exchange process using $\mathrm{FeCl}_{2}$ is compared to that of the same coal subjected to cation 
exchange using an iron acetate solution. Finally, in Figure 3 the room temperature and 12 $\mathrm{K}$ spectra of a sulfated hematite $\left(\mathrm{Fe}_{2} \mathrm{O}_{3} / \mathrm{SO}_{4}^{-2}\right)$ catalyst precursor are compared.

In all cases, (see Table 1) the isomer shifts, quadrupole splittings and magnetic hyperfine fields indicate that the dominant iron species for the initially prepared catalysts is a superparamagnetic ferric oxide. For the ion-exchanged samples and the Shabtai samples, the Mössbauer parameters are similar to those of an oxyhydroxide such as goethite $(\alpha-\mathrm{FeOOH})$. The $\mathrm{Fe}_{2} \mathrm{O}_{3} / \mathrm{SO}_{4}^{-2}$ and $\mathrm{Fe}_{2} \mathrm{O}_{3}$ on carbon black samples have parameters consistent with very fine particle hematite.

All of the spectra exhibit superparamagnetic relaxation effects. This is immediately svident from the fact that both hematite and goethite are antiferromagnetic and would normally exhibit only six-peak magnetic spectra in the bulk state at room temperature. As discussed elsewhere, ${ }^{(17-22)}$ when a magnetically ordered particle is small enough, thermal vibrations may cause the ordered spins of the particle to flip over the magnetic anisotropy energy barrier to a new orientation. To a first approximation, the frequency of spin flipping is given by

$$
f=f_{0} \exp \left(\frac{-K_{a} V}{k T}\right)
$$

where $\mathrm{K}_{2}$ is the magnetic anisotropy constant, $\mathrm{V}$ is the volume of the particle, $k$ is the Boltzman constant and $T$ is the temperature. The frequency factor $f_{0}$ is given by Kundig et al. ${ }^{(17)}$ as

$$
f=K_{\alpha} A / \rho N_{L} h
$$

where $A$ is the molecular weight, $\rho$ is the density, $N_{L}$ is Avogadro's number, and $k$ is the Planck constant. For the magnetic anisotropy constants, we have used the values give by Van der Kraans; ${ }^{(20)} K_{\mathbf{l}}=0.55 \times 10^{5}$ joule $/ \mathrm{m}^{3}$ for hematite, and $K_{\mathrm{a}}=1.67 \times 10^{5}$ joule $/ \mathrm{m}^{3}$ for goethite. Eq. (2) then give $f_{0}=4.2 \times 10^{9} \mathrm{sec}^{-1}$ for hematite and $f_{0}=8.7 \times 10^{9} \mathrm{sec}^{-1}$ for goethite. When $f$ is small compared to the nuclear Larmor precession frequency of the ${ }^{57} \mathrm{Fe}$ nuclear magnetic moment $\left(f_{\mathrm{L}}=5 \times 10^{7} \mathrm{sec}^{-1}\right)$, the particle will exhibit a well-resolved six line magnetic hyperfine spectrum. However, when $f$ becomes comparable to or exceeds $f_{L}$, the magnetic spectrum collapses to a two peak quadrupole doublet. Putting $f$ equal to $f_{\mathrm{L}}$ and rewriting eq. (1), we obtain 


$$
V_{c}=\frac{k T}{K_{a}} \ln \left(\frac{f_{0}}{f_{L}}\right)
$$

Eq. (2) can be viewed as defining a critical volume, $V_{c}$, for temperature $T$. At temperature $T$, to a first approximation, particles of volume $>V_{c}$ will give rise to a six-line magnetic hyperfine spectrum, while particles of volume $<V_{c}$ will exhibit a paramagnetic quadrupole doublet.

The temperature variation of the Mössbauer spectra can be used with Eq. (2) to generate size distributions for the ferric catalyst particles. This is illustrated by Figure 4, which shows the Mössbauer results for a highly dispersed hematite $\left(\mathrm{Fe}_{2} \mathrm{O}_{3}\right)$ on carbon black catalyst. ${ }^{(7)}$ Figure $4 a$ is a plot of the percentage of iron exhibiting a magnetic hyperfine spectrum versus temperature. The spectra are very similar to those shown in Figure 3. Combining Eq. (2) with the magnetic phase percentage temperature dependence, a size distribution histogram can be generated, as shown in Figure 4 b. Here, we have assumed spherical particles in order to derive a mean particle diameter. It is seen that this catalyst has a somewhat bimodal size distribution.

Acquisition of the Mössbauer data required to derive size distributions as detailed as that of Figure $4 \mathrm{~b}$ is quite time consuming, since each spectrum requires $\sim 10-20$ hours of running time. However, less detailed but very informative size histograms can be generated from Mössbauer spectra at only a few temperatures. A number of such histograms are shown in Figure 5 for ferric oxide catalyst precursors synthesized by a variety of methods. For the $\mathrm{Fe}_{2} \mathrm{O}_{3} / \mathrm{SO}_{4}^{-2}$ catalyst we have used the anisotropy and frequency constant of hematite in Eq. 2. All of the other samples exhibited spectra similar to superparamagnetic iron oxyhydroxide, and the anisotropy and frequency constant of goethite, $\alpha-\mathrm{FeOOH}$, seems most appropriate.

It is seen that most of the catalyst particles in all of the catalysts examined are smaller than approximately $65-85 \AA$ in diameter, with from 10 to $70 \%$ of the iron contained in particles less than 20-30 $\AA$ in diameter. Moreover, many of the particles in the smallest size bin may be of molecular dimensions. It is also seen that the samples prepared by the Shabtai $\mathrm{FeCl}_{3}$ impregnation method ${ }^{(3-6)}$ exhibit the smallest size distributions. During the mild hydrotreatment $\left(290^{\circ} \mathrm{C}, 1500 \mathrm{psig} \mathrm{H}_{2}\right.$ hot, 2 hours) used by shabtai and coworkers, ${ }^{(3-6)}$ the iron remains in the form of a ferric oxide or oxyhydroxide and the particle sizes increase somewhat, as seen in Figure 5.

The XAFS data for the as-synthesized catalysts are summarized in Figures 6-9 and Table 2. Figure 6 compares the X-ray absorption near edge structure (XANES) spectra of a 
number of standard compounds to those of several as-dispersed catalysts. It is seen that the XANES spectra of the $\mathrm{FeCl}_{2}$-cation exchanged lignite (PSOC 1482) prepared by our group and the $\mathrm{FeCl}_{3}$-treated Wyodak coal prepared by the Shabtai group are very similar to those of the oxybydraxides, $\alpha-\mathrm{FeOOH}$ and $\gamma-\mathrm{FeOOH}$. Similar XANES were exhibited by all of the Shabtai samples examined, both before and after mild hydrotreatment $\left(290^{\circ} \mathrm{C}, 1500 \mathrm{psig}_{2}\right.$, $2 \mathrm{hrs}$.) and by a lignite cation-exchanged using an iron acetate solution to a level of $8.5 \% \mathrm{Fe}$. A third Fe acetate cation-exchanged lignite reached a level of $10.4 \% \mathrm{Fe}$ and the XANES clearly shows the formation of magnetite. The $\mathrm{Fe}_{2} \mathrm{O}_{3} / \mathrm{SO}_{4}^{-2} \mathrm{XANES}$ is essentially identical to that of $\alpha-\mathrm{Fe}_{2} \mathrm{O}_{3}$.

Fourier transformation of the extended X-ray absorption fine structure (EXAFS) of the XAFS spectrum produces a radial structure function (RSF) that is similar to a radial distribution function. Some typical RSFs from the current samples are shown in Figures 79. As discussed in detail elsewhere, ${ }^{(23)}$ each peak represents a shell of atoms surrounding the central iron atom. The peak positions on the distance axis are shifted slightly downward by about $0.5 \AA$ relative to the true interatomic distances because of phase shifts, and the peak amplitudes are proportional to several factors, one of which is the shell coordination number.

The radial structure functions (Figures 7-9 and Table 2) are more informative than the XANES. In Figure 7, the radial structure function (RSF) of $\alpha-\mathrm{Fe}_{2} \mathrm{O}_{3}$ is compared to that of the $\mathrm{Fe}_{2} \mathrm{O}_{3} / \mathrm{SO}_{4}^{-2}$ catalyst. It is seen that the amplitudes of the peaks arising from the third and fourth nearest neighbor $(\mathrm{nn})$ iron shells in the catalyst are significantly reduced relative to those of the same shells in bulk $\alpha-\mathrm{Fe}_{2} \mathrm{O}_{3}$. A similar effect is seen for the cation-exchanged lignite containing magnetite (Figures 8 ). For the less concentrated cation-exchanged samples and for all of the Shabtai samples, before and after hydrotreatment, the RSFs are similar to that of goethite, but in most cases the third and fourth nn Fe shells are nearly undetectable. This is illustrated by Figure 9.

One explanation of the decreased amplitudes of the third and fourth nn iron shell peaks is that the iron atoms at or near the surfaces of these very small catalyst particles do not have their full complement of third and fourth iron $\mathrm{nn}$. Since it may reasonably be expected that the first nn oxygen coordination of these iron atoms is unchanged, a convenient measure of the decrease in the average number of iron atoms in the third and fourth nn shells is the ratio of those peak heights in the RSF to that of the oxygen first nn shell. These peak height ratios are summarized for all catalyst samples and for the appropriate standard compounds in Table 2. Assuming that these ratios are proportional to the ratios of the coordination numbers, we can deduce the average iron coordination number for the catalysts by comparing their peak height ratios to those of the standard compounds 
for which the coordination numbers are known. These results are also indicated in Table 2. It is of interest to note that the third and fourth nn shell peaks are essentially absent from the RSFs of several of the Shabtai and cation-exchanged samples. However, because of the noise level of the RSFs, the lowest peak height ratio that can be reliably determined from the current data is approximately 0.2 . Therefore, we can only put a lower limit on coordination numbers for these samples.

While this approach is useful for comparing different catalysts on the basis of the RSFs derived from EXAFS, it is clearly a rough approximation. One major source of error is in ignoring the effect of thermal vibration on the RSF peak amplitudes. It is likely that atoms at or near the surfaces of the small catalyst particles will exhibit larger thermal vibrations than atoms in the interior of the particles. Moreover, it is likely that all of the atoms in such small particles will experience larger thermal vibrations than those in the corresponding bulk phases. As discussed elsewhere, ${ }^{(23)}$ the EXAFS signal is proportional both to coordination number and to a Debye-Waller factor that accounts for such thermal vibration. Lytle et al. ${ }^{(24)}$ have discussed the effects of such vibrational factors on the EXAFS of small catalyst particles. Low temperature measurements may determine how important thermal vibration is, since the peak height ratios of the catalysts should change more dramatically than those of the bulk phases as temperature is lowered.

Clearly, it is of interest to determine mean particle diameters from the XAFS data if possible. If we assume that all atoms of the particles have the same thermal vibrational factor and that the ratio of that factor to the bulk vibrational factor at room temperature is $r$, we can write:

$$
\begin{aligned}
& P=H_{3}(F e) / H_{1}(0) \\
& \frac{P_{\text {catalyst }}}{P_{\text {bul }}}=\left\{\left(\frac{R-R_{3}}{R}\right)^{3}+\left[1-\left(\frac{R-R_{3}}{R}\right)^{3}\right] q\right\} r
\end{aligned}
$$

where $q$ is the ratio of the average number of third $n n$ for iron atoms within a distance $R_{3}$ of the surface to that of the bulk phase, $R_{3}$ is the distance from an iron atom to its third $n$ shell, and $R$ is the average radius of the catalyst particles. If we assume reasonable values for $q$ and $r$, particle diameters of 10 to $50 \AA$ are calculated from the peak height ratios in Table 2. Both $q$ and $r$ could be evaluated empirically by XAFS measurements on particles of known size and Eq. 3 could then used to determine the average catalyst particle radii. This will be addressed in future work. 
During liquefaction, the iron oxide particles are partially or completely transformed to pyrrhotite. This has been discussed previously ${ }^{(1)}$ in connection with catalytic species derived from iron pentacarbonyl, which first transforms to a mixture of superparamagnetic ferric oxide and iron carbide, and subsequentiy to pyrrhotite-oxide mixtures. Typical Mossbauer spectra are shown in Figures 10. The top spectrum was obtained from the IOM taken from a coprocessing liquefaction run using Illinois No. 6 coal and Maya ATB with an $\mathrm{Fe}_{2} \mathrm{O}_{3} / \mathrm{SO}_{4}^{-2}$ catalyst. The bottom spectrum was derived from the IOM of a run using Wyodak coal and Maya ATB with an $\mathrm{Fe}(\mathrm{CO})_{6}$ catalyst precursor. All of the peaks in the top spectrum may be assigned to $\mathrm{Fe}_{1 . x} \mathrm{~S}$, while the bottom spectrum shows that part of the iron is contained in $\mathrm{Fe}_{1-\mathrm{x}} \mathrm{S}$ (40\%) and part in a superparamagnetic ferric oxide (60\%). While a systematic study remains to be done, examination of a number of such samples indicates that the highly dispersed ferric oxide readily transforms to pyrrhotite if sufficient sulfur is present in the system.

The size and structure of the pyrrhotite particles are of great interest, since the presence of the pyrrhotite phase is associated with hydrogenation. Figure 11 shows the RSF's of pyrrhotite derived from cation-exchanged iron in lignite during liquefaction in a microautoclave at 385 and $427^{\circ} \mathrm{C}\left(800 \mathrm{psig} \mathrm{H}_{2}\right.$ cold, $\left.1 \mathrm{hr}\right)$. Sufficient dimethyl desulfide was added to ensure that all of the ferric oxide species produced by ion-exchange would be transformed. It is seen that the RSFs of the catalysts are very similar to that of bulk pyrrhotite, although the height of the iron shell peak relative to the sulfur peaks is somewhat reduced in the catalyst RSFs. This reduction in outer shell to inner shell peak height ratio is, however, much less than exhibited by the ferric oxide precursors (Figures 7-9 and Table 2). Similarly, the Mossbauer spectra do not indicate large changes in the pyrrhotite spectra during cooling due to superparamagnetism. These results suggest that significunt pyrrhotite particle growth has occurred during liquefaction. A more thorough study of the extent and kinetics of pyrrhotite particle growth is planned.

\section{Summary and Conclusions}

A variety of iron-based liquefaction catalysts have been investigated by Mössbauer and XAFS spectroscopy. Samples investigated included coals subjected to an $\mathrm{FeCl}_{3}$ impregnation treatment described by Shabtai and coworkers, ${ }^{(3-6)}$ a highly dispersed $\mathrm{Fe}_{2} \mathrm{O}_{3}$ on carbon black catalyst, ${ }^{(7)}$ iron dispersed in a lignite by cation exchange, a sulfated hematite catalyst $\left(\mathrm{Fe}_{2} \mathrm{O}_{3} / \mathrm{SO}_{4}{ }^{-2}\right)$ prepared by Wender and coworkers, ${ }^{(6)}$ and iron catalytic species derived from these forms and from $\mathrm{Fe}(\mathrm{CO})_{6}$ during liquefaction. ${ }^{(1,8)}$ The results may be summarized briefly as follows: 
1. Both Mössbauer and XAFS spectroscopies can determine the structure of the catalysts and provide information on their size. The Mossbauer technique is more accurate but is also a much slower measurement, typically requiring 10 20 hours, while the XAFS measurement normally requires about 30-60 minutes.

2. In all cases, the initial as-dispersed or as-prepared catalyst is in the form of a highly dispersed ferric oxide or oxyhydroxide.

3. Because of superparamagnetic relaxation effects, the Mossbauer spectra exhibit a significant increase in the percentage of iron contributing to magnetic hyperfine patterns as the sample temperature is lowered. These magnetic hyperfine percentages may be converted into particle size distributions which indicate that the size of the as-dispersed ferric oxyhydroxides and oxides range from molecular to particles $\sim 20 \cdot 100 \AA$ in diameter.

4. During a mild hydrotreatment, ${ }^{(3-5)}$ the ferric particles produced in the Shabtai treatment exhibit some growth but no change in atomic structure. During liquefaction, a part or all of the ferric oxyhydroxide/oxide is converted to pyrrhotite $\left(\mathrm{Fe}_{1-2} \mathrm{~S}\right)$, depending on the amount of sulfur available.

Future characterization work on these similar specimens will include scanning transmission electron microscopy (STEM) and magnetization studies using a SQID magnetometer. A detailed study of the kinetics of the transformation and growth of the initial ferric phase to pyrrhotite is also planned, using an in situ sampling probe in conjunction with a one liter stirred autoclave reactor.

\section{References}

1. D. E. Herrick, J. W. Tierney, I. Wender, G. P. Huffman, and F. E. Huggins, Energy and Fuel 4, 231 (1990).

2. G. P. Huffman, B. Ganguly, M. Taghiei, N. Shah, and F. E. Huggins, "Structure and Size Distributions of Highly Dispersed Iron-Based Direct Coal Liquefaction Catalysts," submitted to Energy and Fuel.

3. Shabtai, J. S., Saito, I. U.S. Patent, 4,728,418 (1988).

4. Shabtai, J. S., Zhang, Y. Proc. 1989 Internat. Confer. Coal Science, Tokyo 1989, Vol. II, 807-810.

5. Shabtai, J. S., Skulthai, T. Proc. 1987 Internat. Conference Coal Science, Maastricht, The Netherlands, Elsevier, 761-764.

6. V. R. Pradhan, J. W. Tierney, and I. Wender, American Chemical Society, Division of 
Fuel Chemistry Preprints, Vol 35, No. 3, p. 793, August, 1990.

7. Sample provided by Malvina Farcasiu, Pittsburgh Energy Technology Center

8. D. E. Herrick, I. Wender, and J. W. Tierney,

9. P. S. Walker, Jr., S. Matsumoto, T. Hanzawa, T. Muira, and I. M. K. Ismail, Fuel, 62, 140-149 (1983).

10. R. G. Jenkins, S. P. Nandi and P. L. Walker, Fuel $\underline{52} 288$ (1973).

11. O. P. Mahajan, R. Yarzab, and P. L. Walker, Fuel 57643 (1978).

12. L. R. Radovic, P. L. Walker, and R. G. Jenkins, Fuel $\underline{62} 290$ (9283) ; Fuel $\underline{62} 849$ (1983).

13. H. Yamashita, Y. Oktsuka, S. Yoshida, and A. Tomita, Energy \& Fuels $\underline{3} 686$ (1989).

14. G. P. Huffman and F. E. Huggins Fuel, 592 (1978).

15. G. P. Huffman, M.C. Lin, F. E. Huggins, G. R. Dynmyre, and A. J. Pignocco Fuel $\underline{64}$ 849-86 (1985).

16. F. W. Lytle, R. B. Greegor, D. R. Sandstrom, E. C. Marques, Joe Wong, C. L. Spiro. G. P. Huffman, and F. E. Huggins Nucl. Instrum. \& Methods 226, 542 (1984).

17. W. Kundig, H. Brmmel, G. Constabaris, and R. H. Lindquist, Phys. Rev. 142,327 (1966).

18. H. H. Wickman, "Mössbauer Paramagnetic Hyperfine Structure," pp. 39-66, Mössbauer Effect Methodology, Ed. I. J. Gruverman, Plenum Press, NY, 1966.

19. T. Shinjo, J. Phys. Soc. Japan 21, 917 (1966).

20. A. M. van der Kraan, Phys. Stat. Sol. (a) 18, 215 (1973).

21. A. Vertes, T. C. Huang, J. W. Vanderhoff, K. Lazar, M. Bennetch and W. Reiff, Radiochem. Radioanal. Letters $\underline{59}$ (5-6), 307 (1983).

22. B. Rodmacq, J. Phys. Chem Solds 45,1119 (1984).

23. P. A. Lee, P. H. Citrin, P. Eisenberger, and b. M. Kincaid Rev. Mod. Phys., 1981, 53 769-806.

24. F. W. Lytle, G. H. Via, and J. H. Sinfelt, in H. Winich and S. Doniach (Eds.), Synchrotron Radiation Research, Plenum Press, New York, (1980), pp. 401-424. 


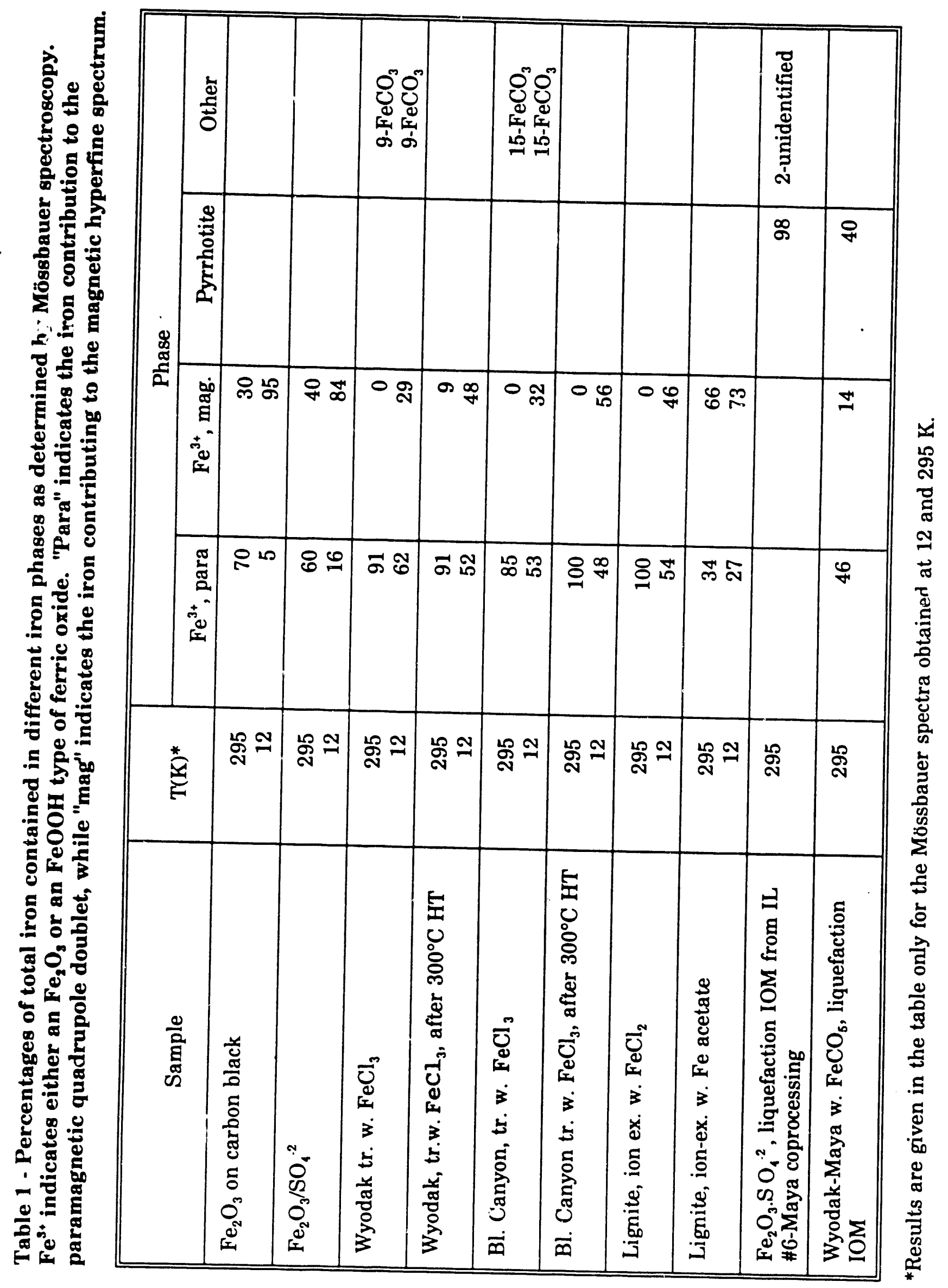


Table 2. Summary of peak height information from RSFs. $H / H_{0 x}$ is the ration of the height of the peak from the iron shell to that of the oxygen nn shell.

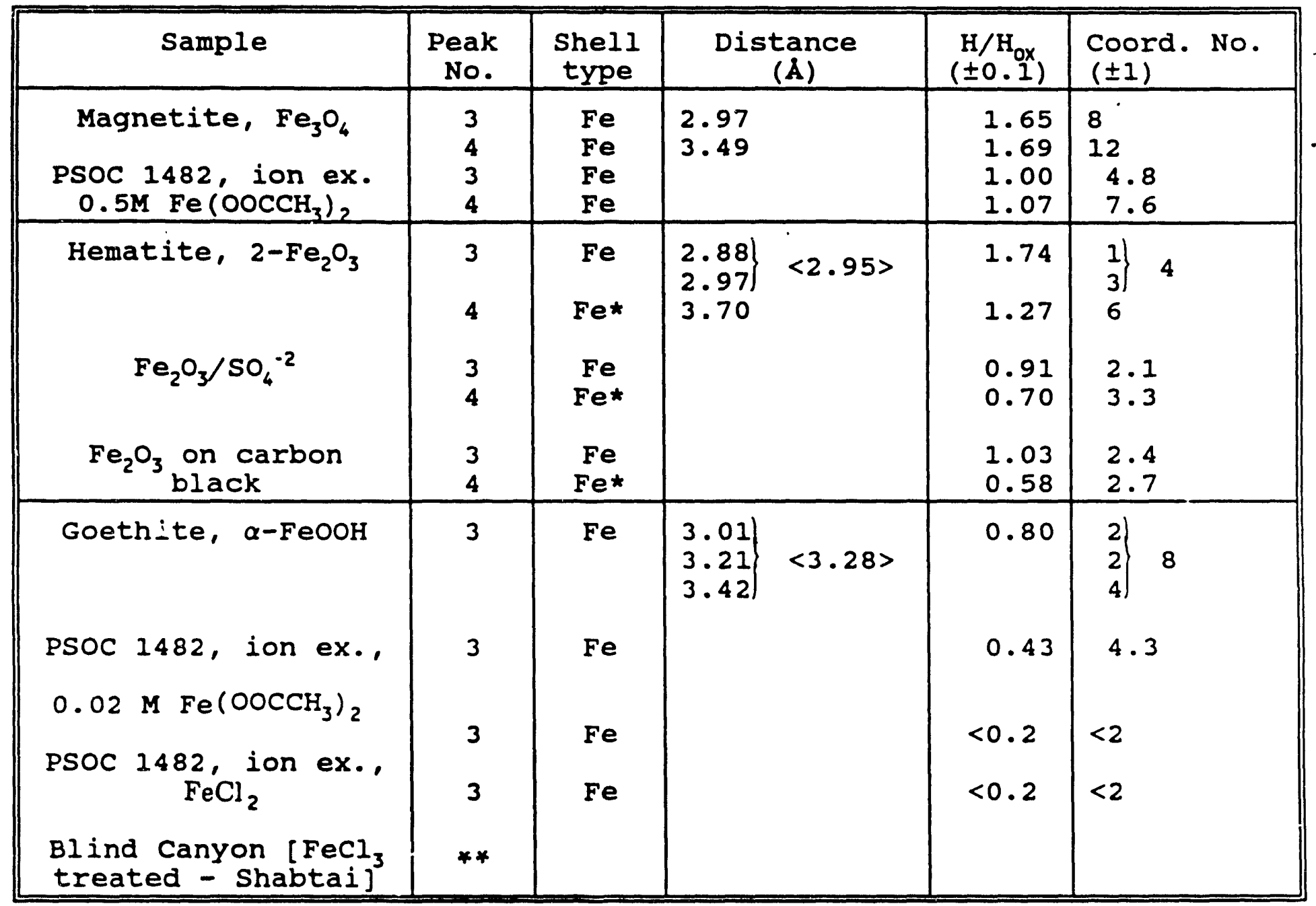

*oxygen neighbor shells also contribute to this peak.

* Peak nos. $3 \& 4$ could not be detected. 

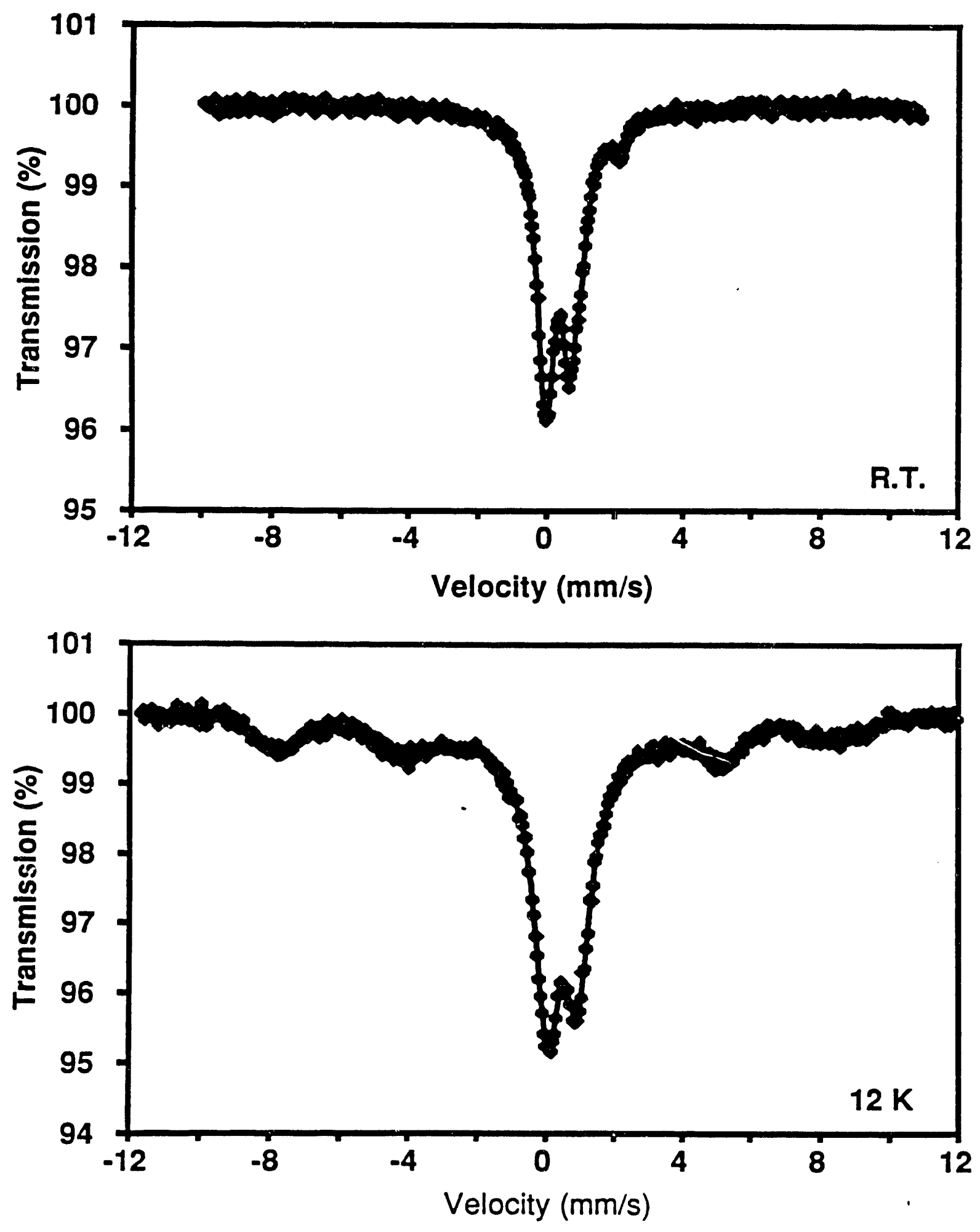

Figure 1 - Room temperature (top) and $12 \mathrm{~K}$ Mössbauer (bottom) spectrum of a Wyodak coal impregnated with iron using the Shabtai treatment. ${ }^{(3-5)}$ 


\section{PSOC 1482 treated with $\mathrm{FeCl} 2$}

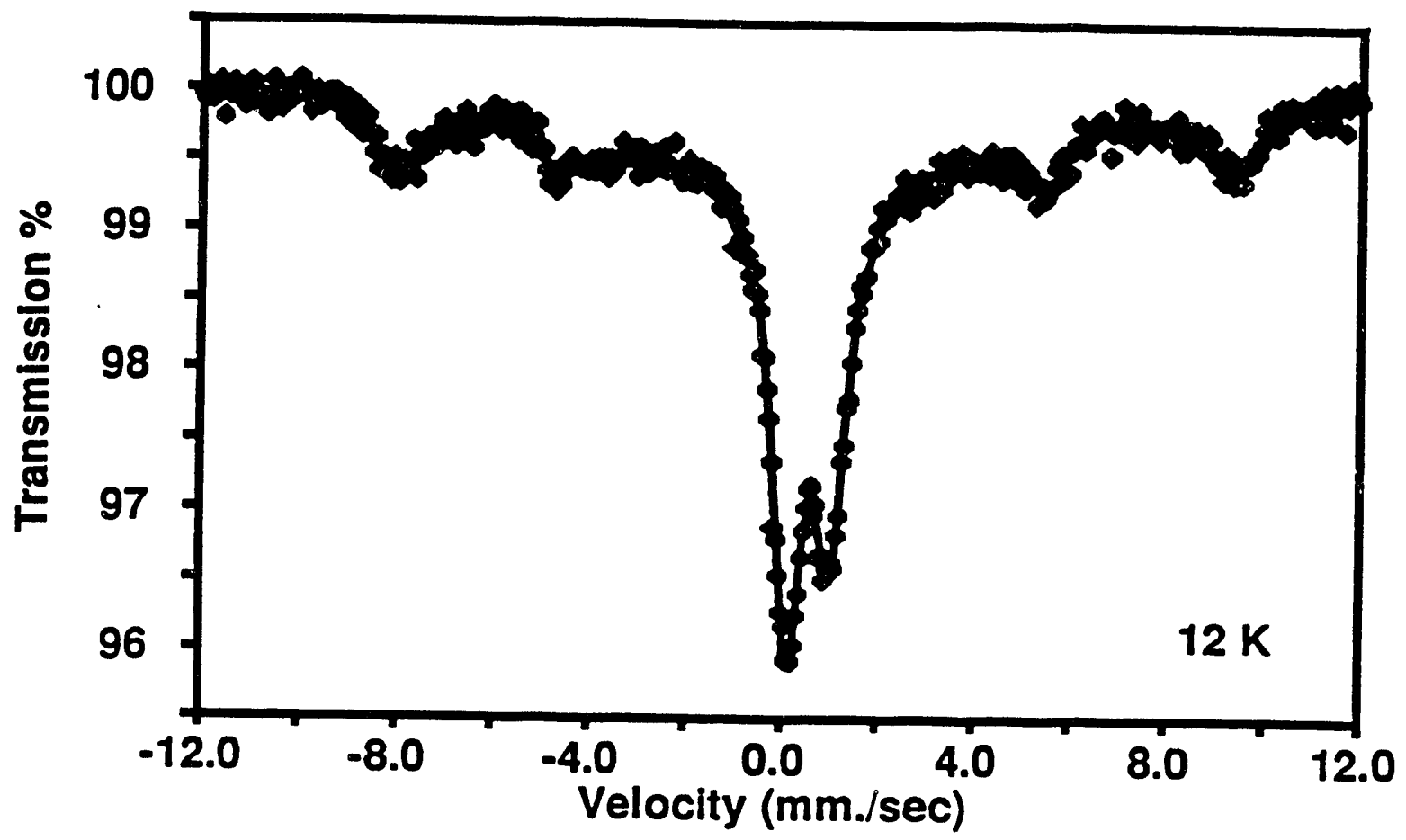

PSOC 1482 treated with $\mathrm{Fe}(\mathrm{OOCCH} 3) 2$

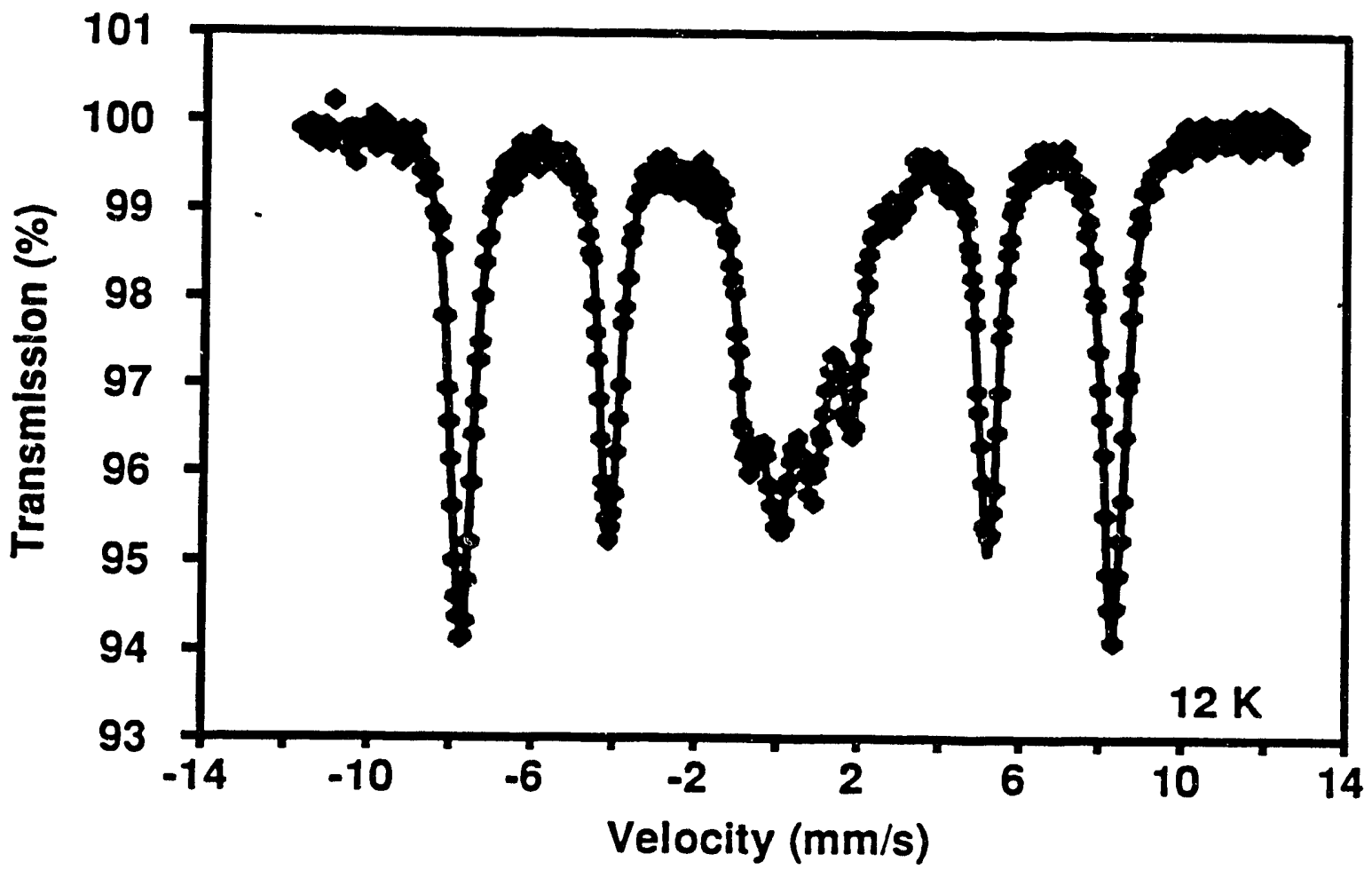

Figure 2 - 12K spectra of lignite after cation exchange in an $\mathrm{FeCl}_{2}$ solutition (îp) and an $\mathrm{Fe}\left(\mathrm{OOCCH}_{3}\right)_{2}$ solution (bottom). 

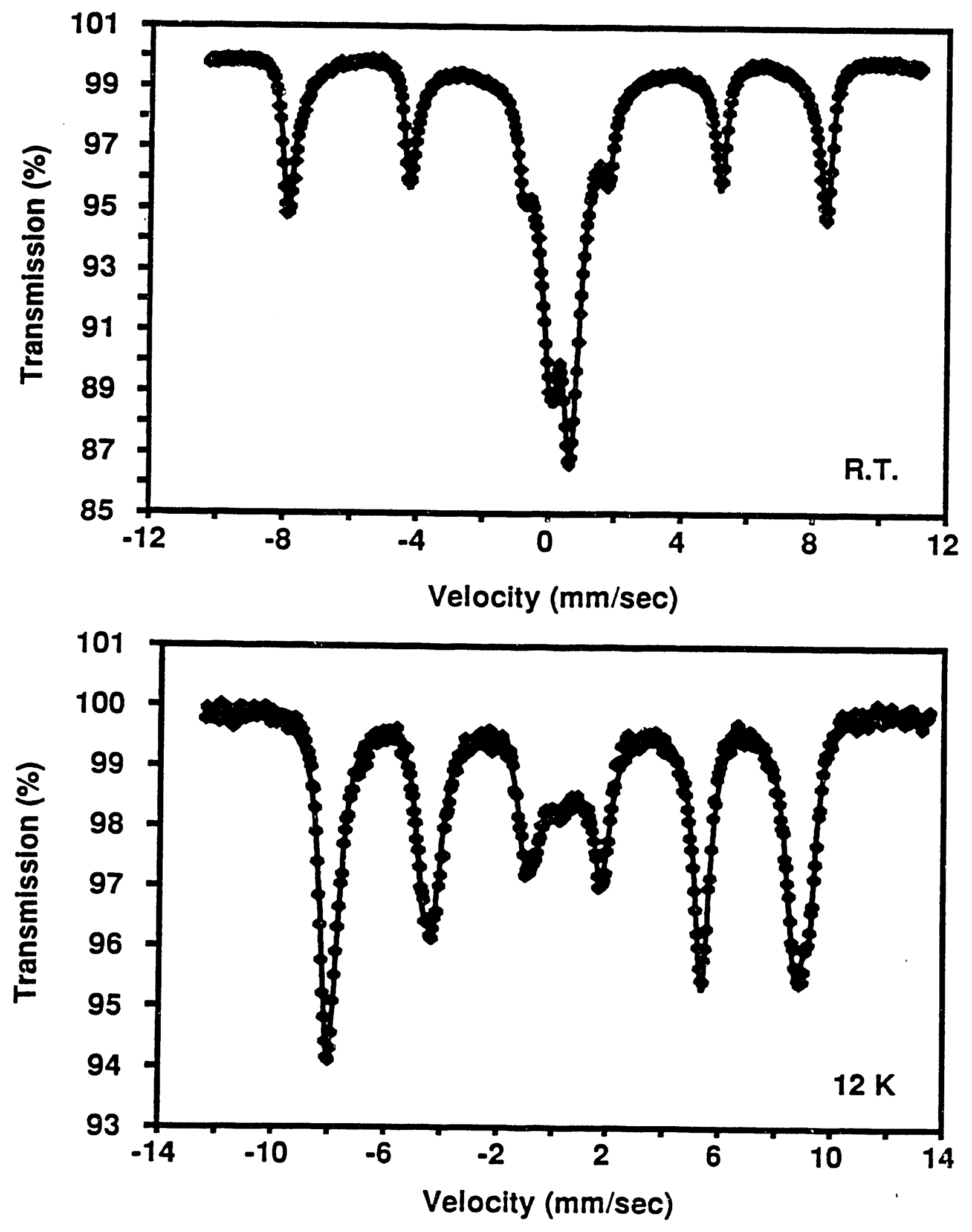

Figure 3 - Room temperature and $12 \mathrm{~K}$ Mössbauer spectra of a sulfated hematite $\left(\mathrm{Fe}_{2} \mathrm{O}_{3} / \mathrm{SO}_{4}{ }^{2}\right)$ catalyst. 


\section{Fe2O3 on carbon black}

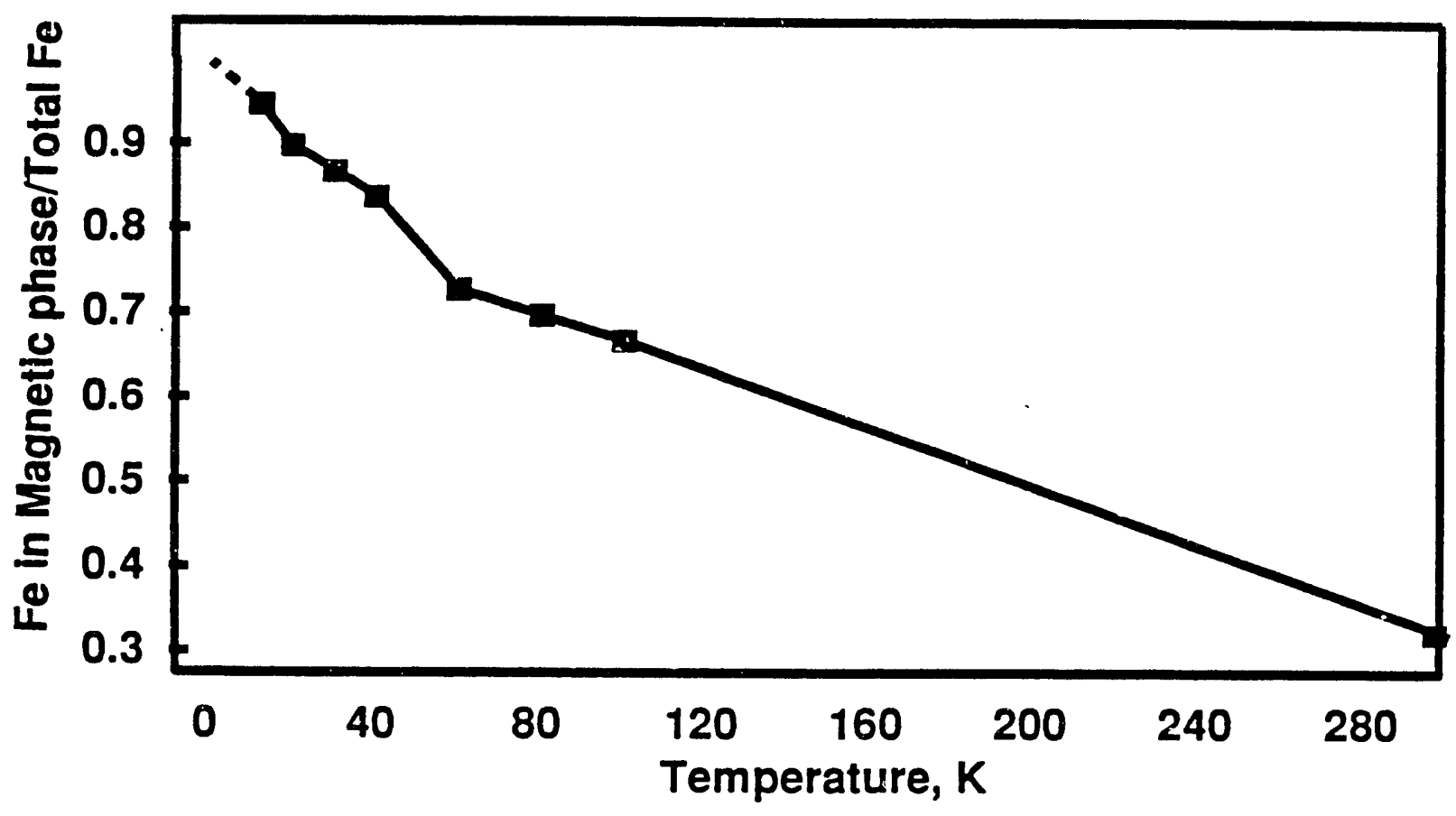

Fe2O3/Carbon Black

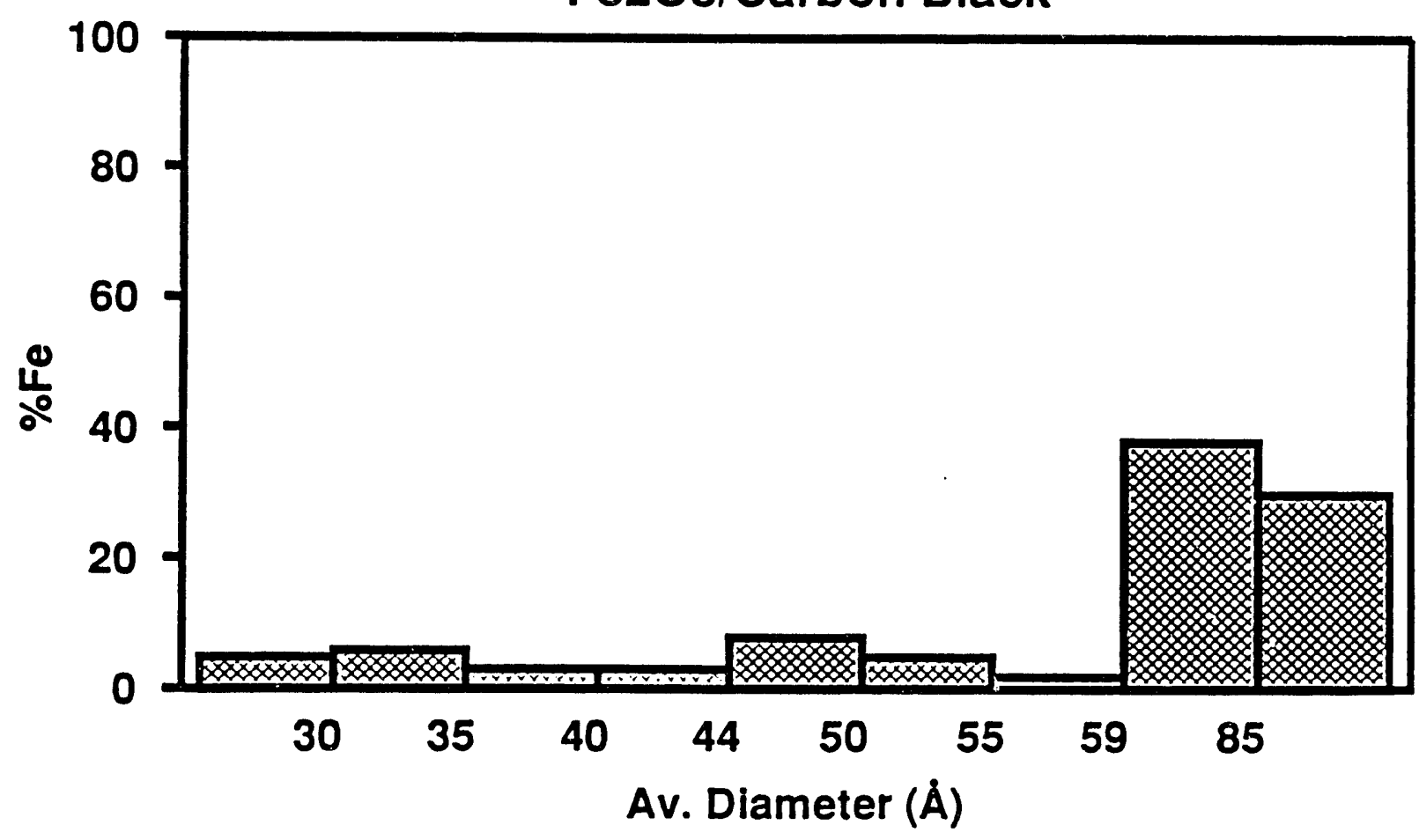

Figure 4 - (4a) Percentage of the iron in an $\mathrm{Fe}_{2} \mathrm{O}_{3}$ /carbon black sample contributing to the magnetic hyperfine pattern as a function of temperature. (14b) Size distribution derived from the data of (4a) ising Eq. 2. 

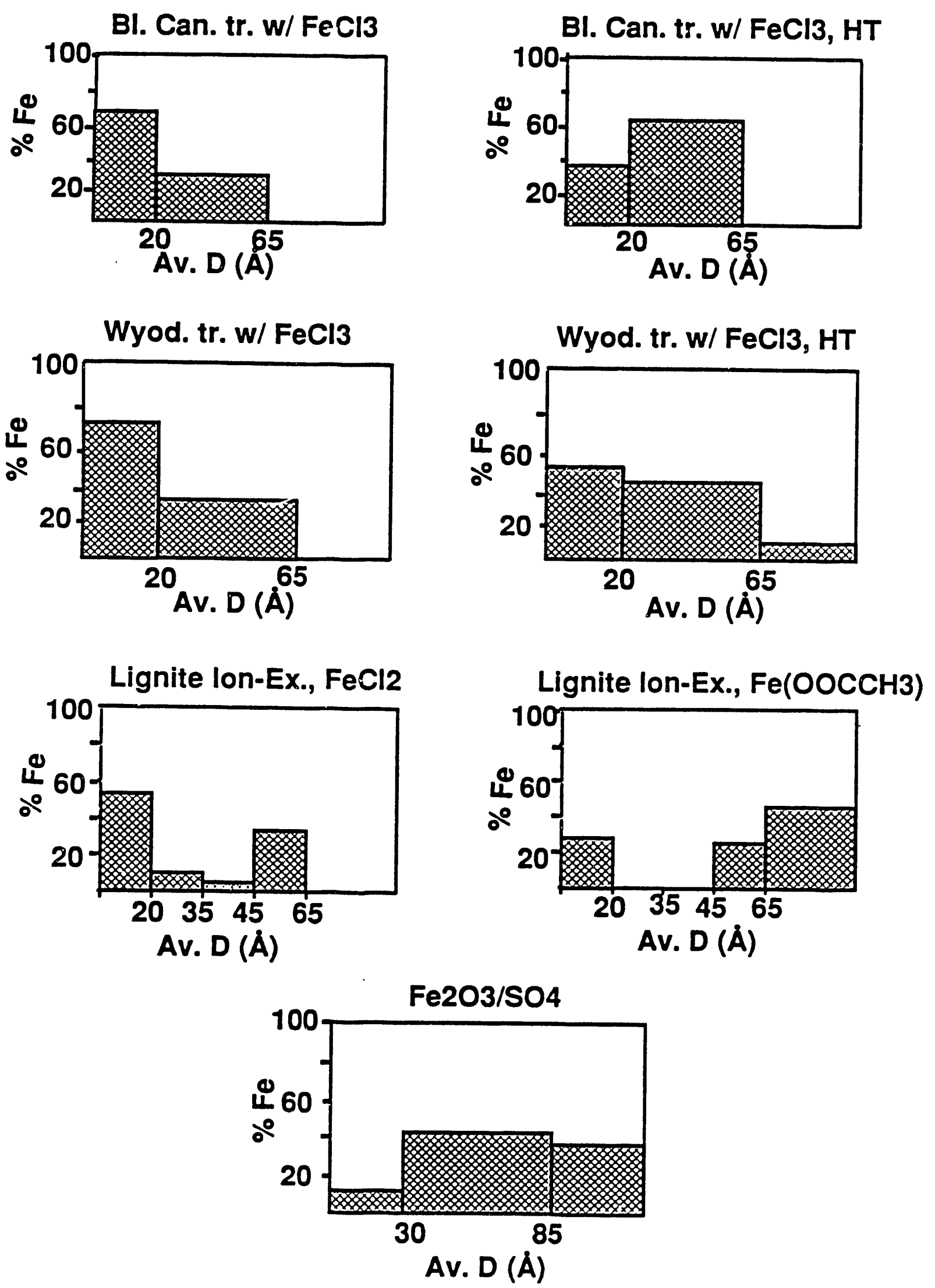

Figure 5 - Size distributions of ferric oxide and oxyhydroxide catalysts prepared by a variety of methods as determined by low temperature Müsstauer spectroscopy. 


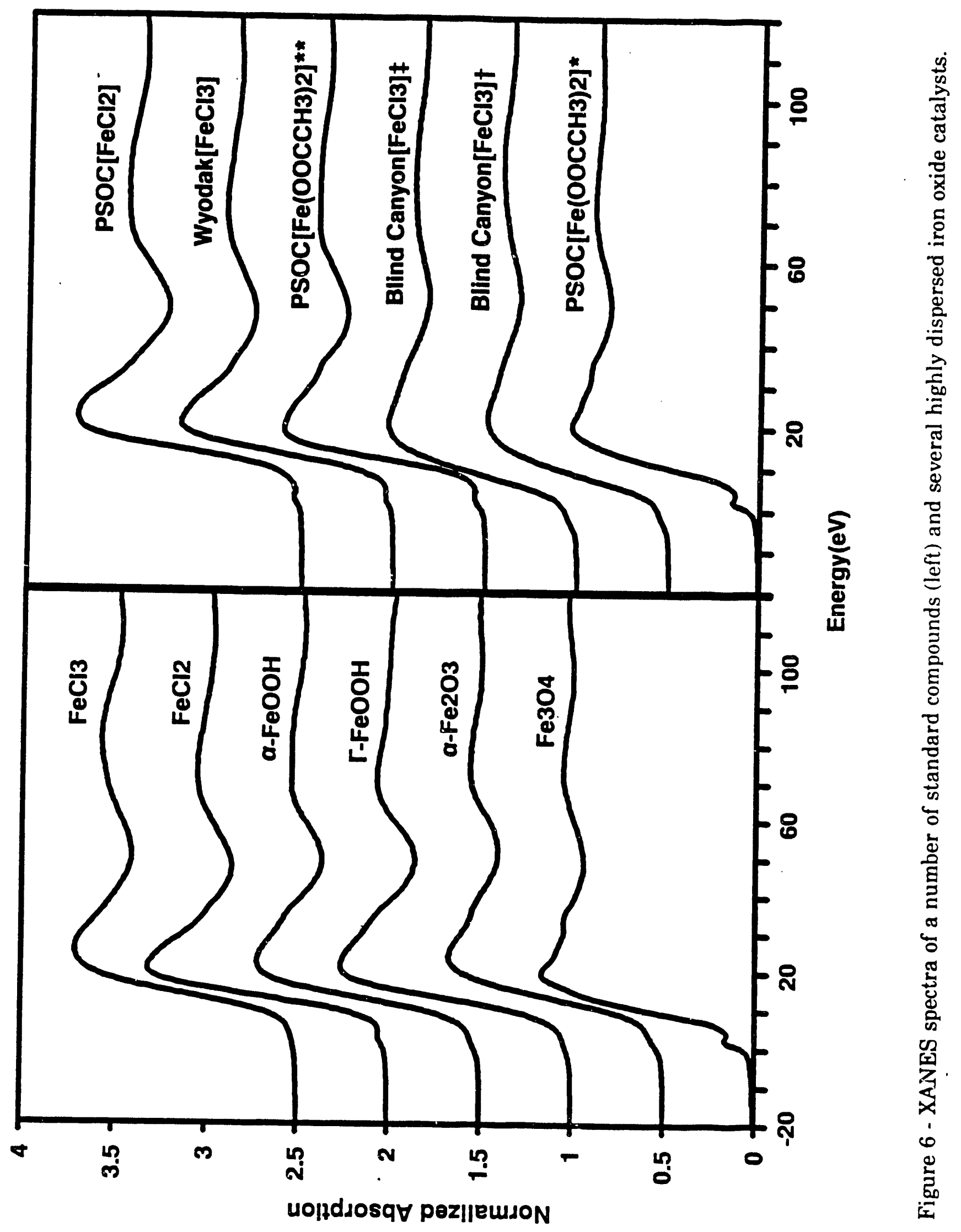




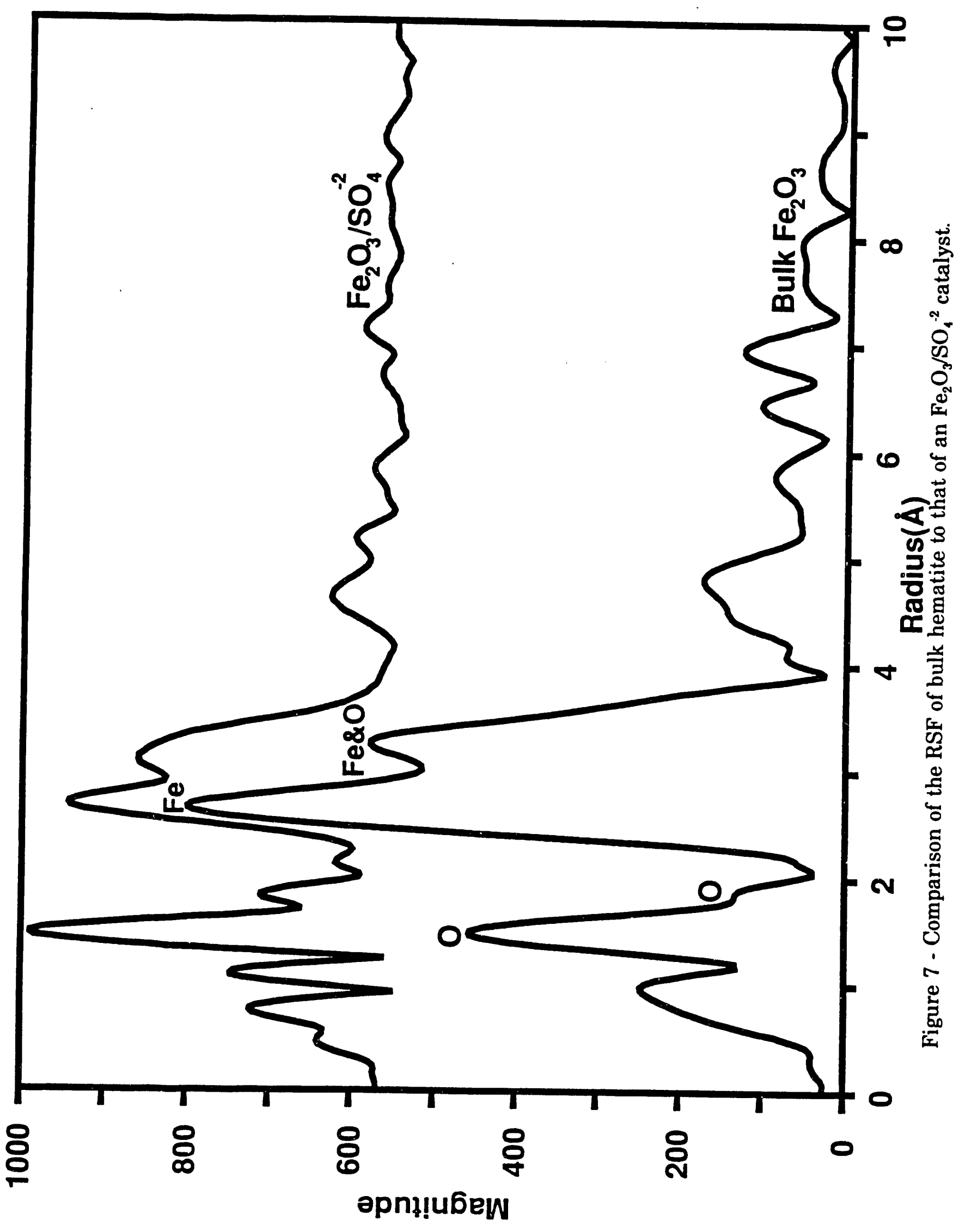




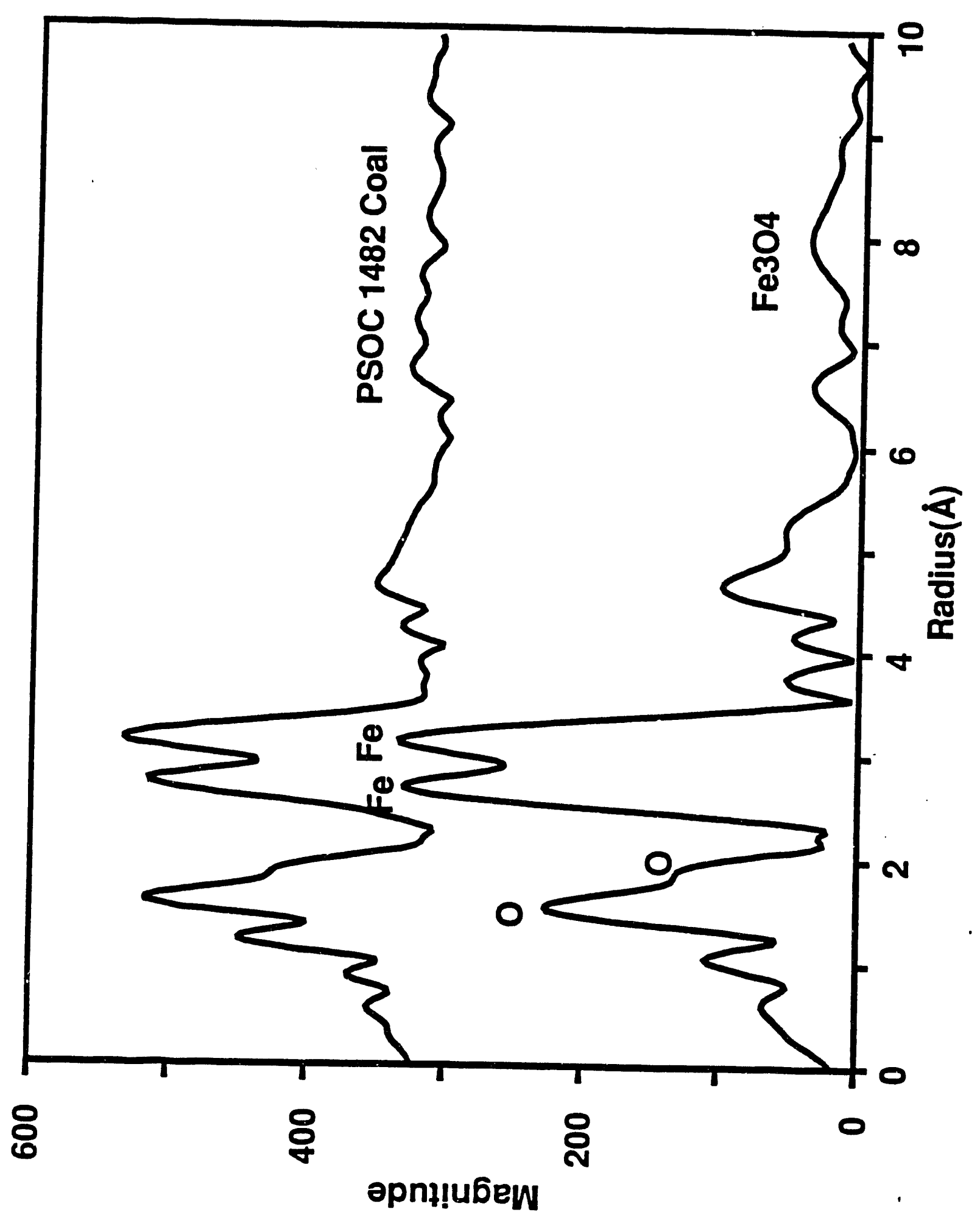

कิ

ชิ

ช

苛

맴

జ 


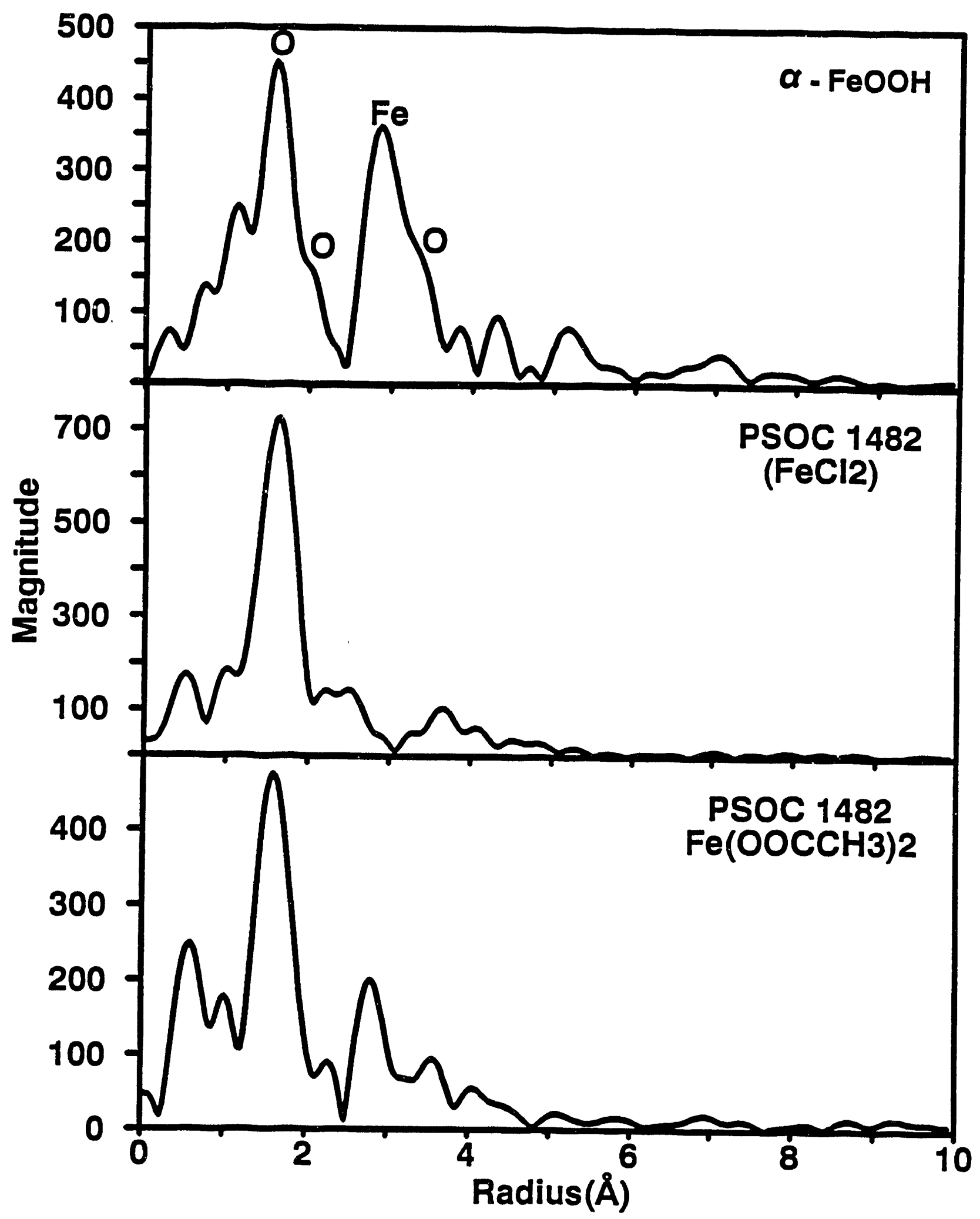

Figure 9 - Comparison of the RSF of goethite ( $\alpha-\mathrm{FeOOH})$ to that of a lignite subjected to iron cation - exchange in $\mathrm{FeCl}_{2}$ and $\mathrm{Fe}\left(\mathrm{OOCCH}_{3}\right)_{2}$ solutions. 

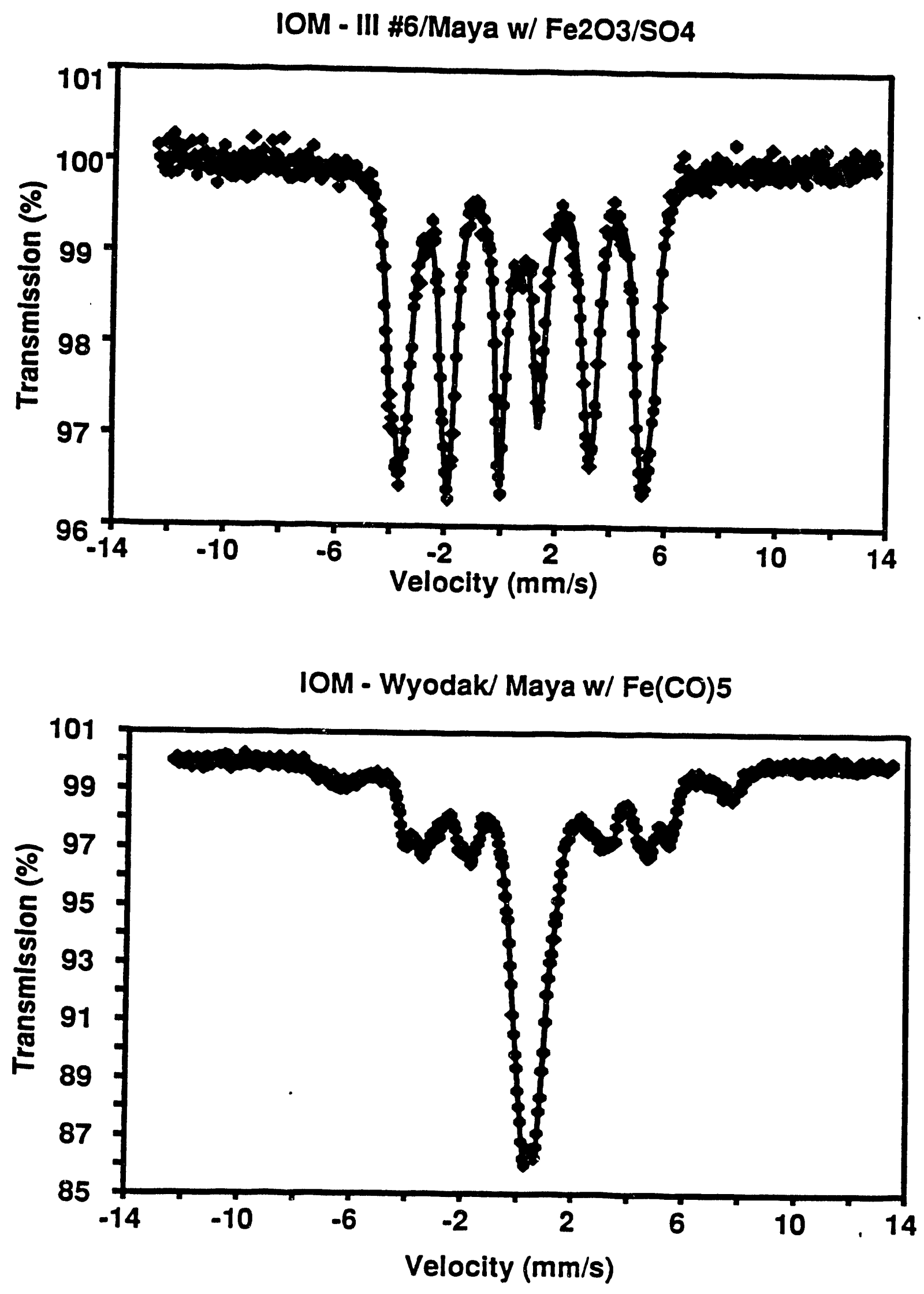

Figure 10 - Mössbauer spectra of IOM samples from liquefaction coprocessing runs eîpioying an $\mathrm{Fe}_{2} \mathrm{O}_{3} / \mathrm{SO}^{-2}{ }_{4}$ and an $\mathrm{Fe}(\mathrm{CO})_{5}$ catalyst precursor. 


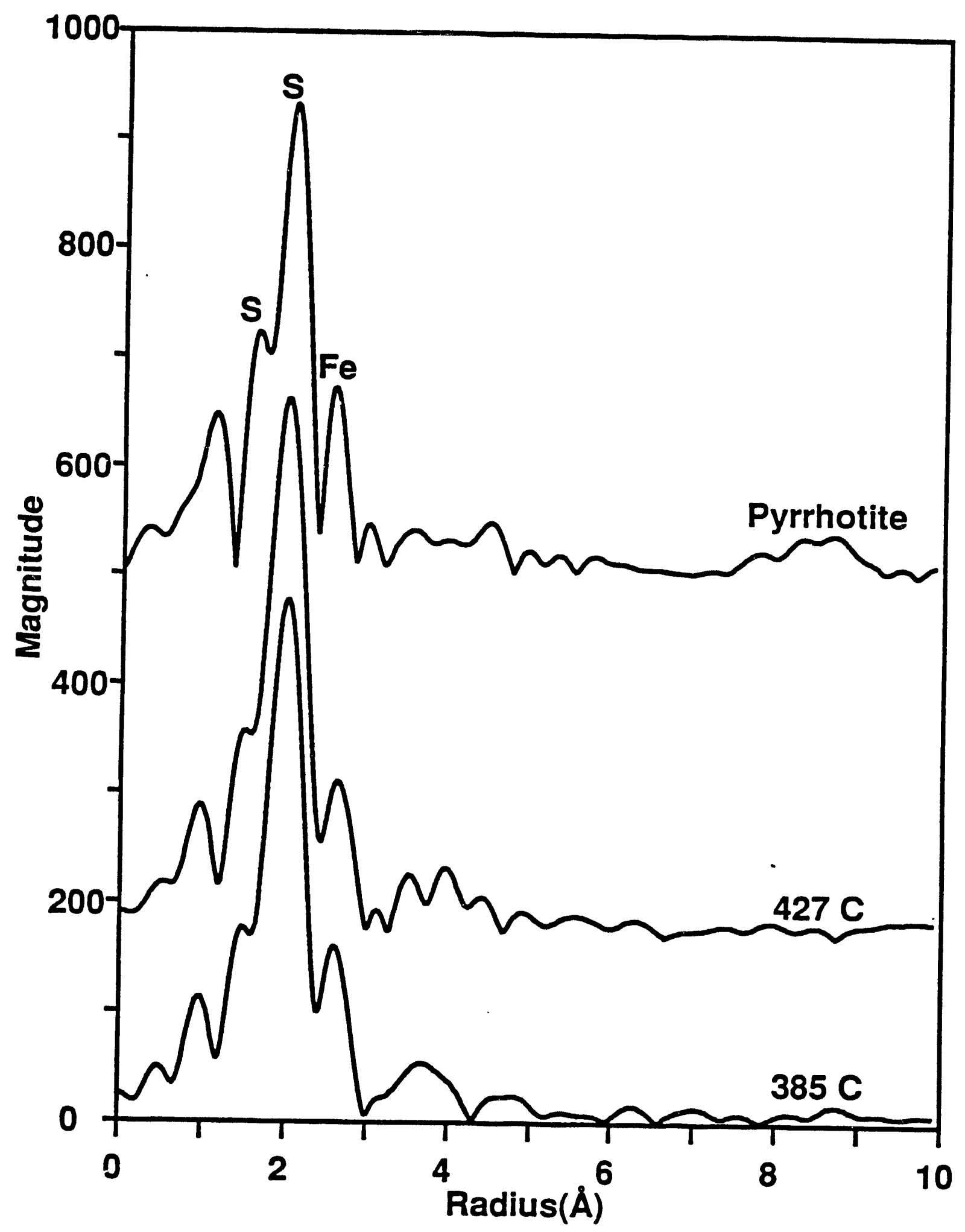

Figure 11 - RSFs of bulk pyrrhotite (top) and of IOM from liquefaction experiments at 385 and $427^{\circ} \mathrm{C}$ using iron cation-exchanged lignite. 


\section{Spectroscopic Studies of the Pyrolysis of Organic Sulfur Compounds}

(Dennis J. Clouthier)

\section{Summary}

Spectroscopic methods for the study of the pyrolysis processes of a variety of organic sulfur compounds have been developed. Thiophenoxy radicals have been detected directly by laser induced fluorescence in the pyrolysis products of phenyl disulfide. A pulsed pyrolysis jet scurce has been built, tested and proven to be a useful apparatus for producing pulses of pyrolysis products which are subsequently cooled by supersonic expansion. A new, precise wavelength calibration method has been developed for use with high resolution pulsed dye lasers in the $500-350 \mathrm{~nm}$ region.

\section{Introduction}

The study of the pyrolytic degradation of organic sulfur compounds has received scant attention in the literature, despite the importance of such processes in coal liquefaction. In particular, the reactive intermediates and transient species involved are largely unknown. We have been attempting to apply sensitive spectroscopic techniques to identify the intermediates so that detailed mechanisms can be explored. Unlike other approaches, we directly probe the primary products of the pyrolysis reaction, rather than analyzing the stable secondary products and inferring the initial event from mechanistic considerations.

The techniques used in this work include laser spectroscopy and a new method we have developed termed pyrolysis jet spectroscopy'. The pyrolysis jet technique consists of diluting the vapor of the compound of interest with a large excess of carrier gas ( $\mathrm{He}, \mathrm{Ar}, \mathrm{N}_{2}, \mathrm{H}_{2}$ etc.), passing the mixture through a heated zone and the then rapidly expanding the pyrolysis products into a vacuum chamber through a small orifice. The expansion produces extensive cooling of the internal degrees of freedom of the pyrolysis products and provides a collision-free environment so that reactive species cannot coalesce to form solid aggregates. The gas pressures used (1-10 atm.) and the pyrolysis temperatures attainable (300-1300 K) are suitable for the study of mosi organic species. The resulting supercooled gas stream is then chemically characterized by laser spectroscopic techniques. The only major limitation of the techaique is that the species being probed must fluoresce in order to be detected.

\section{Pulsed Pyrolysis Jet Spectroscopy}

At the beginning of this project period we set out to develop a pulsed pyrolysis jet 
method, analogous to the continuous jet technique we had been using. The advantages of the pulsed method are that it can give a larger flux of transient species during the time the laser is on, the pumping load on the vacuum system is much less due to the low duty cycle and the internal rotation-vibration cooling is predicted to be substantially better. There is sorse literature precedent for such a technique. Chen et al. ${ }^{2}$ used a pulsed valve and post expansion pyrolysis to produce pulses of methyl radicals which were detected by multiphoton ionization and mass spectrometry.

We began our attempts to develop a pulsed pyrolysis jet apparatus by designing a system based on a General V ilve mini-pulsed valve. After extensive experiments, we were forced to conclude that this valve did not perform according to the manufacturers specifications and was useless for our purposes. We then purchased a Newport Corp. model BV.100 pulsed molecular beam valve which has proven reliable. The apparatus was redesigned around this valve. The major problem to be overcome in the pulsed pyrolysis jet system for laser induced fluorescence work is to prevent the glow from the pyrolysis zone from reaching the photomultiplier which detects the fluorescence. This has been achieved after much experimentation. A schematic diagram of the final design is shown in Figure 1. The main body is water jacketed to protect the beam valve from overheating. The pyrolysis tube is a specially constructed quartz piece with a flange to hold it in place. The heater is a home built nichrome element $1.5 \mathrm{~cm}$ long with a $1 \mathrm{~cm}$ outside diameter.

Tests of the pulsed pyrolysis system show that it is capable of producing high intensity pulses of very cold (10-15 K) transient species with little heater glow, good reproducibility, and long term continuous operation. The pulse intensity and duration can be precisely controlled to match the requirements of a particular experiment.

\section{Thiophenoxy Radical Spectroscopy}

The substantial amount of organic sulfur bound up in coal in aromatic structures motivated us to look at the pyrolytic reactions of phenyl sulfides and disulfides. A literature search revealed that very little work has been done on the spectroscopic detection and characterization of thiophenoxy and substituted thiophenoxy radicals in the gas phase. Some work has been published on trying to establish the role of ArS radicals ir: :oal liquefaction processes ${ }^{3,4}$.

In continuous jet experiments, phenyl disulfide was heated to $340 \mathrm{~K}$ and seeded in two atmospheres of argon. The mixture was passed through the heated zone and expanded into vacuum with a residence time of about $1 \mathrm{~ms}$. At pyrolysis temperatures of $1025 \mathrm{~K}$, a fluorescence excitation spectrum of the thiophenoxy radical was obtained. The spectrum 
consists of 10 sharp ieatures in the $490-520 \mathrm{~nm}$ region which provide a unique and readily identifiable fingerprint of the radical. The fluorescence lifetime was found to be within the time resolution of our detection system, suggesting that it has an upper limit of about $20 \mathrm{~ns}$. Our results are in good agreement with a recent study in which ultravinlet laser photolysis was used to produce the radical in a supersonic jet ${ }^{5}$. Further experiments on substituted thiophenoxy radicals have been delayed by equipment failures in our laser and vacuum systems which are currently being rectified.

\section{Laser Wavelength Calibration System}

Many of the transient species we have been studying absorb laser light at wavelengths shorter than $500 \mathrm{~nm}$. Unfortunately, the standard method of high resolution laser calibration, using laser induced fluorescence (LIF) reference spectra of iodine vapor, is limited to the $800-500 \mathrm{~nm}$ region in which the iodine spectrum has been accurately measured ${ }^{\circ}$. In order to make further progress on our studies of transient species, it became necessary to invent a new calibration method. Our new method has been thoroughly tested and found to have high precision, good accuracy and likely to be widely applicable. A paper on the technique has been accepted for publication in the Review of Scientific Instruments?

The technique relies on Raman shifting the residual laser beam, after it exits the experiment, back into the $800-500 \mathrm{~nm}$ iodine spectrum region. The weak Stokes shifted beam can then be used to excite the iodine LIF spectrum and the original wavelength calculated from precisely known spectroscopic data for hydrogen gas. The apparatus is shown in Figure 2. The Raman shifter is a simple cylindrical cell filled with hydrogen gas at 100-300 psi pressure. The interaction of the gas with the laser beam causes stimulated Raman scattering, producing a multitude of new laser beams, shifted to higher and lower frequencies by multiples of the hydrogen vibrational frequency, 4155 wavenumbers. The beams are separated by a prism and the one of interest used to excite iodine fluorescence which provides the calibration spectrum. Tests have proven the method to be accurate to within 0.007 wavenumbers on a variety of transitions.

\section{References}

1. J. R. Dunlop, J. Karolczak and D. J. Clouthier, Chem. Phys. Lett., 151, 362 (1988).

2. P. Chen, S. D. Colson, W. A. Chupka and J. A. Berson, J. Chem. Phys., 90, 2319 (1986).

3. M. S. Alanjiar and J. A. Franz, Coal. Sci. Technol. 11, 273 (1987).

4. B. Fixari, V. Abi-Khers and P. Le Perchec, Nouv. J. Chem. 8, 177 (1984). 
5. K. Shibuya, M. Nemoto, A. Yanagibori, M. Fukushima and K. Obi, Chem. Phys. 121, 237 (1988).

6. S. Gerstenkorn and P. Luc, Rev. Phys. Appl. 14, 791 (1979).

7. D. J. Clouthier and J. Karolczak, "High Resolution Pulsed Dye Laser Calibration in the 500-350 nm Region Using Iodine Atlas Reference Lines", Rev. Sci. Instr., in press (1990). 


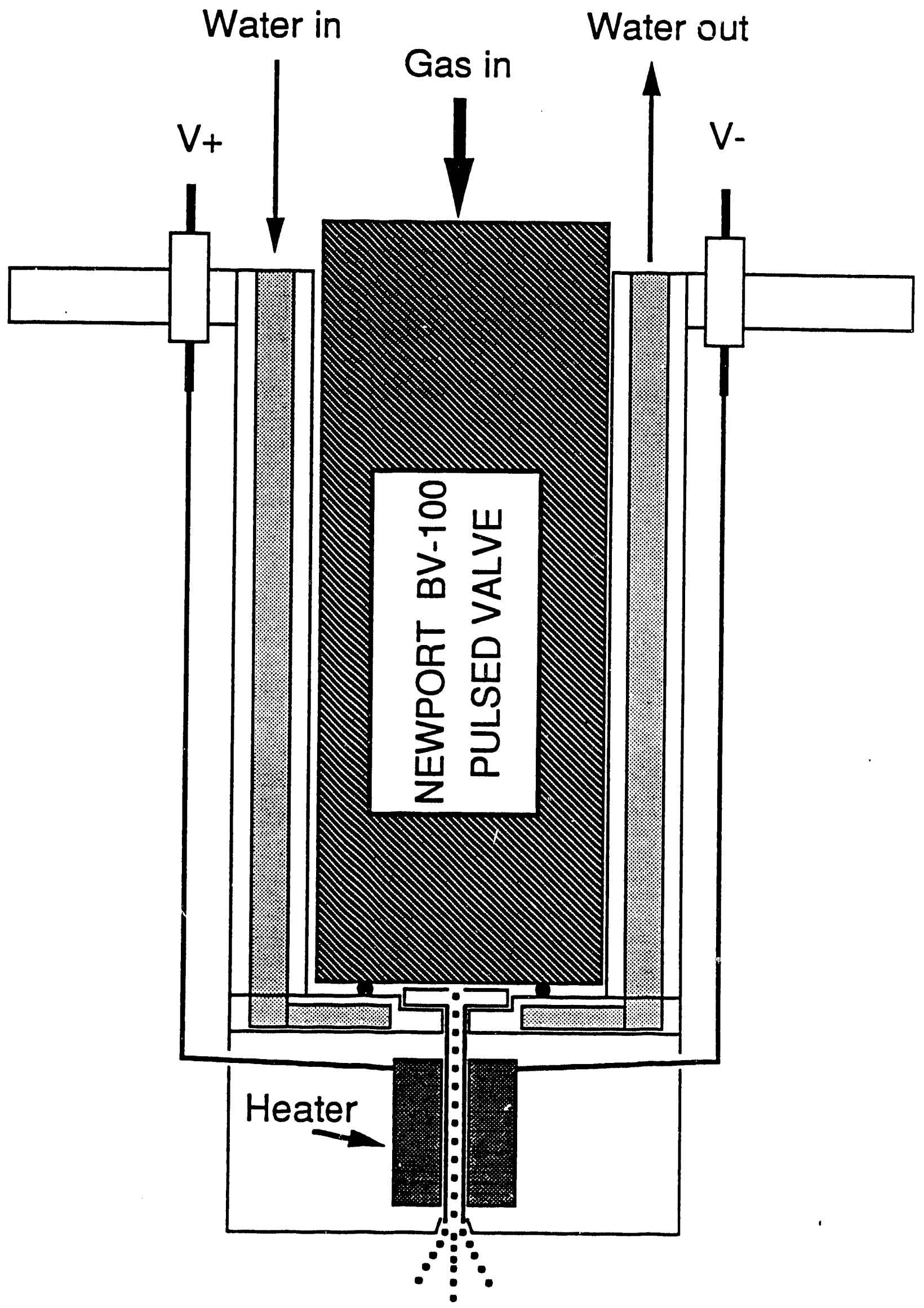

Figure 1: Schematic diagram of the pulsed pyrolysis jet apparatus. 


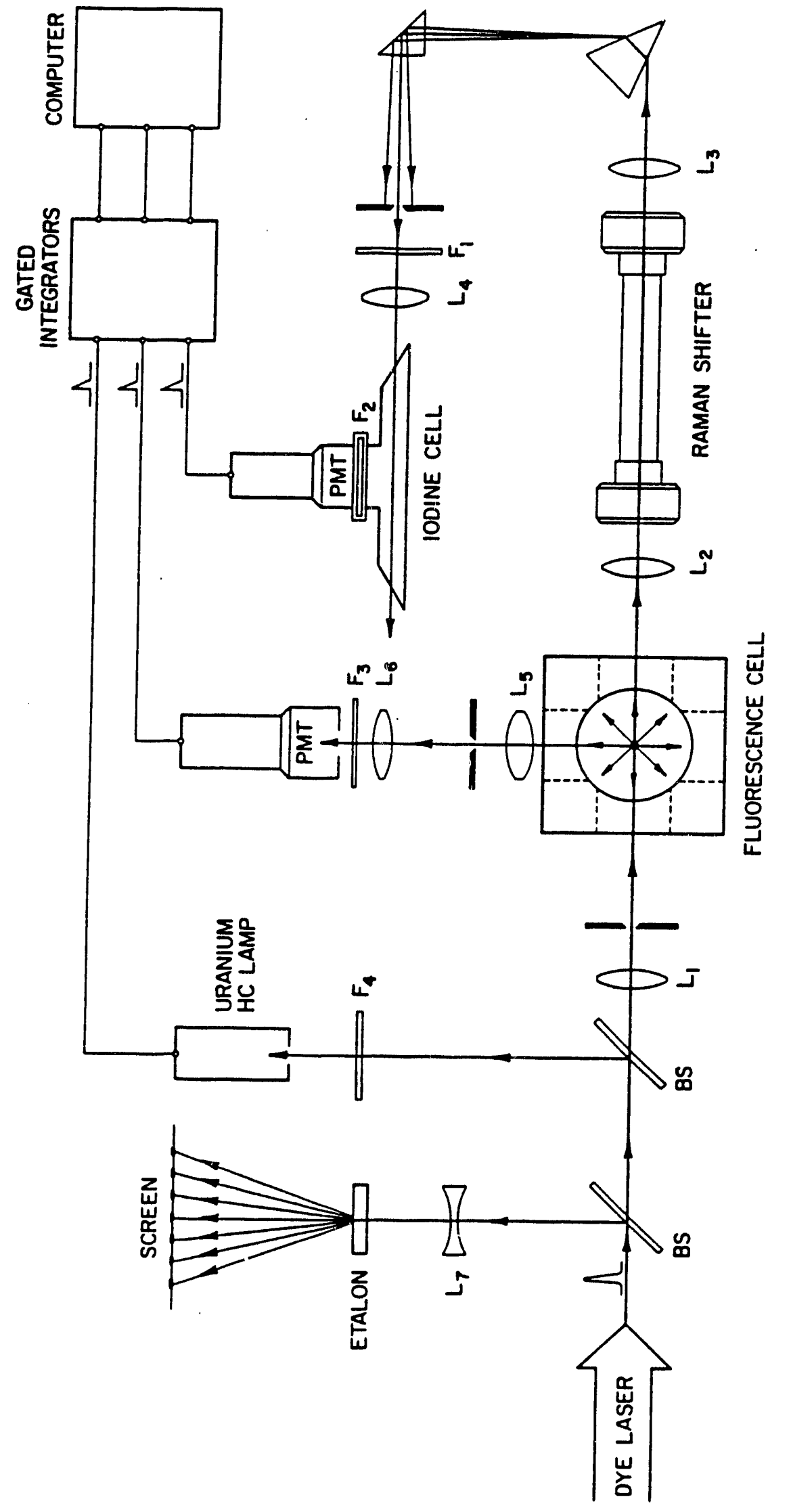

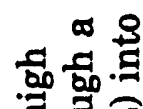

로를

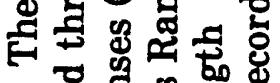

రั

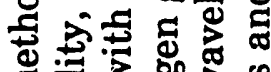

घ: 300

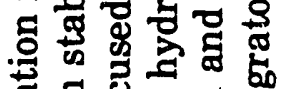

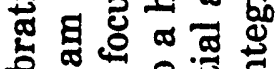

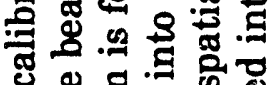

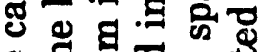

吉娄焉

웡 웡

인.

起完目

की

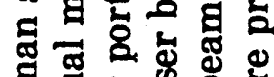

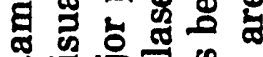

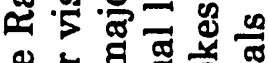

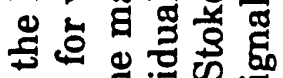

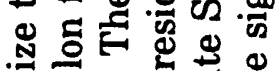

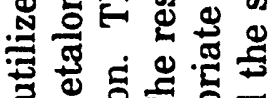

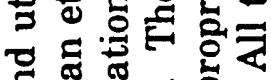

สิ

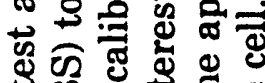

$\$$ صृ के

$S$ 象

马

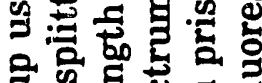

웜웡

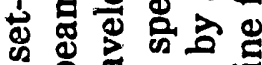

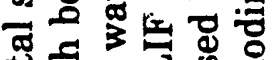

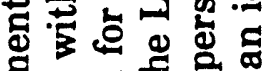

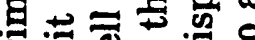

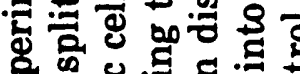
×.

\&

Ч ช

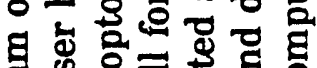

ॠ

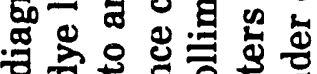

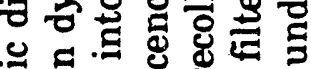

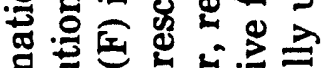

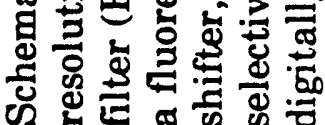

تِ 


\section{Surface Characterization of Coals by XPS}

(P.J. Reucroft and J.Y. Kim)

\section{Introduction}

X-ray photiselectron spectroscopy has been used to study the surface characteristics of coal samples. The technique provides elemental identification and chemical information about the outmost 3-4 $\mathrm{nm}$ of the exposed surface of a solid. The surface composition of the coal samples was measured and compared with the results of bulk analysis. A good correlation with the bulk analysis was obtained for the total carbon and nitrogen using XPS quantitative analysis, although the accuracy of the XPS determinations seems to be lower, in view of uncertainties in determining sensitivity factors. The surface and bulk concentrations differ in the case of sulfur, oxygen, silicon, aluminum and iron. Silicon, aluminum, and oxygen are enriched at the surface, for example, while iron is surface depleted.

Peak deconvoution was conducted to obtain information on the chemical environments of elements, such as sulfur, carbon and nitrogen. These results have been compared with model compound studies and good agreement was observed.

\section{Experimental}

\subsection{Materials}

Two types of coals, 7 KCER samples and 8 APCSB samples, were used in the surface characterization studies. The KCER coal samples were obtained from the Kentucky Center for Energy Research (now Center for Applied Energy Research) Laboratories and are typical Kentucky coals. The APCSB (Argonne Premium Coal Sample Bank) coals were obtained from Argonne National Laboratory. The KCER coals had generally been exposed to air and were thus oxidized.The APCSB coals were stored under nitrogen and showed no significant degree of oxidation. The powdered coal samples were examined in their as-recieved form and were subjected to heat pretreatment at $45^{\circ} \mathrm{C}$ for $8 \mathrm{hrs}$ to remove volatile materials. All particle sizes were less than $5 \mu \mathrm{m}$. Their ultimate chemical analysis data are shown in Tables 1 and 2 (KCER samples) and Tables 3 and 4 (APCSB samples).

In the oxidation studies, an APCSB coal sample (Illinois \#6) was uniformly distributed in trays, with an average layer thickness of $1 \mathrm{~mm}$. The sample was oxidized for 10 days with air at atmospheric pressure and room temperature. After the oxidation treatment the sample was kept under a nitrogen atmosphere until analysis.

\subsection{XPS analysis}

The powdered samples were mounted on the spectrometer probe tip by means of 
double-sided adhesive Scotch insulating tape. The samples were pressed with the aid of a metal spatula and the excess that had not adhered was tapped off. To exclude the possibility of recording contaminants associated with the tape, the tape was also analysed separately. It was found that the constituents of tape were not detected by XPS, and that the photoionization signals are characteristic of the coal samples alone.

The samples were examined by XPS on a Kratos XSAM 800 spectrometer using Mg K $\alpha$ (1253.6 eV) radiation. The spectrometer was run in fixed retarding ratio (FRR) mode at a pass energy of $13 \mathrm{KV}$ and $15 \mathrm{~mA}$. Under these conditions, the full width at half maximum (FWHM) of the Ag $\left(3 d_{6 / 2}\right)$ peak is $1.1 \mathrm{eV}$. Spectra were recorded at $<1 \times 10^{-6}$ torr.

Data acquisition was controlled by a SMS 1000 (DEC PDP 11/73 compatible) microcomputer which was used for spectral analysis and quantification. Long data acquisition times were required to obtain high quality spectra which would allow resolution of the minor elements. For the samples of the lowest sulfur concentration, this amounted to more than $19 \mathrm{hrs}$. of data accumulation in the sulfur peaks region. All data for the minor elements were collected over $0.05 \mathrm{eV}$ increments.

Radiation damage to the sample from long-term exposure to the $x$-ray beam was not observed. All binding energies were referred to carbon (1s) at $285 \mathrm{eV}$ to compensate for sample charging. Elemental concentrations were obtained from peak areas and corrected for atomic sensitivity factors. (O 1s 1.73; S 2p 1.59; Si 2p 1.30; $\mathrm{Al} 2 \mathrm{p} 0.58 ; \mathrm{N}$ 1s $1.49 ; \mathrm{Cl} 2 \mathrm{p}$ 2.13; Fe 2p 7.28; all relative to $\mathrm{C} 1 \mathrm{~s}$ )

The peak was deconvoluted using a peak synthesis procedure which employed a Gaussian line shape at fixed binding energies determined from model compound studies. ${ }^{10,11}$ The data system permitted the intensity of the components and their FWHM to be varied in order to obtain the best fit between the experimental and the synthesized spectrum.

\section{Results and Discussion}

\subsection{Sirface Compositions}

Representative XPS wide-scan spectra for two KCER coal samples are shown in

Figure 1. The spectra show the major elements, carbon and oxygen, as well as the minor components silicon, aluminum, nitrogen, and sulfur. Other elements, known to be present in the coal, can in some cases be detected at the surface but only by extending data acquisition times (4 to 19 hours for each minor element in this study) and degrading resolution to an unacceptable level.

The binding energies of the carbon 1s (C 1s), oxygen $1 \mathrm{~s}(\mathrm{O} 1 \mathrm{~s})$ and other related elements show the expected values and sulfur $2 p$ ( $S$ $2 p$ ) orbital electrons binding energy 
values are given in Tables 5 \& 6 . The carbon and oxygen signals each had one peak, while the sulfur signal had two small background peaks. The $S 2 p$ analysis indicated that two distinct chemical environments of sulphur exist in the coal samples.

\subsection{Sulfur in Coal}

The S 2p XPS spectra of both KCER and APCSB coal samples often showed two peaks with a separation of $5.6 \mathrm{eV}$. This was coincident with a separation of the $\mathrm{Fe} 2 \mathrm{p}_{1 / 2}$ and Fe 2p $p_{3 / 2}$ peaks by $4 \mathrm{eV}$. The existence of two chemically distinct compounds, both containing iron and sulfur was thus indicated. Figure 2 shows the general XPS spectra of sulfur 2p region for two typical KCER coal samples. Two sulfur photolines were observed. A $170 \pm$ $0.5 \mathrm{eV}$ photoline can be ascribed to the oxidized sulfur, organic and inorganic (sulfate). The peak at $164 \pm 0.5 \mathrm{eV}$ corresponds to inorganic sulfide plus the usual organic sulfur forms present in coal (thiophene, sulfides, eq mercaptans). ${ }^{1}$ The binding energy of sulfur $2 p$ in pyrite is $163.5 \mathrm{eV}$ which is very close to the observed peak at $164 \pm 0.5 \mathrm{eV}$. Thus it appears that the bulk of the organic sulfur XPS S 2p peaks are overlapped by the pyrite peak, as described previously. ${ }^{2}$ Pyritic sulfur is not thought to make a significant contribution to the observed sulfur peak at $164 \pm 0.5 \mathrm{eV}$ in the oxygen exposed coals but is mostly found in the form of oxidized sulfur (sulfate) at $170 \pm 0.5 \mathrm{eV}$.

Figure 3 shows the general XPS spectra for two typical APCSB coal samples. It is noteworthy that half of the APCSB coal samples show no evidence for the $170 \pm 0.5 \mathrm{eV}$ sulfate sulfur peak. The sulfate sulfur is also very weak in the rest of the samples (Table 6). Plots of the ratio of the surface $S_{\text {total }}$ concentration to the bulk $S_{\text {total }}$ concentration versus bulk $S_{\text {total }}$ concentration and the ratio of the surface $S_{\text {(organte + pyritic) }}$ concentration to the bulk $S_{\text {(organic + }}$ pyritic) concentration versus bulk $S_{\text {(organic + pyrttc) }}$ are shown in Figures 4 \& 5, respectively for KCER and APCSB coals. Sulfur was depleted in the surface regions in the all coals. From Figure 3, the peak corresponding to $164 \pm 0.5 \mathrm{eV}$ was more intense in the APCSB cuals, the reverse situation appearing in the KCER coals (Fig. 2). The fact that the sulfate sulfur peak for the KCER coals was more intense than the sulfate sulfur peak for the APCSB coals suggested that the KCER coals had been exposed to atmospheric conditions for a long time. Therefore, the possibility of a pyrite oxidation process producing a significant amount of ferrous sulfur is indicated. This observation is in agreement with previously published results. ${ }^{2,3}$

\subsection{The organic sulphur in coal}

To determine the amounts of organic sulfur present in coal, the inorganic forms are 
first determined chemically and then their sum is subtracted from the total sulfur content to yield the organic concentration." This indirect method tends to accumulate the errors of the other analyses, which are not always reliable.

It has been suggested that XPS could be used to determine the organic sulfur in the coal surface. ${ }^{6,6}$ It was originally hoped that organic sulfur would have a unique peak. However the organic sulfur moieties are probably thiol-, thio-ether-, disu phide-, or thiophene- type structures, XPS peaks in the $S 2 p$ region would thus be expected at 163 $165 \mathrm{eV}$ region..$^{6,7,8}$

From Figure 3, no sulfur peak was found at $169-171 \mathrm{eV}$ in APCSB coal samples that had been stored under nitrogen and it can be expected that the organic sulfur peak is overlapped by the pyritic sulfur peak at $164 \pm 0.5 \mathrm{eV}$. This overlap leads to some difficulty in obtaining the exact amount of organic sulfur. Pyritic sulfur must be subtracted to obtain the organic sulfur amount from the overlapped pyritic sulfur peak at $164 \pm 0.5$. Since complete physical removal of the pyrite from the coal is normally not possible, ${ }^{9}$ chemical techniques need to be considered.

It is interesting to note from Figure 6 that the nlinois \#6 coal indicates a possible method for deternining organic sulfur in the $164 \pm 0.5 \mathrm{eV}$ region. This sample was exposed to humid air for 10 days, and the sulfate peak at $170 \pm 0.5 \mathrm{eV}$ increased in intensity as the sulfide peak at $164 \pm 0.5 \mathrm{eV}$ decreased. This observation that the sulfate peak at $170 \pm 0.5$ $\mathrm{eV}$ increases as the coal pyrite oxidation increases may provide the basis for a convenient analysis of organic sulfur in coal by 'shifting' the pyritic sulfur peak to $170 \pm 0.5 \mathrm{eV}$. The peak remaining at $164 \pm 0.5 \mathrm{eV}$ can thus be attributed to organic sulfur. It can be con cluded from Figures 3, 4 and 5 that the pyritic sulfur in the KCER coal samples had been already oxidized by air exposure. The values of $S_{\text {(orzanic + pyritic) }}$ for the KCER coal samples in Table 5 can thus be ascribed to the approximate value of organic sulfur existing on the coal surface.

It is possible to deconvolute the organic sulfur $2 p$ peak into three line shapes with binding energy components at $164.1 \mathrm{eV}, 163.3 \mathrm{eV}$ and $163.7 \mathrm{eV}$. The result is shown in Figure 7 for the nlinois \#6 coal. These binding energy peaks can be compared with the results for several model compounds. ${ }^{10,11}$ The peaks can be ascribed to thiophenic, alkylsulfide and pyritic environments, respectively, as the result of such a comparison. Estimation of relative quantification by peak synthesis gave a $164.1 \mathrm{eV}: 163.3 \mathrm{eV}: 163.7 \mathrm{eV}$ ratio of 8:3.4:1 with a FWHM of $1.7 \mathrm{eV}$. This is in agreement with the results reported by Keleman et al. ${ }^{11}$ The results show that about $2 / 3$ of the surface organic sulfur exists in a thiophenic environment and that organic sulfur is the predominant species in the llinois \#6 
coal.

\subsection{Oxygen in Coal}

The ratio of the surface oxygen concentration to the bulk oxygen concentration versus bulk oxygen concentration for KCER \& APCSB coal samples are presented in Figure 8. XPS cannot distinguish between the oxygen in mineral matter and organic oxygen. The oxygen content, measured by XPS, includes a contribution from the mineral components, such as $\mathrm{SiO}_{2}$ and $\mathrm{Al}_{2} \mathrm{O}_{3}$.

The results from Tables 1-4 showed that surface enrichment of oxygen is 2-3 times higher than the bulk analysis of oxygen. Earlier XPS results ${ }^{12,13}$ have also indicated a surface enrichment of the axygen content compared with that indicated by the bulk ultimate analysis. This can most likely be ascribed to the presence of high concentrations of oxygen groups along the fracture surfaces of coal.

\subsection{Carbon in Coal}

The average $C$ 1s peak binding energy was $285 \pm 0.2 \mathrm{eV}$ for all samples studied. Figure 9 shows the ratio of surface carbon concentration to the bulk carbon concentration versus bulk carbon concentration. A good correlation between surface and bulk analy sis is observed.

The carbon 1s peak was deconvoluted to obtain information about the functional group compositions. According to model compound studies, ${ }^{1,3,12,14}$ the $\mathrm{C}$ 1s peak can be classified into 4 major components; hydrocarbon at $285 \mathrm{eV}$, hydroxyl or ether at $288.6 \mathrm{eV}$, carbonyl at $288 \mathrm{eV}$ and carboxyl at $289.2 \mathrm{eV}$. The results of such peak synthesis are shown in Figure 10, for the KCER 71268 and Wyoming coals, respectively. Carbon 1s peak synthesis data are presented in Table 7. The $C$ 1s peak, for those coals with a low amount of oxygen, shows a low level of carboxyl and carbonyl functional group compositions in both KCER and APCSB coal samples. This is in agreement with the result of Clark et al. ${ }^{12}$ Generally the hydrocarbon peak at $285 \mathrm{eV}$ accounts for $80 \pm 4 \%$ of the total carbon signal.

\subsection{Nitrogen in Coal}

Nitrogen showed a single peak for all coals which could be deconvoluted into two major components at mean binding energies of $400.4 \pm 0.2 \mathrm{eV}$ and $398.8 \pm 0.2 \mathrm{eV}$ (referenced to the $\mathrm{C} 1 \mathrm{~s}$ peak at $285 \mathrm{eV}$ ). From earlier papers, ${ }^{15,16,17}$ these can be ascribed to nitrogen of the pyrrole- and pyridine- types, respectively. Figure 11 shows the result of peak synthesis 
on a fresh North Dakota coal sample. Nitrogen of the pyrrole type contributes $65 \%$ and nitrogen of the pyridine type contributes $32 \%$ to the total nitrogen signal. In general, it is observed that pyrrole- type nitrogen dominates the XPS $N$ is spectrum of all the coal samples.

The ratio of the surface nitrogen concentration to the bulk nitrogen concentration versus bulk nitrogen concentration is shown in Figure 12. A good correlation is observed between the surface and bulk concentration for nitrogen. However, according to the earlier results, ${ }^{1,16}$ nitrogen was depleted on the surface of coal. This discrepancy can possibly be ascribed to the variation in the assumed value of the atomic sensitivity factor.

\subsection{Iron in Coal}

Iron was difficult to detect on most of the APCSB coals and was present only in small amounts in the KCER coals. A comparison between surface versus bulk iron concentration is given in Tables 1 \& 2. A significant amount of surface depletion is observed for iron. On the basis of earlier work, ${ }^{1,11}$ there are two possible explanations for this observation:

1) iron containing particles are surrounded by organic material

2) iron containing particles have a large particle size distribution.

\subsection{Silicon and Aluminum in Coal}

Figure 13 shows the general narrow scan spectrum of the Si and Al region for a typical CER coal sample. Si and Al have binding energies of $100 \pm 0.5 \mathrm{eV}$ and $71 \pm 0.5 \mathrm{eV}$, respectively. These peaks can be ascribed to silicate and aluminosilicate (clays).

Plots of the ratio of the surface concentration to the bulk concentration versus bulk concentration for $\mathrm{Si}$ and $\mathrm{Al}$ are shown in Figures $14 \& 15$, respectively for KCER coals. Si and $\mathrm{Al}$ were also observed in the APCSB coals, but further analysis was not possible because bulk ultimate chemical analysis data for $\mathrm{Si}$ and $\mathrm{Al}$ were not available. The analytical data for APCSB coal samples in Tables 3 and 4 are thus shown on a Si and Al free basis. The results indicate a surface enrichment for each element. According to previous results, ${ }^{1}$ this may be due either to a degree of preferential cleavage along the mineral rich planes in the coal particles or to the presence of fine particles of mineral matter exposing a high external surface area compared with the organic matter.

\section{References}

1. D.L. Perry and A. Grint, Fuel 62, 1983, p1024

2. D.C. Frost, W.R. Leeder and R.L. Trapping, Fuel 53, 1974, p206 
3. J.R. Brown, B.I. Kronberg and W.S. Fyte, Fuel 60, 1981,p439

4. H.N. Lowry, (ED) 'Chemistry of Coal Utilization', Supplemen tary Volume, Wiley, New York, 1963 p218

5. P.H. Given and W.F. Wyss, BCURA Monthly Bull, 1961, 25(5), p165

6. K. Siegbahn, C. Nording, A. Fablman, R. Nordberg, K. Hamrin, J. Hedman, J. Johansson, T. Bergmark, S.E. Karlsson, I. Lindgren, and B. Lindberg, ESCA Atomic, Molecular, and Solid State Struc tured Studied by Means of Electron Spectroscopy, Almquist, Uppsa la, 1967

7. K. Siegbahn, Phil. Trans. R.Soc., London, A 1970, 33, p286

8. H. Hanker and P.M. Sherwood. Phil. Mag. v27, 1973, p 1241

9. H. P. Dibbs and P. Marrieer Methods for removal of Sulphur from coal. Air Pollt. Contr. Directorate Tech. Appraisal Rep. EPS 3-Ap-73-3. Environmental Protection Service, Dept. of Environm., 1973, Ottawa, Canada

10. Handbook of X-ray Photoelectron Spectroscopy (Perkin-Elmer Corporation, Eden Prairie, MN, 1979)

11. S.R. Kelemen, G.N. George, and M.L. IJorbathy, Fuel Process Technology, 1990

12. D.T. Clark, R. Wilson, and J.M.E. Quicke, Chem. Geol. 1983, v39 p.215

13. G. Slodizian, Surface Science, 1975, v48, p161

14. A.R. Gonzalez-Elipe, A. Martinez-Alonso and J.M. Tascon, Surface Interface Analysis, July 1988

15. R.B. Jones, C.B. Mc Court and P. Swift, in Proceeding of International Confererce on Coal Science, Dusseldorf, 7-9, Sep tember 1981, Verlag Gluckauf, Essen, p65\%

16. K.D. Bartle, D.L. Perry and S. Wallace, Fuel Processing Technology, 1987, v15, p351

17. P. Burchill and L.S. Welch, Fuel 1989, v68, p100 


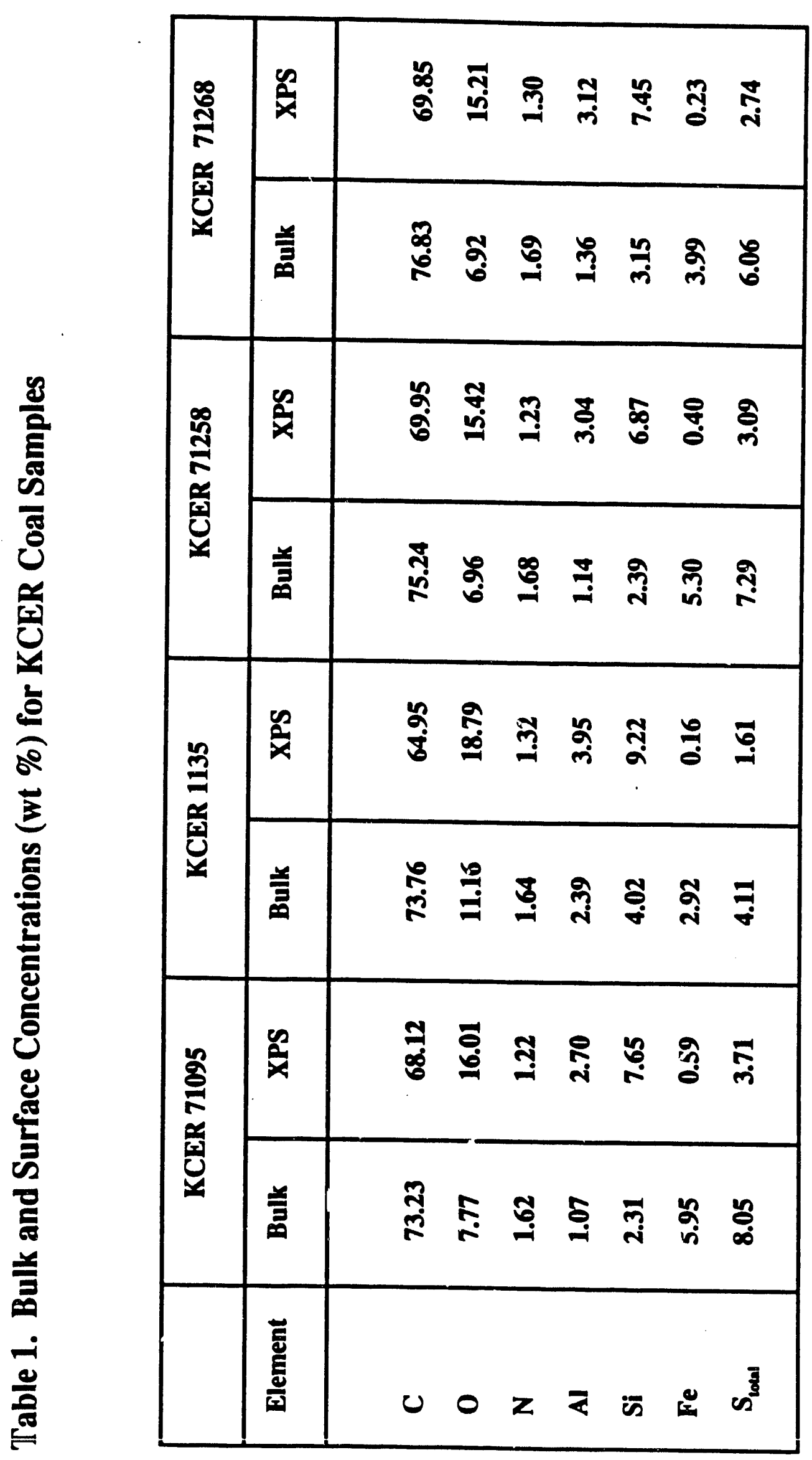




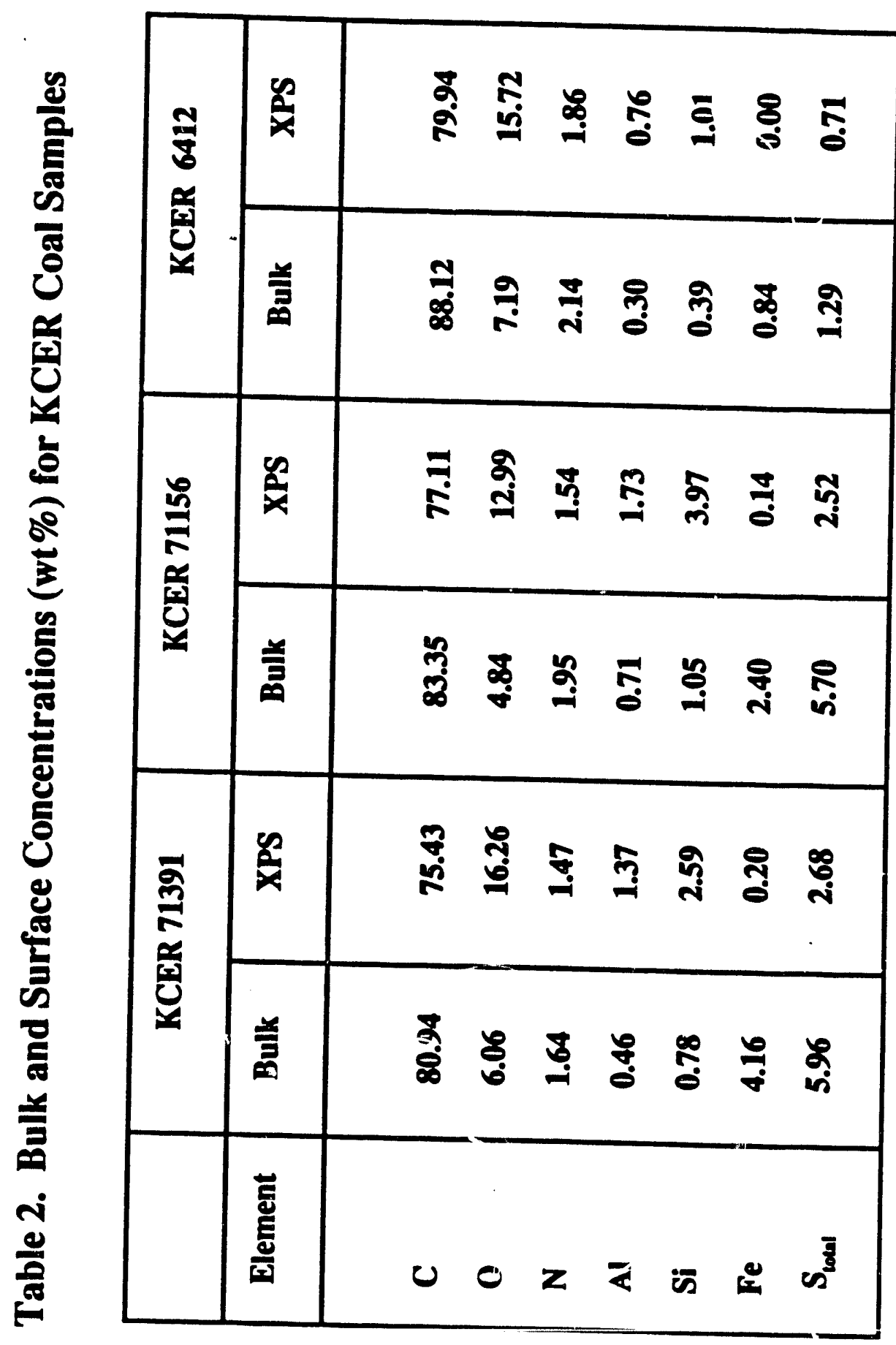




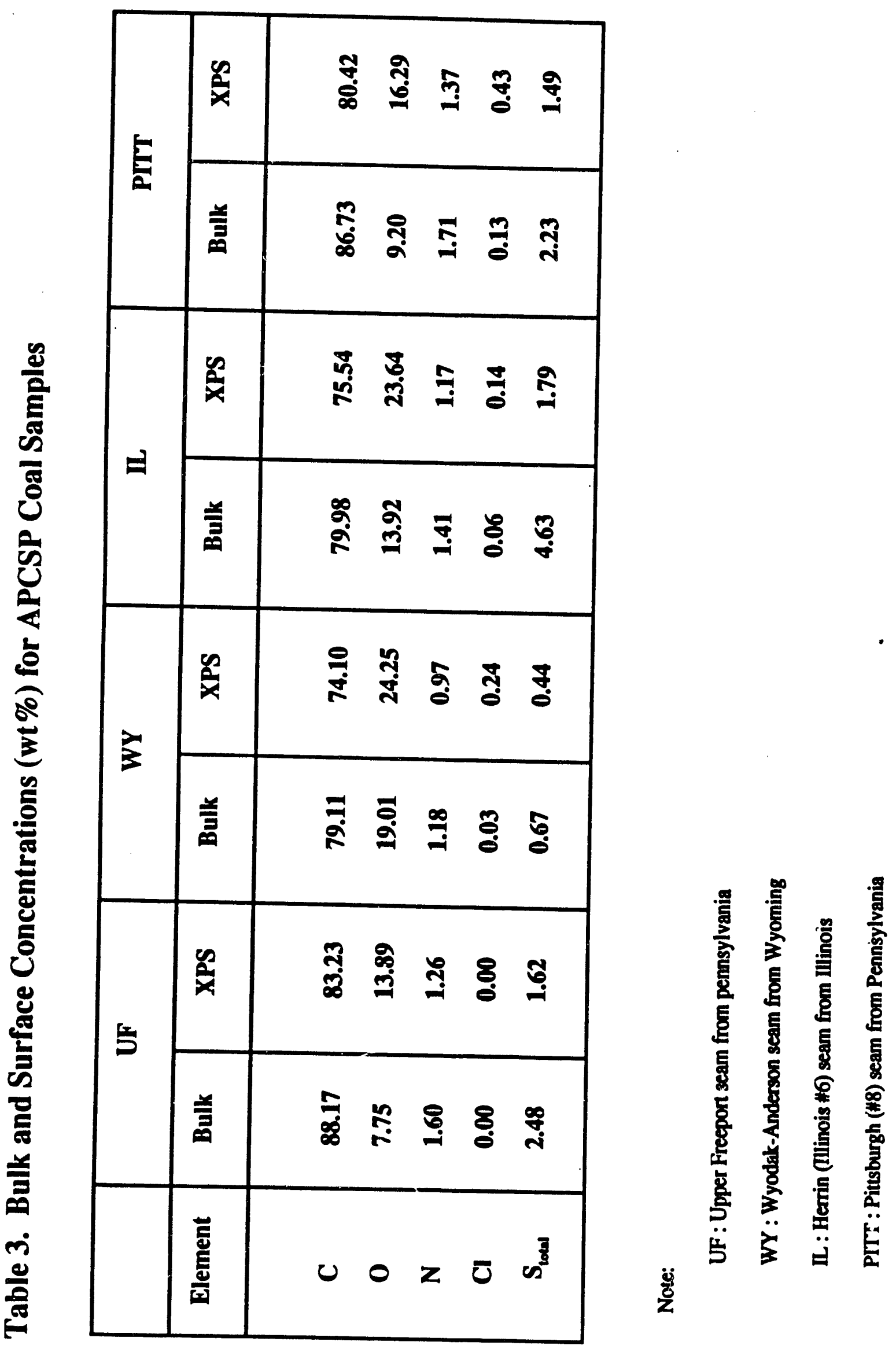




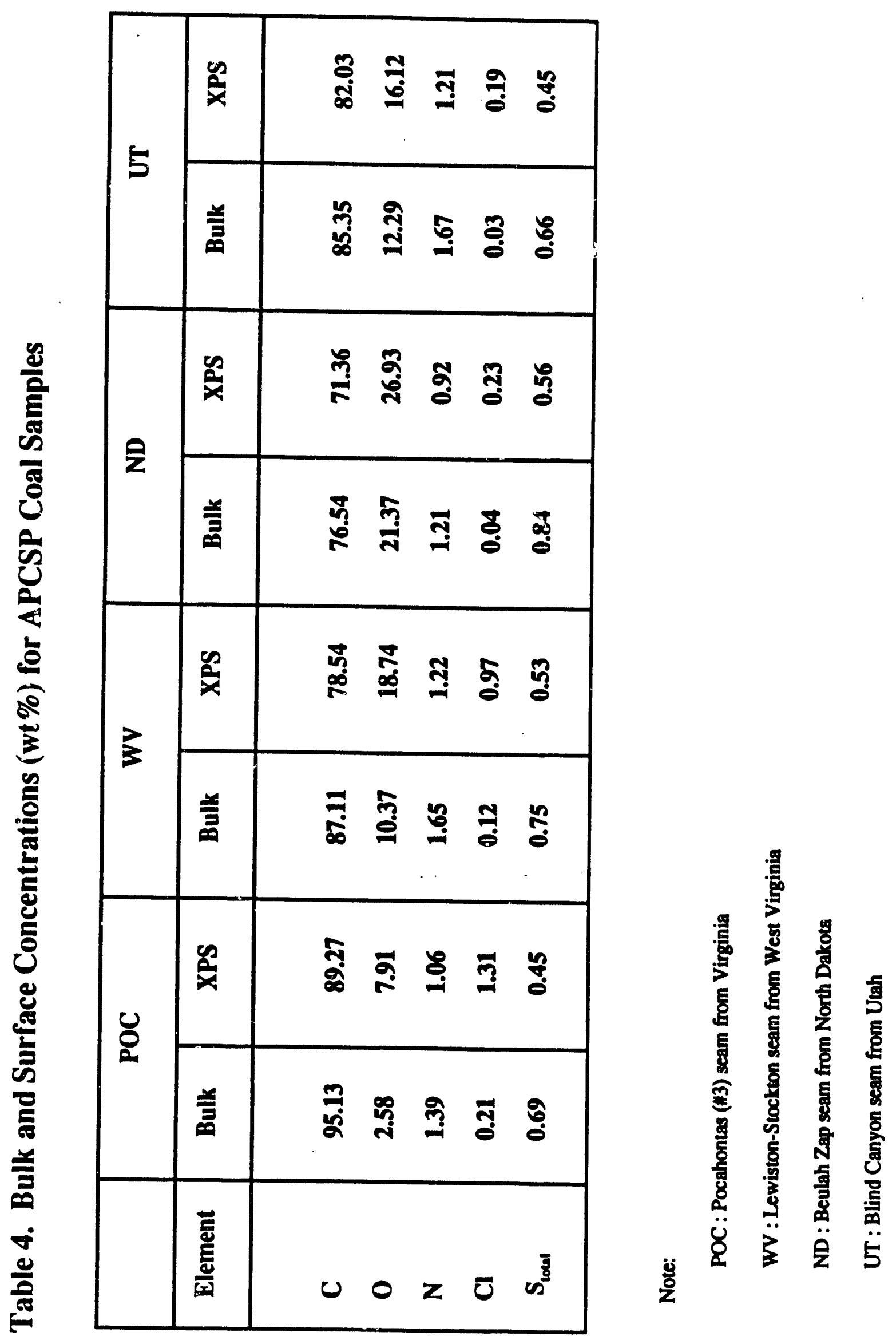




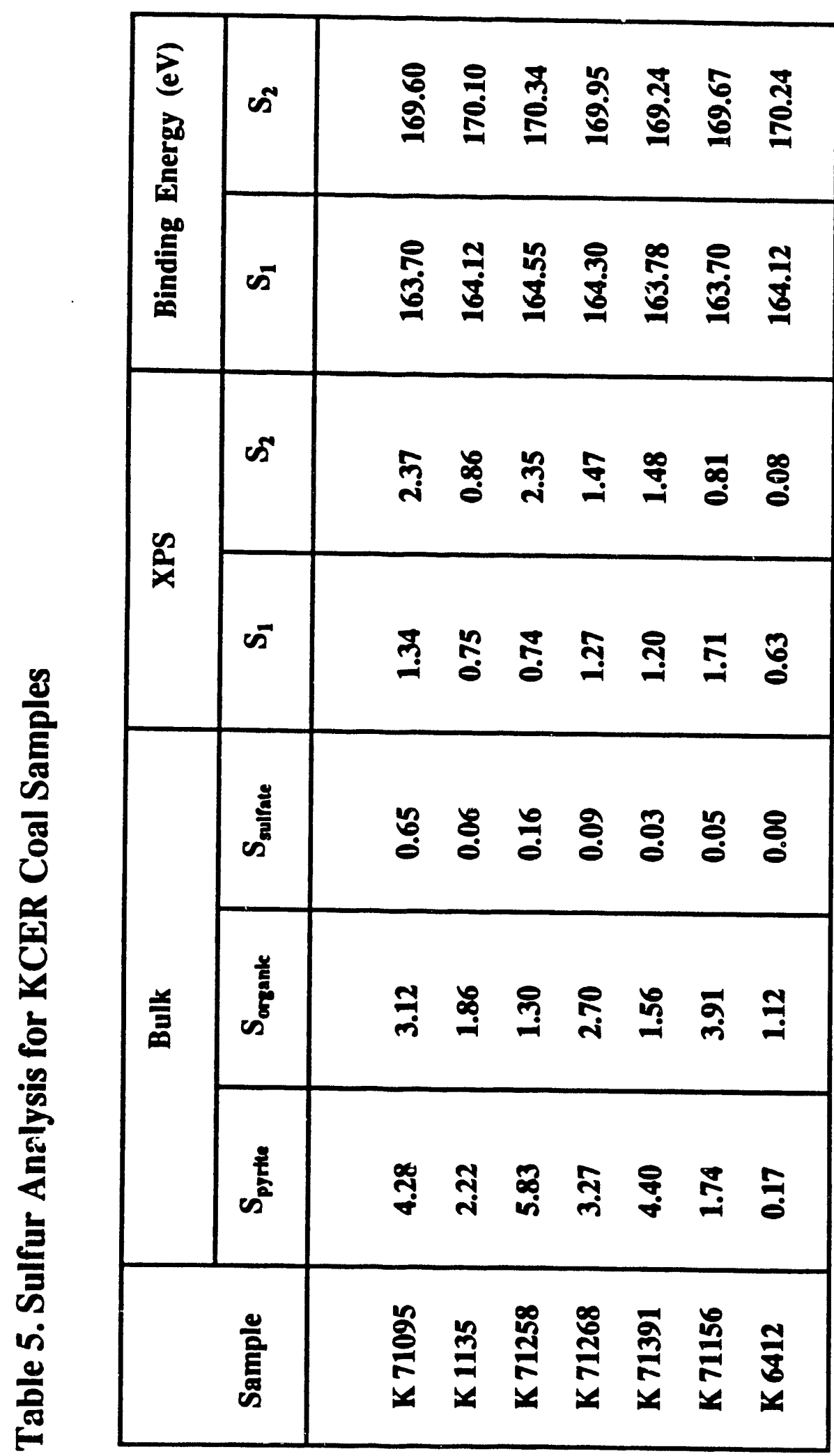

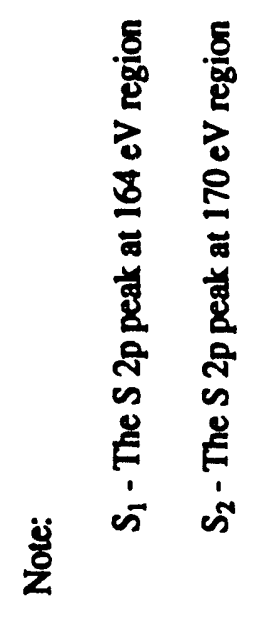




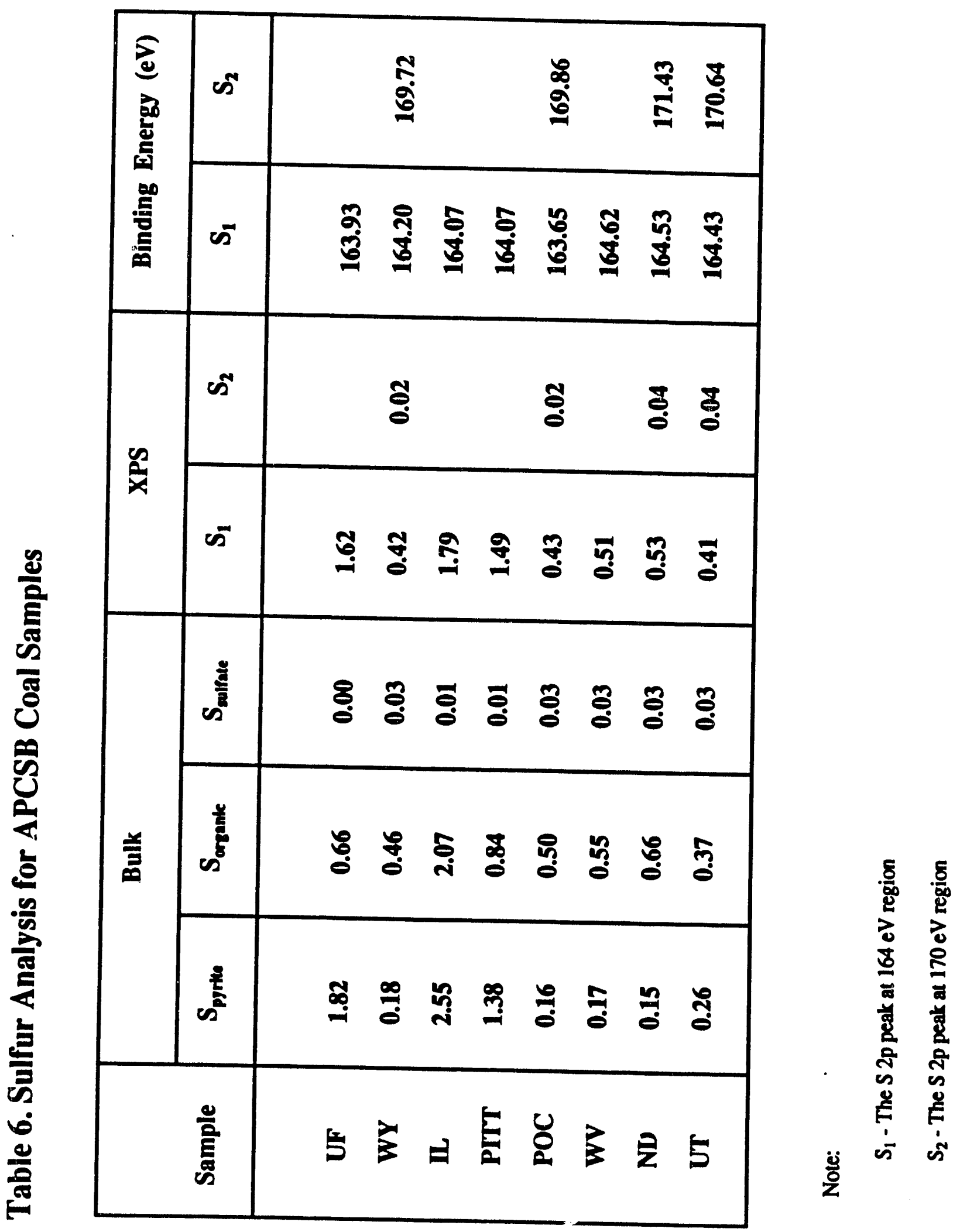


Table 7. Peak Synthesis of C $1 \mathrm{~s}$

\begin{tabular}{|c|c|c|c|c|}
\hline Sample & Hydrocarbon (C-H) & Hydroxyl (C-0) & Carbonyl $(C=0)$ & Carboxyl \\
\hline KCER 71095 & 82.9 & 10.6 & 4.5 & 2.0 \\
\hline KCER 1135 & 77.4 & 10.4 & 7.8 & 4.4 \\
\hline KCER 71258 & 83.3 & 13.1 & 2.5 & 1.1 \\
\hline KCER 71268 & 84.1 & 12.9 & 2.8 & 1.2 \\
\hline KCER 71391 & 83.8 & 12.7 & 2.6 & 0.9 \\
\hline KCER 71156 & 88.4 & 10.5 & 1.1 & \\
\hline KCER 6412 & 82.9 & 13.3 & 2.9 & 0.9 \\
\hline UF & 80.4 & 9.8 & 6.2 & 3.6 \\
\hline WY & 75.1 & 8.3 & 11.7 & 4.9 \\
\hline $\mathbf{n}$ & 80.3 & 10.7 & 5.8 & 3.2 \\
\hline PITT & 82.2 & 11.4 & 5.3 & 1.4 \\
\hline POC & 88.3 & 10.6 & & 1.1 \\
\hline WV & 79.8 & 11.3 & 6.6 & 2.3 \\
\hline $\mathbf{N D}$ & 71.8 & 17.6 & 6.9 & 3.7 \\
\hline UT & 81.3 & 12.2 & 4.1 & 3.4 \\
\hline
\end{tabular}




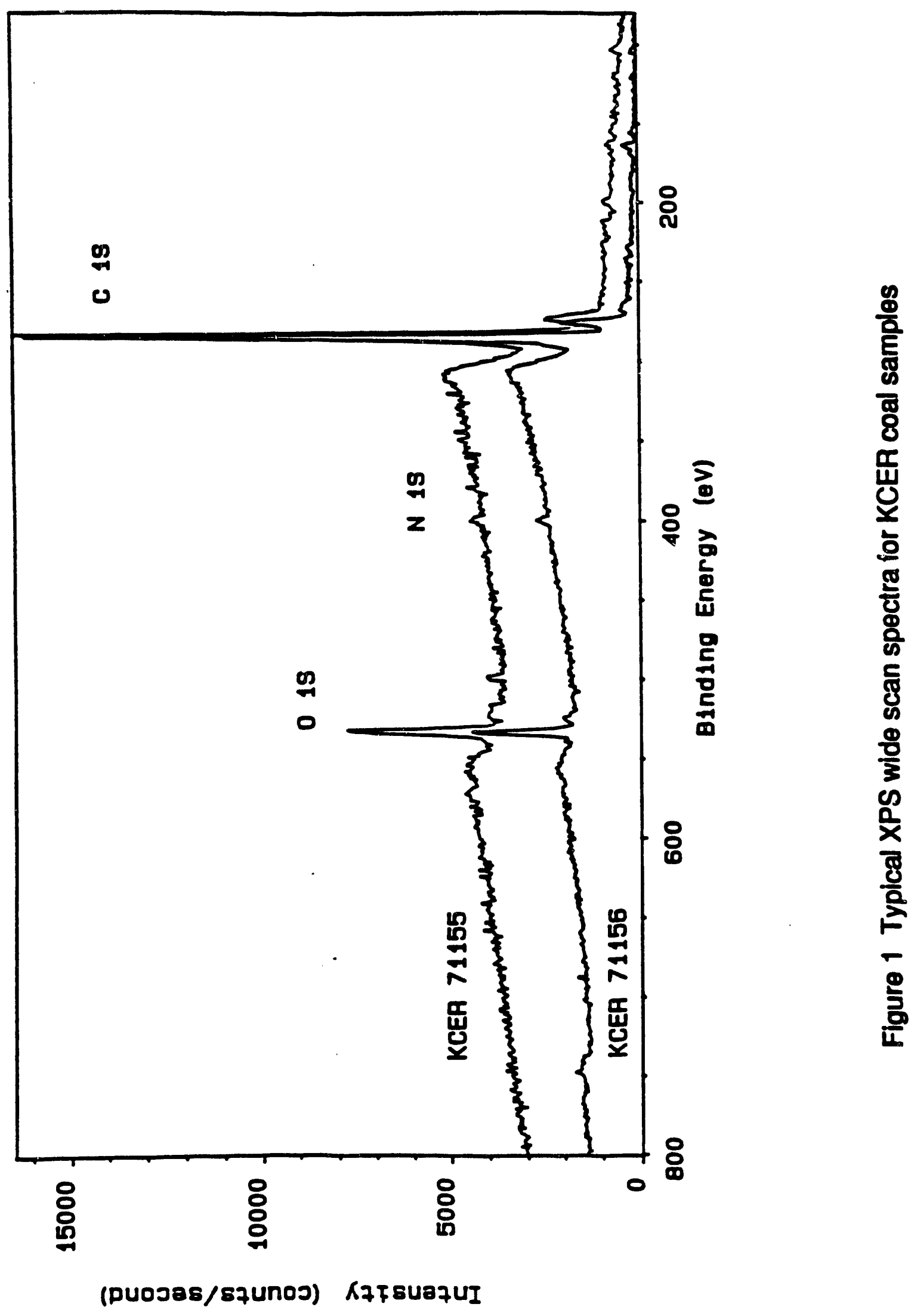




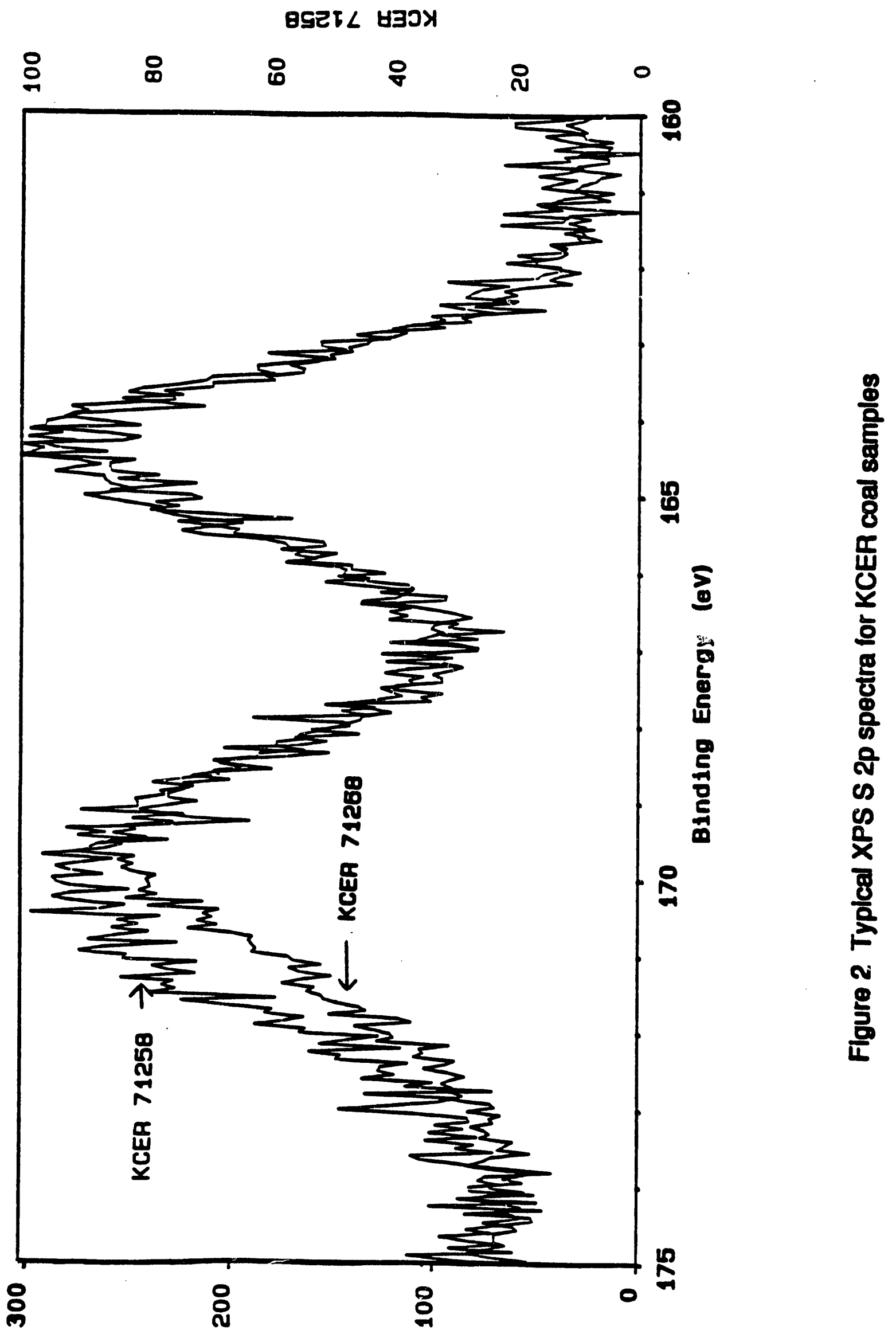

(puose8/8qunos) К7F8uequI 89ETL нコכX 


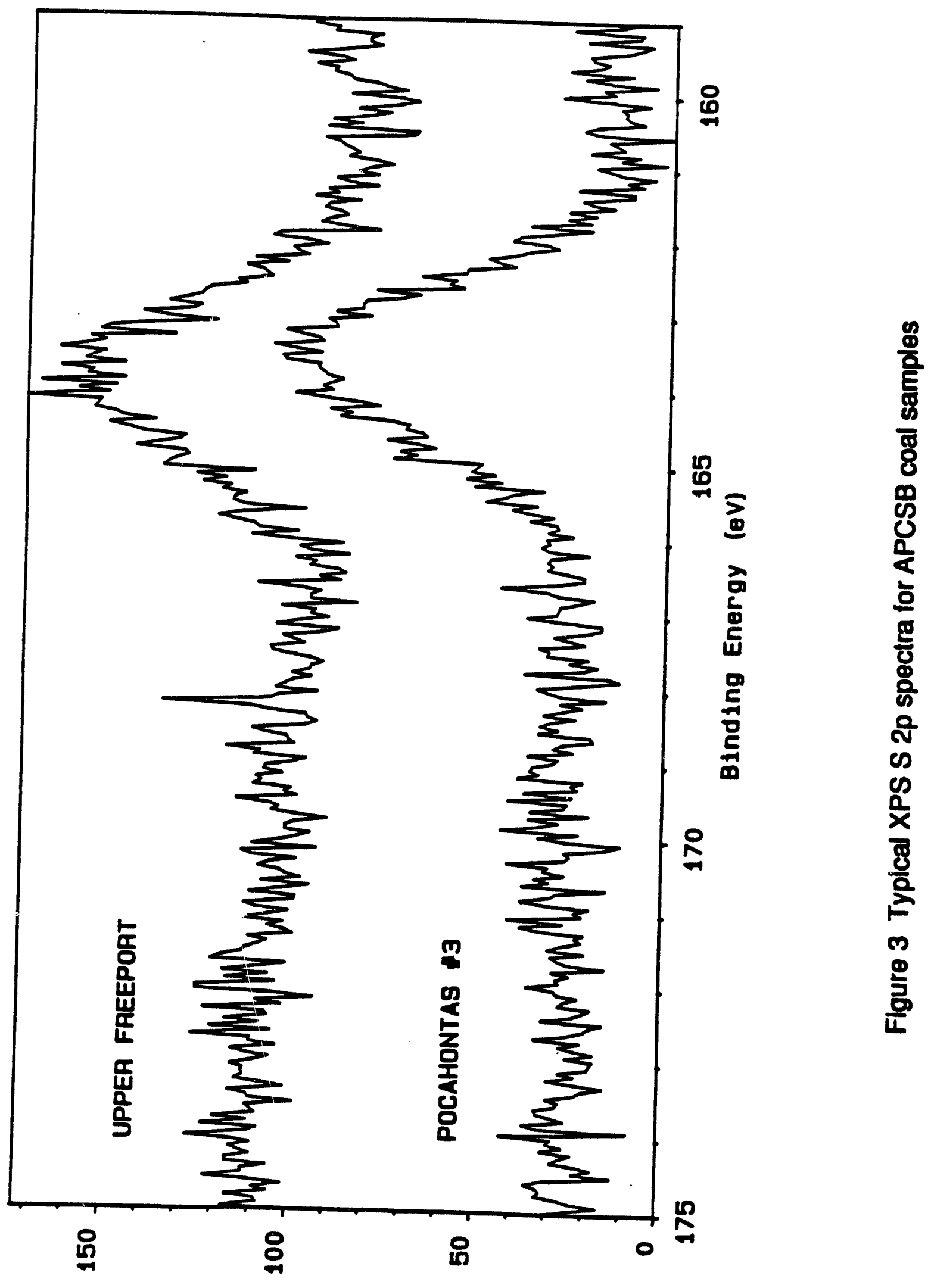

(puosas/squnos) K7FsuazuI 
Fig. 4 Comparison of suriace $S_{\text {total }}$ concentration by XPS with bulk analysis for a range of coals

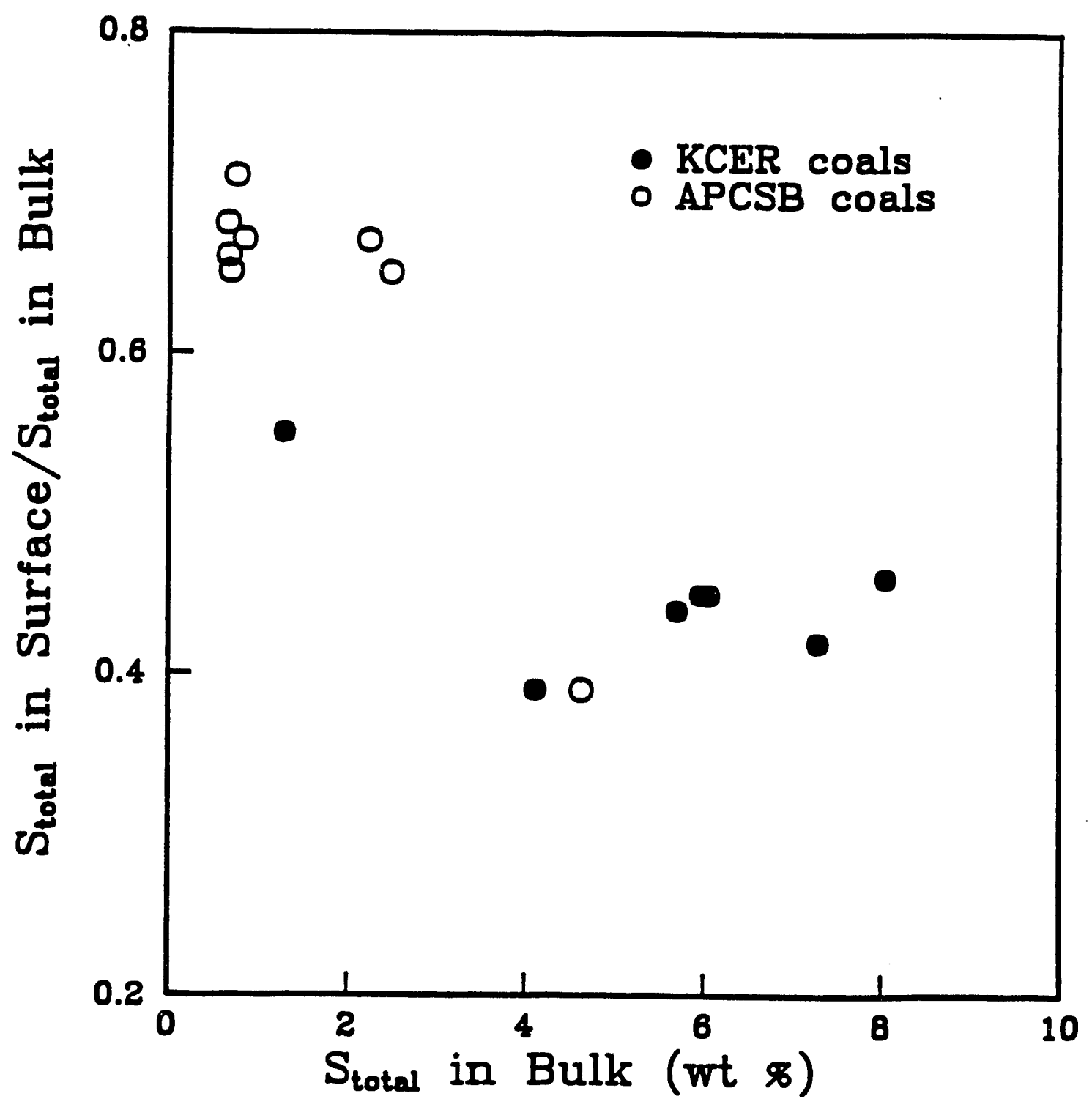


Fig. 5 Comparison of surface S(oranolotppttia) concentration by XPS with bulk analysis for a range of coals

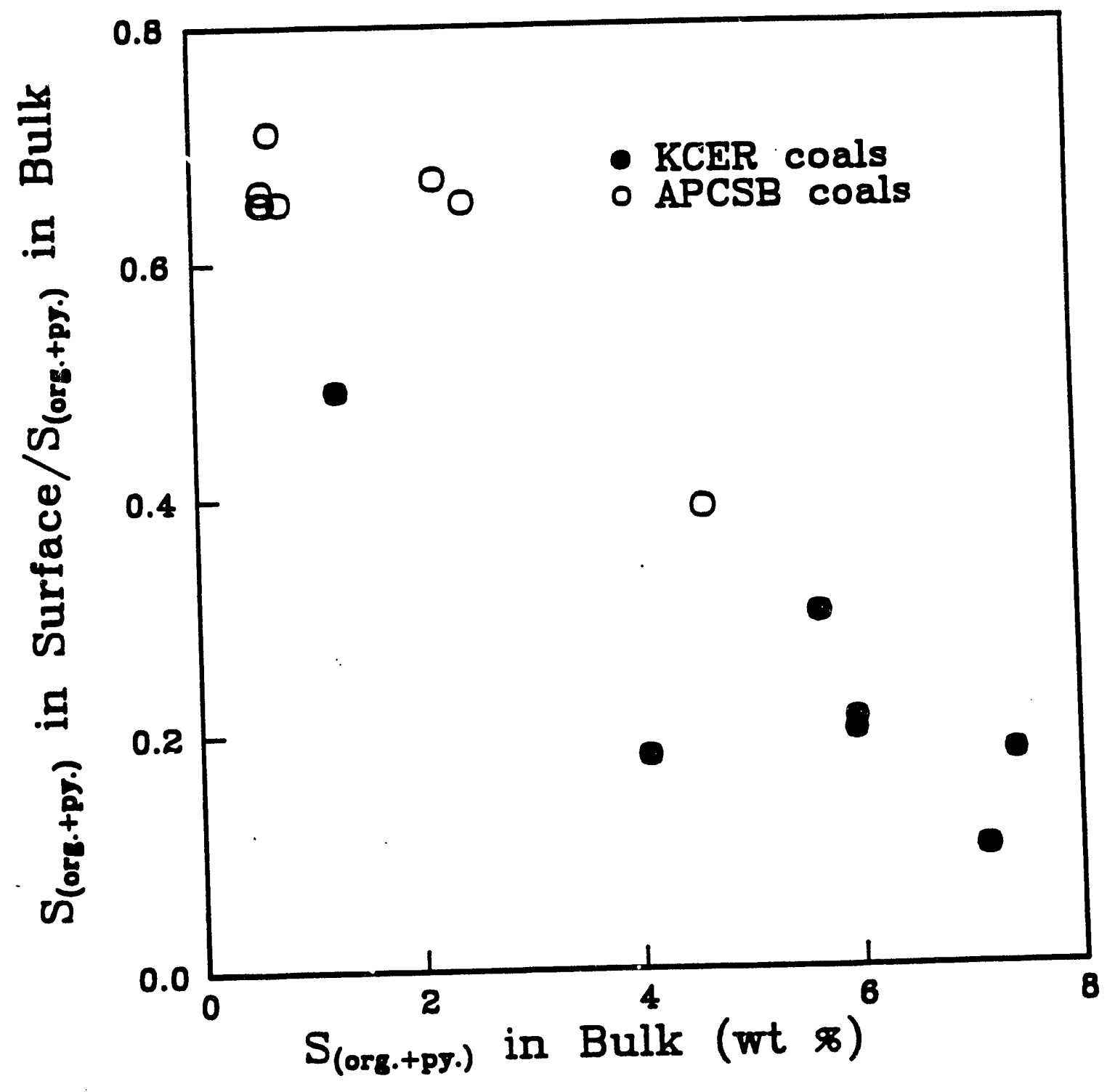




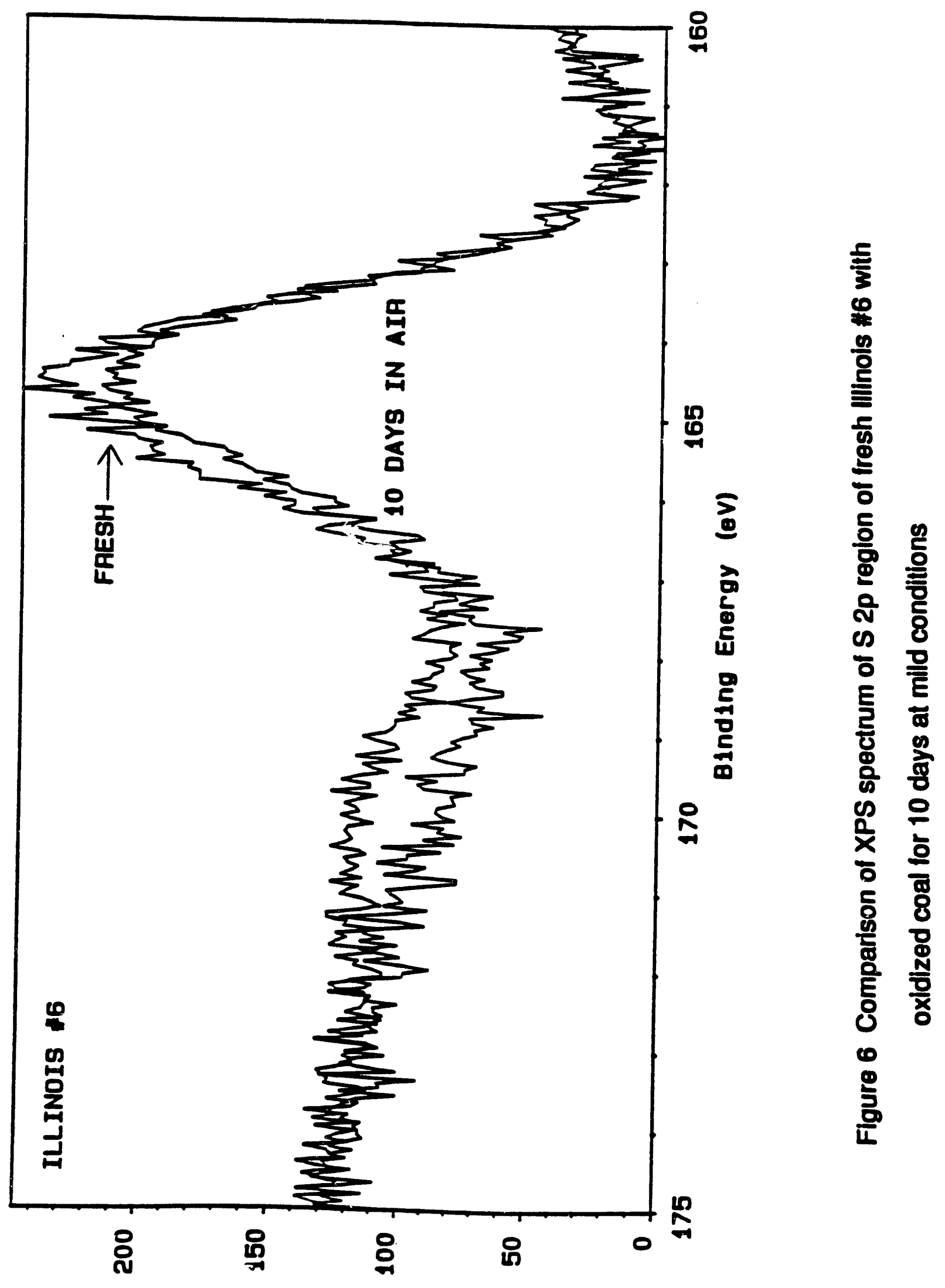

(puosas/s7unos) К7FsuazuI 

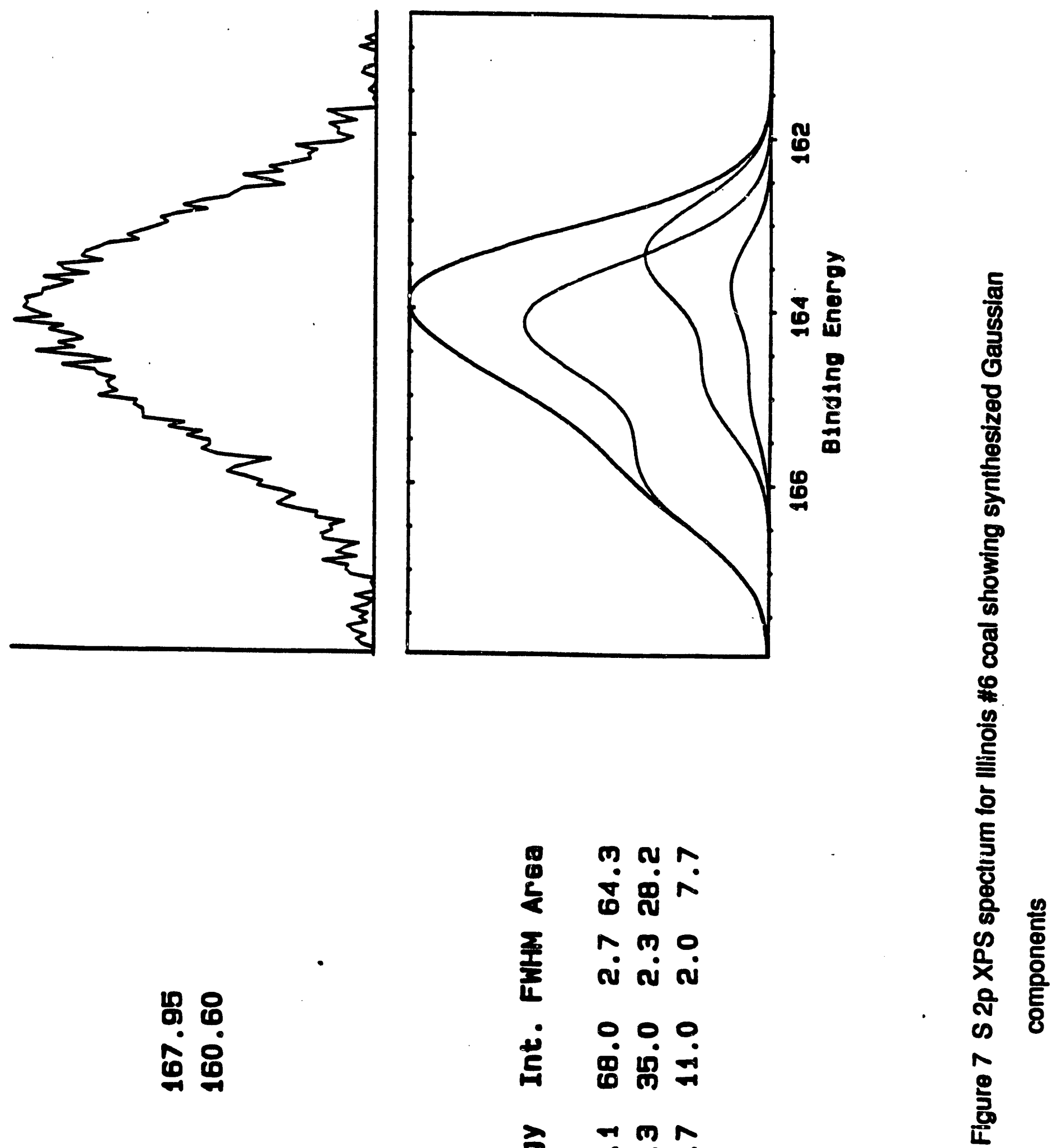

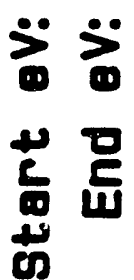

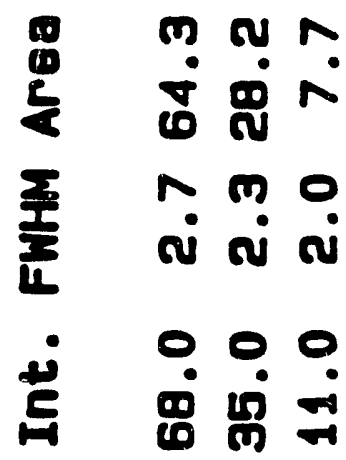

文

兽 क क 
Fig. 8 Comparison of surface 0 concentration by XPS with bulk analysis for a range of coals

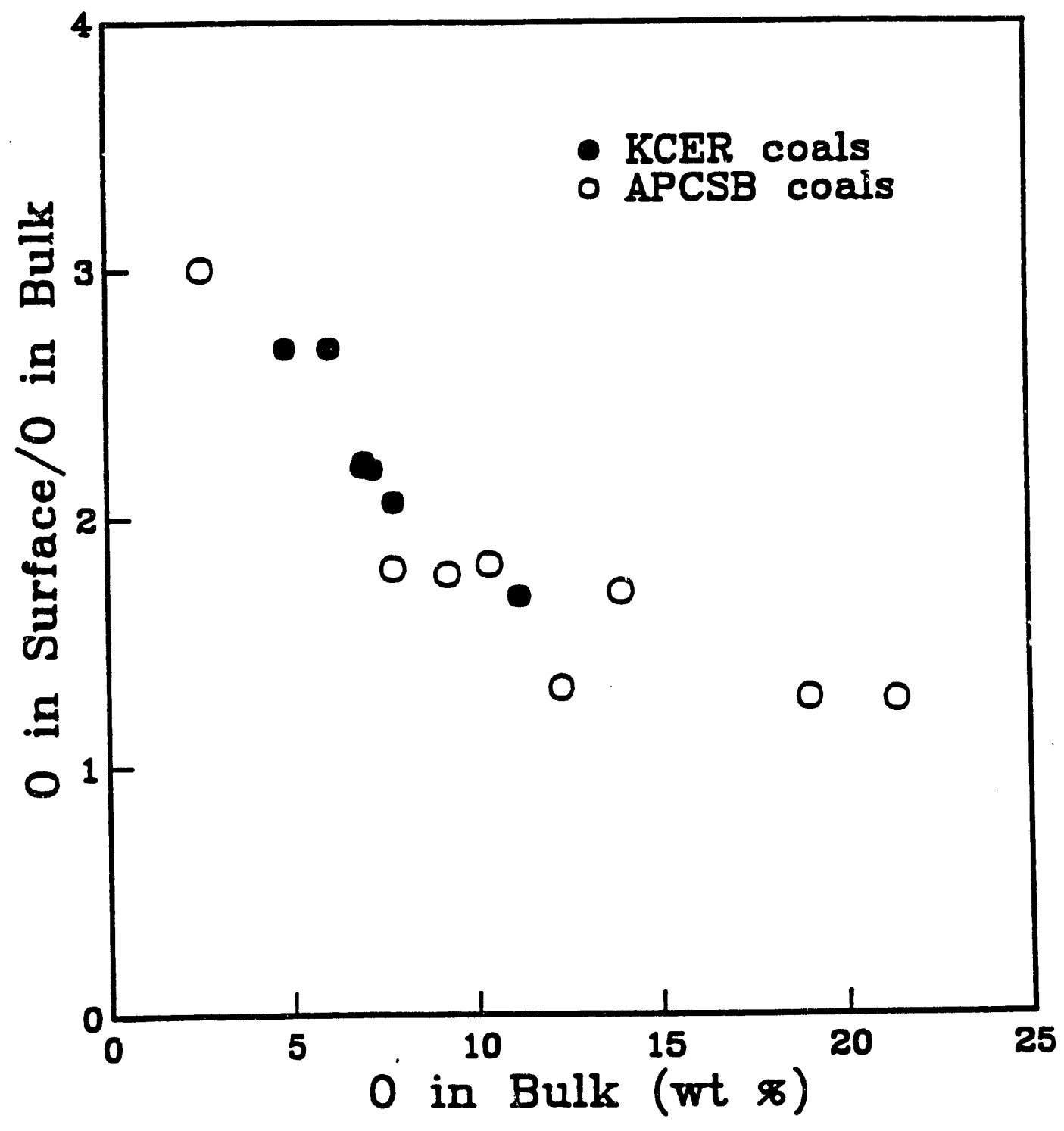


Fig. 9 Comparison of surface C concentration by XPS with bulk analysis for a range of coals

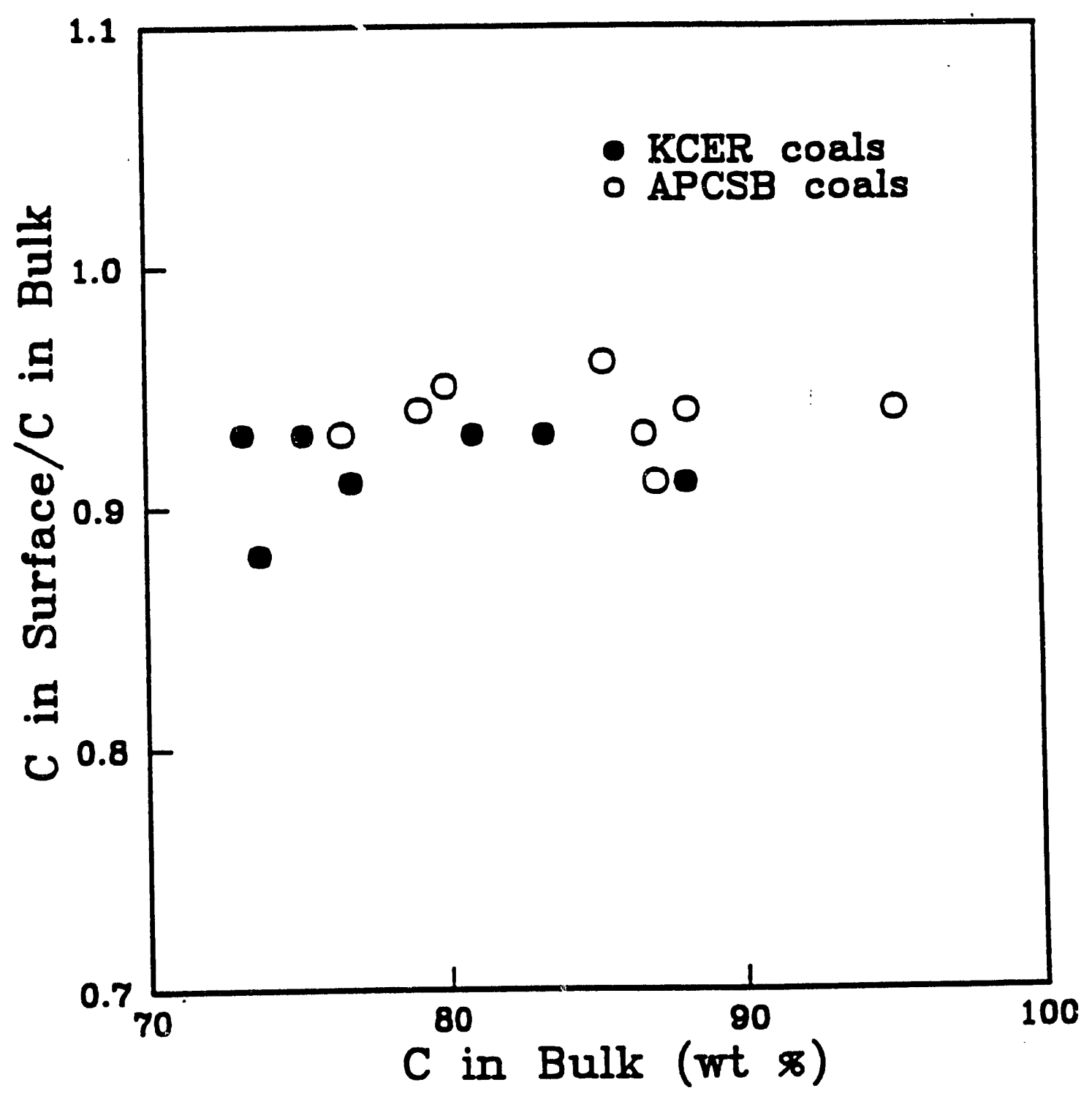



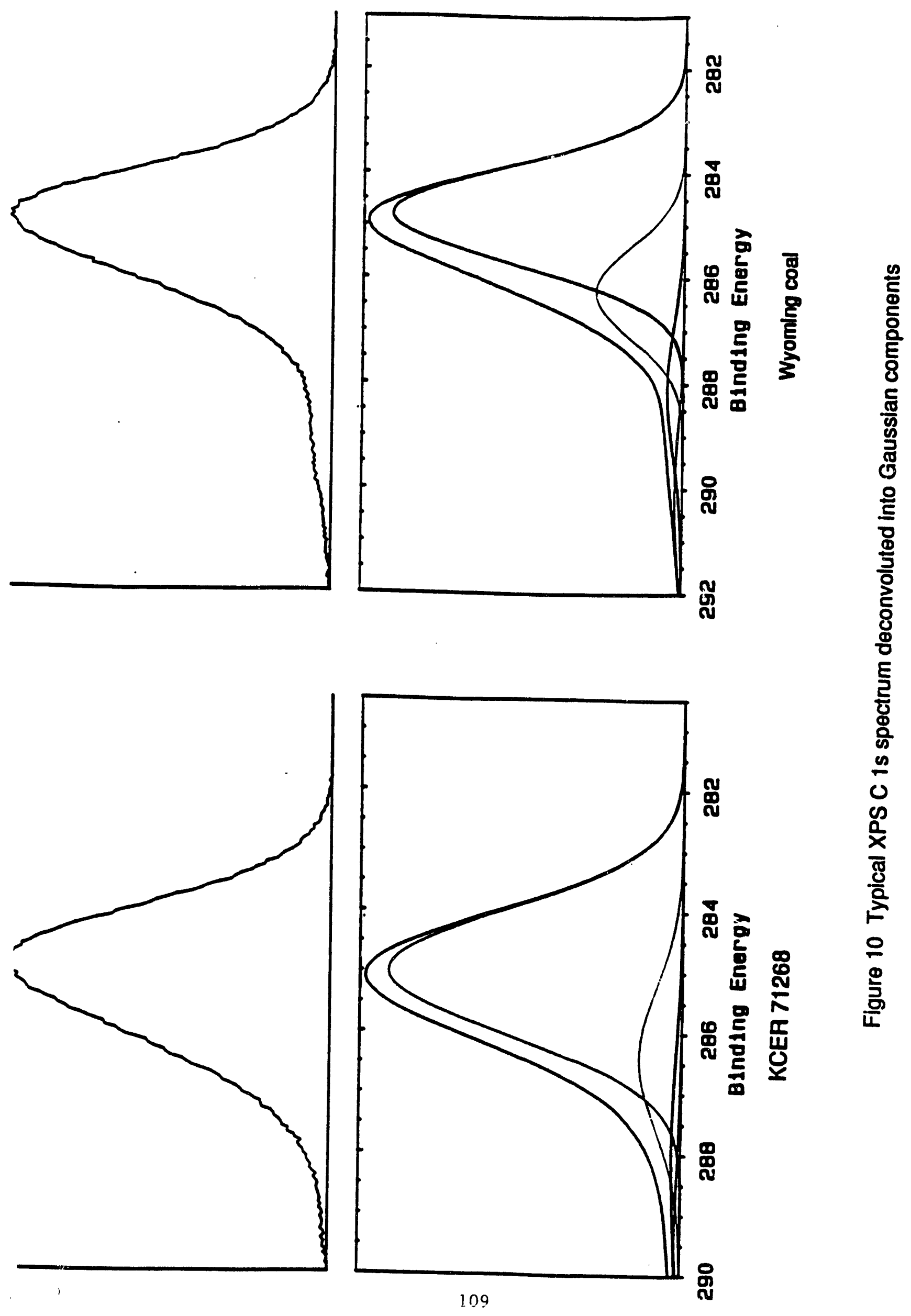

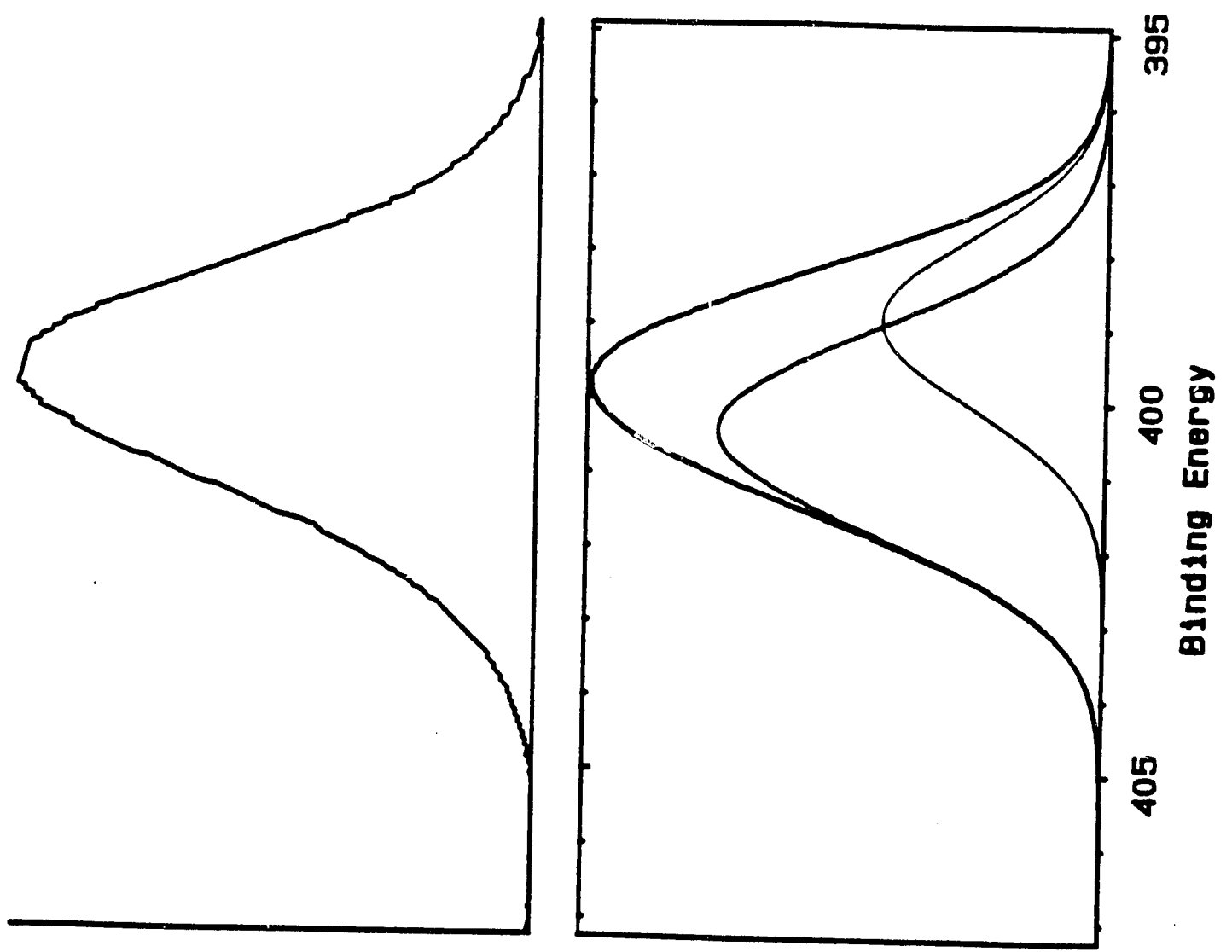

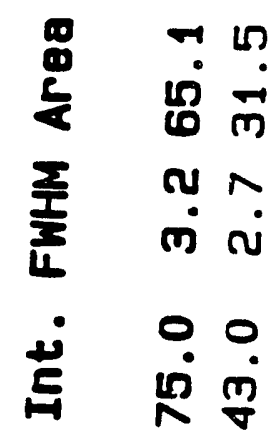

노ํ 욤

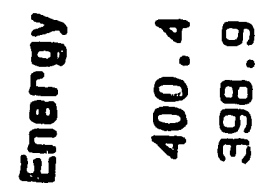
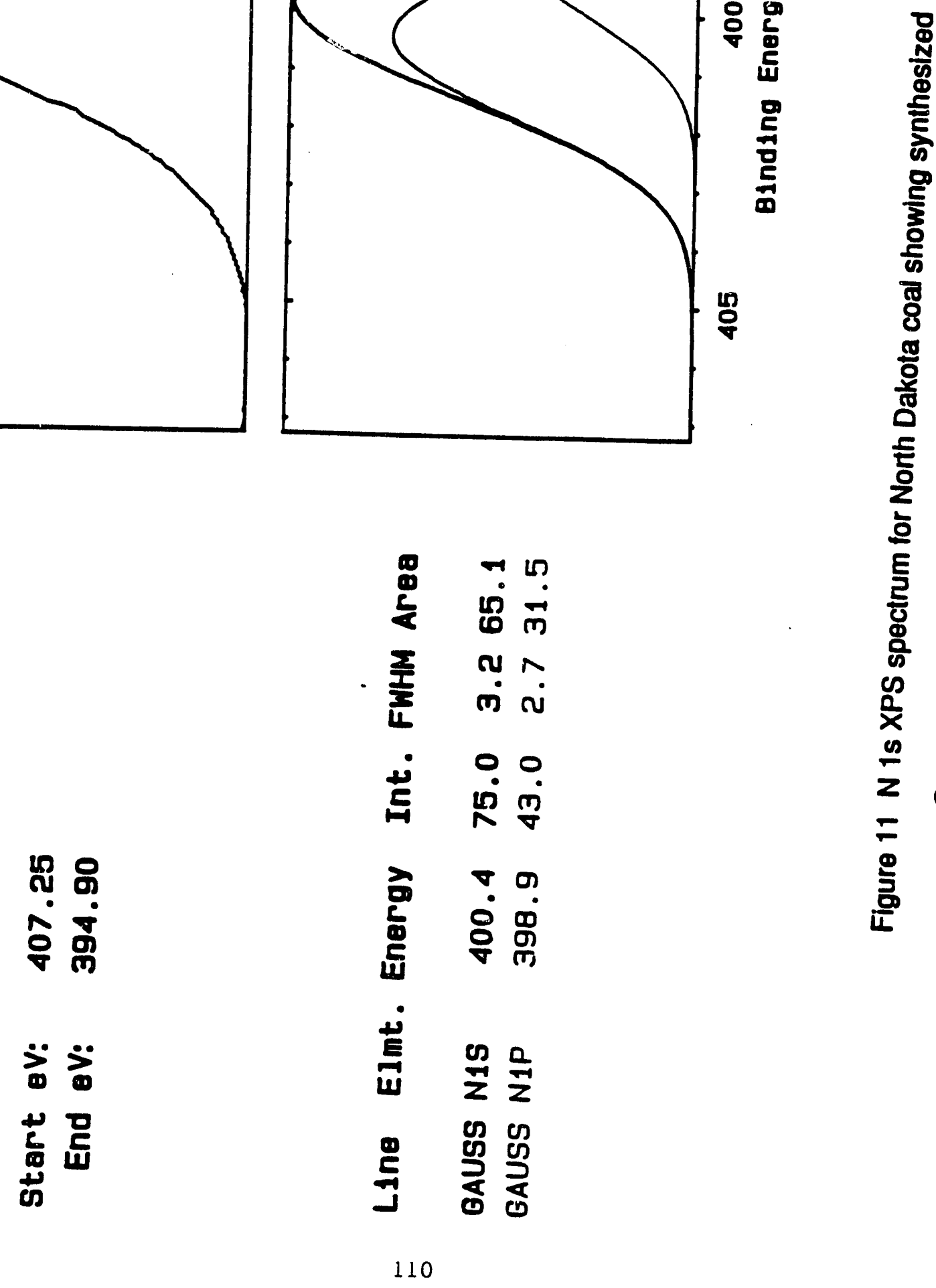

릉

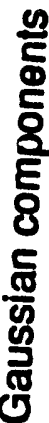

巨ั 
Fig. 12 Comparison of surface $N$ concentration by XPS with bulk analysis for a range of coals

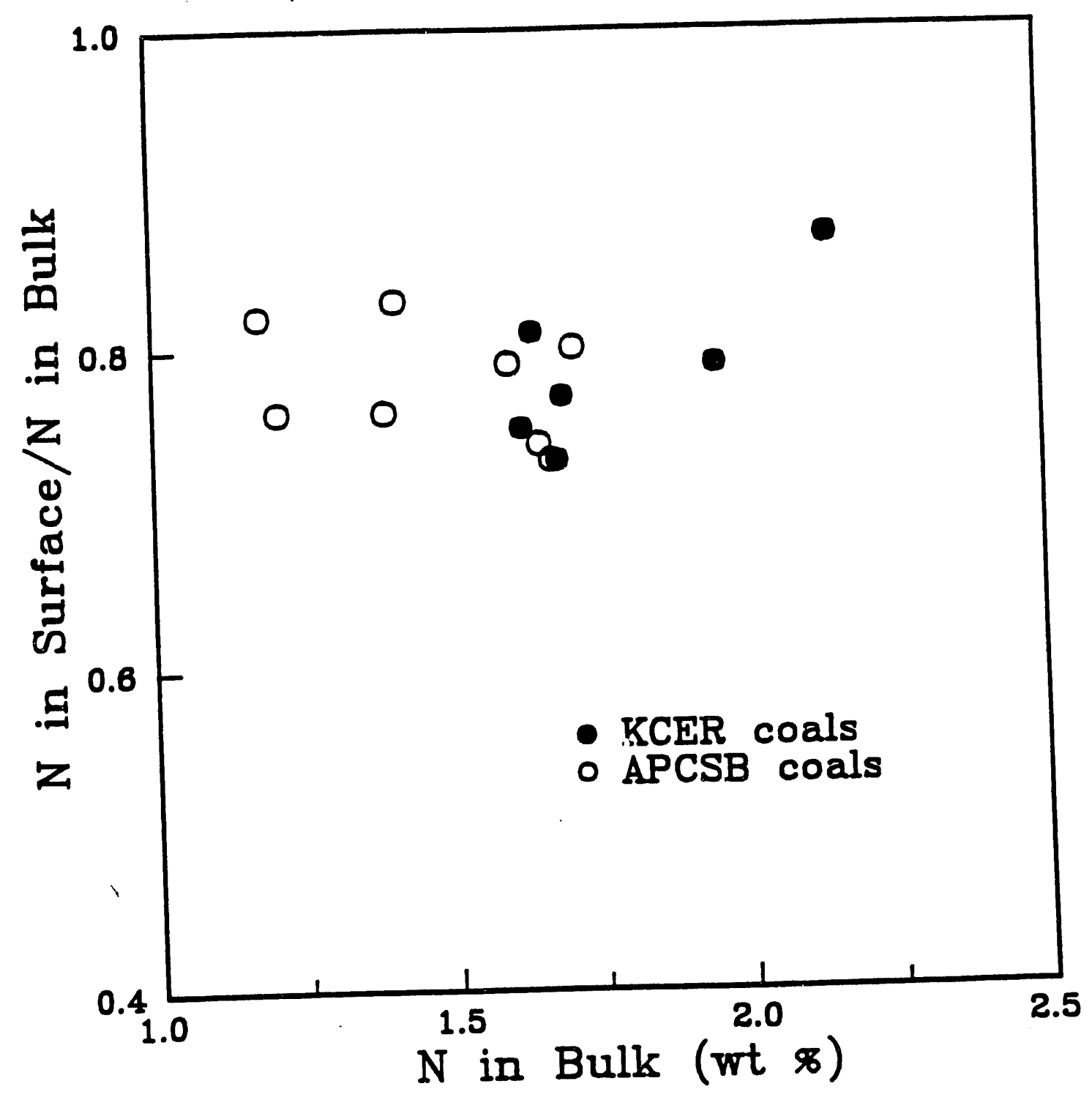




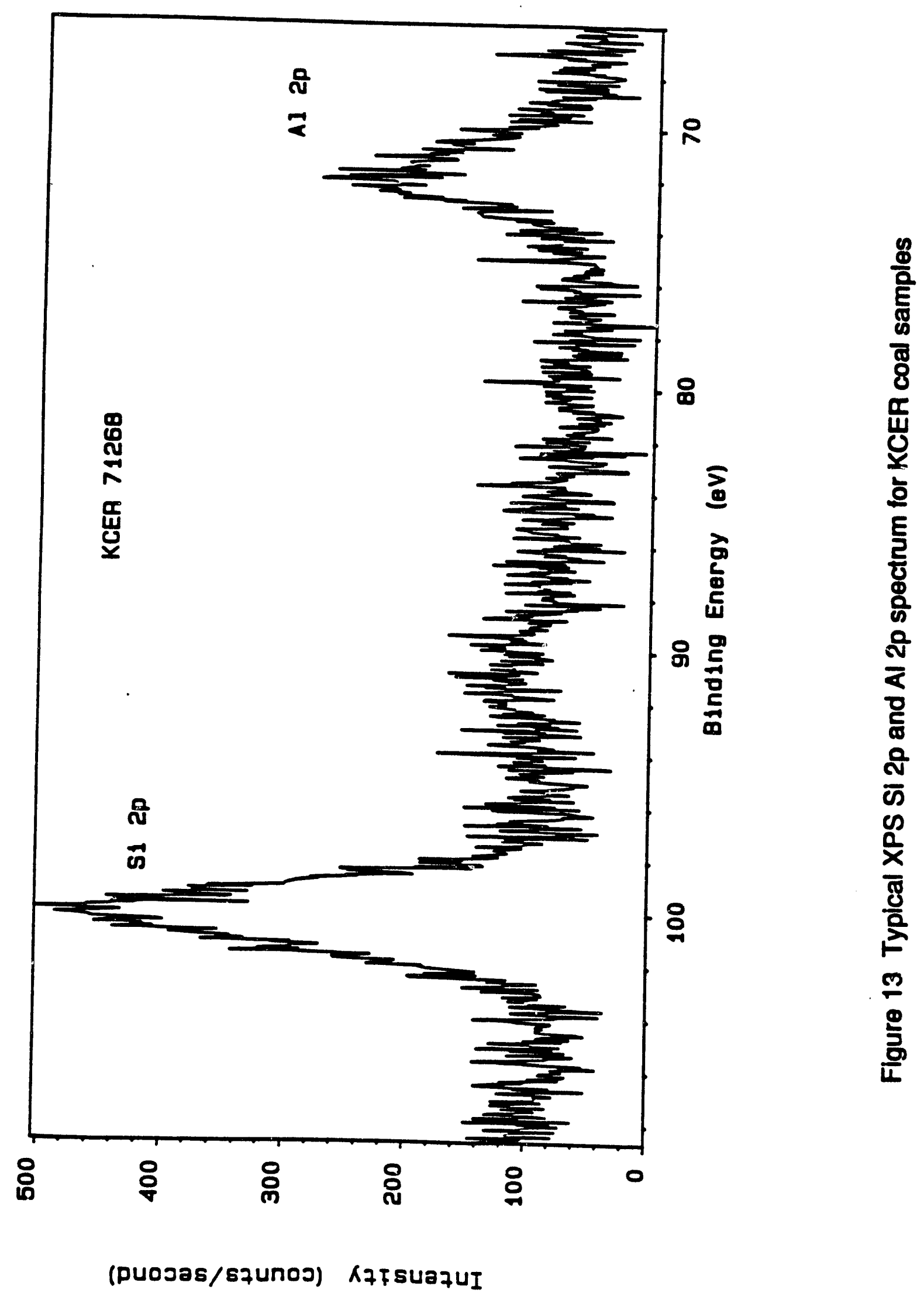


Fig. 14 Comparison of surface $\mathrm{Si}$ concentration by XPS with bulk analysis for a range of KCER coals

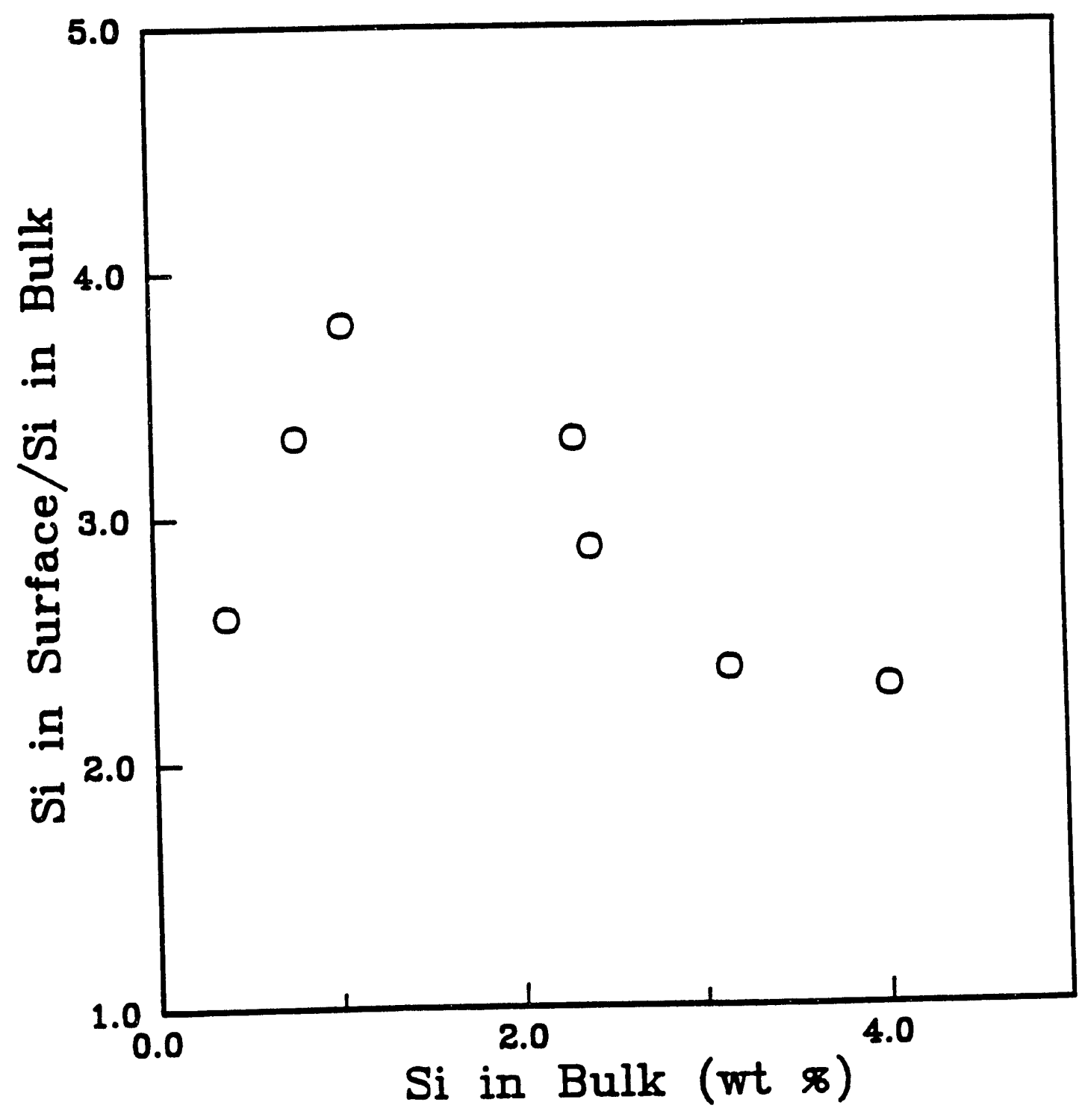


Fig. 15 Comparison of surface Al concentration by XPS with bulk analysis for a range of KCER coals

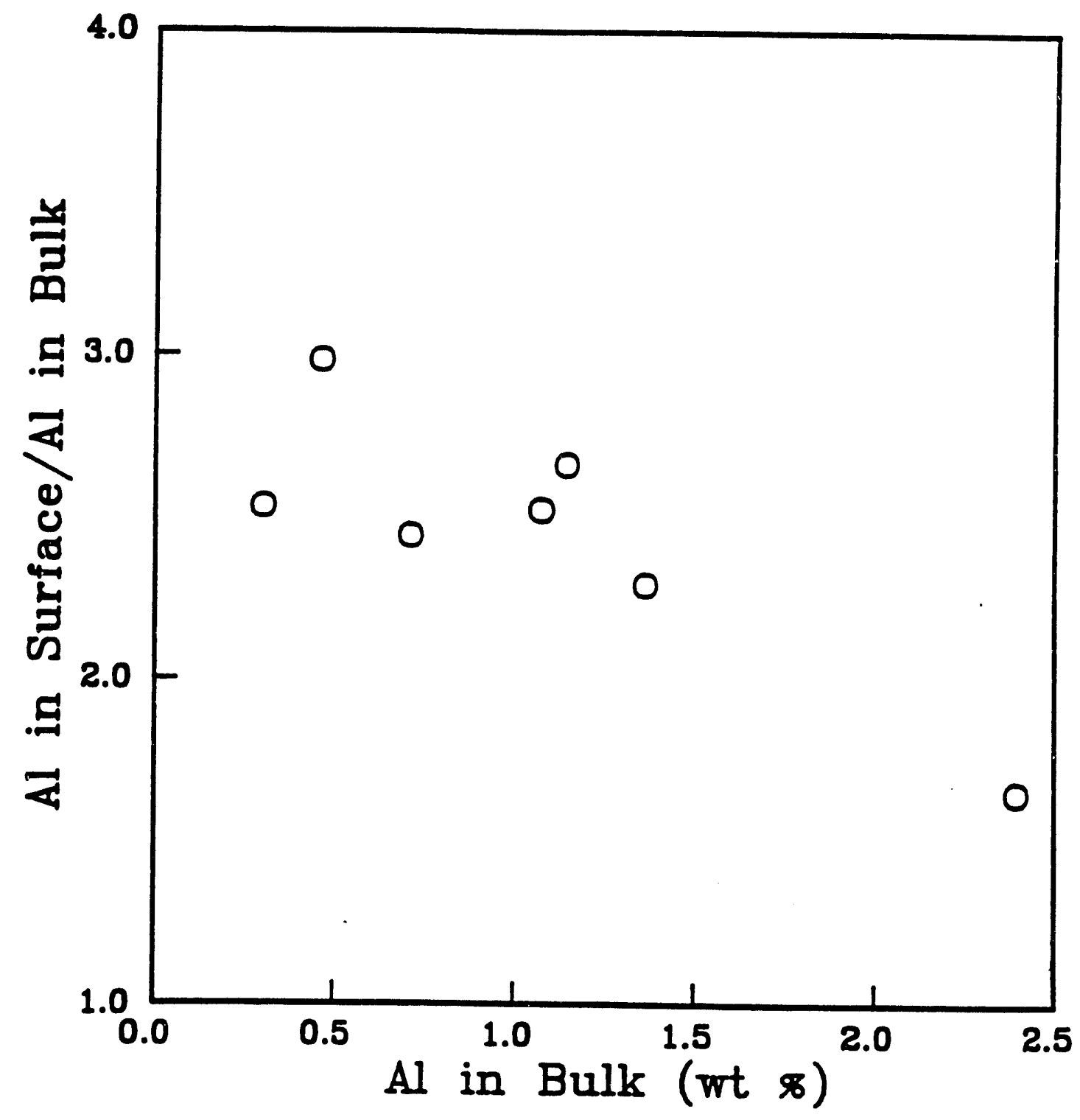




\section{Computer Modeling of Vitrinite}

(K.R. Subbaswamy)

We started the contract year with a study of the Shinn model for vitrinite. ${ }^{1}$ A fragment of the model was studied by the ASED-MO method described below. The geometry, the valence electron density, and bond dissociation energies were studied.

Given the complexity of systems of interest to coal liquefaction, it is very desirable to be able to assess various calculational methods by detailed comparison with experiments where possible. The first step in a coal liquefaction process is the cleavage of covalent bonds, such as the C-C single bonds in the alkylene linkages that connect the polycyclic aromatic portions of the coal structure. Thermal, uncatalyzed cleavage of these covalent linkages requires temperatures so high that undesirable retrograde reactions, leading to the formation of higher molecular weight products, as well as poor cost effectiveness, occur. Therefore, it is of great importance to develop environmentally nonpolluting and cheaper catalysts that allow the liquefaction to occur at lower temperatures at which undesirable side reactions are minimized. It is difficult experimentally to develop bond specific catalysts through the study of bulk coal because of its complexity. But the investigation of model compounds, which contain severa! ditferent kinds of representative linkages and aromatic structures found in bulk coal and are also solids or liquids under the conditions used for coal liquefaction, can more easily allow determination of activation energies, reaction rates and catalytic bond selectivity than in bulk coal.

For any modeling of coal to be significant, first it must be shown that the method employed in the study gives meaningful results for the model compounds for which experimental results are available. We, therefore, turned our attention to performing quantum chemical calculations on 4- (1-naphthylmethyl) bibenzyl (referred to hereafter as Model Compound I), studied recently by Farcasiu and Smith. ${ }^{2}$ In their experiments Farcasiu and Smith demonstrated the bond breaking selectivity of a novel catalyst, carbon black (BP 2000), and measured kinetic reaction rates and bond activation energies for cleavage of the methylene and ethylenc linkages of the compound.

In section 2 the specific semi-empirical method we use is described. Section 3 contains our results for Model Compound I. In the next section our preliminary results for a small fragment of the Shinn model are discussed.

\section{The ASED-MO Method}

Even though computational chemistry has advanced a great deal as a result of 
increasing computer power, the number of atcmic centers and electrons that can be treated reliably in an entirely $a b$ initio manner is rather limited. Thus, for our purposes semi-empirical methods are the only avenue. There is a plethora of methods, each with its own advantages and disadvantages and spheres of applicability. We have started with one of the simplest methods, a variant of Hoffmann's extended Hiickel method. ${ }^{3,4}$

The calculations were done ${ }^{6}$ with Anderson's ASED-MO method, ${ }^{6,7}$ adapted for the IBM 3090 computer by Onwood ${ }^{8}$ in an internal coordinate version. The one-electron ASED-MO (atom superposition and electron delocalization molecular orbital) theory is a modification of Hoffmann's extended Hückel molecular orbital theory (EHMO) which still retains its computational simplicity, ease of interpretation of results and reasonable computational time for calculations on large molecules.

The EHMO method is useful for qualitative predictions for molecules too large to be handled by more rigorous and time intensive methods. In one of the earliest EHMC studies, Hoffmann computed geometric conformations, ionization energies, and internal rotational barriers for a variety of organic molecules. ${ }^{3}$ He found equilibrium C-C single bond distances too long: and rotational energy barriers that were too low compared to experiment.

However, when bond distances were fixed at idealized values similar to experimentally determined values, Hoffmann found that predictions of three-dimensional conformation and relative trends, such as the variation of ionization energies and order of stability in a series of alkanes, were generally adequate.

The EHMO method with charge iteration was successfully used for a study of the absorption of a hydrogen atom in aluminum clusters. ${ }^{9.10}$ The method predicted the most stable absorption site to be an off-center tetrahedral site adjacent to an aluminum vacancy, in agreement with experiment and with the semi-infinite jellium calculations of Larsen and Norskov. "I In that study the Al-Al bond distance was fixed at experimental values and equilibrium Al-H distances were found for various sites of penetration of the hydrogen into the aluminum cluster, both with and without defects. Again the FHMO method appeared to work well at predicting general trends.

The ASED-MO method is an attempt to improve the binding energy curve calculation in EHMO theory. There are two significant modifications. The first one is in the Hamiltonian matrix. In the ASED-MO method, as in the EHMO method, the molecular orbitals are expanded in terms of Slater type orbitals for the valence electrons. The eigenenergies, $\varepsilon_{j}$, and the expansion coefficients are obtained from a solution of the secular equation, 


$$
\left|H_{i j}-\varepsilon_{j} S_{i j}\right|=0
$$

The Hamiltonian matrix $\mathrm{H}$ in ASED-MO is defined by

$$
\begin{gathered}
H_{i j}=-V S I E, \\
H_{i j}=\frac{K}{2}\left(H_{i j}+H_{j j}\right) S_{i j} \exp \left(-\delta R_{i j}\right) .
\end{gathered}
$$

The $S_{y}$ 's are overlap integrals and are calculated explicitly with Slatertype orbitals (STO). The valence state ionization energies (VSIE) and the exponents in the STO's are frequently adjusted slightly from experimental or theoretical norms in order to give numbers that are in closer agreement with experiment. ${ }^{4}$ Note that the off-diagonal matrix elements in (3) are different from the usual EHMO form of the Wolfsberg-Helmholz expression by the inclusion of the exponential factor. The constant, $K$, is taken to be 2.25 in the ASED-MO version, and the exponent $\delta$ is $0.13 \mathrm{au}^{-1}, R_{i j}$ 's are the distances between the various atom centers in the molecule. The practical effect of the exponential factor is to produce a sharper increase in the potential curve between any two atoms than the standard EHMO methods.

The second modification of EHMO in the ASED-MO method is the inclusion of specific pairwise repulsion terms, derived from the consideration of the Hellman-Feynman force theorem. ${ }^{.}$Nuclear repulsion terms, attenuated by nuclear attraction integrals, are included in each pairwise repulsion term. The attraction terms are computed with classical formulae, approximating the density due to $p$ and $d$ electrons by spherical distributions. Electronegativity values, while not used explicitly in the calculations, are used to differentiate between atoms in a pair and affect the calculated pairwise repulsion energy by selecting the atom in the pair whose electron density is used in the nuclear attraction integral. Calzaferri, Forss and Kamber ${ }^{12}$ have suggested an averaging method for calculating repulsion, which seems preferable to Anderson's single center method. The averaging method has not been used in the present calculations. Inclusion of the two modifications of Anderson significantly improves the validity of EHMO calculations, especially for studies involving geometry changes.

The ASED-MO method is best known for its predictions of molecular structure and molecular orbital ionization energies. ${ }^{13}$ Computed bond energies for low atomic number, nonmetallic homonuclear systems are quite good. In heteronuclear systems the bond 
energies must be considered to be approximate, but changes in energy due to geometric distortions and relative orderings of bonding energies in similar systems are believed to be accurate. $^{14}$

ASED-MO theory has been used successfully in such diverse areas as: predicting structure preference energies for $\mathrm{AB}_{2} \mathrm{O}_{1}$ spinels; $^{16}$ determining activation energies of methane on iron, nickel and platinum surfaces; ${ }^{16}$ studying adsorption of acetylene and ethylene on transition metal clusters; ${ }^{17}$ and investigating site stabilities and vibrational frequencies of carbon monoxide on nickel surfaces ${ }^{18}$. Structure and energy predictions with this method are usually superior to those of other semi-empirical methods, but bond energies are generally still too large. Therefore, only the relative magnitudes of these energies should be considered significant.

In the internal coordinate modification of ASED-MO theory, bond lengths, bond angles and dihedral angles of individual pairs may be varied, one by one, until a minimum in the ASED-MO total energy is reached. The input parameters, i.e., Slater exponents and VSIE's, used in the present calculations were those given in Hoffmann's FORTICON program and are listed in Table 1. With these parameters, the equilibrium distance for C-C single bonds is found to be $1.7 \AA$, an over-estimation of about $0.2 \AA$. Attempts to improve this distance by moderate adjustments of VSIE's and STO exponents led to less satisfactory charge distributions and descriptions of other bonds. Ultimately we decided to use the original Hoffmann parameters and the experimental distances for benzene for the aromatic portions of the molecule for the computations.

\section{Results for Model Compound I}

The Model Compound I of Farcasiu and Smith ${ }^{2}$ has the formula

$$
\mathrm{C}_{10} \mathrm{H}_{7} \mathrm{CH}_{2} \mathrm{C}_{6} \mathrm{H}_{4} \mathrm{CH}_{2} \mathrm{CH}_{2} \mathrm{C}_{6} \mathrm{H}_{8}
$$

with the skeletal structure shown in Figure 1. Before bond breaking energies for various bonds in the molecule were calculated, the ASED-MO minimum energy conformation for the molecule was determined. In the beginning, an initial geometric conformation was assumed in which all aromatic $\mathrm{C}-\mathrm{C}$ and $\mathrm{C}-\mathrm{H}$ bonds were assigned bond lengths of $1.394 \AA$ and $1.084 \AA$ respectively, and C-C.C and C-C-H bond angles of $120^{\circ}$. These bond lengths and bond angles were frozen at these values throughout the computations. Aromatic rings were kept planar throughout also. All aliphatic C-H bond lengths were fixed at $1.07 \AA$ and all C-C(sp $\left.p^{3}\right)-\mathrm{H}$ bond angles at $108^{\circ}$. These bond lengths and bond angles are not necessarily the ASED-MO theory equilibrium distances and angles. Bond lengths, bond angles and dihedral angles for all nonaromatic carbon atoms were then allowed to vary, one by one, until the desired 
self-consistent minimum in the ASED-MO total energy and geometry was reached. In general, bond distauces were minimized to $\pm 0.003 \AA$, bond angles to $\pm 2^{\circ}$, and dihedral angles to $\pm 5^{\circ}$. However, it must be remembered that the ASED-MO program used does not automatically find the "true" minimum energy conformation of the molecule. Minimization attempts were terminated when bond breaking energies were not significantly aifected by further changes in the internal coordinates.

The resill of the above minimization procedure is shown in Figure 2. There appear to be several conformations, both folded and extended, that are essentially equal to one another in energy, and differ from one another only in the rotation of the benzene groups about C-C single bonds. This is not surprising since there are no strong structural constraints in the molecule that would prevent fairly free rotation about C-C single bonds. Rotational energy barriers of 1.5 to $2.5 \mathrm{kcal} / \mathrm{mole}$ are calculated for the conversion of one conformation to another. Carlson ${ }^{19}$, using a molecular mechanics minimization program with a Dreiding force field, found a partially folded conformation to be lowest in energy. Other conformations were several kilocalories higher in energy.

Since the ASED-MO method is a one-electron method and neglects bond correlation energy and spin completely, dissociation of bonds into free radival fragments is not always observed in the calculation of the potential curve for dissociation as a function of internuclear separation. Iustead, ionic dissociation frequently occurs for unsymmetric cleavages. To calculate bond breaking energies for dissociation into free radicals, the ASED-MO energy for each free radical fragment, formed from the bond being broken, must be computed. The sum of the energy of the free radical fragments is then compared to the total energy of the parent molecule in its minimum energy conformation. Since no relaxation of the fragments has been allowed, this energy difference can be taken as an upper bound to the activation energy for the breaking of the bond under consideration.

The results of the computations of bond activation energies are summarized in Table 2. The bond labels are given in Figure 2. The energies for the extended and folded conformations are given in columns 1 and 2, respectively. For the neutral molecule in its extended conformation and in the absence of the BP 2000 catalyst, symmetric cleavage of the bibenzyl bond (bond $d$ ) is found, with a bond activation energy of $3.77 \mathrm{eV}$. Bonds $a$ and $b$ are the next easiest to break, requiring 0.32 and $0.12 \mathrm{eV}$ more energy, respectively, than for bond. Bonds $c$ and $e$ require the most energy for cleavage.

The order of the bond activation energies is independent of geometric conformation as can be seen by comparing the results for the extended vs. the folded conformations. However, the activation energy for the breaking of bond $d$ from the folded conformation is about $0.1 \mathrm{eV}$ 
lower in energy than from the extended conformation. The energies for breaking bonds $a$ and $b$ are essentially unchanged on folding and bonds $c$ and $e$ are stabilized on folding. (See Tables 2 and 3.) This can be explained by looking at overlap populations for model compound I. The geometry about bonds $a$ and $b$ is essentially unaffected by the folding process. Therefore, the overlap population between the carbon atoms in these bonds and thus the bond breaking energies are virtually unchanged. In the folding process the bond length of the C-C bond of the bibeizyl portion of the compound increases by $0.012 \AA$ which leads to a decrease in the overlap population and bond breaking energy for bond $d$. There are slight increases in the overlap populations of bonds $c$ and $e$ and, therefore, in the stabilization of these bonds.

Farcasiu and Smith ${ }^{2}$ have studied the decomposition reactions of Model Compound I. In the absence of a hydrogen donor compound and in the absence of the BP 2000 catalyst, cleavage of bond $d$ is favored. Smaller amounts of products from the cleavage of bonds $a, b$ and $c$ are also found. Thus, the results from the ASED-MO calculations are in qualitative agreement with these experimental findings.

In the presence of a hydrogen donor, 9,10-dihydrophenanthrene, and with no catalyst present, bonds $a$ and $d$ are found to cleave to almost the same extent and bond $b$ to a limited extent. 2 The decomposition reaction for bond a follows first order kinetics with an activation energy for cleavage of $60 \mathrm{kcal} / \mathrm{mol}(2.6 \mathrm{eV})$ for bond $a$. The activation energy for the cleavage of bond $d$ is $70 \mathrm{kcal} / \mathrm{mol}(3.0 \mathrm{eV})$ linder the same conditions. No attempt has yet been made to model the effect of a hydrogen donor with the ASED-MO rnethod.

This model compound has also been studied by Meuzelaar and Huai ${ }^{20}$ with the low voltage (12 eV) Py-MS method. From mass spectrometry data, ions from the cleavage of bond $d$ are found to be the most numerous. There are also ions present from the cleavage of bonds $b, a$ and possibly $c$, with those from bond $b$ being slightly more numerous than those from bond $a$.

In the absence of catalyst and hydrogenating agent, Farcasiu and Smith ${ }^{2}$ found the product distribution to be about $64 \%$ from the breaking of bond $d, 17 \%$ from bond $a, 3 \%$ from bond $b$ and $16 \%$ from bond $c$. If one uses a Boltzmann distribution

$$
N_{i} / N_{0}=\exp (-\Delta E / k T)
$$

to estimate prcjuct distributions from the computed ASED-MO energies, the distribution predicted at $400^{\circ} \mathrm{C}$ gives $88-98 \%$ from bond $d, 0.07-0.4 \%$ from bond $a, 2-11 \%$ from bond $b$ and $0.001-0.03 \%$ from bond $c$, depending on whether or not the molecule is in the extended or folded conformation. In comparing these to the expeximental distributions, it should be zemembered that the latter are affected by kinetics not taken into account in the ASED-MO 
theory. Our calculations are in closer agreement with the mass spectrometry data than with the diroct thermolysis data.

When the catalyst, carbon black (BP 2000), is added to the model compound in the absence of a hydrogen donor, Farcasiu and $S_{m i t h}{ }^{2}$ find that products resulting from the cleavage of bond $a$ now predominate, accompanied by large decreases in products from the cleavage of bonds $d$ and $c$. There is an increase in the amount of reactant conversion at a given temperature in the presence of the catalyst also. In the presence of the hydrogen donor, bond $a$ is cleaved almost exclusively, with an activation energy for cleavage between 17 and $25 \mathrm{kcal} / \mathrm{mol}$ (i.e., 0.74 to $1.1 \mathrm{eV}$ ), depending on the amount of catalyst present. Since the same preference for bond cleavage is found both in the presence and in the absence of the hydrogen donor, a mechanism other than a "solvent mediated hydrogenolysis" , such as that proposed by McMillen, et $a .^{21}$ for cleavage of bonds between methyl groups and polycyclic aromatic radicals in the presence of hydrogen donors, is most likely. One of the possibilities proposed by Farcasiu ${ }^{23}$ is the formation of a positive radical ion from the compound as a result of the catalytic activity of carbon black (BP 2000).

We have examined this proposed mechanism with the ASFD-MO method by placing a +1 charge on Model Compound I. The electron removed comes from the $\pi$ orbitals of the naphthyl functional group. This is an expected result since it is known from UV-photoelectron spectroscopy that naphthalene has a lower first ionization energy $(8.15 \mathrm{eV})$ than benzene $(9.25 \mathrm{eV}$ ) or aliphatic carbon atoms (about $12 \mathrm{eV}){ }^{22}$ ASED-MO results are also consistent with this experimental observation. Bond separation energies for the positive radical ion are calculated as before. No relaxation of the molecule after the removal of the electron nor relaxation of the fragments after rupture of the bonds has been allowed. The bond breaking energies are calculated by always placing the +1 charge on the fragment containing the naphthyl group (see Figure 1). Thus, the energies of the species that occur after ionization (the positive radical ion and the positively charged fragment containing the naphthyl group) increase by the energy of the HOMO of the corresponding neutral parent.

The other fragment is considered to be a free radical. Dissociation energies calculated in this manner for the positive ion are shown in Table 3. Once again, values for both the extended and the folded conformations are shown.

For the positive radical ion, the ASED-MO method predicts that bond lt will be easier to break than bond $d$. The activation energies for cleavage of all bonds have also been lowered, which would lead to greater conversion of reactants at a given temperature. It can also be seen, in Table 3 , that the conformation of the radical ion again does not greatly affect bond breaking energies and certainly not the ordering of the energies. 
As can be seen in Table 3, all bonds are easier to break when the +1 charge is placed on the molecule, not just the bonds closest to the naphthyl ring. The reason for this, in the context of our calculation may be understood from the following argument. The electron removed in the formation of the positive radical ion comes from the highest occupied molecular orbital (HOMO) of the model compound, essentially centered on the naphthyl portion of the compound. In the ionization process, the largest bond order changes occur in the vicinity of the naphthyl moiety. All bonds that are not adjacent to this naphthyl group are virtually unaffected, as can be seen in the bond orders for the neutral compound and its ion in Table 4. As a result, the total energies of fragments not containing the naphthyl portion are unchanged in the transition from the neutral to the positive Model Compound I. With no relaxation allowed, the change in bond energy for any bond when going from dissociation of the neutral molecule to that of the positive radical ion, is simply the difference between the HOMO energy of the neutral model compound and the HOMO energy of the fragment containing the naphthyl moeity after the bond is broken. The former is always more negative than the latter, thus explaining why this change in bond dissociation energy is negative for all bonds in our calculation.

One might then question why the HOMO of the fragment containing the naphthyl moeity should change as the various bonds are cleaved. This is easy to understand when one notes that cleavage of bonds $b, d$ and $e$ results in the fragment containing the naphthyl group having a terminal alkyl radical, while the remaining cases ( $\alpha$ and $c$ ) give rise to fragments containing terminal aryl radicals. With the computed molecular orbitals of benzene and the phenyl radical and of toluene and the benzyl radical, one can examine the rearrangement of the orbitals after breaking of the $\mathrm{C}-\mathrm{H}$ bond and formation of the free radical. In benzene and toluene the HOMO's are $\pi$ molecular orbitals. In the radicals, $\sigma$ MO's on the phenyl radical and $\sigma$ MO's centered on the $\mathrm{CH}_{2}$ group in the benzyl radical become HOMO's. The HOMO of the benzyl radica] is lower in energy than the HOMO of the phenyl radical. In other words, the electron in a $\sigma$ HOMO of an aryl radical is not as tightly bound as an electron in the $\sigma$ HOMO of the alkyl radical. A similar observation is found when examining naphthalene, 1-methyl naphthalene and the related radicals.

An examination of the MO's of the neutral molecule shows that many of the energy levels, even deep-lying levels, have a mixing of atomic orbitals from all parts of the molecule. Thus, cleavage of any bond leads to a quite drastic rearrangement of the electron density over the whole molecule. This makes it very difficult to give a simple explanation as to why the ordering of the bond dissociation energies change in going from cleavage of the neutral molecule to cleavage of the positive radical ion. 
In the presence of the catalyst, the product distribution observed by Farcasiu and Smith is about $34 \%$ from bond $d, 51 \%$ from bond $a, 7 \%$ from bond $b$ and $8 \%$ from bond $c$. For the same conditions the present wark indicates a distribution of 3-26\% from bond $d$, 73-94\% from bond $a, 0.7-0.8 \%$ from bond $b$ and 0.4-3\% from bond $c$, depending on the conformation. Again the ASED-MO method predicts the general trends observed in the experiment. It should be remembered that the observed product distribution is affected by kinetics, which are not taken into account in the theory.

\section{Results for the Shinn Model Fragment}

A seventy-six atom fragment of the Shinn model has been investigated thus far with the ASED-MO method (see Figure 3). A partial geometric conformation minimization has been carried out and bond breaking energies computed. These results are summarized in Table 5. In a departure from results on the model compound, the ASED-MO method predicts an unsymmetric cleavage of the ethylene linkage of the "bibenzyl" portion of the fragment. In the Shinn fragment, a hydrogen atom on the phenanthrene portion of the molecule interacts unfavorably with the ethylene linkage, creating a repulsion that is absent in a true bibenzyl moiety. Unsymmetric cleavage of bibenzyl has been observed in model experimental studies. For instance, Buchanan, et al. ${ }^{24}$ examined the thermolysis of surface immobilized bibenzyl $\left(\mathrm{C}_{8} \mathrm{H}_{5} \mathrm{CH}_{2} \mathrm{C}_{6} \mathrm{H}_{5}\right)$ a process that models the more limited mobility of groups in coal, and observed a unsymmetric cleavage of $-\mathrm{CH}_{2} \mathrm{CH}_{2}$ - linkage upon this change in local environment. Therefore, structure, reactivity, and local environment may well be closely related.

\section{Conclusion}

We have used the ASED-MO method to calculate bond dissociation energies in a compound studied recently as a model for coal fragments. The method has yielded results in qualitative agreement with experiment and appears to be promising for studying coal liquefaction processes. General trends appear to be reproduced, such as product distribution comparisons, but not absolute numbers. The study of the model compound, as well as a fragment of the Shinn model, clearly show the interplay of structure and reactivity, and underscore the importance of such fundamental studies in the development of novel catalysts or processes. Further testing and improvements of the method are underway.

\section{Acknowledgments}

The ASED-MO programs were originally formulated for the University of Kentucky IBM 3090/300E computer by the late David P. Onwood. This research was supported by USDOE 
contract DE-FC22-89PC89851 to the Consortium for Fossil Fuel Liquefaction Science. We are grateful to the University of Kentucky Center for Computational Sciences for partial support. We are grateful to Dr. Malvina Farcasiu for suggesting the study of Model Compound I, and for providing experimental data. We also thank Dr. H. Meuzelaar for sending us the mass spectrometry data.

\section{References}

1. Shinn, J. Proceedings of the International Conference on Coal Science, 1985, 738-741.

2. Farcasiu, M.; Smith, C. Prepr., Div. Fuel Chem., Am. Chem. Soc. 1990, 35, 404.

3. Hoffman, R. Chem. Phys. 1963, 39, 1397.

4. Howell, J.; Rossi, A.; Wallace, D.; Haraki, K; Hoffmann, R. Quantum Chemistry program Exchange No. 344 (Indiana University Bloomington).

5. Ades, H. F.; Companion, A. L.; Subbaswamy, K R. Submited to J. Phys. Chem. (1990).

6. Anderson, A. B. J. Chem. Phys. 1974, 60, 2477.

7. Anderson, A. B. J. Chem. Phys. 1975, 62, 1187.

8. Onwood, D. P. (1985, unpublished).

9. Ades, H. F.; Companion, A. L. Sol. State Comm. 1983, 48, 337.

10. Ades, H. F.; Companion, A. L. Surf. Scie. 1986, 177, 553.

11. Larsen, D.S.; Nфskov, J. K. J. Phys, F 1979, 9. 1975.

12. Calzaferri, G.; Forss, L; Kamber, I. J. Phys. Chem. 1989, 93, 5366.

13. Anderson, A. B. J. Chem. Phys. 1975, 63, 4430.

14. Anderson, A. B. J. Chem. Phys. 1976, 65, 1729.

15. Grimes, R. W.; Anderson, A. B; Heuer, A. H. J. Am. Chem. Soc. 1989, 111, 1.

16. Anderson, A. B.; Maloney, J. J. J. Phys. Chem. 1988, 92, 809.

17. Kang, D. B.; Anderson, A. B. Surf. Sci. 1985 155, 639.

18. Maruca, R.; Kusuman, T.; Hicks, V.; Companion, A. (Surf. Sci., in press).

19. Carlson, G. A. (private communication).

20. Meuzelaar, H. L. C.; Huai, H. (private communication).

21. McMillen, D. F.; Malhotra, R.; Chang, S. J.; Ogier, S.; Nigenda, S. E.; Fleming, R. H. Fuel 1987, 66, 1611.

22. Turner, D. W.; Baker, C.; Baker, A. D.; Brundle, C. R. Molecular Photoelectron Spectroscopy Wiley-Interscience: London, 1970.

23. Farcasiu, M; Smith, C. Energy and Fuels (in press).

24. Buchanan, A.C. III; Dunstan, T. Don J.; Douglas, E. C.; Poutsma, M.L. J. Am. chem. Soc. 1986 108, 7703. 


\begin{tabular}{lccc}
\hline \hline Atom & Orbital & $\begin{array}{c}\text { VSIE } \\
(\mathrm{eV})\end{array}$ & $\begin{array}{c}\text { Exponent } \\
\mathrm{au}^{-1}\end{array}$ \\
\hline $\mathrm{H}$ & $1 \mathrm{~s}$ & -13.6 & 1.300 \\
$\mathrm{C}$ & $2 \mathrm{~s}$ & -21.4 & 1.625 \\
& $2 \mathrm{p}$ & -11.4 & 1.625 \\
\hline \hline
\end{tabular}

Table 1: Atomic orbital parameters used.

\begin{tabular}{ccc}
\hline \hline Bond & \multicolumn{2}{c}{ Energy (eV) } \\
& Extended & Folded \\
\hline $\mathrm{a}$ & 4.09 & 4.11 \\
$\mathrm{~b}$ & 3.89 & 3.90 \\
$\mathrm{c}$ & 4.23 & 4.35 \\
$\mathrm{~d}$ & 3.77 & 3.68 \\
$\mathrm{e}$ & 4.18 & 4.29 \\
\hline \hline
\end{tabular}

Table 2: Bond breaking energies for Model Compound I in the absence of a catalyst. The bond labels are shown in Figure 1. The second column is for the extended conformation, while the last one is for the folded conformation.

\begin{tabular}{lcc}
\hline \hline Bond & \multicolumn{2}{c}{ Energy (eV) } \\
& Extended & Folded \\
\hline $\mathrm{a}$ & 2.80 & 2.82 \\
$\mathrm{~b}$ & 3.08 & 3.09 \\
$\mathrm{c}$ & 3.01 & 3.13 \\
$\mathrm{~d}$ & 3.00 & 2.88 \\
$\mathrm{e}$ & 3.40 & 3.59 \\
\hline \hline
\end{tabular}

Table 3: Bond cleavage energies for Model Compound I after a +1 charge is placed on the molecule. The naphthyl portion retains the +1 charge after the bond is broken. The bond labels are shown in Figure 1. 


\begin{tabular}{cccccc}
\hline \hline Bond & \multicolumn{2}{c}{ Bond } & Order & Bond & \multicolumn{2}{c}{ Bond Order } \\
& Neutral & Positive & & Neutral & Positive \\
\hline $1-2$ & 1.0445 & 1.0738 & $10-11$ & 0.6264 & 0.6361 \\
$2-3$ & 1.1092 & 1.0645 & $11-12$ & 0.6080 & 0.5978 \\
$3-4$ & 1.0322 & 1.0331 & $12-13$ & 1.1020 & 1.1024 \\
$4-5$ & 1.0220 & 1.0221 & $13-14$ & 1.0718 & 1.0702 \\
$5-6$ & 1.0294 & 1.0344 & $14-15$ & 1.1000 & 1.1003 \\
$1-6$ & 1.1091 & 1.0621 & $15-16$ & 1.1004 & 1.1004 \\
$4-7$ & 1.0318 & 1.0308 & $16-17$ & 1.0712 & 1.0696 \\
$7-8$ & 1.1090 & 1.0589 & $15-18$ & 0.6330 & 0.6321 \\
$8-9$ & 1.0416 & 1.0749 & $18-19$ & 0.5752 & 0.5751 \\
$9-10$ & 1.1322 & 1.0773 & $19-20$ & 0.6231 & 0.6229 \\
$5-10$ & 1.0548 & 1.0494 & & & \\
\hline \hline
\end{tabular}

Table 4: Bond orders for bonds in Model Compound I. The first column is for the neutral molecule, the second for the positive radical ion. The atom numbers in the bond designations are shown in Figure 1.

\begin{tabular}{lc}
\hline \hline Bond & Energy (eV) \\
\hline $\mathrm{a}$ & 4.32 \\
$\mathrm{~b}$ & 3.80 \\
$\mathrm{c}$ & 3.53 \\
\hline \hline
\end{tabular}

Table 5: Bond dissociation energies for the Shinn model fragment. The bond labels are identified in Figure 3. 


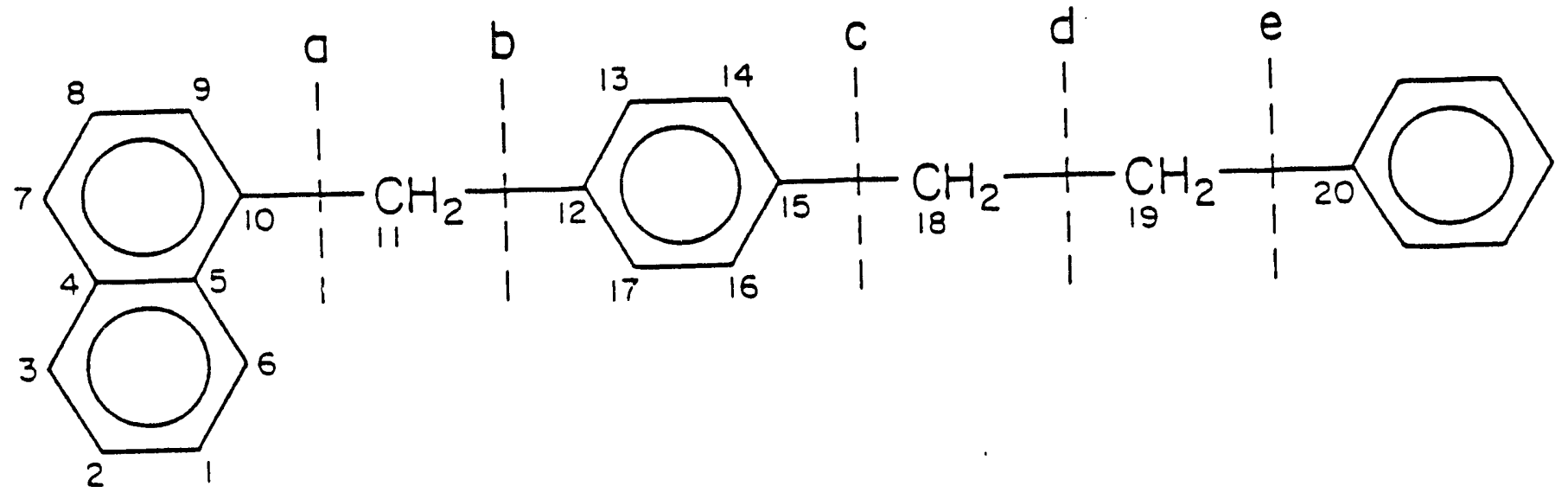

Figure 1: Skeletal structure of Model Compound I. Bonds whose cleavage are considered in this study are indicated and labeled. 

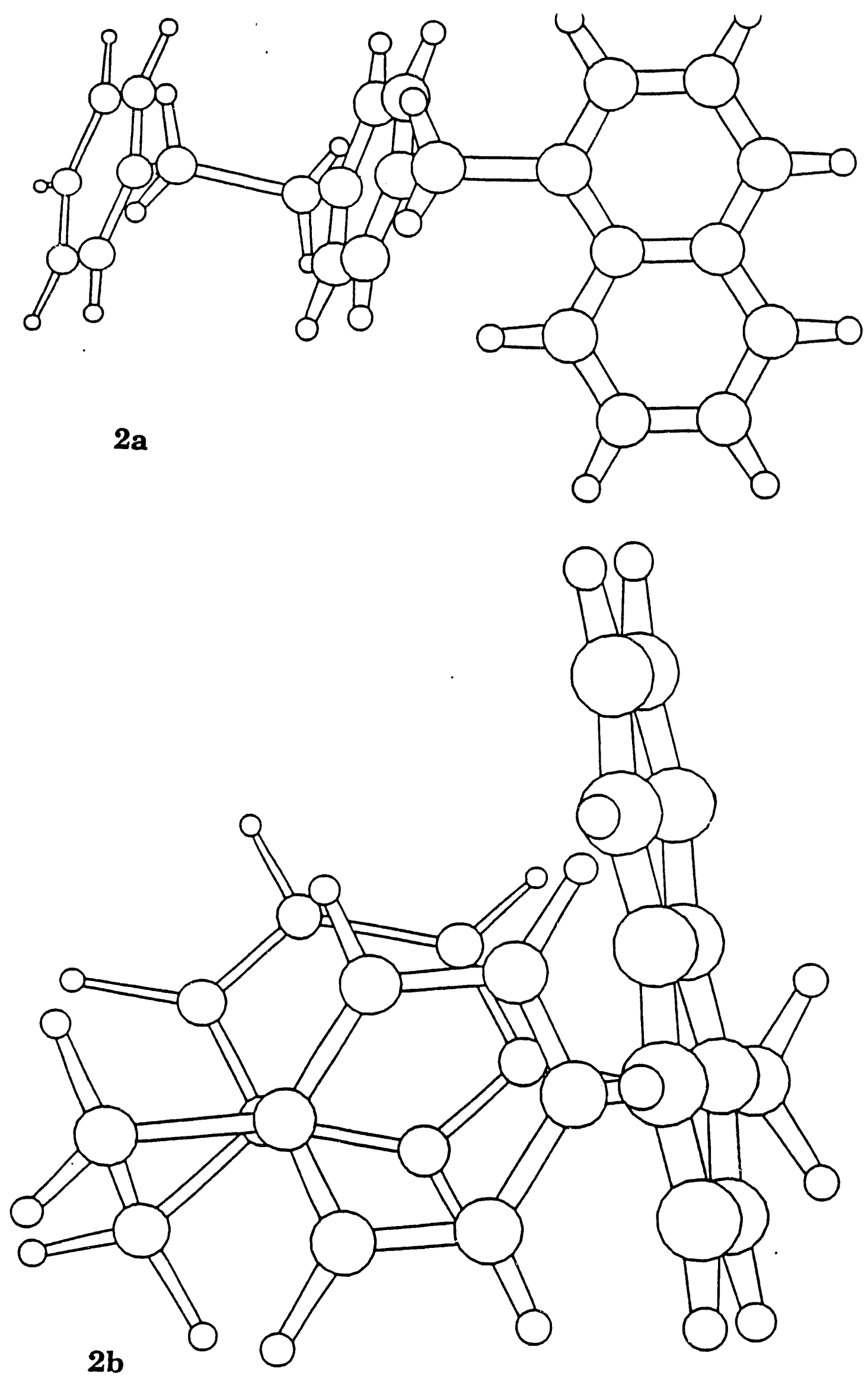

Figure 2: Three dimensional structure of Model Compound I. The extended conformation shown in (a) and folded conformation, such as the one in (b), are close in energy. 

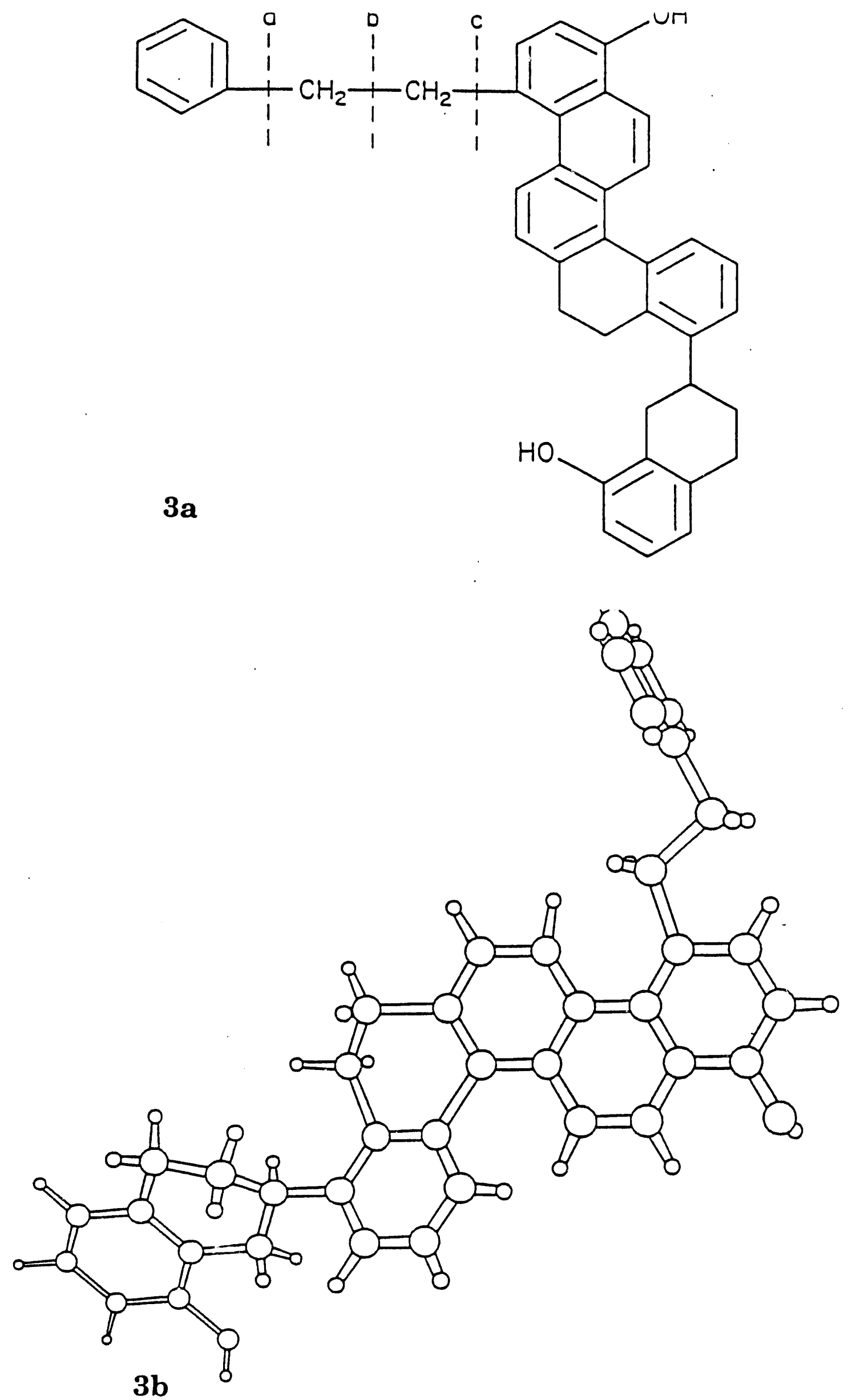

Figure 3: Fragment of the shinn model studied in this paper. The cleaved bonds are indicated and labeled in (a). The three dimensional structure of the fragment of the Shinn model is shown in (b). 
Task II

\section{University of Pittsburgh}




\section{TASK II}

\section{NOVEL APPROACHES TO CATALYSIS IN COPROCESSING}

\section{AND IN THE DIRECT LIQUEFACTION OF COAL}

- Prs:

Report Coordinator:

Phone:

Report Period:
I. Wender and J. W. Tierney

I. Wender

(412) 624-9644

May 1, 1989 - April 30, 1990

\section{TASK II.1 CATALYSIS BY SOLUBLE IRON AND MOLYBDENUM PRECURSORS FOR COPROCESSING OF ARGONNE PREMIUM COALS}

\section{SUMMARY}

The development of improved catalysts for the direct liquefaction of coal is generally recognized as a promising route towards lowering the cost of producing synthetic liquid fuels from coal. ${ }^{1}$ One attempt to improve coal liquefaction catalysis involves the use of soluble catalyst precursors which decompose under reaction conditions to form very small catalyst particles. ${ }^{2}$ These precursors have the ability to make a high degree of coal-catalyst interaction possible. ${ }^{3,4}$ The use of soluble precursors containing iron has been studied extensively. Examples of such precursors are ferrocene, ferric acetylacetonate and carbonyl complexes of iron such as $\mathrm{Fe}(\mathrm{CO})_{5}{ }^{5,6}$ all of which produce highly dispersed liquefaction catalysts. Water-soluble ammonium molybdate, ${ }^{7}$ and oil-soluble molybdenum naphthenate ${ }^{8}$ have been found to have high activity for hydrogenation of coal and production of oils at low concentrations of metal. The high activity of these catalysts at relatively low concentrations is attributed in part to their high dispersion. Combinations of iron and molybdenum catalysts have been studied to determine the benefits of using the two metals in combination. Garg and Givens, ${ }^{9}$ Suzuki and others ${ }^{5}$ found that addition of very small amounts of molybdenum to iron results in increased conversion of asphaltenes to oils. The presence of sulfur was also found to be important.

We have previously reported ${ }^{2}$ reference the extent of dispersion of the catalyst produced from $\mathrm{Fe}(\mathrm{CO})_{5}$ and its relationship with catalyst composition and activity at various stages of coprocessing reactions. This report concentrates mainly on the work done using $\mathrm{Fe}(\mathrm{CO})_{5}$ for 
coprocessing reactions employing three Argonne premium coals of varying rank to determine if the catalyst was effective in the conversion of coals of different properties. The effect of adding very small amounts of molybdenum precursors (either $\mathrm{Mo}(\mathrm{CO})_{8}$ or molybdenum naphthenate) to iron was evaluated. Molybdenum catalysts usually possess a stronger hydrogenation ability than do iron catalysts, but they are relatively expensive compared to iron catalysts. It was expected that a combination of an iron catalyst with a small amount of molybdenum could achieve high conversions and oil yields without excessive catalyst cost.

\section{RESULTS AND DISCUSSION}

The $\mathrm{Fe}(\mathrm{CO})_{5}$ precursor was used with three coals in a coprocessing mode to determine how the activity of this catalyst was affected by coals of different rank. Upper Freeport medium volatile bituminous, nlinois No.6 high volatile bituminous, and Wyodak-Anderson subbituminous coals from the Argonne coal sample bank were each reacted with Maya ATB at $425 \mathrm{C}$ and $1000 \mathrm{psig}$ (cold) $\mathrm{H}_{2}$. The effect of adding $0.5 \mathrm{wt} \% \mathrm{Fe}$ as $\mathrm{Fe}(\mathrm{CO})_{5}$ was compared to the no-catalyst run in each case. The results, shown in Table 1, indicate that, in each case, large increases in conversions to methylene chloride soluble products were obtained with this catalyst.

Table 1. Effect of Coal Type on the Activity of Fe[CO $]_{5}$ as a Catalyst Precursor on Coprocessing of Coals with Maya ATB at 425 C

\begin{tabular}{|c|c|c|c|c|c|c|}
\hline Coal & $\begin{array}{l}\mathrm{Fe}(\mathrm{CO})_{5} \\
\text { wt\% } \mathrm{Fe}\end{array}$ & Conv. & \%IOM & \%Asph & \%Oils & $\begin{array}{c}\% \text { GAS } \\
\text { And Losses }\end{array}$ \\
\hline $\begin{array}{l}\text { Maya ATB } \\
\text { only }\end{array}$ & $\cdots$ & $\ldots$ & 5.8 & 14.9 & 73.4 & 5.9 \\
\hline $\begin{array}{l}\text { Upper Freeport } \\
\text { Medium Volatile } \\
\text { Bituminous }\end{array}$ & $\begin{array}{r}0 \\
0.5\end{array}$ & $\begin{array}{r}-11 \% \\
35 \%\end{array}$ & $\begin{array}{l}19.7 \\
11.4\end{array}$ & $\begin{array}{l}11.9 \\
19.7\end{array}$ & $\begin{array}{l}61.1 \\
60.3\end{array}$ & $\begin{array}{l}7.3 \\
8.6\end{array}$ \\
\hline $\begin{array}{l}\text { nlinois \#6 } \\
\text { High Volatile } \\
\text { Bituminous }\end{array}$ & $\begin{array}{r}0 \\
0.5\end{array}$ & $\begin{array}{l}33 \% \\
75 \%\end{array}$ & $\begin{array}{r}10.9 \\
4.3\end{array}$ & $\begin{array}{l}17.2 \\
22.6\end{array}$ & $\begin{array}{l}63.0 \\
66.5\end{array}$ & $\begin{array}{l}8.9 \\
6.6\end{array}$ \\
\hline $\begin{array}{l}\text { Wyodak } \\
\text { Subbituminous }\end{array}$ & $\begin{array}{r}0 \\
0.5\end{array}$ & $\begin{array}{l}12 \% \\
72 \%\end{array}$ & $\begin{array}{r}12.5 \\
3.9\end{array}$ & $\begin{array}{l}14.0 \\
19.0\end{array}$ & $\begin{array}{l}70.4 \\
71.0\end{array}$ & $\begin{array}{l}3.1 \\
6.1\end{array}$ \\
\hline
\end{tabular}

\%Gas calculated by difference; Conv. = Conversion to $\mathrm{CH}_{2} \mathrm{Cl}_{2}$ solubles; $20 \%$ coal/80\% Maya ATB, $425 \mathrm{C}, 1000 \mathrm{psi}_{2}$ initial; $50 \mathrm{~min}$., $300 \mathrm{cc}$ autoclave

*to $\mathrm{CH}_{2} \mathrm{Cl}_{2}$ solubles 
The negative conversion found for the non-catalyzed Upper Freeport run was due to the low conversion of coal to soluble products coupled with retrogressive reactions of the residuum to form insoluble products.

The activity of small amounts (500 ppm or less) of molybdenum used alone and with

- $\mathrm{Fe}(\mathrm{CO})_{5}$ in coprocessing reactions was investigated. The effectiveness of the Mo catalysts was determined by measuring conversion to methylene chloride soluble products, the product slate produced from the coprocessing reactions, and elemental analysis of the $\mathrm{CH}_{2} \mathrm{Cl}_{2}$ soluble products. The conversions and product distributions of the coprocessing reactions carried out at various levels of $\mathrm{Fe}$ and Mo are shown in Table 2. These results indicate several interesting effects. The activity of Mo catalysts produced from either carbonyl or naphthenate precursors is quite high even at $500 \mathrm{ppm}$ of Mo. Both Mo precursors also increase the pentane-soluble oil yield over that with the Fe catalyst without Mo. The results of elemental analysis of methylene chloride solubles of the coprocessing product from using the Fe-Mo precursors are listed in Table 3.

The effect of simultaneous addition of $\mathrm{Fe}(\mathrm{CO})_{5}$ and $\mathrm{Mo}(\mathrm{CO})_{6}$ precursors on coprocessing gave conversions greater than that of either catalyst alone, but the benefit of adding one catalyst is decreased at higher concentrations of the other. This point is illustrated in Figures 1 and 2. As seen in Figure 1, the point corresponding to $0.5 \mathrm{wt} \% \mathrm{Fe}$ and $50 \mathrm{ppm}$ Mo gives the highest conversion of Illinois No.6 to methylene chloride solubles. This effect will be investigated further.

Table 2. Coprocessing Reaction Results: nlinois No. 6 Coal, $\mathrm{Fe}[\mathrm{CO}]_{5}$ and Mo-precursors

\begin{tabular}{|c|c|c|c|c|c|c|c|}
\hline $\begin{array}{l}\mathrm{Fe}(\mathrm{CO})_{5} \\
(\mathrm{wt} \% \mathrm{Fe})\end{array}$ & $\begin{array}{l}\text { Molybdenum } \\
\text { Precursor }\end{array}$ & ppm Mo & Conv. & \%IOM & \%Asph & \%Oils & \%Gas \\
\hline 0 & $\ldots$ & 0 & $\overline{32.6 \%}$ & $\overline{10.9}$ & $\overline{17.2}$ & 62.9 & 8.9 \\
\hline 0 & $\mathrm{Mo}(\mathrm{CO})_{6}$ & 500 & $76.5 \%$ & 3.8 & 20.6 & 73.4 & 2.2 \\
\hline 0 & Mo Naph. & 500 & $71.0 \%$ & 4.6 & 18.4 & 72.6 & 4.3 \\
\hline 0.2 & $\ldots$ & 0 & $52.4 \%$ & 7.7 & 16.9 & 66.2 & 9.2 \\
\hline 0.2 & $\mathrm{Mo}(\mathrm{CO})_{6}$ & 500 & $83.1 \%$ & 2.7 & 21.2 & 69.5 & 6.6 \\
\hline 0.5 & -...... & 0 & $73.7 \%$ & 4.3 & 22.6 & 66.5 & 6.6 \\
\hline 0.5 & $\mathrm{Mo}(\mathrm{CO})_{6}$ & 50 & $83.3 \%$ & 2.7 & 22.7 & 71.6 & 3.0 \\
\hline 0.5 & $\mathrm{Mo}(\mathrm{CO})_{6}$ & 100 & $80.4 \%$ & 3.2 & 20.3 & 70.9 & 5.6 \\
\hline 0.5 & $\mathrm{Mo}(\mathrm{CO})_{6}$ & 500 & $85.5 \%$ & 2.3 & 21.8 & 74.3 & 1.6 \\
\hline 0.5 & $\ldots$ & 0 & $73.7 \%$ & 4.3 & 22.6 & 66.5 & 6.6 \\
\hline 0.5 & Mo naph. & 100 & $77.4 \%$ & 3.7 & 20.2 & 69.4 & 6.7 \\
\hline 0.5 & Mo naph. & 500 & $82.7 \%$ & 2.8 & 19.3 & 77.4 & 0.5 \\
\hline
\end{tabular}

20\% Illinois \#6 coal (Argonne)/80\% Maya ATB, $425 \mathrm{C}, 1000 \mathrm{psi} \mathrm{H}_{2}$ initial, $60 \mathrm{~min}$. batch reaction, $300 \mathrm{cc}$ autoclave 


\section{Table 3. Elemental Analyses of $\mathrm{CH}_{2} \mathrm{Cl}_{2}$ Solubles from}

Fe-Mo Catalytic Systems

\begin{tabular}{|c|c|c|c|c|c|c|c|c|}
\hline \multirow{2}{*}{$\begin{array}{l}\mathrm{Fe}(\mathrm{CO})_{5} \\
(\mathrm{wt} \% \mathrm{Fe})\end{array}$} & \multicolumn{3}{|c|}{ Molybdenum } & \multicolumn{5}{|c|}{$\mathrm{CH}_{2} \mathrm{Cl}_{2}$ Solubles } \\
\hline & Precursor & ppm Mo & Conv. & $(\mathrm{H} / \mathrm{C})$ & $\% \mathrm{C}$ & $\% \mathrm{H}$ & $\% \mathrm{~N}$ & $\% \mathrm{~S}$ \\
\hline 0 & -. & 0 & $\overline{32 .}$ & 1.49 & 83.0 & 10.30 & 0.25 & 3.18 \\
\hline 0.5 & - & 0 & 73. & 1. & & 10 & 0.33 & 3.01 \\
\hline 0.5 & Mo napl & 500 & 82. & 1. & & 10.50 & 0.40 & 2.89 \\
\hline 0.5 & $\mathrm{Mo}(\mathrm{CO})_{6}$ & 500 & $85.5 \%$ & 1.57 & 82.53 & 10.79 & 0.43 & 2.94 \\
\hline
\end{tabular}

All runs with Ilinois \#6 Coal, $425 \mathrm{C}, 1000 \mathrm{psig}_{2}$ initial pressure 60 minutes, $300 \mathrm{cc}$ autociave, $20 \mathrm{wt} \% \mathrm{coal} / 80 \mathrm{wt} \%$ Maya ATB.

X-ray diffraction studies and transmission electron microscopy were carried out to determine the phases present in the liquefaction residues and to characterize their size distribution. The XRD results showed that pyrrhotite was formed from the $\mathrm{Fe}(\mathrm{CO})_{5}$, but there was no evidence of the formation of Mo compounds, probably due to the low concentration Mo $(500 \mathrm{ppm})$. There was no evidence that the Mo compounds interfere with the formation of $\mathrm{Fe}_{1-\mathrm{r}} \mathrm{S}$ from the iron precursor. TEM/SEM images of the molybdenum on activated carbon revealed that the Mo-containing particles were very small, on the order of $50 \mathrm{~nm}$ or less. Figure 3 displays the larger carbon support decorated with smaller Mo catalyst particles. Energy dispersive X-ray analysis confirmed that the smaller, dark particles contained molybdenum, while the more diffuse carbon matrix did not.

\section{CONCLUSIONS}

The $\mathrm{Fe}(\mathrm{CO})_{5}$ precursor was effective at increasing the conversions of all three types of coal tested in coprocessing at $425 \mathrm{C}$. The use of Illinois No.6 high volatile bituminous and Wyodak subbituminous coals resulted in conversions of greater than $70 \%$ using 0.5 wt\% Fe. The Upper Freeport medium volatile bituminous coal had a low conversion even with a catalyst, but there was still a significant increase over the negative conversions obtained without catalyst. Each of these conversions represented a large increase over the uncatalyzed reaction. The addition of small amounts of Mo from soluble precursors increased conversions when used alone and with $\mathrm{Fe}(\mathrm{CO})_{5}$, but conversions greater than $90 \%$ were not obtained. The effect of the Mo catalyst was to increase conversions while also increasing the hydrogen content of the liquid products. The images of the Mo and Fe-Mo catalysts obtained using TEM show a highly dispersed catalyst, with particle sizes below $50 \mathrm{~nm}$. 


\section{REFERENCES}

1. Derbyshire, F. J., Catalysis in Coal Liquefaction: New Directions for Research, IEA CR-08, London. U.K, IE.A Coal Research, Jan. 1988.

2. Herrick, D.E., Tierney, J.W., Wender, I., Huffman, G.P., and Huggins, F.E., Energy and Fuels 4, 231 (1990).

3. Watanabe, Y., Yamada, O., Fujita, K, Takegami, Y., and Suzuki, T., Fuel, 63, 752 (1984).

4. Kamiya, Y., Nobusawa, Y., and Futamura, S., Fuel Proc. Tech. 18, 1 (1988).

5. Suzuki, T., Yamada, H., Sears, P., and Watanabe, Y., Energy and Fuels, 3, 707 (1989).

6. Cook, P.S., and Cashion, J.D., Fuel, 66, Góy (1987).

7. Weller, S., and Pelipetz, M.G., Ind. Eng. Chem. 43, 1243 (1951).

8. Curtis, C.W. Tsai, K.J., and Guin, J.A., Ind. Eng. Chem. Res. 26, 12 (1987).

9. Garg, D., and Givens, E., Ind. Eng. Chem. Proc. Des. Dev., 21, 113 (1982). 


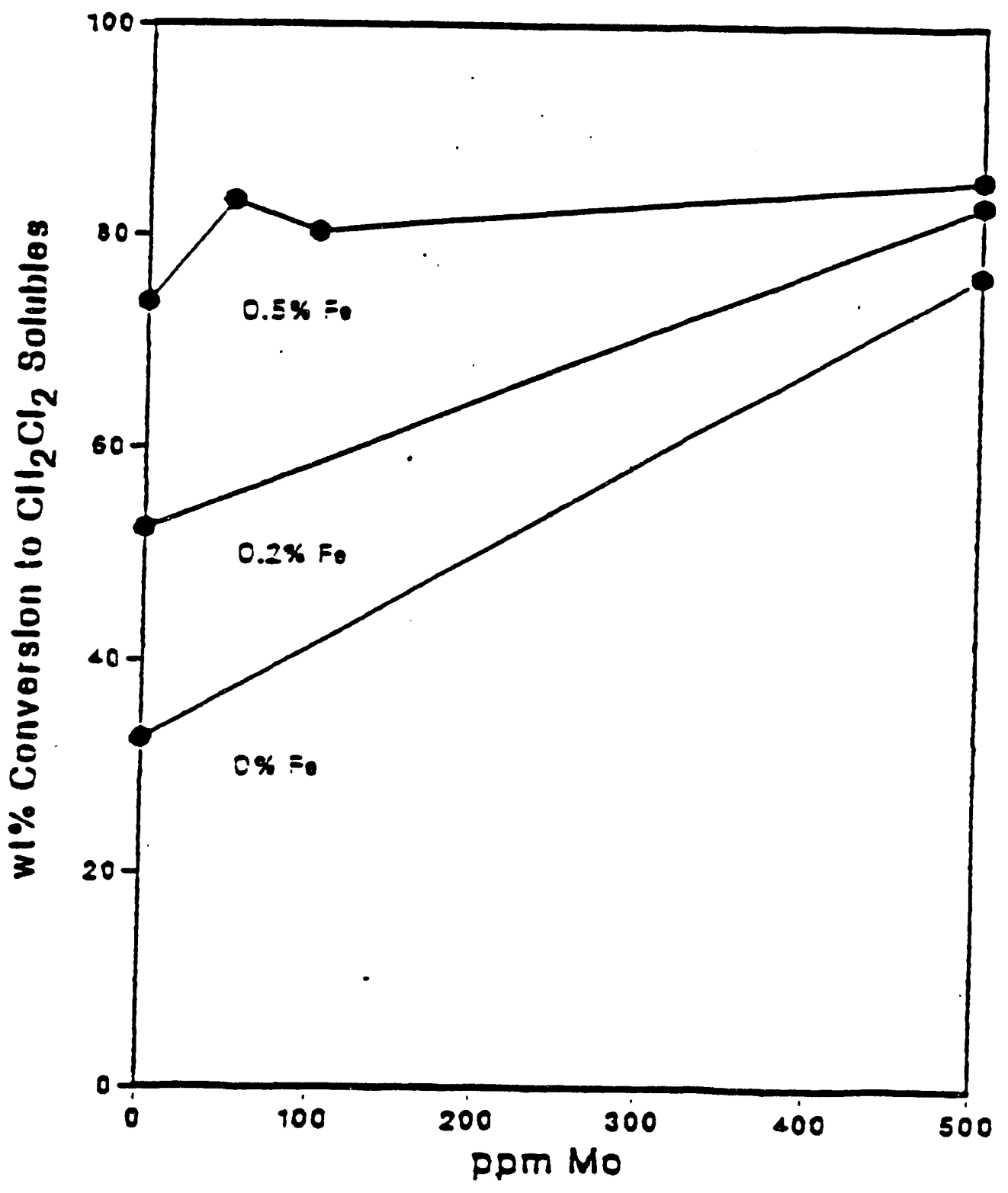

Figure 1. Coprocessing Conversions vs. Mo Loadings at Three Fe Loadings for Illinois No. 6 Coal and Maya ATB at $425^{\circ} \mathrm{C}$ 


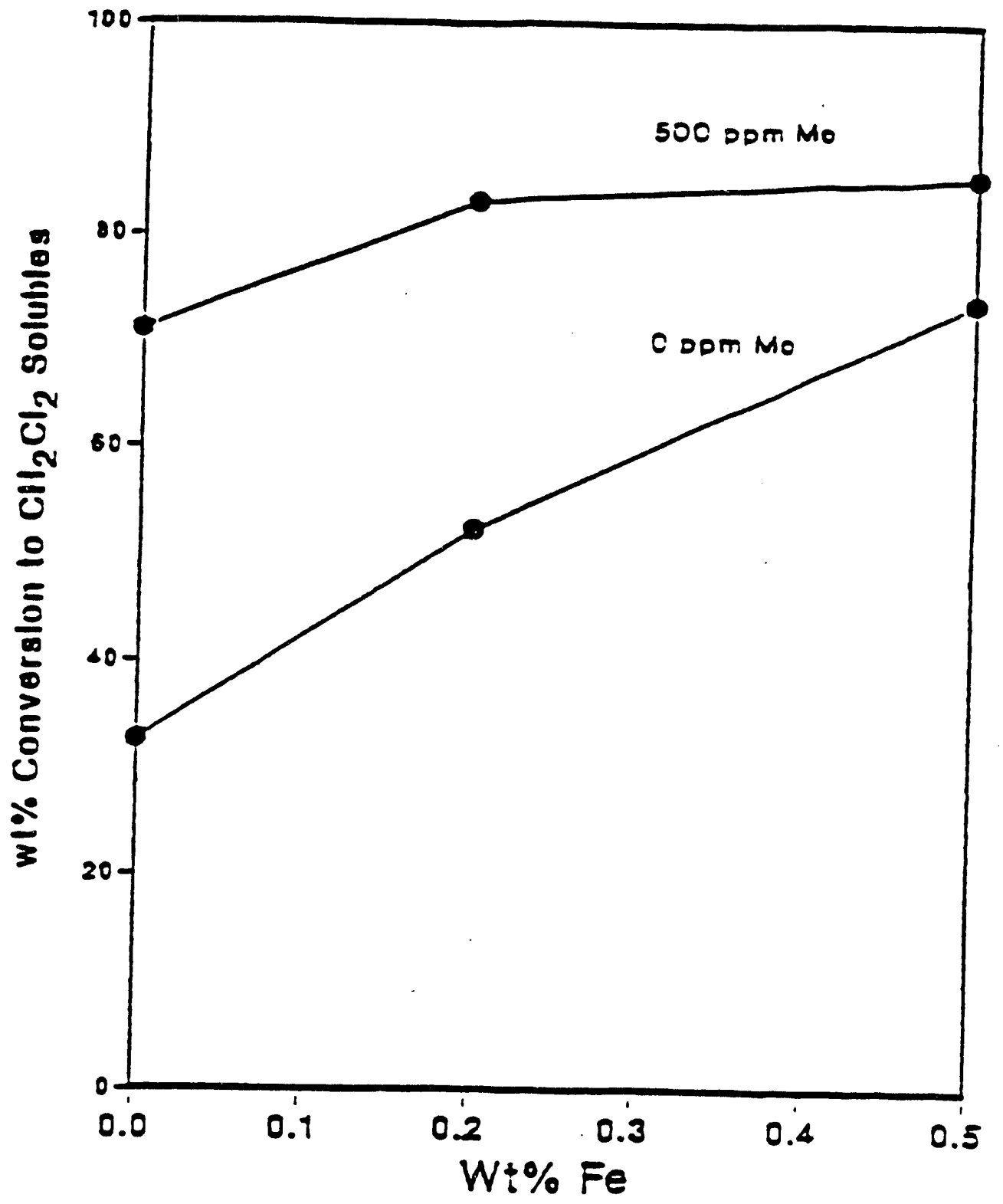

Figure 2. Coprocessing Conversions vs. Fe Loadings at Two Mo loadings for Illinois No. 6 Coal and Maya ATB at $425^{\circ} \mathrm{C}$ 


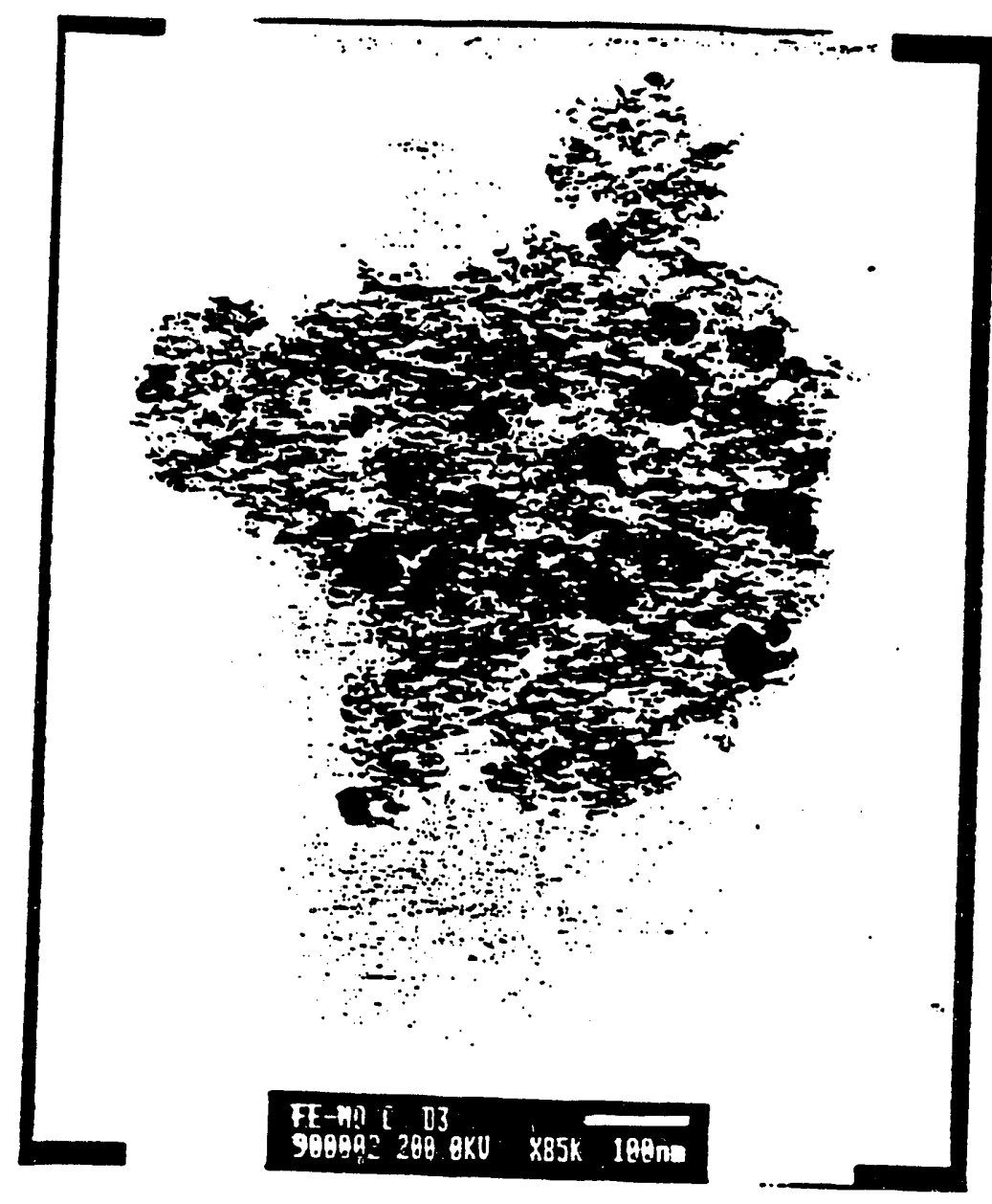

Figure 3. TEM Image of Mo Catalyst Particles on an Activated carbon support with Toluene as solvent at $300^{\circ} \mathrm{C}$ 


\section{TASK II.2 COAL LIQUEFACTION UNDER MULD CONDITIONS USING TRIFLIC ACID, IODINE, AND THEIR COMBINATION}

\section{SUMIMARY}

The concept of using a superacid coal depolymerization catalyst with a balancing hydrogenation/hydrogenolysis function to prevent retrogressive reactions forms the basis of this work. In an efficient coal dissolution process, macromolecular fragments of the coal structure should be selectively cleaved. While thermal cracking is non-selective and requires elevated temperatures, catalytic cracking can allow the use of less severe conditions. However, owing to the wide variety of the types of linkages existing in the coal structure, a single catalyst may not be sufficiently effective for bond breakage at critical linking points to allow for efficient coal conversion. Acid catalyzed coal depolymerization is a widely studied subject. ${ }^{10,11,12}$ Olah et al. ${ }^{2}$ treated Illinois No.6 coal with a superacid system $\left(\mathrm{H}_{0}=-16\right)$ consisting of $\mathrm{HF}$ and $\mathrm{BF}_{3}$ at about $105 \mathrm{C}$ to obtain over $90 \%$ pyridine extractability. We chose to study the effect of triflic acid, a raild superacid $\left(\mathrm{H}_{\mathrm{o}}=-14.2\right)$, on the hydroliquefaction of coal with or without small amounts of iodine as a hydrogenation co-catalyst ${ }^{13}$.

\section{RESULTS AND DISCUSSION}

We have previously reported (at the 1989 CFFLS annual meeting at Auburn University) the effects of different reaction variables such as reaction time and reaction temperature along with the effects of iodine and triflic acid concentration on coal conversion. During the last year, the effects of reaction solvent, coal rank and hydrogen pressure were investigated. Tetralin and toluene were used as solvents in these reactions. As shown in Table 1 tetralin gave a conversion of about $80 \%$, whereas a relatively inert solvent, toluene, afforded a $66 \%$ conversion of Wyodak coal. The asphaltenes yield followed the same trend as the overall coal conversion. This supports the idea that better contact between the coal and the catalyst and a stronger solubilization medium tend to enhance the primary liquefaction (to asphaltenes) without greatly affecting secondary, (mainly hydrogenation/hydrogenolysis) pathways to give gas and oils.

In the course of a separate series of experiments with Illinois No.6, methylcyclohexane, a source of hydride ions, was compared with tetralin as a solvent using only triflic acid (10\% wt. coal) as catalyst at $300 \mathrm{C}$. The results are listed in Table 2. 
Table 1. Effect of Solvent on Wyodak Coal (W-1) in the Presence of 25 wt\% TFA/0.5 wt\% Iodine at $300 \mathrm{C}$ and 1000 psig (cold) Hydrogen

Conversions wt\%

\begin{tabular}{|c|c|c|c|c|}
\hline Solvent & Asphaltenes & $\underline{\text { Oils }}$ & Gases and Losses & Tota \\
\hline None & 21 & 18 & 10 & 49 \\
\hline Toluene & 36 & 23 & 7 & 66 \\
\hline Tetralin & 44 & 29 & 6 & 7 \\
\hline
\end{tabular}

Table 2. Acid Catalyzed Conversion of Argonne minois No. 6 Coal*

\begin{tabular}{|c|c|c|c|c|c|c|}
\hline Expt. No. & Solvent $^{b}$ & Catalyst & $\mathrm{Gas}^{\mathrm{c}}$ & $\begin{array}{l}\text { Pressure } \\
\text { Drop MPa }\end{array}$ & Conv.\% & $\begin{array}{l}\% \mathrm{CH}_{2} \mathrm{Cl}_{2} \\
\text { Solubles }\end{array}$ \\
\hline ILA3 & Tet. & TFA-0.2g & $\mathrm{H}_{2}$ & 0.94 & 54 & 48 \\
\hline R1 & $\mathrm{MCH}$ & TFA-0.2g & $\mathrm{H}_{2}$ & 1.19 & 68 & 64 \\
\hline $\mathrm{R} 2$ & Tet & $\begin{array}{l}\mathrm{H}_{2} \mathrm{SO}_{4} \\
(98 \%-0.14 \mathrm{~g})\end{array}$ & $\mathrm{H}_{2}$ & 0.58 & 42 & 39 \\
\hline R3 & Tet & TFA $0.2 \mathrm{~g}$ & $\mathrm{~N}_{2}$ & -0.11 & 35 & 30 \\
\hline $\mathrm{R} 4$ & Tet & TFA-0.2g & $\begin{array}{l}\mathrm{H}_{2} / \mathrm{N}_{2} \\
(3: 7)^{\mathrm{d}}\end{array}$ & 0.71 & 44 & 39 \\
\hline R5 & Tet & TFA-0.2g & $\left(\mathrm{H}_{2}\right)^{\prime}$ & 1.50 & 59 & 52 \\
\hline
\end{tabular}

a. $2 \mathrm{~g} ; 300 \mathrm{C}, 1.5 \mathrm{~h}$

b. $8 \mathrm{~g}$; Tet=Tetralin; $\mathrm{MCH}=$ Methylcyclohexane

c. Total initial pressure (cold, 6.8 $\mathrm{MPa}$ (1000 psi), except in R5)

d. Pressure ratio

e. $10.2 \mathrm{MPa}$ (cold)

An appreciable increase in conversion, from 54 to $68 \%$, was achieved with methylcyclohexane indicating an apparent role of a hydride donor and the predominance of a carbocation mechanism which is promoted by the acid. The effect of coal rank on the activity of triflic acid and iodine catalyst for coal conversion was also determined in a separate series of experiments (Figure 1). The effect of hydrogen partial pressure on coal conversion using the triflic acid/iodine combination was studied by using different partial pressures of hydrogen in the gas phase at the beginning of the reactions. Table 2 also lists the results obtained in reactions carried out with varying hydrogen pressure. The effect of increasing the hydrogen pressure from about 6.8 $\mathrm{MPa}$ to $10.2 \mathrm{MPa}$ (all cold) has been shown for Illinois No.6 coal in tetralin at $300 \mathrm{C}$ using $10 \mathrm{wt} \%$ triflic acid as catalyst. Under nitrogen pressure only a $35 \%$ 
conversion was obtained along with a negative pressure drop probably due to gas produced during the reaction.

Five alkyl-aromatic compounds containing types of linkages hypothesized to be present in coal were chosen to study the effect of the triflic acid/iodine catalyst system. They were used together as well as separately for the hydrogenolysis/hydrocracking reactions of these model compounds. Diphenylmethane was chosen to represent methylene groups present in coal between clusters of aromatic rings. Benzyl and phenyl ethers represented ether types of linkages present in coal. Phenyl sulfide was also studied as a model compound representing a sulfide linkage in the coal structure.

Triflic acid and iodine were both found to be effective catalysts for model compounds reactions under mild reaction conditions. Details of the reactions with different model compounds and catalysts are tabulated in Tables 3 to 7.

TABLE 3. Hydrocracking of Diphenylmethane

Model Compound: $10 \mathrm{~g}, \mathrm{H}_{2}: 800$ psig., TFA: $0.5 \mathrm{~g}, \mathrm{I}_{2}: 0.05 \mathrm{~g}$, Reaction Time: $60 \mathrm{~min}$.

\begin{tabular}{lcccc}
\hline Catalyst & Temp., ${ }^{\circ} \mathrm{C}$ & \% Conversion & \% Yield,Light Prod. \% Yield,Heavy Prod. \\
None & 300 & 4.3 & 21.1 & 78.9 \\
TFA & 250 & 71.6 & 72.6 & 27.4 \\
Iodine & 300 & 14.4 & 42.4 & 57.6 \\
Both & 300 & 84.8 & 89.6 & 10.4 \\
\hline
\end{tabular}

TABLE 4. Hydrocracking of Benzyl Ether Model Compound: $10 \mathrm{~g}, \mathrm{H}_{2}: 800$ psig., TFA:0.5 g, $\mathrm{I}_{2}: 0.05 \mathrm{~g}$, Reaction Time: $60 \mathrm{~min}$.

\begin{tabular}{lcccc}
\hline Catalyst & Temp., ${ }^{\circ} \mathrm{C}$ & \% Conversion & \% Yield, Light Prod. \% Yield,Heavy Prod. \\
None & 300 & 16.6 & 94.1 & 5.9 \\
TFA & 250 & 94.2 & 41.6 & 58.4 \\
Iodine & 300 & 93.5 & 54.5 & 45.5 \\
Both & 300 & 99.8 & 97.4 & 2.6 \\
\hline
\end{tabular}


TABLE 5. Hydrocracking of Phenyl Sulfide

Model Compound: $10 \mathrm{~g}, \mathrm{H}_{2}: 800$ psig., TFA:0.5 g, $\mathrm{I}_{2}: 0.05 \mathrm{~g}$, Reaction Time: $60 \mathrm{~min}$.

\begin{tabular}{lcccc}
\hline Catalyst & Temp., ${ }^{\circ} \mathrm{C}$ & \% Conversion & \% Yield,Light Prod. & \% Yield, Heavy Prod. \\
None & 300 & 4.6 & 78.8 & 21.2 \\
TFA & 250 & 36.1 & 68.9 & 31.1 \\
Iodine & 300 & 11.4 & 95.1 & 4.9 \\
Both & 300 & 42.3 & 94.2 & 5.8 \\
\hline
\end{tabular}

TABLE 6. Hydrocracking of Phenyl Ether

Model Compound: $10 \mathrm{~g}, \mathrm{H}_{2}: 800$ psig., TFA:0.5 g, $\mathrm{I}_{2}: 0.05 \mathrm{~g}$, Reaction Time: $60 \mathrm{~min}$.

\begin{tabular}{lcccc}
\hline Catalyst & Temp., ${ }^{\circ} \mathrm{C}$ & \% Conversion & \% Yield,Light Prod. & \% Yield,Heavy Prod. \\
None & 300 & 2.55 & 91.1 & 8.9 \\
TFA & 250 & 4.32 & 54.8 & 45.2 \\
lodine & 300 & 3.51 & 78.4 & 21.6 \\
Both & 300 & 9.88 & 68.4 & 31.6
\end{tabular}

TABLE 7. Hydrocracking of Pyrene

Model Compound: 2 g, Solvent: 4 g, $\mathrm{H}_{2}: 800$ psig., TFA:0.5 g, $\mathrm{I}_{2}: 0.05 \mathrm{~g}$, Reaction Time: $60 \mathrm{~min}$.

\begin{tabular}{lclc}
\hline Catalyst & Temp. C & Solvent & \% Conversion \\
None & 300 & tetralin & 3.4 \\
TFA & 250 & toluene & 8.2 \\
Iodine & 300 & tetralin & 45.0 \\
Both & 300 & toluene & 31.0 \\
\hline
\end{tabular}

As indicated in Table 3, hydrogenolysis of diphenylmethane was carried out at $250 \mathrm{C}$, in the absence of iodine and at $300 \mathrm{C}$, in the presence of iodine. Ten grams of diphenylmethane were treated with small amounts of triflic acid and iodine $(0.5 \mathrm{~g}$ and $0.05 \mathrm{~g}$ respectively) under 800 psig of hydrogen for one hour. Benzene, toluene, and xylene were among the main products identified using external standards. The conversions by weight were calculated based on the weight percent of these products formed. The area ratios of the peaks of these compounds seen on gas chromatograms were proportional to their weight ratios in the mixtures. 
As shown, a thermal reaction carried out in the absence of any catalyst results in very poor hydrocracking of diphenylmethane. Triflic acid alone is an effective cracking catalyst; it converted 72 percent of diphenylmethane to products, $72 \%$ of which were lighter than diphenylmethane itself. Iodine, on the other hand, is not as active a catalyst for the cleavage of methylene type of linkages, giving only about 14 percent conversion when used alone. When both these catalysts were used, a type of synergistic effect was observed on the overall conversion of diphenylmethane to various products. Also, good selectivity to lighter products was obtained.

Interestingly, results in Table 4 indicate that iodine is as effective a catalyst as triflic acid in the cracking of ether linkages present in benzyl ether. In all cases, iodine seems to improve selectivity to lighter products over the value of that obtained with only triflic acid as catalyst. This can be explained by the idea that iodine, by virtue of its ability to form radicals, stabilizes primary products of the bond-cleavage reactions by making hydrogen atoms available (iodine itself must be undergoing a radical type reaction with molecular hydrogen). The results of phenyl sulfide hydrogenolysis shown in Table 5 makes the same point regarding the roles of triflic acid and iodine in converting certain types of linkages thought to exist in coal. Phenyl ether is fairly unreactive under the reaction conditions employed here, probably due to difficulty in the formation of a phenyl cation, making linkages such as Ar-O-Ar difficult to cleave under these mild reaction conditions with either iodine or triflic acid as a catalyst.

Pyrene, representative of a model compound for coal-derived polycyclic aromatic compounds, when reacted with iodine in presence of tetralin,gives about $45 \%$ conversion to hydrogenated derivatives of pyrene. With triflic acid alone, conversion was small, limited to only about $7 \%$. However, a $31 \%$ conversion was obtained with the combination of both triflic acid and iodine in toluene as solvent. These reactions constitute an exploratory effort to identify the roles or the functions of the two different catalysts, iodine and triflic acid in coal liquefaction reactions. From the results of these reactions, it can be inferred that triflic acid by itself is not a good catalyst for hydrogenation of polycyclic aromatic clusters, while iodine is an efficient catalyst for hydrogenation of such compounds. This observation substantiates our view that a combined system of two or more catalysts, having different functions, could improve the

- efficiency of coal conversion to liquids.

\section{CONCLUSIONS}

A catalytic system based on the combination of triflic acid and iodine was found to be 
effective for three types of coal: Illinois No.6, Wyodak, and Pittsburgh seam. Weaker acids such as p-toluenesulfonic acid and $98 \%$ sulfuric acid were less effective than triflic acid in this catalytic coal conversion. From the dependence of the product selectivity on the catalytic system and reaction conditions, and from the elemental analyses of liquids and residues and ${ }^{1} \mathrm{H},{ }^{13} \mathrm{C}$ NMR measurements, it is concluded that the main function of the acid is to enhance coal depolymerization to asphaltenes while the main role of iodine in the combined catalytic system is to hydrogenate and hydrocrack the asphaltenes to oils. Finally, the combined catalytic system removed more than $50 \%$ of the nitrogen and over $90 \%$ of the sulfur from the original coal. Triflic acid by itself was found to be a very efficient denitrogenation catalyst. Strong acids are known to be active in the removal of nitrogen compounds from model compounds. ${ }^{14}$

\section{REFERENCES}

10. Ouchi, K., Imuta, Kl, and Yamashita, Y., Fuel, 52, 156 (1973).

11. Olah, G.A., Bruce, M.R., Edlelson, S.H., and Husain, F., Fuel, 63, 1130 (1984).

12. Heredy, L.A., Kostyo, A.E., and Neuworth, M.B., Fuel, 42, 182 (1963).

13. Fraenkel, D, Pradhan, V.R., Tierney, J.W., Wender, I., Liquefaction of Coal under Mild Conditions: Catalysis by Strong Acids, Iodine and their Combinations, Submitted for publication to Fuel.

14. Hirschon, A.S., Laine, R.M. Fuel, 68, 1416 (1989). 


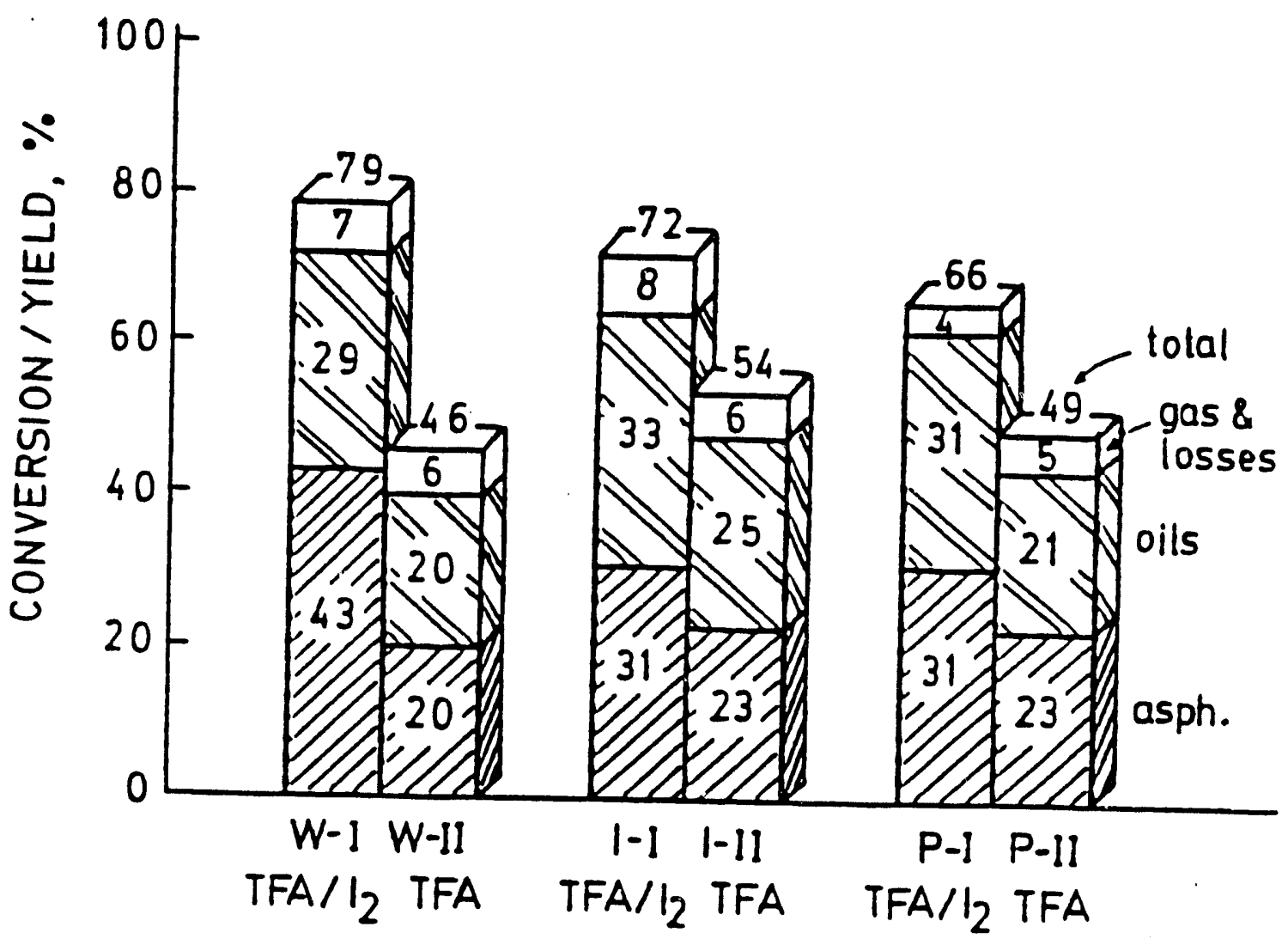

Figure 1. Effect of coal Rank and catalyst combinatiobn on Coal Conversion at $300^{\circ} \mathrm{C}, 0.5 \mathrm{wt}$ Iodine, I. PETC coal, II. APCS coal 25 wt $\%$ TFA for Wyodak [W] and 10 wt? TFA for Pitt. seam [P], and III. \#6 [I] 


\section{TASK 11.3 ON THE COLIQUEFACTION OF COAL WTHH LIGNIN}

\section{SUMMARY}

A short study was made to investigate the possible advantages of the coliquefaction of coal and lignin. The main reasons for looking into this subject were: (a) lignin is the second most abundant polymeric organic product found in nature; (b) native lignin is a complex 3dimensional polymer which contains about 0.3 wt\% sulfur and essentially no nitrogen; addition of lignin (in significant amounts) in coal liquefaction would cut down unwanted emissions; and (c) lignin-coal coliquefaction may increase distillate yields. A problem immediately became apparent: not only does the word "lignin" mean different things to different people but the difficulty and expense of obtaining pure "native" lignin with low sulfur and nitrogen is too great for an investigation of coliquefaction to be worthwhile. A few experiments (without a catalyst) were run before the study was halted.

\section{DISCUSSION}

First, there is the so-called native lignin. Although present in large amounts in vascular plants, it is difficult to separate from the cellulose and hemicelluloses in the plant. Another material, also called a "lignin," consists of the residues from pulp and paper manufacture; over 22 million tons per year of this material, often termed INDTILIN, is available in the U.S. But this product contains about 2.26 wt\% of sulfur and more than 3 wt\% cf ash.

A third source of "lignin" is part of the residue from the hydrolysis of cellulose to sugars for fermentation to ethyl alcohol. The Tennessee Valley Authority (TVA) is operating a small pilot plant providing a by-product residue, supposedly containing large amounts of lignin, from their process for producing grain alcohol from wood.

When a plant falls, microorganisms attack the cellulose rapidly, leaving the lignin behind. This has led to a widespread belief that coal is mainly derived from lignin. On this premise, material from petrified wood has also been used as a type of lignin.

Coughlin et al. ${ }^{1-4}$, on the assumption that the conversion of hardwood lignin did not change on the addition of Mlinois No. 6 coal, obtained results that suggested that the coliquefaction of coal and lignin produced an effect greater than a mere additive effect. Lignin to coal ratios varied from 1:4 to 1:1. It seemed probable that the lignin aided coal liquefaction since the bonds in lignin are less stable than those in coal but inorganic minerals in coal could catalyze the liquefaction of lignin and even radicals formed from coal could attack the lignin. 
These workers found that lower temperatures could be used for the depolymerization of coal in the presence of lignin. For example, by adding 35g. of Iotech hardwood lignin to $15 \mathrm{~g}$. of nlinois No. 6 coal and $200 \mathrm{ml}$. of tetralin with $5 \mathrm{~g}$ of $\mathrm{SiO}_{2} / \mathrm{Al}_{2} \mathrm{O}_{3}$ and 36 atm of $\mathrm{H}_{2}$ at $300 \mathrm{C}$ for three hours, a synergistic effect was claimed. More coal seemed to be liquefied than would be obtained in the absence of the lignin. These authors suggested that thermal decomposition of the lignin formed phenoxy radicals which could cause cleavage of aliphatic carbon-carbon bonds in the coal.

Interestingly, these favorable effects on the above (filterable) products were obtained using lignin at $300 \mathrm{C}$ but did not occur at $350 \mathrm{C}$; perhaps more extensive polymerization or radical recombination took place at $350 \mathrm{C}$.

In contrast to these results, Rudnicks found that addition of only small amounts of lignin to a subbituminous coal increased the yield of solubles in the product as measured by the amount of product soluble in THF, with little impact on coal conversion. Addition of only 1\% of lignin (type unspecified) substantially improved selectivity of the process (427 C, 1000 psi of $\mathrm{H}_{2}$ for 6 minutes) to liquid products. But addition of $10 \%$ of lignin had a detrimental effect on selectivity and a substantial negative effect on the conversion of the coal (Belle Ayr subbituminous).

It is important that lignin, in all cases, be added as such; lignin that has first been converted to liquids and then added to coal does not show any so-called synergistic effects. This seems at variance with the idea that phenoxy radicals aid in coal conversion for the hydrogenation/hydrocracking of lignin yields liquids with high contents of phenols.

Kruse and Knudson ${ }^{6}$ are studying the use of lignin obtained from the TVA process to provide phenolics for solvent balance in the ChemCoal Process tested at tha University of North Dakota Energy and Environmental Research Center mainly on western low rank coals. This coal liquefaction process uses phenolic solvents (here supposedly provided partly from the lignin) in the presence of carbon monoxide and aqueous sodium ions at 340-350 C and 1800 to 2000 psig.

We have carried out a few reactions of Wyodak coal and Iotech (native) lignin in the absence of an added catalyst. The yield of pentane solubles (oils) was increased at a 1:1 lignincoal ratio at $300 \mathrm{C}$ but there was no significant effect at higher temperatures (Figure 1). There also was no significant effect on the yield of $\mathrm{CH}_{2} \mathrm{Cl}_{2}$ solubles (Figure 2). 


\section{REFERENCES}

1. Coughlin, R.W. and F. Davoudzadeh, Nature, 303, 789 (1983).

2. Coughlin, R.W. and F. Davoudzadeh, Fuel, 65, 95 (1986).

3. Coughiin, R.W. and P. Altieri, Energy and Fuel, 1, 253 (1987).

4. Coughlin, R.W. and P. Altieri, Chapt. 6, p. 219, inergy Rrecovery from Lignin, Peat and Lower Rank Coals, ed. by D.J. Trantolo and D.L. Wise, Elsevier (1986).

5. Rudnick, L.R., U.S. Patent 4,409,089, October, 1983 (to Mobil Oil Corp.).

6. Kruse, C.W. and C.L. Knudson, Center for Research on Sulfur in Coal, Carterville, II, Technical Report, Dec. 1989 through Feb., 1990. 


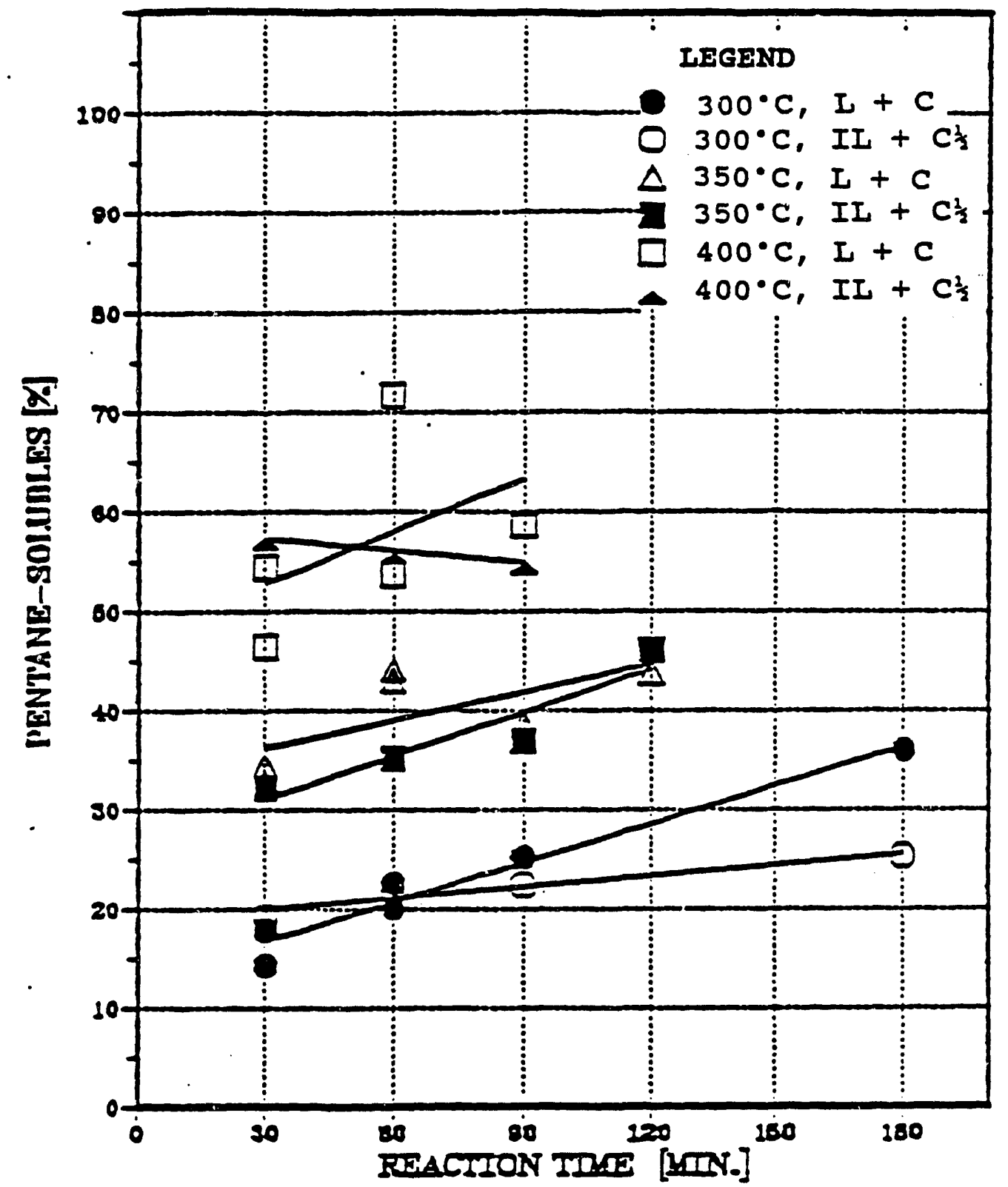

$I+C \quad$ Reaction of Coal and Iignin Mixturé

II $+C^{\frac{1}{2}}$ Mean Value of the Resonance of Coal and Iignin Alone.

Figure 1. Effect of Time and Temperature on overall Conversion of Wyodak Coal plus Iignin [1:1] and the yean value of the Reaction of myodak Coal alone and Iignin alone on the yield of n-pentane soluble products 


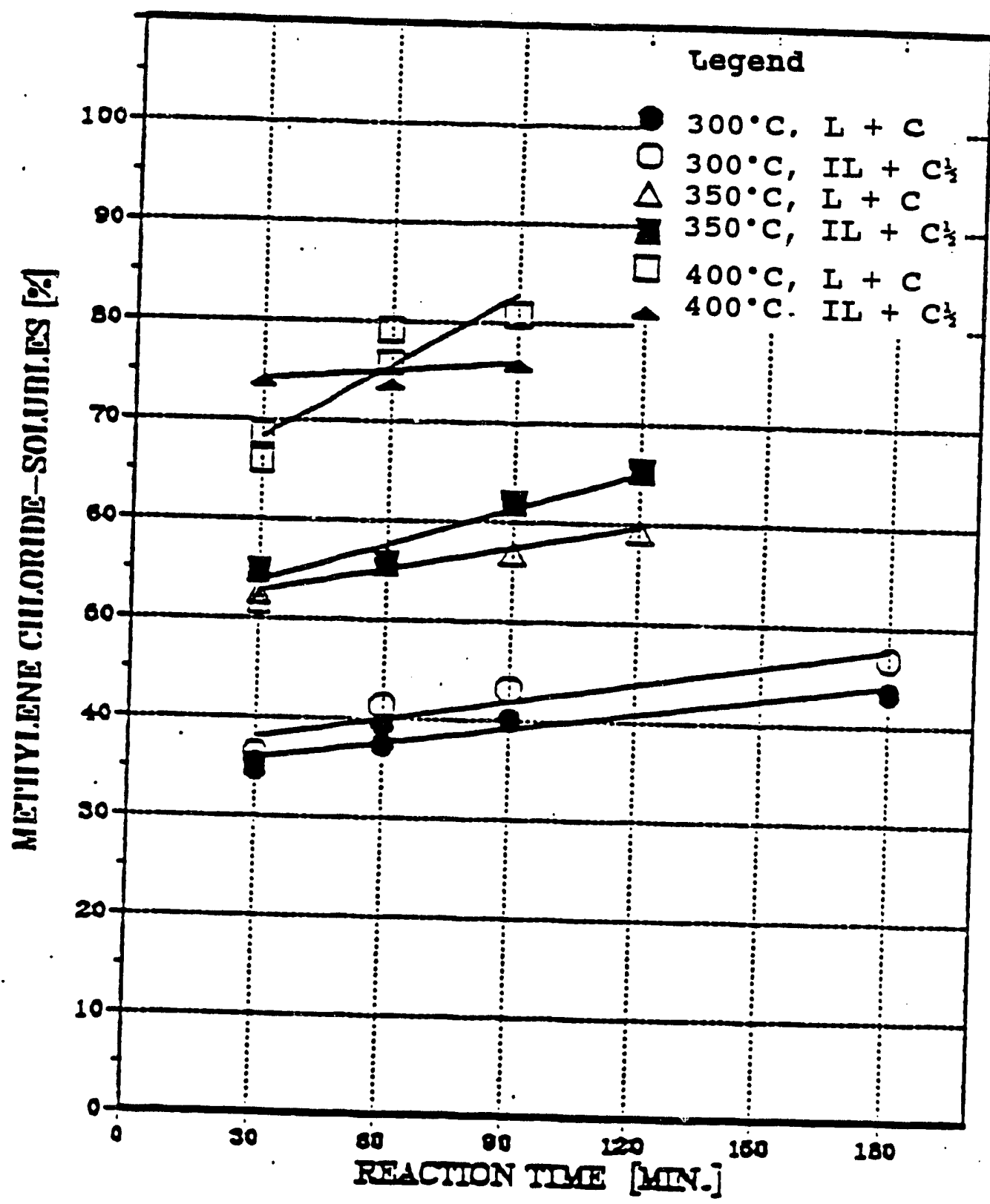

$\begin{array}{ll}L+C & \text { Reaction of Coal and Lignin Mixture }\end{array}$

IL $+C$ Mean Value of the Resonance of Coal and Lignin Alone

Figure 2. Effect of Time and Temperature on overall Conversion of Wyodak Coal plus Iignin [1:1] and the Mean Value of the Reaction of Fyodak Coal alone and Iignin alone on the Yield of Methylene Chloride Soluble Products 


\section{Task III}

\section{West Virginia University}




\section{Liquefaction Research in Pyrolysis, Catalysis and Coal Dissolution}

PI's:

Richard A. Bajura
Naresh Dalal
Mohindar Seehra
John W. Zondlo
Alfred Stiller
Wallace Whiting
James L. Clements

Report

Coordinator: Richard A. Bajura

Phone Number: (304) 293-2867

Period:

May 4, 1989 - May 3, 1990

\author{
John Penn \\ Jeffrey P. DeLooze \\ Manjula M. Ibrahim \\ Peter Stansberry \\ Shishir Gadam \\ Dady Dadyburjor
}

\section{Subtask III.1: Chemical Routes to Breaking Bonds: New Approaches to Low Temperature Liquefaction (John H. Penn, Chemistry Department)}

During the past year, we have completed our evaluation of the abilities of quinones to enhance THF extractability of five different coals. These quinones include benzoquinone (BQ) and 2,3-dichloro-5,6-dicyanoquinone (DDQ). In addition to evaluating the potential of quinones to enhance extractability, we have also explored the use of the counterpart of benzoquinone, (i.e., dihydroquinone, $\mathrm{DHQ}$ ) for its capabilities to induce enhanced extractability or liquefaction. The results of these studies show that $B Q$ enchances liquefaction for only one coal out of the five coals tested, while, in contrast, DHQ gives dramatically enhanced extractability at low temperatures for all five coals used in this study.

\section{Results and Discussion}

\section{- General Methodology}

Our general methodology for evaluating the effect of a given reagent on coal has been - described in previous reports. A solution of the coal (ca. $0.3 \mathrm{~g}$ ) and the desired reagent (ca. $0.3 \mathrm{~g}$ ) in $10.0 \mathrm{~mL}$ of freshly distilled acetonitrile (from $\mathrm{CaH}_{2}$ ) are degassed by three freeze-pump-thaw degassing cycles to remove oxygen from the sample. The glass tube is sealed by a flame and inserted into a steel tubing bomb reactor. The excess volume of the tubing bomb reactor is filled with acetonitrile for equilization of the pressure internal and external to the glass ampule. After sealing, the container is placed in an oil bath at the indicated temperature. After the 
indicated length of time, the sample is removed from the oil bath and placed in a Soxhlet extractor for 24 hours with THF as the solvent. The weights of the residue in the Soxhlet extractor and the THF extracts are determined to examine the mass balance. Control experiments showed that $\mathrm{BQ}$ and $\mathrm{DHQ}$ can be quantitatively recovered in the THF extract, while only $45 \%$ of the DDQ is THF extractable under the reaction conditions.

The liquid enhancements are defined as shown in eq. 1. In this equation, L.E. is the

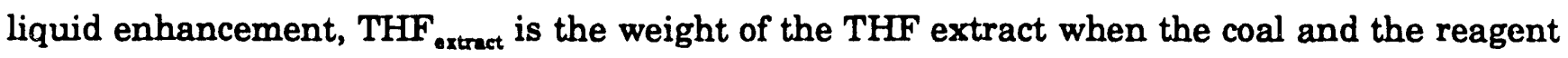
are allowed to react together, $T H F_{\text {cal }}$ and $T H F_{R}$ are the weights of the THF extract for coal and the reagent alone, respectively, and $\mathrm{Wgt}_{\text {coal }}$ is the starting weight of the coal sample. The THF $_{\text {coal }}$ runs were determined under the reaction conditions and are given in Table 1.

L.E. $=\frac{\mathrm{THF}_{\text {oxtract }}-\left(\mathrm{THF}_{\text {coal }}+\mathrm{THF}_{\mathrm{R}}\right)}{\mathrm{Wgt}_{\text {cool }}} \times 100 \%$

\section{BQ-Enhanced Liquefaction of Coal}

We have previously shown that $B Q$ will enhance the amount of THF extractables from Bakerstown coal [1]. During the current contract year these results have been reproduced by additional co-workers and exhaustively tested. As seen in Table 2, the amount of BQ used relative to the amount of coal plays a large role in determining the THF extractable yield. When using small amounts of $B Q$ relative to the amount of coal (i.e., coal/BQ ratio $=3$ ), a $45 \%$ enhancement of the THF extractable yields was obtained relative to the situation when no $B Q$ is added to the Bakerstown coal. Interestingly, as the ratio of $B Q$ to coal is increased, the liquid enhancements go down. For example, a 1:1 weight ratio of BQ to coal results in a ca. $30 \%$ liquid enhancement while a BQ to coal ratio of 2 gives only a 7\% liquid enhancement. The same trend is observed for Sewell coal in which increasing the amount of BQ relative to the weight of the coal results in decreased THF extractable yields. However, in the case of Sewell coal, the addition of $B Q$ results in a decrease in the THF extractable yields relative to that obtained for the extraction of the coal itself.

The finding that small amounts of $B Q$ give large THF extractable enhancement encouraged us to examine the potential of $B Q$ to act as a catalyst for enhancing liquid yields in a variety of coals selected to represent a variety of ranks. However, we found that $B Q$ was unable to enhance the THF extractable yield for any of the other coals selected for this study. In fact, use of $1: 1$ weight ratios of $B Q$ to the coal resulted in negative enhancements (i.e., the yield of THF extractables was less than observed for just the coal itself). The observation of positive liquefaction enhancements for Bakerstown coal may suggest that BQ is useful for coals which do not depolymerize well by themselves.

A further observation is that the enhancement in THF extractable yields is greatest for 
smaller amounts of $B Q$ relative to the coal. Since the amount of the liquid enhancement is greatest at the lowest concentration, we believe that $B Q$ does indeed have some catalytic effect which is operative in all coals. However, the negative enhancement of the THF extractable yields for the majority of coals indicates that $\mathrm{BQ}$ may actually play a negative role in enhancing THF extractable yields and counterbalance the beneficial effects of $B Q$ in higher rank coal. Therefore, $\mathrm{BQ}$ seems to be ruled out as a reagent for enhancing commercially useful coal liquefaction.

\section{DDQ Enhanced Liquefaction:}

A second quinone was evaluated for its potential to enhance the THF extractable yield in coal. This quinone was DDQ and it was selected for evaluation purposes because it gave the fastest rates of bond cleavage reactions for a series of benzylic ethers [2]. However, in contrast to the $B Q$ enhanced liqufaction, as can be seen in Table 2, the experiments with DDQ lead to a marked decrease in the amount of THF extractables. Presumably, this decrease results from enhanced dehydrogenation, which is well known to be enhanced by DDQ. This result led to abandonment of DDQ as a liquefaction reagent.

\section{DHQ Enhanced Liquefaction}

Studies on the dihydroquinone enhanced liquefaction were undertaken because of the early initial success in the BQ enhanced liqufaction of Bakerstown coal. The DHQ molecule is the reduced form of $\mathrm{BQ}$, containing one molecule of hydrogen for each molecule of $\mathrm{BQ}$. This extra molecule of hydrogen has the potential to allow DHQ to react in a fashion similar to known hydrogen-donor solvents (e.g., tretralin). The strategy of using DHQ has the potential advantage that the $\mathrm{BQ}$ formed in the reaction may act synergistically by inducing further bond cleavage or depolymerizations than can be obtained by using hydrogen-donor solvents.

This strategy has been found to be amazingly effective as seen in Table 2. DHQ induces a dramatic enhancement of liquid yields for ali five coals in this study. In fact, the lowest enhancement observed here is with Sewell coal where the enhancement in THF extractables is - only $33 \%$. The maximum enhancement is for Bakerstown Coal where a ca. $66 \%$ enhancement is observed. These results are particularly significant when it is considered that the

- depolymerization reactions are occurring at the relatively mild temperature of $200^{\circ} \mathrm{C}$. Although the reaction times are still somewhat long ( 48 hours), the low temperature pathway gives great promise for novel low temperature liquefaction conditions.

\section{Discussion}

The results presented here allow several conclusions concerning the $\mathrm{BQ}$ induced 
enhanced liquefaction to be made. First, maleic anhydride (MA) has been shown to be an effective liquefaction reagent by breaking of pi stacking interactions in the coal. Since MA has been shown to be an effective liquefaction reagent, then $B Q$ should behave somewhat similarly since $\mathrm{MA}$ is known to react only slightly faster than $B Q$ in model compound reactions. Since $B Q$ functions very poorly as a liquefaction reagent and, in fact, acts better in lower concentrations, it is unlikely that $B Q$ acts to break up pi stacking interactions through Diels-Alder reactions with the coal. Therefore, the role of $B Q$ must be as a hydrogen atom donor.

A second result which can be determined from the results described above is that $D H Q$ serves as an excellent liquefaction reagent at very low temperatures. DHQ gives remarkable liquid enhancements, presumably through hydrogen donation. Experiments are currently underway to verify that $B Q$ is formed in these reactions and would indicate that the enhanced liquefaction is due to the hydrogen donation mechanism.

Table 1

Coal Coal Rank \%

Bakerstown

Sewell

Pittsburgh \#8

Utah Blind Canyon

North Dakota Beulah

Zap

Lignite
\% THF Number of

Extractable Experiments

19.9 ( )

$26.2(1.1) \quad 2$

High Volatile Bituminous

High Volatile Bituminous
34.4 (3.0)

$31.0(1.1)$

72.5 (3.8)
2

4

4 
Tablo 2

\begin{tabular}{|c|c|c|c|c|c|c|c|}
\hline Coal & $\begin{array}{l}\text { Coal/ } \\
\text { Reagent }\end{array}$ & Reagent & $\begin{array}{l}\text { Liquid } \\
\text { Enhancement }\end{array}$ & $\begin{array}{l}\text { Temp } \\
\left({ }^{\circ} \mathrm{C}\right)\end{array}$ & $\begin{array}{l}\text { Time } \\
\text { (h) }\end{array}$ & $\begin{array}{l}\text { Mass } \\
\text { Balance }\end{array}$ & $\begin{array}{l}\text { Number of } \\
\text { Experiments }\end{array}$ \\
\hline & Ratio & & & & & & \\
\hline Bakerstown & 0.5 & $\mathbf{B Q}$ & $7.1(3.8)$ & 200 & 48 & $106(3.5)$ & 3 \\
\hline & 1 & $\mathbf{B Q}$ & $29.6(0.8)$ & 200 & 48 & $112(14)$ & 3 \\
\hline & 1 & $B Q$ & $14.6(4.9)$ & 200 & 24 & $111(1)$ & 4 \\
\hline & 3 & $\mathrm{BQ}$ & $45.2(2.4)$ & 200 & 48 & $120(5.7)$ & 4 \\
\hline & $?$ & $\mathrm{DHQ}$ & $65.9(3.2)$ & 200 & 48 & $138(2.8)$ & 2. \\
\hline Sewell & 0.5 & $\mathbf{B Q}$ & $-40.3(4.9)$ & 200 & 48 & $125(5.8)$ & 4 \\
\hline & 1 & $\mathrm{BQ}$ & $-21.6(8.7)$ & 200 & 48 & $130(5.1)$ & 4 \\
\hline & 3 & $\mathrm{BQ}$ & $-7.8(1.7)$ & 200 & 48 & $123(7.9)$ & .4 \\
\hline & 1 & DHQ & $32.3(4.2)$ & 200 & 48 & $128(2)$ & 2 \\
\hline Pittsburgh \#8 & 1 & $B Q$ & $-27.7(3.5)$ & 200 & 48 & $124(2.5)$ & 4 \\
\hline & 1 & $D D Q$ & $-91.7(2.9)$ & 200 & 48 & $136(2)$ & 2 \\
\hline & 1 & DHQ & $41.8(8.4)$ & 200 & 48 & $132(4.1)$ & 3 \\
\hline Blind Canyon & 1 & $\mathbf{B Q}$ & $-8.6(3.0)$ & 200 & 48 & $130(5.5)$ & 4 \\
\hline & 1 & DHQ & $45.5(4.6)$ & 200 & 48 & $130(2.8)$ & 2 \\
\hline North Dakota & 1 & BQ & $-12.7(5.1)$ & 200 & 48 & $127(1)$ & 2 \\
\hline
\end{tabular}

\section{References}

1. J.H. Penn and P. Yassinni, 3rd Annual CFFLS Technical Meeting, Auburn, AL, August, 1989.

2. J.H. Penn and Z. Lin, J. Org. Chem. 1990, 55, 1554. 
Role of Free Radicals and Catalysis-active Sites in Coal

Liquefaction (Naresh Dalal and Jeffrey P. DeLooze, Department of Chemistry)

\begin{abstract}
Summary
The lack of information about the chemical structure of coal-based radicals has been a major obstacle in the understanding of the structural-chemical reactivity relationship. Our past research has indicated that the electron nuclear double resonance (ENDOR) technique has

- the potential of providing the most accurate structural information on the coal-based radicals. Unfortunately, the sensitivity of the best available ENDOR instrumentation was too low for liquefaction studies. During the current year, however, we have developed an ENDOR probe which yields an increase in the sensitivity of the technique that enables us to investigate the structural-chemical reactivity patterns of coal-based free radicals under mild conditions suitable for liquefaction. We have used this methodology to examine chemically characterized mlinois \#6 at temperatures up to $200^{\circ} \mathrm{C}$. A methodology was developed for the analysis of the resulting ENDOR signals in which three different types of protons were identified. Analyses were also performed on the temperature dependence of the ENDOR signal intensity. Efforts are currently being made to enhance the measurement methodology's sensitivity above $200^{\circ} \mathrm{C}$.
\end{abstract}

\title{
Introduction
}

It is well established that free radicals must play a dominant role in coal liquefaction. Thus, a detailed understanding of the reaction mechanism(s) of the free radicals in coal is thought to be essential for the further improvement in the efficiency of coal liquefaction. However, a lack of knowledge concerning the chemical structure(s) of these same coal-based radicals has been a major hinderance in the development of the possible reaction mechanism(s) and, hence, an impediment to the improvement of liquefaction technology. The difficulty in this aspect has been that the structure(s) of the radicals is (are) complex and, hence, standard methods of radical structure determination, such as ESR, are not adequate. Our working hypothesis has been that the high resolution possible with the electron nuclear double resonance (ENDOR) technique would enable us to provide the much needed structural information on the radicals.

Commercially available ENDOR instrumextation does not provide the sensitivity for the required measurements at temperatures of interest to coal liquefaction scientists. Thus, our first task was the development of a new probe that would enable us to make measurements at $200^{\circ} \mathrm{C}$ and higher. We have developed such a probe, as described in our first quarterly report this year, and the results obtained with it in the studies of Illinois \#6 coal are summarized below. Illinois \#6 was chosen because it has been accepted by many researchers as a model coal 
for liquefaction studies. Thus, it is possible to obtain large amounts of uniformly processed coal along with a detailed analysis of the chemical constituents of each sample.

\section{Experimental Results}

The ENDOR measurements were made with a Bruker ER-200-D ENDOR spectrometer, using an ENDOR probe designed in our laboratory, as described in our earlier report. The ENDOR spectral lineshape was found to be quite sensitive to the instrumental parameters as well as sample preparation. A most important factor was the removal of oxygen from the sample via evacuation: the lower the overall pressure in the sample tube, the stronger became the ENDOR signals, especially those corresponding to the larger couplings. In order to find the experimcatal parameters which would lead to the optimum signal-to- noise ratio, we carried out systematic ENDOR measurements on the llinois \#6 coal, by varying the microwave power, radio frequency (rf) power, and sample temperature.

The critical parameters for obtaining maximum signai to noise ratio in the ENDOR response from the nlinois \#6 coal above room temperature were as follows:

\begin{tabular}{|c|c|}
\hline microwave power: & 13 \\
\hline rf power: & $95 \mathrm{~W}$ \\
\hline FM depth: & $200 \mathrm{kHz}$ \\
\hline scan range: & $10-35 \mathrm{MHz}$ \\
\hline temperature: & $150 \mathrm{~K}$ \\
\hline magnetic field: & $3,385 \mathrm{G}$ \\
\hline lock-in phase: & $0 / 90^{\circ}$ \\
\hline time constant: & $100 \mathrm{~ms}$ \\
\hline
\end{tabular}

Figure 1 is a typical plot of the ENDOR spectrum of protons in an Arkwright, West Virginia, coal as reported earlier [1] with the original, non-optimized probe under the previously stated operating parameters. The spectrum shows quite high baseline noise and a poor signal-to-noise ratio. Figure 2 shows a typical ENDOR spectrum of the llinois \#6 coal, obtained with the new probe; the greatly reduced noise level and, hence, the much higher signal-to-noise ratio is readily apparent. The improvement in the signal-to-noise ratio was found to be at least a factor of 200. This improvement in signal-to-noise also made it possible to obtain still higher spectral resolution via second harmonic detection, as demonstrated by the lower plot in Figure 2. Figure 3 is an expanded scale view of the optimized spectra in Figure 2. Note that the apparent discrepancies in the measured ENDOR frequencies is due to the re-tuning of the cavity for optimum response. 
Figure 4 shows typical ENDOR spectra of Illinois \#6 as a function of temperature. It can be clearly seen that the new probe allows not only for a significant improvement in the room temperature signai, but also provides spectra at previously unheard of temperatures higher than that ever achieved before. Figure 5 shows a plot of the intensity of the ENDOR response as a function of temperature. It is clearly seen that the ENDOR intensity drops as a near-quadratic power function above room temperature to the point that the signals become undetectable above $-425 \mathrm{~K}\left(-150^{\circ} \mathrm{C}\right)$.

\section{Analysis Procedure}

The measured ENDOR frequencies, $v_{\mathrm{E}}$, were analyzed in terms of the corresponding hyperfine coupling, $A_{4}$, via the following effective spin Hamiltonian [2,3]:

$$
\mathcal{H}=\langle S\rangle \underline{A}_{H} \cdot \hat{I}-g_{n} B_{n} \hat{H} \cdot \hat{I},
$$

where $\langle S\rangle$ is the effective electron spin; $\hat{I}$, the electron spin; $\hat{H}$, the applied magnetic field; and $g_{a} B_{n}$, the magnetic moment of the nucleus under consideration, in this case, protons. The general solution of equation (1) will give the energy levels as a function of the applied magnetic field $\hat{H}$ and the properties of the hyperfine coupling tensor, $\hat{A}_{H}$. The ENDOR transitions are then given by the differences in the energy levels, with the selection rules $\Delta m_{1}=0, \Delta m_{1}= \pm 1$, where $m_{1}$ and $m_{1}$ are the electronic and nuclear spin quantum numbers. In the high-field approximation, i.e., when $\hat{H}>\underline{A}_{H}$ (in Gauss), and along the principal directions, the ENDOR transition frequencies, $v_{E}$, are related to $\underline{A}_{H}$ and $\hat{H}$ via:

$$
v_{E}=A_{H} / 2+g_{a} B_{n} H
$$

For the spectrum shown in Figure $3, \mathrm{~g}_{a} B_{n} \hat{H}$ was equal to $14.492 \mathrm{MHz}$, and thus equation (2) provides a direct method of calculating hyperfine couplings from the measured ENDOR frequencies.

The hyperfine couplings calculated via equation (2) are listed in Table 1. It is clear that the ENDOR measurements show that there are essentially three types of protons in the struct re of the free radicals in Dlinois \#6 coal. The majority of the protons, labeled \#1 in the table (ref. Figure 3), exhibit no measurable isotropic hyperfine coupling with the unpaired electron. These protons are assigned to the coal matrix, although some of these might belong to the radical itself but located at positions of negligible spin density. The second set of protons,

labeled \#2, exhibits a coupling of about $3 \mathrm{MHz}$. The third set of protons (labled \#3) exhibits the largest hyperfine coupling $(=10.6 \mathrm{MHz})$.

The above results indicate that the free radicals imbedded in the matrix of the Ilinois \#6 coal are essentially highly delocalized aromatic structures: protons of type \#3 being located at the positions of high spin density, i.e. ortho- to the free electron center. Protons of type \#2 might be located at either the meta- or para- positions. 


\section{Theoretical Model}

While exact details of the model explaining the rapid decrease in the ENDOR intensity upon heating the Hlinois \#6 coal have not yet been worked out, it is well established [3] that the ENDOR response is determined by an effective electron-nuclear spin-lattice relaxation time $\left(T_{10}\right)$. In general, one then sees that the temperature dependence of the ENDOR signals simply reflects the decrease in $T_{10}$. For simple model compounds, $T_{10}$ is a sensitive function of the molecular motion of the radical, given by the autocorrelation function, $J\left(\omega_{L}\right)$, as:

$$
\begin{aligned}
& \left(T_{1}\right)^{-1}=B_{1} J\left(\omega_{L}\right), \text { where } \\
& J\left(\omega_{L}\right)=2 / \tau\left(1+\omega_{L}^{2} \tau^{2}\right) \\
& \tau=\tau_{0} \exp \left(E / k_{B} T\right)
\end{aligned}
$$

Where $\tau$ is the correlation time for the relevant molecular motion, $\tau_{0}$ the pre-exponential factor, and $\underline{E}$ the activation energy. From these relationships, it is seen that $T_{10}$ will decrease rapidly with increase in temperature when $\omega^{2} \tau^{2}<1$ (i.e., fast motion limit).

We are now carrying out a detailed analysis of the temperature dependence of the ENDOR intensity as shown in Figure 4 as assuming different values for $\tau_{0}$ and E. A satisfactory set of these parameters fitting ihe data in Figure 4 will provide us with further insight into the chemical structure and, more importantly, the relevant molecular motions in the radical entities in the Illinois \#6 coal, and in all probability, in other coals.

\begin{tabular}{|c|c|c|c|}
\hline Proton \# & $\mathrm{v}_{\mathrm{E}} \mathrm{MHz}$ & $\mathrm{A}_{0}{ }^{\circ} \mathrm{MHz}$ & Assignment \\
\hline 1 & 14.448 (two) & 0.0 & coal matrix \\
\hline 2 & 15.448 & 2.87 & meta-, para- \\
\hline 3 & 19.765 & 10.63 & ortho-, para- \\
\hline
\end{tabular}

Table 1: Proton ENDOR transition frequencies, $v_{B}$ and hyperfine couplings, $\underline{A}_{H}$, for the free radicals in nlinois \# 6 Coal.

\section{References}

1. N. S. Dalal and J. P. DeLooze, "ENDOR Characterization of the Chemical Structures of Free Radicals in Coal," paper presented at the Third Annual Technical Meeting of the Consortium for Fossil Fuel Liquefaction Science, Auburn, Alabama, 1-3 August 1989.

2. See, for example, Electron Spin Double Resonance, by L. Kevan and L. D. Kispert, Wiley Interscience, New York, 1976.

3. N. S. Dalal, Advances in Magnetic Resonance 10, 119-218 (1982). 


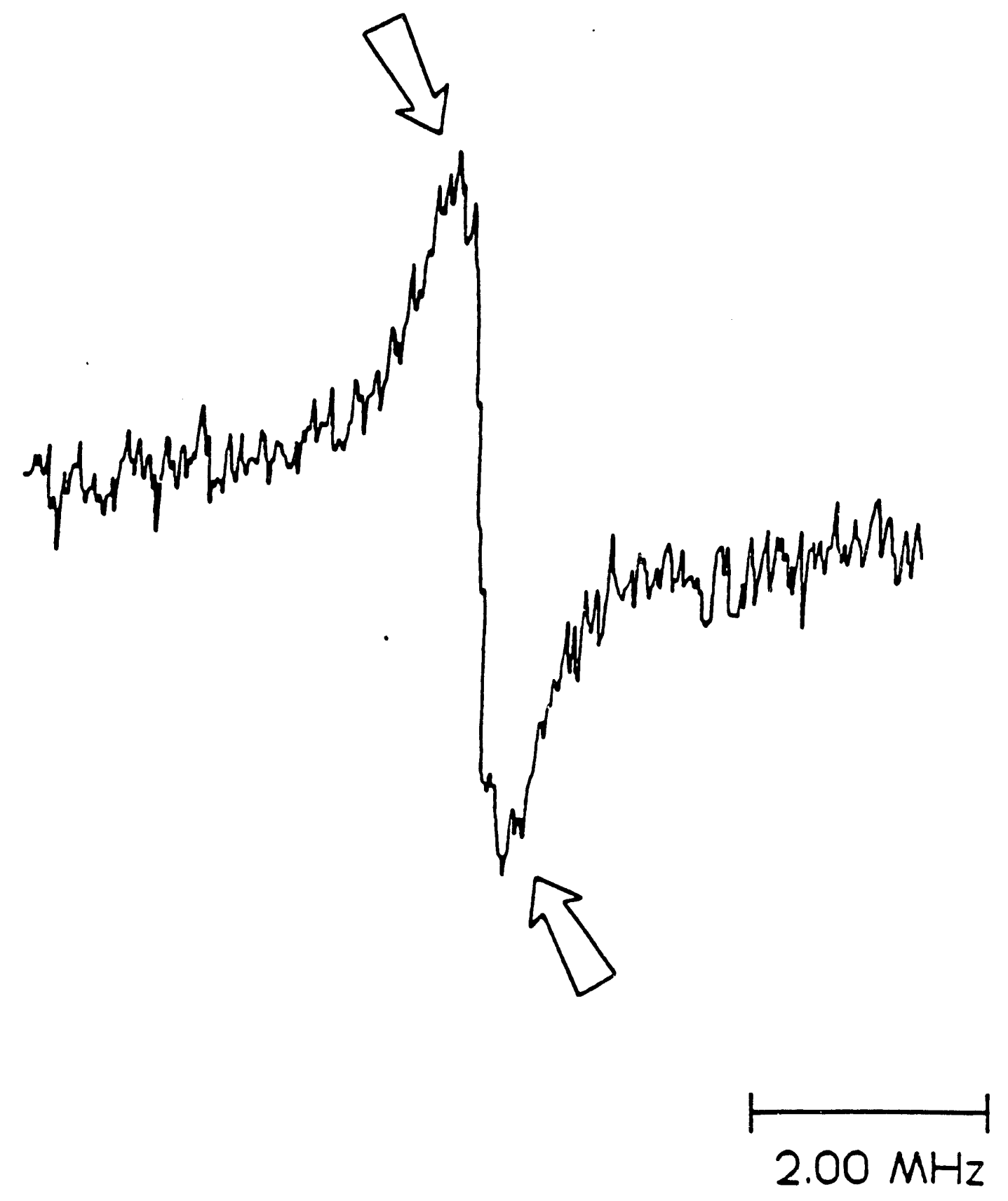

Figure 1: $\quad$ ENDOR spectrum of Arkwright, West Virginia coal sample Arrows indicate the sidebands separated by approximately $750 \mathrm{kHz}$ 


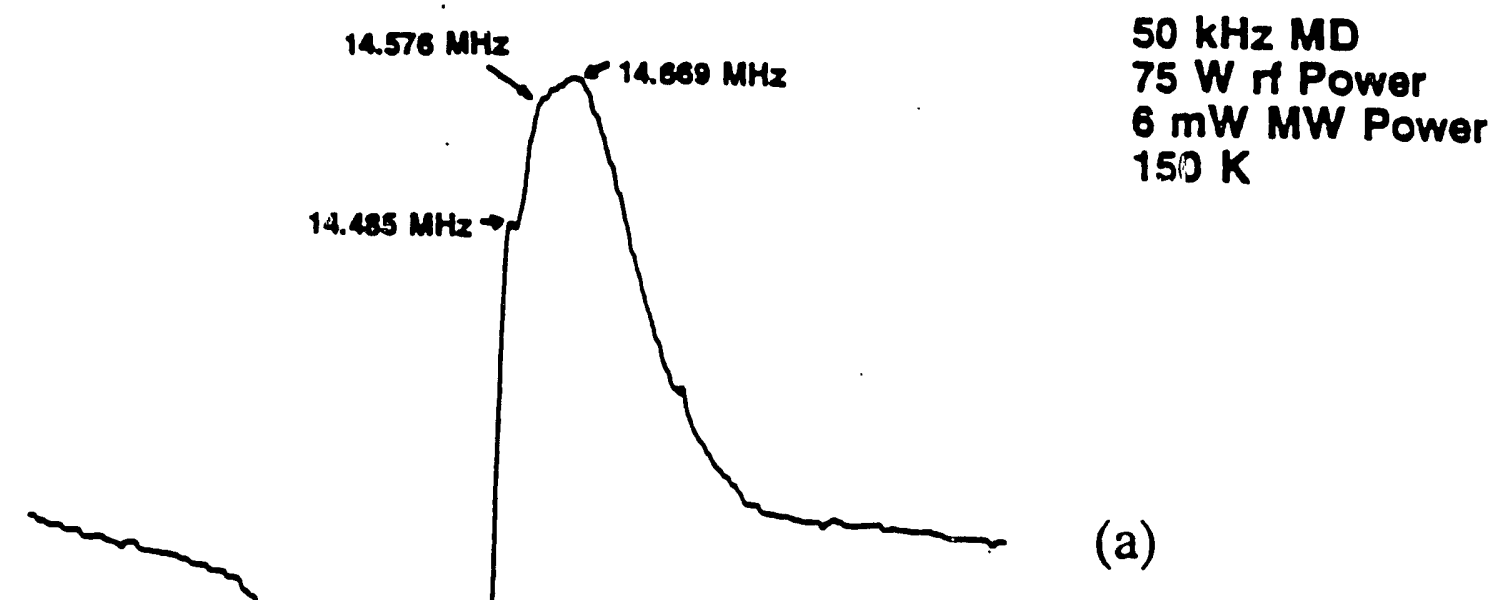

$50 \mathrm{kHz}$ MD

75 W if Power $6 \mathrm{~mW}$ MW Power $150 \mathrm{~K}$

(a)

(b)
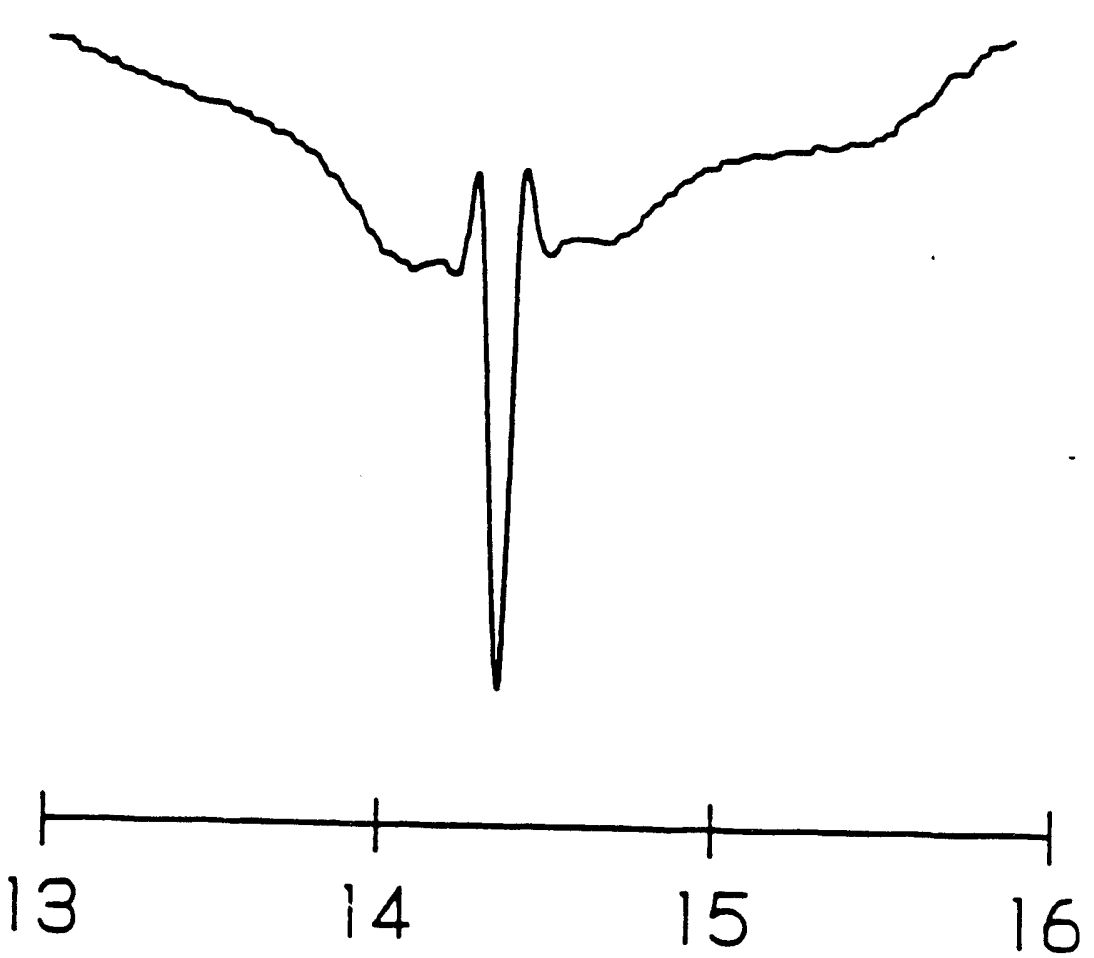

$M H z$

Figure 2: $\quad$ ENDOR spectra of Illinois \#6 coal (a) first derivative and (b) ser.ond derivative The higher resolution of the second-derivative presentation (as compared to the first-derivative presentation) can be noted from a comparison of (a) and (b). 


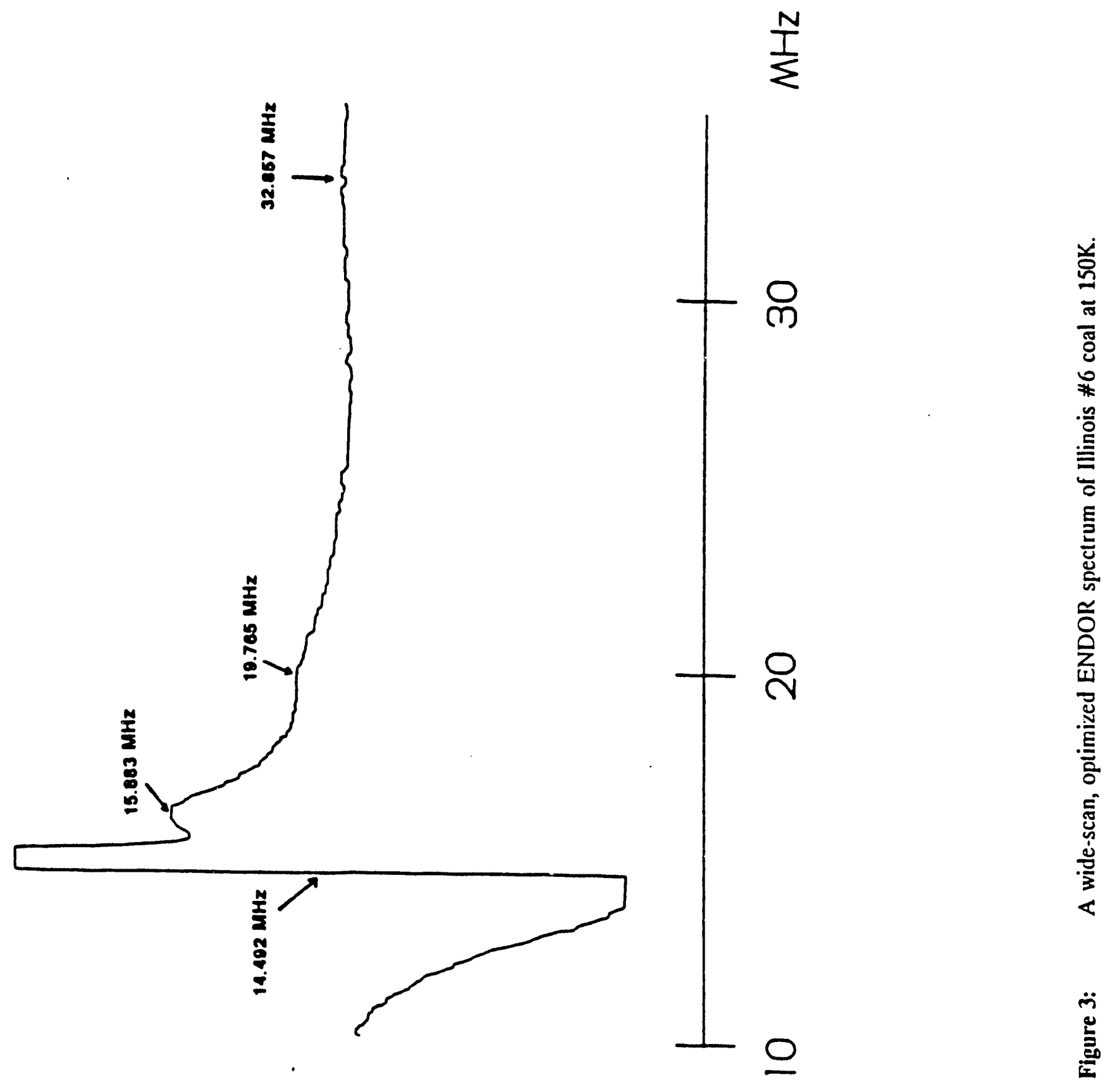



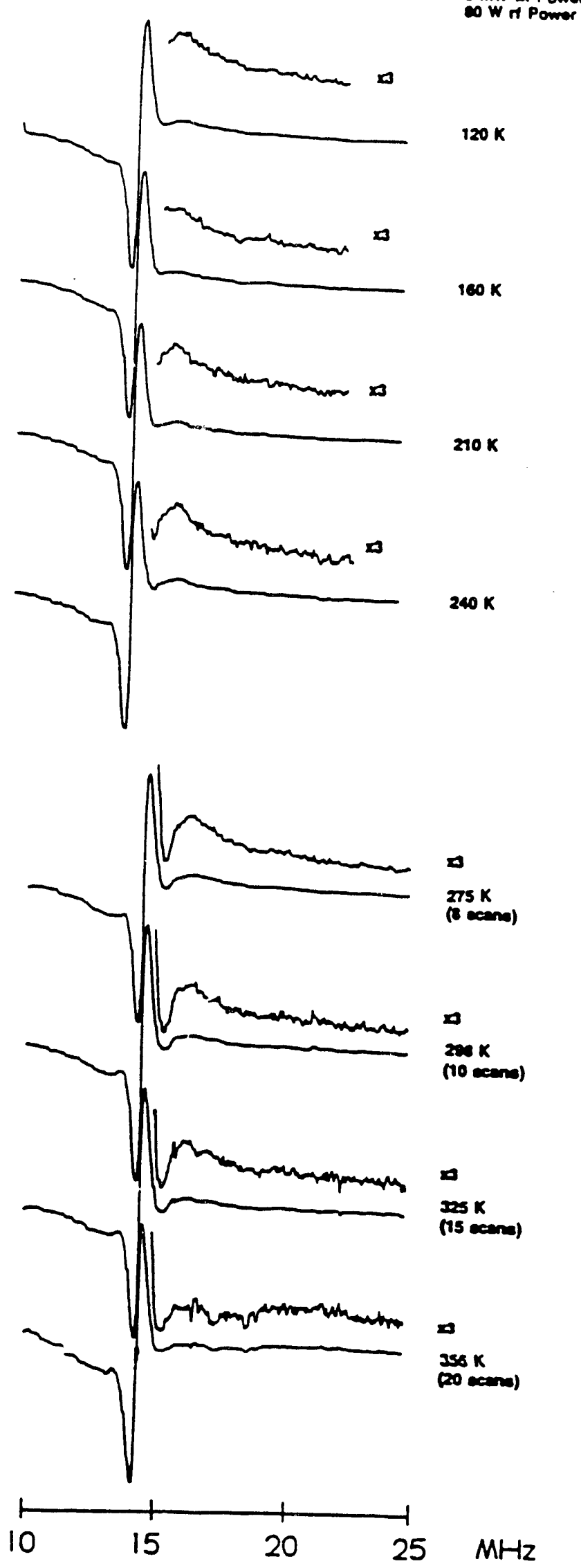

Figure 4: Temperature dependence of the ENDOR spectra of Illinois \#6 coal. 


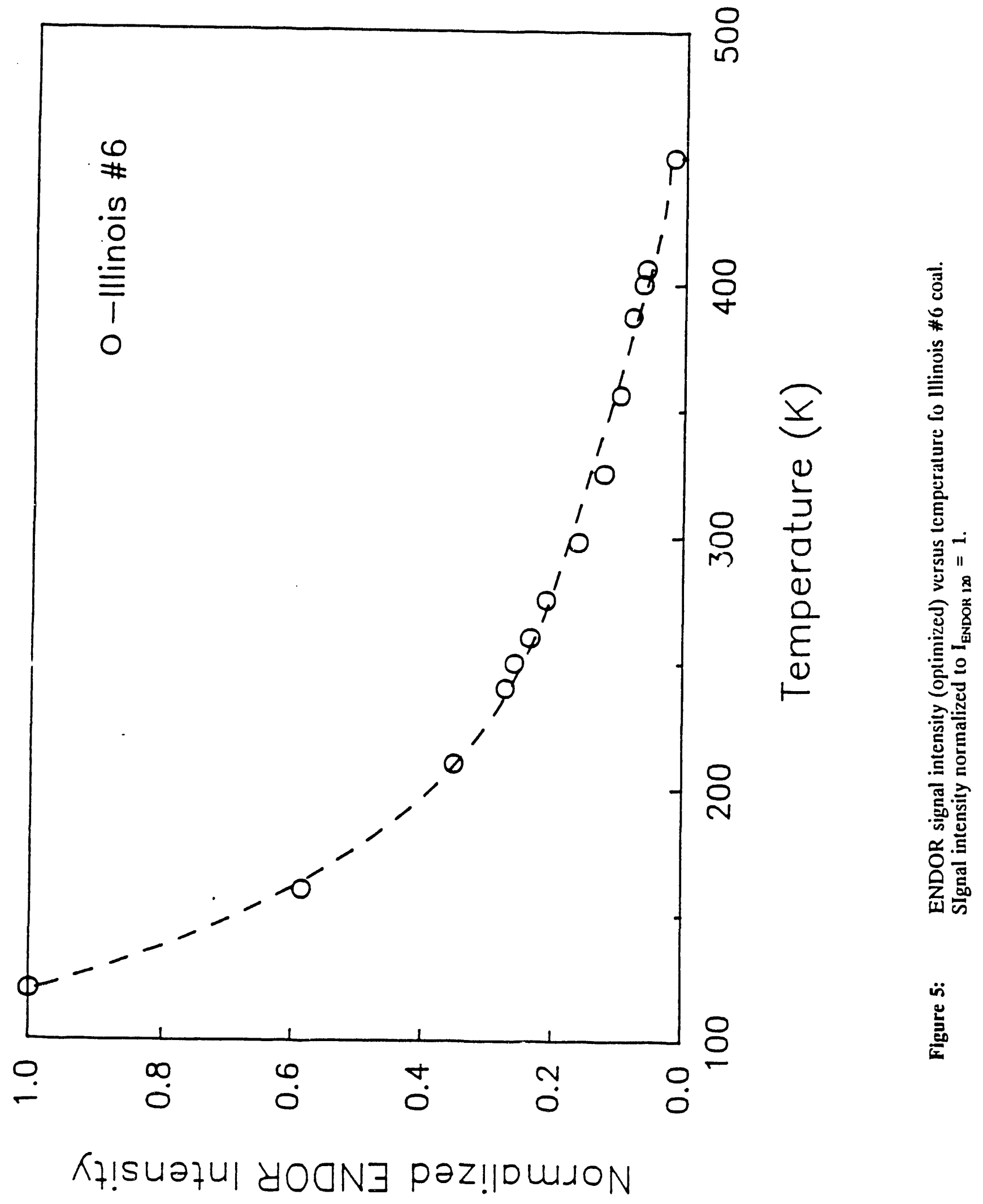




\section{SubTask III.3: Kinetics of Coal Hydrogenation and Cracking with Lewis}

Acids (Mohindar S. Seehra and Manjula M. Ibrahim,

Physics Department)

\section{Objectives}

The objectives of this research were to investigate the effect of Lewis acids $\left(\mathrm{ZnCl}_{2}\right.$, $\mathrm{ZnBr}_{2}, \mathrm{ZnI}_{2}$...) on the cracking and hydrogenation of coals, with particular attention to temperatures below $300^{\circ} \mathrm{C}$, since the earlier studies were limited to higher temperatures. The proposal included the use of in-situ ESR (electron spin resonance) spectroscopy of free radicals generated in cracking and the use of TG/DTG (thermogravimetry/differential thermogravimetry) for measuring the mass loss and rate constants.

\section{Summary of Results}

Our measurements show that at temperatures as low as 150 to $175^{\circ} \mathrm{C}$, zinc halides promote approximately a $30 \%$ mass loss due to release of volatiles. A corresponding increase in the free radical density is observed from ESR spectroscopy in flowing nitrogen gas. These observations are interpreted in terms of coal cracking catalyzed by the zinc halides. The effect of $\mathrm{H}_{2}$ gas is to lower the free radical density, presumably because of quenching of the free radical. The practical implications of these results are that significant liquefaction of coals at these low temperatures may be possible and that at these temperatures, the corrosive nature of zinc halides may not pose as severe a practical problem as encountered at higher temperatures.

\section{Introduction}

One of the thrust areas of research in the direct liquefaction of coals is to find a suitable catalyst or a combination of catalysts to effect conversion under milder conditions of temperature and pressure. Lewis acids ${ }^{12}$ and in particular zinc halides ${ }^{3}$ have been used as test catalysts for lowering the operating conditions and to increase product selectivity. Struck and Zielke ${ }^{3}$ reviewed the status of zinc chloride as a catalyst for coal liquefaction in bench scale experiments. These experiments showed that when used in 1:1 coal to zinc chloride ratio, it yielded superior product distribution and selectivity. These experiments were done at $420^{\circ} \mathrm{C}$ and 17.3 MPa hydrogen pressure. There are a number of other studies available on the catalytic activity of zinc halides in coal liquefaction and in various model compounds..$^{3-6}$ However these studies were done either at high pressures of hydrogen or at temperatures above $300^{\circ} \mathrm{C}$. A disadvantage of reacting coal at elevated temperatures (besides additional costs) is that thermal cracking makes substantial contributions to the reaction and there is a loss of full catalytic control and with it the gains in selectivity it can offer. On the other hand, at lower 
temperatures, a good balance of hydrogenation and cracking can be achieved to get better

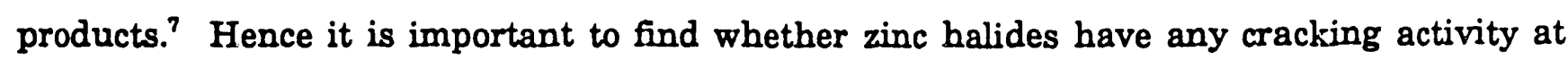
lower temperatures.

Here, we report substantial catalytic cracking of coals with zinc halides at temperatures below $200^{\circ} \mathrm{C}$. The evidence of the low temperature cracking with zinc halides is obtained by using thermogravimetric (TG) and in-situ electron spin resonance (ESR) experiments. We have observed substantial mass loss below $200^{\circ} \mathrm{C}$ when a coal is mixed with a zinc halide $\left(\mathrm{ZnCl}_{2}\right.$, $\mathrm{ZnBr}_{2}, \mathrm{ZnI}_{2}$ ). In addition we observe generation of additional free radicals at temperature using in-situ ESR spectroscopy. These observations are strong evidences of catalytic cracking of coals by zinc halides, at temperature as low as $150^{\circ} \mathrm{C}$, since in our recent experiments ${ }^{8}$ we have reported a strong correlation of the liquefaction yield of coals with the rate of mass loss in TG experiments and to a lesser extent with the thermally generated free radicals. Also we demonstrate that the effect of hydrogen is to lower the density of free radicals, perhaps by quenching. Results of these experiments are presented below.

\section{Experimental Section}

The coals used in our experiments are the same as used in our recent experiments on liquefaction and TG/ESR experiments. They are from Kentucky mines and their proximate, ultimate and petrographic analyses are given in Ref. 8. In Table 1, some of the important features of these coals are listed to aid comprehension of this report. The halides, viz. $\mathrm{ZnCl}_{2}$, $\mathrm{ZnBr}_{2}$ and $\mathrm{ZnI}_{2}$, were obtained from Alfa Products. The coals and the halides, taken in the appropriate ratios, were dry-mixed in a mortar. TG measurements were carried ont in a Mettler system, Model TA3000, using $10 \mathrm{mg}$ of the sample, heating rate of $10^{\circ} \mathrm{C} / \mathrm{min}$ and a gas flow rate of $100 \mathrm{~cm}^{3} / \mathrm{min}$.

ESR experiments were carried out at $\mathrm{X}$ band $(9 \mathrm{GHz})$ frequencies using a reflection-type spectrometer with a $\mathrm{TE}_{102}$ mode cavity. Details of the experimental procedures for in-situ measurements to $600^{\circ} \mathrm{C}$ and for calculations of the free radical density, $\mathrm{N}$, have been reported previously. ${ }^{2-11}$ All our earlier measurements ${ }^{8-11}$ were carried out on ramples that were vacuum sealed in ESR tubes. To carry out the experiments in a flowing gas, the flow chamber shown in Fig. 1 was used. ESR measurements were done from ambient temperatures up to $450^{\circ} \mathrm{C}$ in the presence of nitrogen or hydrogen gas at the flow rate of $10 \mathrm{~cm}^{3} / \mathrm{min}$.

\section{Experimental Results}

The changes in the weights of zinc halides, and coal $F$ mixed with zinc halides in the ratio $1: 1$ as a function of temperature and under the flow of nitrogen gas are shown in Fig. 2. $\mathrm{ZnCl}_{2}$ and $\mathrm{ZnBr}_{2}$ are quite stable for temperatures up to $500^{\circ} \mathrm{C}$ whereas $\mathrm{ZnI}_{2}$ shows a rapid 
weight loss above $350^{\circ} \mathrm{C}$ probably because of thermal decomposition. The small losses observed for $\mathrm{ZnCl}_{2}$ and for $\mathrm{ZnBr}_{2}$ below $350^{\circ} \mathrm{C}$ are probably due to the release of adsorbed water. These losses are taken into consideration when calculating the weight loss of coal in the presence of a halide.

In Fig. 2, we also show the weight loss of a coal F (after correcting for the weight loss of the halide) when mixed with $\mathrm{ZnCl}_{2}, \mathrm{ZnBr}_{2}$ and $\mathrm{ZnI}_{2}$. Between 20 and $30 \%$ of the weight is lost, presumably because of the release of volatiles.

To demonstrate that the phenomenon of weight loss in coal $\mathrm{F}$ below $200^{\circ} \mathrm{C}$ in the presence of halides is not restricted to one coal only, we also carried out experiments with the remaining three coals of Table 1. The results using $\mathrm{ZnCl}_{2}$ are shown in Fig. 3. Although the weight loss and the temperature at which the first stage of the weight loss is completed varies slightly from coal to coal, the observations are qualitatively similar in all coals. The weight loss occurring above $350^{\circ} \mathrm{C}$ is probably due to thermal cracking as it is also observed in coals without the presence of zinc halides.

To measure the effect of halide loading on the weight loss, we show in Fig. 4 the remaining weight of coal $\mathrm{C}$ as a function of temperature for different loadings of $\mathrm{ZnCl}_{2}$. [The percentage loading is defined as (weight of $\mathrm{ZnCl}_{2}$ /weight of coal) $\times 100$ ]. The weight loss of coal at $175^{\circ} \mathrm{C}$ vs $\%$ loading is shown in Fig. 5 and it shows that the weight loss is non-linear, approaching saturation for $100 \%$ loading.

In Fig. 6 we have plotted the rate of mass loss normalized to the room temperature mass viz. (dm/dt)/mo vs temperature for coal $C$ and for coal $C$ mixed with the different halides at $100 \%$ loading. There is a peak in this reaction rate also at temperatures between 150 and $175^{\circ} \mathrm{C}$ in the presence of the halides and there is no change in this quantity in this temperature range for coal alone. Recent measurements have shown ${ }^{9}$ that this reaction rate correlates well with \% conversion, thereby indicating a possible significant effect of the halides on coal liquefaction at these low temperatures.

ESR spectra of all four coals at room temperature consist of a broad and a narrow component, reflecting the different macerals present in these coals. Following the studies of Retcofsky et $\mathrm{al}^{12}$ and Silbernagel et $\mathrm{al},{ }^{13}$ we infer that the broad component is due to vitrinites and exinites and the narrow component is due to inertinites. In our earlier ESR studies on the pyrolysis of coals, ${ }^{8-11}$ we have reported on the changes in the g-values, linewidths and free radical density, $\mathrm{N}$, as a function of temperature. Major changes occur in $\mathrm{N}$ only (after allowing for the Curie law variation of $N$ ). In Fig. 7, we have plotted $N$ vs temperature for coal $C$ and for coal $\mathrm{C}$ mixed with the three zinc halides at $100 \%$ loading. It is evident that above about $150^{\circ} \mathrm{C}$, the magnitude of $\mathrm{N}$ is considerably higher in the presence of a halide. Also, the four stages observed in the pyrolysis using ESR spectroscopy ${ }^{8-11}$ are now shifted to lower 
temperatures. The third stage, where major cracking is found to occur, is especially affected, as it is shifted by hundreds of degrees. Above about $450^{\circ} \mathrm{C}$, the ESR signal essentially disappears. These observations are consistent with the catalytic cracking of coals at lower temperatures due to zinc halides, as discussed later.

The effect of a flowing hydrogen gas on the free radical density $\mathrm{N}$ is shown in Fig. 8 , where we have plotted $\mathrm{N}$ vs temperature for coal $\mathrm{C}$ in $\mathrm{N}_{2}$ flow, for coal $\mathrm{C}+\mathrm{ZnCl}_{2}$ (100\% loading) in $\mathrm{N}_{2}$ flow, and for coal $\mathrm{C}+\mathrm{ZnCl}_{2}$ in $\mathrm{H}_{2}$ flow. It is quite clear that the effect of $\mathrm{H}_{2}$ lowers the observed free radical by quenching some of the free radicals density.

\section{Discussion}

The results presented above using TG, DTG (differential thermogravimetry) and ESR spectroscopy show that the zinc halides promote catalytic cracking of coals at temperatures between 150 and $175^{\circ} \mathrm{C}$. This is the lowest temperature at which cracking of coals using zinc halides has been reported. The extensive liquefaction experiments of Anderson and Miin ${ }^{1}$ using a variety of catalysts, including zinc halides, were all carried out at $300^{\circ} \mathrm{C}$ and the earlier bench scale liquefaction studies with $\mathrm{ZnCl}_{2}$ were carried out at $420^{\circ} \mathrm{C}$ and $17.3 \mathrm{MPa}$ of $\mathrm{H}_{2}$ pressure. We believe that observations reported here are important because they indicate significant cracking of coals at much lower temperatures. However, the present study alone is not sufficient to establish that high conversion of coals will occur with zinc halides at $150-175^{\circ} \mathrm{C}$. Also, the thermodynamics of the reaction may be different at higher pressures of hydrogen, than at lower pressures. Nevertheless, the prominent increase in the generation of free radicals, their stabilization by molecular hydrogen, and accompanying mass loss due to volatiles all point to catalytic cracking of coals at these low temperatures.

Our results support of some earlier published findings with zinc halides. For example, Jolly et al. ${ }^{14}$ studied the evolution of hydrogen and methane in the presence of $\mathrm{ZnCl}_{2}$ and observed an increase in the amount of these evolved gases when loading was increased from $1 \%$ to 25\%. The measurements of Yokono et al..$^{16}$ using in-situ ESR spectroscopy and pressures of $10 \mathrm{MPa}$ showed that, in the presence of nitrogen, $\mathrm{ZnCl}_{2}$ effects an increase in $\mathrm{N}$, whereas in the presence of $\mathrm{H}_{2}, \mathrm{~N}$ decreases. These observations, however, were made at temperatures above $300^{\circ} \mathrm{C}$, whereas we have observed similar phenomena at lower temperatures. From a study of model compounds in the presence of a donor solvent (tetralin) and hydrogen at high pressures, Vernon ${ }^{16}$ concluded that both molecular hydrogen and a source of free radicals, generated either catalytically or thermally, are necessary for good conversion. Our results show that zinc halides are effective in promoting an increase in free radicals and that there is quenching of the free radicals in the presence of $\mathrm{H}_{2}$.

The mechanism of the cleavage of linkages and cracking of aromatic structures by zinc 
halides has been investigated by Bell et al..$^{4,17}$ According to these studies, scission by zinc halides eventually leads to a formation of a carbonium ion and a smaller hydrocarbon. The studies reported here do not yield any new information on the mechanism since it has not been possible to resolve the free radicals observed in ESR spectroscopy. We are now in the process of developing a program to deconvolute the ESR spectra at various temperatures. If successful, this procedure may provide further insight into the nature of catalytically generated free radicals and of the free radicals quenched by hydrogen.

The practical implications of the results reported here are obvious if direct liquefaction experiments using zinc halides as catalysts were to show significant conversion at the low temperatures of $150-175^{\circ} \mathrm{C}$ useo here. It is therefore hoped that this work will stimulate such experiments because, at these lower temperatures, the corrosive nature of zinc halides ${ }^{3}$ may not be as severe a practical problem as at higher temperatures.

\section{References}

1. Anderson, L. L.; Miin, T. C. Fuel Proc. Tech. 1986, 90, 165-174.

2. Nomura, M.; Sakashita, H.; Miyaka, M.; Kikkawa, S. Fuel 1983, 62, 73-77.

3. Struck, R. T.; Zielke, C. W. Fuel 1981, 69, 796-

4. Mobley, D. P.; Bell, A. T. Fuel 1979, 58, 661-666.

5. Yokono, T.; Iyama, S.; Sanada, Y.; Makino, K. Fuel 1985, 64, 1014-1016.

6. Salim, S. S.; Bell, A. T. Fuel 1982, 61, 745-754.

7. Sulimma, A.; Leonhardt, P.; van Heek, K. H.; Juentgen, H. Fuel 1986, 65, 1457-1461.

8. Ibrahim, M. M.; Seehra, M. S.; Keogh, R. Fuel Proc. Tech. 1990(in press).

9. Seehra, M. S.; Ghosh, B.; Mullins, S. E. Fuel 1986, 65, 1315-1316.

10. Seehra, M. S.; Ghosh, B. J. Anal. Appl Pyralysis 1988, 13, 209-220.

11. Seehra, M. S.; Ghosh, B.; Zondlo, J. W.; Mintz, E. A. Fuel Proc. Tech.1988, 18, 279-286.

12. Retcofsky, H. L.; Stark, J. M.; Friedel, R. A. Anal. Chem. 1968, 40, 1699-1704.

13. Silbernagel, B. G.; Gebhard, L. A.; Dyrkacz, G. R.; Bloomquist, C. A. A. Fuel 1986, 65, 558-565.

14. Jolly, R.; Charcosset, H.; Boudou, J. P.; Guet, J. M. Fuel Proc. Tech. 1988, 20, 51-

15. Yokono, T.; Iyama, S.; Sanada, Y.; Shimokawa, S.; Yamada, E. Fuel 1986, 65, 1701-

16. Vernon, L. W. Fuel 1980, 59, 102-106.

17. Frederick, T. J.; Bell, A. T. J Catalysis 1984, 87, 196-209, 210-225, 226-237. 
Table 1. Weight $\%$ of selected constituents of the four coals used in these experiments. For more complete analysis; see Ref. 8.

\section{Other}

$\underline{\text { Coal Volatile Matter }} \underline{\mathrm{C}} \underline{\mathrm{H}} \quad \underline{\text { Vitrinite }} \underline{\text { Exinite }}$ Macerals

$\begin{array}{rrrrrrr}\text { C } & 36.03 & 81.54 & 5.72 & 83.9 & 6.3 & 9.8 \\ \text { D } & 25.64 & 83.66 & 4.95 & 22.0 & 10.4 & 67.6 \\ \text { E } & 30.68 & 87.74 & 5.13 & 41.0 & 12.6 & 46.4 \\ \text { F } & 38.65 & 84.36 & 5.55 & 60.0 & 12.0 & 28.0\end{array}$




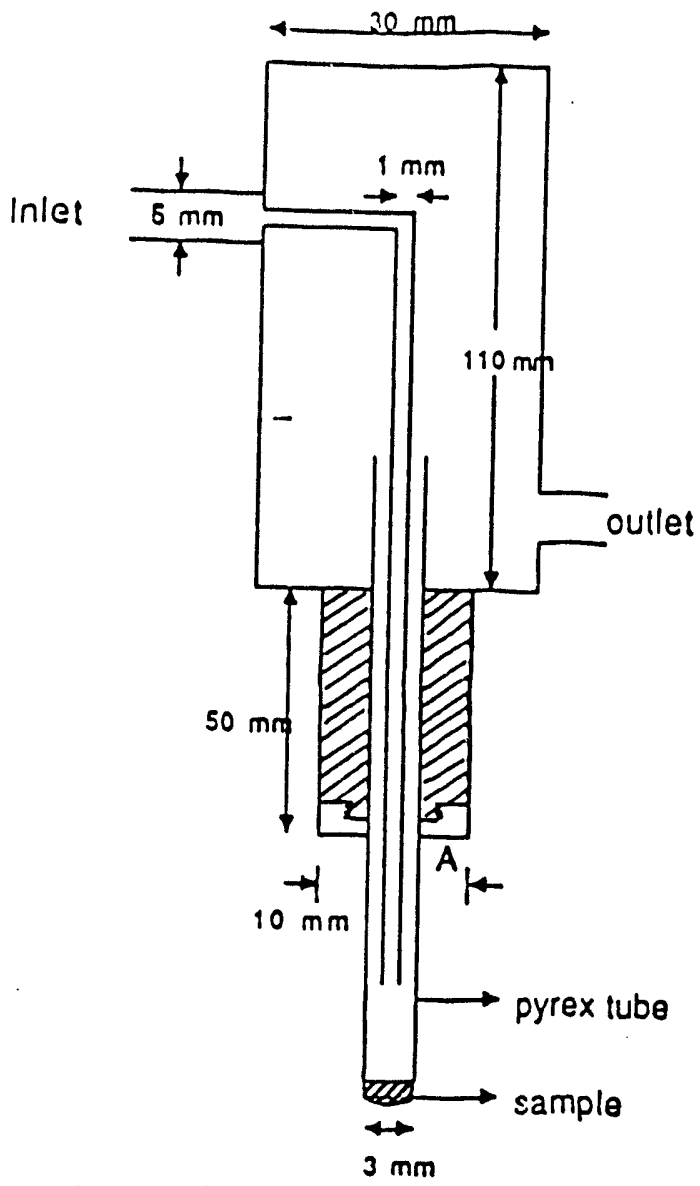

Fig. 1. Schematic diagram (not to scale) of the flow chamber used in the ESR experiments. The joint at $A$ allows the pyrex ESR tube to be adjustable in height below $A$.

Fig. 2. Percenatage remaining weight of coal $F$ plotted against temperature. Coal $F$ and the zinc halides are mixed in the $1: 1$ ration and correction for the weight loss due to the halides is applied.

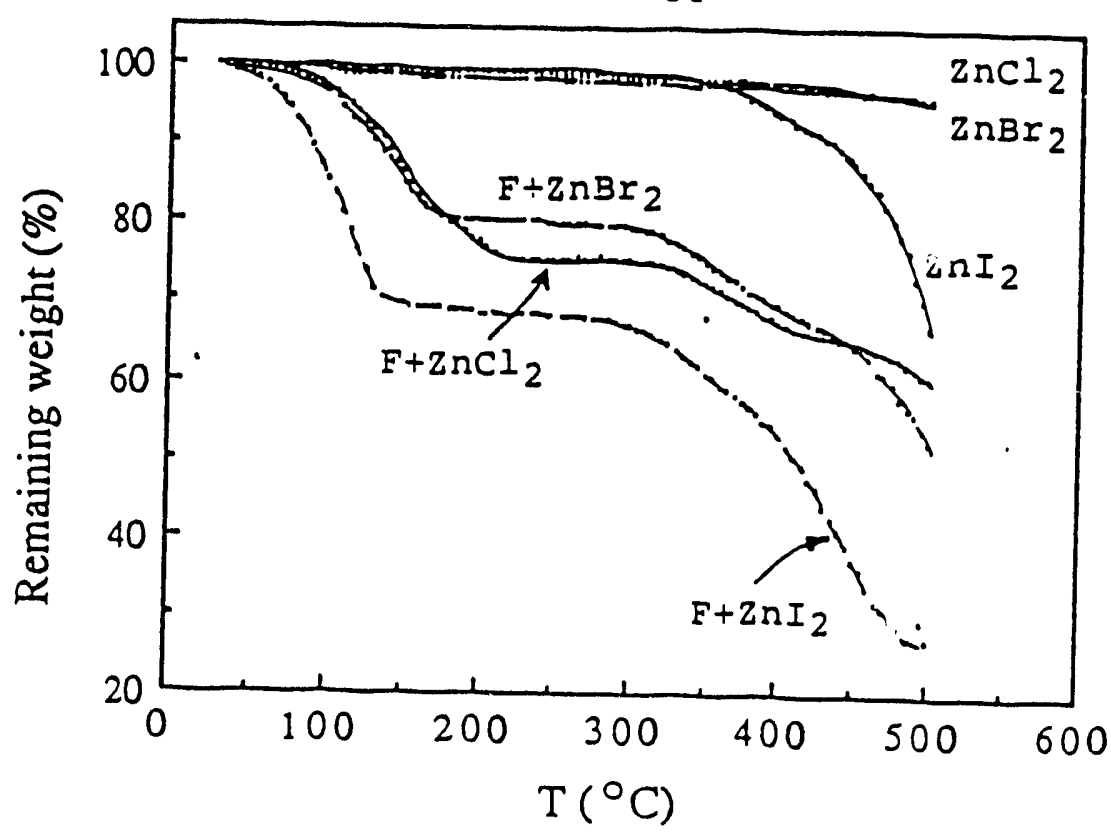




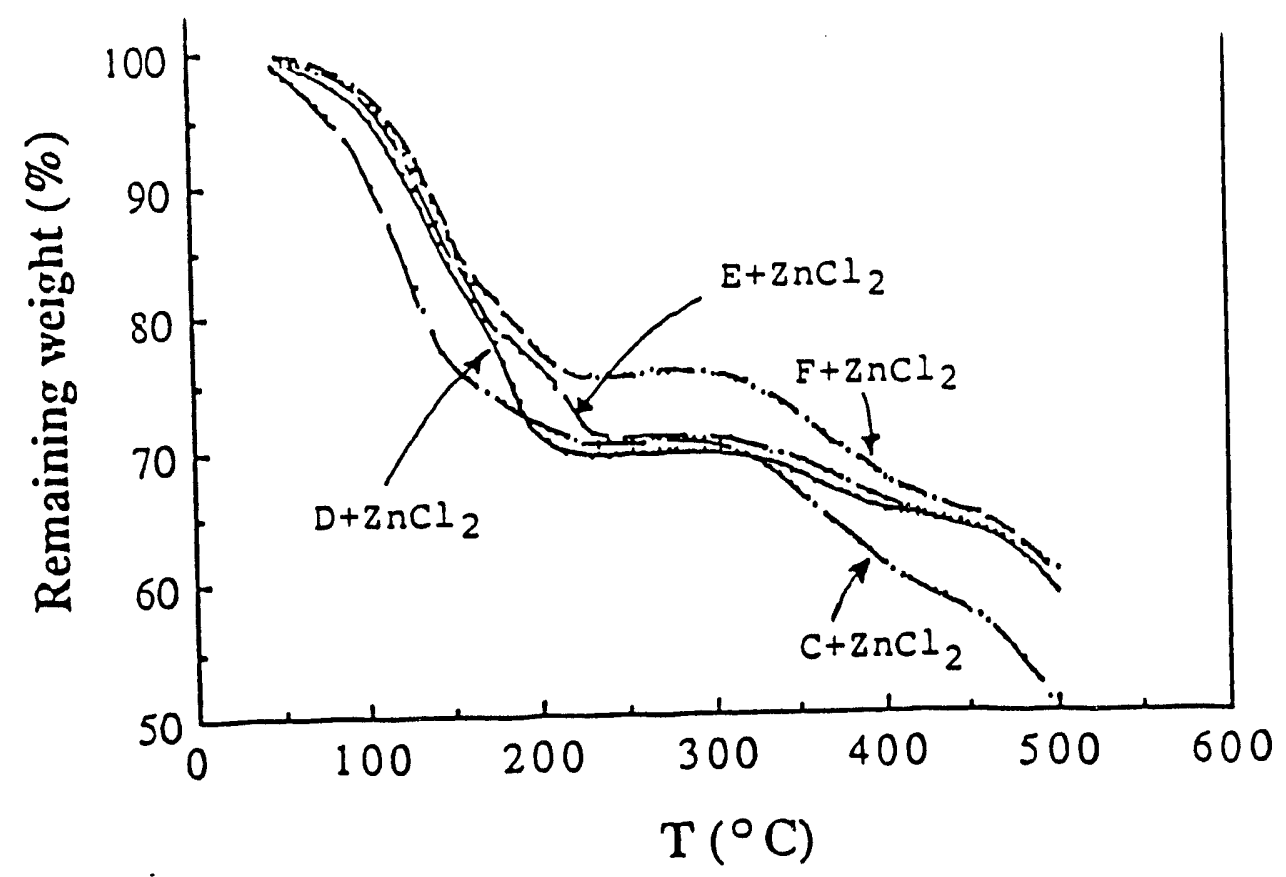

Fig. 3. Percentage remaining weight for coals $C, D, E$ and $F$ of Table 1 plotted against temperature when mixed with
$\mathrm{ZnCl}_{2}$ in the $1: 1$ ratio.

Fig. 4. Percentage remaining weight of coal $c$ for different loadings of $\mathrm{ZnCl}_{2}$ plotted against temperature. $100 \%$
loading represents $1: 1$ mixing.

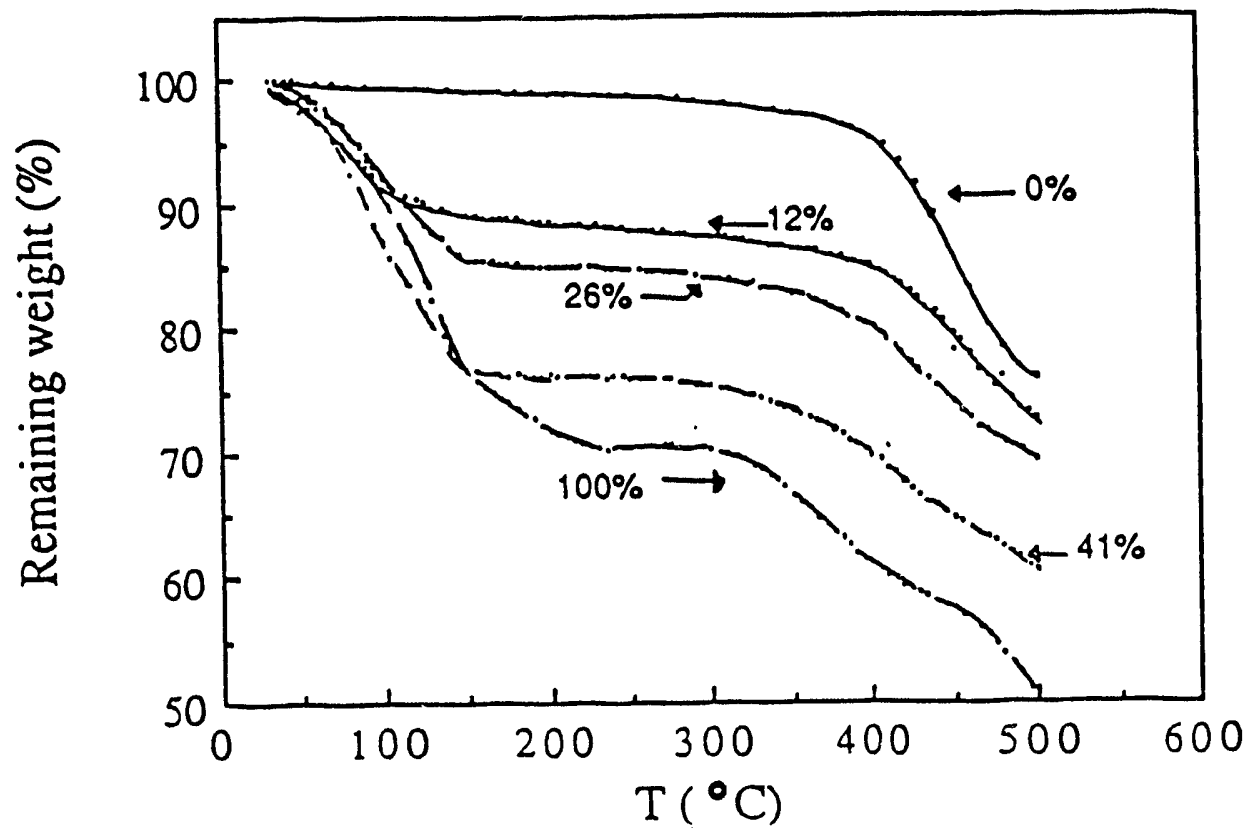




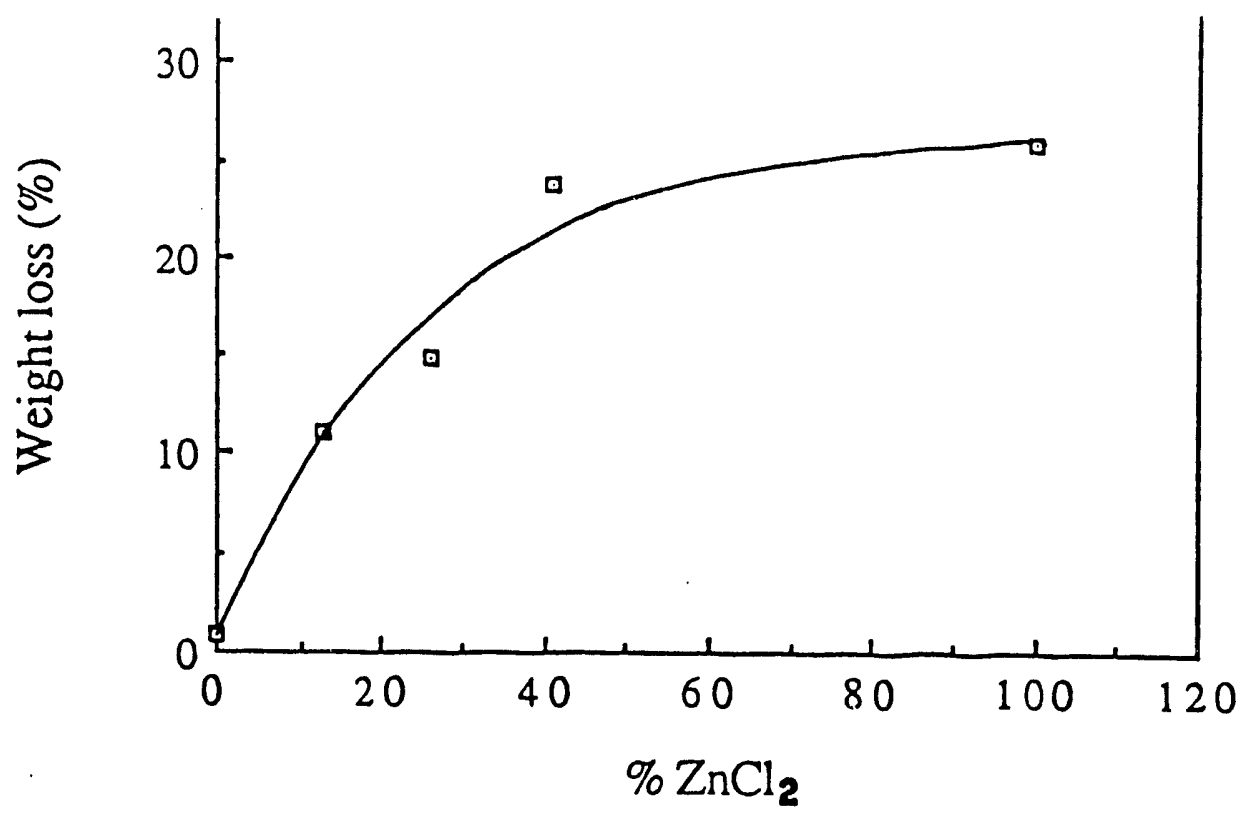

Fig. 5. Weight loss vs $\% \mathrm{ZnCl}_{2}$ for coal C. $100 \%$ loading represents $1: 1$ mixing of coal and $\mathrm{ZnCl}_{2}$. The solid line is drawn as a guide for the eye.

Fig. 6. The measured reaction rate $(\mathrm{dm} / \mathrm{dt}) / \mathrm{m}_{0}$ for coal $C$ and for coal $C$ mixed with the halides in the $1: 1$ ratio, plotted against temperature. plote the peak in the reaction rate near $150^{\circ} \mathrm{C}$ due to halides.

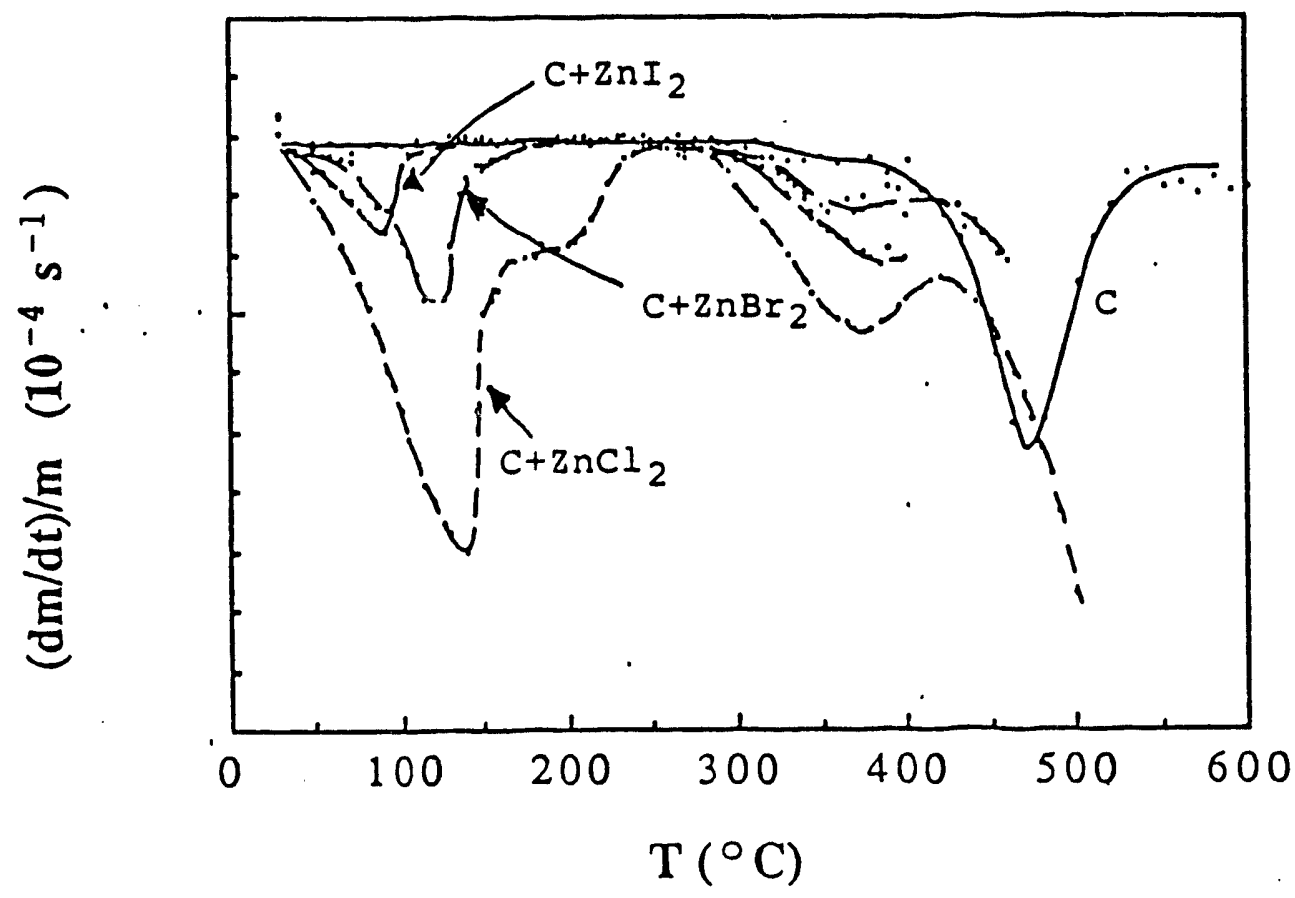




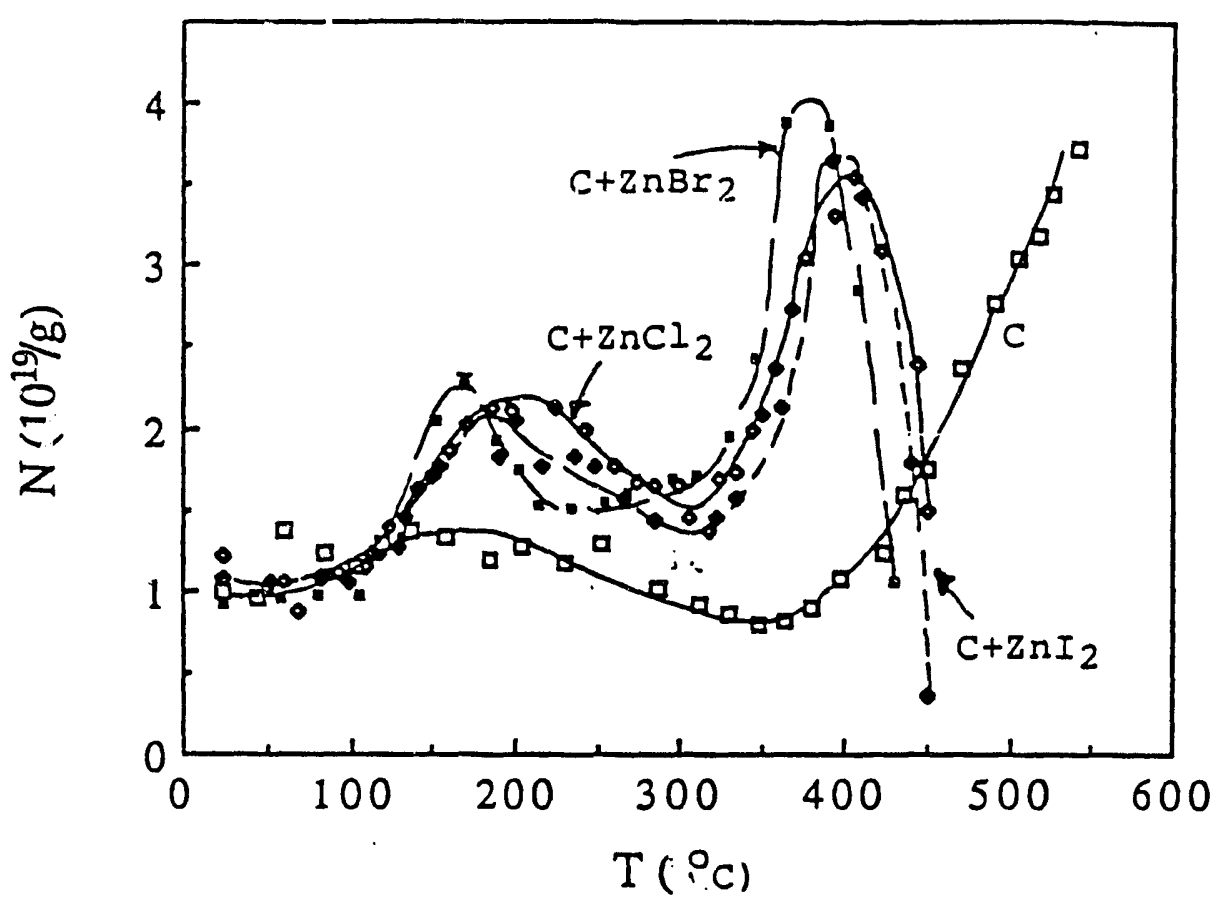

Fig. 7. The free radical density $N$ (after allowing for the Curie law contribution) plotted against temperature for coal $C$ and for coal $C$ mixed with the halides in the $1: 1$ ratio. The experiments were carried out in flowing $\mathrm{N}_{2}$ gas.

Fig. 8. Comparison of the effect of flowing $\mathrm{N}_{2}$ gas and flowing $\mathrm{H}_{2}$ gas on the free radical density at different temperatures for coal $C$ and coal $C$ mixed with $\mathrm{ZnCl}_{2}$ in the $1: 1$ ratio.

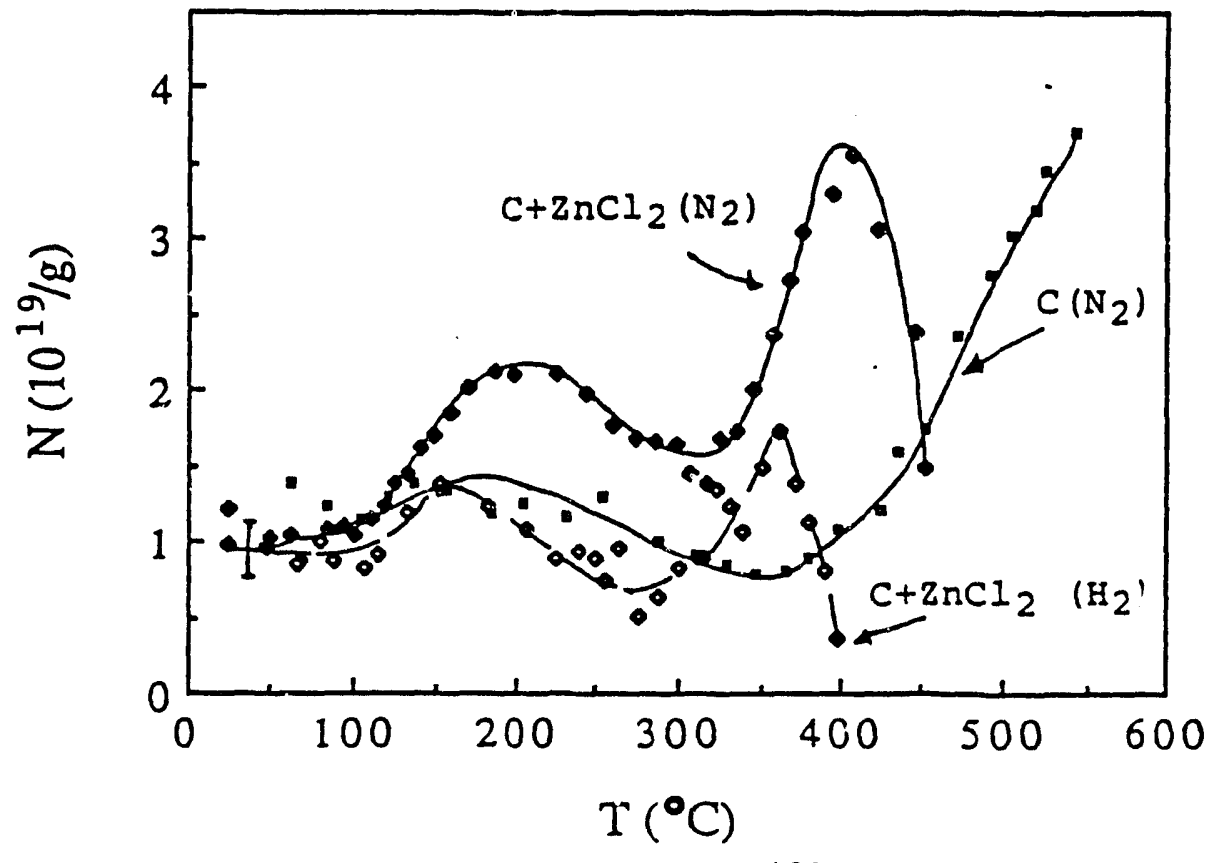


Subtask III.4: Low Temperature Extraction with Supersolvents (John W. Zondlo, Peter Stansberry, Alfred Stiller, Chemical Engineering Department)

\section{Summary}

Extraction kinetics were measured for three different bituminous coals that exhibited distinct extraction behavior and characteristics. These kinetic data were modeled by a newly-developed reversible kinetic model. The model simulates not only the behavior of the extraction at short times, but can also be used to predict the ultimate extraction yield of each coal at any desired temperature.

$h$ ?moval of organic sulfur species was attempted using a metal : metal-salt redox couple. When applied to the raw coal, organic sulfur in the extract dropped from $2.38 \%$ down to $0.84 \%$. Model compound studies showed the method to be applicable to thiol type linkages but not effective in dibenzothiophenic bonds.

Hydrogenation experiments using NMP both as a solvent during hydrogenation and as a catalyst impregnation solvent in pretreatment showed some effects on the overall product yield and distribution. Shifts in the product slate towards the heavier asphaltois indicated that the iron chloride catalyst promoted condensation reactions rather than further ring cleavage and hydrogenation. However, such experiments may be useful in the future for determining the effects of iron-based catalytic hydrogenation since the coal extracts as prepared do not contain any iron species.

\section{Kinetics of Coal Extraction}

The kinetics of coal extraction have been studied for three different types of coals. All coals were of bituminous rank, but exhibited different extraction yields and behaviors. These coals and their extraction characteristics are given below:

Coal

1. Upper Eagle (West Virginia)

2. Firecreek (West Virginia)

3. KCER 6402 (Kentucky)
Extraction Characteristics

High extraction yields, fast extraction

High extraction yields, very slow extraction

Low extraction yields, very fast extraction

The kinetic data were collected over a temperature range of 30 to $200^{\circ} \mathrm{C}$ for the Upper Eagle coal and 70 to $200^{\circ} \mathrm{C}$ for the Firecreek and KCER 6402 coals. An extraction model based on the assumption of first-order irreversible kinetics was developed. Previously, the initial rate data for the Upper Eagle and Firecreek coal were fitted very well by this model. The pseudo-firstorder rate constant was determined at each temperalure for both the coals. The dependence of the rate constant on temperature was studied and the activation energy of extraction was estimated. 
The drawback of this model, however, is that it utilizes only the initial rate of extraction and therefore it predicts the extraction behavior of coal only for short times. It can not predict the ultimate extraction yield at any given temperature.

A generalized model which can predict the extraction yield at any time and temperature was then developed. This model is based on pseudo-first-order reversible kinetics and it utilizes the kinetic data over the entire duration of extraction and not just the initial data. From the kinetic data collected for the KCER 6402 coal, it was observed that the extraction yield leveled off at a different extraction extent for each of the temperatures tested. Also, as temperature increased, the ultimate extraction yield increased and the time required to attain the ultimate yield decreased. This model provides a very reasonable mathematical framework and throws light on the kinetics as well the thermodynamics of the extraction process.

The integrated rate equation for such a first-order reversible extract process is given as:

$$
\text { en }\left[\frac{C_{a-\infty}-C_{e}(t)}{C_{\infty}}\right]=-\left(k_{1}+k_{2}\right) t
$$

where

$$
\begin{aligned}
& C_{0}=\text { equilibrium concentration of the extract }(\mathrm{g} / \mathrm{cc}) \\
& \mathrm{C}_{0}(\mathrm{t})=\text { concentration of extract at any time } t \text {. }
\end{aligned}
$$

Here $k_{1}$ and $k_{2}$ are the rate constants for the forward and reverse reaction $\left(\min ^{-1}\right)$.

The plot of

$$
\left[\frac{C_{a-\infty}-C_{e}(t)}{C_{\infty}}\right]
$$

vs. time $t$ should yield a straight line with a slope $-\left(k_{1}+k_{2}\right)$. The kinetic data were fitted to this equation and the ixtercepts were forced through the origin in order to correct for the concentration of extract at zero time. Typical raw data and a fit of the data to the model in Equation 1 are shown in Figures 1 and 2, respectively. Furthermore, the equilibrium constant, $K$, is defined as:

$$
K=\frac{k_{1}}{k_{2}}=\frac{C_{\infty}}{C_{R_{0}}-C_{o m}}
$$


where,

$$
\mathrm{C}_{\mathrm{R}_{0}}=\text { initial concentration of raw coal. }
$$

By making use of Equations 1 and 2, $k_{1}$ and $k_{2}$ are uniquely determined. This procedure was repeated for each temperature. The Arrhenius plots describing the dependence of rate constant on temperature for $k_{1}$ and $k_{2}$ are shown in Figure 3. The activation energy for the forward reaction was found to be $7.46 \mathrm{kcal} / \mathrm{mol}$ and for the backward reaction to be $4.91 \mathrm{kcal} / \mathrm{mol}$.

The relationship between the equilibrium constant and temperature is given by the thermodynamic expression,

$$
\ln K=-\frac{\Delta H^{0}}{R T}+\frac{\Delta S^{0}}{R}
$$

where,

$$
\begin{aligned}
& \Delta H^{\circ}=\text { heat of reaction at standard conditions } \\
& \Delta S^{\circ}=\text { entropy of reaction at standard conditions }
\end{aligned}
$$

From the data collected in the laboratory, $\Delta \mathrm{H}^{\circ}$ was estimated to be $+2.55 \mathrm{kcal} / \mathrm{mol}$ and $\Delta S^{\circ}$ was estimated to be $0.0035 \mathrm{kcal} / \mathrm{mol}$.

From Equations 2 and 3, the relationship between equilibrium concentration and temperature can be expressed as,

$$
\frac{C_{\omega}}{C_{R_{\circ}}}=\frac{\exp \left[\frac{\Delta S^{\circ}}{R}-\frac{\Delta H^{\circ}}{R T}\right]}{1+\exp \left[\frac{\Delta S^{\circ}}{R}-\frac{\Delta S^{\circ}}{R T}\right]}
$$

Least-squares fits were done on the kinetic and thermodynamic data and the values of the appropriate parameters were determined. The model was then used to predict the extraction Dehavior of concentration vs. time for a selected temperature $\left(154^{\circ} \mathrm{C}\right)$. This prediction was then compared to the actual data and the degree of agreement was found to be excellent as shown in Figure 4. With the model in hand, similar plots can be made for any temperature within the range of 70 to $200^{\circ} \mathrm{C}$. In addition, the equilibrium thermodynamics allows the prediction of the ultimate extraction yield at any temperature between 70 and $200^{\circ} \mathrm{C}$. The predictions are compared to the actual measured ultimate extraction yields in Figure 5.

Similar extraction behavior has been observed previously for the Upper Eagle coal and hence it appears that the model can be applied to other coals as well. Of course, the values of the various fitted parameters will be different. The above model will be of tremendous benefit in not only providing a mathematical framework for the process but also allowing researchers to 
gain some insight into the nature of the extraction mechanism itself.

\section{Desulfurization Studies}

Experiments were conducted to characterize and quantify the in-situ removal of organic sulfur from extracted coal materials. Previously, extraction of coal in N-methyl-2-pyrrolidone (NMP) at $202^{\circ} \mathrm{C}$ and $1 \mathrm{~atm}$ pressure for 1 hour showed that all the inorganic sulfur remains in the residual material, but the organic sulfur is split nearly equally between the residue and the extract. The organic sulfur is typically present in the form of thiols, sulfides, disulfides, and thiophenes.

Attempts were nade to remove the organic sulfur from coal extracts by treatment with active metal/metal saits. The effect of different treatment conditions on Ilinois \#6 coal and its extract is shown in Taible 1 . Note that treatment at room temperature appears to be more effective for sulfur removal than that at $200^{\circ} \mathrm{C}$. Iii addition, experiments were carried out to effect the extraction and desulfurization simultaneosusly in the same step. To this end, a coal: $\mathrm{FeCl}_{2}$ ratio of 5:1 (wt:wt) was used with NMP as the solvent. Upon product work up, the extract was found to be contain only $0.84 \%$ (wt) of organic sulfur compared to $2.38 \%$ (wt) in the untreated extract. Moreover, the overall sulfur balance for the raw coal, residue, and extract was found to within $1 \%$.

In order to provide insight into the desulfurization reaction, studies with model compounds were undertaken. Dibenzothiophene and benzenethiol were chosen as the model compounds, since they are believed to simulate some of the organic sulfur functionalities found in coal.

The desulfurization reaction was carried out using the $\mathrm{Fe} / \mathrm{FeCl}_{2}$ couple (1:1 ratio by weight) at $200^{\circ} \mathrm{C}$ in the solvent NMP. The experiments using dibenzothiophene did not show any evidence of desulfurization under these treatment conditions. However it was found that at the same treatment conditions, significant desulfurization was observed with benzenethiol.

A preliminary quantitative analysis showed that benzene was formed as primary reaction product in the benzenethiol reaction. Under otherwise identical conditions of temperature and time, $40 \%$ conversion of benzenethiol was observed with the use of the $\mathrm{Fe} / \mathrm{FeCl}_{2}$ couple, whereas in the purely thermal treatment only trace conversions were noted. Moreover, an overall sulfur balance was performed as an independent check on the conversion and these results agreed to within $10 \%$ of those found using a calibrated GC.

\section{Hydrogenation Studies}

Some experiments were conducted to determine if NMP couid be used for coal extraction at hydrogenation conditions near the softening point of coal (near $350^{\circ} \mathrm{C}$ ). Gas chromatographic analysis of NMP after it had been treated at $350^{\circ}$ with 1000 psig hydrogen (initial cold) showed 
that under these conditions NMP alone was quite stable. A series of experiments was then carried out between 200 and $350^{\circ} \mathrm{C}$ and under $1000 \mathrm{psig}$ hydrogen with a bituminous coal KCER 91864. The yield of extract at the normal boiling point was $39.6 \%$ (daf). Table 2 shows the results at higher temperatures and pressures. Increasing the temperature from 200 to $350^{\circ} \mathrm{C}$ does result in an increase in coal solubility, but the excessive mass balance indicates that NMP is reactive under these conditions and incorporates into the coal products.

Experiments were performed in order to determine if NMP could act in a manner to convey a soluble iron catalyst to the reactive centers inside the matrix of the residue to aid in further increasing the carbon utilization of the raw coal. Residues from coal KCER 6402 were prepared using the NMP extraction technique. Two methods were used to impregnate the residue with iron catalyst $\mathrm{FeCl}_{2}$ (ferrous chloride was selected because it is soluble in both NMP and water). The first procedure entailed impregnation with iron from an aqueous solution to give an iron loading of 1 wt \% Fe based on the organic weight of the residue. The other procedure impregnated the residue with iron from an NMP solution at the same loading level. In both cases the mixtures were slurried for 12 hours and the excess solvent was then removed by vacuum drying at $150^{\circ} \mathrm{C}$ to constant weight.

The impregnated residues were liquefied in tetralin using a $450 \mathrm{~mL}$ stirred Parr reactor for 1 hour at $375^{\circ} \mathrm{C}$ under 500 psig initial cold hydrogen pressure ( $60 \mathrm{~mL}$ tetralin: 20 grams charge). Following reaction, the products were washed out with $600 \mathrm{~mL}$ of NMP into a beaker and slurried for 1 hour at $200^{\circ} \mathrm{C}$. The insoluble materials were separated from the liquid products by hot, vacuum filtration. Excess NMP and tetralin were removed from the converted material by vacuum rota-evaporation at about $130^{\circ} \mathrm{C}$. Product distributions were then determined based on solvent partitioning. Asphaltols are defined here as those materials soluble in NMP but insoluble in benzene; asphaltenes are those materials soluble in benzene but insoluble in hexane; oils are those materials soluble in hexane. The reproducibility of the liquefaction experiments varied between $2.5 \%$ and the average of duplicate runs are shown in Table 3 . Liquefaction of the raw coal gave a total conversion of about $92 \%$ with the bulk of the products appearing as asphaltenes. Liquefaction of the residue alone produced a conversion of about $83 \%$, again with the majority of the products appearing as asphaltenes. High conversion of the residue is surprising because one would think that the liquefaction yield would be greatly reduced after removal of nearly $25 \%$ of the original organic matter. More interesting, though, are the shifts to the heavier asphaltols in the presence of $\mathrm{FeCl}_{2}$. Although the overall conversion of each of the residues was the same within experimental error, the generation of significantly more asphaltols at the expense of asphaltenes suggests that the iron chloride promoted condensation reactions. In this instance, it is probable that the dispersed iron was not in its active form. Although there is still some debate on the form of iron that is active in direct 
liquefaction, it is most likely some sulfided species. It is also noted in Table 3 that the NMP impregnation gave slightly better results than that of the water impregnation, a fact most likely due to the ability of NMP to swell the residue significantly.

Table 1. Results of Desulfurization Studies for Hlinois \#6 Extract

\section{Treatment Conditions}

1. Raw. coal (Ilinois \# 6)

2. Residue

3. Extract (untreated)

4. $\mathrm{Cu}(-100 \mathrm{mesh})$ treated extract $\left(200^{\circ} \mathrm{C}, 4 \mathrm{hrs}\right)$

5. $\mathrm{Cu}$ (10 micron) treated extract $\left(200^{\circ} \mathrm{C}, 4 \mathrm{hrs}\right)$

6. Fe: $\mathrm{FeCl}_{2}$ : extract (5:1:5), room temp., $4 \mathrm{hrs}$., rotavap

7. Fe:FeCl ${ }_{2}$ : extract (5:1:5), room temp., $4 \mathrm{hrs}$, precipitation

8. Fe: $\mathrm{FeCl}_{2}$ : extract $(5: 1: 5)$, $200^{\circ} \mathrm{C}, 4 \mathrm{hrs}$., precipitation
Organic S wt\%

2.55

2.05

2.38

2.27

2.09

0.67

0.36

0.65
\% Reduction in Organic S

Table 2. Hydrogenation of Kentucky Coal KCER 91864 in the Solvent NMP at Various Conditions

\begin{tabular}{lcc}
\hline Reaction Conditions & $\begin{array}{l}\text { \% Yield } \\
(\text { daf })\end{array}$ & \% Mass Balance \\
\hline $200^{\circ} \mathrm{C}, 1$ hour 1000 psig H2 & 4.3 & +3.2 \\
$300^{\circ} \mathrm{C}, 1$ hour, 1000 psig H2 & 45.9 & +9.7 \\
$350^{\circ} \mathrm{C}, 1$ hour, 1000 psig H2 & 86.6 & +16.5 \\
\hline
\end{tabular}


Table 3. Liquefaction of a Western Kentucky Coal (KCER 6402) and Its NMP-Extracted Residue (3:1 (wt) Tetralin:charge; 500 psig $\mathbf{H}_{2}$ initial cold; 1 hour at $375^{\circ} \mathrm{C}$ )

Run ID Conversion Asphaltols $\frac{\% \text { Yields (dry basis) }}{\text { Asphaltenes Oils }}$

Whole coal

P168-172

91.7

29.7

67.4

3.5

Residue

P160-174

83.1

22.6

53.7

0.5

$\mathrm{FeCl} 2 / \mathrm{H} 2 \mathrm{O}$

P163-165

79.6

52.8

29.4

2.4

$\mathrm{FeCl}_{2} / \mathrm{NMP}$

P170-176 85.3

48.8 32.2 1.4

$$
\text { Conversion }=\frac{\text { wt dry charge }- \text { wt dry insolubles }}{\text { wt dry charge }} 100
$$

$$
\text { Asphaltols, asphaltenes, oils }=\frac{\text { wt material }}{\text { wt dry charge }} 100
$$




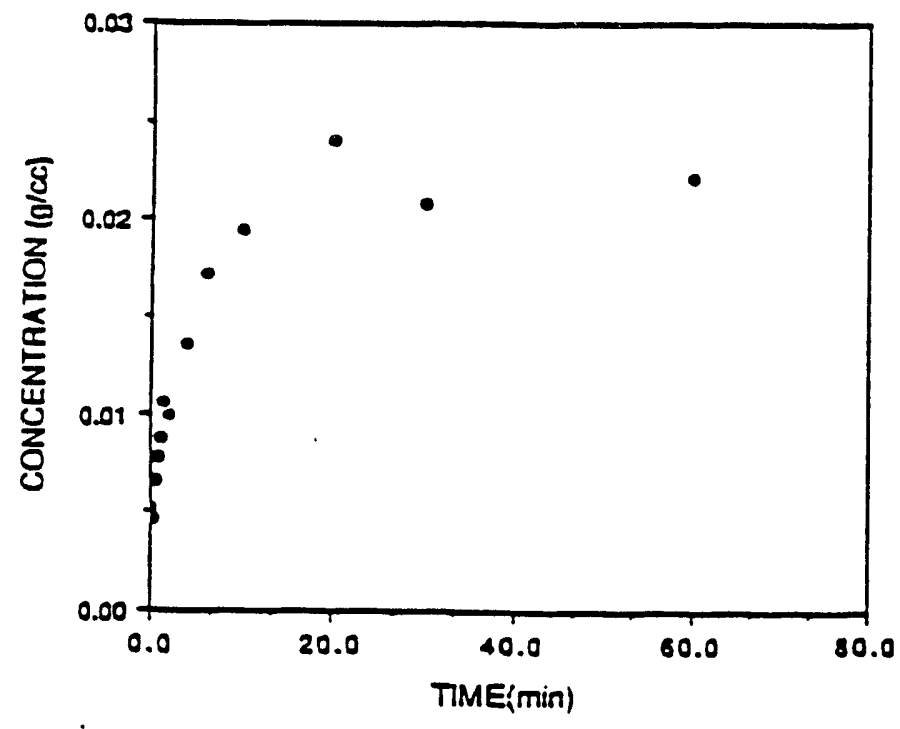

FIGURE 1

KINEIIC DATA AT $154^{\circ}$ C FOR KCER 6402 COAL

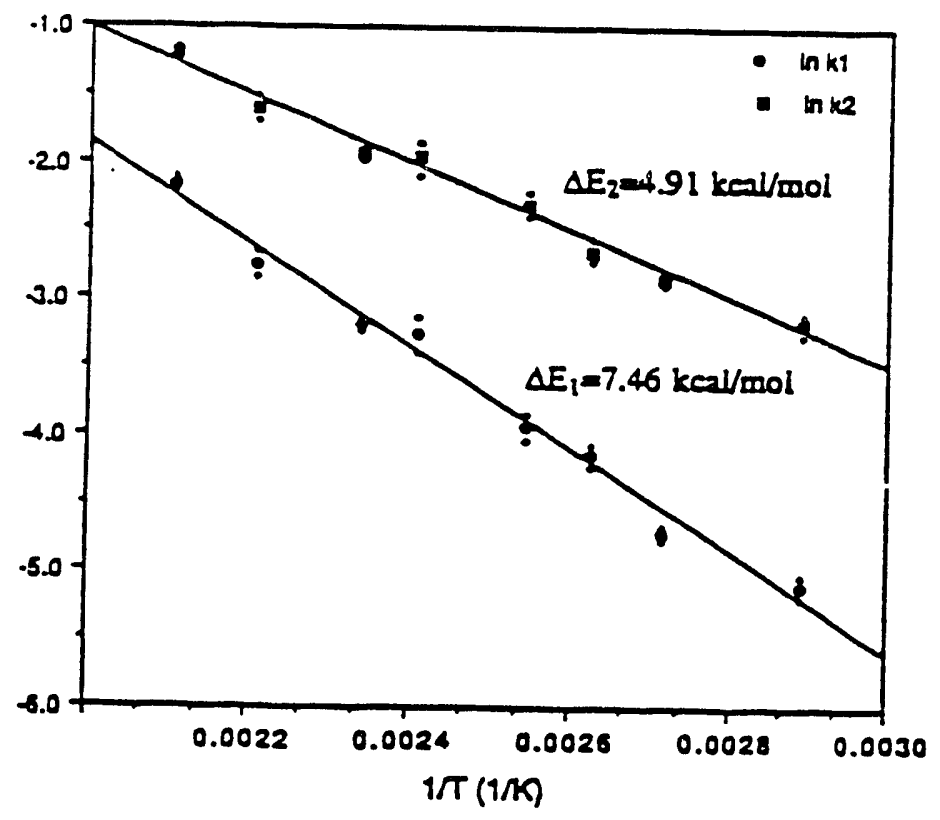

FTURE 3

ARRHENTUS PLOTS FOR FORWARD AND BACKWARD REACIICN

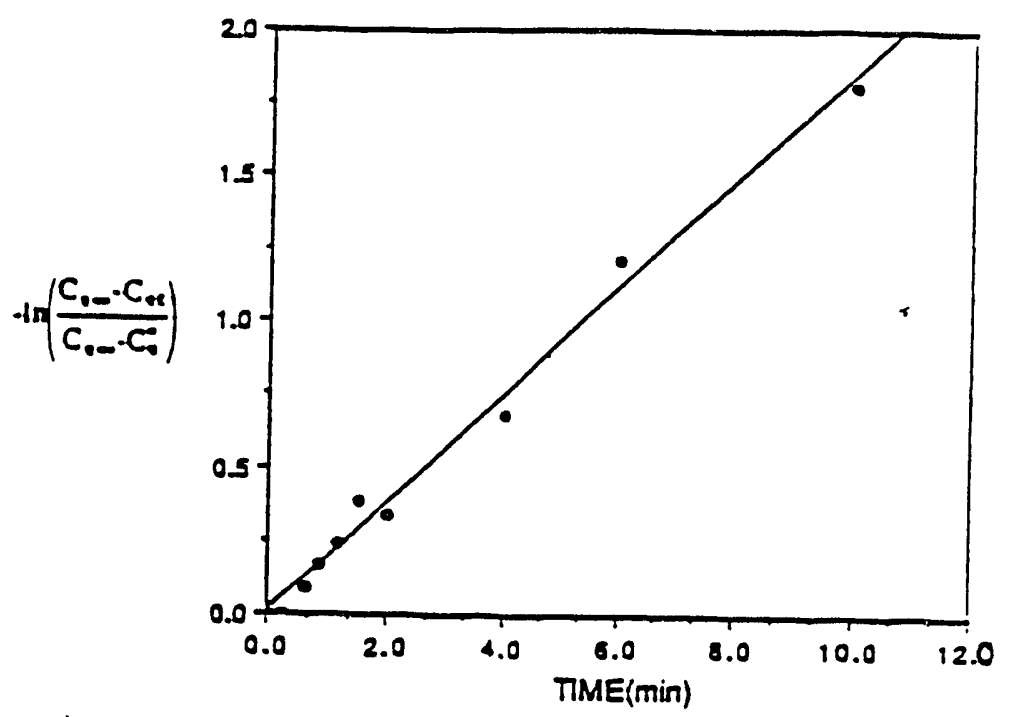

FGURE 2 FIRST ORDER REVERSLBLE KTNETICS AT IS4 C FOR KCR 6402 COAL

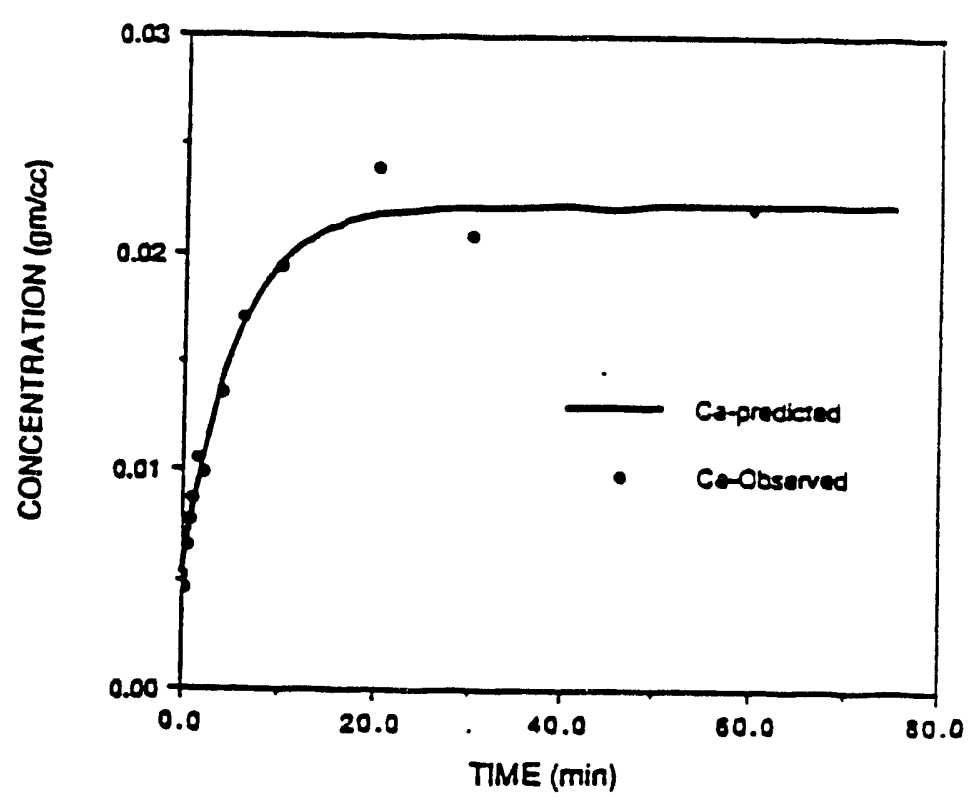

FIGURE 4

COMPARISON OF UBSERVED AND PREDICTED KNNETICDATA AT $154^{\circ} \mathrm{C}$ 


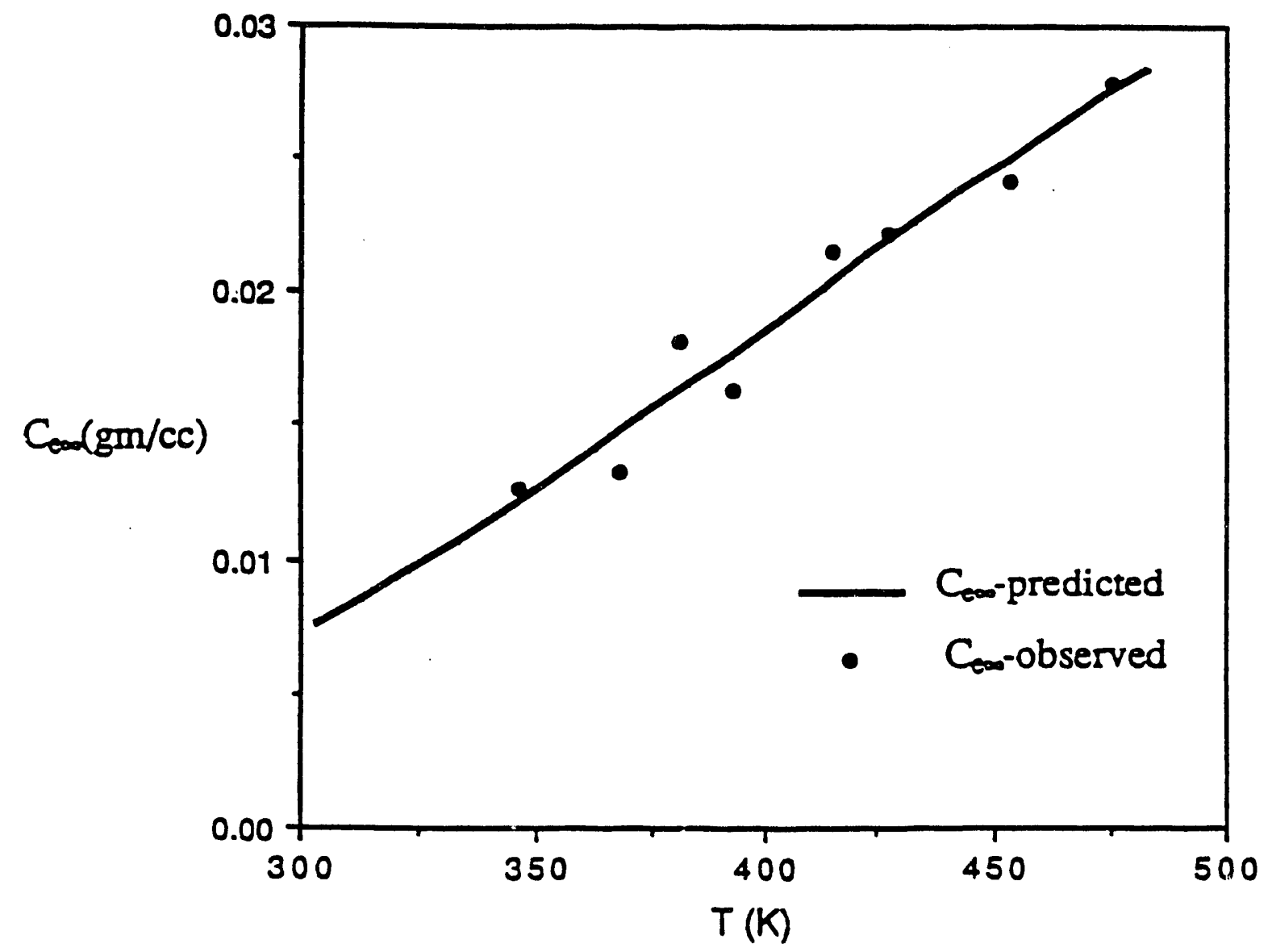

FIGURE 5

COMPARISON OF OBSERVED AND PREDICTED EQUILIBRIUM CONCENTRATIONS AT DIFFERENT TEMPERATURES 


\section{Subtask III.5: Calculation of Physical Properties for Complex Fluids in}

Coal Liquefaction (Wallace B. Whiting)

\section{Summary of Results Obtained This Year}

We have tested our group-contribution equation of state and found it to be more flexible, more widely applicable, and more internally consistent than other available models. Our adaptive algorithm for continuous-thermodynamics phase-equilibrium calculations has been tested and published. Five papers describing the details of our work have been published and two are under review for publication. We have developed contacts with industry to try to obtain more data for representative systems. Our conclusion is that the available experimental data are insufficient to test our model fully.

\section{Overview}

Process simulation and design of coal-liquefaction facilities require accurate physical-property-estimation techniques (esp. for vapor-liquid equilibrium) that are compatible with computer-aided-design process simulators. However, the fluids encountered in coal liquefaction and in the processing of coal fluids are more complex than those encountered in other chezuical processes; thus, they are not well described by existing physical-propertyestimation packages. We have developed models, algorithms, and computer software to address these requirements.

Coal-derived fluids contain very many different species and characterization of the fluids is, therefore, complicated. The data that are the easiest to obtain (boiling-point curves and liquid densities) do not indicate the identity of the species information something that is required for thermodynamic models used in process simulators. Also, if all the individual species could be identified, the calculations for thermodynamic properties in the simulations would overload even the fastest supercomputers.

To address this problem in a way that offers the efficiency and accuracy required for process simulation, we use the new technique of continuous thermodynamics. With this approach, we model the fluid as a continuous distribution of species according to one or more characterization variables. We have used molecular weight, normal boiling point, density, and PNA analyses as - the characterization variables. These inspection data are the data most likely to be available for complex fluids, although as new and more sophisticated analyses become common, we can include these in our models.

Another problem with coal fluids is that they contain molecules, the structures of which dictate an activity-coefficient approach to vapor-liquid equilibria, rather than an equation-ofstate approach. As previous work had dealt with equations of state, we extended continuous 
thermodynamics to activity- coefficient models. Specifically, we use the UNIFAC model, which allows one to calculate activity coefficients of systems for which no experimental data exist.

For systems at high pressure or those containing low boiling-point species, an equation-of-state approach is desirable. However, for complex continuous fluids, binaryinteraction parameters are needed for all possible binary pairs. We are developing a group-contribution equation of state that allows the calculation of these parameters for many systems.

Algorithm development is an important aspect of our work. We developed efficient continuous-thermodynamics algorithms based on our models.

\section{Distribution Functions for Calculation of Phase Equilibria of Continuous Mixtures}

Ill-defined mixtures such as coal liquids with a very large number of components are traditionally handled by a pseudo-component method for the prediction of thermodynamic properties. Alternatively, these mixtures can be regarded as continuous distributions of one or more families of chemical species (continuous mixtures). To perform phase-equilibrium calculations for continuous mixtures, it is necessary to describe quantitatively the continuous distributions of chemical species by mathematical functions.

We compared the pseudo-componer.t method, the method of moments, the Lobatto quadrature method, and our new method in which cubic-spline approximations are incorporated. Although each method has advantages for specific types of systems and calculations, the cubic-spline method was found to be most accurate and adaptive, with only slight increases in computation times.

We have studied various functions for describing continuous distributions. The cubic-spline procedure has the best fit in each case. An illustration of the differences between the various methods is given in Figures 1 and 2, which show the vapor- and liquid-phase distributions for a coal liquid. Further details are given elsewhere (Wang and Whiting, 1988a).

\section{Parameters for the Perturbed-Hard-Chain Theory from Characterization Data for Coal Liquids}

We have developed correlations for pure-component parameters for the Perturbed-HardChain equation of state in terms of conventional characterization data (molecular weight, normal-boiling point, and specific gravity). These correlations are comparable to and often better than correlations using molecular-structure information in predicting saturated liquid densities and vapor pressures for coal liquids. This is especially important because such structural-property data require elaborate measurements and are, thus, usually unavailable.

Further details are available elsewhere (Wang and Whiting, 1988b). 


\section{Group-Contribution Binary Interaction Parameters for Equations of State}

It is not possible to measure all of the binary vapor-liquid equilibria required to determine the binary-interaction parameters for equations of state for coal liquids. To overcome this problem, we have developed a procedure for determining these parameters through group contribution.

Whereas there are only approximately 40 functional groups in our method, these groups can represent the many thousands of compounds that might exist in a typical coal liquid. Our technique uses the UNIFAC group-contribution model for liquid-state activity coefficients. This model is widely recognized and used for low-pressure vapor-liquid equilibrium calculations for systems at moderate temperatures. Our model eliminates these severe constraints by incorporating the group-contribution concept into an equation of state.

The equation of state that we have used in our development is the Mathias version of the Soave-Redlich-Kwong cubic equation of state, but any equation of state can be used in our method. We generate synthetic vapor-liquid equilibrium data within the limited range of applicability of the UNIFAC model and regress binary-interaction parameters for the equation of state from these synthetic data. The equation of state can then be used over a much wider range of temperatures and pressures. Details are given elsewhere (Wang and Whiting, 1987; Hou et al., 1989).

We have tested our group-contribution equation of state agairst the only two other such models (GC-EOS and UNIWAALS). Our method appears to have significant advantages; it is more flexible, more widely applicable, and more internally consistent. It is also substantially easier to incorporate in process- simulation software systems. A model similar to ours has been developed independently by Schwartzentruber et al. (1989).

\section{The Area Algorithm}

With our collaborators at Texas A \& M University, we developed an improved algorithm for calculation of phase equilibria. This procedure is especially useful in the critical and retrograde regions, where conventional algorithms often break down. Coal-fluid processing will involve such conditions with thermodynamic models more sophisticated than those used in petroleum processing. Thus, the demands on the efficiency and robustness ri: the phase-equilibrium algorithms are increased.

The essence of the algorithm is to maximize the area enclosed by the Gibbs energy of mixing curve and the line connecting the two assumed phase compositions. This method is quite promising as it is superior to various tangent-plane algorithms. Sample calculations for critical, cricondentherm, and cricondenbar points have demonstrated the versatility of this algorithm. Details are provided elsewhere (Barrufett et al., 1989). 


\section{Sensitivities of Model Parameters to Thermodynanic Data}

With our collaborators at the University of Connecticut, we have begun studies of the sensitivities of model parameters to thermodynamic data. We have found that small errors in phase-equilibrium measurements can have dramatic effects on the regressed values of parameters in thermodynamic models. In fact, we found that for many systems the $90 \%$ confidence regions for binary-interaction parameters regressed from different data sets did not overlap. And the phase equilibria calculated from the extrema of these riggions are far from within experimental error.

We are developing heuristics for evaluating the sensitivity of model parameters to thermodynamic data and will be publishing these results next year. Such a procedure will be very useful in defining the important phase-equilibrium experiments needed to evaluate thermodynamic models for coal fluids. This is especially important in light of our confirmation that inadequate experimental phase-equilibrium data available for such evaluations.

With the help of ENSR Consulting and Engineering and that of Dr. Curtis White (PETC), and others, we attempted to obtain additional (complete) coal-liquid phase-equilibrium data with wiveh to test thermodynamic models. However, very few such data are available.

We obtained some data for heavy oil-shale fluids from Davy-Dravo Engineers, but these data were not sufficiently complete to test our models rigorously.

\section{Collaborators}

Thomas F. Anderson, Professor of Chemical Engineering, University of Connecticut, Storrs Hui-Min Hou, former M.S.Ch.E. student, West Virginia University (now at NUS Corporation) Ming-Jing Hwang, former Postdoctoral fellow, West Virginia University (now at Biosym, Inc.)

Donald J. Klein, Principal Process Engineer, Davy-Dravo Engineers and Constructors

Panthulu Ramanna, former M.S.Ch.E. student, West Virginia University (now at BOC Group, Inc.)

Michael E. Reed, M.S.Ch.E. student, West Virginia University

Shao-Hwa Wang, former Ph.D. student, West Virginia University (now at SRI International)

Charles W. White, Adjunct Professor of Chemical Engineering, West. Virginia University, and Research Engineer, EG\&G Washington Analytical Services

\section{Publications}

1. "A Group-Contribution, Continuous-Thermodynamics Framework for Calculation of Vapor-Liquid Equilibria," Proceedings of the World Congress III of Chemical Engineering, Tokyo, Japan, September 1986, Vol. II, p. 172, with S.-H. Wang. 
2. "Studies in Continuous Thermodynamics," Shao-Hwa Wang, Ph.D. Dissertation, 1986, West Virginia University.

3. "Fluid Phase Stability and Equations of State," Fluid Phase Equilibria, 34, 101 (1987), with I. F. Radzyminski.

4. "A Group-Contribution, Continuous-Thermodynamics Framework for Calculation of Vapor-Liquid Equilibria," Canadian J. Chem. Eng., 65, 651 (1987), with S.-H. Wang.

5. "A Comparison of Distribution Functions for Calculation of Phase Equilibria of Continuous Mixtures," Chemical Engineering Communications, 71, 127 (1988), with S.-H. Wang.

6. "Parameters for the Perturbed-Hard-Chain Theory from Characterization Data for Heavy Fossil Fuel Fluids," Ind. Eng. Chem. Research, 27, 1058 (1988), with S.-H. Wang.

7. "Group-Contribution Equation of State," Hui-Min Hou, M.S.Ch.E. Thesis, 1989, West Virginia University.

8. "Group-Contribution Binary-Interaction Parameters for Equations of State," submitted for publication (1S39), with H.-M. Hou and S.-H. Wang.

9. "The Area Algorithm," submitted for publication (1989), with M. Barrufett, E. Hassad, and P. T. Eubank.

\section{Presentations}

1. "Continuous Thermodynamics: A Group-Contribution Approach," American Institute of Chemical Engineers Spring National Meeting, New Orleans, LA, April 1986, with S.-H. Wang.

2. "Determination of Parameters for the Perturbed-Hard-Chain Equation of State from Inspection Data," American Institute of Chemical Engineers Annual Meeting, Miami Beach, FL, November 1986.

3. "Group-Contribution Binary-Interaction Parameters for Equations of State," American Institute of Chemical Engineers Spring National Meeting, Houston, TX, March 1987.

4. "Calculation of Physical Properties for Complex Fluids in Coal Liquefaction," First Annual Technical Meeting of the Consortium for Fossil Fuel Liquefaction Science, Lexington, KY, June 1987.

5. "Physical Properties for Coal Liquefaction," Second Annual Technical Meeting of the Consortium for Fossil Fuel Liquefaction Science, Morgantown, WV, August 1988. 


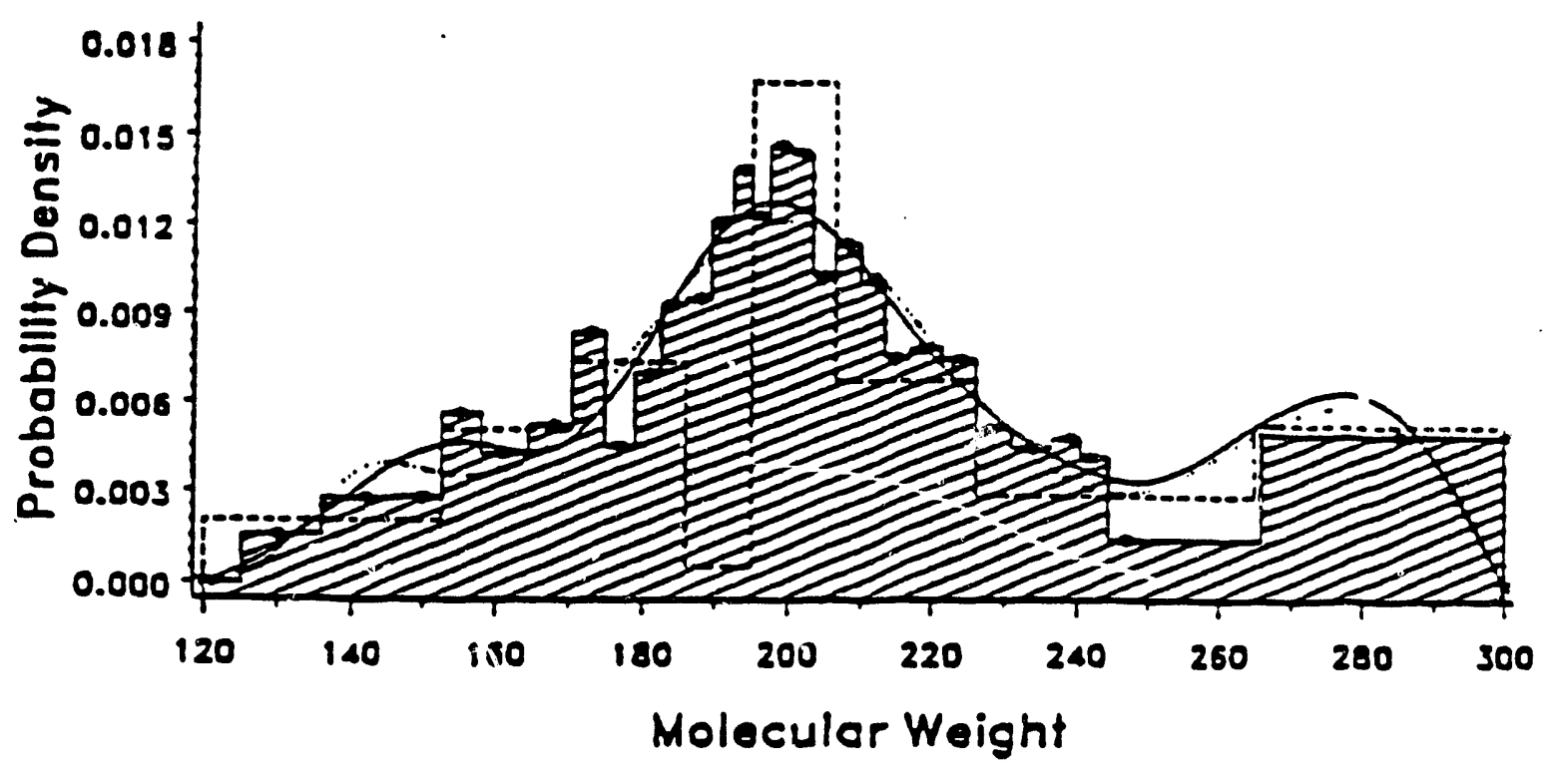

28-eomponent (date). ___ cuble spline

-- - - pseude-somponent, ...... quedrature

FIGURE 1. Comparison of flash calculations: vapor phase molar distribution of a coal liquid.
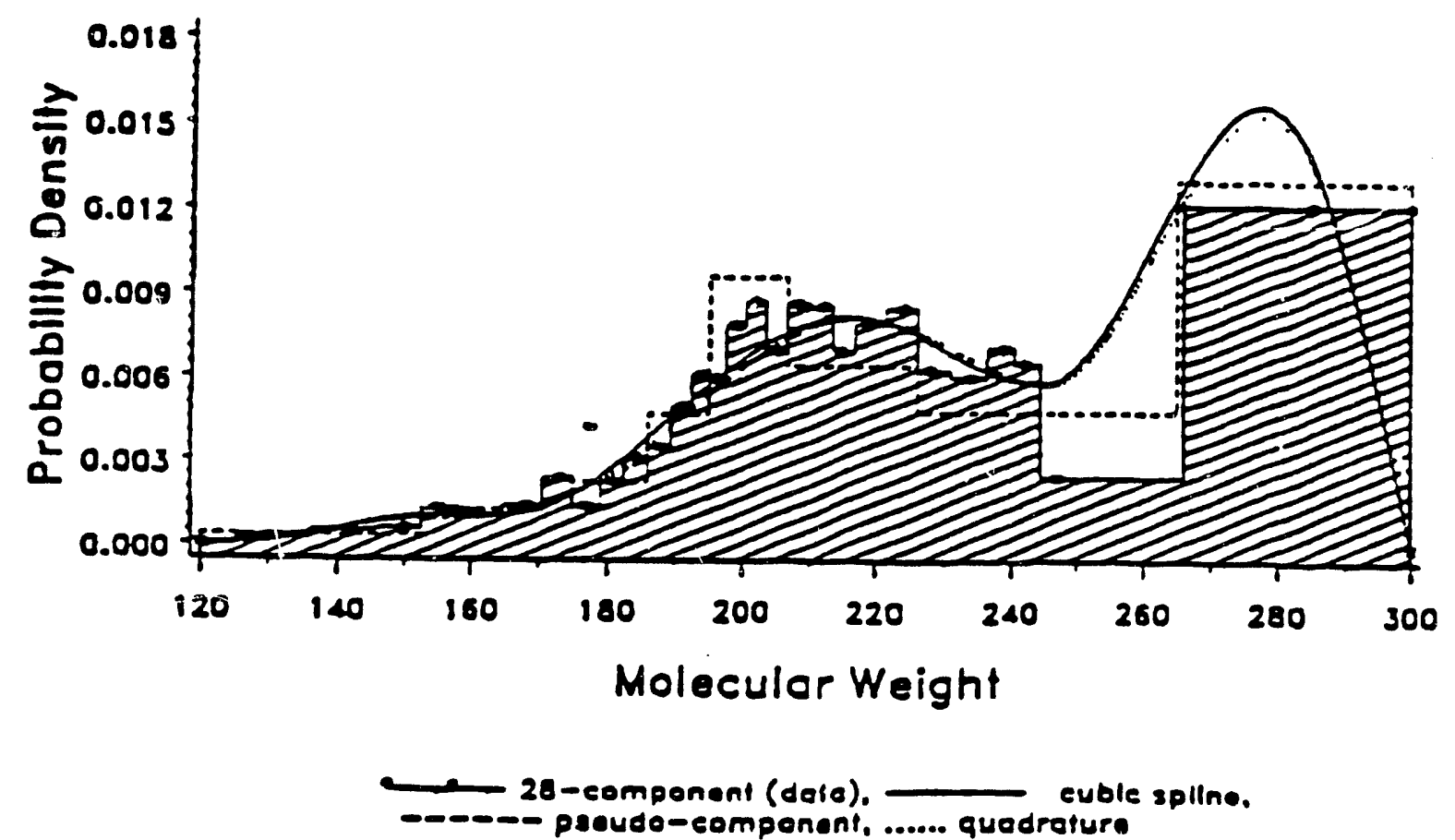

FIGURE 2. Comparison of flash ealculations: liquid phase molar distribution of a coal liquid. 
Subtask III.6: Evaluation of Residue from the WVU Coal Extraction Process As A Disposable Hydrotreatment Catalyst (Dady B. Dadyburjor, Chemical Engineering Department; James L. Clements Research Post Doctorate)

\section{Objectives}

The objective of this project was to study hydrodenitrification reactions (HDN) under - conditions similar to those used commercially and in the previous HDS project. Specific goals of the project are:

1. To obtain reaction rates of model compounds (quinoline) for HDN reactions under standard hydrotreatment conditions using feed coal for the WVU coal extraction process and the corresponding residue as disposable catalysts. Tests were also performed using a Houdry $\mathrm{CoMo} / \mathrm{Al}_{2} \mathrm{O}_{3}$ commercial catalyst as a control.

2. To obtain reaction rates for combined $\mathrm{HDN}$ and $\mathrm{HDS}$ reactions proceeding competitively under standard hydrotreatment conditions using both feed coal and its WVU Coal Extraction Process residue as catalysts.

\section{Introduction}

The project has been studied in three phases. In the first phase, HDN tests were performed using a $30 \mathrm{ml}$ bomb reactor under mild conditions $\left(200^{\circ} \mathrm{C}, 2\right.$ hours reaction time and $200 \mathrm{psig}$ initial pressure). In the second phase, tests were performed using a $300 \mathrm{ml}$ Parr autoclave and stronger reaction conditions $\left(350^{\circ} \mathrm{C}, 2\right.$ hours, $600 \mathrm{psig}$ initial hydrogen pressure).

Under phase 2 conditions, conversion of the model compound, quinoline to tetrahydroquinoline (a saturated product) was observed for the presulfided forms of all the catalysts studied (a Houdry Commercial Catalyst, KCER \#91864 feed coal and KCER \#91864 coal extraction process residue). However, the formation of nitrogen-free products only occurred with the commercial catalyst.

A final phase of $\mathrm{HDN}$ tests is currently being performed to improve production of the nitrogen free products and the conversion of quinoline to its HDN products. There tests are being performed in the $300 \mathrm{ml}$ Parr autoclave at $400^{\circ} \mathrm{C}, 4$ hours of reaction time and 600 psig initial hydrogen pressure.

\section{Results and Discussion}

The literature shows that HDN of quinoline should proceed following the reaction system shown below (1). 
Detail of the Hydrodenitrification Reactions

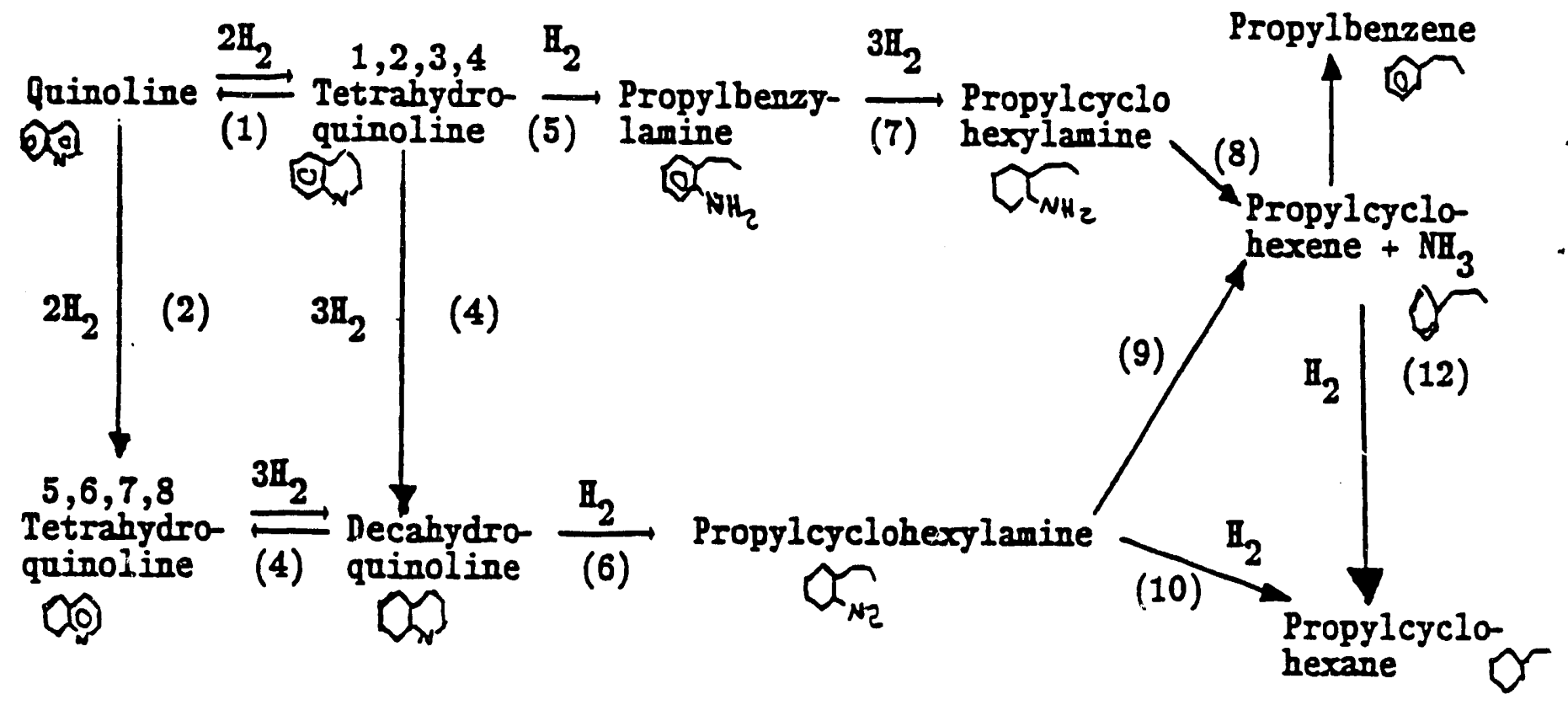

Reactions $1,2,3$, and 4 are considered to be reversible while reactions $5,6,7,8,9,10,11$, and 12 are considered to be irreversible. Reactions 8,9 , and 10 are considered to be fast compared to the other reactions. As a result little or no propylcyclohexylamine has been observed in our reaction products and in those reported by other investigators $(1,2,3)$. Other investigators also have shown that equilibrium between quinoline and 1,2,3,4, Tetrahydroquinoline occurs quickly even at relatively low temperatures $(2,3)$.

\section{Phase One}

Phase one tests were performed by placing six $\mathrm{ml}$ of a $1 \%$ quinoline in hexadecane solution in a stainless steel bomb reactor. The reactor was sealed, charged with 200 psig of hydrogen gas and then placed in a $200-225^{\circ} \mathrm{C}$ oil bath for $1-2$ hours. After the reaction period was completed, the reactor was cooled to room temperature, then the residual hydrogen gas was released and the reactor was opened and the solvent saved for analysis. Analytical results showed no formation of the expected reaction products.

\section{Phase Two}

It was felt that the $200-225^{\circ} \mathrm{C}$ reaction temperature used in the phase one tests was below the threshold temperature required for the $\mathrm{HDN}$ of quinoline. As a result, additional HDN tests were performed using the $300 \mathrm{ml}$ Parr autoclave. These phase two tests were performed by 
placing $50 \mathrm{ml}$ of a $1 \%$ quinoline in hexadecane solution and 0.138 grams of catalyst in a $300 \mathrm{ml}$ Parr autoclave. The autoclave was then sealed, charged with 600 psig of hydrogen gas, and heated to $350^{\circ} \mathrm{C}$, without stirring. When the autoclave reached $350^{\circ} \mathrm{C}$, stirring was started and the hydrogen gas was allowed to react with the quinoline. After a two hour reaction period the autoclave was cooled to room temperature, the residual hydrogen gas was released and the solvent was separated from the spent catalyst by filtration. Analytical results for this phase showed the production of HDN products (tetrahydroquinoline) for the coal, residue and a Houdry $\mathrm{CoMo} / \mathrm{Al}_{2} \mathrm{O}_{3}$ commercial catalyst. Production of propylcyclohexane, a nitrogen-free product was also observed for the commercial catalyst (see Table 1 ).

Table 1. Analytical Results for HDN Tests Using Quinoline as a Model Compound

Catalyst

Kentucky Coal KCER \#91864

Residue KCER \#91864

Houdry $\mathrm{CoMo} / \mathrm{Al}_{2} \mathrm{O}_{3}$

\section{Reactant/Product}

Quinoline

Tetrahydroquinoline

Quinoline

Tetrahydroquinoline

Quinoline

Tetrahydroquinoline

decahydroquinoline

propylcyclohexane

Results

\section{$\underline{\text { Percent }}$}

88.26

11.74

85.22

14.78

5.24

91.13

1.30

2.33
Feed coal

residue

Houdry
$11.74 \%$ conversion of quinoline to $\mathrm{HDN}$ products $00.00 \%$ conversion of nitrogen free products

$14.78 \%$ conversion of quinoline to $\mathrm{HDN}$ products $00.00 \%$ conversion of nitrogen free products

94.76\% conversion of quinoline to $\mathrm{HDN}$ products $2.33 \%$ conversion to nitrogen free products

\section{Phase Three}

While phase 2 tests showed some definite progress in conversion of quinoline to $\mathrm{HDN}$

- products, the conversion of quinoline to nitrogen free products was far too low. It was determined that propylcyclohexane production could be increased by making the following changes in the reaction conditions:

1. longer reaction times

2. higher reaction temperatures

3. decreasing the particle size of the catalyst for the coal and residue 
4. increasing the catalyst : model compound ratio (adding more catalyst)

In the phase 3 tests, options 1 and 2 were emphasized: the reaction time was increased from 2 to 4 hours and the reaction temperature was increased to $400^{\circ} \mathrm{C}$. The procedure used in phase 2 was also used to perform the phase 3 tests with the above changes. HDN tests were also performed using ground residue as a catalyst. The analytical results for these HDN tests are shown in Table 2.

\section{Table 2. Analytical Results for Phase 3 HDN Tests Using Quinoline on a Model}

\section{Compound}

Catalyst

Houdry CoMo/ $/ \mathrm{Al}_{2} \mathrm{O}_{3}$

Residue $\left(400^{\circ} \mathrm{C}\right)$

$\left(400^{\circ} \mathrm{C}\right.$, ground)

$\left(375^{\circ} \mathrm{C}\right)$

$\left(400^{\circ} \mathrm{C}\right)$
Reactant/Product

Quinoline

Tetrahydroquinoline

Propylbenzylamine

Propylcyclohexane

Quinoline

Tetrahydroquinoline

Propylbenzylamine

Propylbenzene

Propylcyclohexane

Quinoline

Tetrahydroquinoline

Quinoline

Tetrahydroquinoline

Propylbenzylamine

Propylcyclohexane $\frac{\text { Percent }}{6.76}$

46.03

12.44

34.78

9.38

36.98

7.38

12.62

33.21

59.48

40.52

43.69

33.52

22.53

0.27

\section{Results}

Houdry

$375^{\circ} \mathrm{C}$

93.24\% conversion of quinoline to HDN products $34.78 \%$ conversion to nitrogen-free products

$400^{\circ} \mathrm{C}$

$90.62 \%$ conversion of quinoline to $\mathrm{HDN}$ products 45.83\% conversion to nitrogen-free products

Residue $\quad 400^{\circ} \mathrm{C}$

$400^{\circ} \mathrm{C}$, ground

$40.52 \%$ conversion of quinoline to $\mathrm{HDN}$ products $00.00 \%$ conversion to nitrogen-free products

$56.31 \%$ conversion of quinoline to $\mathrm{HDN}$ products $0.27 \%$ conversion to nitrogen-free products 
These results show production of propylcyclohexane increased with the increasing reaction temperature and reaction time. Production of HDN products also increased for the residue. In addition production of a nitrogen-free product by the residue was also observed.

Rate constants were obtained for the phase 2 results and the $400^{\circ} \mathrm{C}$ unground residue results by assuming that the conversion of quinoline to HDN products approximates a first order irreversible reaction. As such, the reaction is approximated by the expression:

$$
\mathrm{dC}_{\alpha} / \mathrm{dt}=-\mathrm{kC}_{\mathbf{2 0}} \mathrm{g}
$$

where $\quad C_{a}$ is the concentration of quinoline

$t$ is the time in minutes

$\mathrm{C}_{\infty}$ is the initial concentration of quinoline

$g$ is the weight of catalyst used in grams

As such

$$
\mathrm{d} \mathrm{C}_{\mathrm{z}} / \mathrm{C}_{\mathrm{wo}}=-\mathrm{kg} \mathrm{dt}
$$

or

$$
k=-\left(\ln C_{2} / C_{20}\right) /(g \cdot t)
$$

Using this equation, rate constants were calculated for the phase 2 results and the $400^{\circ} \mathrm{C}$ unground residue results. The kinetics for the other phase 3 results can not be approximated by a pseudo 1st order irreversible reaction. They will be solved using a computer method.

\section{Table 3. Pseudo First Order Reaction Rate Constants for the HDN Reactions}

Catalyst

\section{Concentration of Quinoline Reaction Rate Constant}

$$
\text { (moles/liter) }
$$
Coal
0.068
0.754
Residue
$350^{\circ} \mathrm{C}$
0.066
0.947
$400^{\circ} \mathrm{C}$
0.046
1.463
Houdry
0.0041
17.41
- $\left(\mathrm{C}_{\mathrm{an}}=0.077\right.$ moles/iter $)$

As expected the commercial catalyst has the fastest rate. However, increasing the reaction temperature increased the rate constant for the residue from $0.947 \mathrm{~min}^{-1} \mathrm{~g}^{-1}$ to $1.463 \mathrm{~min}^{-1} \mathrm{~g}^{-1}$ a $54 \%$ increase. 
These constants were obtained assuming a single HDN reaction as shown below:

$$
\text { Quinoline } \stackrel{2 \mathrm{H}_{2}}{\longrightarrow} \text { tetrahydroquinoline }
$$

The reaction rate constants for the remaining phase three tests will be calculated assuming the following reaction sequence

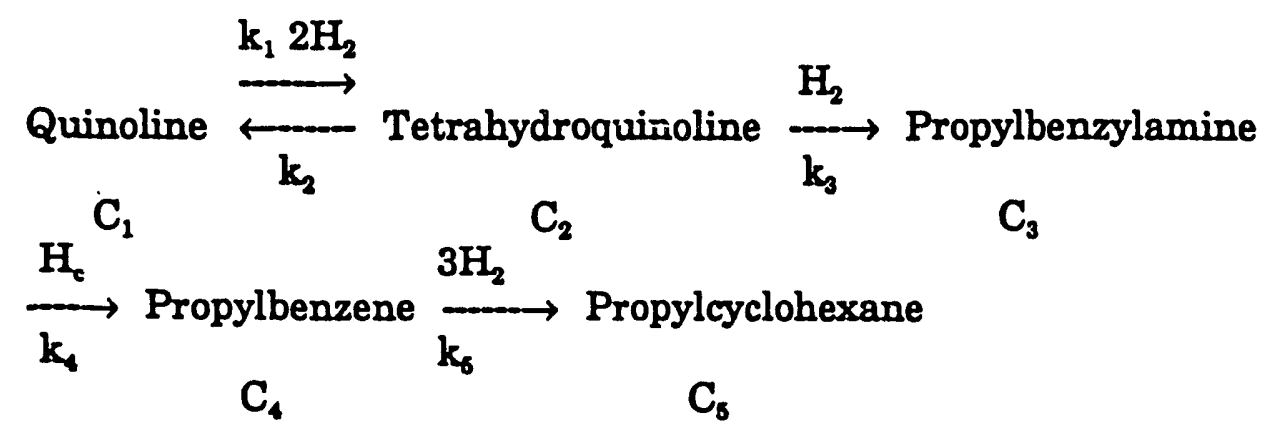

As such the kinetics for the sequence will be

$$
\begin{aligned}
& \mathrm{dC}_{1} / \mathrm{dt}=\left(-\mathrm{k}_{1} \mathrm{C}_{1}+\mathrm{k}_{2} \mathrm{C}_{2}\right) \\
& \mathrm{dC}_{2} / \mathrm{dt}=\left(\mathrm{k}_{1} \mathrm{C}_{2}-\mathrm{k}_{2} \mathrm{C}_{2}-\mathrm{k}_{3} \mathrm{C}_{2}\right) \\
& \mathrm{dC}_{3} / \mathrm{dt}=\left(\mathrm{k}_{3} \mathrm{C}_{3}-\mathrm{k}_{4} \mathrm{C}_{3}\right) \\
& \mathrm{dC_{4 }} / \mathrm{dt}=\left(\mathrm{k}_{3} \mathrm{C}-\mathrm{k}_{6} \mathrm{C}_{4}\right) \\
& \mathrm{dC}_{6} / \mathrm{dt}=\left(\mathrm{k}_{6} \mathrm{C}_{4}\right)
\end{aligned}
$$

This system of equations will be solved using a computer technique.

A pseudo-activation energy estimate was made for the residue using the following aquation:

$$
k_{1}=A e^{-E / R T} \text { or } \frac{d \ln \left(k_{1}\right)}{d 1 / T}=-E_{\alpha} / R
$$

The natural log of the rate constant was plotted as a function of the reciprocal temperature in degrees Kelvin for the $350^{\circ} \mathrm{C}$ and $400^{\circ} \mathrm{C}$ rate constants. The slope of the line generated by these two points was used to calculate a pseudo activation energy for the residue $H D N$ reaction. The activation energy was $7.208 \mathrm{Kcal} / \mathrm{mole}$. While the value is low for a chemical reaction controlled condition, it is reasonably close to the value reported other investigators for the reaction of quinoline to tetrahydroquinoline (3).

\section{References}

1. Satterfield, Charles N., and Yang, Shan Hai, Catalytic Hydrodenitrogenation of Quinoline in a Trickle-Bed Reactor. Comparison With Vapor Phase Reaction, I \& EC Process Design 
and Development, 1984 23, 11-19.

2. Satterfield, Charles N., and Cocchetto, Joseph F., Reaction Network and Kinetics of the Vapor-Phase Catalytic Hydrogenation of Quinoline, I \& EC Process Design and Development, 1981, 20, 53-62.

3. Satterfield, Charles N., Modell, Michael, Hites, Ronald A., and Declerck, Claude J., Intermediate Reactions in the Catalytic Hydrogenation of Quinoline, I \& EC Process Design and Development, Vol. 17, No. 2, 1979, 141-148. 


\section{Task IV}

Auburn University 


\section{Task IV}

\section{Enhanced Reactivity and Selectivity in Coal Liquefaction and Co-Processing Systems}

\begin{tabular}{lll} 
Prs: & $\begin{array}{l}\text { Christine Curtis } \\
\text { James Guin }\end{array}$ & Ray Tarrer \\
$\begin{array}{l}\text { Report } \\
\text { Coordinator: }\end{array}$ & A. R. Tarrer \\
Phone Number: & $(205) 844-4827$ \\
Period: & May 4, 1989-May 3, 1990 \\
\hline
\end{tabular}

Task IV.I. Cyclic Olefins: New Donors for Coal Liquefaction

\section{Introduction}

A new set of hydrogen donors, cyclic olefins, has been evaluated to determine their effectiveness as hydrogen donors to coal. These cyclic olefins, as well as their conventional hydrogen donor analogues, were reacted with Western Kentucky No. 9 coal under a variety of conditions. Also performed was a series of MNDO molecular orbital calculations to investigate the dehydrogenation pathway of isotetralin and tetralin. A comparison was made of three initial theoretical calculations to previous experimental work involving the thermal reactivity of isotetralin and tetralin in a nitrogen atmosphere.

\section{Experimental}

The reactions of both CLO's and three conventional hydroaromatic donor compounds with Western Kentucky No. 9 were performed. For this study the following reaction conditions were employed: 30 minute reaction time, 1250 psig nitrogen or hydrogen atmosphere at ambient temperature, $380^{\circ} \mathrm{C}$ reaction temperature, $2.0 \mathrm{~g}$ coal, $4.0 \mathrm{~g}$ total solvent mixture including 0.1-0.5 weight percent donable hydrogen of the model compound with the balance being either fluorene or hexadecane as the diluent solvent, $700 \mathrm{cpm}$ vertical agitation rate using stainless steel tubing bomb reactors of approximately $50 \mathrm{~cm}^{3}$ volume.

The hydrogen donors and their products from the coal liquefaction reactions were analyzed by gas chromatography using a Varian 3400 gas chromatograph equipped with a HT-5 column and FID detection. The internal standard method was used for quantitation with biphenyl as the internal standard. The peaks were identified by comparing retention 
times with authentic compounds and by analysis with a VG 70EHF mass spectrometer.

The tetrahydrofuran soluble (THFS) fraction for each reaction was then further analyzed utilizing a liquid chromatographic separation procedure based upon that developed by Boduszynski $(1,2)$. This procedure consists of the elution of a series of solvents through a column of inert packing coated with the sample to be analyzed. The eluant then passes into a column of basic alumina which performed the separation. The four fractions obtained are hydrocarbons, nitrogen heterocycles, hydroxyl aromatics, and polyfunctional compounds. The solvents employed are hexane, toluene, chloroform, chloroform/methanol, and THF.

For this work coal couversion is defined as conversion $=1-[$ IOM (maf) $/$ coal charge (maf) $] \times 100$

where maf means moisture and ash free. In order to use this definition of coal conversion, both the moisture and ash contents were needed. The moisture content of Western Kentucky No. 9 coal was $4.5 \pm 0.6 \%$ and the ash, $10.16 \pm 0.14 \%$.

\section{Reaction of Hydrogen Donors with Western Kentucky No. 9 Coal}

\section{Thermal Reactions.}

A series of thermal reactions using cyclic olefins (CLO's), isotetralin (ISO) and hexahydroanthracene (HHA), and their hydroaromatic analogues, tetralin (TET), octahydroanthracene (OHA), and dihydroanthracene (DHA) with Western Kentucky No. 9 coal in a nitrogen atmosphere was performed. The solvent used was fluorene. The amount of coal conversion to tetrahydrofuran solubles (THFS) was determined in at least duplicate reactions.

Two important results are presented in Table I: the ranking of the five donor species at the equivalent loading of 0.5 weight percent donable hydrogen and a comparison of HHA and OHA at three different levels of donable hydrogen, $0.5,0.2,0.1$ weight percent. At the 0.5 weight percent level of donable hydrogen, the two CLO's, HHA and ISO, yielded more coal conversion than did their respective conventional hydroaromatic analogues, OHA, DHA, or TET. HHA produced an increase in coal conversion of approximately $12 \%$ more than its nearest analogue, DHA, and 13\% more than OHA. ISO yielded approximately $14 \%$ more conversion than TET. At the various donable hydrogen levels, HHA consistently converted more coal than did either DHA or OHA, albeit, in decreasing differential amounts as the level of donable hydrogen decreased. This result implies that a deficiency of donable hydrogen occurred at these lower levels of donable hydrogen.

Coal reactions were also performed at three different thermal reaction conditions: $410^{\circ} \mathrm{C}$ and $380^{\circ} \mathrm{C}$ in nitrogen and $380^{\circ} \mathrm{C}$ in hydrogen. The amount of coal conversion obtained 
for each added donor species is presented in Table II. Each reaction was at least duplicated. The solvent used for these reactions was fluorene and the level of donable hydrogen was 0.5 weight percent for each reaction.

Substantial differences in the amount of coal conversion achieved with the different - added donor species were observed in nitrogen. A greater difference was observed between HHA and ISO and their respective analogues at $380^{\circ} \mathrm{C}$ than at $410^{\circ} \mathrm{C}$. The reactions the donors experienced at $410^{\circ} \mathrm{C}$ were probably more rapid, resulting more quickly in the production of aromatic species that did not serve as donors to coal.(3) A high level of reactivity was observed in these systems regardless of donor addition. The reaction at $380^{\circ} \mathrm{C}$ in nitrogen with just fluorene as the solvent present converted nearly $60 \%$ of the coal, indicating that fluorene provided a good solvating medium for coal.

The coal reactions with added donor in hydrogen also showed high levels of reactivity but no differentiation among the added donor species. The reaction with fluorene alone yielded nearly $79 \%$ conversion of coal. Fluorene apparently was an excellent solvent for coal and even perhaps participated in hydrogen shuttling reactions that promoted coal dissolution. Further examination of the role of fluorene is planned.

For each of the reactions presented in Table II, the THF soluble fraction was then further analyzed by gas chromatography to determine the effect of the coal reaction on the model hydrogen donor present. The product distributions from the reacted added donor species are presented in Table III. The coal conversions obtained in the corresponding reactions are presented in Table II.

Even in the presence of coal a substantial difference in the reactivity of the CLO's (ISO and HHA) and the hydroaromatic donors (TET, DHA, OHA) was observed; the CLO's were completely converted, whereas all the hydroaromatic donors remained partially unconverted. In comparing the two studies at $380^{\circ} \mathrm{C}$, the reactions in a nitrogen atmosphere produced more aromatic products than those obtained in a hydrogen atmosphere except for the tetralin reaction. At $410^{\circ} \mathrm{C}$, substantially more aromatic species were produced from - each donor regardless of type than at $380^{\circ} \mathrm{C}$. Also, under hydrogen the model compounds HHA and DHA were hydrogenated to produce a significant amount of OHA (40.6 and 63.8\%, respectively). In a hydrogen atmosphere OHA remained virtually unreacted.

Also calculated for the three sets of reactions was the net amount of hydrogen released. This amount was determined as the difference in the total amount of hydrogen released and the amount of hydrogen required to form other species. For example, when HHA reacted and formed products of DHA, THA and OHA, hydrogen was released when DHA and THA were formed bui was consumed when OHA was 
formed. The amount of net hydrogen released to the system was dependent upon the amount of HHA converted and the relative amounts of each product produced. Table IV presents the net hydrogen released for the three studies at different reaction conditions using 0.5 weight percent donable hydrogen.

Figures 1 and 2 present a graphical representation of this data. For the two sets of reactions under a nitrogen atmosphere, the general trend is that as coal conversion increased, the net amount of hydrogen released also increased. The solid line on the figures is the linear best fit to the data.

The coal reaction in fluorene and in a hydrogen atmosphere showed no significant difference in the amount of coal conversion obtained with or without any of the donor compounds added (Figure 3). It is postulated that the consistent coal conversions obtained ( 77\%) were caused by the diluent solvent, fluorene, shuttling hydrogen from the gas phase to the coal matrix, thereby masking any effect the hydrogen donor compound may have had. In order to determine the effect of the donor compounds in a hydrogen atmosphere without the influence of fluorene, the reactions were repeated using an inert solvent, hexadecane, and the coal conversion to THF solubles determined as presented in Table V.

Coal conversions obtained thermally in a hydrogen atmosphere with additional hydrogen donors in hexadecane showed substantial changes that depended upon the particular hydrogen donor species added (Table V). Coal conversion in hexadecane was low at $-26 \%$ indicating that the solvent itself had little reactivity and did not provide a good solvating medium for coal. ISO produced $\sim 50 \%$ coal conversion, almost $20 \%$ more than TET. Also, HHA produced $65 \%$ coal conversion which was approximately $18 \%$ more than OHA. DHA produced a slightly higher coal conversion than HHA yielding $\sim 68 \%$. Thus, the addition of ISO resulted in more coal conversion than TET and HHA more than OHA. DHA in hydrogen was as efficient a donor to coal as HHA, once the effect of the diluent solvent was eliminated.

The reaction products from the added donors from these thermal reactions are also presented in Table V. In a hydrogen atmosphere without a catalyst, HHA reacted completely and formed nearly an equivalent amount of OHA and DHA (47.5 and 45.7\%, respectively). DHA showed a slightly higher coal conversion than HHA; DHA converted more than $75 \%$ yielding $61 \%$ OHA and $14.5 \%$ ANT as products. OHA, which only converted about half the amount of coal as the other two donors, remained $-85 \%$ unreacted yielding $-15 \% \mathrm{DHA}$ as product. The net hydrogen released from these reactions is given in Table $\mathrm{V}$ and shows that HHA released nearly twice as much hydrogen as did OHA and that DHA appeared to incorporate molecular hydrogen from the gas phase into the production of OHA. 
ISO in hydrogen reacted completely yielding $17.8 \%$ TET and $82.2 \%$ NAP as products, while TET only converted $\sim 20 \%$ to produce NAP. The amount of hydrogen released for TET was more than four times that released from TET. The reactivity and amount of hydrogen released from ISO corresponded to the higher coal conversion obtained from ISO. Lower coal conversion along with lower reactivity and hydrogen released was observed from TET.

\section{Catalytic Reactions.}

The catalytic reactions using $\mathrm{NiMo} / \mathrm{Al}_{2} \mathrm{O}_{3}$, also presented in Table $\mathrm{V}$, showed that the solvent hexadecane yielded the same amount of coal conversion as in the thermal reactions. ISO produced more coal conversion than TET but the amount of coal conversion for both decreased compared to the thermal reaction as did the difference between them. In the three ring series, DHA converted $\sim 71 \%$ of the coal to THF solubles while HHA converted -59\% of the coal and produced $20 \%$ more coal conversion than OHA. The coal conversion of the conventional hydroarumatic donors OHA and DHA increased in the catalytic system compared to the thermai system. However, the CLO's, ISO and HHA, produced less coal conversion in the catalytic system. One possible explanation for this phenomenon is that the CLO's released their donable hydrogen in the catalytic system faster than the acceptor sites of the coal matrix could utilize the hydrogen. Also, ISO and HHA may have heen rapidly converted to conventional hydrogen donor species thereby losing their reactive edge toward converting coal.

The reaction products from the added donors in the catalytic reactions in Table $V$ showed more conversion of DHA and HHA to OHA and more OHA remaining than in the thermal reactions. Also less ANT and slightly less DHA were produced in the catalytic reactions compared to the thermal. More hydrogen was released from HHA than from OHA, but DHA consumed molecular hydrogen in its production of OHA and in its conversion of coal (Table VI). In the catalytic environment, rehydrogenation of the reacted donor is possible because of involvement of the catalyst and molecular hydrogen so that the net amount of hydrogen released calculated from the reaction products may not be a true indicator of all of the reactions that are involved.

- ISO completely converted in the catalytic reaction yielding nearly $85 \%$ TET and $15 \%$ NAP (Table V). By contrast, no conversion of TET was observed. The net amount of hydrogen released from ISO did not seem to contribute substantially to coal conversion. No hydrogen was released from TET and, hence, no net increase in coal conversion over the solvent was observed. These ISO/coal and TET/coal reactions were repeated and the results verified. 


\section{Liquid Chromatographic Analysis of Coal Products.}

Further analysis of the coal liquefaction products by liquid chromatography has been initiated. Liquid chromatographic analyses of the THF soluble fractions have been completed for the coal reactions at $380^{\circ} \mathrm{C}$. The technique employed was an adaptation of the liquid chromatographic separation of liquefaction products based upon the method developed by M.M. Boduszynski $(1,2)$ and used previously in these laboratories. Table VI presents the results of this analysis.

For the reactions with lower coal conversion the percentage fraction of the obtained products appeared to be composed of a larger amount of hydrocarbons and a smaller amount of hydroxyl aromatic compounds. For the compounds producing higher coal conversion, i.e. ISO and FHA, the fractional percentage of the obtained products was lower in hydrocarbons and higher in hydroxyl oromatics. The higher conversions obtained with the CLO's probably occurred because of increased conversion of hydroxyl aromatics to the THF soluble fraction from the IOM or unconverted coal. By contrast, the iess efficient hydrogen donor compounds, TET, OHA and DHA, did not convert this part of the coal matrix to THF solubles. It also appears that the CLO's converted more nitrogen heterocycles from the coal to the THF soluble fraction than did the conventional hydrogen donors.

Table VII presents a comparison of the liquid chromatographic separation results for the reaction involving DHA at three levels of donable hydrogen. For these reactions, a higher fractional percentage of the product as hydrocarbons was obtained from the higher level of donable hydrogen. The liquid chromatographic separation will be performed on future reactions to monitor the changes in the different fractions resulting from varying the reaction conditions.

\section{Molecular Modeling of ISO and TET}

\section{Objective.}

The objective of this research was to use the molecular orbital calculation, MNDO, to examine the energies involved in the dehydrogenation of ISO and TET, to predict the most likely dehydrogenation mechanism, and to compare the calculated results to those obtained experimentally. The first step toward achieving these goals was to perform MNDO calculations on the basis of a free radical mechanism. These calculations consisted of geometry optimization and MNDO calculations for ISO and 1,4-DHN, as well as their single radical and bi-radical transition state intermediates. Because the actual dehydrogenation mechanism is not known a priori, cationic and anionic dehydrogenation mechanisms were also calculated and compared to the free radical mechanism.

When these calculations were completed, additional MNDO calculations were 
performed to investigate the probability of a reaction pathway from 1,4-DHN to 1,2-DHN and NAP that employed a free radical, cationic and/or anionic mechanism based upon the results obtained from the work heretofore described. A summary of these calculations is presented next.

\section{MNDO Calculations.}

The approach to the MNDO calculations for the first part of the theoretical work was as follows. The first step was to assume that the molecules were fixed in a plane, while allowing the bond lengths and bond angles to be optimized. These results were then used in the next calculational sequence where the dihedral angles were optimized. The heat of formation for TET with no dihedral angle optimization was $6.36 \mathrm{kcal} / \mathrm{mole}$, while the heat of formation with optimization was $2.59 \mathrm{kcal} /$ mole. The more favorable geometry for TET is a "puckered" configuration, as indicated by the lower heat of formation obtained with dihedral angle optimization since a lower heat of formation results in a more stable molecule. When the same calculations were performed for ISO, the heat of formation with no dihedral angle optimization was $20.51 \mathrm{kcal} /$ mole; with optimization, $19.45 \mathrm{kcal} / \mathrm{mole}$. Therefore, ISO remained essentially a "planar" molecule, as indicated by the small change in the heat of formation with dihedral angle optimization.

The next step was to remove a hydrogen from each molecule forming a radical species, by employing the keyword Doublet in the MNDO calculation. The doublet state has a single unpaired electron in tb.e outer orbital. Then an additional hydrogen from each radical species was removed to form a bi-radical. In turn, both Singlet and Triplet keyword configurations were employed. The configurations correspond, respectively, to the singlet and triplet excited electronic states. The singlet state has the outer pair of electrons in opposite spins while the triplet state has the outer pair of electrons in the same spin. The final step was to perform the MNDO calculation on DHN.

The results of the series of MNDO calculations are presented in Table VIII for the ground state reactants (ISO and TET), the transition states (single or bi-radical), and the ground state product (DHN). In the ground state TET is more stable than ISO, as indicated - by its lower heat of formation $\left(\mathrm{H}_{4}\right)$. This result is not surprising, since it is expected that conjugated double bond systems particularly in aromatic rings should be more stable than non-conjugated systems. The single radical species obtained by the removal of a hydrogen atom from TET is more stable (i.e. lower heat of formation) than that obtained from ISO. In performing the MNDO calculations on the bi-radical species derived from TET and ISO, both the triplet state and the singlet state were employed. The singlet state conformation for ISO 
is the more energetically favorable state, yielding a lower heat of formation. However, for TET the triplet state conformation has the lower heat of formation and is more stable. In the transition of TET through a radical (doublet) and then a bi-radical (triplet), the energy barrier is much higher than the for ISO. A graphical representation of the heat of formation data for the transition of TET and ISO to DHN is presented in Figure 4.

Since the transition of ISO to DHN by a radical mechanism is more favorable than that for TET, ISO should more readily donate its hydrogen and be a more reactive hydrogen donor species than TET. This conclusion agrees with the experimental results obtained in the thermal reactivity studies of ISO and TET where ISO completely reacted while TET did not under liquefaction conditions. Therefore, MNDO calculations appear to be a useful tool to explain, and possibly predict, experimental observations in comparing CLO's to their hydroaromatic analogues.

Additional work was performed to test the possibility of a cationic or anionic dehydrogenation mechanism as presented in Table VIII. The heat of formation $\left(\mathrm{H}_{f}\right)$ and ionization potential (Ip) obtained for ground state 1,4-DHN were $\mathrm{H}_{\mathrm{f}}=25.27 \mathrm{kcal} / \mathrm{mole}$ and Ip $=9.17 \mathrm{kcal} / \mathrm{mole}$, respectively. Graphical representations of the data in Table VIII are presented in Figures 4, 5, and 6 (radical, cationic, and anionic mechanisms, respectively). For both the cationic and anionic mechanisms, the pathways for ISO have a higher energy barrier than does the pathway for TET. These pathways are in contrast to the free radical mechanism that showed a higher energy barrier for TET than it did for ISO. Also previously described experimental results showed that ISO was much more reactive than was TET and, therefore, should have a lower energy barrier than TET.

If the reaction pathway from ISO passes through 1,4-DHN, ISO should also have a lower energy barrier in its dehydrogenation pathway to 1,4-DHN. These molecular orbital calculations, in conjugation with the experimental results, suggest that the free radical mechanism is a more likely mechanism for the dehydrogenation of ISO and TET to 1,4-DHN than an anionic or cationic mechanism. $\mathrm{H}_{f}$ is higher for both the cationic and anionic pathways as compared to the free radical mechanism. This result also implies that the cationic and anionic mechanisms are not as thermodynamically favorable as the free radical pathway.

In the previously discussed MNDO calculations, the initial input geometry was determined by making a physical model, and then determining connectivity, bond lengths, and dihedral angles (internal coordinates), and then using this matrix to perform the MNDO calculations. A new procedure was also employed utilizing the software package SYBYL (Tripos Associates, Inc.) which allows building and manipulating molecules. The first step 
used SYBYL to draw the desired molecular species; then the structures were optimized utilizing a force field energy minimization procedure. The force field minimization procedure consisted of a series of line searches until a minimum in energy was reached. The searches were performed by the Conjugate Gradient method, which provides a convergence method based upon information acquired from one iteration to the next. The resulting optimized structures were then in turn configured for the AMPAC version available in SYBYL, with the input geometry computed in the form of cartesian coordinates. These input files were then downloaded from SYBYL and installed on a VAX 6320 computer. Once installed, the MNDO calculations were performed as before. The only difference in this and the previous procedures was the manner by which the input files were generated.

The first series of calculations performed was to determine if the two calculational methods produced the same results. To accomplish this objective, both ISO and TET were built with SYBYL and their geometry optimized using the force field minimization procedure. Then MNDO calculations were performed on the optimized geometries. As a check, MNDO calculations were also performed utilizing the non-optimized geometries. As can be seen in Table $\mathrm{IX}$, there is excellent agreement for heat of formation and ionization potential results with the conventional input geometries (internal coordinates) and the structures generated from SYBYL (cartesian coordinates). Therefore, no anomalies, caused by the use of the SYBYL software package, are apparent. The next results generated can be compared to or utilized with the work that has been previously performed.

The next step in this project was to investigate the isomerization pathway of 1,4$\mathrm{DHN}$ to 1,2-DHN by employing a free radical, cationic, or anionic mechanism. Calculations for the cationic and anionic mechanisms were performed for comparison purposes. The intermediate for this isomerization was a single radical or charged species of 1,4-DHN formed by the removal of a hydrogen from carbon number 4 (IUPAC numbering conventional for the naphthalene parent compound). Table X presents the results obtained for the MNDO calculations performed.

The transition from 1,4-DHN to 1,2-DHN has a lower heat of formation in the anionic mechanism. But when examined in the larger context of the transition of ISO and TET to , 1,4-DHN and then to 1,2-DHN, the anionic mechanism is still not energetically favorable compared to the free radical mechanism.

This unfavored state is even more dramatically illustrated when the bi-radical or charge $=+2$ or -2 species is considered, e.g. the transition of 1,4-DHN to NAP. Therefore, the next set of MNDO calculations performed was to investigate the dehydrogenation of 1,4DHN to NAP. Table X presents the results that were obtained for the transition of ISO or 
TET to 1,4-DHN and then to NAP via a free radical, anionic, or cationic mechanism. Higher energy barriers were observed for both the cationic and anionic pathways than for the free radical mechanism. Since both cationic and anionic pathways for the transition of 1,4-DHN to NAP exhibited a very high energy barrier, the free radical mechanism is the more likely pathway.

The free radical pathway for the transition of ISO or TET to 1,4-DHN, and the transition of 1,4-DHN to 1,2-DHN or NAP is more energetically favorable than either a cationic or anionic mechanism. Also, the free radical mechanism predicts a lower energy barrier for these transitions for ISO than for TET. These results are in agreement with the experimental results showing ISO to be much more reactive than TET. Therefore, it can be concluded that molecular orbital calculations can be utilized as a good model and a predictive tool to compare the reactivity of cyclic olefins to their conventional hydrogen donor analogues.

\section{Summary and Conclusions}

Thermal liquefaction reactions in nitrogen with added CLO's resulted in a substantial increase in coal conversion in comparison with their respective hydroaromatic analogues. More hydrogen was correspondingly released from the CLO's than from the hydroaromatic donors. Likewise, substantial differences were observed among the CLO's and their hydroaromatic analogues in hydrogen in an inert solvent, hexadecane. The CLO's converted substantially more coal and released more hydrogen than their hydroaromatic analogues.

Catalytic reactions with presulfided $\mathrm{NiMo} / \mathrm{Al}_{2} \mathrm{O}_{3}$, hydrogen, hexadecane as solvent, and added donors resulted in ISO converting more coal than TET and HHA more than OHA; however, DHA converted more coal than either and appeared to incorporate molecular hydrogen from the coal reaction system. The major reaction product from DHA was OHA. The reason for high reactivity of DHA under these conditions has not yet been determined but may in part result from steric or configural interactions between DHA and the $\mathrm{NiMo} / \mathrm{Al}_{2} \mathrm{O}_{3}$ catalyst. The other three-ring donor species formed the same reaction products but did not form DHA or show the high reactivity level of DHA.

Theoretical calculations using MNDO predict that ISO dehydrogenated to 1,4-DHN and subsequently to NAP via a free radical mechanism. The calculated heats of formation for the required transitions were less than with either an anionic or a cationic mechanism. MNDO calculations with steric considerations also predicted that the ground state of ISO is less stable and more reactive than that of TET because ISO has a higher heat of formation. 


\section{References}

1. Boduszynski, M.M.; Hurtubise, R.J.; Silver, H.F.; "Separation of Solvent-Refined Coal into Solvent-Derived Fractions"; Anal. Chem.; 1982, 54, 372-375.

2. Boduszynski, M.M.; Hurtubise, R.J.; Silver, H.F.; "Separation of Solvent-Refilled Coal into Compound-Class Fraction"; Anal. Chem.; 1982, 54, 375-381.

3. Curtis, C.W.; Guin, J.; Kwon, K; "Coal Solvolysis in a Series of Model Compound Systems"; Fuel; 63, 1989.

\section{Table I. Coal Conversion of Kentucky No. 9 for Different Levels of Donable Hydrogen}

Donor

Compound

HHA

ISO

DHA

OHA

TET

none

\section{$\underline{0.5 w t \% \mathrm{DH}}$}

$88.6(0.04)$

$81.0(0.9)$

$76.6(0.8)$

75.5(0.7

$66.8(0.9)$

$57.4(2.3)$
Conversion* at

$\underline{0.2 w t \% ~ D H}$

78.2(0.5)

73.8(1.1)

70.2(2.7)

$57.4(2.3)$ $\underline{0.1 w t \% ~ D H}$

64.2(2.5)

62.5(1.2)

$60.3(0.7)$

$57.4(2.3)$

conversion defined as 1 - IOM (maf)

and $\mathrm{DH}$ refers to donable hydrogen

All reactions were performed at $380^{\circ} \mathrm{C}, 1250 \mathrm{psig}$ (cold) $\mathrm{N}_{2}$ atmospheres, $700 \mathrm{cpm}$, and 30 minute reaction time.

In the pairs of numbers above, the first is the conversion obtained. The second, in parenthesis, is the standard deviation for that data point.

Table II. Coal Conversion for Kentucky No. 9 Coal at Different Reaction Conditions with Fluorene as the Solvent

\begin{tabular}{lccc} 
Donor* & Coal Conversion & Coal Conversion & Coal Conversion \\
Compound & at $410^{\circ} \mathrm{C}$ & at $380^{\circ} \mathrm{C}$ & at $380^{\circ} \mathrm{C}$ \\
& (Nitrogen) & (Nitrogen) & (Hydrogen) \\
\hline
\end{tabular}

HHA

DHA

84.3(1.1)

$80.9(0.5)$

$88.6(0.04)$

76.6(0.8)

75.5(0.7)

$80.8(0.6)$

ISO

78.1(0.6)

TET

70.1(0.2)

$81.0(0.9)$

$66.8(0.9)$

None

NP**

57.4(2.3)

76.7(0.1)

76.6(1.0)

77.6(0.8)

$76.3(0.8)$

79.0(0.3)

78.8(3.4)

*Using 0.5 weight percent donable hydrogen. $\quad$ **NP = not performed 
Table III. Reaction Products from Model Donors for Thermal Coal Reactions Using Fluorene as the Solvent

\begin{tabular}{|c|c|c|c|c|}
\hline $\begin{array}{l}\text { Model } \\
\text { Compound } \\
\text { Added } \\
\end{array}$ & $\begin{array}{l}\text { Reaction } \\
\text { Conditions } \\
\end{array}$ & OHA & $\frac{\text { Products ( }}{\text { DHA }}$ & ANT \\
\hline HHA & $\begin{array}{l}\mathrm{N}_{2}, 380^{\circ} \mathrm{C} \\
\mathrm{N}_{2}, 410^{\circ} \mathrm{C} \\
\mathrm{H}_{2}, 380^{\circ} \mathrm{C} \\
\end{array}$ & $\begin{array}{l}18.5(0.5) \\
14.3(1.1) \\
40.6(8.7) \\
\end{array}$ & $\begin{array}{l}45.2(0.2) \\
24.2(2.5) \\
47.1(2.7) \\
\end{array}$ & $\begin{array}{l}36.3(0.6) \\
61.5(3.5) \\
12.3(6.3) \\
\end{array}$ \\
\hline OHA & $\begin{array}{l}\mathrm{N}_{2}, 380^{\circ} \mathrm{C} \\
\mathrm{N}_{2}, 410^{\circ} \mathrm{C} \\
\mathrm{H}_{2}, 380^{\circ} \mathrm{C} \\
\end{array}$ & $\begin{array}{l}51.2(0.6) \\
19.3(1.2) \\
89.7(0.3) \\
\end{array}$ & $\begin{array}{l}42.4(0.4) \\
44.0(0.9) \\
10.3(0.3) \\
\end{array}$ & $\begin{array}{l}6.4(0.3) \\
36.7(1.4) \\
\text { None } \\
\end{array}$ \\
\hline \multirow[t]{2}{*}{$\mathrm{DHA}$} & $\begin{array}{l}\mathrm{N}_{2}, 380^{\circ} \mathrm{C} \\
\mathrm{N}_{2}, 410^{\circ} \mathrm{C} \\
\mathrm{H}_{2}, 380^{\circ} \mathrm{C} \\
\end{array}$ & $\begin{array}{l}18.1(0.6) \\
14.5(0.9) \\
63.8(0.9) \\
\end{array}$ & $\begin{array}{l}21.5(0.3) \\
15.3(0.4) \\
25.1(0.7) \\
\end{array}$ & $\begin{array}{l}60.4(0.8) \\
70.1(1.0) \\
11.1(0.3) \\
\end{array}$ \\
\hline & & TET & NAP & \\
\hline ISO & $\begin{array}{l}\mathrm{N}_{2}, 380^{\circ} \mathrm{C} \\
\mathrm{N}_{2}, 410^{\circ} \mathrm{C} \\
\mathrm{H}_{2}, 380^{\circ} \mathrm{C} \\
\end{array}$ & $\begin{array}{l}14.4(0.7) \\
4.9(0.5) \\
96.1(0.3) \\
\end{array}$ & $\begin{array}{l}85.6(0.7) \\
95.1(0.5) \\
3.9(0.4) \\
\end{array}$ & \\
\hline TET & $\begin{array}{l}\mathrm{N}_{2}, 380^{\circ} \mathrm{C} \\
\mathrm{N}_{2}, 410^{\circ} \mathrm{C} \\
\mathrm{H}_{2}, 380^{\circ} \mathrm{C} \\
\end{array}$ & $\begin{array}{l}68.5(0.6) \\
36.6(0.6) \\
21.1(1.1)\end{array}$ & $\begin{array}{l}31.5(0.6) \\
63.4(0.6) \\
78.9(1.1)\end{array}$ & \\
\hline
\end{tabular}


Table IV. Net Hydrogen Released from Model Donors

Coal Study I: Kentucky No. $9,30 \mathrm{~min}, 380^{\circ} \mathrm{C}, \mathrm{N}_{2}$, Fluorene as Solvent

Donor

Added

HHA

ISO

DHA

OHA

TET

None
Coal

Conversion

88.6(0.04)

81.1(0.9)

$76.6(0.8)$

75.5(0.7)

$66.8(0.9)$

57.4(2.3)
Net $\mathrm{H}(\mathrm{g})$

Released

0.01824

0.01294

0.00265

0.01138

0.00462

Coal Study II: Kentucky No. 9, $30 \mathrm{~min}, 410^{\circ} \mathrm{C}, \mathrm{N}_{2}$

\begin{tabular}{lll}
$\begin{array}{l}\text { Donor } \\
\text { Added }\end{array}$ & $\begin{array}{l}\text { Coal } \\
\text { Conversion }\end{array}$ & $\begin{array}{l}\text { Net H(g) } \\
\text { Released }\end{array}$ \\
\cline { 2 - 2 } & $84.3(1.1)$ & 0.02203 \\
DHA & $80.9(0.5)$ & 0.00879 \\
OHA & $80.8(0.6)$ & 0.02076 \\
ISO & $78.1(0.6)$ & 0.01439 \\
TEr & $70.1(0.2)$ & 0.00958 \\
\hline
\end{tabular}

Coal Study III: Kentucky No. 9, $30 \mathrm{~min}, 380^{\circ} \mathrm{C}, \mathrm{H}_{2}$

$\begin{array}{lll}\begin{array}{l}\text { Donor } \\ \text { Added }\end{array} & \begin{array}{l}\text { Coal } \\ \text { Conversion }\end{array} & \begin{array}{l}\text { Net H(g) } \\ \text { Released }\end{array} \\ \text { TET } & 79.0(0.3) & 0.01200 \\ \text { None } & 78.8(3.4) & \text { NM }^{*} \\ \text { OHA } & 77.6(0.8) & 0.00246 \\ \text { HHA } & 76.7(0.1) & 0.00921 \\ \text { DHA } & 76.6(1.0) & -0.05314 \\ \text { ISO } & 76.3(0.8) & 0.00060\end{array}$

*NM = none measured 
Table V. Reaction Products from Model Donors from Thermal and Catalytic Coal Reactions Using Hexadecane as the Solvent

Coal Study IV: Thermal, Kentucky No. $9 \mathrm{H}_{2}, 380^{\circ} \mathrm{C}$, Hexadecane as Solvent

\begin{tabular}{|c|c|c|c|c|c|}
\hline $\begin{array}{l}\text { Donor } \\
\text { Added }\end{array}$ & $\begin{array}{l}\text { Coal } \\
\text { Conversion }\end{array}$ & $\begin{array}{l}\text { Net H(g) } \\
\text { Released } \\
\end{array}$ & \multicolumn{3}{|c|}{ Reaction Products, wt\% } \\
\hline $\begin{array}{l}\text { OHA } \\
\text { HHA } \\
\text { DHA }\end{array}$ & $\begin{array}{l}37.2(0.4) \\
65.0(3.3) \\
68.3(1.0)\end{array}$ & $\begin{array}{c}0.00324 \\
0.00659 \\
-0.04970\end{array}$ & $\begin{array}{l}85.4(0.1) \\
47.5(0.3) \\
61.0(2.3)\end{array}$ & $\begin{array}{l}14.6(0.1) \\
45.7(0.1) \\
24.6(2.4)\end{array}$ & $\begin{array}{l}\text { none } \\
6.8(0.3) \\
14.5(1.5)\end{array}$ \\
\hline & & & TET & NAP & \\
\hline $\begin{array}{l}\text { ISO } \\
\text { TET } \\
\text { None }\end{array}$ & $\begin{array}{l}49.7(1.0) \\
29.8(0.2) \\
25.6(1.8)\end{array}$ & $\begin{array}{l}0.01248 \\
0.00298 \\
0\end{array}$ & $\begin{array}{l}17.8(1.3) \\
79.8(0.7) \\
0\end{array}$ & $\begin{array}{l}82.2(1.3) \\
20.2(0.7) \\
0\end{array}$ & \\
\hline
\end{tabular}

Coal Study V: $\mathrm{NiMo} / \mathrm{Al}_{2} \mathrm{O}_{3}$, Kentuclay No. $9, \mathrm{H}_{2}, 380^{\circ} \mathrm{C}$, Hexadecane as Solvent

\begin{tabular}{|c|c|c|c|c|c|}
\hline $\begin{array}{l}\text { Douror } \\
\text { Added }\end{array}$ & $\begin{array}{l}\text { Coal } \\
\text { Conversion } \\
\end{array}$ & $\begin{array}{l}\text { Net H(g) } \\
\text { Released } \\
\end{array}$ & \multicolumn{3}{|c|}{ Reaction Products, wt\% } \\
\hline $\begin{array}{l}\text { OHA } \\
\text { HHA } \\
\text { DHA }\end{array}$ & $\begin{array}{l}39.8(2.4) \\
58.9(2.9) \\
71.6(2.1)\end{array}$ & $\begin{array}{c}0.00237 \\
0.00392 \\
-0.06097\end{array}$ & $\begin{array}{l}89.3(0.2) \\
56.3(0.8) \\
71.0(0.7) \\
\text { TET }\end{array}$ & $\begin{array}{l}10.7(0.2) \\
43.7(0.8) \\
22.4(0.5) \\
\text { NAP }\end{array}$ & $\begin{array}{l}\text { none } \\
\text { none } \\
6.6(0.2)\end{array}$ \\
\hline $\begin{array}{l}\text { ISO } \\
\text { TET } \\
\text { None }\end{array}$ & $\begin{array}{l}28.6(0.3) \\
20.8(0.2) \\
25.6(2.0)\end{array}$ & $\begin{array}{l}0.00230 \\
0.0 \\
0\end{array}$ & $\begin{array}{l}100.0 \\
0\end{array}$ & $\begin{array}{l}84.8(0.4) \\
\text { trace } \\
0\end{array}$ & $15.2(0.4)$ \\
\hline
\end{tabular}

Table VI. Boduszynski Analysis Summary for Coal Study I*

\begin{tabular}{|c|c|c|c|c|c|}
\hline \multirow[b]{2}{*}{$\begin{array}{l}\text { Donor } \\
\text { Added }\end{array}$} & \multirow[b]{2}{*}{$\begin{array}{l}\text { Coal } \\
\text { Conversion }\end{array}$} & \multicolumn{4}{|c|}{ Compound Classes of Reaction Products (wt\%) } \\
\hline & & $\mathrm{HC}^{1}$ & $\mathrm{NHC}^{2}$ & $\mathrm{HA}^{3}$ & $\mathrm{PFC}^{4}$ \\
\hline HHA & $88.6(0.04)$ & $73.4(3.0)$ & $1.8(1.6)$ & $9.2(1.8)$ & $15.6(2.4)$ \\
\hline ISO & $81.0(0.9)$ & $84.8(1.7)$ & $0.7(0.8)$ & $9.4(0.8)$ & $5.2(1.3)$ \\
\hline OHA & $75.5(0.7)$ & $80.1(2.4)$ & trace & $4.8(1.5)$ & $15.2(0.9)$ \\
\hline DHA & $76.6(0.8)$ & $91.3(0.6)$ & $0.5(0.7)$ & $4.5(0.2)$ & $3.7(0.1)$ \\
\hline TET & $66.8(0.9)$ & $93.2(1.2)$ & trace & $2.8(1.7)$ & $4.0(2.3)$ \\
\hline
\end{tabular}

* Conditions: Western Kentucky No. 9 coal, $\mathrm{N}_{2}, 380^{\circ} \mathrm{C}, 30 \mathrm{~min}, 0.5$ wt\% donable hydrogen

1 hydrocarbons

2 nitrogen heterocycles

${ }^{3}$ hydroxyl aromatics

4 polyfunctional compounds 
Table VII. Boduszynski Analysis Summary for DHA*

Level of

Donable Coal

Hydrogen Conversion $\mathrm{HC}$

Compound Classes of Reaction Products (wt\%)

$\begin{array}{llllll}0.5 & 76.6(0.8) & 91.3(0.6) & 0.5(0.7) & 4.5(0.2) & 3.7(0.1) \\ 0.2 & 73.8(1.0) & 80.0(4.1) & 1.6(1.4) & 9.9(3.0) & 8.6(4.4) \\ 0.1 & 62.5(1.2) & 82.3(4.0) & 2.3(2.0) & 9.9(4.1) & 5.5(0.9)\end{array}$

* Conditions: Western Kentucky No. 9 coal, $\mathrm{N}_{2}, 380^{\circ} \mathrm{C}, 30 \mathrm{~min}$

Table VIII: Heats of Formation and Ionization Potentials Obtained for the Dehydrogenation of TET and ISO

STATE
radical (doublet)
bi-radical (singlet)
bi-radical (triplet)
charge $=-1$
charge $=-2$
charge $=+1$
charge $=+2$
ground state

\begin{tabular}{|c|c|}
\hline \multicolumn{2}{|c|}{ ISO } \\
\hline $\mathrm{H}^{*}$ & $\underline{I p^{*}}$ \\
\hline 40.14 & 7.75 \\
\hline 25.40 & 9.18 \\
\hline 73.85 & 2.89 \\
\hline 1.71 & -3.39 \\
\hline 140.40 & -4.57 \\
\hline 209.69 & 13.42 \\
\hline 545.48 & 18.06 \\
\hline 19.45 & 9.32 \\
\hline
\end{tabular}

\begin{tabular}{|c|c|}
\hline \multicolumn{2}{|c|}{ TET } \\
\hline 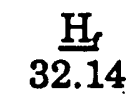 & $\frac{\text { Ip }}{7.62}$ \\
\hline 104.27 & 7.34 \\
\hline $\begin{array}{r}59.88 \\
1.95\end{array}$ & 3.68 \\
\hline $\begin{array}{r}99.17 \\
\end{array}$ & -3.17 \\
\hline 499.18 & 19.15 \\
\hline 2.59 & 9.23 \\
\hline
\end{tabular}

*Units are kcal/mole.

Table IX: Heats of Formation and Ionization Potentials Obtained for Ground State TET and ISO

\begin{tabular}{|c|c|c|c|c|}
\hline \multirow[b]{2}{*}{ STATE } & \multicolumn{2}{|c|}{ ISO } & \multicolumn{2}{|c|}{ TET } \\
\hline & $\underline{\mathrm{H}}_{r}^{*}$ & $\underline{I p}^{*}$ & $\underline{\mathrm{H}}$ & Ip \\
\hline AMPAC Only & $19 . \overline{45}$ & $9 . \overline{32}$ & 2.59 & 9.2 \\
\hline SYBYL, Optimization & 19.44 & 9.3 & 1.5 & 9.22 \\
\hline SYBYL, NO Optimizatio & n19.55 & 9.32 & 1.64 & 9.2 \\
\hline
\end{tabular}

*Units are $\mathrm{kcal} / \mathrm{mole}$.

Table X: Summary of Heat of Formation and Ionization Potentials Obtained for the Excited Transition State Species for the Transition of 1,4-DHN to NAP

\section{STATE}

radical (doublet)

bi-radical (singlet)

bi-radical (triplet)

charge $=-1$

charge $=-2$

charge $=+1$

charge $=+2$
ISO

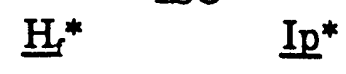

$\begin{array}{rr}49.48 & 7.51 \\ 38.85 & 8.57 \\ 81.65 & 3.26 \\ 11.72 & 2.03 \\ 117.92 & -3.82 \\ 212.54 & 13.90 \\ 524.49 & 18.98\end{array}$

*Units are kcal/mole. 


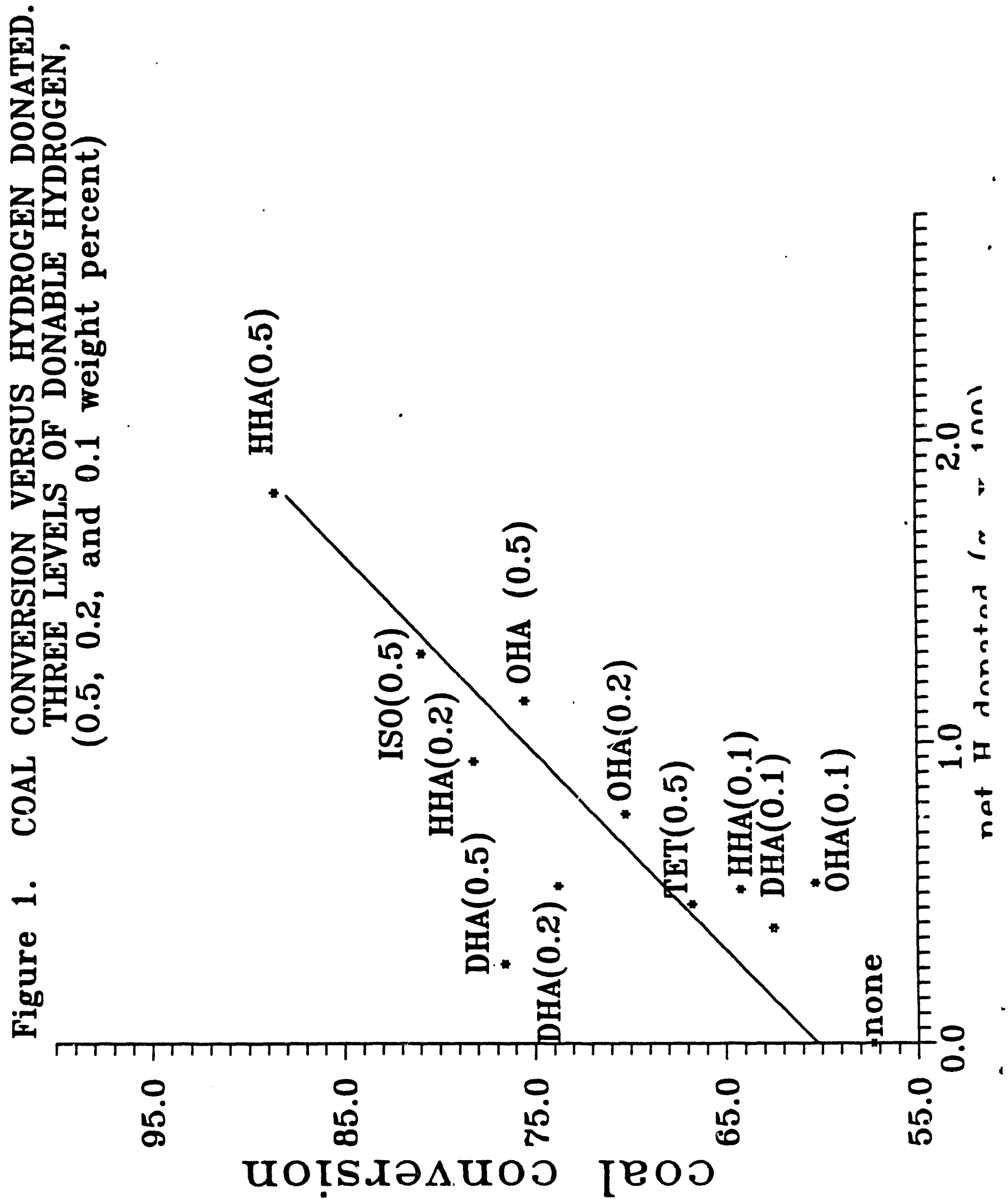




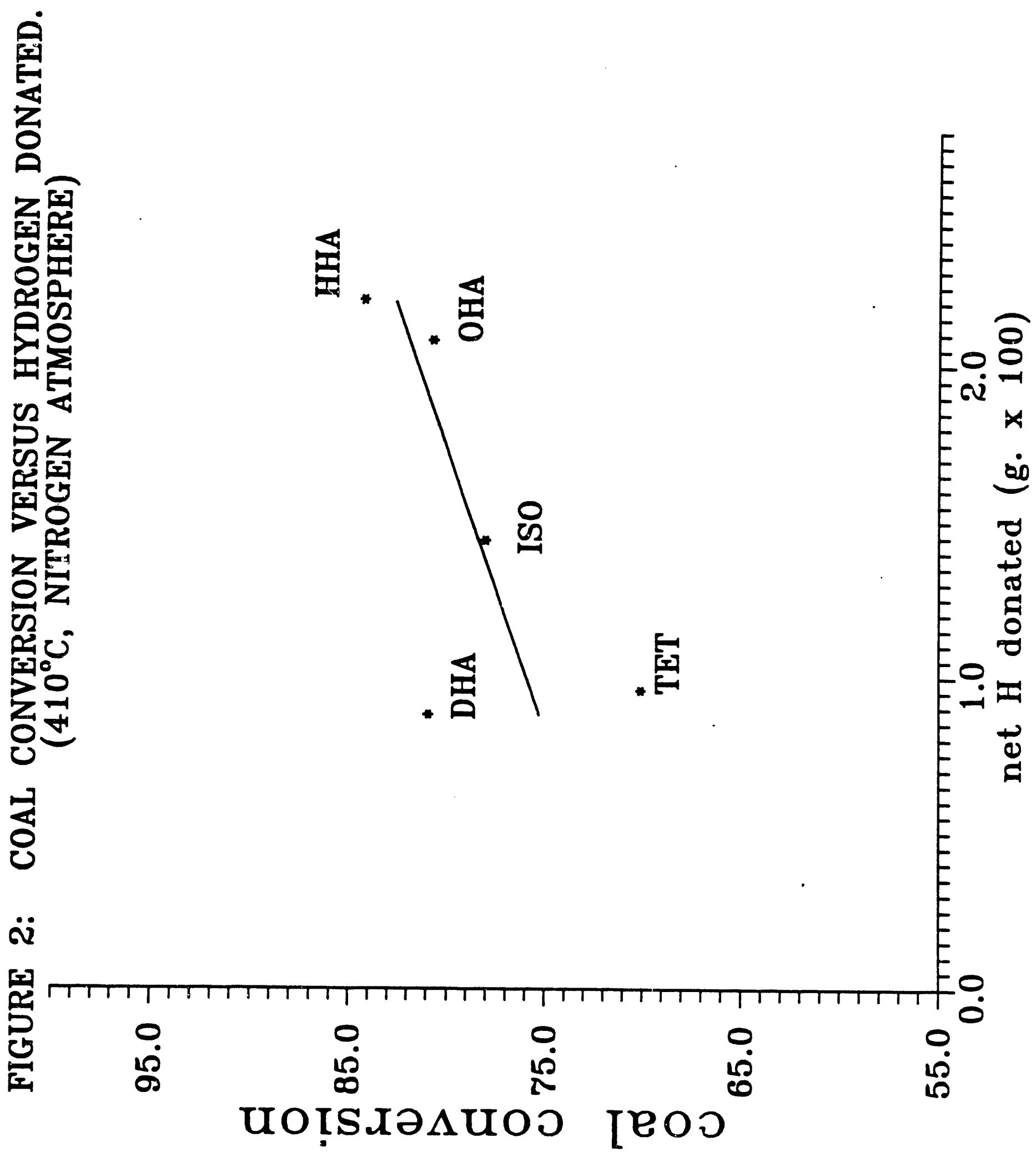




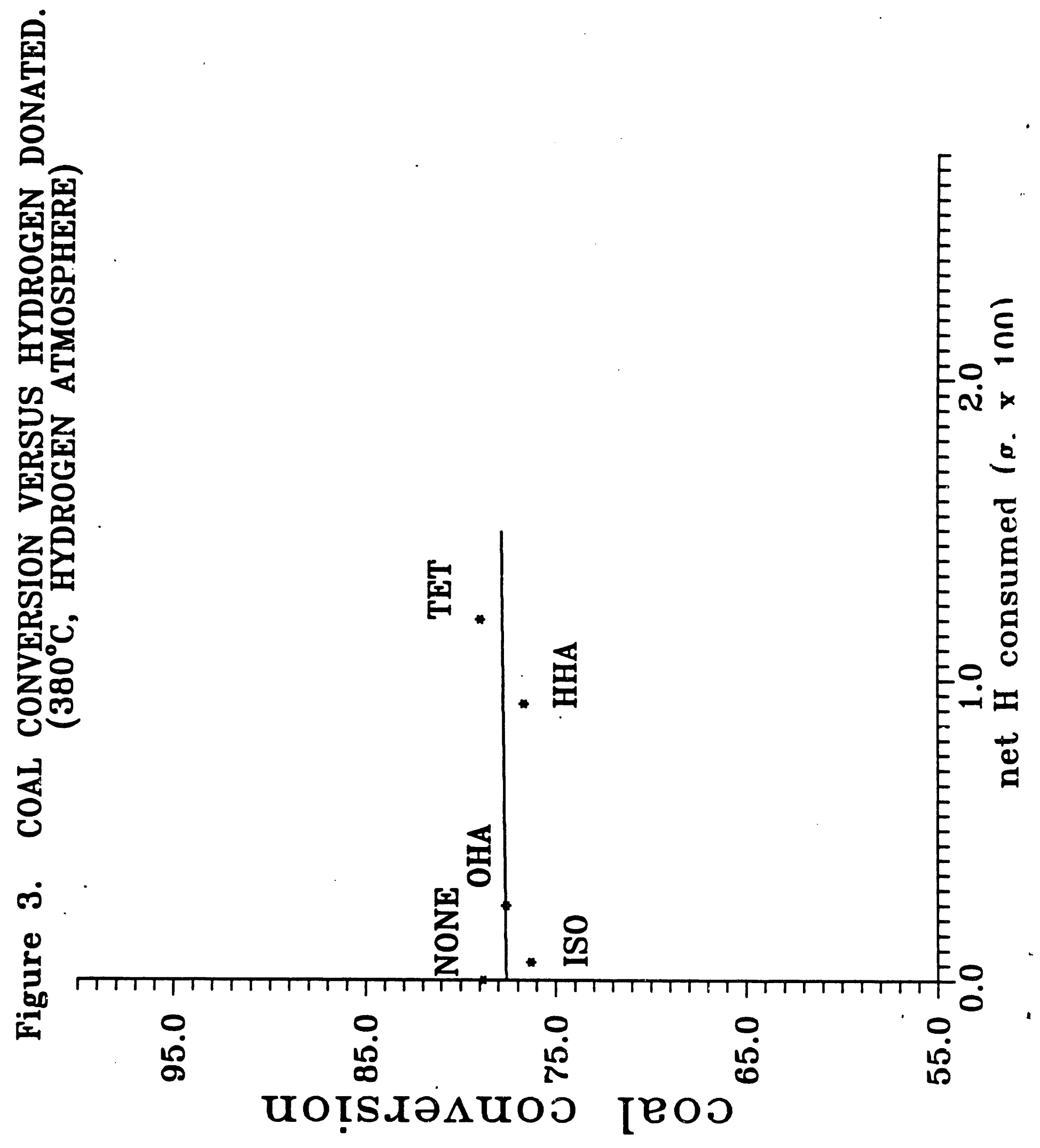



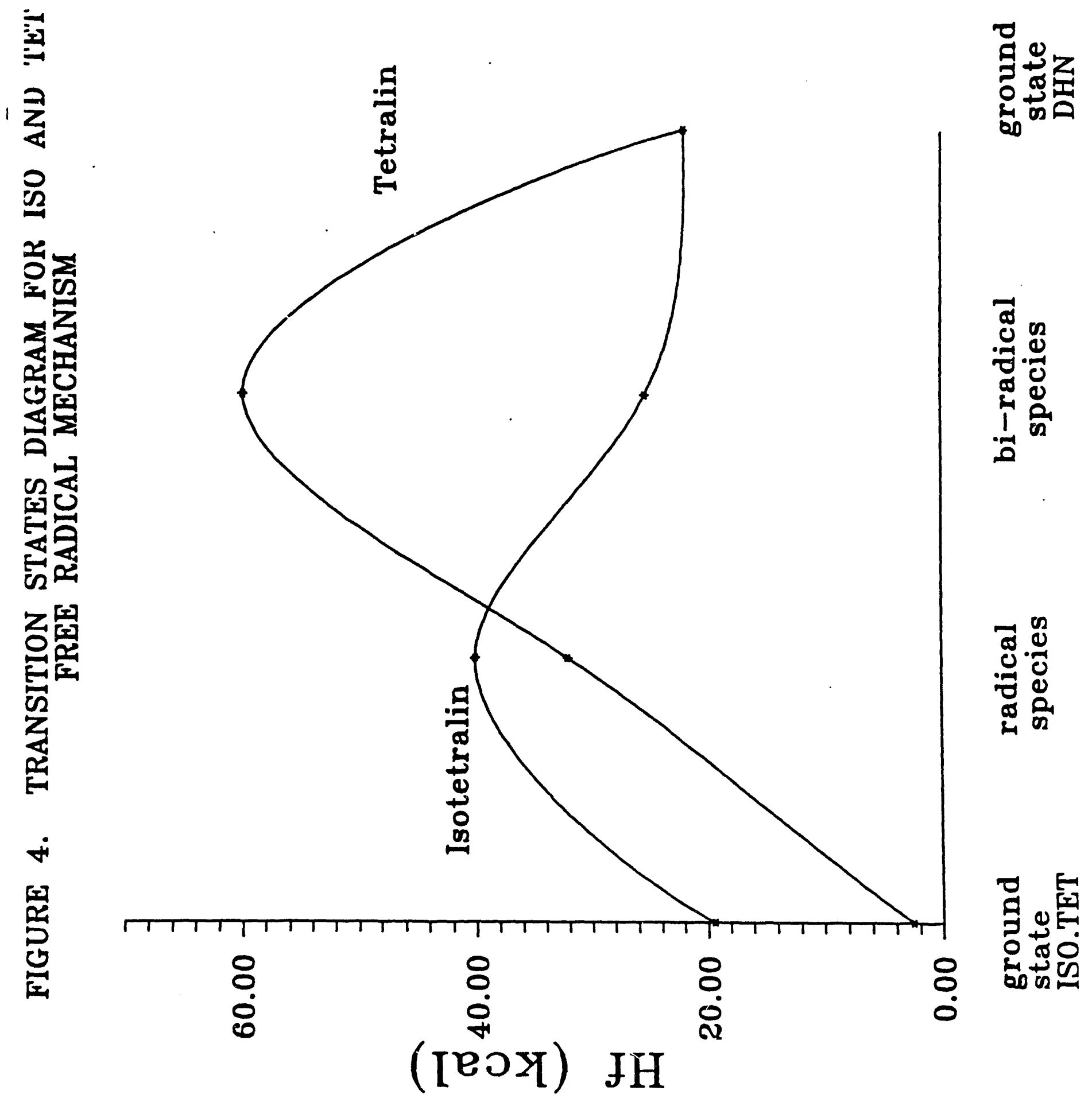
눈

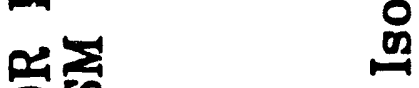

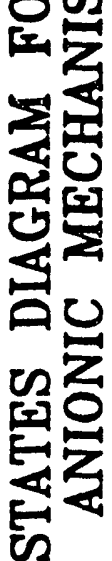

z

م่

$\sum_{0}^{5}$
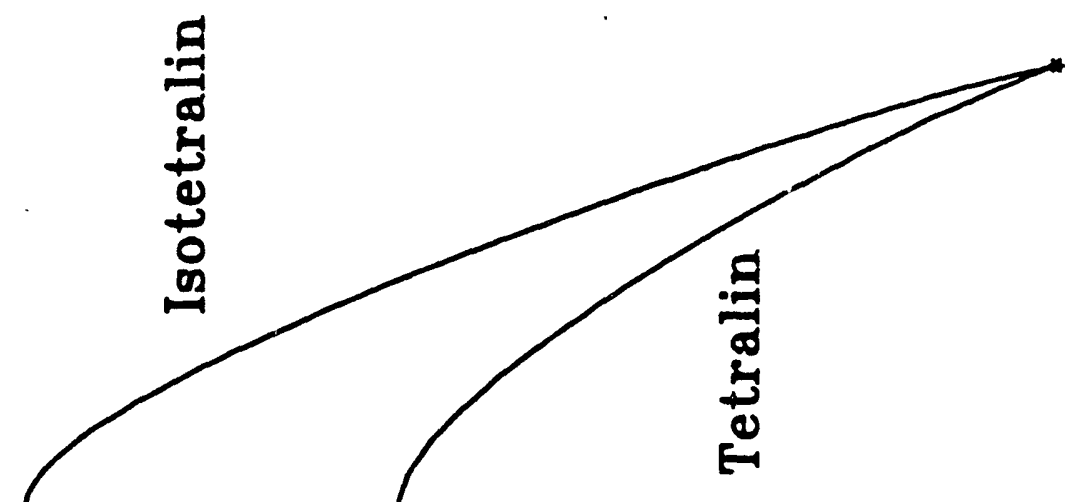

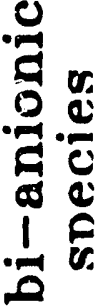

量

2

里

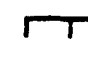

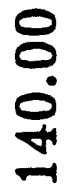

:

$\stackrel{8}{\dot{9}}$

([воY) JH

هُ

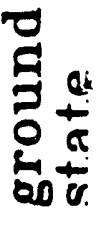




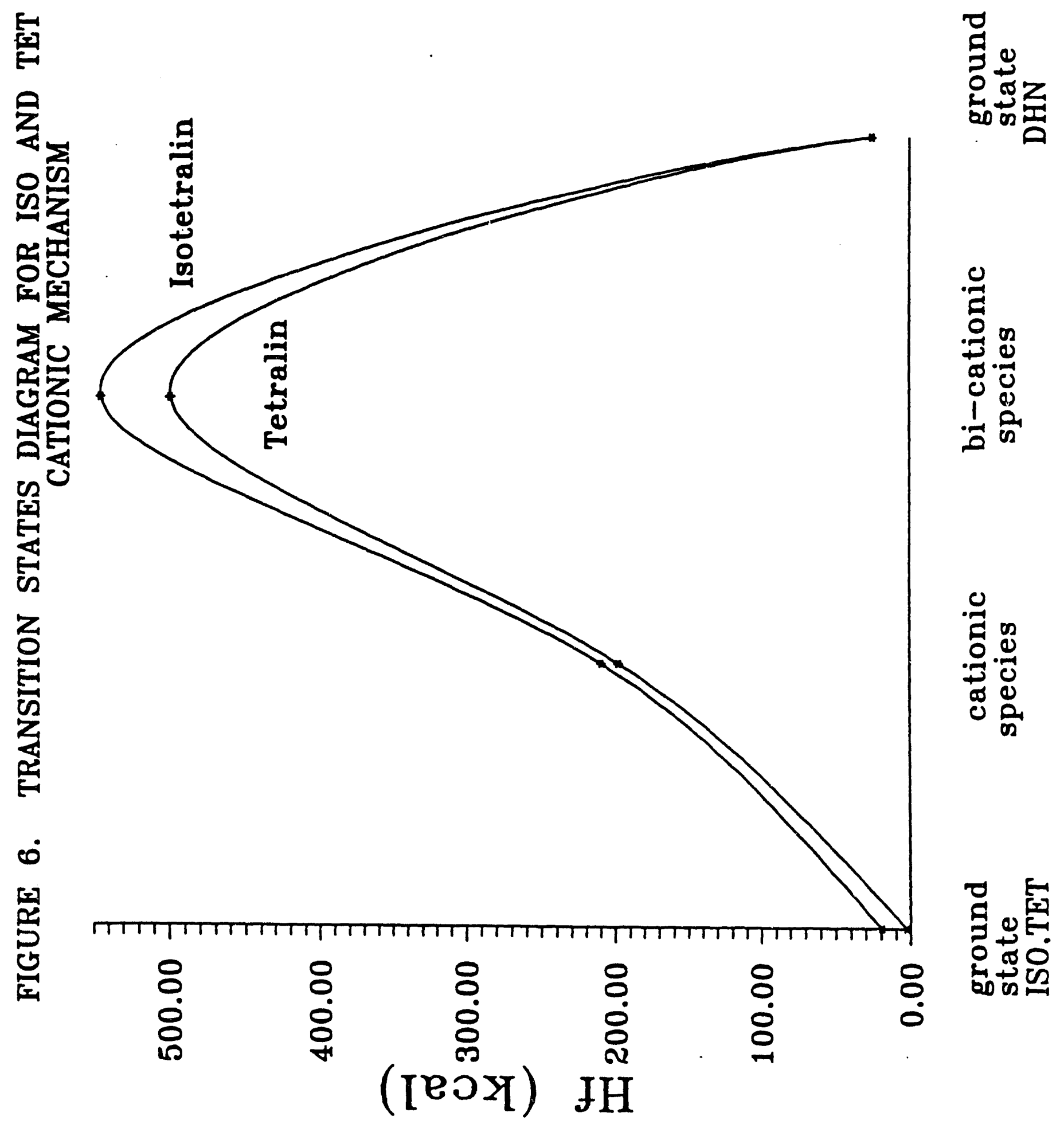




\section{TASK IV.2. CATALYTIC COPROCESSING STUDY USING PROBE REACTANTS}

\section{Introduction}

Four model probe compounds were introduced to investigate the complex chemistry of coal-oil coprocessing reactions in this study. These four probe reactants served as monitors for evaluating the catalytic inhibitory effects of different hydrocarbonaceous substances. The four probe systems used were dibenzofuran for hydrodeoxygenation, dibenzothiophene for hydrodesulfurization, quinoline for hydrodenitrogenation, and anthracene for hydrogenation. The four model compounds were reacted individually with resid, coal, two different resid fractions and two prehydrogenated residuum, respectively, in order to discover how different compositional factors affected the reactivity of the individual probe reactions.

\section{Experimental}

The reactions were conducted in $20 \mathrm{~cm}^{3}$ stainless steel tubing bomb microreactors (TBMR). The reaction conditions were set at $380^{\circ} \mathrm{C}, 1250$ psi hydrogen pressure at ambient temperature, $550 \mathrm{rpm}$ for horizontal agitation, and $860 \mathrm{rpm}$ for vertical agitation. The catalyst used for each reaction was presulfided $\mathrm{NiMo} / \mathrm{Al}_{2} \mathrm{O}_{3}$, Shell 324, which was ground into $+250-325$ mesh.

The chemicals, dibenzofuran, dibenzothiophene, quinoline, anthracene, and hexadecane, used as reactants and solvent, respectively, in all probe reactions, were obtained from Aldrich Chemical Company and rated at 99 percent purity. Western Kentucky No. 9 coal was ground to -100 mesh, stored under an inert atmosphere, prior to being used in the reactions. The resid was produced from a blend of different crudes and was obtained from Amoco.

The products from the probe reactant reactions were analyzed by gas chromatography using a Varian Model 3700 gas chromatograph equipped with a $30 \mathrm{~m}, 0.32$ mm ID DB-5 fused silica column and FID detection. Quantitation was performed using the internal standard method with p-xylene as the internal standard.

The resid was pretreated in several ways by obtaining the hexane soluble fraction, the hydrocarbon fraction, and prehydrogenation of the resid. The hexane soluble fraction

- was obtained by extracting resid with hexane and collecting that fraction. the hydrocarbon fraction was obtained by using a chromatographic separation based on the methods of Boduszynski. Prehydrogenation of the resid at 30 minutes and 3 hours at $380^{\circ} \mathrm{C}$ with 1250 psi at ambient temperature followed by extraction with tetrahydrofuran was performed. 


\section{Results and Discussion}

The reactions were performed using probe reactants in the presence of coal and resid individually and in combination. The products from the probe reactants were analyzed by gas chromatography. To summarize and compare the data, the terminology presented in the following was used: percent hydrodeoxgenation (HDO), percent hydrodesulfurization (HDS), percent hydrodenitrogenation (HDN), percent hydrogenation (HYD) and percent recovery. Percent hydrodeoxygenation (HDO), percent hydrodesulfurization (HDS), and percent hydrodenitrogenation (HDN) are the summations of the mole percents of the liquid reaction products that do not contain oxygen, sulfur, or nitrogen, respectively. Percent hydrogenation (HYD) is the number of moles required to produce the observed liquid product distribution from a given reactant as a percentage of the moles of hydrogen required to obtain the final most hydrogenated liquid product.

\section{The Four Model Reactions.}

Four probe reactions, HDO, HDS, HDN, and HYD, with dibenzofuran, dibenzothiophene, quinoline, and anthracene serving as the four model probes, were performed under catalytic and thermal liquefaction conditions. The thermal reactions served as a base line for the catalytic reactions so that the effect of the catalyst at liquefaction conditions could be discerned. Without a catalyst at $380^{\circ} \mathrm{C}$, no reaction of dibenzofuran, dibenzothiophene, or anthracene was observed. Only quinoline reacted with about 30 mole percent 1,2,3,4-tetrahydroquinoline being produced.

The four model compounds were then reacted with a presulfided $\mathrm{NiMo} / \mathrm{Al}_{2} \mathrm{O}_{3}$ catalyst at the same conditions as thermal reactions. $\mathrm{NiMo} / \mathrm{Al}_{2} \mathrm{O}_{3}$ proved highly effective for hydrogenating and removing heteroatoms from the probe reactants when neither coal nor resid was added.

Dibenzofuran and dibenzothiophene have similar chemical structures and, although they had different ratios of the products present in the product distribution after catalytic reactions, these compounds after heteroatom removal had the same types of products. In the presence of $\mathrm{NiMo} / \mathrm{Al}_{2} \mathrm{O}_{3}$, both species underwent complete heteroatom removal, leaving only hydrocarbons as products. The products of the dibenzofuran HDO catalytic reaction were bicyclohexyl, methylcyclopentylcyclohexane, methylcyclopentane and cyclohexane which was the major product. The products for dibenzothiophene reaction were bicyclohexyl, cyclohexylbenzene, methylcyclopentane, and cyclohexane. In these two probe reactions the most hydrogenated product was cyclohexane.

To establish the reaction pathways occurring for both the $\mathrm{HDO}$ and HDS reaction 
pathways, the observed reaction products as well as the postulated intermediates were reacted under the same conditions. The compounds, biphenyl, cyclohexylbenzene and bicyclohexyl, showed similar product distributions as the dibenzofuran and dibenzothiophene reactions. The exceptions were that bicylohexyl did not produce cyclohexane as a product and none of the compounds produced methylcyclopentane.

Quinoline at these reaction conditions and in the presence of $\mathrm{NiMo} / \mathrm{Al}_{2} \mathrm{O}_{3}$ underwent total denitrogenation. The products of the quinoline HDN catalytic reaction were methylpropylpentane, propylbenzene and the primary product, propylcyclohexane with the most hydrogenated product being propylcylclohexane. Reactions were performed with decahydroquinoline (DHQ), 1,2,3,4-tetrahydroquinoline (THQ), and 5,6,7,8tetrahydroquinoline (THQ) to establish if these compounds were feasible intermediates for quinoline HDN. Under these conditions, DHQ and 5,6,7,8-THQ produced propylbenzene (PB), methylpropylcyclopentane (MPCP), propylcyclohexane (PCH) and trace amounts of other hydrocarbons (HC) while 1,2,3,4-THQ did not produce PB and produced more HC. Further reactions with $\mathrm{NiMo} / \mathrm{Al}_{2} \mathrm{O}_{3}$ showed that $\mathrm{PB}$ and propylaniline (PA) were readily converted to $\mathrm{PCH}$ and small amounts of $\mathrm{MPCH}$ while $\mathrm{PCH}$ remained largely unconverted but produced measurable quantities of MPCP and PB.

Anthracene readily hydrogenated under these reaction conditions. The products of the anthracene HYD catalytic reaction were six isomers of perhydroanthracene. All aromatic rings were saturated but no ring was broken.

The reaction pathways for these reactions show that the oxygen and sulfur heteroatoms were removed from dibenzofuran and dibenzothiophene in HDO and HDS reactions prior to hydrogenating the aromatic rings. The evidence for this is the production of bicyclohexyl and cyclohexylbenzene in the reaction products of the HDO and HDS reactions, respectively. By contrast, in quinoline $\mathrm{HDN}$ reactions nitrogen removal occurred only from quinoline after saturation of the aromatic rings. No nitrogen containing species were present after 30 minutes of reaction. All of the hydrocarbon species present were composed of one ring structures, indicating that the nitrogen containing aromatic rings were saturated and hydrogenolyzed prior to nitrogen removal.

\section{The Model Reactions with Coal, Resid and Coal-Resid Mixture.}

Coal and resid were added both individually and in combination to the four probe reactions. The presence of these species lowered the amount of hydrogenation and heteroatom removal observed with each of the probe reactants. All four probe reactions showed that the coal and resid mixture lowered the amount of hydrogenation or heteroatom 
removal reactions occurring more than did either coal or resid alone. Coal alone was more inhibitory than resid alone. Figure 1 presents a comparison of these added species on the HYD, HDO, HDS, and HDN reactions.

When coal or a coal-resid mixture was added to the catalytic reactions containing dibenzofuran, the HDO reaction was completely inhibited. When untreated resid was added to dibenzofuran the HYD and HDO reactions decreased from 100 mole\% to 3.8 and $4.8 \%$, respectively. However, when these substances were added to quinoline, quinoline still underwent a substantial amount of HYD and HDN and totally converted to other products. The inhibitory effect was greatest with coal plus resid but even then the HYD and HDN were only lowered by -30 to $40 \%$ to 68 and $58 \%$, respectively.

Dibenzothiophene also showed a high level of HDS; untreated resid lowered the HDS from $100 \%$ to $80 \%$ while the coal reaction lowered it $66 \%$ and the coal/resid combination to 53\%. The HYD reaction experienced by dibenzothiophene was lowered from $\sim 76 \%$ for no addition to $\sim 25 \%$ for resid, $\sim 17 \%$ for coal and $\sim 15 \%$ for the coal-resid mixture. When the HYD of anthracene at $100 \%$ from the catalytic reaction without any additives was compared to that with additives the HYD of anthracene became lower: $\sim 53 \%$ for added resid, $\sim 45 \%$ for coal and $43.5 \%$ for the coal-resid mixture.

\section{The Model Reactions with Hexane Soluble Resid Fraction.}

In fulfillment of the purpose of this work, hydrocarbonaceous substances with different character were prepared from resid and then tested for their catalytic inhibition on the four probe reactants as presented in Table 1. These procedures were performed: resid was fractionated into hexane soluble and hydrocarbon fractions and was also hydrogenated at $380^{\circ} \mathrm{C}$ and $1250 \mathrm{psi}$ in a tubing bomb microreactor.

The hexane soluble resid, when introduced to each of the four model compounds, showed less catalytic inhibition of all probe reactions than the untreated resid, coal or the combination of coal and resid. The hexane soluble residuum was composed of $87.7 \mathrm{wt} \%$ of original residuum and had no asphaltenes present. This material had a detrimental effect on dibenzofuran HYD and HDO reactions where only $9 \% \mathrm{HYD}$ and $12 \% \mathrm{HDO}$ occurred compared to $67 \%$ HYD and $100 \%$ HDO without resid, but showed slightly greater dibenzofuran reactivity than the untreated resid. Quinoline reactions maintained a high degree of HYD (93.7\%) and HDN (92.6\%) in the presence of the hexane soluble resid while dibenzothiophene desulfurized readily yielding $90.5 \%$ HDS but did not undergo substantial of HYD reaction (30\%). This amount of HYD was only slightly higher than the untreated resid. Added hexane soluble fraction of resid had a moderate effect on the anthracene HYD 
reaction (58\%) compared to the $52 \%$ of the untreated resid.

\section{The Model Reactions with the Resid Hydrocarbon Fraction.}

A hydrocarbon fraction from the hexane soluble resid was collected by the modified Boduszynski method. Since only a small amount could be collected within a reasonable period of time, the hydrocarbon fraction was only used with HDS and HDO reactions. These reactions were chosen because the presence of residuum showed the most effect on their reactivity while little effect on the quinoline $\mathrm{HDN}$ reaction and only moderate effects on the anthracene reaction were observed. In comparison with the other resid reactions, the probe reactions with added hydrocarbon fraction resid were less inhibited than those with added hexane soluble fraction resid or added untreated resid. Even with this, a substantial influence of the resid hydrocarbon fraction was observed on the HDO and HYD reactions of dibenzofuran. Only $25 \%$ of the dibenzofuran reacted. Dibenzofuran reactivity with the hydrocarbon fraction showed only slightly greater reactivity than with the untreated or hexane soluble fraction. By contrast, dibenzothiophene was able to undergo substantial sulfur removal yielding $93 \%$ HDS; this compared to $79 \%$ with untreated resid and $91 \%$ with hexane soluble resid. The HYD reaction of dibenzothiophene was still affected only $32 \%$ HYD occurred compared to $\sim 76 \%$ without any added resid or resid fraction.

\section{The Model Reactions with Prehydrogenated Residuum.}

In order to test whether the catalytic inhibitory nature of the resid, as measured by the reactivity of probes, could be altered by chemical changes occurring during hydrogenation, the resid was hydrogenated in thirty minute and three hour reactions. Eighteen grams of resid were prehydrogenated in two large-sized tubing bomb microreactors at $380^{\circ} \mathrm{C}$ at each reaction time. The effect of the prehydrogenated resids on the different probe reactants varied according to the length of reaction time and the probe used. The 3 hour prehydrogenated resid inhibited the reactivity of the probe reactants less than the 30

- minute reaction.

The 30 minute prehydrogenated resid had little effect on quinoline $\mathrm{HDN}$ reaction and

- HYD reaction. Neither the 30 minute nor 3 hour prehydrogenated resid substantially affected quinoline HDN or HYD in comparison with the catalytic probe reactions without any resid. In contrast to quinoline high reactivity, dibenzofuran scarcely reacted, with $91 \%$ dibenzofuran remaining unreacted. The level of dibenzofuran reactivity was less than the reaction with the hydrocarbon fraction of the resid and slightly greater than the hexane soluble or untreated resid. The dibenzofuran HDO reaction increased from $6.1 \%$ with 30 - 
minute prehydrogenated resid to $8.5 \%$ with 3 -hour prehydrogenated resid and the HYD reaction improved from 4.6 to $6.4 \%$.

Dibenzothiophene showed substantial HDS reaction but a low HYD reaction in comparison to reactions without resid for both prehydrogenated resids. The anthracene HYD reaction showed moderate inhibition by the prehydrogenated residua. The amount of anthracene HYD observed was 54\% with the 30 minute treated resid, $56 \%$ with the 3 hour pretreatment $\sim 58 \%$ with the hexane soluble fraction, and $53 \%$ with the untreated resid. These HYD were reduced from $100 \%$ without any resid. Hence, the presence of any resid reduced the reactivity of the probe anthracene by nearly one-half.

\section{Summary}

The results of these probe reactions indicate that when resid coal or a combination of coal plus resid was added to all probe reactions the HYD reaction as well as HDO, HDS and HDN were decreased. The resid participated in competitive catalytic reactions with the model reactions where the resid molecules compete for the active catalytic sites, perhaps even poiscning the active site thereby inhibiting the catalytic activity of the sites. A comparison of the heteroatom removal reactions of the probe reactions given in Figure 1 showed that adiled untreated resid, coal, and coal plus resid had little effect on sulfur and nitrogen removal from dibenzothiophene and quinoline, but inhibited oxygen removal from dibenzofuran. All HYD reactions were affected; the most affected was the HYD of dibenzofuran, followed by benzothiophene, anthracene and quinoline in that order.

In the detailed reactions using hydrocarbon fractions, hexane soluble and prehydrogenated resid, prehydrogenated resid inhibited reactions more than did hexane soluble resid which in turn was more inhibitory than hydrocarbon fraction residua as shown in Figures 2 and 3. The addition of untreated resid showed the greatest inhibitory effects on model compound reactions in comparison with the addition of different specified resid. The four model compounds had the same trend of reaction with the different added resids. The added resids and coals, in order of their inhibitory effect on the probe reactions, are: coalresid mixture > coal > untreated resid > thirty-minute prehydrogenated resid $>$ three-hour prehydrogenated resid $>$ hexane soluble resid $>$ hydrocarbon fraction resid.

Compared with reactions with untreated resid, any additional pretreatment of resid to remove heavy materials or prehydrogenation of the resid contributed to some improvement in the reactivity of the four probe reactions. In a comparison of the four model compound reactions, quinoline consistently showed more HYD reaction than other model compounds and a high mole percent of HDN reaction while anthracene did not show much 
variation in HYD reactions with addition of the different resids.

\section{References}

1) Boduszynski, M.M.; Hurtubise, R.J.; Silver, H.F.; "Separation of Sovlent-Refined Coal into Solvent-Derived Fractions"; Anal. Chem.; 1982. 54. 372-375.

2) Boduszynski, M.M.; Hurtubise, R.J.; Silver, H.F.; "Separation of Solvent-Refilled Coal into Compound-Class Fraction"; Anal. Chem.; 1982, 54, 375-381. 
If

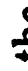

$\$$

.$\Xi$

疍

过

ర్๊

㺃

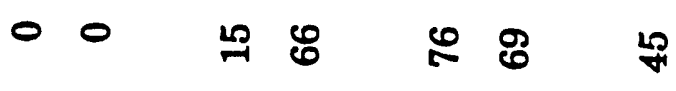

ஜำ ニ

แั่

을

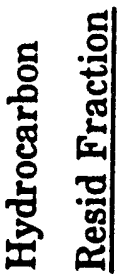

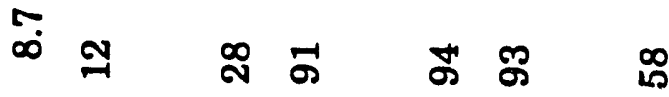

‡

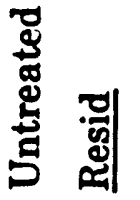

$\stackrel{\infty}{\infty} \stackrel{\infty}{\sim}$

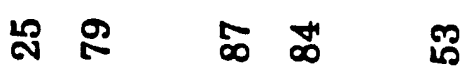

总

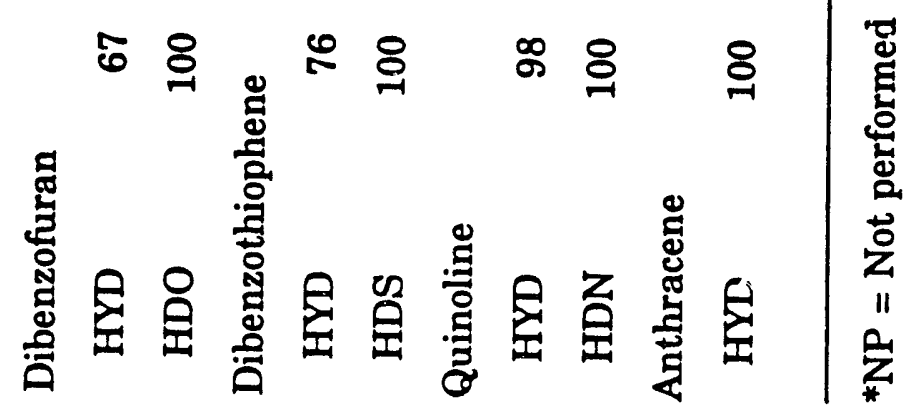




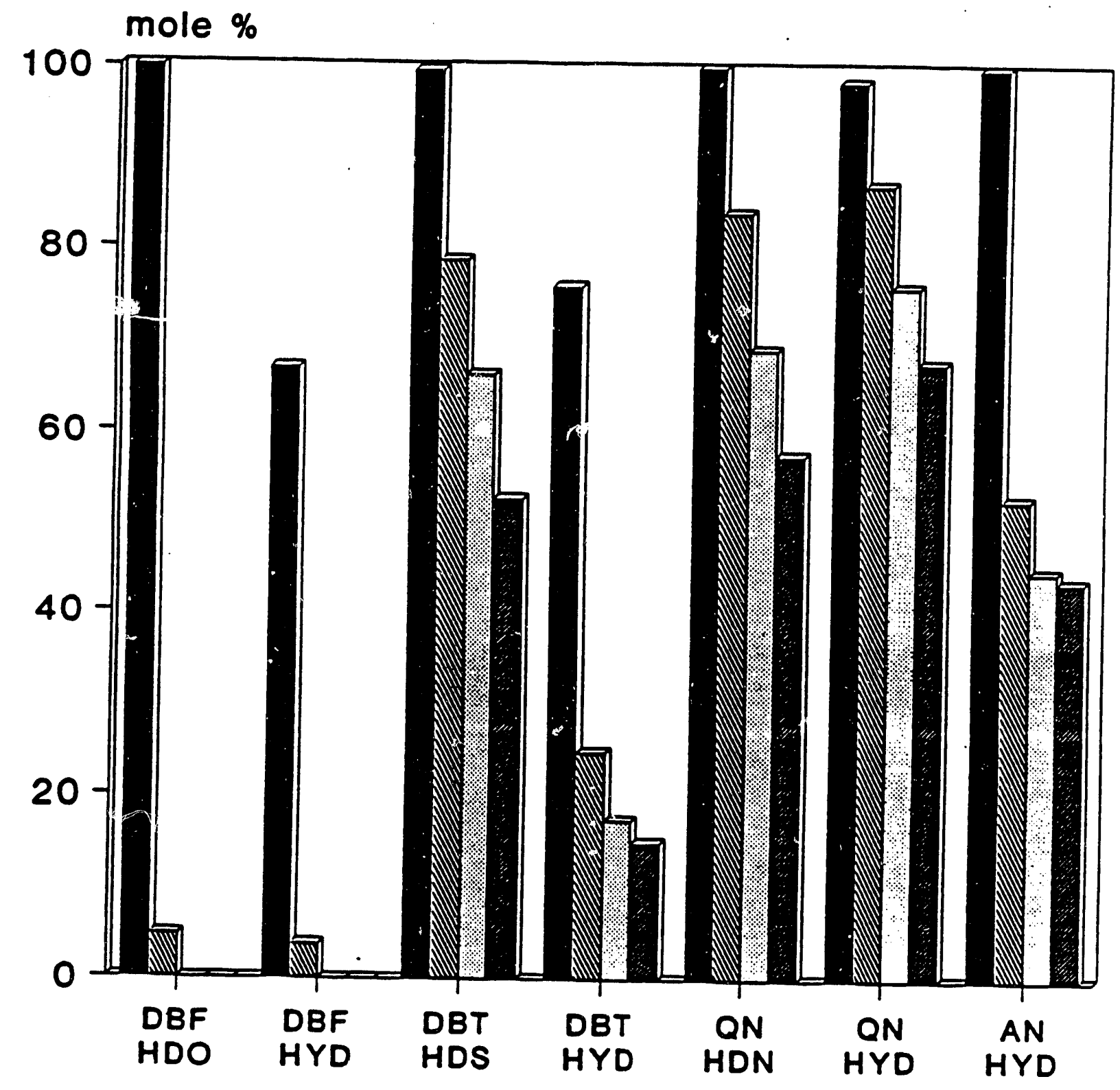

CAT. RESID COAL C +

Figure 1 Comparison of Catalytic/Resid/Coal/ Coal-Resid Mixture Reactions 


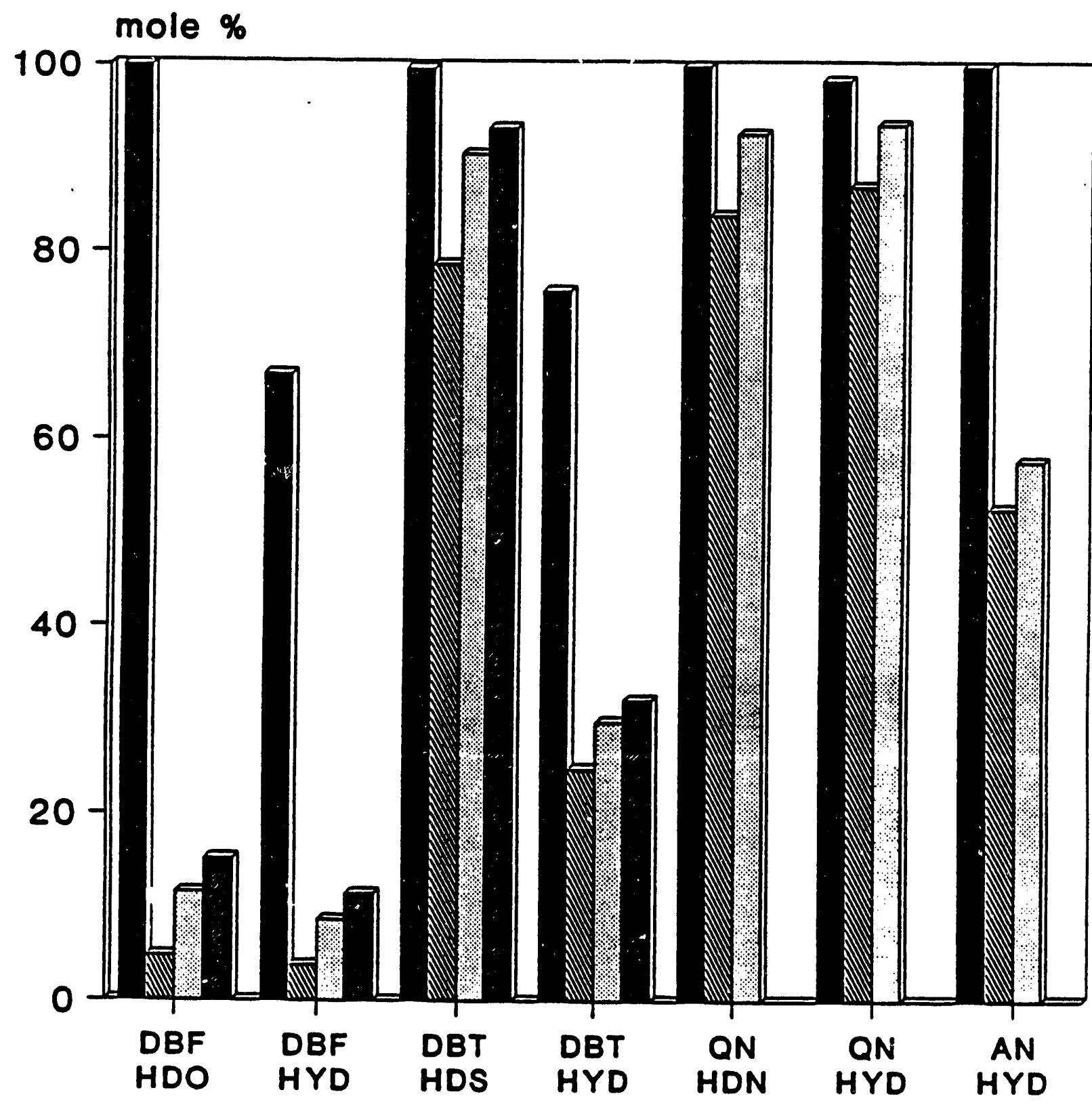

$\square$ CAT. WHW RESID HCNS

Figure 2 Comparision of Catalytic/Resid/Hydrocarbon Fraction/Hexane Soluble Resid Reactions 


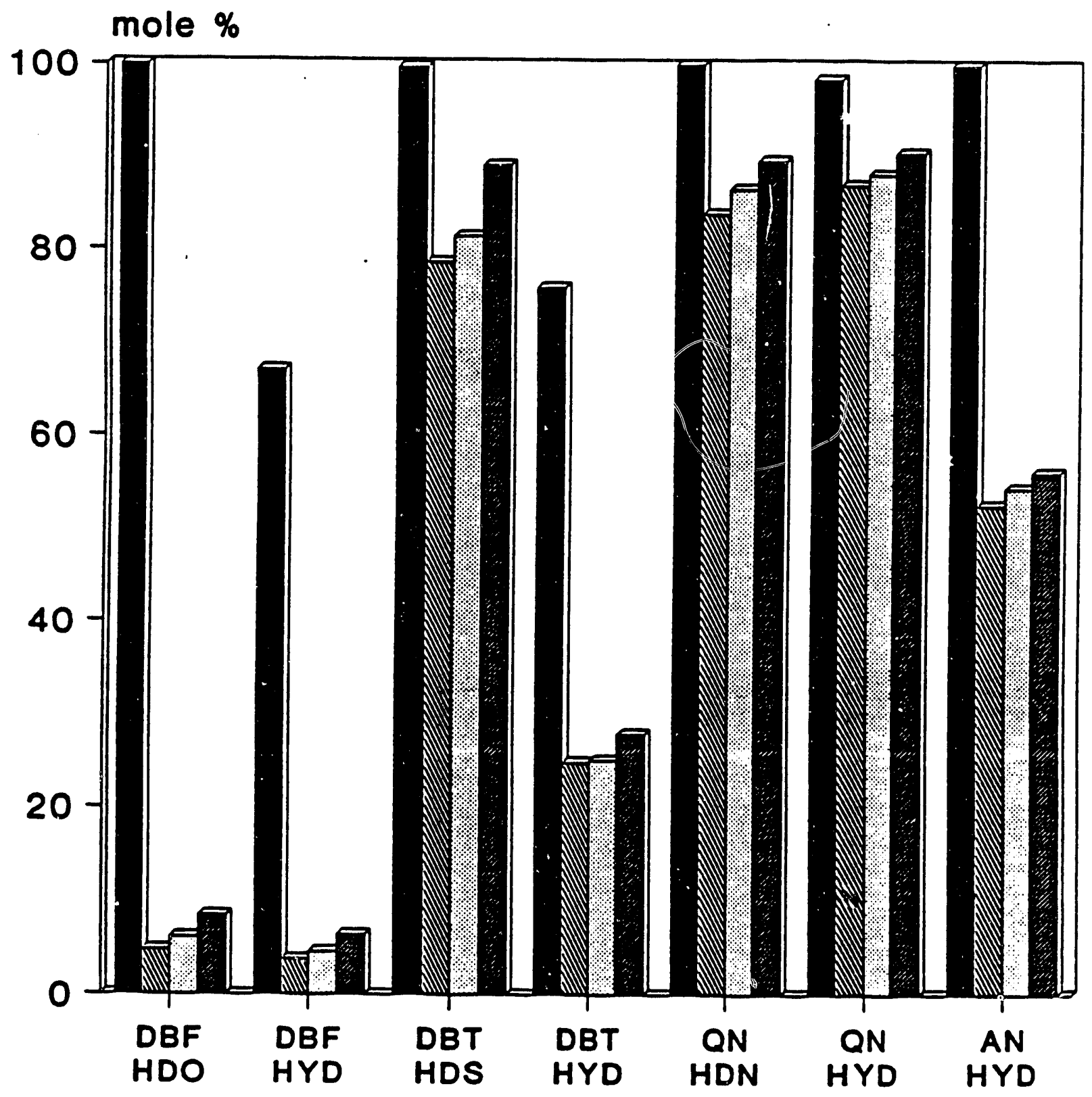

CAT. 30 Mins Resid 3 Hrs

Figure 3 Comparison of Catalytic/Resid/Prehydrogenated Resid(30 mins/3 hrs) Reactions 


\section{Subtask IV.3. Improved Catalysts for Coal Liquefaction and Coprocessing}

This year we have tested new bimodal and unimodal catalysts with a suite of additional coals from the DOE Argonne Coal Data Bank. We have also used the continuous CDS reactor to study the deactivation of these catalysts with model compound hydrogenation/demetallation reactions. In addition we have performed pore diffusion experiments to obtain effective diffusivities for these catalysts. Our results in these areas are summarized in the following. Complete details are given by Rhee (19) and Wang (18).

\section{Introduction}

Supported extrudate catalysts such as Ni-Mo or Co-Mo on $\mathrm{Al}_{2} \mathrm{O}_{3}$ are often used to accelerate reactions in direct coal liquefaction. Because of limited diffusional accessibility in small pores, large asphaltene type molecules often have low overall conversion rates in such catalysts. Furthermore, smaller pores may become plugged with coke and/or metallic deposits, thereby exacerbating this problem. In an attempt to circumvent this dilemma, the use of bimodal catalysts has been suggested. Presumably, the macroporous regions of the extrudate permit easier access to the microporous subregions of higher surface area and activity, especially for larger molecules. Recently, we have evaluated a series of laboratory catalysts for their initial activity for residuum desulfurization and coal liquefaction (1). As a result of this initial study it was found that bimodal pore structures offered an indication of higher activity as compared to their unimodal counterparts. Because this initial work used only a single hvcb coal, Ilinois No. 6, it was felt worthwhile to examine the performance of the same catalysts for a suite of well characterized coals of varying ranks, in order to see if the trend observed earlier could be generalized.

Thus, in the present study, a suite of five well characterized coals obtained from the DOE Argonne Laiooratory Premium Coal Sample Bank have been liquefied with a series of specially prepared laboratory catalysts. To provide benchmarks, two commercial catalysts were utilized as well. Generally speaking, the bimodal catalysts evidenced higher activity for every coal examined.

\section{- Previous Studies.}

The importance of diffusional limitations in coal liquefaction and resid upgrading has been shown experimentally by Shah and Paraskos (2) and Curtis et al. (3). The importance of catalyst pore size distribution in coal liquefaction has been demonstrated by several investigators including Ho and Weller (4) Shimada, et al. (5), and Shimura, et al. (6). Indications of the possible advantages of the bimodal catalyst pore structure relative to the 
unimodal pore structure have been found by Tischer and other researchers at the Pittsburgh Energy Technology Center (7-9) and at the Amoco Oil Company (10). Nalitham and coworkers (11) at the Wilsonville Coal Liquefaction Facility have also found indications of a lower catalyst replacement being required with a bimodal catalyst. Hardin and coworkers $(12,13)$ have conducted a significant research program in the development of high porosity catalysts and have considered diffusional limitations of macromolecules experienced in such pore structures. Chantang and Massoth (14) have also examined the diffusional restrictions encounted by large molecules as they negotiate catalysts having varying pore structures.

The present research is an extension of the work reported earlier by Rhee et al. (1), which was limited to a single coal. In the present study we have studied the liquefaction of a suite of five Argonne Premium Coal Samples (15). The liquefaction behavior of these coals has been studied by other investigators including Baldwin, et al. (16) who reported that the high volatile bituminous coal (Illinois \#6) was the most reactive, followed by the subbituminous (Wyodak) and the medium and low volatile bituminous coals. They also tested effect of various solvents on the coal liquefaction and found an increase of 20 to $30 \%$ in the conversion to toluene solubles when the liquefaction solvent was switched from a nondonor to a hydrogen donor solvent (tetralin).

\section{Experimental}

\section{Materials}

The following chemicals were used: tetralin (Fisher, purified), tetrahydrofuran (Fisher, certified) and pentane (Fisher, certified). Ultra high purity hydrogenation was supplied by Air Products and Chemicals. The coals include an nlinois No. 6 which had been stored in our laboratory for several years (denoted as $I L-A U$ ) and a set of five pristine coal samples from the Argonne Premium Coal Collection as follows:

mlinois No. 6 (II), high volatile bituminous

Pittsburgh No. 8 (PITT), high volatile bituminous

Upper Freeport (UF), medium volatile bituminous

Pocahontas (POC), low volatile bituminous

Wyodak (WY), subbituminous

The Argonne coals had been ground to 100 mesh and contained in amber ampoules under nitrogen. More extensive characterization is available for these coals (15). Catalyst characterization data are given in Wang (18).

\section{Tubing Bomb Microreactor System}

Tubing bomb microreactors (TBMR's) were used to study the initial activity of 
catalysts in coal liquefaction and the liquefaction reactivity of the suite of Argonne coal samples. A typical TBMR reactor apparatus consists of two tubing reactors attached to a shaft for high speed agitation and immersed in a fluidized sand bath to maintain constant temperature. The tubing bomb is made of 1 inch O.D. 316 stainless steel tubing and 1 inch Swagelok fittings with a 45 cc capacity. The TBMR is run in horizontal orientation to minimize gas liquid mass transfer limitations compared with the vertical orientation (17). The agitation stroke length is about $3.8 \mathrm{~cm}$ and the shaking speed is fixed at $850 \mathrm{CPM}$. In order to prevent the catalyst pellets from being pulverized under high speed agitation, a cylindrical stainless steel screen with 420 micron mesh was fashioned into a basket to contain catalyst pellets and glass beads. The outer holder of the basket fit tightly within the tubing bomb so attrition of the catalyst did not occur.

\section{Methods}

Batch coal liquefaction experiments were carried out in 45 cc TBMR's. The TBMR's were cleaned with a strong acidic solution before reaction to eliminate residual materials (coke and metal sulfides) from the inside surfaces and obviate any wall "memory effect". Because the water content of the coals was variable, all coal samples were dried in a vacuum oven at $80^{\circ} \mathrm{C}$ for 8 hours before reaction. For example, as received Wyodak coal contains $28 \%$ water while Pocahontas coal contains only $0.65 \%$ water. Reactants in all experiments were: $10 \mathrm{~g}$ tetralin, $3 \mathrm{~g} \mathrm{mf}$ coal, and $1250 \mathrm{psig} \mathrm{H}_{2}$ gas (charged at room temperature). Tetralin was selected as a solvent to provide uniformity and because of the preponderance of research results available using this solvent. One gram of catalyst pellets mixed homogeneously with glass beads were contained in the basket inside the TBMR.

Experiments were performed in a fluidized sandbath (Techne SBL-2D) maintained at $425^{\circ} \mathrm{C}$ or, in a few cases, $400^{\circ} \mathrm{C}$. After 60 minutes reaction time, TBMR's were removed and quenched in water.

The pentane soluble oils formed by coal liquefaction were measured and used as an indication of catalyst activity and liquefaction reactivity for the coal samples. Pentane soluble oils formation is one of the more sensitive criteria for indicating the degree of coal liquefaction and upgrading. After reaction, the liquid products in the TBMR were washed, centrifuged, and decanted three times with pentane in a $250 \mathrm{ml}$ Teflon FEP centrifuge bottle to extract the pentane soluble oils. The pentane insolubles then were dried and weighed and the maf pentane soluble yield after liquefaction calculated from the following equation:

Pentane Solubles \% = $\frac{(\mathrm{mf} \text { Coal }-\mathrm{mf} \text { Pentane Insolubles) }}{m f} \times 100 \%$ mf Coal x (1- Dry Ash \%) 


\section{Results and Discussion}

Figure 1 presents the pentane soluble liquefaction results for the $I L$ - AU coal which had been stored for several years in our laboratory. A few preliminary runs were conducted at $400^{\circ}$ instead of the $425^{\circ} \mathrm{C}$ used for all later runs. Generally, the commercial catalysts BC and UC evidenced somewhat higher activity than the laboratory prepared catalysts. This could be attributed to manufacturing differences and/or additional promoter trace elements in the commercial catalysts. Our objective was not to prepare the highest activity catalyst possible, but to compare the performance of a set of catalysts prepared in the same way, but with different pore structures. Thus we are mainly interested in the relative ranking of laboratory catalysts F-40, F-20, and F-00. With regard to the commercial catalysts, it is interesting that the unimodal catalyst (UC) is more active at $400^{\circ} \mathrm{C}$ while the bimodal variety $(B C)$ has a higher activity at $425^{\circ} \mathrm{C}$. One would expect diffusional limitations to be more severe at the higher temperature, and hence this is one possible explanation for the improved relative performance of the bimodal catalyst at the higher temperature although certainly not the only one. The increase in pentane soluble activity in going from $400^{\circ} \mathrm{C}$ to $425^{\circ} \mathrm{C}$ is quite impressive, especially for catalyst $\mathrm{BC}$. With regard to the laboratory prepared catalysts there is a slight, but statistically significant, increase in activity as the bimodality increases, i.e., F-40 > F-20 > F-00.

Pentane soluble yields for the suite of Argonne coal samples are shown in Figures 2 -

\section{Figures 2 and 3 show the relative ease of catalytic and thermal (noncatalytic)} conversion of the Argonne coals to pentane soluble oils with commercial and laboratory catalysts respectively. It is interesting to note that, with a few exceptions, the ease of liquefaction of the Argonne coals with every catalyst is WY $>\mathrm{IL}>$ PITT $>$ UF $>$ POC. The thermal (no catalyst) results approximate this same trend. Baldwin (16) has also studied the liquefaction of these same coals and reports a ranking similar to that observed here. Although the comparison between individual numbers are close, in every coal tested, the bimodal catalysts give higher pentane soluble yields than the unimodal varieties, i.e. BC > UC and F-40 > F-20 > F-00. Because of the large number of experiments and coals utilized, this evidence is believed to be statistically significant in showing that the inclusion of macropores offers an improvement in initial catalytic activity. This trend is easier seen by cross-plotting the data as in Figures 4 and 5.

\section{Diffusion Experiments}

We have used the batch method (19) to measure the diffusion coefficients of octane in decane solvent in the pores of several laboratory catalysts. Figures 6 and 7 show typical 
experimental diffusivity results for two catalysts $F-00$, and $F-40$, respectively used, in the preceeding for coal liquefaction runs. In these figures the abcissa is a dimensionless time variable (theta) containing the pore diffusivity $D_{e}$, while the ordinate is the ratio of the octane concentration in the batch at time $t$ to the same value at an "infinite" time. By following the modeling procedure as shown by Rhee (19), the pore diffusivity $D_{0}$ can be found. Figures 6 and 7 show that the theoretical line fits the data points well and that the

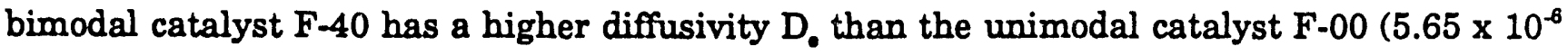
vs. $\left.2.92 \times 10^{-6} \mathrm{~cm}^{2} / \mathrm{s}\right)$. This difference could be due to the higher porosity of the bimodal catalyst and shows the potential of macroporous catalysts in increasing reactant accessibility.

\section{Catalyst Deactivation during Hydrogenation and Hydrodemetallation in the CDS Trickle Bed Reactor System}

The deactivation of different pore-structured catalysts during hydrogenation and hydrodemetallation was investigated in a CDS trickle bed reactor system. In this work, the flow rate of feedstock and hydrogen are set at $200 \mu \mathrm{g} / \mathrm{min}$ and $100 \mathrm{sccm}$ (flowrates converted to superficial velocities are $0.0026 \mathrm{~cm} / \mathrm{sec}$ and $1.32 \mathrm{~cm} / \mathrm{sec}$ respectively). This is a typical trickle flow corresponding to the low flow rate of gas and liquid usually used in laboratory studies. The liquid phase is evenly dispersed in the flowing gas.

The system is comprised of two basic modules, a reactor system and a control system, as shown in Figure 8. Feed preparation consists of receiving, metering and mixing a combination of gases and liquids. The mixer/vaporizer is employed to create the homogeneous stream. The mixture enters a trickle bed reactor (ID 1/2", length 18") where catalyst is packed into the bed and supported with glass wool. The rest of the reactor is filled with $3 \mathrm{~mm}$ glass beads to avoid radical temperature gradients. After reaction the products pass through a gas/liquid separator where liquids go to an autosampler and gases pass through the back pressure regulator then vent outside. A control system provides programming for reactor temperature (initial time and temperature, ramp rate, and final time and temperature), feed flowrate, pressure and reaction methods. All the parameters appear on the monitor screen to be monitored. Experiments were performed by chaining select methods for automatic, sequential execution.

\section{Materials}

Ultra high purity hydrogen and nitrogen were supplied by Air Products and Chemicals. Mineral oil with high viscosity and boiling point from Humco Laboratory Inc. was used as the solvent. The model compounds, tetraphenylporphyrin (TPP) and vanadyl(IV)- 
etioporphyrin (VO-etio), were from Midcentury Chemicals. The following chemicals (source, purity) were used: naphthalene (Fisher, purified), tetralin (Aldrich, 99\%), cisdecahydronaphthalene (Aldrich, 99\%), trans-decahydronaphthalene, 99\%), decane (Fisher, certified), p-xylene (Fisher, certified), tetrahydrofuran (Fisher, certified), and di-tertiary nonyl polysulfide (Pennwalt Corporation).

Mineral oil was used as a solvent because it was relatively stable under the reaction condition $\left(400^{\circ} \mathrm{C}\right)$. However, it showed a little cracking in the beginning of the reaction owing to the initial activity of the catalyst. Naphthalene and VO-etio were served as model compounds to represent the highly complex reactions in coal liquefaction.

There were four feedstocks prepared for these experiments:

(1) 2 wt\% naphthalene in mineral oil.

(2) 2 wt\% naphthalene and 2.7 wt\% di-tertiary nonylpolysulfide (contains $37 \%$ sulfur) in mineral oil.

(3) 2 wt\% naphthalene and $100 \mathrm{ppm}$ of TPP in mineral oil.

(4) 2 wt\% naphthalene and $40 \mathrm{ppm}$ of VO-etio in mineral oil.

Because the solubility limit of VO-etio in mineral oil is about $40 \mathrm{ppm}$, it is not easily dissolved at room temperature. The detailed procedure for preparing the feeds is given by Wang (18).

\section{Method}

Five catalysts, with different pore structures containing nickel and molybdenum supported on alumina as described in Wang (18), were chosen to study catalyst deactivation. It normally would take more than one year to completely deactivate the catalyst under normal reaction conditions. In order to obtain more efficiency in running experiments, severe conditions to accelerate the deactivation of the catalyst are needed. Our approach was to alternate 24 hour periods at reaction conditions $\left(400{ }^{\circ} \mathrm{C}\right.$ with $\mathrm{H}_{2}$ gas) with similar periods of more severe conditions $\left(450-510^{\circ} \mathrm{C}\right.$ with $\mathrm{N}_{2}$ gas) in order to accelerate coke formation (see Figure 9). At the end of each reaction period a liquid sample (about $3 \mathrm{cc}$ ) was taken for analysis to determine the catalytic activity remaining. A quantity $(0.3 \sim 0.4 \mathrm{~g})$ of sample was diluted to about $3 \mathrm{~g}$ with $\mathrm{p}$-xylene and analyzed with a rapid scanning UV. visible spectrophotometer (Varian DMS 100S) in conjunction with Beer's law to determine 
the concentration of TPP or VO-etio. The rest of the sample was combined with about 1 wt\% decane as an internal standard and analyzed with a gas chromatograph (Varian model 3700) equipped with a $30 \mathrm{~m} \times 0.32 \mathrm{ID}$ fused silica capillary column (J \& W Scientific).

\section{Comparison of Long Term Activity of Catalysts in Hydrogenation and Hydrodemetallation}

The hydrodemetallation of VO-etio is a consecutive reaction as follows (20):

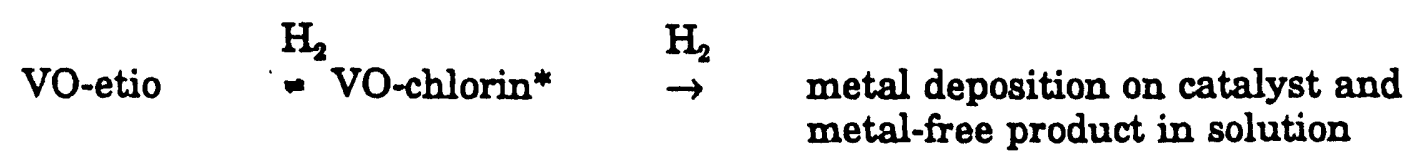

The relative activities of a catalyst for hydrogenation $\left(A_{H 1}\right)$ and hydrodemetallation $\left(A_{\mathrm{HDM}}\right)$ were utilized as indications for the extent of deactivation of the catalyst. $A_{H}$ is defined as moles of $\mathrm{H}_{2}$ consumed for products (tetralin and decalins) divided by the moles of $\mathrm{H}_{2}$ required to produce the most hydrogenated product (decalins):

$$
A_{H}=\text { mole\% Decalins } \times 1.0+\text { mole\% Tetralin } \times 0.4
$$

$A_{\mathrm{HDM}}$ is defined as metal removal divided by original concentration of VO-etio (40 ppm).

$$
A_{\mathrm{HDM}}=\frac{(40-\text { Conc. of VO-etio at the reactor outlet) }}{40}
$$

\section{Evaluation of Laboratory Prepared Catalysts}

The approach to examine the performance of three experimental catalysts during longterm reactions followed the procedures given by Wang (18). The operating conditions and the results of all runs are listed in reference (18). A typical weight percent-time curve for naphthalene hydrogenation is shown in Figure 10 as Run NV-14. The highest catalyst selectivity for tetralin occurred at the reaction time of 264 hours. Figures 11 and 12 show the trend of catalyst deactivation during the reaction in terms of the catalyst relative activities: $A_{F D M}$ for hydrodemetallation and $A_{H}$ for hydrogenation. Both results indicated the performance of three catalysts in the following order: F-40 $>$ F-20 $>$ F-00, i.e. the more macropores, the longer the lifetime of the catalyst. They are encouraging results in that

*Vanadyl Chlorin - partially hydrogenated form of VO-etio (21). 
they show that the presence of macropores may help to decrease the rate of catalyst deactivation in coal liquefaction.

\section{Literature Cited}

1. Rhee, Y.W.; Guin, J.A.; Curtis, C.W., Energy \& Fuels, 1989, 3(3), 391-397.

2. Shah, Y.T.; Cronauer, D.C.; Mclivried, H.G.; Paraskos, J.A., Ind. Eng. Chem. Process Des. Dev. 1978, 17, 288.

3. Curtis, C.W.; Guin, J.A.; Kamajian, B.L.; Moody, T.E., Fuel Processing Technol. 1986, 12, 111-125.

4. Ho, P.N.; Weller, S.W., Fuel Processing Technol. 1981, 4, 21-29.

5. Shimada, H.; Kurita, M.; Sato, T.; Yoshimura, Y.; Kawakami, T.; Yoshitomi, S.; Nishijima, A., Bull. Chem. Soc. Jpn. 1986 59, 2885.

6. Shimura, M.; Shiroto, Y.; Takeuchi, C., Ind. Eng. Chem. Fundam. 1986, 25, 330-337.

7. Tischer, R.E.; Narain, N.K.; Stigegel, G. J.; Cillo D. L., U.S. DOE Report No. DOE/PETC/TR-86/1, 1985.

8. Ticher, R.E., J. Cat. 1981, 72, 255-265.

9. Ticher, R.E.; Narain, N.K. Steigel, G.J.; Cillo, D.L., J. Cat. 1985, 95, 406-413.

10. Amoco Oil Company, Report No. DOE/ET/14803-T8 "Coal Liquefaction Catalyst Development" Contract No. AC22-79ET14803, August, 1982. Also see "Catalyst Development for Coal Liquefaction" AF-1233, EPRI Project No. 408-1,2 Final Report, November, 1979.

11. Nalitham, R.V.; Lee, J.M.; Lamb, C.W., Fuel Processing Technol. 1987, 17, 13-27.

12. Hardin, A.H.; Packwood, R.H.; Ternan, M., Preprints, Div. Petroleum Chem., Amer. Chem. Soc. 1978, 23 (4), 1450.

13. Hardin, A.H.; Ternan, M., Preprints, Second World Congress on Chemical Engineering, $1981,3,134$.

14. Chantong, A.; Massoth, F.E., AIChE J, 1983, 29 (5) 725-731.

15. Vorres, K.S., Users Handbook for the Argonne Preminum Coal Sample Program, 1989.

16. Baldwin, R.M.; Shin, S-C; Miller, R.L., Preprints, Am. Chem. Soc., Div. Fuel Chem. 1978, 32 (4), 352-358.

17. Rhee, Y.W.; Guin, J.A.; Curtis, C.W., Fuel Processing Technol. 1989, 22, 97-105.

18. Wang, W.P., M.S. Thesis, Auburn University, AL (1990).

19. Rhee, Y.W., Ph.D. Thesis, Auburn University, AL (1989).

20. Agrawal, R. and Wei, J., Ind. Eng. Chem. Process Des. Dev. 23, 505 (1984).

21. Dongen, R. H.; Bode, D; Eijk, H.; Klink, J.; Ind. Eng. Proc. Des. \& Dev., Vol. 19, 630, (1980). 


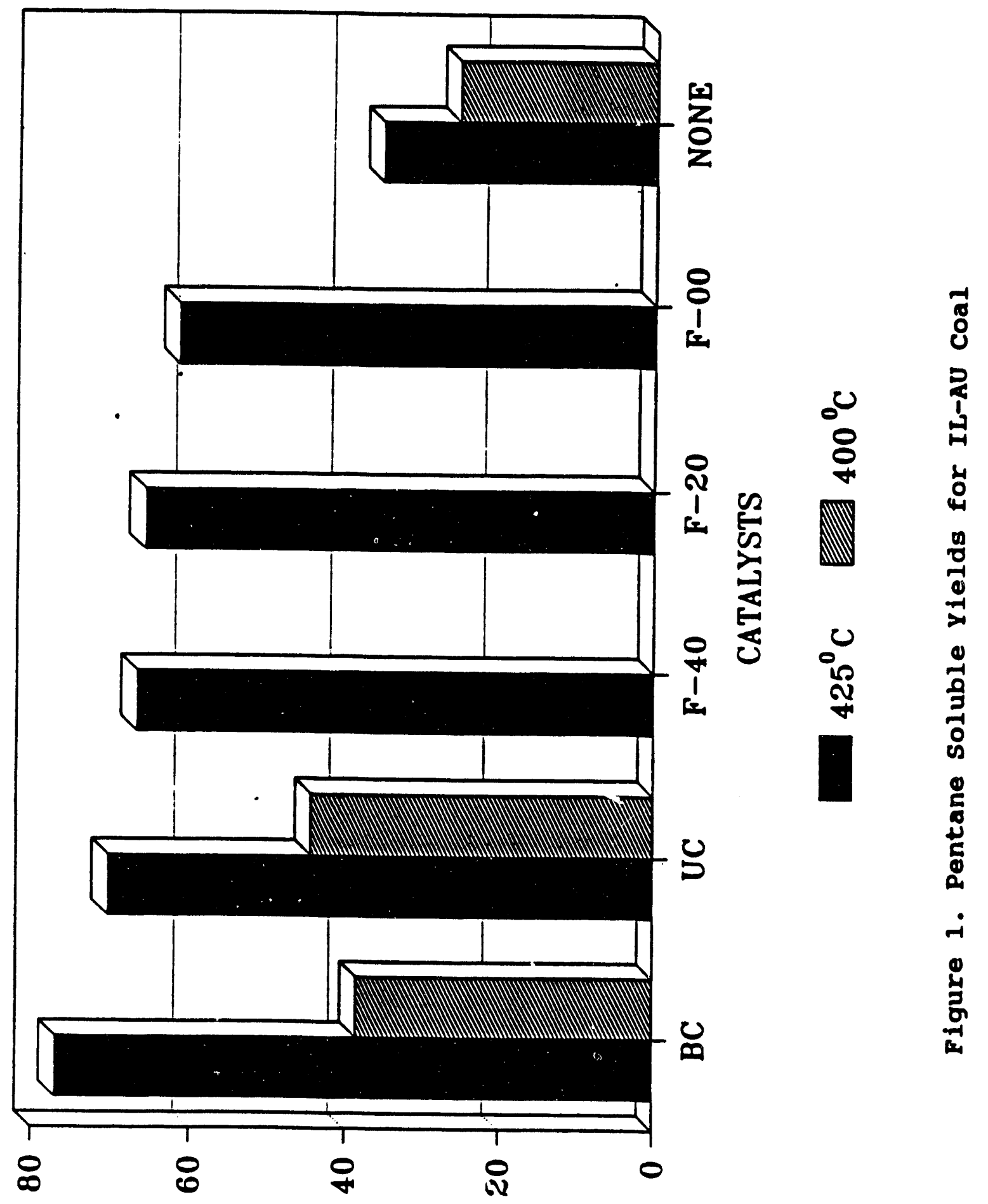

Q. 


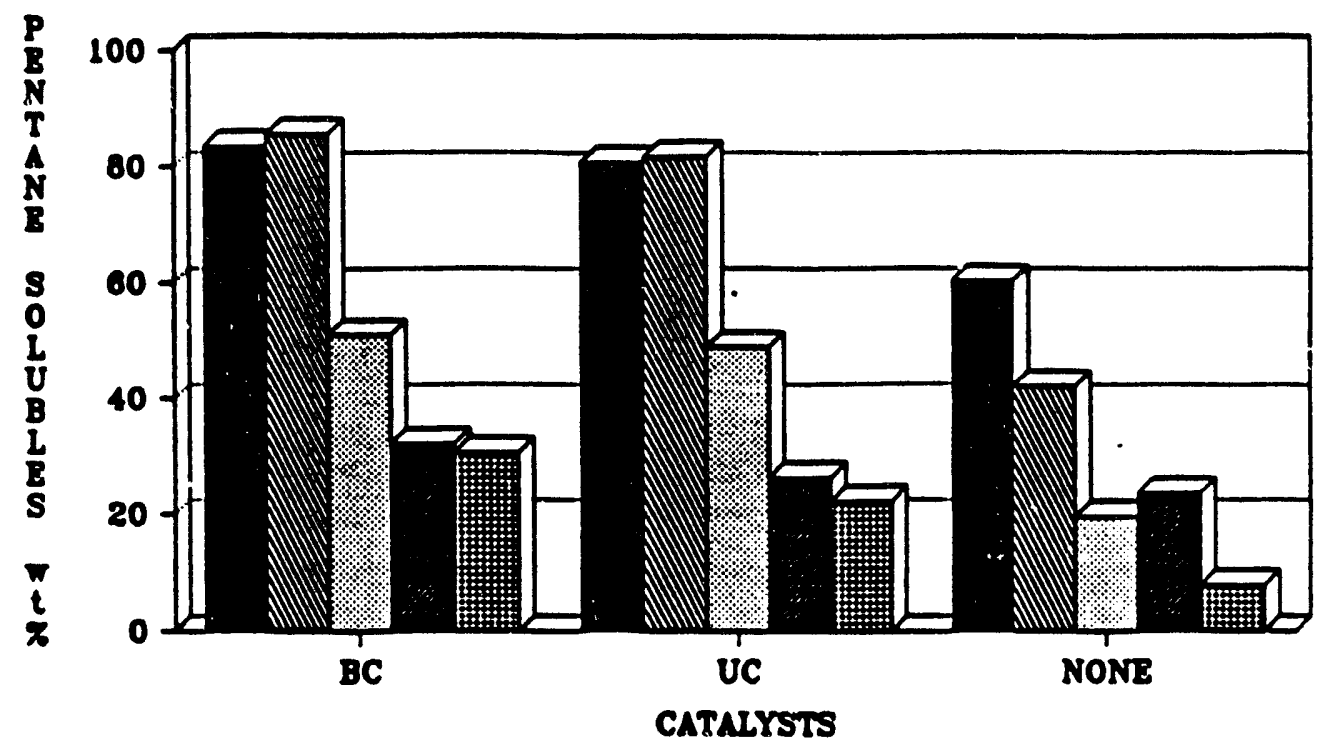

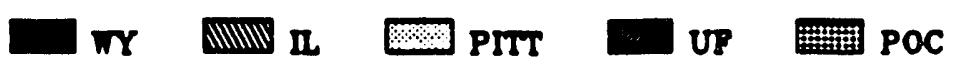

Figure 2. Pentane Soluble Yields for Argonne Coals with Commercial Catalysts

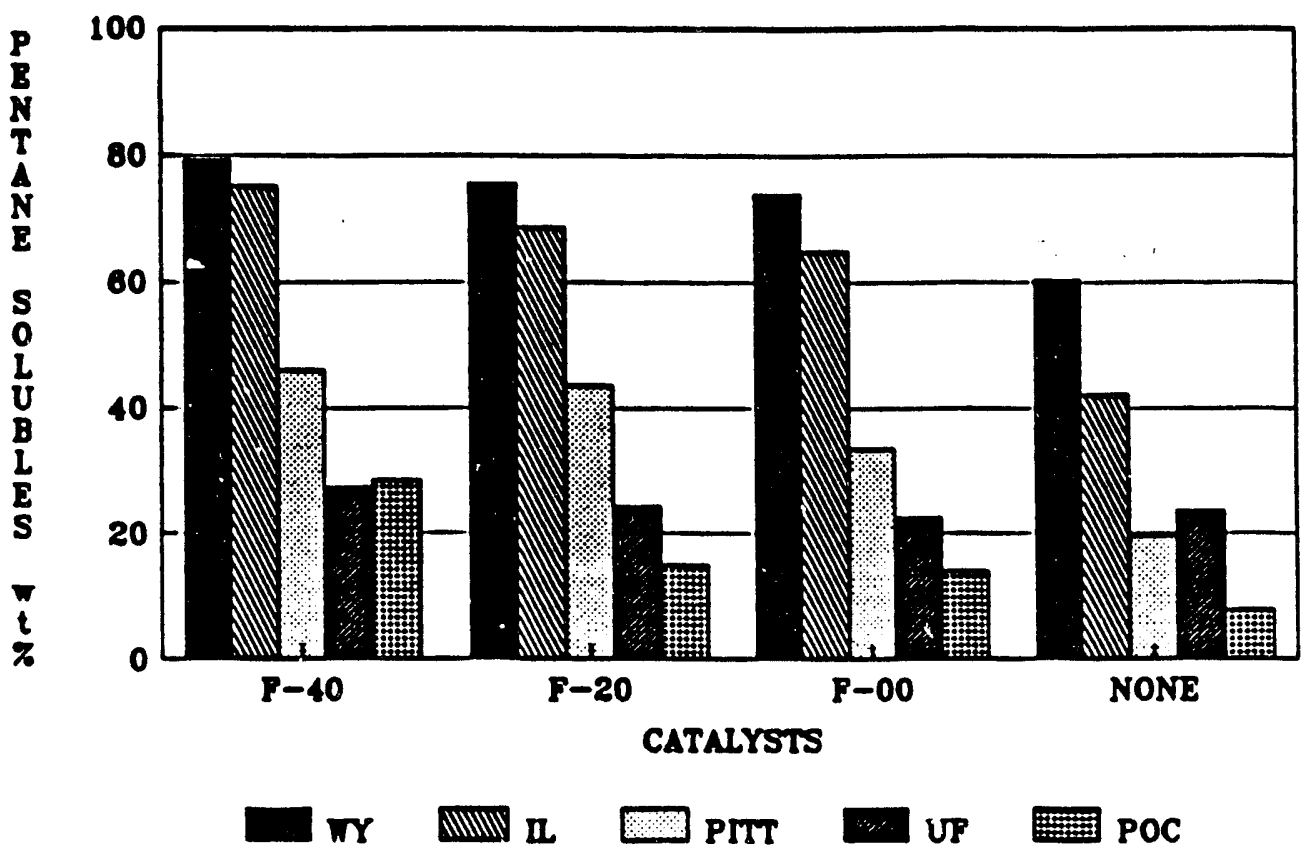

Figure 3. Pentane Soluble Yields for Argonne Coals with Laboratory Catalysts 


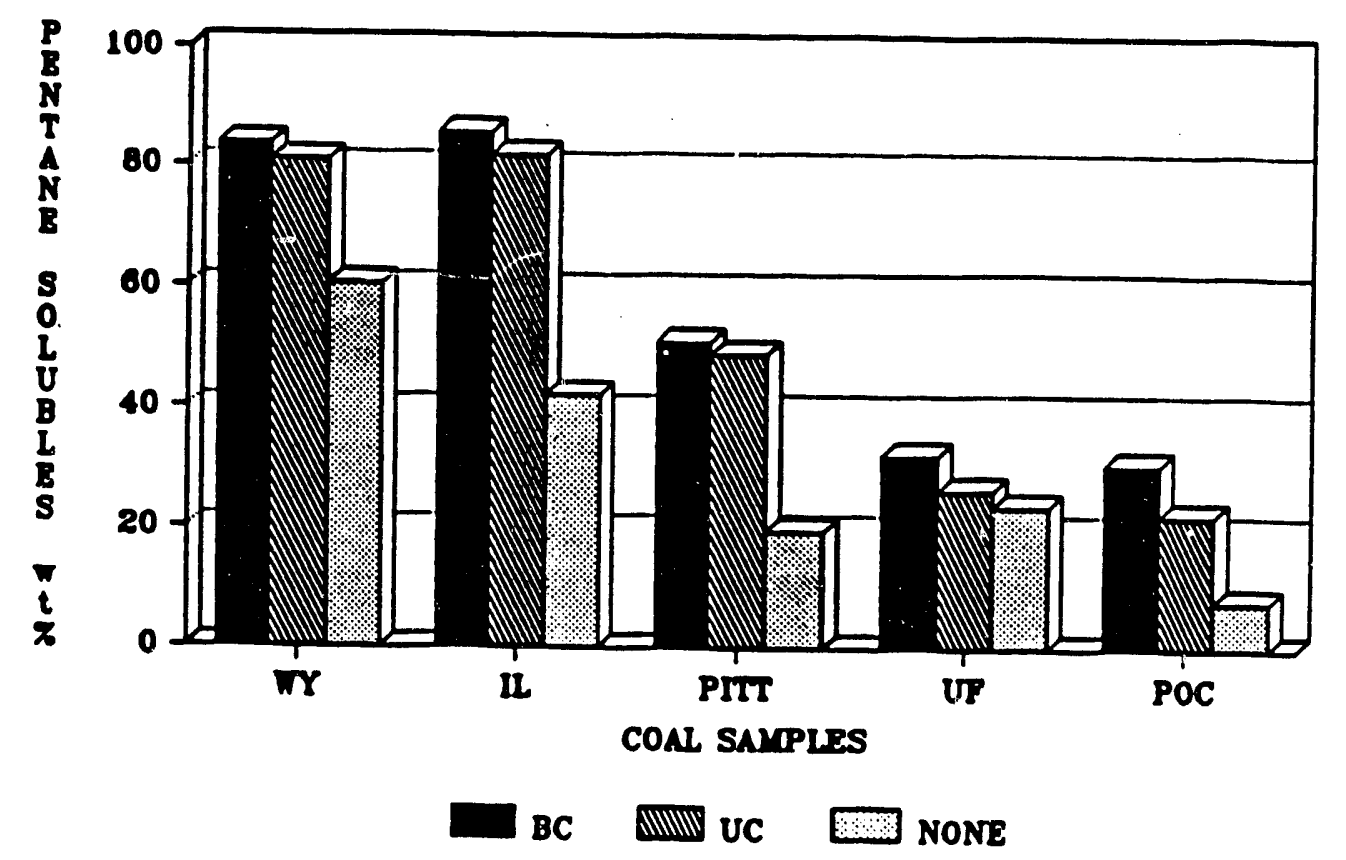

Figure 4. Pentane Soluble Yields for Argonne Coals with Commercial Catalysts

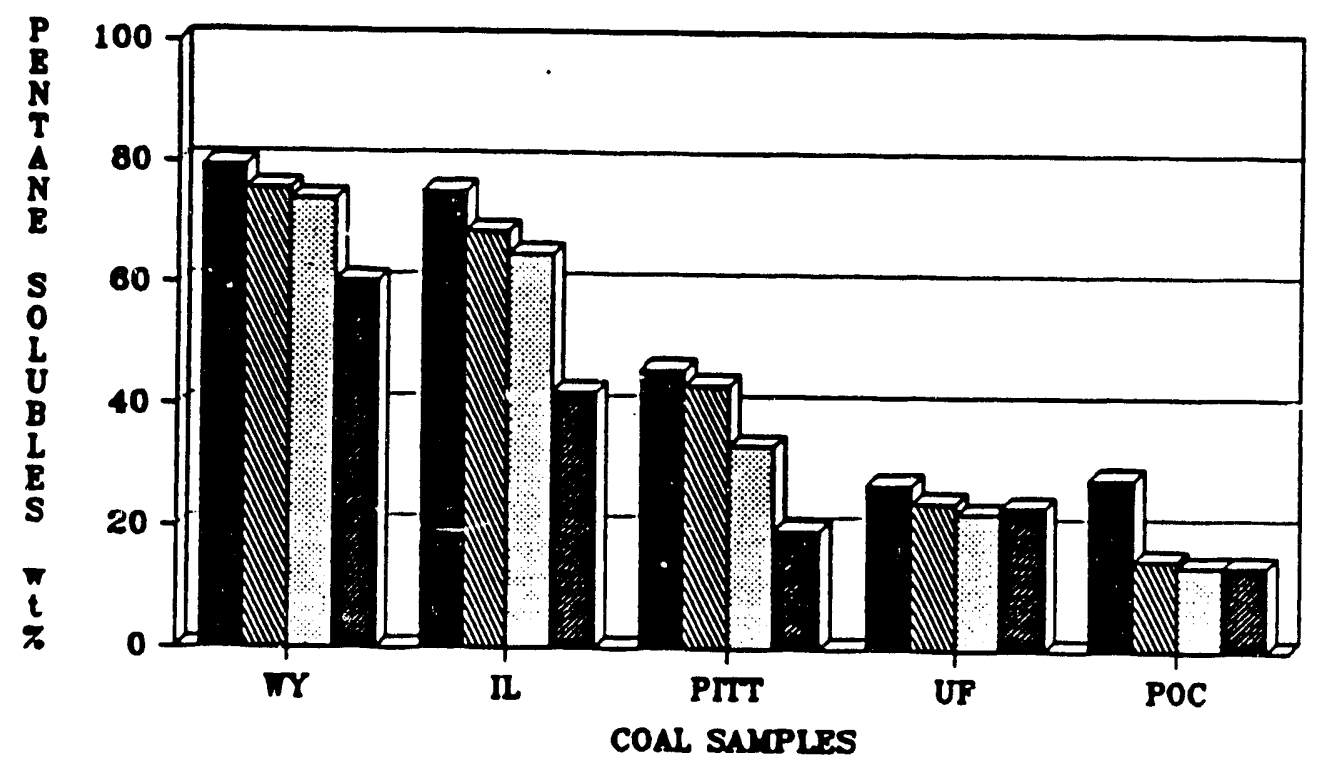

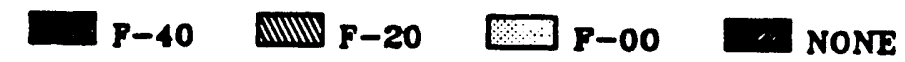

Figure 5. Pentane Soluble Yields for Argonne Coals with Laboratory Catalysts 


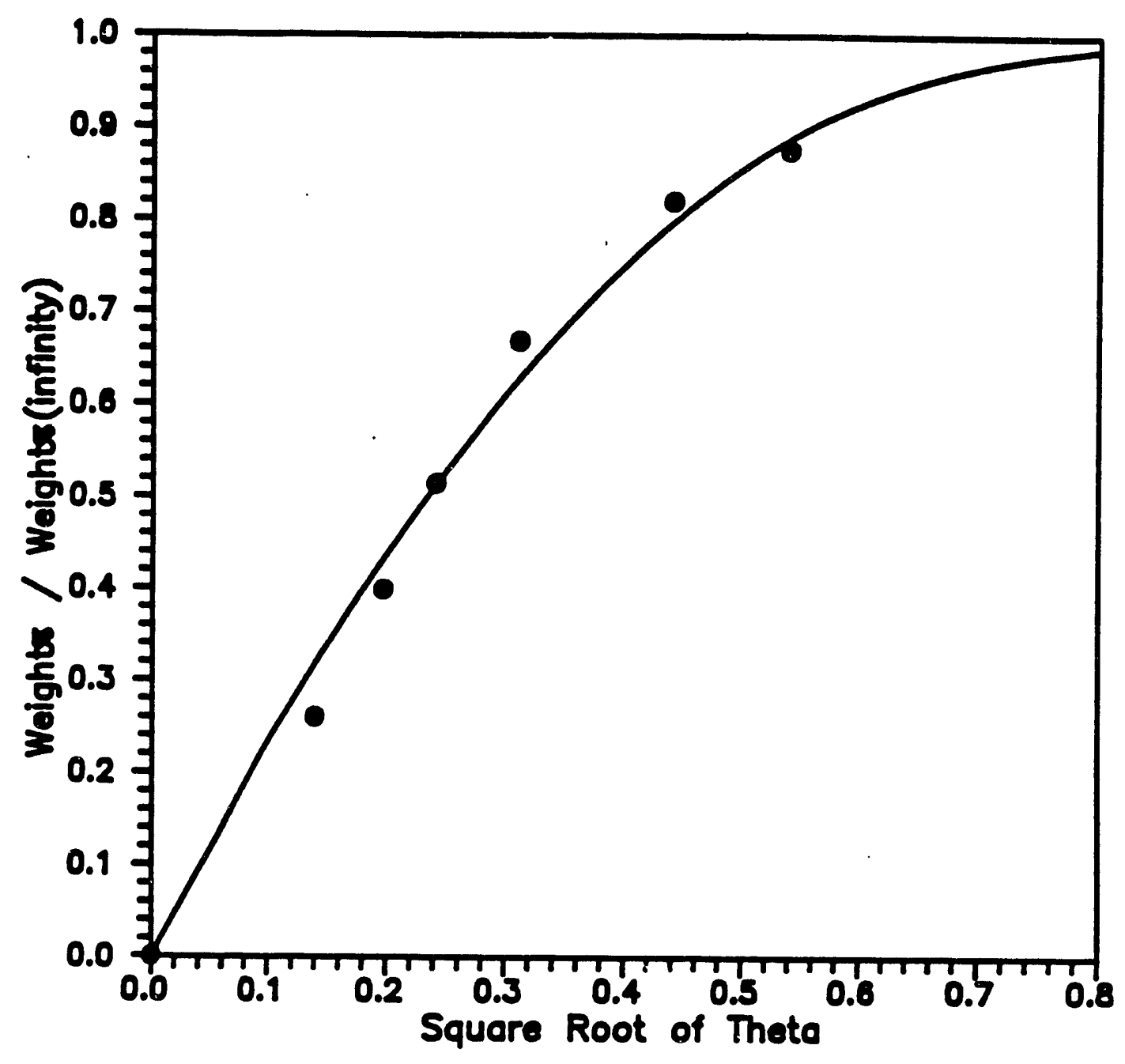

Figure 6. Typical Result of Octane/Decane Diffusion Experiment with Fresh Catalyst F-00. (De $=2.92 \times 10^{-6} \mathrm{~cm}^{2} / \mathrm{s}$ ) 


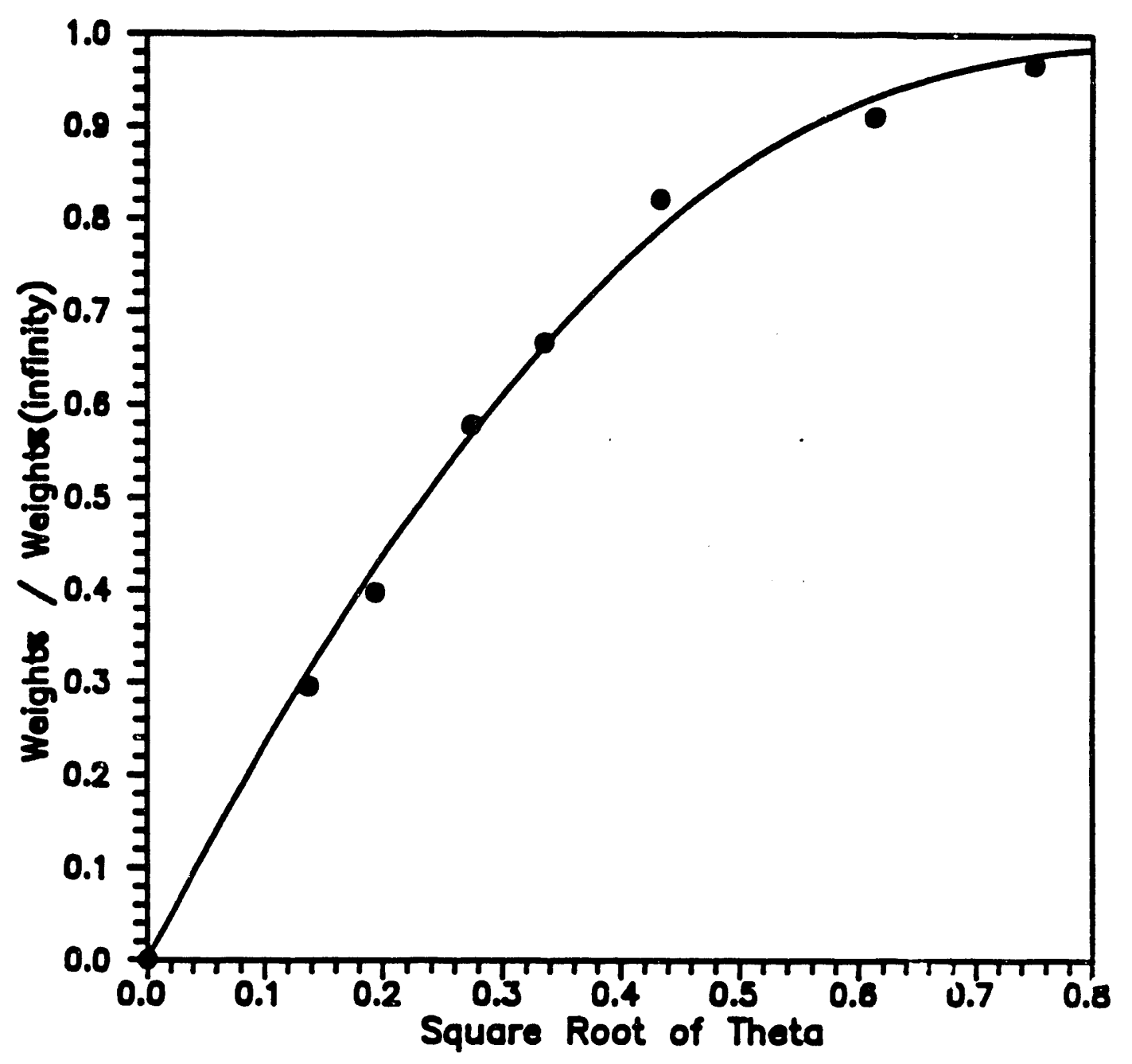

Figure 7. Typical Result of Octone/Decane Diffusion Experiment with Fresh Cotalyst F-40. (De $=5.65 \times 10^{-1} \mathrm{~cm}^{2} / \mathrm{s}$ ) 


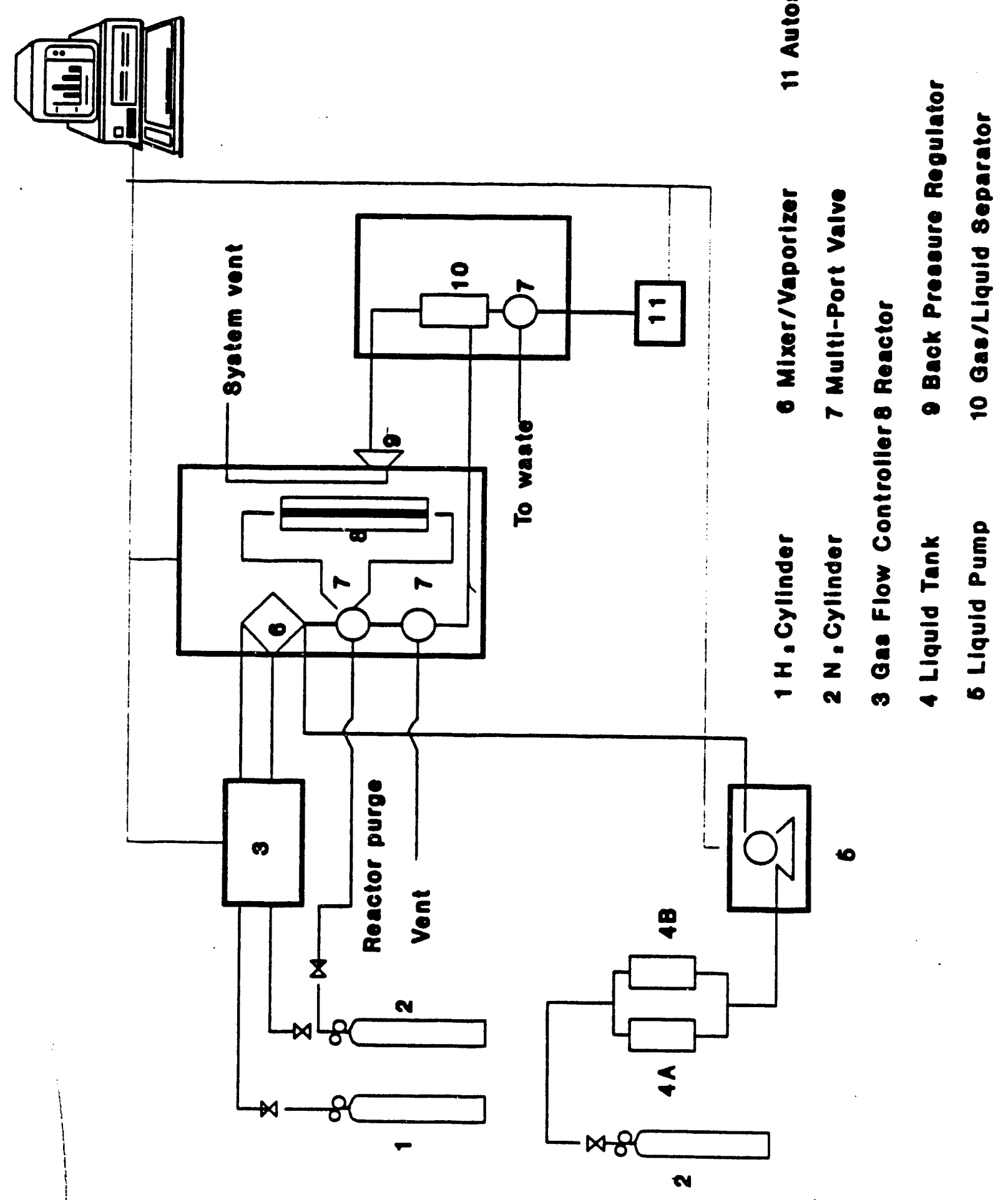

Figure 8. Schematic of CDS Reactor System 
TIMS

(days)

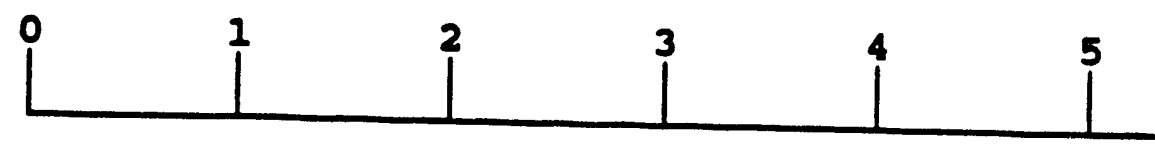

100 (SCAX)

HYDROGEN

0

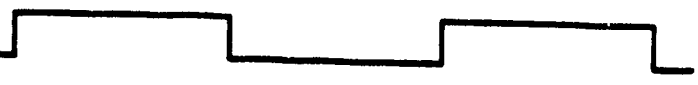

25 (SCCY)

NITROGEN

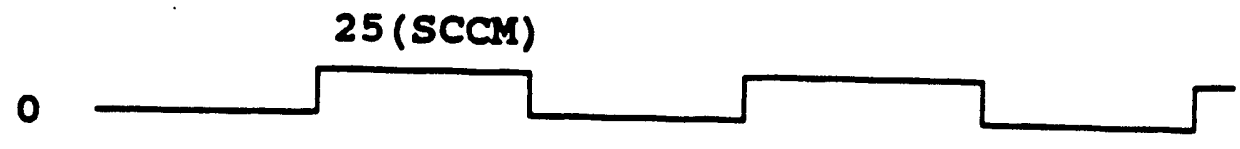

PURE

MINERAL OIL 0

$200 \mu e$

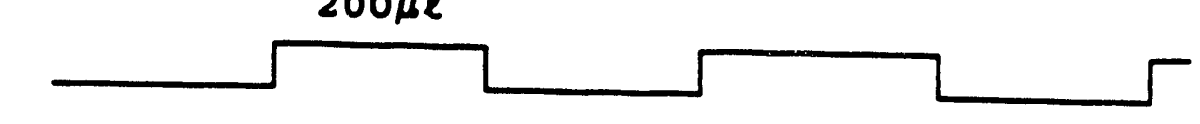

2 Wt? HAPPHTHAIENE

4OPPM VO-ITIO $200 \mu \mathrm{K}$

In

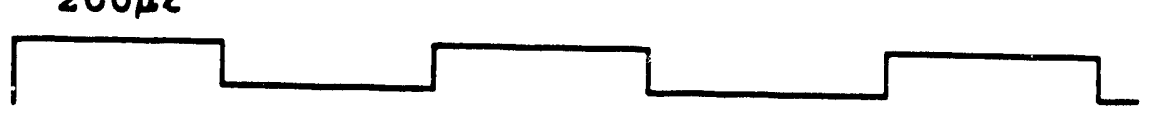

1000 PSI

TRESSURE 0

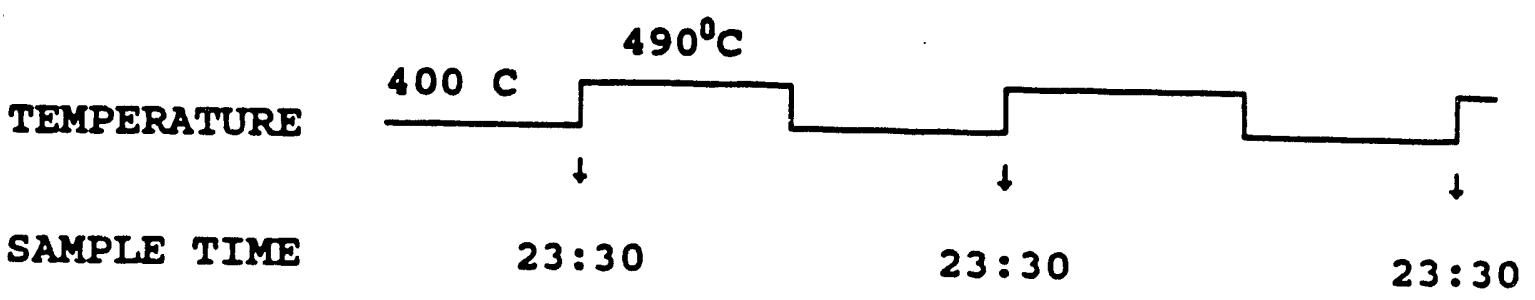

Figure 9. Graphic Layout of Experimental Parameters in CDS Reactor System for Alternating 24-Hour Reaction and Deactivation Time Periods 


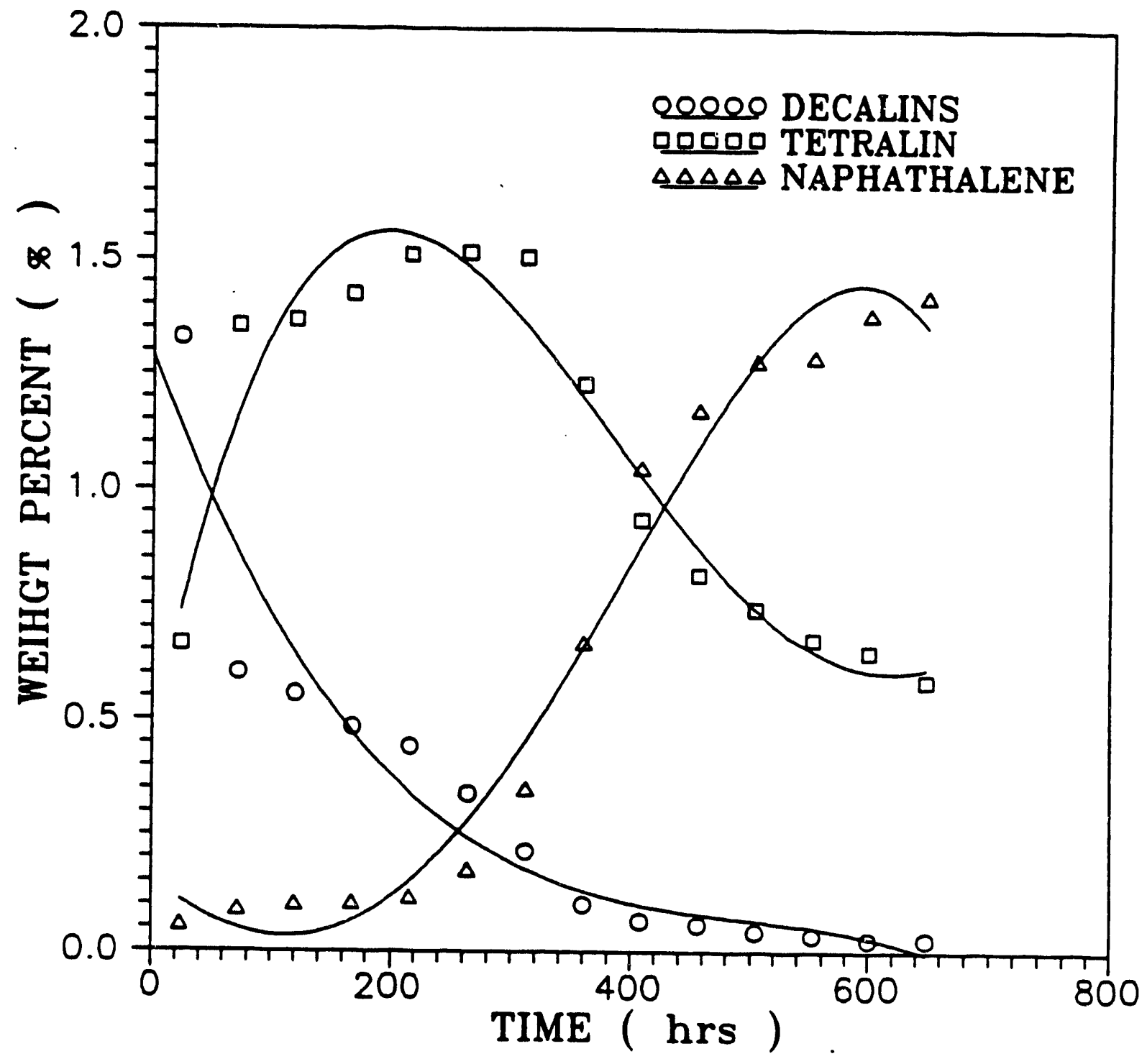

Figure 10. Product Distributions of Naphthalene Hydrogenation Using F-40 Catalyst: Run. Nv-14 


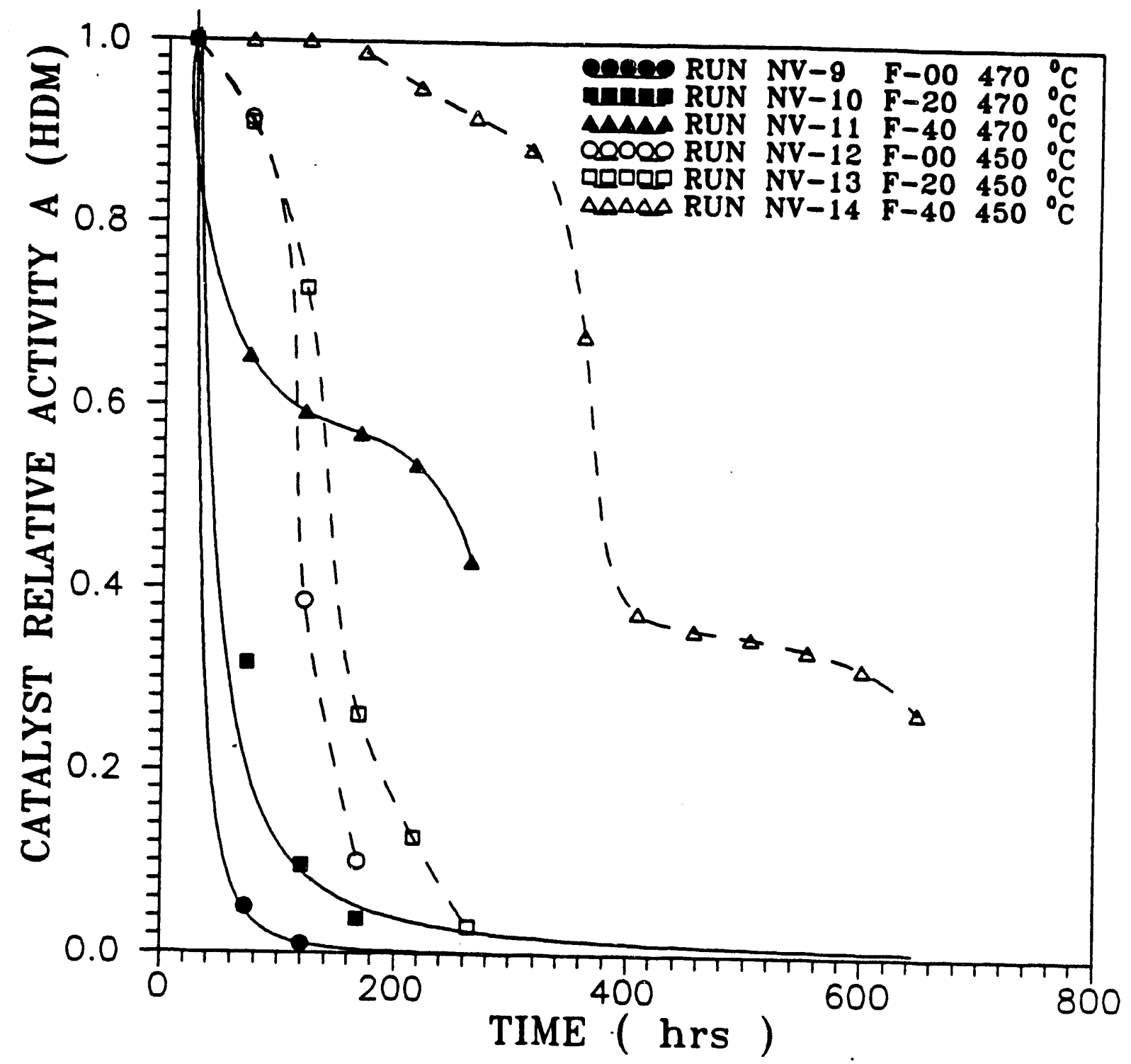

Figure 11. Catalyst Deactivation during Hydrogenation and Hydrodemetallation in Terms of Catalyst

Relative Activity $A_{\text {HON }}$ : Runs NV-9 to 14 


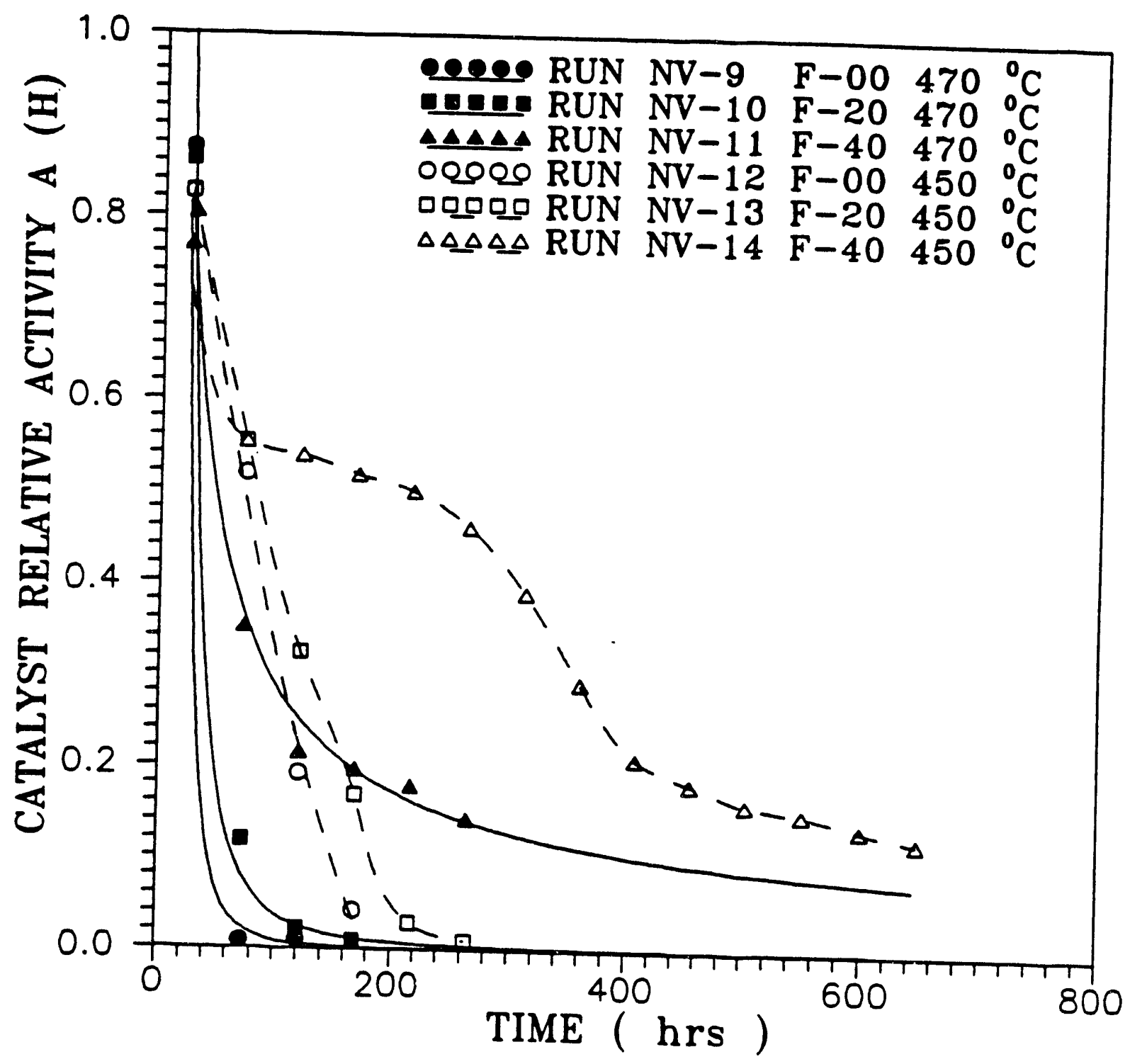

Figure 12. Catalyst Deactivation during Hydrogenation and Hydrodemetallation in Terms of Catalyst

Relative Activity $A_{4}$ : Runs NV-9 to 14 


\section{Task IV.4 Reduced Coal Liquefaction Process Severity through Better Hydrogen Gas-Liquid Mass Transfer}

\section{Summary}

A slurry phase reactor system was developed during this past year that offers sufficient versatility in reaction rate controllability and the necessary mass transfer/heat transfer characteristics to prevent retrogressive reactions from occurring due to $\mathrm{H}_{2}$ starvation, while attaining a very high conversion of reactants. During the initial stages of liquefaction, it was hypothesized that free-radical reactions occur rapidly and reaction rates increase almost exponentially. As a result, no practical, commercial gas-liquid contactor was postulated to be capable of providing $\mathrm{H}_{2}$ at a sufficiently high enough rate to prevent $\mathrm{H}_{2}$ starvation, and thus retrogressive reactions, from occurring. This was considered to be true as long as the reaction temperature was held constant and free-radical propagation was allowed to proceed at an uncontrollable rate. Previous experimental evidence supported this hypothesis.

Rather than attempt to seek some way solely of satisfying the $\mathrm{H}_{2}$ transfer rate requirements of uncontrollable free-radical propagation reactions via exceptionally high mass transfer rates, an alternative design approach was used. The reactor system was designed in order to control the rate of free-radical propagation so that only practical, achievable $\mathrm{H}_{2}$ mass-transfer rates would be required.

It was felt that, with proper reaction rate control, $\mathrm{H}_{2}$ mass transfer rates could be made sufficiently high (via gas-liquid contactor design) so that retrogressive reactions could be kept at a minimum. The more the $\mathrm{H}_{2}$ mass-transfer rate is raised for each unit of reactor volume via the gas-liquid contactor design, the further the reactor's operational range can be extended over the period in which the rate of retrogressive reactions is low. The approach taken in developing the reactor system was, therefore, two-fold: 1) a reactant flow pattern was selected which would allow versatility in reaction rate controllability and 2) a gas-liquid contactor design was sought that provided the highest mass-transfer rates per unit volume

- without significantly affecting reaction rate controllability.

Basically, a bang-bang control strategy was implemented to regulate reaction rate. That is, a cyclic switching from high to low reaction temperatures was used to control reaction rate. The adjustable control parameter selected was the frequency of the cyclic switching in reaction temperature. Physically this was accomplished using a recycle looptype reactor in which the temperature in part of the loop was kept high (the reaction zone) and that in the remainder of the loop was kept low (the quenching zone). This reactor flow arrangement has been commonly used with jet-type reactors which have been utilized 
commercially when reaction rates are very high (as is the case in the initial stages of liquefaction). The frequency of cyclic switching in reactant temperature can be varied simply by changing the reactant recycle flow rate. And by sufficiently increasing the recycle flowrate, the $\mathrm{H}_{2}$ mass-transfer requirements per reaction cycle could be kept low within a range that we felt could be satisfied via practical, gas-liquid contactors.

A variety of designs for gas-liquid contactors were evaluated: gas-liquid jet injectors, motionless mixers, column packings, and high-shearing mechanical agitators. Of the contactors studied to date, each type was evaluated not only in terms of its relative effect on gas-liquid mass transfer but also on the feasibility of its use in the proposed reactor system. The effective mass transfer coefficients for the contactors in a low-temperature sodium sulfite oxidation system and in a slurry-phase catalytic naphthalene hydrogenation system were determined.

Naphthalene hydrogenation was used as a model reaction to direct developmental efforts. For these experimental studies a simple packed reactor was used. The reactor design was improved during the year to the point that this model reaction could be carried out to completion without mass transfer being limiting under a base set of conditions. The $\mathrm{H}_{2}$ mass-transfer rate per unit volume in the reactors was shown to depend directly on the liquid velocity through the reactor. The initial system, as well as other conventional reactors, exhibited significant gas-liquid mass-transfer related limitations under the same base conditions.

A mathematical model for the reactor system was developed. The model was used extensively in interpreting experimental observations and parameterizing the system. Using the model in conjunction with experimental measurements, the current reactor design was shown to not be strictly mass-transfer limited. The $\mathrm{H}_{2}$ mass-transfer coefficient in the current system was determined to be only about 0.13 per second, which is about six times higher than that of a bubble column ( 0.02 per second). But even with this low order of improvement in the $\mathrm{H}_{2}$ mass-transfer rate, a significant hydrogen concentration appeared to be maintained throughout the reaction zone, and the overall reaction was observed to not be strongly mass-transfer limited. This was true because the reaction rate was controlled, which allowed the available amount of $\mathrm{H}_{2}$ to be sufficient.

Immediate plans are to evaluate the reactor system using tetralin oxidation as a model reaction. This reaction has a very rapid reaction rate due to free-radical propagation. The ability of the reactor to control product selectivity for this model reaction will provide a tangible measurement of its effectiveness to control a rapid reaction, such as those that occur in the initial stages of liquefaction. A mechanical high-shearing gas-liquid contactor 
now under construction will be used in these studies. Upon completion of these limited developmental studies, coal liquefaction studies will then be performed to observe the responses in product distribution to well-controlled liquefaction under high $\mathrm{H}_{2}$ mass transfer rate conditions.

Overall these studies should reveal how to modify the preheater for the dissolver in the conventional coal liquefaction process train so as to minimize retrogressive reaction product formation. By so doing, downstream liquefaction product upgrading can be simplified significantly.

\section{Background and Rationale}

\section{Coal Liquefaction}

The major goal of most coal conversion processes is the removal of sulfur and minerals from the coal by conversion of the coal into a clean, solid or liquid fuel. The two major problems in coal liquefaction are the difference in the hydrogen content between the raw materials and the desired products and the need for removal of heteroatoms, such as oxygen, sulfur, and nitrogen.

\section{Chemistry}

The chemical structure of coal has been identified as a combination of multibenzene ring structures (1). The number of rings can vary from one to as many as six or seven rings condensed together, with an average size unit of about three rings. The first step in conversion of such a multi-ring structure to materials which would be gaseous or liquid at room temperature involves breaking the connecting bridges to produce free radicals (2) . These ring fragments, in turn, must be stabilized by the addition of hydrogen to the unsaturated bond of the free radical. The amount of hydrogen required is dependent on the hydrogen content of the coal. The atomic hydrogen content of a typical coal (1) is on the order of 0.82 hydrogen atom for each carbon atom, while typical transportation fuels show hydrogen to carbon ratios of 2:1. Thus, liquefaction of a typical coal without the addition of hydrogen would theoretically allow only 42 percent of the carbon present to be converted into transportation fuels.

In addition, hydrogen combines with heteroatomic sulfur and oxygen in the coal to form hydrogen sulfide and water (1). These by-products can then be removed as gases. Therefore, most liquefaction processes require the addition of large quantities of hydrogen to the coal, usually by a combination of donor solvent and molecular hydrogen, to increase the hydrogen-to-carbon ratio and remove impurities. The resulting liquid products can then be 
used as transportation fuels.

\section{Kinetics}

Conversion of coal to a soluble form has been shown to consist of a series of very fast reactions followed by slorrer reactions (2). The fast initial reactions have been proposed to involve the thermal disruption of the coal structure to produce free radical fragments. This similarity between coal liquefaction and coal pyrolysis has been previously demonstrated (3): Earlier research by Petrakis (4) has shown that large quantities of free radicals $(~-0.1$ molar solutions) are produced when suspensions of coal in various solvents are heated to $450^{\circ} \mathrm{C}$.

For the purpose of this discussion, it is assumed that the thermal formation of free radicals is an autocatalytic reaction. The reaction can be represented as follows:

$$
A+R \rightarrow R+R
$$

where $A$ equals the concentration of coal and $R$ is the concentration of free radicals. In such a reaction scheme, the reaction rate is slow at first because few free radicals will be present. However, the rate increases to a maximum as free radicals are formed, and then drops to a low value as the coal is consumed.

The next step in the conversion of coal is the hydrogenation of the free radicals to saturated compounds. If hydrogen is available to the free radicals, product formation occurs. The rate of this reaction is dependent on the amount of hydrogen available in the bulk liquid. If insufficient hydrogen is available, the radicals are not stabilized and undergo a variety of undesirable reactions where high molecular weight solids are formed. These reactions can be represented by:

$$
\begin{aligned}
& \mathrm{R}+\mathrm{H}_{2} \rightarrow \mathrm{P}_{1} ;(\mathrm{H}: \mathrm{C})_{1} \\
& \mathrm{R}+\mathrm{R} \rightarrow \mathrm{P}_{2} ;(\mathrm{H}: \mathrm{C})_{2}
\end{aligned}
$$

The hydrogen-to-carbon ratio, $(\mathrm{H}: \mathrm{C})_{1}$, of the products from reaction 1 is higher than the hydrogen-to-carbon ratio of the products from reaction 2. So, the $\mathrm{H}: \mathrm{C}$ ratio of the overall product mixture will be equal to:

$\frac{(\mathrm{H}: \mathrm{C})_{1}\left(\text { moles } \mathrm{P}_{1}\right)}{\left(\text { moles } \mathrm{P}_{1}+\mathrm{P}_{2}\right)}+\frac{(\mathrm{H}: \mathrm{C})_{2}\left(\text { moles } \mathrm{P}_{2}\right)}{\left(\text { moles } \mathrm{P}_{1}+\mathrm{P}_{2}\right)}=\frac{\mathrm{H}: \mathrm{C} \text { of coal }}{\text { products }}$

Therefore, the $\mathrm{H}: \mathrm{C}$ ratio of the coal products is a measure of the number of desirable reactions and an indication of the amount of hydrogen available.

The kinetics is further complicated by a competing reaction which also utilizes hydrogen. As discussed earlier, the removal of heteroatoms requires hydrogen. There is evidence that 
this reaction is a preferential one and that hydrogen used for heteroatom removal is at the expense of product formation. Longer residence times at reaction temperature will allow more removal of heteroatoms at the expense of the $\mathrm{H}: \mathrm{C}$ ratio of the products. This effect has been confirmed. Whitehurst (2) concluded that "long contact time thermal processes have the intrinsic disadvantage of poor selectivity for light hydrocarbon gas formation relative to heteroatom removal." Figure 1, where the removal of oxygen from the products represents conversion of heteroatoms, demonstrates this effect.

The production of large quantities of free radicals without hydrogen leads to an unstable condition. In addition, there is evidence that this step of the conversion involves a catalyst. Test results (5) indicate that pyrite or pyrrhotite (derived from pyrite during coal liquefaction) catalyzes coal liquefaction, increasing the conversion to liquid and gaseous products and decreasing the sulfur content of the products. Also, in some processes, a commercial catalyst is used in an attempt to improve yield.

This catalytic effect further increases the demand for hydrogen. Since hydrogen is sparingly soluble in the reaction medium and the reaction is a three phase catalytic type, it is very similar to reactions such as fermentation and catalytic oxidation. These other reactions are known to be gas-liquid mass transfer limited $(6,7)$ and it is theorized that the main resistance to coal liquefaction lies also in gas-liquid mass transfer.

\section{Gas-Liquid Mass Transfer}

The assumption that the hydrogenation of free radical fragments from the thermal disruption of coal is gas-liquid mass transfer limited leads to the conclusion that the reaction rate is strongly dependent on the movement of hydrogen through the gas-liquid interface. The hydrogenation reaction will take place very fast and consume large quantities of hydrogen quickly because the free radicals are unstable. The inventory of dissolved hydrogen is relatively small, so it must be continuously added to the reaction mixture to maintain the reaction rate. This is not a simple task since the low hydrogen solubility

- guarantees that the concentration difference which drives the transfer of hydrogen from one zone to another is always very small.

The two equilibrated interfacial concentrations, $c_{g i}$ and $c_{l i}$ on the gas and liquid sides shown in Figure 2, may typically be related through a linear partition-law relationship such as Henry's law:

$$
M c_{\mathrm{lj}}=\mathrm{c}_{\mathrm{gi}}
$$

At steady state, the hydrogen transfer rate to the gas-liquid interface equals its transfer rate through the liquid side film. This can be written as follows: 


$$
\begin{aligned}
\text { Hydrogen flux } & =\operatorname{mol~} \mathrm{H}_{2} /\left(\mathrm{cm}^{2}-\mathrm{sec}\right) \\
& =k_{8}\left(c_{8}-c_{81}\right) \text { gas side } \\
& =k_{1}\left(c_{1}-c_{11}\right) \quad \text { liquid side }
\end{aligned}
$$

where $c_{z}$ and $c_{1}$ are the hydrogen concentrations in the bulk gas and liquid sides, as shown in Figure 2.

The interfacial concentrations are difficult to measure experimentally. An overall mass transfer coefficient, $K_{1}$, and overall concentration driving force, $\left(c^{*}-c_{1}\right)$, are more easily determined. The liquid phase concentration, $c_{1}^{*}$, is in equilibrium with the bulk gas phase:

$$
M c_{1}^{*}=c_{8}
$$

Therefore, the hydrogen flux in the liquid phase is given by:

$$
\text { Hydrogen flux }=K_{1}\left(c_{1}^{*}-c_{1}\right)
$$

The overall mass-transfer coefficient, $K_{1}$, then becomes:

$$
\frac{1}{K_{l}}=\frac{1}{k_{l}}+\frac{1}{M k_{l}}
$$

For a sparingly soluble gas species like hydrogen, $M$ is much larger than unity. In addition, $k_{z}$ is usually considerably larger than $k_{4}$. In this case, $k_{4}$ becomes approximately equal to $k_{4}$. Essentially, then, all the resistance to mass transfer lies on the liquid side of the gas-liquid interface.

The hydrogen-transfer rate per unit reactor volume,

$$
\begin{aligned}
\mathbf{Q}_{\mathrm{H}_{2}} & =\text { hydrogen absorption rate } \\
& =\frac{(\text { flux }) \text { (interfacial area) }}{\text { reactor liquid volume }} \\
& =k_{1}\left(c_{1}^{*}-c_{1}\right) \frac{A}{V} \\
& =k_{1} a^{\prime}\left(c^{*}-c_{1}\right)
\end{aligned}
$$

where $a^{\prime}=A V$ is the gas-liquid interfacial area per unit liquid volume. The approximation $\mathrm{K}_{1}=\mathrm{k}_{1}$ has also been incorporated. The symbol, a, often used in other transfer rate expressions is the gas-liquid interfacial area per unit volume of reactor (gas + liquid) contents. The two areas are related by the gas holdup volume, $\mathrm{H}$ :

$$
a^{\prime}(1-H)=a
$$

where $\mathrm{H}$ is the volume of gas (total bubble volume) per reactor volume. The head space gas is not included in either expression. 
The hydrogen consumption rate, defined above, is the local volumetric rate at a specific point. The average volumetric absorption rate (moles of hydrogen per time per volume) in a volume, V, is given by:

$$
\overline{\mathrm{Q}}_{\mathrm{H}_{2}}=\int_{\mathrm{V}}^{0} \frac{1}{\mathrm{~V}} \mathbf{Q}_{2} \mathrm{dV}
$$

Typically, $\bar{Q}_{\mathrm{H}_{2}}$ is eciual to $\mathrm{Q}_{\mathrm{B}_{2}}$ only if the hydrodynamic conditions are uniform throughout the vessel. These hydrodynamic conditions include interfacial area per reactor volume and hydrogen concentration.

\section{Hydrogen Deficit}

The maximum concentration of gas at the gas-liquid interface, $c_{1}^{*}$, is dependent on the solubility of hydrogen in the reaction solvent (usually tetralin) at the reaction temperature. The solubility will determine the maximum driving force, $\left(c_{1}^{*}-c_{1}\right)$, across the gas-liquid interface. This maximum driving force will, in turn, provide a maximum hydrogen supply rate to the reaction.

The maximum gas transfer rate could possibly be achieved by setting $c_{1}$ equal to 0 and $c_{1}^{*}$ equal to the solubility of hydrogen in the reaction solvent at the reaction temperature. In this scenario, all the hydrogen entering the bulk liquid solution through the liquid film is immediately consumed by the reaction. This would occur when the reaction rate is very fast and where the hydrogen concentration, $c_{i}^{*}$, approached the solubility of hydrogen.

If the hydrogen concentration is assumed to be at the saturation point, then $c_{1}^{*}$ would equal the saturation concentration of hydrogen at the temperature and pressure. This value can be determined from published or experimental data. Assuming that the local hydrogen consumption rate is equal to the average consumption rate $\left(\bar{Q}_{H_{2}}\right.$ equal to $\left.\mathrm{QH}_{2}\right)$, a hydrogen supply rate can be calculated from the following:

$$
\bar{Q}_{\mathrm{H}_{2}}=Q_{\text {oupply }]}=c_{1}^{*}\left[k_{1} a^{\prime}\right]
$$

Hyd̈rogen supply rates with time for various $k_{q} a^{\prime}$ values are shown in Figure 3.

For the purposes of this discussion, it is assumed that the hydrogen demand rate is dependeni only on the conversion of free radicals to product and no additional reactant is fed after time equals zero. In this scenario, the hydrogen demand will be proportional to the formation of free radicals with time, where the hydrogen requirements for heteroatom removal and the effects of the catalyst on reaction rate have been ignored. The reaction rate 
for a typical autocatalytic reaction (8) is:

$$
\mathbf{r}=\mathbf{k}[\mathrm{A}][\mathrm{R}]=\frac{\mathrm{d}[\mathrm{R}]}{\mathrm{dt}}
$$

where $k=k_{0} e^{-E R T}$. The demand for hydrogen will be proportional to the rate of formation of free radicals, using the aforementioned assumptions. Therefore, $Q_{\text {(domand }}$ is proportional to $\mathrm{d}[\mathrm{R}] \mathrm{dt}$. Free radical formation does not occur until the reaction mixture is above a threshold temperature. Time was set equal to zero at this point in the reaction and $Q_{\text {[demand] }}$ was calculated using a constant reaction temperature. As shown in Figure 3, the demand rate reaches a maximum and then drops to zero as the reactant is consumed. The difference in the hydrogen demand and supply at any residence time is equal to the hydrogen deficit.

As discussed earlier, the $\mathrm{H}: \mathrm{C}$ ratio of the coal products is a measure of the number of undesirable reactions and an indication of the magnitude of the hydrogen deficit. The hydrogen deficit will affect the number of moles of product 1 formed. At the end of the residence time in the reactor, the reaction mixture temperature is lowered to quench the reaction. Since a hydrogen deficit will exist at all but the longest residence times, the number of moles of free radicals at the end of the residence time will convert to moles of product 2. The number of moles of product 1 and 2 determines the $\mathrm{H}: \mathrm{C}$ ratio of the products.

Therefore, if the assumptions made are correct, Figure 3 predicts that the $\mathrm{H}: \mathrm{C}$ ratio of the overall products will decrease with residence time until the reactant is depleted. At this point, the $\mathrm{H}: \mathrm{C}$ ratio of the coal products will start to increase.

Whitehurst (2) performed tests where the $\mathrm{H}: \mathrm{C}$ ratio of the coal products was determined with residence time. This data is represented in Figure 4. As predicted, the H:C ratio of the coal products in this reactor system reached a minimum at a residence time of approximately 95 minutes and then increased.

It is concluded, then, that residence time determines the concentration of free radicals (maximum conversion) and hydrogen concentration determines the conversion of these free radicals into the desired product (product selectivity).

Figure 3 predicts that the magnitude of the hydrogen deficit is related to the reactor design. The hydrogen demand is related to residence time in the reactor and the reaction temperature. The hydrogen supply is dependent on the mass transfer coefficient, reaction temperature, and residence time.

\section{Reactor Design}

Previous work in coal liquefaction has involved attempts to influence yield by the addition of catalyst and/or increasing temperature and residence time. These attempts have 
met with only limited success. The kinetics of the reaction illustrate the reason for the difficulties. As residence time increases at temperatures where thermal disruption occurs, the free radical concentration increases exponentially. However, the hydrogen supply is insufficient to saturate all the free radicals, so less desirable heavier hydrocarbons are formed.

Selection of a reactor is a two step process; (1) choice of the type of reactor, and (2) design of reaction conditions and geometry of the reactor system. The best reactor design will optimize productivity and selectivity.

The production of free radicals determines the productivity. Autocatalytic reactions are more efficient in mixed reactors for low conversions, while plug flow reactors are preferred for high conversions (8). A repetitive low conversion is planned for the current reactor scheme and, therefore, it is concluded that thermal degradation would be more efficient in a mixed reactor.

On the other hand, selectivity is dependent on the availability of hydrogen to supply consecutive reactions after free radical formation. The principal factors affecting selectivity are:

- Solubility of gas in liquid

- Relation between the reaction rate and mass transfer rate

- Temperature of the reaction mixture

- Gas pressure

- Interfacial area related to unit volume of liquid

- Intensity of axial mixing in both phases

- Arrangement of the flows of gas and liquid in the reactor (co- or counter-current)

There are two extreme cases which can be used to compare reactor designs in relation to their suitability for product selectivity. The relative magnitude of the effect of mass transfer on the reaction rate $\left((\mathrm{H}: \mathrm{C})_{1}, \mathrm{p} .7\right)$ is used to distinguish the two cases.

First, it is assumed that the reaction is instantaneous and irreversible. Therefore, the 1 reaction rate is controlled by the transport of gas to the reaction. The productivity of such a reactor is determined primarily by the extent of the interfacial area and not by the liquid hold-up volume. The reaction rate can be thought of as an accelerating factor for the rate of physical absorption of the gas due to its affect on the concentration driving force, as discussed earlier. Thus, high liquid velocity and turbulence would be preferred. Designs which are used for physical absorption, such as packed columns, would be adaptable for this case. 
In the second case, it is assumed that the reaction is very slow. For this condition, the concentration of dissolved gas, $c_{1}$, is controlled by phase equilibrium (solubility). A relatively long residence time is desirable in order to allow sufficient contact time. Thus, a reactor with a large liquid hold-up volume and low velocity would be preferred. Mass transfer has no effect on the reaction rate in this case. However, the mass transfer does affect product selectivity. For example, if the desired reaction product undergoes one or more consecutive reactions, an insufficient rate of mass transfer in the liquid phase may cause a decrease in the selectivity.

Free radical conversion to product in coal liquefaction falls into the first case. Therefore, a packed column with its small liquid hold-up volume and high mass transfer would be preferred for hydrogenation. So, even though conversion can be optimized in an agitated reactor a packed column is better for product selectivity. It was concluded that product selectivity was the more important criteria for reactor selection and therefore a packed column design was used.

The hydrogen supply to the reaction can be increased through equipment modifications. The gas concentration at the gas-liquid interface, $c_{1}{ }^{*}$, is determined by the gas transfer rate. Factors which affect the average gas transfer rate include power input per unit volume, fluid and dispersion rheology, gross flow patterns in the reactor, coalescence and redispersion rates, bubble size distribution, total bubble surface area, holdup (gas volume fraction), and gas and liquid residence time distributions. These factors, in turn, are dependent on equipment design. In fact, the equipment design is the most influential factor in determining the gas concentration, $c_{1}{ }^{*}$, at the gas-liquid interface. Therefore, an improvement in yield or reaction time with a mass-transfer-limited reaction from modification in equipment design would indicate that the design provided better gas-liquid mass transfer.

The maximum value for $c_{1}{ }^{*}$ is equal to the solublity of the gas in the reaction mixture. This maximum value is only dependent on temperature and pressure. The solubility of hydrogen in tetralin increases with increasing temperature (9). Therefore, an increase in temperature will increase $q^{*}$ and result in a higher hydrogen supply rate. However, the magnitude of the increase is small.

The hydrogen demand rate is determined by the reaction kinetics. As discussed earlier, the formation of free radicals will take place if the mixture is above a threshold temperature. The concentration of free radicals (related to $Q_{\text {demand }}$ as shown on Figure 3) with time will follow an exponential curve until a maximum free radical concentration is reached. 
There are several factors which could reduce the hydrogen deficit. Hydrogen solubility can be improved by using higher pressures or temperatures, thus increasing the maximum value of $c_{1}^{*}$. Dilution of reactant in the bulk liquid will increase $c_{1}$. However, the magnitude of these changes is too small to completely eliminate the deficit.

This scenario suggests that while hydrogenation at a constant temperature for long residence times will produce high conversion, it will not provide the product selectivity needed. One option is to slow the formation of free radicals by controlling residence time when the temperature is above the threshold for free radical formation. A very short reaction time combined with good mass transfer (high hydrogen supply rate) will allow operation of the reactor in a zone at the left side of Figure 3, where the hydrogen deficit is minimized. In addition, by limiting the time the reaction mixture is exposed to a hydrogen deficit, catalyst deactivation is prevented and product selectivity is obtained.

One is left with the dilemma that short contact thermal time is desired from the standpoint of product selectivity and controlling heteroatom removal, but product conversion with short residence times is small and therefore not viable economically. Reactor selection must balance these competing forces. Recycle of the reaction mixture through a reactor system containing a short contact thermal time with a quench cycle will provide high conversions with product selectivity.

The current reactor system utilizes this concept to continuously cycle the reaction mixture. The cycle is made up of a series of residence times under various operating conditions.

\section{MODEL REACTION STUDIES}

\section{Introduction}

Coal liquefaction, as discussed earlier, involves a complex set of reactions. Major reactions known to occur (1) include decarboxylation, desulfurization, denitrogenation,

a deoxygenation, and hydrogenation of aromatic rings. The complexity of the reaction mechanism does not lend itself to experimental testing of equipment designs. Therefore, several model reactions, known to be limited by gas liquid mass transfer, were used to evaluate equipment designs for their potential to improve mass transfer. The model reaction selected was hydrogenation of naphthalene to tetralin.

\section{Hydrogenation of Naphthalene}

In the hydrogenation of naphthalene to tetralin, free radicals will be formed if the 
reaction mixture is at temperature $\left(>90^{\circ} \mathrm{C}\right)$. Product formation will occur only if hydrogen is available with sufficient catalyst. It has been reported (9) that in catalytic liquid phase hydrogenation with suspended powder catalysts the reaction is zero order with respect to the hydrogenated product. The hydrogenation of naphthalene has been shown to be gas-liquid mass transfer limited when sufficient catalyst is present. In gas-liquid mass transfer, the most influential factor is equipment design. Therefore, improvements in yield or reaction time would indicate that the reactor design provided better gas-liquid mass transfer.

Different equipment designs were investigated and evaluated for mass transfer improvements. in the current work.

In naphthalene bydrogenation, the bulk chemical reaction rate would equal $k_{1} a^{\prime} c_{1}{ }^{*}$, if it is assumed that $c_{1-} 0$. There is negligible total reaction rate in the film compared to the mass transfer rate, $k_{1}\left(c_{1}^{*}-c_{1}\right)$. When gas is continually added to the reaction mixture, a hydrogen mass balance on the gas phase determines the maximum consumption rate, $Q_{\mathrm{H}}$ :

$$
\begin{aligned}
\mathrm{Q}_{\mathrm{B}_{2}}= & {\left[\mathrm{F}_{\mathrm{z} \text { inlet }}\left(\mathrm{P}_{\mathrm{B}_{2}}\right)_{\text {inlet }}\right] \text { VRT } } \\
= & \text { average volumetric rate of } \\
& \text { hydrogen utilization } \\
= & \text { moles hydrogen/volume-time }
\end{aligned}
$$

where $F_{g}$ is the volumetric gas flow rate, $P_{\mathrm{H}_{2}}$ is the partial pressure of hydrogen, and hydrogen is assumed to behave as an ideal gas.

If the hydrogen concentration is assumed to be at the saturation point, then $c_{1}{ }^{*}$ would equal the saturation concentration of hydrogen at the temperature and pressure. This value can be determined from published or experimental data. If the local hydrogen consumption rate is assumed to be equal to the average consumption rate, then $Q_{\mathrm{H}_{2}}$ would equal ${ }^{Q} \mathrm{H}_{2}$. Therefore, a minimum $k_{1} a^{\prime}$ value for the hydrogenation reaction system can be calculated from the following:

$$
\mathbf{k}_{1} \mathbf{a}^{\prime}=\overline{\mathrm{Q}}_{\mathrm{H}_{2}} / \mathrm{c}_{1}^{*}
$$

The average hydrogen consumption rate is a measurable quantity in the current system and minimum $k_{1} a$ ' values can be determined for each experiment.

The overall objective of these model reaction studies was to develop a reactor system that would besi allow reactant gas mass transfer to the solid-catalyst-liquid reactant slurry at a sufficient rate to minimize retrogressive reactions and resultant catalyst deactivation. These efforts have been successful as is indicated in Figures 5 and 6 . Firure 5 shows the 
rates of naphthalene conversion in the current system, and Figure 6 shows those in the reactant system used at the beginning of the work year. Eighty weight percent conversion was obtained in about 15 minutes as opposed to about 25 minutes in the original system. Furthermore, a lower reaction temperature was used in the current system $\left(156^{\circ} \mathrm{C}\right.$ as opposed to $162^{\circ} \mathrm{C}$ ). Even when a reaction temperature of only $147^{\circ} \mathrm{C}$ was used, $80 \mathrm{wt} \%$ conversion was obtained in only about 21 minutes. These improvements have been the result of improved mass transfer and reaction-rate control in the reaction system.

\section{Physical Description of Reactor System}

The hydrogenation reactor system can be divided into three interrelated subsystems. These are: 1) the basic system consisting of a packed column reactor unit and liquid pump; 2) a gas recirculation unit; and 3) a liquid heating system and temperature control.

In the basic system, a packed column reactor unit is employed which consists of seven stainless steel reactor tubes, each 8" L x 1" O.D., and connected in series by 0.5" O.D. tubing. The reactor tubes are oriented vertically and each is filled with stainless steel packing. The entire packed column reactor unit is immersed in a fluidized sand bath which is connected to a PID controller.

The slurry is pumped through the closed-loop system by a positive displacement metering pump (other types of pumps are being evaluated for suitability as well). As the slurry leaves the pump, it flows first through a preheater which increases the temperature by approximately $25^{\circ} \mathrm{C}$. The slurry then flows through the packed column reactor unit, through a chiller into a gas-liquid decoupling chamber, and finally back to the liquid pump. Both the preheater and chiller are composed of a length of 0.5 " O.D. tubing surrounded by a water jacket.

The gas recirculation unit consists of a gas-liquid decoupling chamber, three condensation tubes (gas-liquid separators in Figure 7), and an air-driven gas compressor. The gas is drawn from the decoupling chamber into each condensatior abe in succession.

- Any vapors in the gas condense into liquid and are drained after each run. The gas is then fed to the gas compressor and pumped back into the slurry at a point in the discharge side of the liquid pump.

The liquid heating system is composed of a custom-made cylindrical tank with three electric water heaters, a centrifugal pump, and associated tubing. Ethylene glycol is preheated to a certain temperature. It is then circulated to the preheater and back to the tank.

The liquid heating system together with the chiller and heated fluidized sand bath are 
responsible for temperature control. As the slurry flows through the reactor system, it is first heated to a certain temperature by the preheater. It then flows through the packed column reactor unit which is immersed in the heated fluidized sand bath. The sand bath, which has been preheated to a selected temperature, increases the temperature of the slurry to the level necessary to support hydrogenation reactions.

After leaving the packed column unit, the slurry flows through the chiller which then cools it to a level at or below that which is necessary to sustain the chemical reaction. Ordinary tap water at room temperature flows through the chiller's water jacket.

The external tubing connecting the liquid pump, preheater, packed column reactor unit, chiller, and decoupling chamber is $0.5 "$ in diameter. The tubing used in the gas recirculation unit is $0.25^{\prime \prime}$ O.D.

These three areas - the preheater, the heated fluidized sand bath, and the chiller offer very good temperature control of the slurry and also allow the investigation of the effects of different temperature parameters within the limits of the system.

\section{Modeling of the Reactor System}

The system was modeled as a plug flow reactor in that it was assumed to consist of five consecutive well-mixed reactors (CSTR) in series. Mass balance equations for the two components in each reactor, naphthalene and hydrogen, resulted in ten ordinary differential equations. Heat transfer equations for each reactor added five additional differential equations. The 15 ordinary differential equations were solved simultaneously using System Simulation Language (SYSL). The fourth order Runge-Kuita fixed step size method was used to solve the equations.

The purpose of the modeling was to describe the system mathematically and predict the performance of the system for various operating conditions. A knowledge of the heat and mass transfer coefficients of the system is essential in order to simulate its performance. The heat transfer coefficient (UA) was obtained by using the actual inlet and outlet temperatures for the reactor. This coefficient was assumed to be the same in each reactor tube. The results obtained from the simulation and the experiments compared well, when a mass transfer coefficient $\left(K_{L_{e}}\right)$ of $5 \mathrm{~min}^{-1}$ was used. This value is higher than the $K_{\mathrm{La}}$ value in a bubble column $\left(1.2 \mathrm{~min}^{-1}\right)$.

The conversion versus time plots for various catalyst loadings are shown in Figures 811. A comparison between the experimental runs and the simulations shows the best agreement at a catalyst loading of 5\%. The slope of the conversion versus time plot is an indication of the rate of the reaction. The slopes from the experimental plot and from the 
simulation results compared well at catalyst loading of $5 \%, 7.5 \%$, and $15 \%$ while those at a $10 \%$ catalyst loading did not agree. The model has to be improved in order to predict the performance of the system at various catalyst loadings.

The rate constant and the activation energy for the reaction were obtained from earlier work at Auburn University. These values are given in Table 1. The frequency factor in the Arrhenius equation was determined using the rate constant at one particular temperature. The factor was then used to calculate the rate constants at various temperatures in the reactor. The notation used in the program is given in Table 1. The results from a simulation run for a catalyst loading of 5\% are shown in Figures 15 and 16 . The concentration of hydrogen in the reactors does not go below $2.800 \times 10^{-5} \mathrm{gm}$-moles/cc. The saturation concentration is $4.5706 \times 10^{-5} \mathrm{gm}$-moles/cc. The temperature of the liquid coming out of the fifth reactor was $149^{\circ} \mathrm{C}$ which compared well with an outlet temperature of $152^{\circ} \mathrm{C}$ in the actual run.

The mass transfer coefficient $\left(K_{Y} a\right)$ in the system was determined experimentally by running a series of reactions using various catalyst loadings and by mathematically simulating the system. A plot of $1 /$ rate vs 1 /catalyst loading allows computation of the mass transfer coefficient. Figure 12 compares the results from the experiment and the simulation for catalyst loadings of 5-15\%. The values obtained for the $k_{1}$ a were $7.52 \mathrm{~min}^{-1}$ using model simulation and $8.87 \mathrm{~min}^{-1}$ from the experimental results.

The model was used to simulate the performance of the system at higher catalyst loadings. The results from these simulations then were used to draw a plot of $1 /$ rate versus 1/catalyst loading at catalyst loadings between 5-30\% (Figure 13). The experimental results for catalyst loadings between $5-15 \%$ are also shown in Figure 13. The simulation curve indicates two regions of operation: a completely mass transfer controlled region and a region in which both mass transfer and kinetics are important. It is evident from the experimental curve that, at the base line operating conditions ( $10 \%$ catalyst loading), the present system is operating in the region in which both mass transfer and kinetics are of consequence. The gas consumption rate, which is proportional to the rate of the reaction, is shown for catalyst loadings (5-15\%) in Figure 14. The reactor system design has been improved sufficiently so that, for reactions occurring at rates comparable to that of naphthalene hydrogenation, it should not be mass transfer controlled. We will now proceed to examine faster reaction systems. 


\section{Gas-Liquid Contactor Evaluation}

\section{Introduction}

A literature review was performed to investigate different equipment designs for their potential to increase gas-liquid mass transfer. Three general areas were selected for review. These areas were (1) modifications for packed columns, (2) mechanical agitation, and (3) gasliquid nozzles.

\section{Reactors}

Several reactors are typically used for bench scale coal liquefaction. These reactors include tubing bomb microreactors, stirred autoclaves, and bubble columns. Tubing bomb microreactors utilize vertical oscillations of the entire reactor for mixing of the reactor contents. An impeller and baffles are used in stirred autoclaves for mixing. However, in bubble columns gas-liquid mixing occurs primarily from dispersion of the gas phase in the slurry. Typically, the gas is bubbled through the slurry, containing a solvent and suspended solids, for gas-liquid contact.

\section{Packed Columns}

A series of packed columns were employed as reactors in the hydrogenation of naphthalene to tetralin. Stainless steel packing rings of varying sizes were used in the columns to evaluate the effect on the gas-liquid mass transfer coefficient. Two sizes (0.4" and $0.625 "$ ) of packing were tested first with a sodium sulfite solution and then in the hydrogenation reaction.

\section{Mechanical Agitator}

Another reactor design to be investigated is a continuous stirred-tank reactor. A one gallon Autoclave Engineers reactor was modified with a sleeve and multiple impellers. The sleeve will contain four baffles plus feed and discharge lines. The baffles will be spaced onehalf the baffle width from the vessel wall to prevent accumulation of solids based on standard design conventions (11). The baffle width will be equivalent to one tenth the vessel inside diameter based on standard ratios (12). Industrial stirred tank reactors typically have impeller-to-tank diameter ratios of 0.1 to 0.5 (13). The impeller-to-tank diameter ratio to be used in this design is 0.5 .

\section{Gas-Liquid Nozzles}

Gas-liquid nozzles are used to form the initial gas-liquid interface. This initial contact 
step is a critical one since gas bubble size throughout the reactor system is dependent on the initial gas bubble size formed in this contactor. The parameters affecting the gas-liquid contactor efficiency are gas velocity, liquid velocity, and coalescence of bubbles in the reaction slurry. The coalescence is, in turn, influenced by the solids content and salt concentration of the reaction mixture. The presence of solids has been shown(14)to increase coalescence and therefore lower mass transfer. However, this effect does not become significant until a solids content over $10 \%$ is reached. Salt concentration in the reaction mixture will also affect coalescence. Tests $(14,15)$ indicate that low sodium chloride concentrations favored mass transfer in a slot injector.

\section{Modified Slot Injector}

The literature review uncovered a slot injector design (Figure 17) which had provided higher gas-liquid mass transfer coefficients than venturis and orifices when tested in waste treatment systems. Waste treatment reactions typically have many similarities to hydrogenation and other mass transfer limited reactions. Both reactions involve a threephase slurry with a slightly soluble gas. The oxidation of waste treatment streams has been demonstrated to be mass transfer limited. Since this reaction has similar limitations, the results and equipment designs can be extrapolated. The slot injector was modified for use in the hydrogenation reaction piping system. The equipment used for initial contact of the gas and liquid is a critical parameter. Theory indicates that the initial contact under unsteady state conditions can affect the gas-liquid mass transfer more than any other factor.

\section{Packed Column Modifications}

It has been demonstrated (Figure 13) that liquid velocity in a packed column is a more influential factor than gas velocity on the average mass transfer coefficient. Once the gas velocity exceeds a relatively low threshold value, an increase in the liquid velocity increases the average mass transfer coefficient linearly. Thus, a higher pump rate in the - current reactor system would be expected to increase the mass transfer and provide a higher conversion and/or faster reaction time.

\section{Point Gas-Liquid Contactors (Motionless Mixers)}

A variety of saturators having different designs and sizes were procured and evaluated (refer to Table 5 for a list of saturators purchased). The mass transfer coefficients of some of these saturators were measured in a cold-flow test bench system using the standard $\mathrm{O}_{2}-\mathrm{Na}_{2} \mathrm{SO}_{3}$ reaction system. The mass transfer coefficients ranged from 0.1 to 0.02 
$\left(\mathrm{s}^{-1}\right)$. This is comparable to that expected in a bubble column, as shown in Table 2. This is about the highest rate of mass transfer that can be achieved in a standard Autoclave Engineers reactor (Figure 19), as is shown in Table 3. It is only at the highest stirring rate $(2,150 \mathrm{rpm})$ that the mass transfer rate becomes comparable to that of a bubble column $\left(k_{\mathrm{L}} \mathrm{a}\right.$ $0.02 \mathrm{~s}^{-1}$ ). It should be noted, however, that in a tubing bomb reactor (Figure 20), much higher mass transfer rates were attained (as high as $3.8 \mathrm{~s}^{-1}$, Table 4). And even in a tubing bomb reactor, mass transfer related problems have been experienced in the past. With the control system under development, even though the actual saturator efficiency is not as high as desired, it is felt that excellent overall mass transfer rates can be attained. This can be accomplished by properly adjusting the extent of reaction per cycle, that is, reaction rate, as has been discussed in earlier reports.

Using the reactor system in place at the beginning of the year, different saturators were evaluated. Generally, it was found that the motionless-mixer type saturators performed as well as, if not better than, others for saturating the reactant liquid at a specific point in the recycle loop. When a $1 / 2$ inch diameter saturator was used, there were no apparent significant plugging problems due to catalyst collecting in the saturator, as indicated by the relatively small degree of standard deviation among different reaction runs. The mechanical saturator had the least amount of standard deviation, and presumably, the least amount of plugging $( \pm 0.15$ versus \pm 0.46 and \pm 0.30 for the $1 / 2$ inch and $1 / 4$ inch motionless mixer type saturators, respectively, Table 5).

Table 6 shows the response observed in the rate of naphthalene conversion to the number of motionless mixers used. Originally the contactors were evenly distributed across the length of the reactor. Separate reactions were performed, each having a different number of contactors distributed across the reactor.

No apparent difference in reaction rate was observed as the number of contactors was reduced from five to none. Apparently the packing in the reactors, for the liquid flow rates used, was having a dominant mixing effect. The hydrogen solubility is so low that point saturation with $\mathrm{H}_{2}$, as occurs with the contactors such as motionless mixers, has little or no effect on overall reaction rates.

\section{BIBLIOGRAPHY}

1. Crow, M., B. Bozeman, W. Meyer, and R. Shangraw, Jr., "Synthetic Fuel Technology Development in the United States," Praeger Publishers, New York (1988). New York (1973). 
2. Whitehurst, D. D., A New Outlook on Coal Liquefaction through Short-Contact-Time Thermal Reactions: Factors Leading to High Reactivity, p. 133 in "Coal Liquefaction Fundamentals," D. D. Whitehurst, ed., American Chemical Society, Washington D.C. (1980).

3. Wiser, W., Fuel, 47, 475 (1968).

4. Petrakis, L., D. W. Grandy, p. 147 in Fuel Div. Preprints, 146th ACS Meeting, Miami, FL (November, 1978).

5. Given, P. H., W. Spackman, A. Davis, and R. G. Jenkins, Some Proved and Unproved Effects of Coal Geochemistry on Liquefaction Behavior with Emphasis on U.S. Coals, p. 3 in "Coal Liquefaction Fundamentals," D. D. Whitehurst, ed., American Chemical Society, Washington D.C. (1980).

6. Van't Riet, K, Mass Transfer in Fermentation, Trends in Biotechnology, 1, 113 (1983).

7. Bailey, J. E. and D. F. Ollis, "Biochemical Engineering Fundamentals," 2nd ed., McGrawHill, New York (1986). 8. Levenspiel, O., "Chemical Reaction Engineering,: 2nd ed., John Wiley \& Sons, New York
(1972).

9. Simnick, J. J., C. C. Lawson, H. M. Lin, and K. C. Caho, Vapor-Liquid Equilibrium of Hydrogen/Tetralin System at Elevated Temperatures and Pressures, AIChE Journal, 23, 469 (1977).

10. Horak, J., J. Pasek, "Design of Industrial Chemical Reactors from Laboratory Data," Heyden \& Son Ltd., London (1978).

11. Perry, R. F. and C. H. Chilton, "Chemical Engineer's Handbook,: 5th ed., McGraw-Hill, New York (1973).

12. Treybal, R. E., "Mass Transfer Operations," McGraw-Hill, New York (1968).

13. Michel, B. J. and S. A. Miller, Power Requirements of Gas-Liquid Agitated Systems, AIChE Journal, 8, 262, (1962).

14. Zlorkarnik, M., Sorption Characteristics of Slot Injectors and Their Dependency on Coalescence Behavior of the System, Chem. Eng. Sci., 34, 1265 (1979).

15. Jackson, M. L., Aeration of Bernoulli Types of Devices, AIChE Journal, 10, 836 (1964). 


\section{Table 1: SYSL PROGRAM NOTATION}

\section{CONSTANTS:}

Volume of each reactor tube (V1, V2, V3, V4, V5)

$$
=67 \mathrm{cc}
$$

Volume of the system (other than the reactors) (VH)

$=407 \mathrm{cc}$

Saturation Concentration of Hydrogen in the Solvent (CS)

$=4.57065 \mathrm{E}-5$

Liquid density (RO)

$=1.145 \mathrm{gm} / \mathrm{cc}$

Liquid flow rate (Q)

$=4911.7 \mathrm{cc} / \mathrm{min}$

Specific heat of liquid (CP)

$=0.42$

Activation energy (E)

$=17000 \mathrm{cal} / \mathrm{min}$

Frequency factor (KO)

$=2.825 \mathrm{E} 12 \mathrm{cc} / \mathrm{gm}-\mathrm{moles} \mathrm{min}$

Gas Constant (R)

Heat transfer coefficient (UA)

$=1.987$

Mass transfer coefficient $\left(K_{L} a\right)$

$=488.63 \mathrm{cal} / \mathrm{min}^{\circ} \mathrm{C}$

Inlet temperature to preheater (TPI)

$=5.0 \mathrm{~min}^{-1}$

$=79^{\circ} \mathrm{C}$

Outlet temperature from preheater (TPO)

$=110^{\circ} \mathrm{C}$

Average temperature of sand bath (TH)

$=174.3^{\circ} \mathrm{C}$

VARIABLES:

Temperaure in each reactor tube: $\mathrm{T} 1, \mathrm{~T} 2, \mathrm{~T} 3, \mathrm{~T} 4, \mathrm{~T} 5$

Reaction rate constant in each reactor tube: KS1, KS2, KS3, KS4, KS5

Gas transfer from gas to the liquid phase: N1, N2, N3, N4, N5

Concentration of hydrogen in the liquid phase: $\mathrm{C}_{\mathrm{lr}}{ }^{*}(\mathrm{r}=$ reactor $)$

Concentration of Naphthalene in the liquid phase: $\mathrm{C}_{2 \mathrm{r}}{ }^{*}$

Conversion of Naphthalene: $\mathrm{X}$

*Concentrations are in gm-moles/cc. 
Table 2: Typical Volumetric Mass Transfer Coefficients in Bubble Columns as Reported in the Literature

Reference

System

Approximate Range

of $\mathrm{K}_{\mathrm{L}} \mathrm{a}, \mathrm{sec}^{-1}$

Ostergaard and Fosbol, 1972

$\mathrm{O}_{2}$-water

0.008-0.05

Ostergaard, 1978

Wang and Fan, 1978

$\mathrm{CO}_{2}$-carbonate buffer

0.01-0.03

$\mathrm{O}_{2}$-water

0.03-0.1

Deckwer et al., 1974

$\mathrm{O}_{2}-\mathrm{Na}_{2} \mathrm{SO}_{4}$

0.005-0.1

Greenhalgh et al., 1975

$\mathrm{O}_{2}-\mathrm{Na}_{2} \mathrm{SO}_{3}$

0.01-0.11

Botton et al., 1980

-

$$
0.02-0.12
$$

Mashelkar and Sharma, 1970

$\mathrm{CO}_{2}$-carbonate buffer

0.05-0.13

Hagberg and Krupa, 1976

Air-water

0.01-0.15

Reith and Beek, 1968

$\mathrm{O}_{2}-\mathrm{Na}_{2} \mathrm{SO}_{3}$

0.02-0.15

$\mathrm{Air}-\mathrm{Na}_{2} \mathrm{SO}_{3}$

0.005-0.16

$\mathrm{CO}_{2}$-water

0.01-0.16

Towell et al., 1965

$\mathrm{O}_{2}$-sodium dithionate

0.04-0.12

Juvekar and Sharma, 1973

Table 3: Mass Transfer Coeffients for Oyrgen Absorption in the Sulfate Solution

Impeller Speed

$\frac{\text { rpm }}{60}$

.250

625

775

1050

1650

- 2150

$$
\mathrm{CO}_{2}: \mathrm{k}_{\mathrm{L}} \text { a } \times 10^{4}
$$

$\mathrm{s}^{-1}$

1.59

2.25

3.96

30.91

99.26

688.0

1300.0
$\mathrm{O}_{2}: \mathrm{k}_{\mathrm{L}} \mathrm{a} \times 10^{4}$ *

$\mathrm{s}^{-1}$

1.95

2.75

4.85

37.88

121.6

843.3

1593

*The oxygen coefficients wre obtained from the following relation

$$
k_{L} a_{1}=k_{L} a_{2} \times\left(D_{1} / D_{2}\right)^{2 / 3}
$$

Experimental Conditions:

Temperature $\quad=26^{\circ} \mathrm{C}$

Autoclave volume $=300 \mathrm{cc}$

Liquid volume $\quad=150 \mathrm{cc}$

$\mathrm{D}$ (oxygen) $\quad=1.9 \times 10^{-6} \mathrm{~cm}^{2} / \mathrm{s}$

$\mathrm{D}$ (carbon dioxide) $=1.4 \times 10^{-5} \mathrm{~cm}^{2} / \mathrm{s}$ 


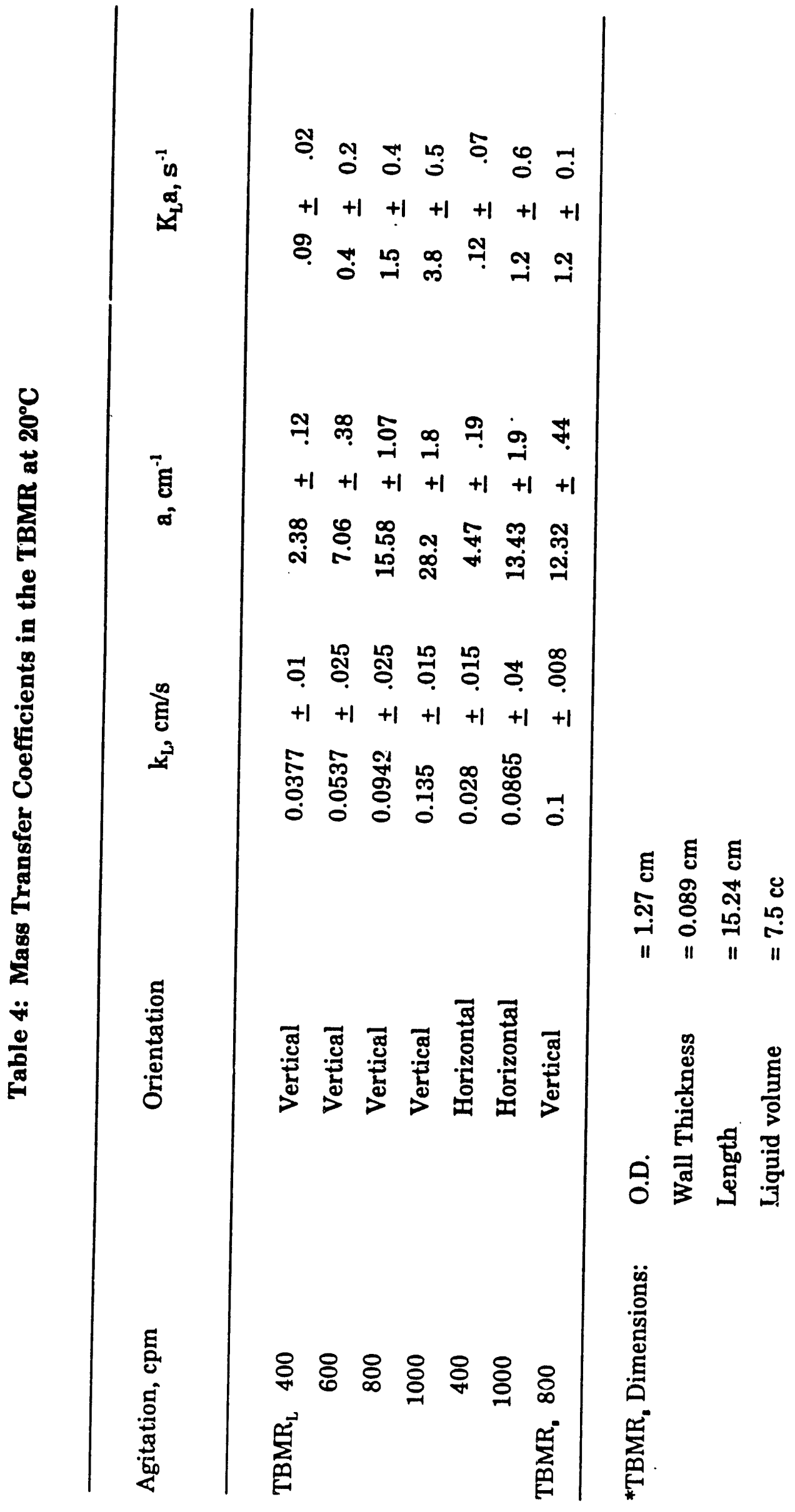


Table 5: Rate of Naphthalene Conversion for Different Motionless Mixers

\begin{tabular}{|c|c|c|c|c|}
\hline $\begin{array}{l}\text { Vendor } \\
\text { and Size }\end{array}$ & Length & $\begin{array}{l}\text { Number of } \\
\text { Elements }\end{array}$ & \multicolumn{2}{|c|}{$\begin{array}{l}\text { Rate of Naphthalene Conversion } \\
\text { (\% conversion / min) }\end{array}$} \\
\hline $\begin{array}{l}\text { EMI } 1 / 4^{\prime \prime} \\
\text { Komax } 1 / 4^{\prime \prime}\end{array}$ & $\begin{array}{l}5.75^{\prime \prime} \\
4.0^{\prime \prime}\end{array}$ & $\begin{array}{l}18 \\
21\end{array}$ & \multicolumn{2}{|c|}{$\begin{array}{l}2.25 \pm 0.29 \\
2.07 \pm 0.30\end{array}$} \\
\hline $\begin{array}{l}\text { EMI } 1 / 2 " \\
\text { Ross } 1 / 2^{\prime \prime}\end{array}$ & $\begin{array}{r}12.75^{\prime \prime} \\
4.25^{\prime \prime}\end{array}$ & $\begin{array}{l}18 \\
6\end{array}$ & \multicolumn{2}{|c|}{$\begin{array}{l}2.23 \\
2.93 \pm 0.46\end{array}$} \\
\hline Koch 1" & $6.0^{\prime \prime}$ & 6 & \multicolumn{2}{|c|}{$1.71 \pm 0.08$} \\
\hline Mean Reactio & $\begin{array}{l}\text { ditions: } \\
\text { Temperature } \\
\text { Catalyst Loadin } \\
\text { Pressure }\end{array}$ & $\begin{array}{l}=102.7 \mathrm{C} \\
\mathrm{g}=7.5 \% \\
=110 \mathrm{psi}\end{array}$ & $\begin{array}{l}\text { Liquid Flow } \\
\text { Gas Flow }\end{array}$ & $\begin{array}{l}=0.22 \mathrm{gpm} \\
=96.5 \mathrm{cu} . \mathrm{in} . / \mathrm{min}\end{array}$ \\
\hline
\end{tabular}

Table 6: Response in Rate of Naphthalene Conversion to the Number of Point Gas -liquid Contactors Used

\begin{tabular}{|c|c|c|}
\hline Number of Mixers* & $\begin{array}{r}\text { Rate } \\
(\%\end{array}$ & $\begin{array}{l}\text { Naphthalene Conversion } \\
\text { onversion / } \mathrm{min} \text { ) }\end{array}$ \\
\hline 5 & & 4.10 \\
\hline 4 & & 4.11 \\
\hline 3 & & 4.30 \\
\hline 2 & & 3.93 \\
\hline 1 & & 4.09 \\
\hline 0 & & 4.25 \\
\hline $\begin{array}{l}\text { Mean Reaction Condit } \\
\text { Temperature } \\
\text { Catalyst Loading } \\
\text { Pressure }\end{array}$ & $\begin{array}{l}\text { 10ns: } \\
=161.2 \mathrm{C} \\
=10.0 \% \\
=110 \mathrm{psi}\end{array}$ & $\begin{array}{ll}\text { Liquid Flow } & =0.22 \mathrm{gpm} \\
\text { Gas Flow } & =96.5 \mathrm{cu} . \mathrm{in} . / \mathrm{min}\end{array}$ \\
\hline
\end{tabular}

- *Original contactors were evenfy distributed across the reactor length. 


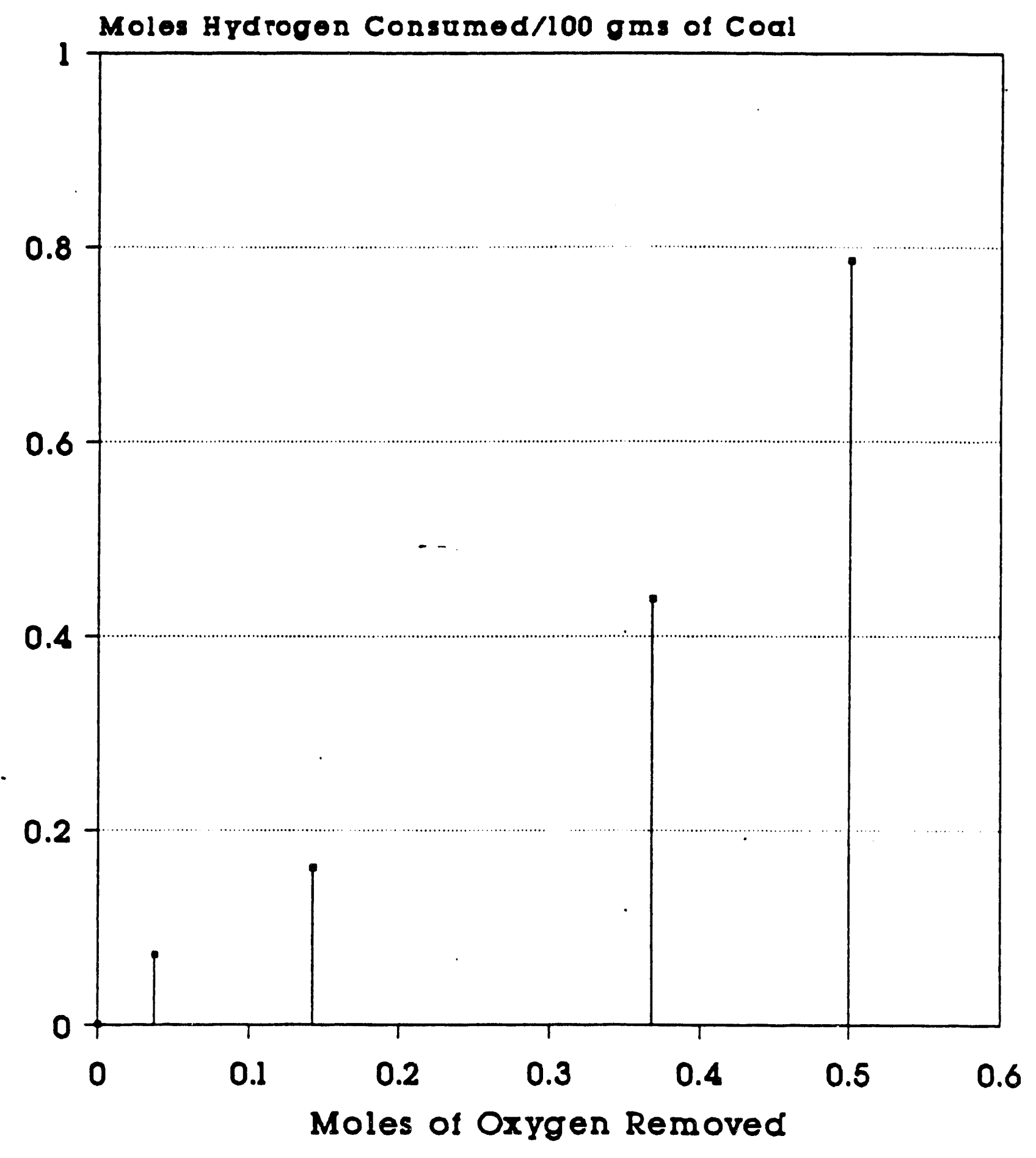

Figure 1: Hydrogen Consumption Related to Heteroatom Removal 


$$
\text { Hydrogen flux } \begin{aligned}
& -m o 1 \mathrm{H}_{2} /(\mathrm{cm}-\mathrm{sec}) \\
& -k_{g}\left(c_{g}-c_{g 1}\right) \text { gas s1de } \\
& =k_{1}\left(c_{1}-c_{11}\right) \quad \text { 11quid side }
\end{aligned}
$$

where $c_{g}$ and $c_{1}$ are the hydrogen concentrations in the bulk gas and IIquid sides, as shown in Figure 2.

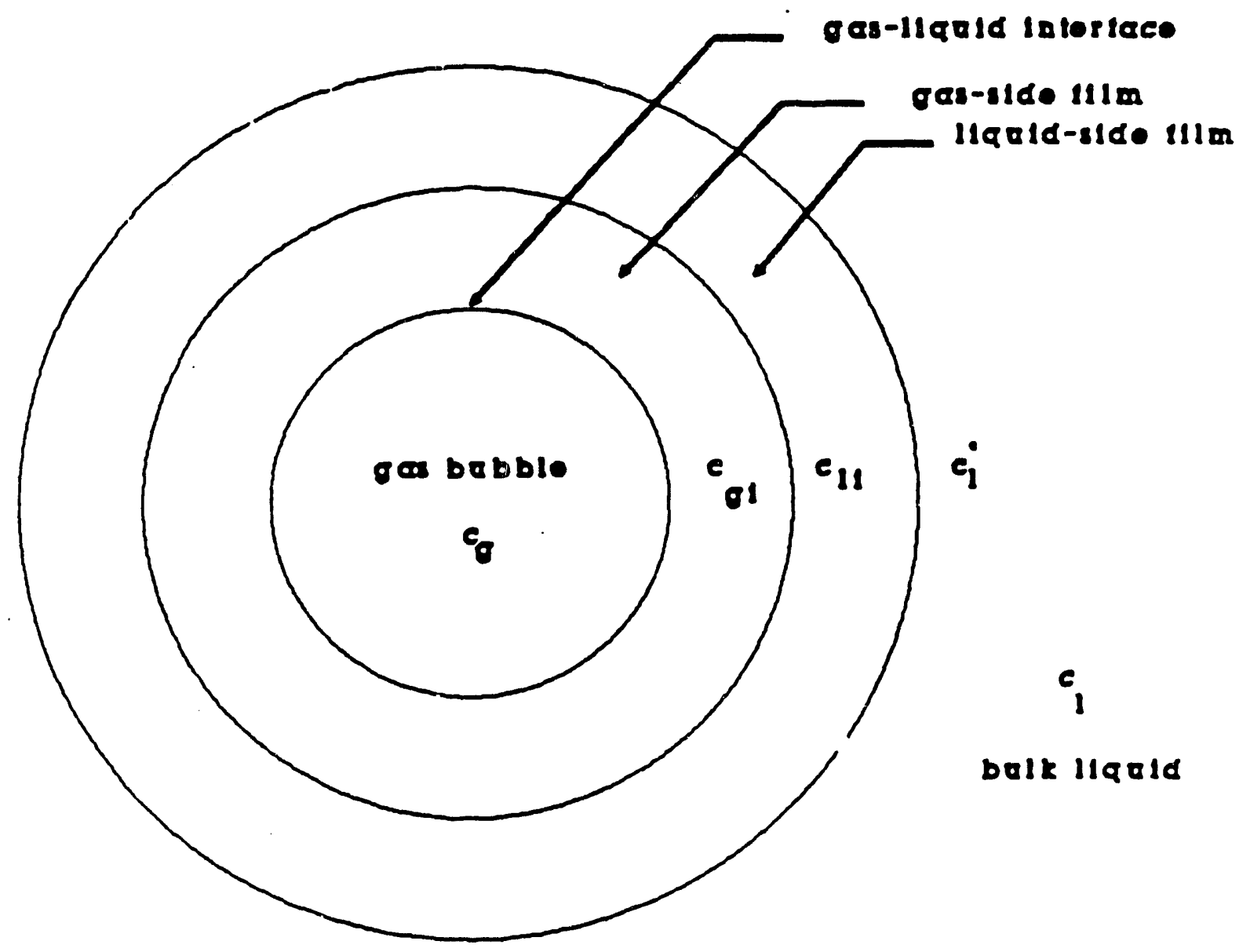

Figure 2: Diagram of Transport of Gas from Gas Bubble to Bulk Llquid 


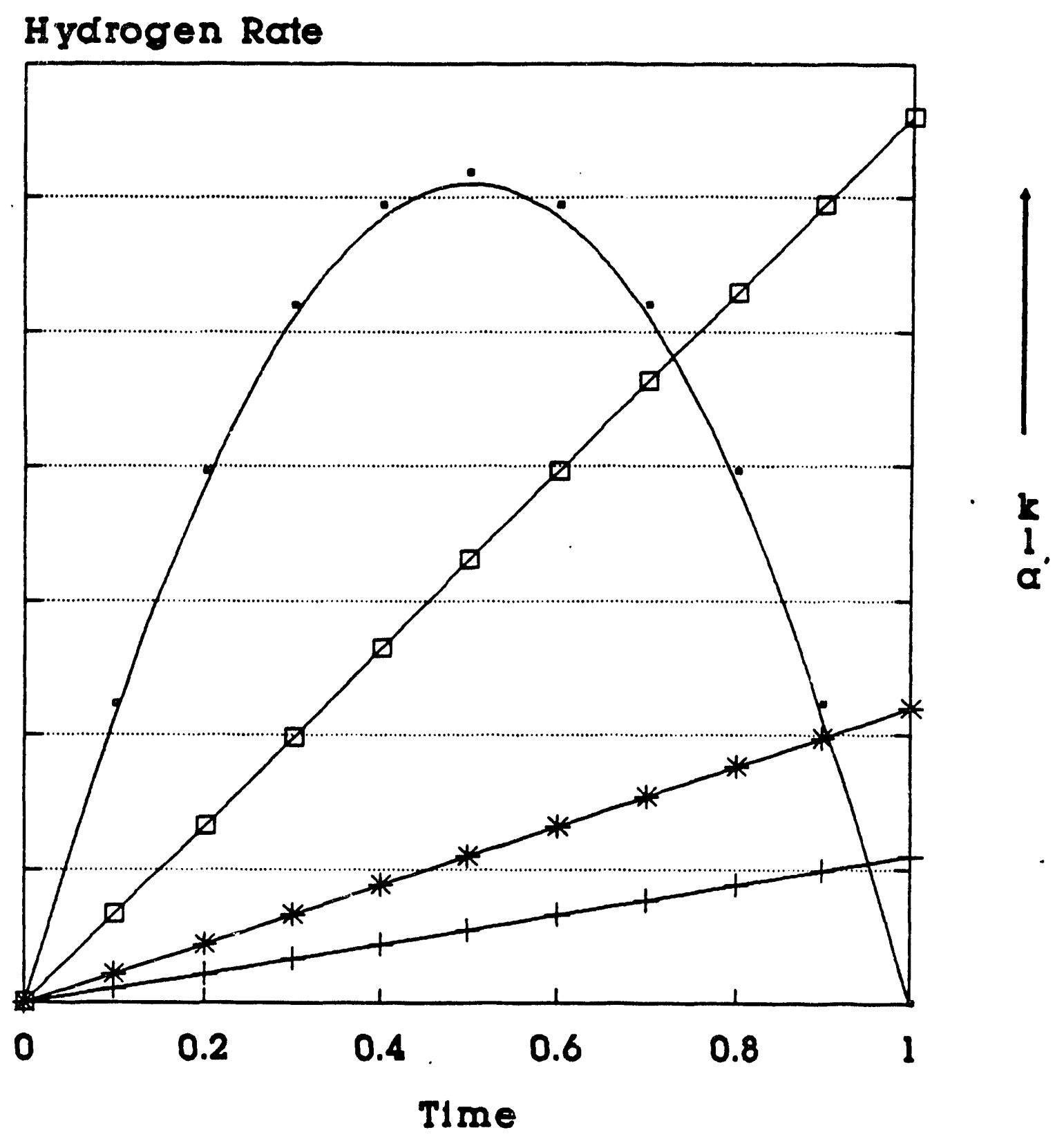

\section{Q Q Demand + Low Q supply \\ * Mod Q Supply $\quad$ High Q Supply}

Figure 3: Hydrogen Deficit 


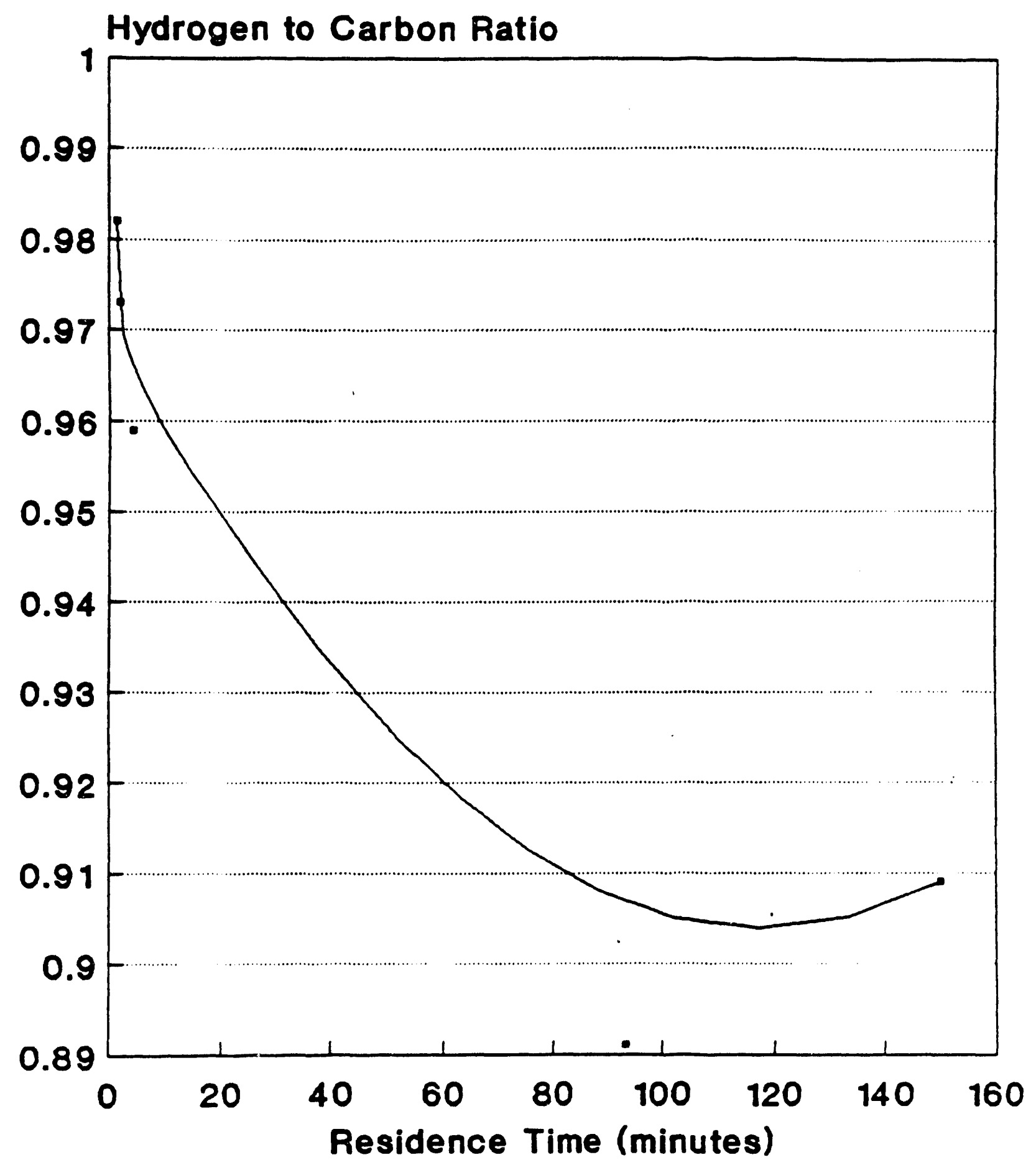

Figure 4: H:C Mole Ratio of Coal Products with Time 


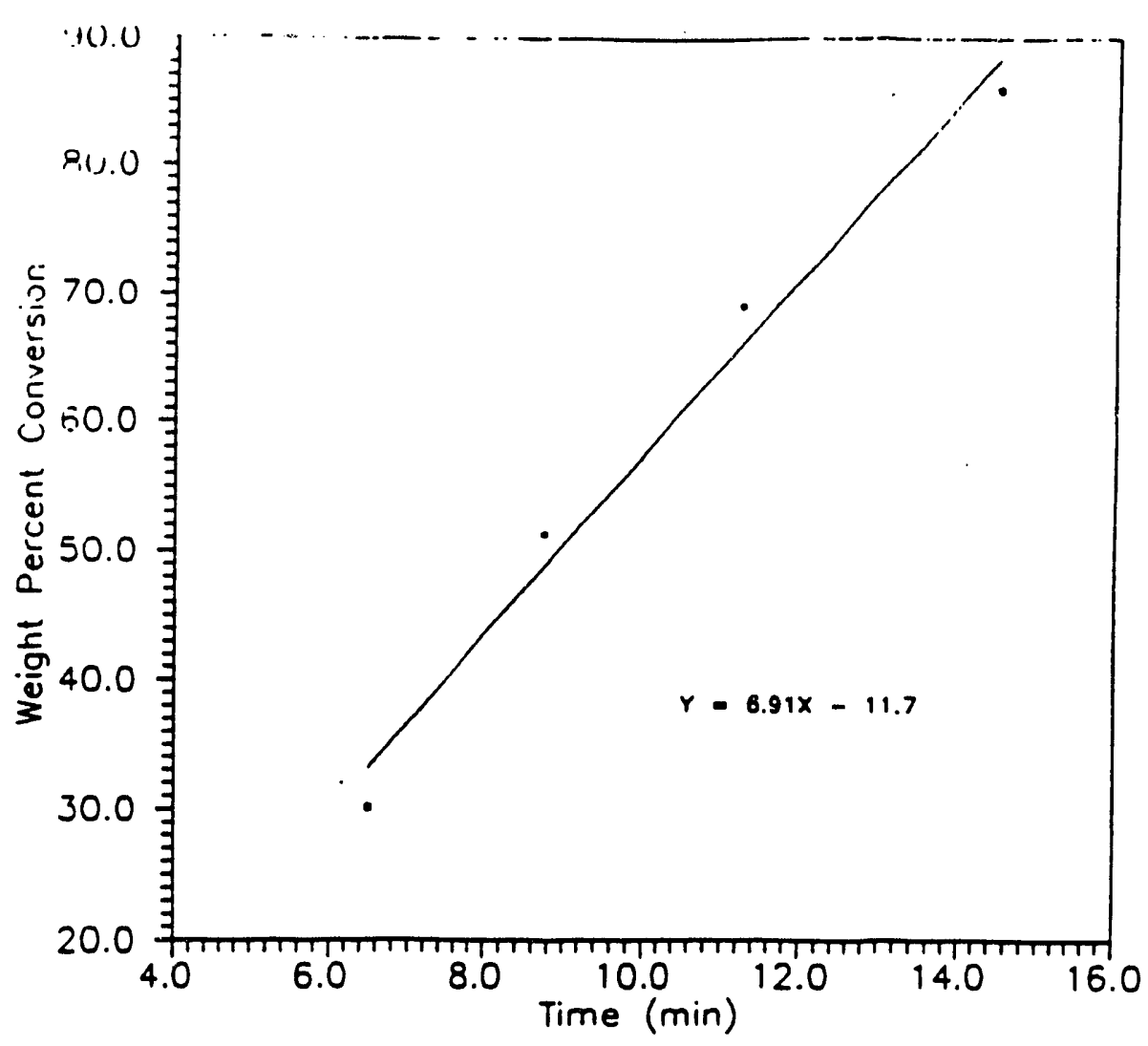

Figure 5: Experimental Determination of Nophthalene Conversion verses Time for Reoctor System in Use ot the End of the Work Yeor

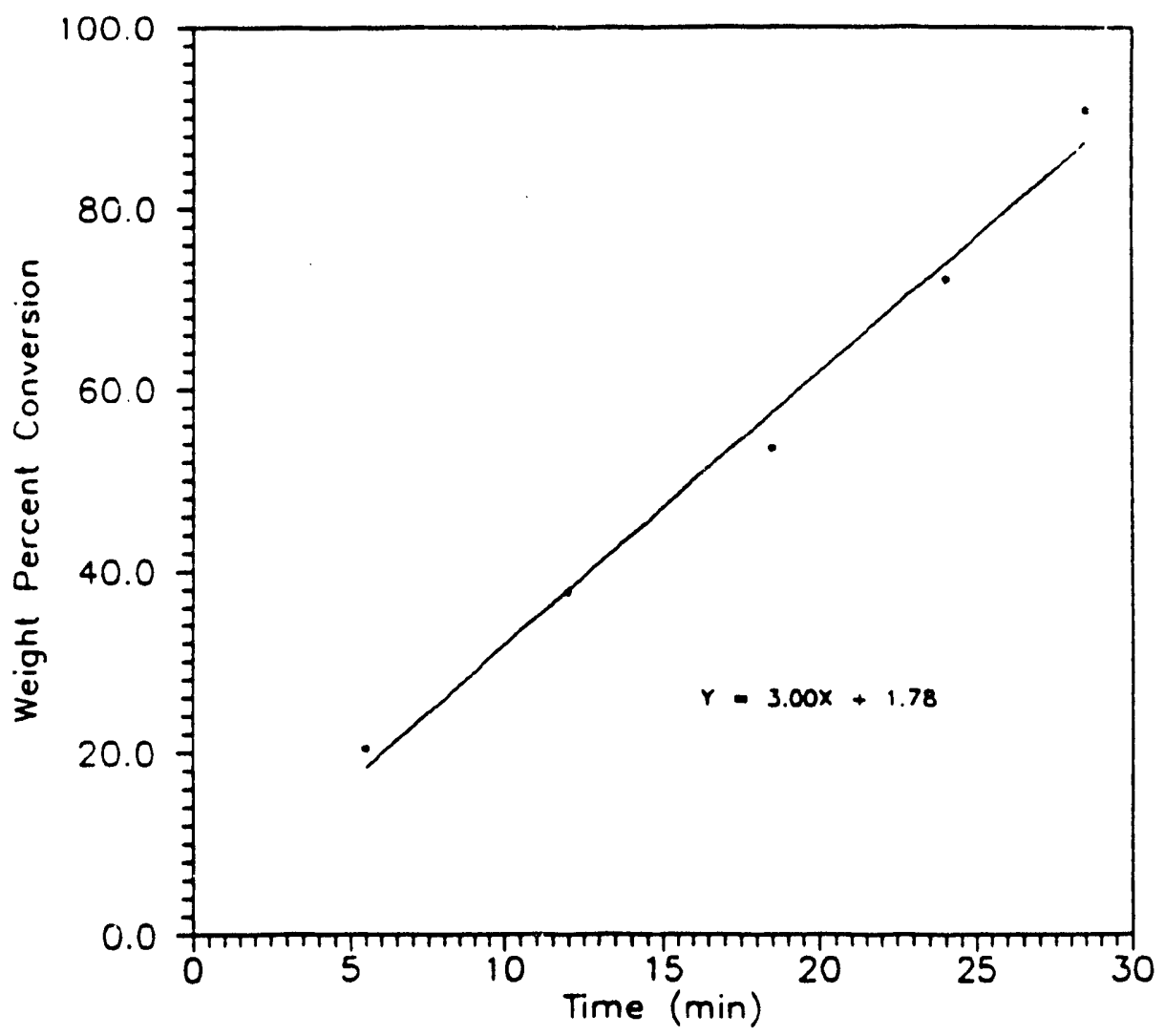

Figure 6: Experimental Determination of Nophtholene Conversion verses Time for Reoctor System in Use ot the Beginning of the Work Ieor 


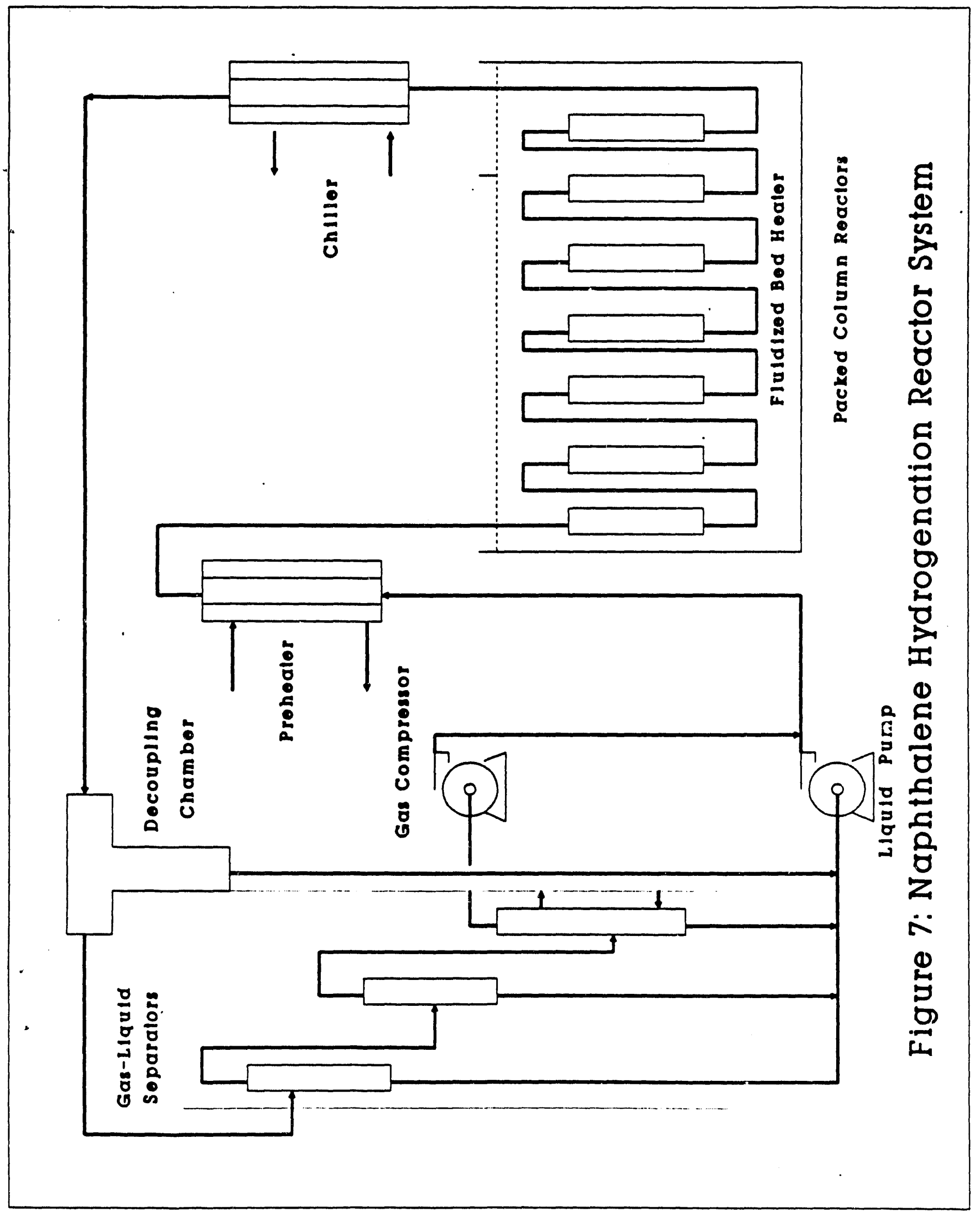




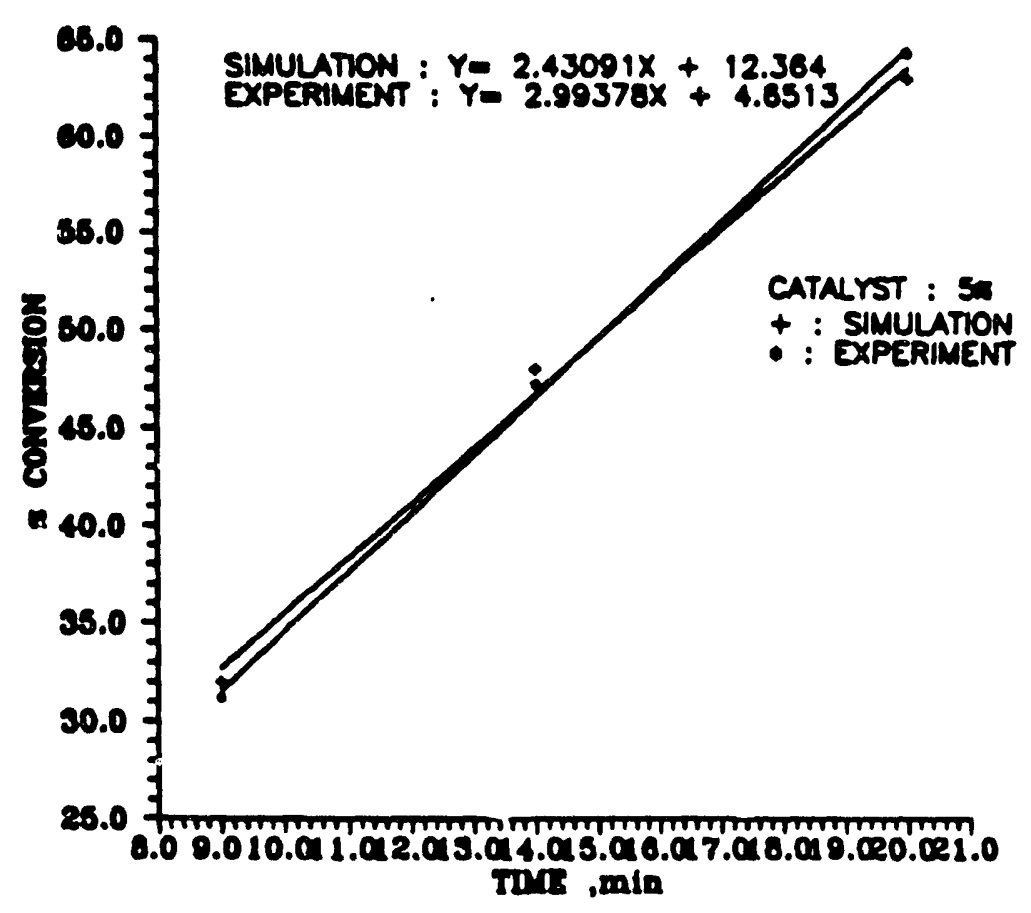

Fig. 8: COMMERSION Vs TMEE (AT 5A CATNLYST lOADING)

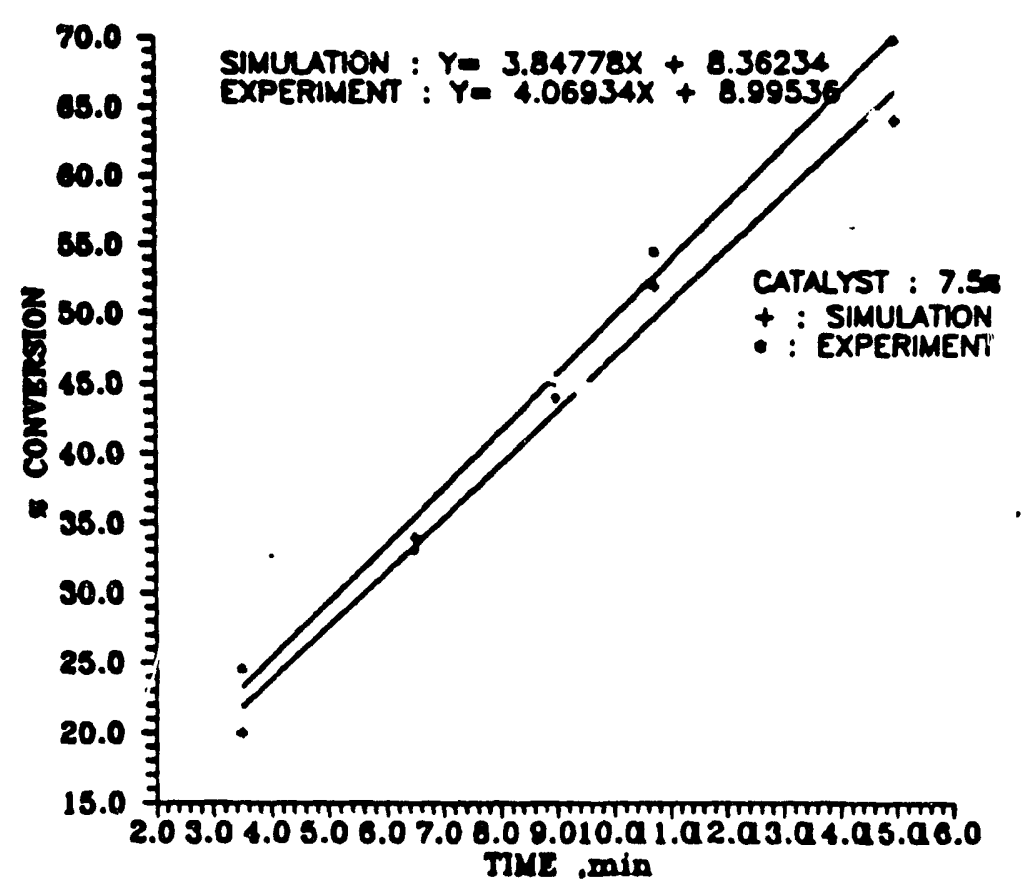

Fig. 9: CONVERSION Va TIME (AT 7.5a CATALYST LOADING) 


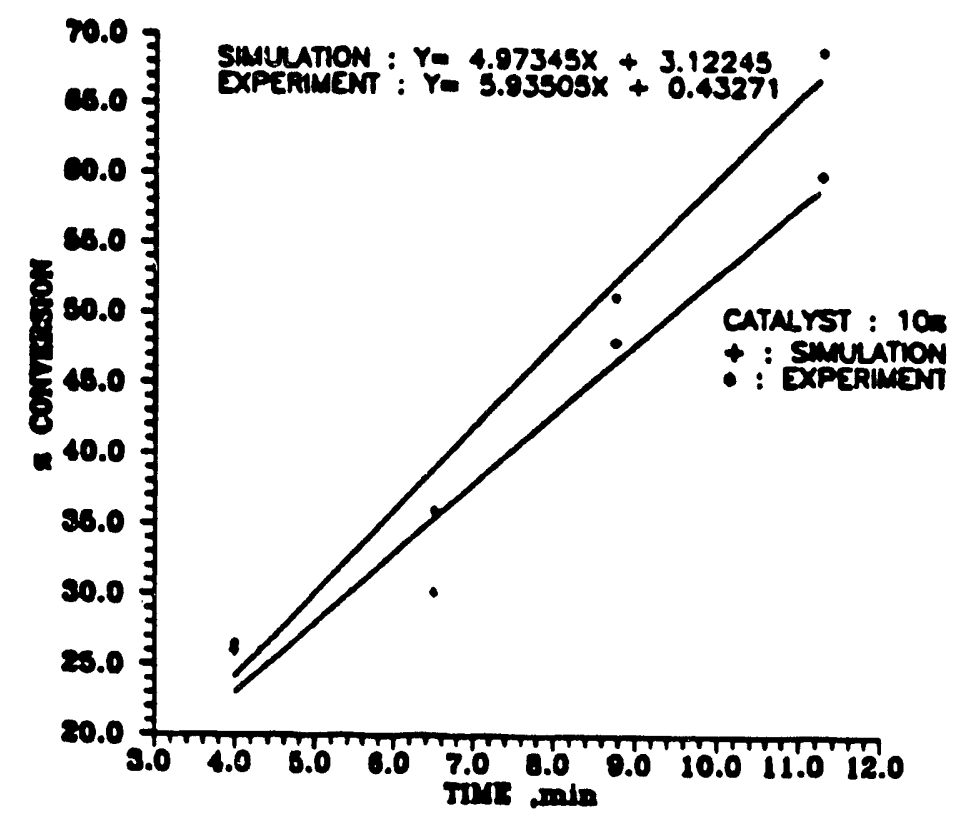

Fig. 10: CONVERSION VS TME (AT 10 CATALYST LOMDING)

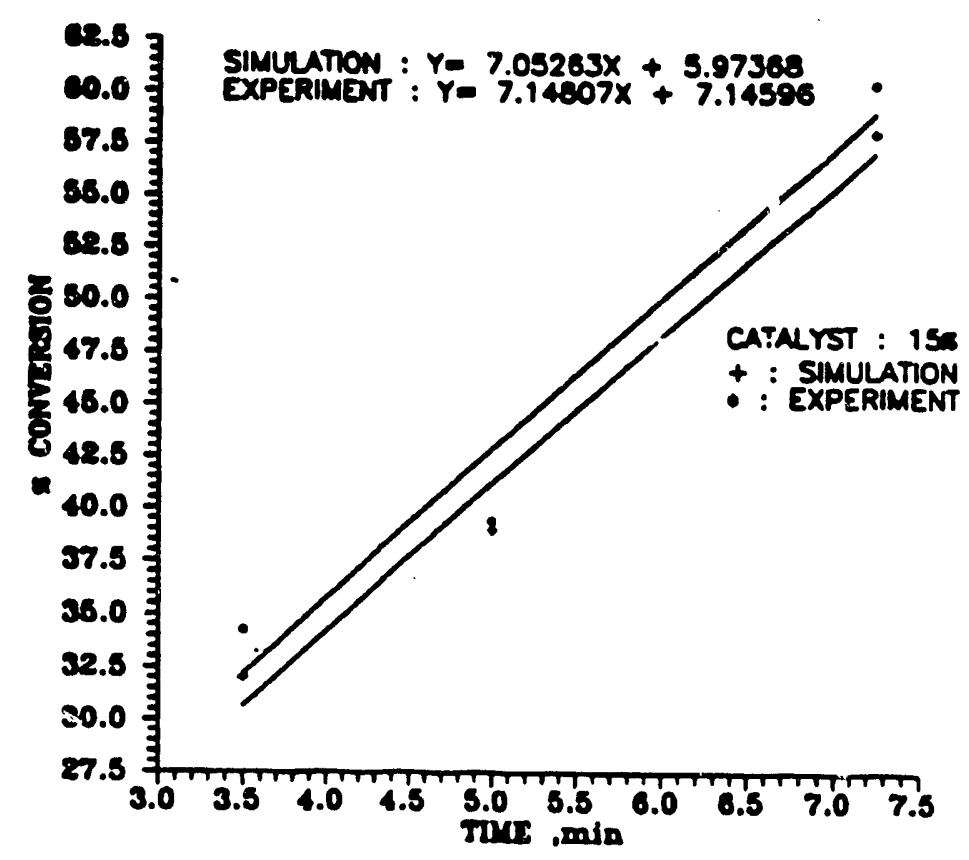

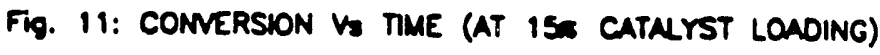




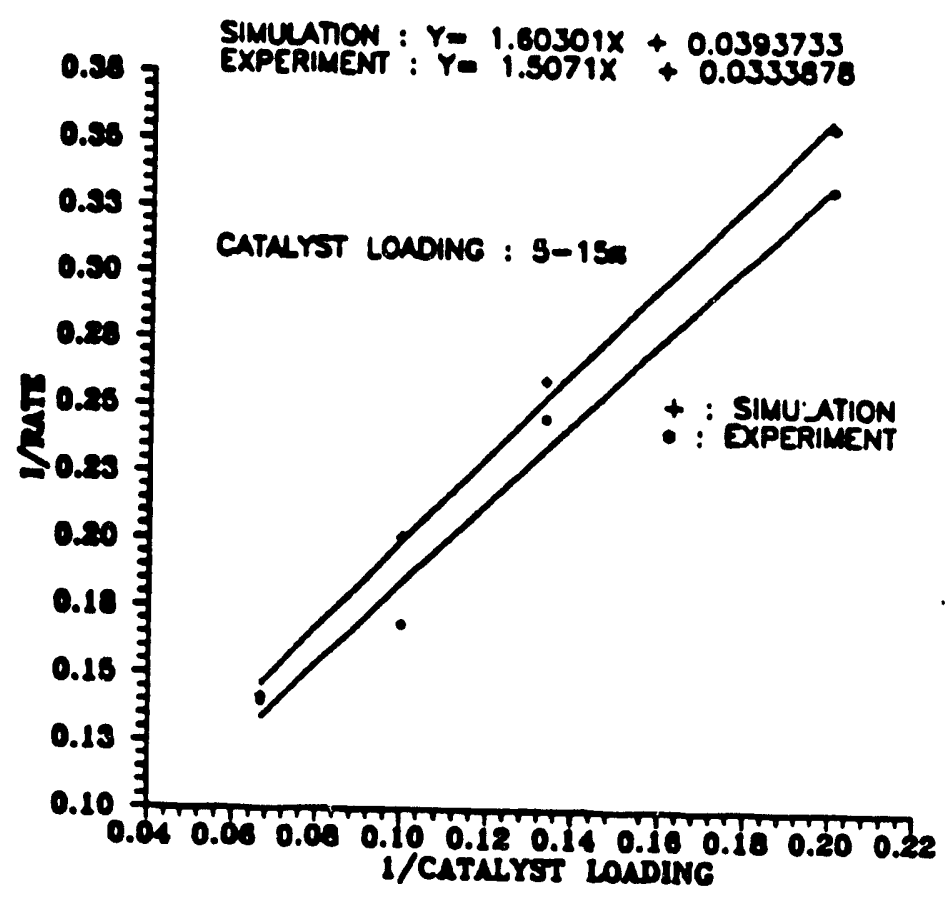

Fig. 12: (1/RATE) ve (1/CATALYST LONDING)

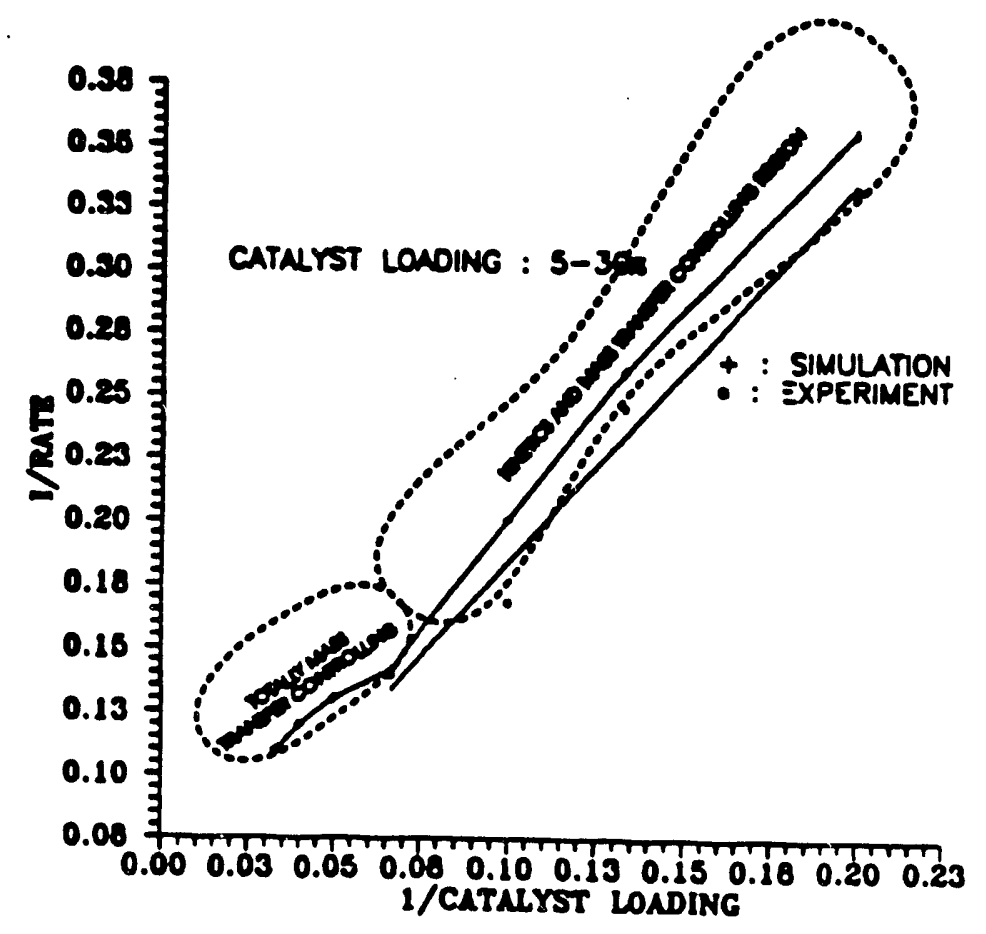

FIg.13: (1/RATE) VE (1/CATALYST LOADING; 


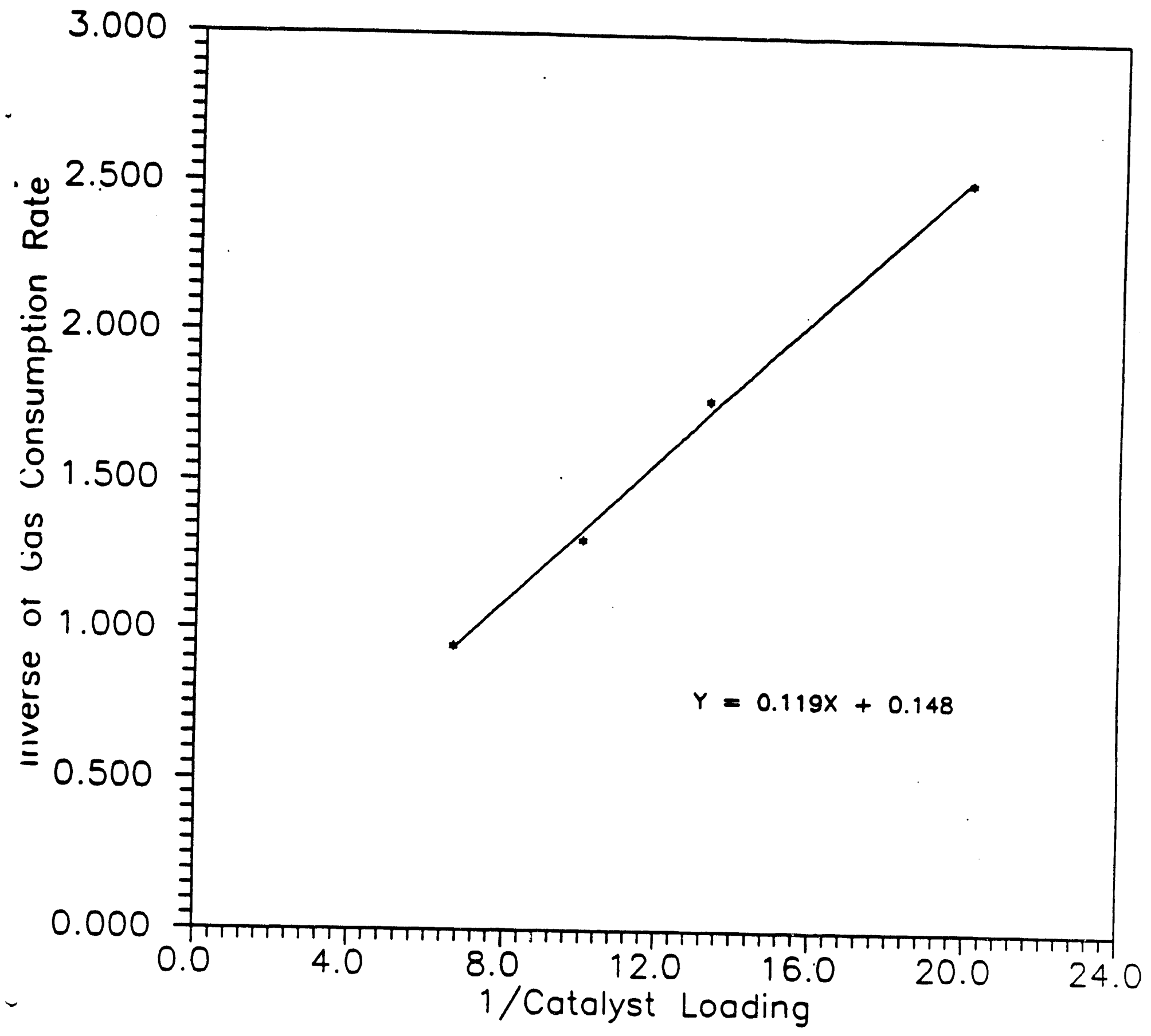

Figure 14: Inverse Reaction Rate as Determined by Niean Gas Consumption Rate Versus Inverse Catalyst Loading. 

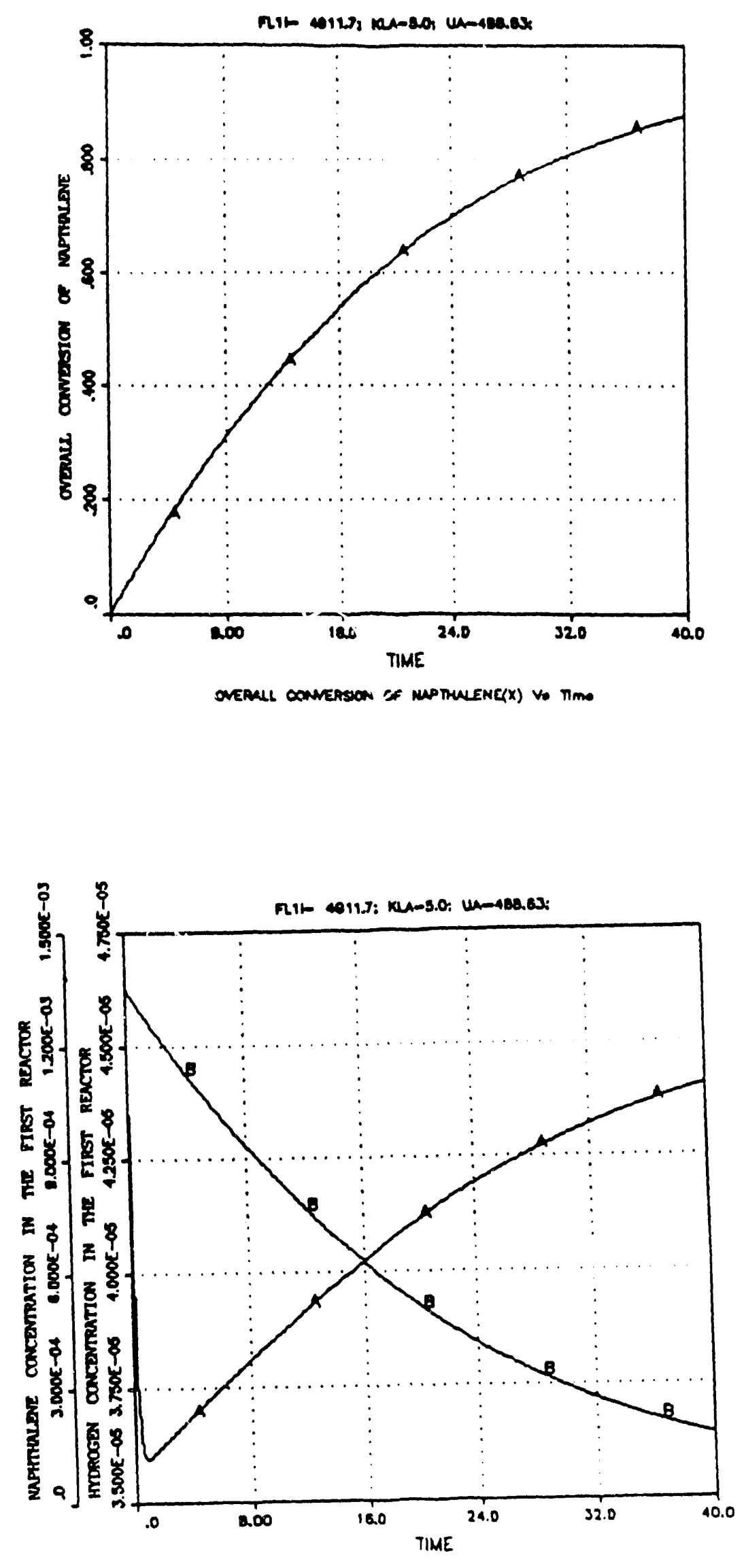

concentration ve rome protes in the lat reoctive

FIGURE 15: RESULTS FROA THE STMUIATTON 


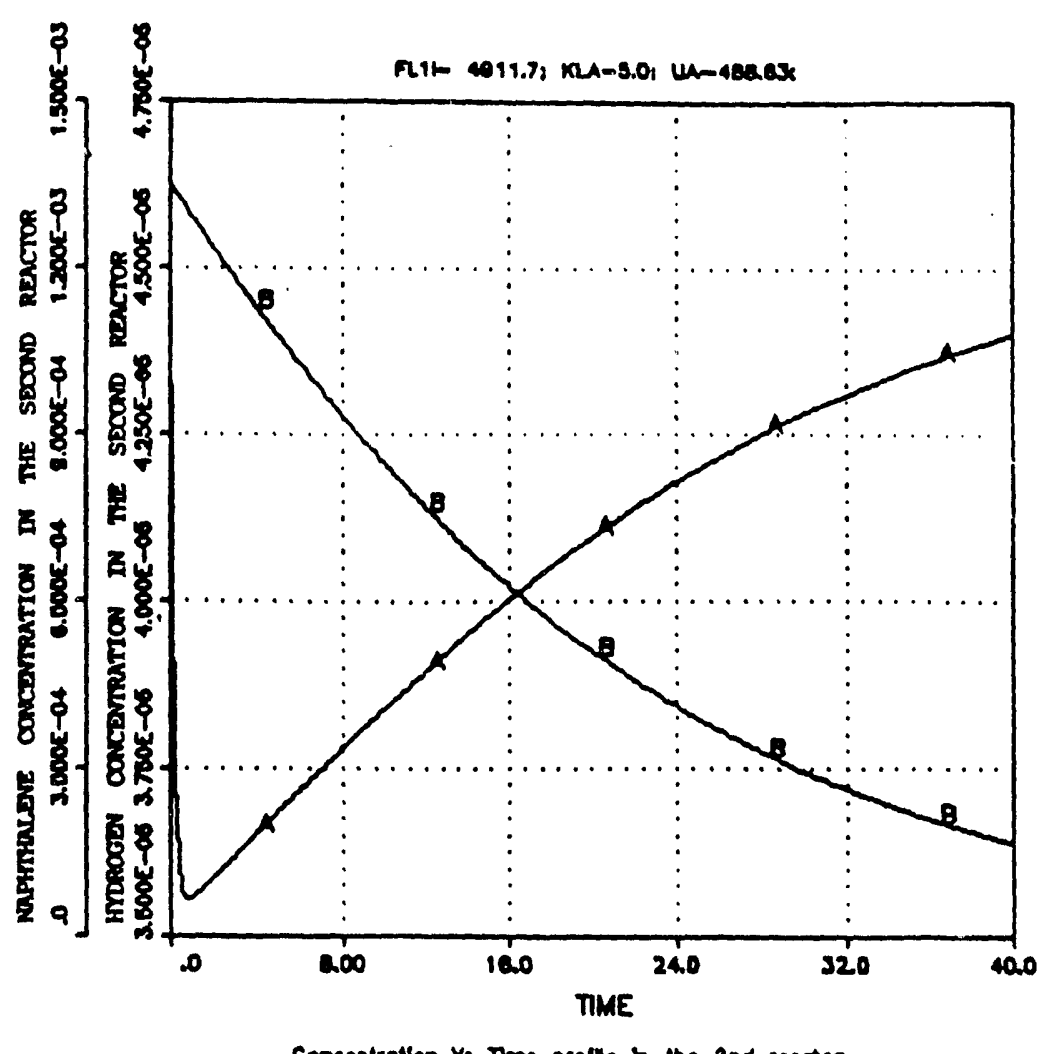

Comeentrotion Ve Trme profles in the 2nd reector

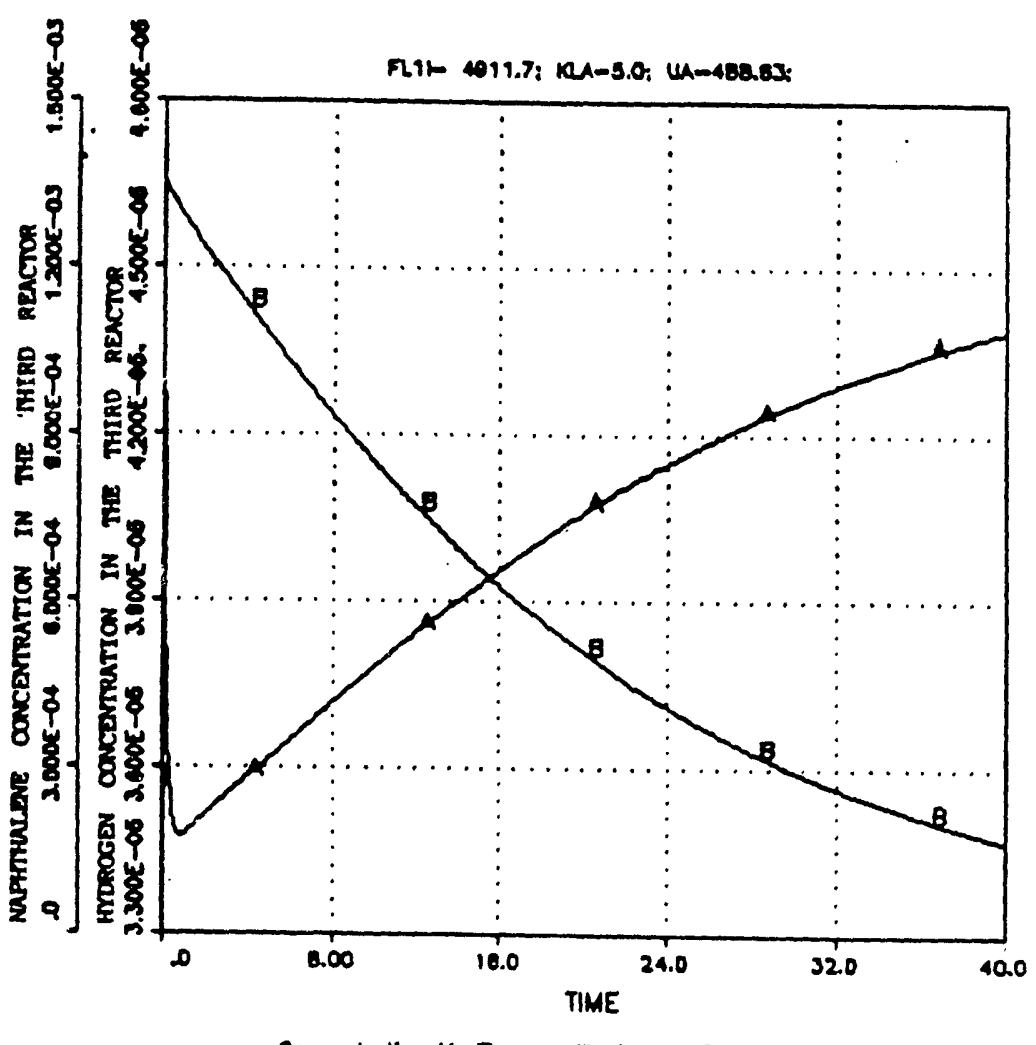

Concentretion ve nme profle in the 3rd reseter

FIGURE 16: RESULTS FROM THE STMULATION 

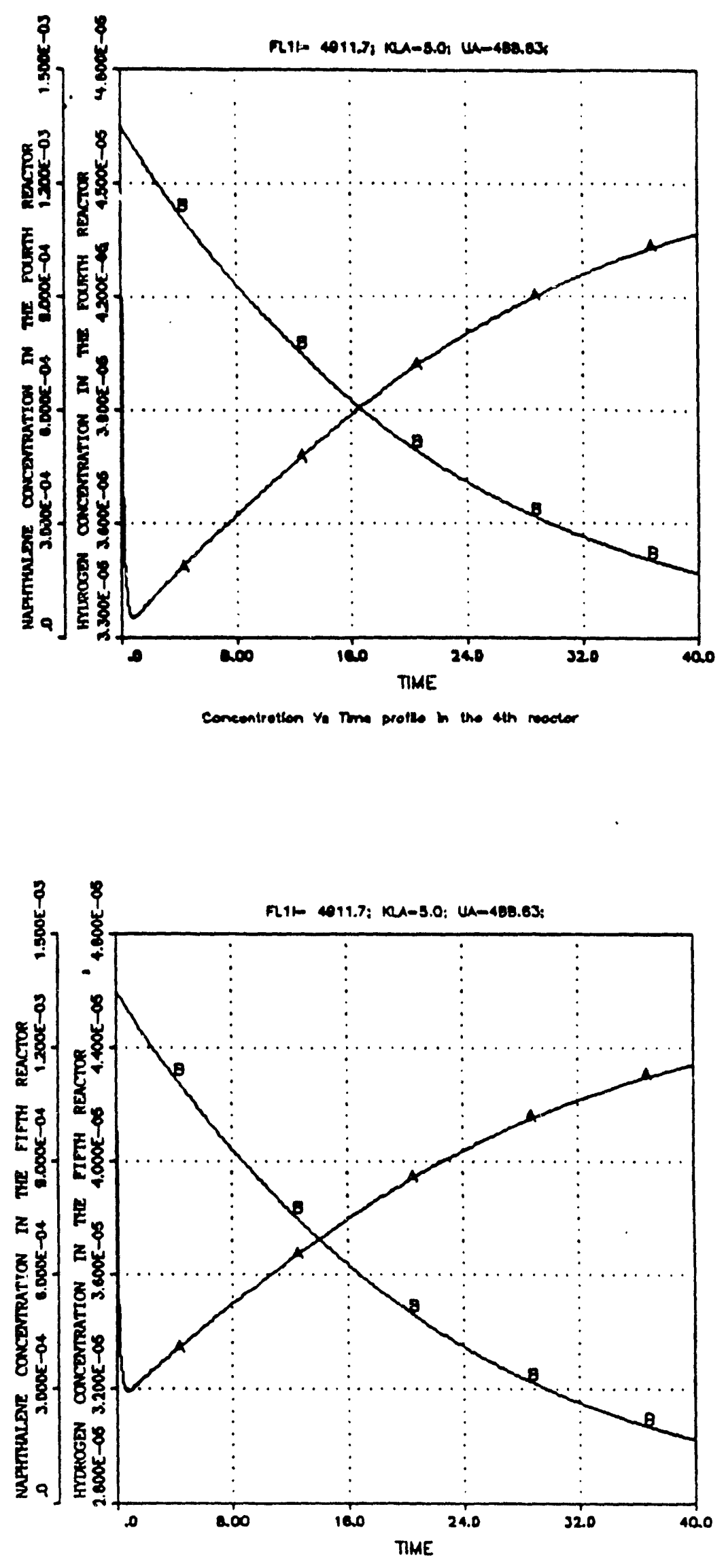

Concentration Ve Trme prollse in the Sth neoctor

FIGURE 16a: RESULTS FROM THE SMMLATION 


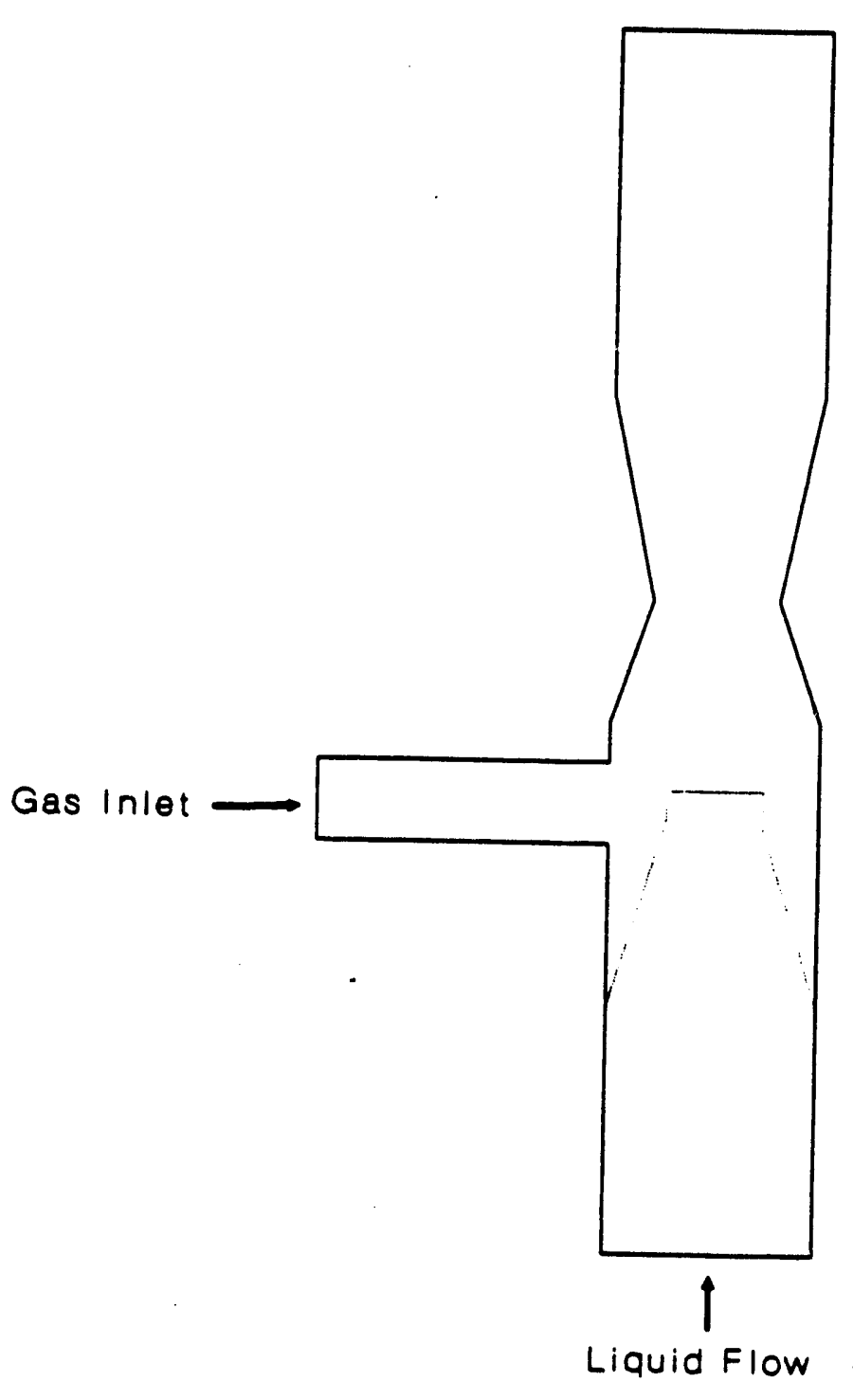

Figure 17: Modified Slot Injector 


\section{Figure 18 \\ Effect of Liquid Velocity on Gas- Liquid Mass Transfer Coefficient}

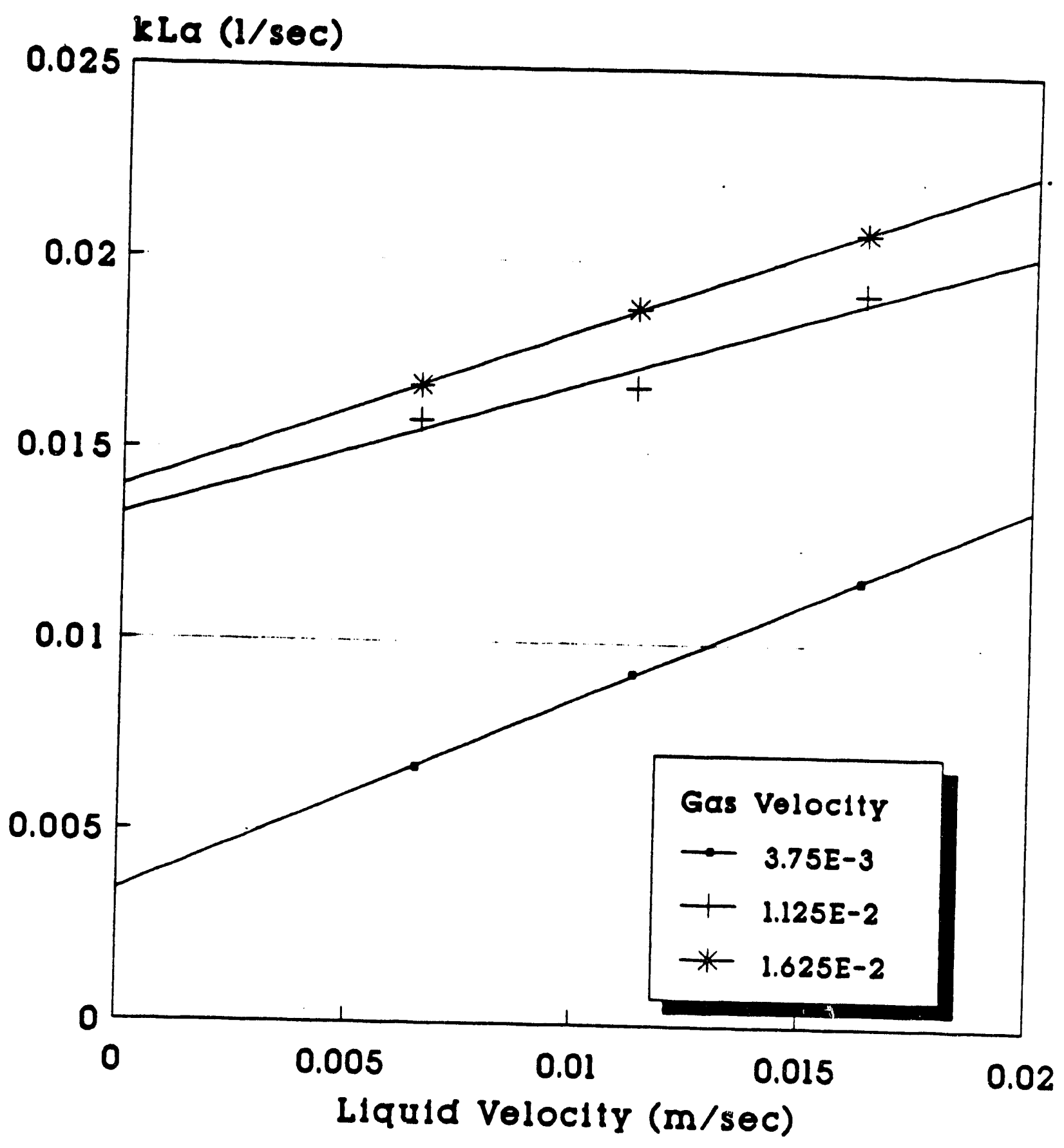

Packed Column Diameter $=0.064 \mathrm{~m}$ 


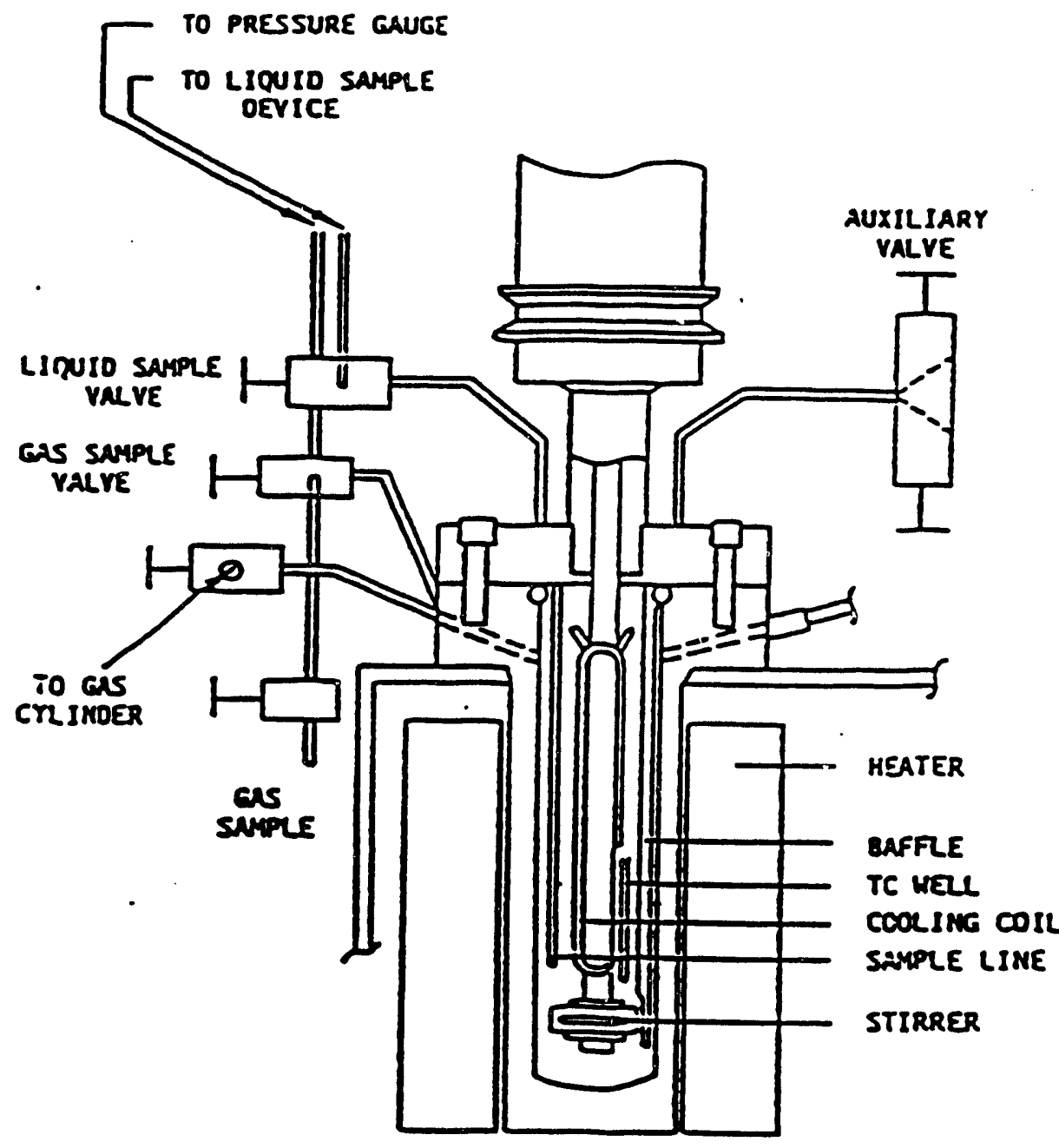

Figure 19: Autoclave Assembly 


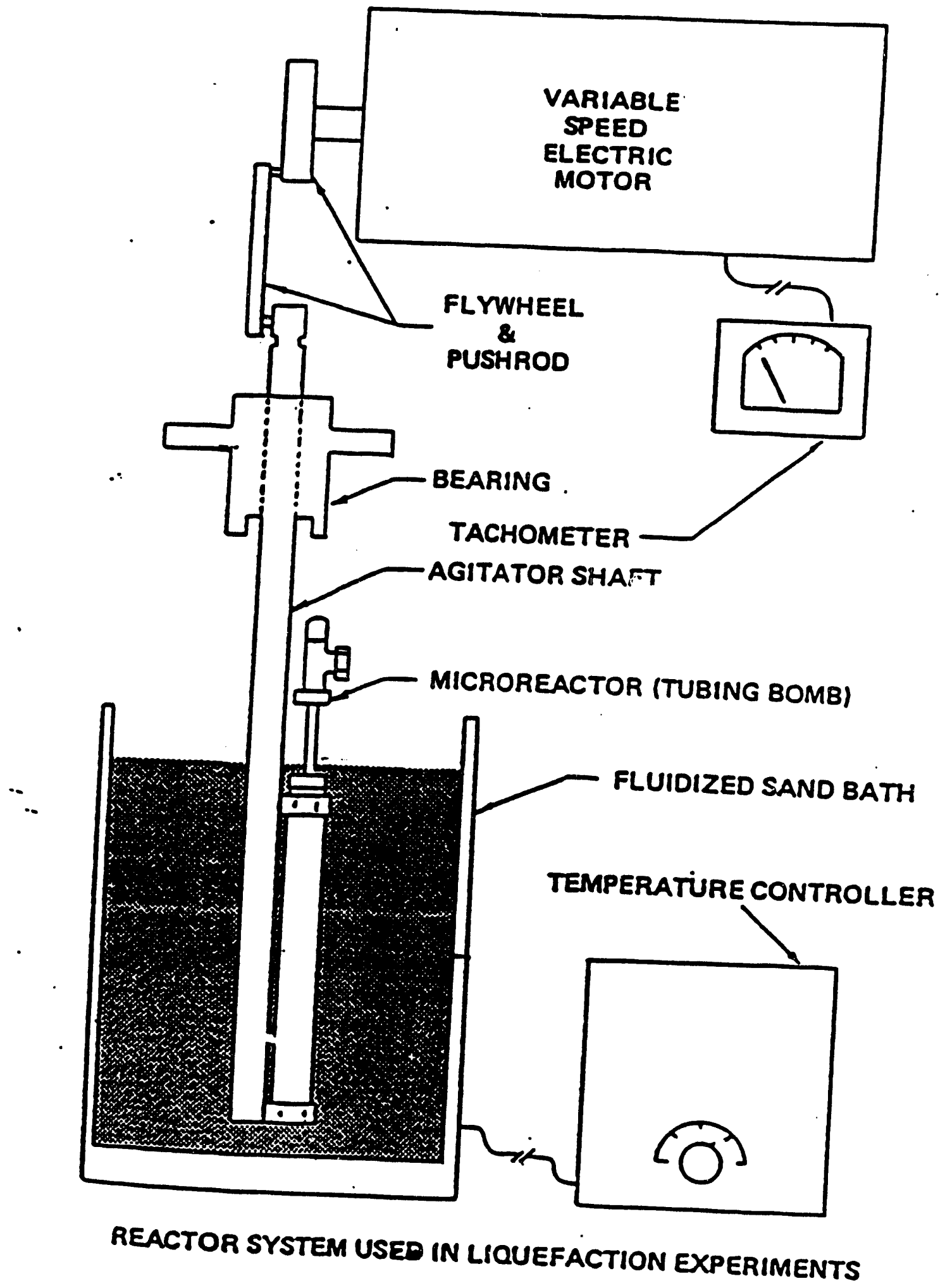

Figure 20 


\section{Task V}

\section{University of Utah}




\section{Task V \\ Integrated Coal Liquefaction/Characterization \\ University of Utah}

P.I.:

Co. Prs:

Report

Coordinator:

Phone No.:

Period:
Henk I.C. Meuzelaar

Larry L. Anderson

Ronald J. Pugmire

Henk L.C. Meuzelaar

(801) $581-8431$

May 1, 1989 - April 30, 1990
Edward M. Eyring

Joseph S. Shabtai

\section{Objectives}

1. To study the details of the structure and composition of coals by means of advanced spectroscopic techniques, including NMR, MS and FTIR and to provide average structural models at a near-molecular level for selected premium U.S. coals. Such models could be very useful as fingerprints for differentiation of various coals by indicating (a) the degree of condensation and aromaticity of the aromatic-hydroaromatic cluster units, and (b) the chemical nature and distribution of intercluster linkages, as a function of the coal source and rank.

2. To extend our understanding of the relations between maceral and other compositional data and the quantity and quality of liquefaction products that can be obtained by differential liquefaction methods, i.e., procedures that produce one or more economically valuable products (gas, char, resin) in addition to coal liquids.

3. To elucidate the mechanisms and kinetics of coal liquefaction chemistry by means of modern spectroscopic techniques. Special attention will be paid to the effects of catalysts and of changes in liquefaction conditions (heating rate, sample size, pressure) as well as of differences in coal chemistry (rank, maceral composition, weathering status).

4. To provide fundamental, laboratory-scale data on the feasibility of a recently developed new processing concept for stepwise, low-temperature depolymerization-liquefaction of premium U.S. coals, leading to their conversion into light liquid hydrocarbon fuels.

5. To develor coal liquefaction models based on spectroscopic measurements of the relative abundance of chemically recognizable coal "maceral" components as well as of statistical structural parameters. 


\section{Task V.1 COAL STRUCTURE/LIQUEFACTION YIELD CORRELATION BY MEANS OF ADVANCED NMIR TECHNIQUES (R. J. Pugmire and A. Orendt)}

\section{Overview}

The work on this task has focused on developing procedures that increase the amount of information available on coals. Research activities included efforts to (1) use 2-dimensional NMR techniques for structure determination in liquids; and (2) the use of solid state NMR techniques to prove the structure of coals. A lengthy report on (1) was provided in the last quarter Progress Report and will not be duplicated as part of the Annual Report.

Our w rork on solid NMR techniques has been primarily in examination of ${ }^{13} \mathrm{C}$ chemical shielding anisotropy. The report provides a summary of the work carried out in this laboratory and a brief review of the work in the field that is guiding our future activities.

\section{NMR Structure studies}

There have been a number of techniques developed in nuclear m:agnetic resonance spectroscopy that can be used to obtain ihemical shielding anisotropy (CSA) information from solid samples. In this report the various methods will be described, alorig with the application of some of the techniques to both coal and substituted polycyclic aromatic compounds whose structures may be important in the understanding of the structure of coal. In the following, all the various experimental methods will be discussed in terms of the ${ }^{13} \mathrm{C}$ chemical shielding even though the techniques can be applied to any nuclei.

The methods for obtaining the CSA that will be discussed include measurement and analysis of the static powder lineshape [1], analysis of the variable angle sample spinning lineshapes [2,3] and analysis of the intensity pattern obtained from spinning at the magic angle at a rotation rate less than the width of the anisotropy $[4,5]$. Techniques that require more elaborate equipment and control over the orientation of the sample with respect to the magnetic field include the magic angle hopping experiment [6] and the dynamic angle spinning technique $[7,8]$. In both of these two dimensional (2-D) experiments the orientation of the sample is changed during each scan. A related experiment is the stop and go technique [9] in whirh the sample rotation rate is rapidly changed from a stationary state to a high spinning rate during each scan. Another technique for studying the CSA involves spinning at the magic angle and using rf pulses to regain the chemical shift anisotropy information that is averaged by the spinning [10]. Finally, the application of a 2-D chemical shift-chemical shift correlation technique [11] to coal samples will be discussed.

Before presenting the description of the experimental methods used to measure the CSA, 
a few words must be given about the information that can be obtained from such experiments. The CSA is a reflection of the interaction of the electron environment of the nuclei with the external magnetic field. Depending on the orientation of a given molecule in the field, a different value of the chemical shielding is observed. If one has a sample that can simultaneously exist in all possible orientations, i.e. a random powder sample, a superposition of all the possible shielding values for the nuclei in the molecule is observed. The pattern observed is the so-called "powder pattern". The frequencies obtained from the breakpoints or discontinuities of the powder pattern are the principal values of the chemical shielding tensor [1]. For a nucleus (e.g., ${ }^{13} \mathrm{C}$ ) that is in an anisotropic environment one can observed three distinct values correspond to the three elements $\left(\sigma_{11}, \sigma_{22}\right.$, and $\left.\sigma_{33}\right)$ found when the chemical shielding tensor is diagonal. From the study of a single crystal both the principal values of the shielding tensor and their orientation in the molecular framework can be determined [1]. From single crystal studies of arc natic compounds it is known that $\sigma_{33}$ is always perpendicular to the plane of the aromatic ring [12], and its value in the aliphatic region reflects its independence of the $\pi$ system. The other two tensor components, $\sigma_{11}$ and $\sigma_{22}$, are oriented in the plane of the aromatic system. Theoretical studies have placed $\sigma_{11}$ nearly perpendicular to the C-C bond with the largest $\pi$-bond character in a number of polycondensed aromatic hydrocarbons [13].

In most of the experimental methods to be described, only the aromatic region of the coal spectrum will be analyzed. For convenience, the aromatic carbons in coal can be grouped into four categories: protonated, substituted (having an alkyl carbon substituent), phenolic (having an oxygen substituent) and bridgehead or condensed. In Figure 1 ideal lineshapes for the four types of carbons are shown. These ideal lineshapes were obtained by tabulating literature values for the shield tensor components for aromatic carbons in simple organic compounds [1419] measured by a variety of methods (and includes the variable angle sample spinning results from this laboratory). These results are presented in Table 1. The number in parentheses after the carbon type is the number of measurements considered in the analysis. For protonated aromatic carbons a highly asymmetric tensor with an isotropic chemical shift of 121 ppm relative to TMS is expected. The substituted aromatic carbons have an average isotropic chemical shift of $134 \mathrm{ppm}$ while the phenolic aromatic carbons have an average isotropic chemical shift of $150 \mathrm{ppm}$. It is noted that the downfield isotropic shift observed due to the electronegativity of oxygen is almost entirely due to the shift in the value of $\sigma_{33}$. Furthermore, the tensors of both substituted and phenolic carbons are slightly less assymetric than that of the protonated carbons (i.e., the difference between $\sigma_{11}$ and $\sigma_{22}$ is smaller in both cases than is 
observed for the protonated carbons). Finally, for the condensed aromatic carbons an average isotropic chemical shift of $131 \mathrm{ppm}$ is observed, with a shielding tensor that is nearly axially symmetric $\left(\sigma_{11} \sim \sigma_{22}\right)$ due to the local symmetry (approaching $C_{3}$ ) in the plane of the molecule at the bridgehead carbons.

\section{Static Powder Samples}

The most straightforward experimental method to obtain the chemical shielding information in a solid is to tale the ${ }^{13} \mathrm{C}$ spectrum under static conditions [1]. The powder sample contains all orientations with respect to the magnetic field and as such gives a broad lineshape from which one can obtain the principal values of the shielding tensor. Experimentally, one can either do a simple FT experiment with high power ${ }^{1} \mathrm{H}$ decoupling to remove the ${ }^{1} \mathrm{H}-{ }^{1} \mathrm{H}$ and the ${ }^{1} \mathrm{H}-{ }^{13} \mathrm{C}$ dipolar interactions or a cross polarization experiment $[20]$ with ${ }^{1} \mathrm{H}$ decoupling. The cross polarization technique is generally used due to the four fold enhancement in the ${ }^{13} \mathrm{C}$ signal that can be realized.

In Figure 2 a static powder pattern for 1,2,3,6,7,8-hexahydropyrene, along with its best SIMPLEX fit using the POWDER method [21] is shown. The principal values of the shielding tensor obtained from this spectrum are given in Table 2. This example clearly illustrates the limitations of the technique. The upfield components of the aromatic carbon shielding tensors are often obscured by the signal from the aliphatic carbons in the sample. With samples that have many carbons with similar isotropic chemical shifts, it becomes very difficult to determine the breakpoints. A practical limit for this experiment is two to three aromatic tensors.

Earlier work on the analysis of static powder patterns from coal samples by Wemmer, Pines, and Whitehurst [14] used a set of data from aromatic carbons in a large number of compounds to derive ideal lineshapes for aromatic, condensed aromatic, alkoxy, and aliphatic carbons. Static coal spectra were then fit to obtain the composition in terms of these four predetermined tensors.

In the work pres mnted below, no assumptions were made about the value of the tensor components of the various types of aromatic carbons. Due to the problem of overlap of the aromatic $\sigma_{33}$ component and the aliphatic carbon tensor patterns, this technique is only useful on coals whose aromaticities are high, e.g., greater than 95\%. In these cases the small amount of aliphatic signal, when spread over its CSA will usually be lost in the baseline noise. A representative static spectrum of a coal sample is shown along with the results of the SIMPLEX fit in Figure 3. In the spectral fitting routine all the tensor components as well as the relative amount of each type of carbon were allowed to vary. The results obtained to date on anihracite coals studied in this laboratory are summarized in Table 3. Given the inherent errors 
associated with the experiment, the correlation with the elemental analysis data (atomic H/C) is remarkably good. It is important to note that the tensor components in these three coals are remarkably similar to those given in Table 1 for model compounds.

\section{Variable Angle Sample Spinning}

The technique of variable angle sample spinning (VASS) $[2,3]$ spinning at an angle other than the magic angle, has been shown to be very useful in the study of model compounds and appears to be useful for the study of coal. The expression that governs the averaging of the CSA during rapid spinning (spinning speeds greater than the CSA) is given in Equation 1.

$$
W(\theta, \alpha, \beta)=W_{0}\left(\sigma_{1}+1 / 2\left(3 \cos ^{2} \theta-1\right)\right.
$$

$$
\left.\left[1 / 2\left(3 \cos ^{2} \beta-1\right)\left(\sigma_{33}-\sigma_{11}\right)+1 / 2\left(3 \sin ^{2} \beta \cos 2 \alpha\right)\left(\sigma_{22}-\sigma_{11}\right)\right]\right]
$$

For $\theta$, the angle the spinning axis makes with respect to the magnetic field, such that $\left(3 \cos ^{2} \theta\right.$ $1)=0$, i.e., at the magic angle, this expression reduces to the isotropic chemical shift, $\sigma_{i}$. For angles less than the magic angle a partially averaged and scaled powder pattern is cbtained, whereas for angles greater than the magic angle the pattern is inverted as well as scaled. Experimentally, five spectra are generally recorded; one at the magic angle, two at larger angles, and two at smaller angles. Each of the four spectra recorded off of the magic angle have their own distinctive lineshape; they are not just scaled versions of the same lineshape. The four off angle spectra can then be fit simultaneously with the isotropic chemical shifts locked to the values obtained from the spectrum at the magic angle.

VASS removes some of the difficulties associated with the analysis of static powder patterns. By both the partial averaging of the CSA and the inversion at angles larger than the magic angle, this technique removes the overlap observed between the aromatic and aliphatic powder patterns, thereby allowing for the analysis of samples with substantial aliphatic components. It also takes less time to obtain a spectrum with satisfactory signal to noise due to the scaling of the CSA. Due to the number of spectra that are being fit and the different lineshapes, the limitation of the number of aromatic carbon tensors that can be analyzed (relative to a static powder pattern) increases to about four or five. In terms of the study of model polycondensed aromatic compounds, one is still restricted to symmetrically substituted compounds. The results of applying this method to several model compounds are given in Table 4. The set of VASS spectra for 1,2,3,6,7,8-hexahydropyrene along with the simulations are shown in Figure 4. The comparison of Figures 2 and 4 shows the advantages of the VASS technique as compared to the analysis of static powder patterns.

This technique has also been applied to several coals, with the results shown in Table V.1.5. The aromaticities given in the table are from variable contact time CP/MAS experiments. 
A representative set of two VASS spectra along with the best fit is shown in Figure 5. The fitting of the spectra is complicated due to the lack of clear breakpoints. Hence there is no unique solution to the SIMPLEX fitting routine (i.e. there were many locals minimums on the surface). Many of the solutions could be eliminated by the fact that the fitted values for the shielding tensor components and isotropic shift values were quite different from those observed in model compounds and in the anthracite coals. Other solutions could be eliminated because the percentages of the protonated and nonprotonated shielding tensors were obviously wrong, the tensor components were not in the expected range, or the sum of squares was lowered by adding extensive broadening in the simulation. Using information from CP/MAS and dipolar dephasing studies on the percenta je of the aromatic carbons that are protonated as a starting point (but not a locking point) in the fitting process, a reasonable solution was obtained for each of the coals. Therefore, by using information obtained from CP/MAS and dipolar dephasing experiments, the VASS technique yields principal values of the shielding tensor for the aromatic carbons in coal that are consistent with those observed in model compounds.

\section{Slow Magic Angle Spinning}

If the rate of spinning at the magic angle is much less than the CSA then the resultant spectrum will be a narrow line at the isotropic chemical shift with sidebands spaced at integer numbers of the spinning frequency for the width of the CSA [4,5]. Analysis of the peak intensities of this sideband pattern yields the principal values of the shield tensor. The analysis procedure is difficult, and the procedure has not been used in many cases. In addition, it requires that the spinning speed be constant, e.g., $\pm 10 \mathrm{~Hz}$, during the entire experiment; if the spinning speed varies the linewidths of the sidebands are increased and the peak intensities become unreliable. If a compound contains more than about three inequivalent carbons with isotropic shifts in the same region, the spectrum becomes very complicated, making it difficult to determine the connectivity between the central peak and its associated sidebands. Morgan and Newman [19] recently used this approach to obtain the tensor componenis of a series of twelve benzene compounds with oxygenated substituents. On: group has extended this technique to a two dimensional method [25] in which the various sideband patterns are separated as discussed in the next section.

The slow spinning method has been applied to the study of several Australian coals by Burgar [26,27]. In this work, average tensor components for three types of aromatic carbons (protonated, substituted and bridgehead) taken from the work of Wemmer, Pines, and Whitehurst [14] were used and the percentage composition was varied to obtain the best fit. The percentage of aliphatic and alkoxy carbons were also determined with a reported accuracy 
of $5 \%$.

\section{Magic Angle Hopping, Stop and Go, and Dynamic Angle Spinning}

The three techniques discussed in this section all share the feature of separating the CSA information of the individual inequivalent carbons. In addition, the mechanical aspects of the experiments are very demanding. The methods of magic angle hopping (MAH) [6] and dynamic angle spinning (DAS) [7] both require that the sample be reoriented with respect to the magnetic field. The stop and go (STAG) method [9] requires that the sample be taken from a high spinning speed to rest during each acquisition. The basic pulse sequences used in these three techniques are shown in Figure 6. In the MAH experiment, discrete jumps are made between three orientations, related to each other by $120^{\circ}$ rotations about the magic angle, with equal time being spent at each orientation. In the DAS experiment the rapidly spinning sample is reoriented from the magic angle to any other angle; the choice of the second angle determines the degree of scaling observed in the tensor patterns obtained in the second dimension. Finally, in the STF,G sequence the experiment is done under stationary conditions except for the detection period. The major program with all three of the techniques is the mechanical difficulties encountered in moving the sample (either stop and start or reorienting a spinning sample) in a very short period of time. In addition, all three metbods require much more time for acquisition than any of the one dimensional methods in order to get reasonable signal to noise in the slices of the 2-D plot. There are now commercially available DAS probes which promise reorientation times in the neighborhood of $20 \mathrm{~ms}$.

All of the experiments give the same result in the final spectrum. The two dimensional spectra have the MAS spectrum of the isotropic chemical shift in one dimension, while the second dimension contains all the anisotropic information in the form of separated powder patterns, which may or may not be scaled. This separation into the second dimension removes the limitation of the number of inequivalent carbons that can be studied. If the carbon has an isotropic chemical shift that can be resolved from all the other isotropic shifts the powder pattern in the second dimension will be of only one carbon. Therefore, these techniques will definitely be powerful tools in the study of more complicated and less symmetrical model compounds. An example of the power of the DAS technique in resolving a large number of tensor patterns is shown in Figure 7 [8]. In this spectrum of cholic acid there are 14 carbons with isotropic chemical shifts between 10 and 50 ppm, and all are completely separated in the slices of the 2-D plot.

It has yet to be seen how valuable these techniques will be in the study of the coal. The short carbon $T_{1}$ 's reported in coals [28] make a short reorientation time a necessity. In addition, 
since one does not resolve the inequivalent carbons, it remains to be seen what information can be obtained from the overlapping carbons contained in the slices in the second dimension and/or how difficult the analysis of these slices may be.

\section{MAS with Symchronous Application of of Pulses}

This method retrieves the CSA information from a MAS experiment by the application of a train of $\pi$ pulses which are synchronized with the sample rotor speed, a technique first introduced by Lippmaa and coworkers [10]. A $6 \pi$ pulse version of this experiment [30] is shown in Figure 8. It has been shown that this type experiment is very sensitive to pulse imperfections. The experiment is further complicated by the need to carefully synchronize the pulse train with the sample rotation. Since its introduction, several groups have made modifications to minimize the effects of pulse imperfections [29], and to extend the method into a 2-D technique [30]. More recentiy, Tycko et al. [31] have modified the basic method in order to render the CSA patterns obtained in the second dimension undistorted, making it easier to obtain reliable tensor components.

To date, no work on coal has been reported. It should be noted that this method does not suffer from the problem of relaxation as do the methods discussed in the previous section. However, the questions concerning the time required for acquisition and the interpretation of the slices in the second dimension still remain.

\section{Chemical Shift-Chemical Shift Correlation Spectroscopy}

A new technique for measuring ${ }^{13} \mathrm{C}$ chemical shielding tensors in powdered solid samples has great potential for providing new information about coals and similar materials. It is a modification of the well known 2-D exchange NMR experiment [32] where the sample is rapidly reoriented during the mixing time of a 2-D experiment (Flipper NMR). The spectra have projections onto the two chemical shift axes which are merely the conventional ${ }^{23} \mathrm{C}$ solid state chemical shift spectra in each of the two orientations of the sample relative to the external field. The complete spectrum, however, contains more detailed, correlative information about the orientational distribution of ${ }^{13} \mathrm{C}$ atoms in the sample, and as such, perhaps the most promising aspect of this experiment is its ability to characterize order in partially ordered samples [33]. This technique has several unique advantages over conventional 1-D ${ }^{13} \mathrm{C} N \mathrm{NM}$ experiments.

One surprising result is the ability of this experiment of sort out overlapping ${ }^{13} \mathrm{C}$ chemical shielding patterns in powdered polycrystalline samples such as coronene [34]. A powder has randomly distributed ${ }^{13} \mathrm{C}$ atoms, so the chemical shielding patterns in any orientation with the external field are identical. This gives a 2-D Flipper spectrum that is 
symmetrical across its diagonal. The unique representation of chemical shielding tensors in this experiment allows all of the principal values of one tensor to be measured by connecting peaks in the spectrum in triangular (for axially asymmetric shielding tensors) or hexagonal (for - neral shielding tensors) patterns, as shown in Figure 9. Each unique chemical shielding censor in the sample has its own unique hexagonal or triangular pattern, and the ac'ded spectral space and inherent redundancy of the second frequency dimension permits good resolution of different tensors in cases where conventional 1-D spectroscopy might fail.

Figure 10 shows the spectrum obtained on a Pennsylvania anthracite coal (SUI 613a) using a sample that was preserved in its original bedded form. The NMR sample was cut from that sample and oriented such that F1 represents the chemical shielding pattern where the external field is perpendicular to the bedding plane and F2 the chemical shielding pattern with the external field parallel to the bedding plane. There are two important features in this spectrum. The first is the large, triangular signal in the aromatic region, indicating that the aromatic carbons are nearly axially symmetric in this high rank coal. Notice that there is no overlap of $\sigma_{33}$ and the aliphatic signal. Secondly, note the small, isotropic peaks that are on the diagonal in the aliphatic region. The narrowness of these signals is indicative of either a small CSA or of rapid isotropic molecular motion. In this case the signal is from the epoxy used to hold the sample; however, they do serve as a good demonstration of the separation between aromatic and aliphatic signals. As shown by the MAS spectrum in Figure 10, this coal has no aliphatic signal. The spectrum shows some small degree of ordering, but it is difficult to characterize due to the low signal to noise ratio and other subtle spectral features. Additional data is required in order to adequately assess the full magnitude of the contribution offered by this experiment.

\section{Summary and Conclusions}

A number of techniques exist for the determination of the CSA in solids. The choice of a method is often limited by the availability of the necessary equipment and by the difficulty of the experiment in terms of the required pulse sequences and the mechanical aspects of sample spinning and/or reorientations. Furthermore, the complexity of the compound can rule out some of the experiments due to the complexity of the resultant spectrum.

From the limited number of coal samples on which the tensor information has been obtained several conclusions can be reached. The shielding values obtained (both tensor components and isotropic chemical shifts) are consistent with those obtained in the substituted polycyclic aromatic compounds that are often used as model compounds. The majority of the results are within one standard deviation of the average taken from aromatic carbons in a large 
number of compounds. In addition, the treakdown of the aromatic carbons into the four classes outlined in the first section as determined by both static or VASS lineshape analysis is consistent with the results of both dipolar dephasing experiments and elemental analysis.

There are also several advantages in study of the CSA in coal instead of doing only the more common CP/MAS experiments. Most obviously, one is determining not only the isotropic chemical shift but the three principal values which provide more information on the electronic environment of the nucleus. The information of the percentages of bridged and phenolic aromatic carbons obtained in the fitting of the shielding pattern may be more reliable than those from CP/MAS and dipolar dephasing in which it is assumed that all the intensity in a specific frequency range is due to a certain type of aromatic carbon.

Finally, the 2-D techniques which separate the individual static or scaled lineshapes are very powerful tools and have the potential of being extremely useful. The usefulness of DAS for the stucly of complex compounds has already been demonstrated [8]. The full potential of this method, as well as the other 2-D techniques, in the study of coal still remains to be explored. The 2-D chemical shift - chemical shift correlation method may also prove to be a valuable tool in the study of coal. Refinements of the experimental procedure are currently under study.

\section{Acknowledgement}

Portions of this work was supported by the National Science Foundation through the Advanced Combustion Engineering Research Center and the Chemical Sciences Division, Office of Basic Energy Science, DOE. 


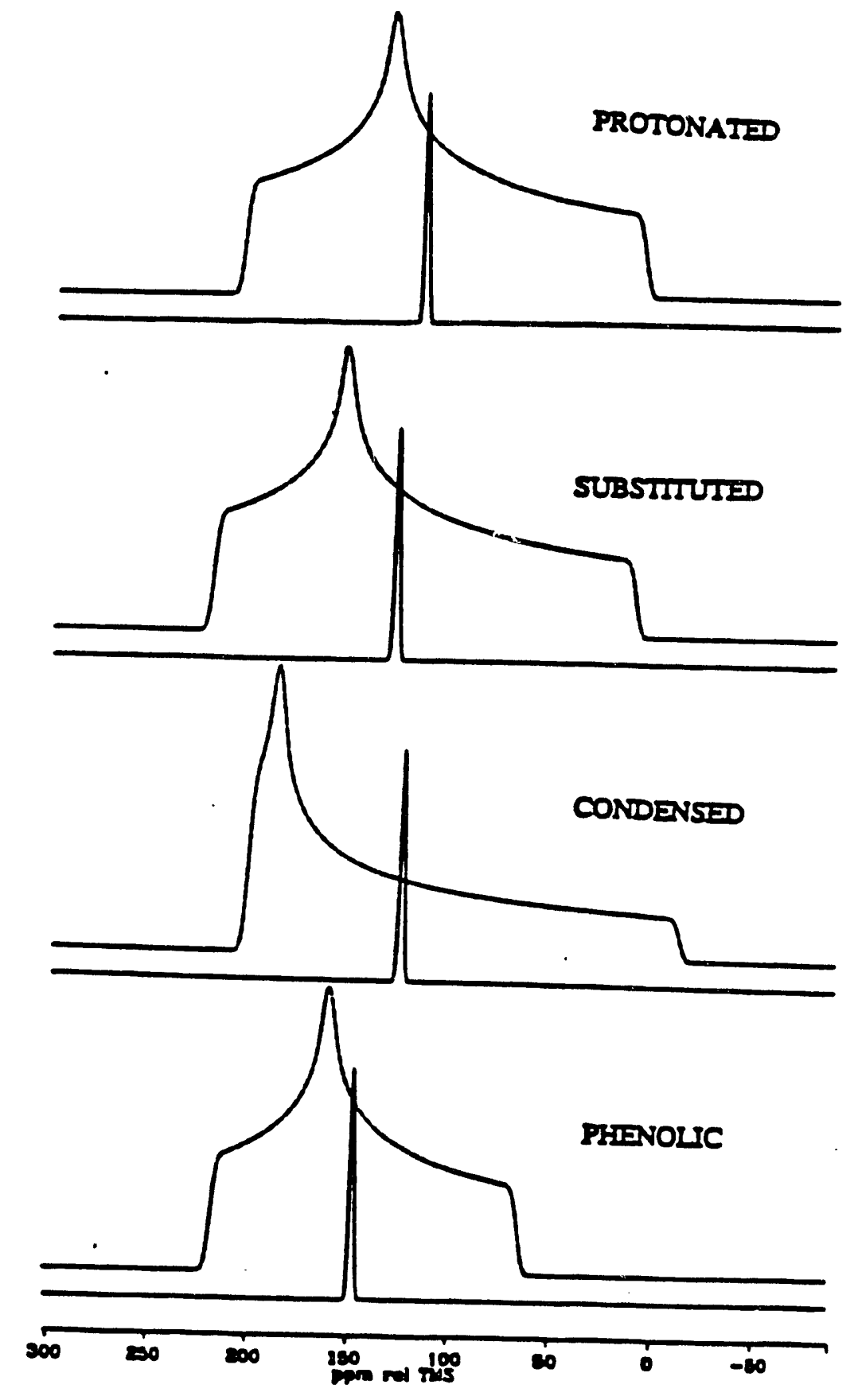

Figure V.1.1. Ideal tensor pattems for the four types of aromatic carbons in coal. 
Table 1 : Average Chemical Shielding Tensor Values for Aromatic Carbons

\begin{tabular}{lllll}
\hline Carbon Type & $\sigma_{11}$ & $\sigma_{22}$ & $\sigma_{33}$ & $\sigma_{\text {avg }}$ \\
\hline Protonated (73) & $209 \pm 21$ & $138 \pm 14$ & $15 \pm 10$ & $121 \pm 11$ \\
Substituted (32) & $223 \pm 8$ & $160 \pm 17$ & $18 \pm 8$ & $134 \pm 7$ \\
Phenolic (33) & $219 \pm 17$ & $163 \pm 9$ & $69 \pm 5$ & $150 \pm 7$ \\
Condensed (10) & $206 \pm 6$ & $193 \pm 7$ & $-4 \pm 11$ & $131 \pm 5$ \\
& & & & \\
\hline
\end{tabular}

Table 2: Tensor Components for Aromatic Carbons in $1,2,3,6,7,8$-hexahydropyrene

\begin{tabular}{lccccc}
\hline Carbon type & $\sigma_{11}$ & $\sigma_{22}$ & $\sigma_{33}$ & $\sigma_{\text {avg }}$ & $\%$ \\
\hline Protonated & 225 & 128 & 22 & 125 & 40 \\
Substituted & 231 & 168 & 9 & 136 & 40 \\
Condensed & 203 & 197 & -6 & 131 & 20 \\
\hline
\end{tabular}



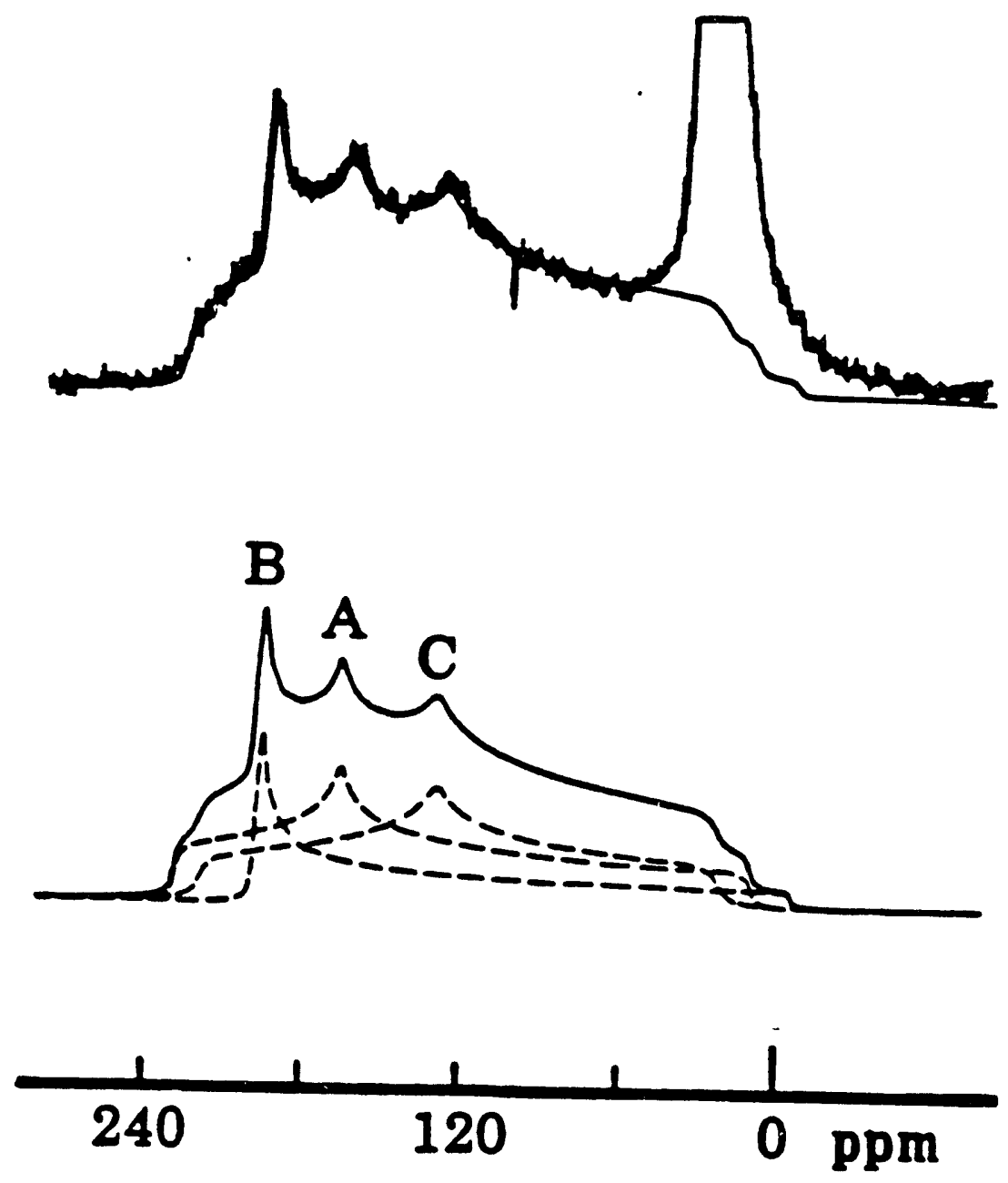

Figure 2. Static lineshape with best fit of aromatic region for $1,2,3,6,7,8$-hexahydropyrene. A indicates the pattem for the substituted aromatic carbons, $B$ for the bridgehead or condensed aromatic carbons, and $C$ for the protonated aromatic carbons. Reproduced from ACS Fuel Division
Preprints 1987, 32, 155. 


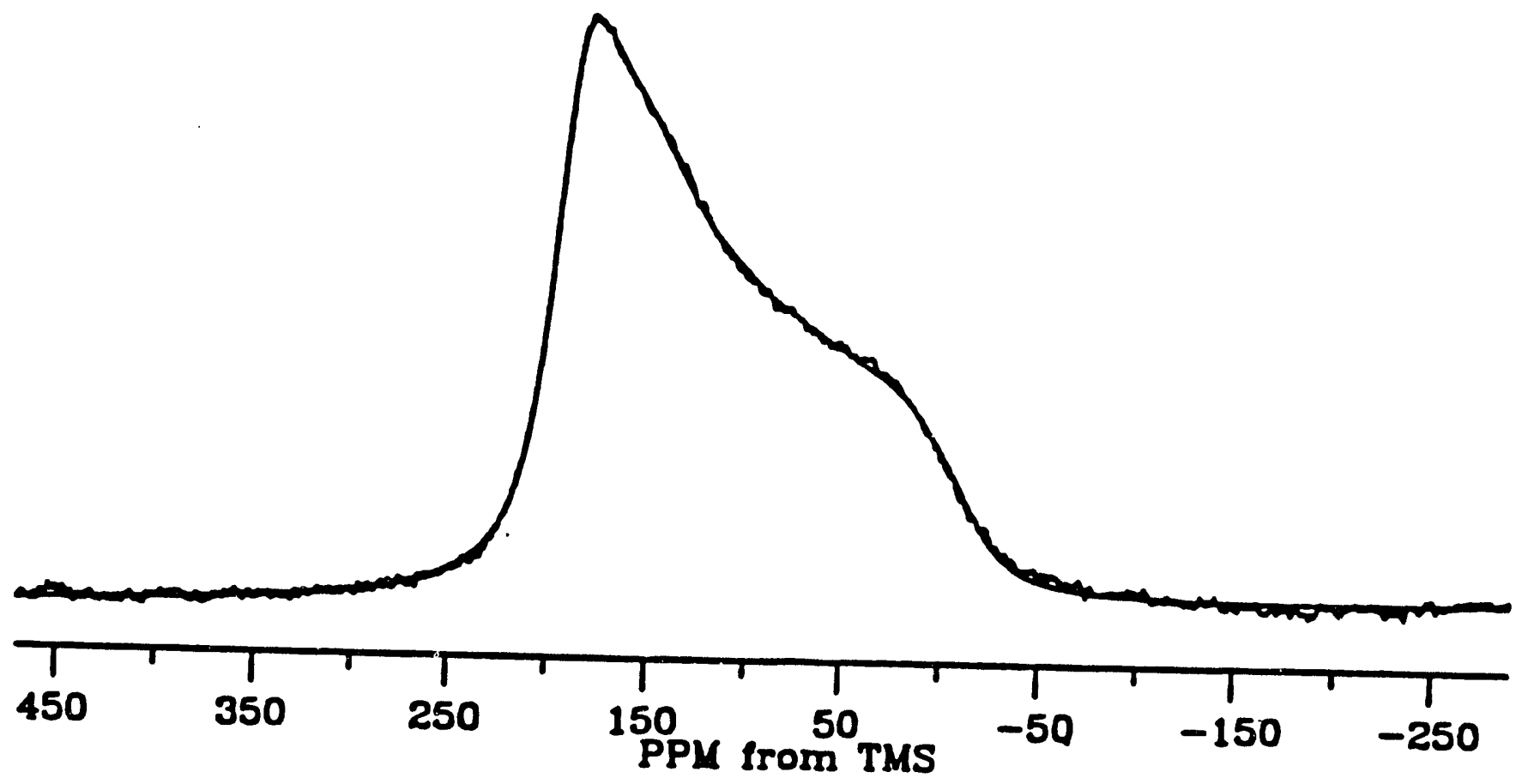

Figure 3. Static lineshape with best fit for PSOC-1468. 
Table 3: Tensor Components for Aromatic Carbons in Coals

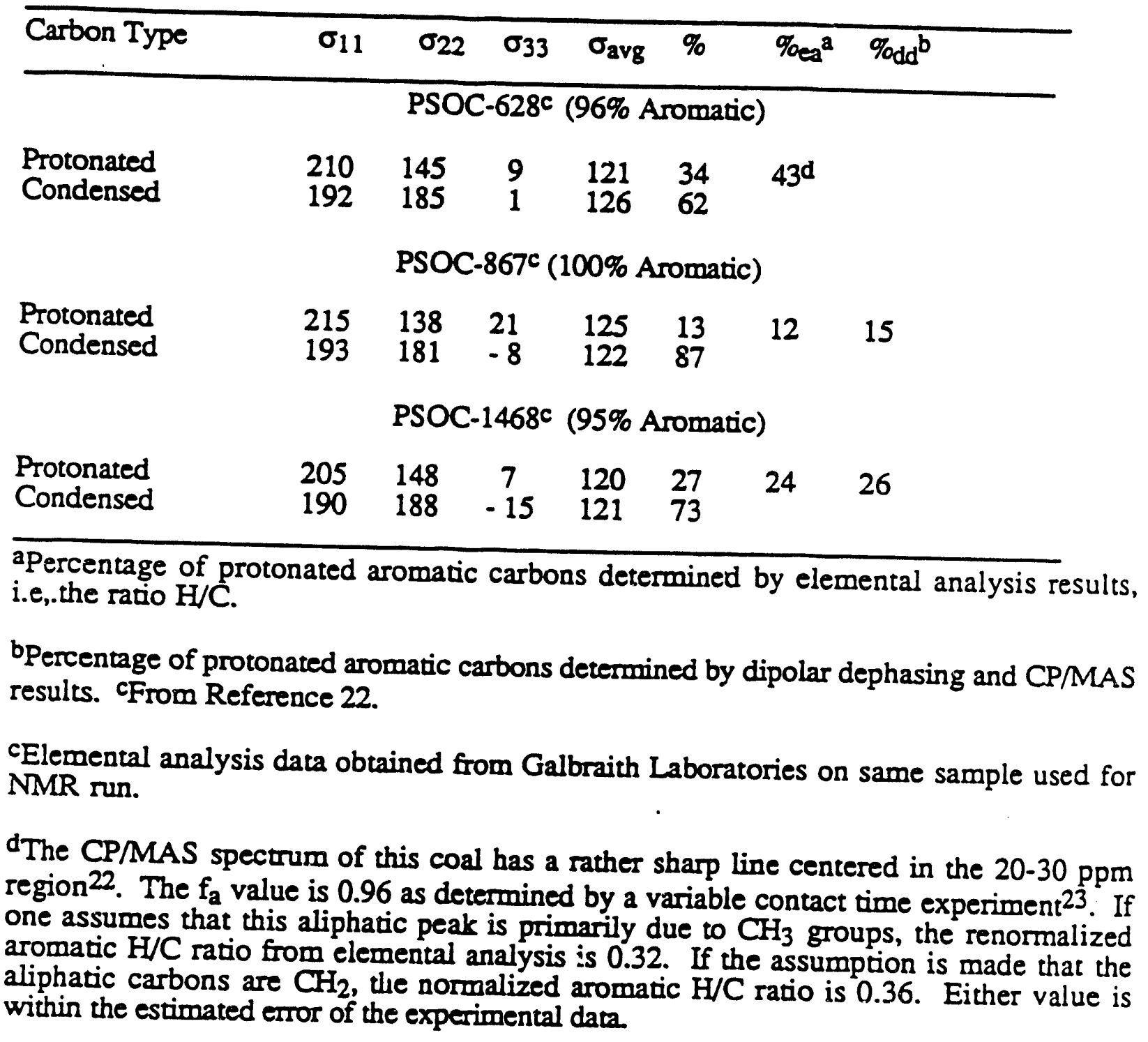


Table 4: Tensor Components for Model Compounds

\begin{tabular}{|c|c|c|c|c|c|}
\hline Carbon Type & $\sigma_{11}$ & $\sigma_{22}$ & $\sigma_{33}$ & $\sigma_{\text {avg }}$ & $\%$ \\
\hline \multicolumn{6}{|c|}{$1,2,3,6,7,8$-hexahydropyrene } \\
\hline $\begin{array}{l}\text { Protonated } \\
\text { Substituted } \\
\text { Condensed }\end{array}$ & $\begin{array}{l}225 \\
231 \\
203\end{array}$ & $\begin{array}{l}128 \\
168 \\
197\end{array}$ & $\begin{array}{r}22 \\
9 \\
-6\end{array}$ & $\begin{array}{l}125 \\
136 \\
131\end{array}$ & $\begin{array}{l}40 \\
40 \\
20\end{array}$ \\
\hline \multicolumn{6}{|c|}{ Pyracene } \\
\hline $\begin{array}{l}\text { Protonated } \\
\text { Substituted } \\
\text { Condensed }\end{array}$ & $\begin{array}{l}209 \\
226 \\
202\end{array}$ & $\begin{array}{l}142 \\
166 \\
192\end{array}$ & $\begin{array}{l}19 \\
36 \\
24\end{array}$ & $\begin{array}{l}124 \\
142 \\
139\end{array}$ & $\begin{array}{l}40 \\
40 \\
20\end{array}$ \\
\hline \multicolumn{6}{|c|}{ 1,4,7,8-tetramethyinaphthalene } \\
\hline $\begin{array}{l}\text { Protonated } \\
\text { Substituted } \\
\text { Condensed }\end{array}$ & $\begin{array}{l}235 \\
231 \\
204\end{array}$ & $\begin{array}{l}123 \\
161 \\
202\end{array}$ & $\begin{array}{r}32 \\
14 \\
1\end{array}$ & $\begin{array}{l}130 \\
135 \\
135\end{array}$ & $\begin{array}{l}40 \\
40 \\
20\end{array}$ \\
\hline \multicolumn{6}{|c|}{ 9,9,10,10-tetramethyl-9,10-dihydroanthracene } \\
\hline $\begin{array}{l}\text { Protonated } \\
\text { Protonated } \\
\text { Substituted }\end{array}$ & $\begin{array}{l}229 \\
224 \\
.219\end{array}$ & $\begin{array}{l}147 \\
143 \\
186\end{array}$ & $\begin{array}{r}7 \\
17 \\
20\end{array}$ & $\begin{array}{l}128 \\
128 \\
142\end{array}$ & $\begin{array}{l}33 \\
33 \\
33\end{array}$ \\
\hline
\end{tabular}




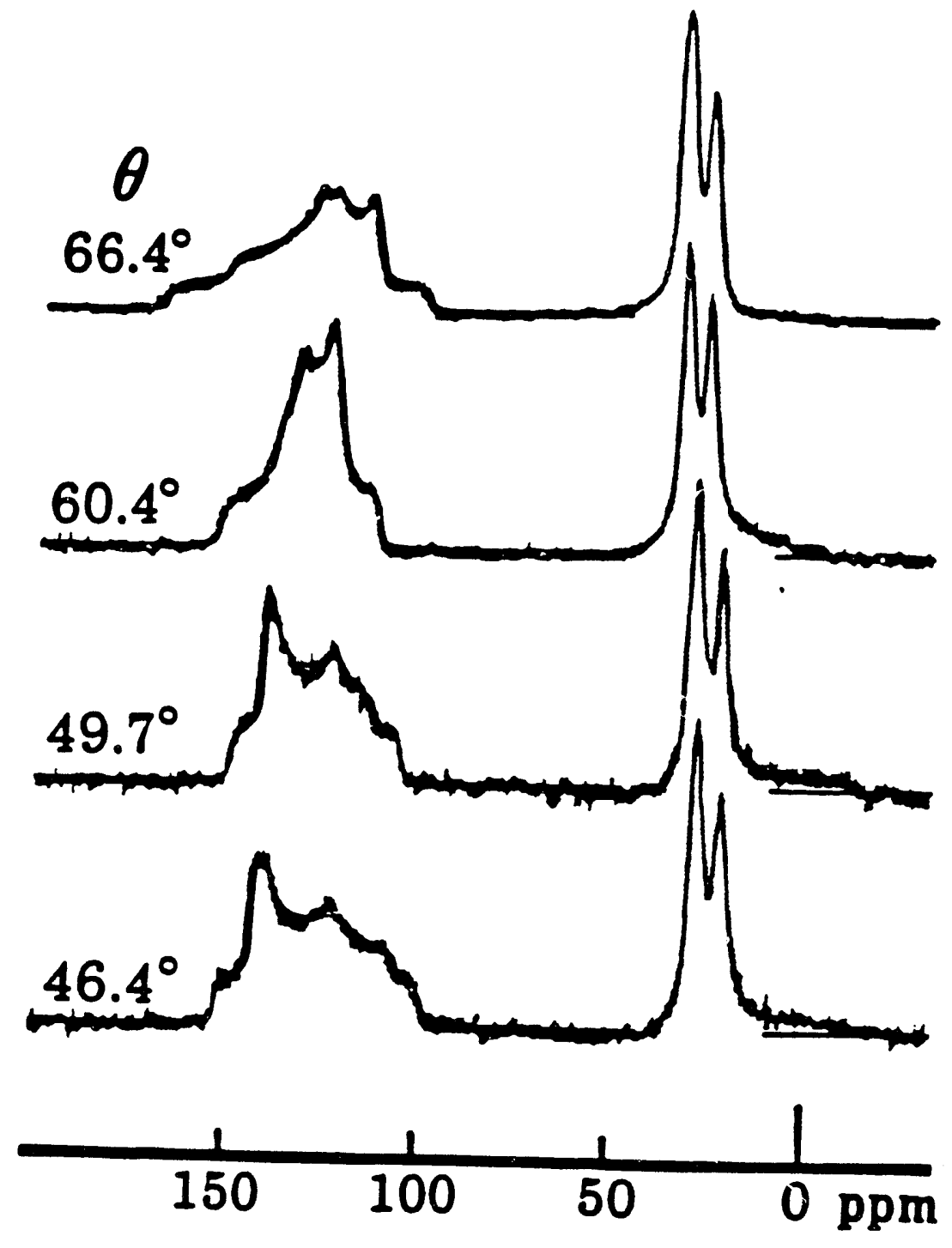
Figure 4. VASS spectra of 1,2,3,6,7,8-hexahydropyrene with best fits. Reproduced
from ACS Fuel Division Preprints 1987, 32,155. 
Table 5: Tensor Components and Population Factors for Coals Obtained by VASS

\begin{tabular}{lllllll}
\hline Carbon Type & $\sigma_{11}$ & $\sigma_{22}$ & $\sigma_{33}$ & $\sigma_{\text {avg }}$ & $\%$ & $\%_{\text {dd }}{ }^{2}$ \\
\hline
\end{tabular}

Illinois \#6 (72\% arornatic) $)^{b}$

$\begin{array}{lrrrrrr}\text { Protonated } & 204 & 142 & 32 & 126 & 27 & 26 \\ \text { Substiuted } & 231 & 161 & 46 & 146 & 18 & 18 \\ \text { Condensed } & 212 & 184 & -9 & 129 & 22 & 22 \\ \text { Phenolic } & 215 & 155 & 80 & 150 & 5 & 6\end{array}$

Upper Freeport (81\% aromatic)

$\begin{array}{lrrrrrr}\text { Protonated } & 208 & 141 & 20 & 123 & 28 & 28 \\ \text { Substituted } & 217 & 158 & 33 & 136 & 21 & 20 \\ \text { Condensed } & 203 & 189 & -11 & 127 & 30 & 29 \\ \text { Phenolic } & 229 & 159 & 71 & 153 & 2 & 2\end{array}$

Pocahontas ( $86 \%$ aromatic)

$\begin{array}{lllrrrr}\text { Protonated } & 215 & 145 & 20 & 127 & 33 & 33 \\ \text { Substinted } & 220 & 162 & 31 & 138 & 17 & 17 \\ \text { Condensed } & 203 & 191 & -10 & 128 & 34 & 34 \\ \text { Phenolic } & 225 & 158 & 73 & 152 & 2 & 2\end{array}$

Aldwarke Silkstone Fusinite ( $88 \%$ aromatic)

$\begin{array}{lrrrrrr}\text { Protonated } & 223 & 149 & 17 & 130 & 34 & 34 \\ \text { Substituted } & 231 & 161 & 46 & 146 & 6 & \\ \text { Condensed } & 204 & 192 & -30 & 122 & 48 & \\ \text { Phenolic } & - & - & - & - & 0 & \end{array}$

apercentages from the result of dipolar dephasing experiments. bPercent aromatic and $\%_{d d}$ values taken from Reference 23 . cPercent aromatic and percent protonated from Reference 24. 


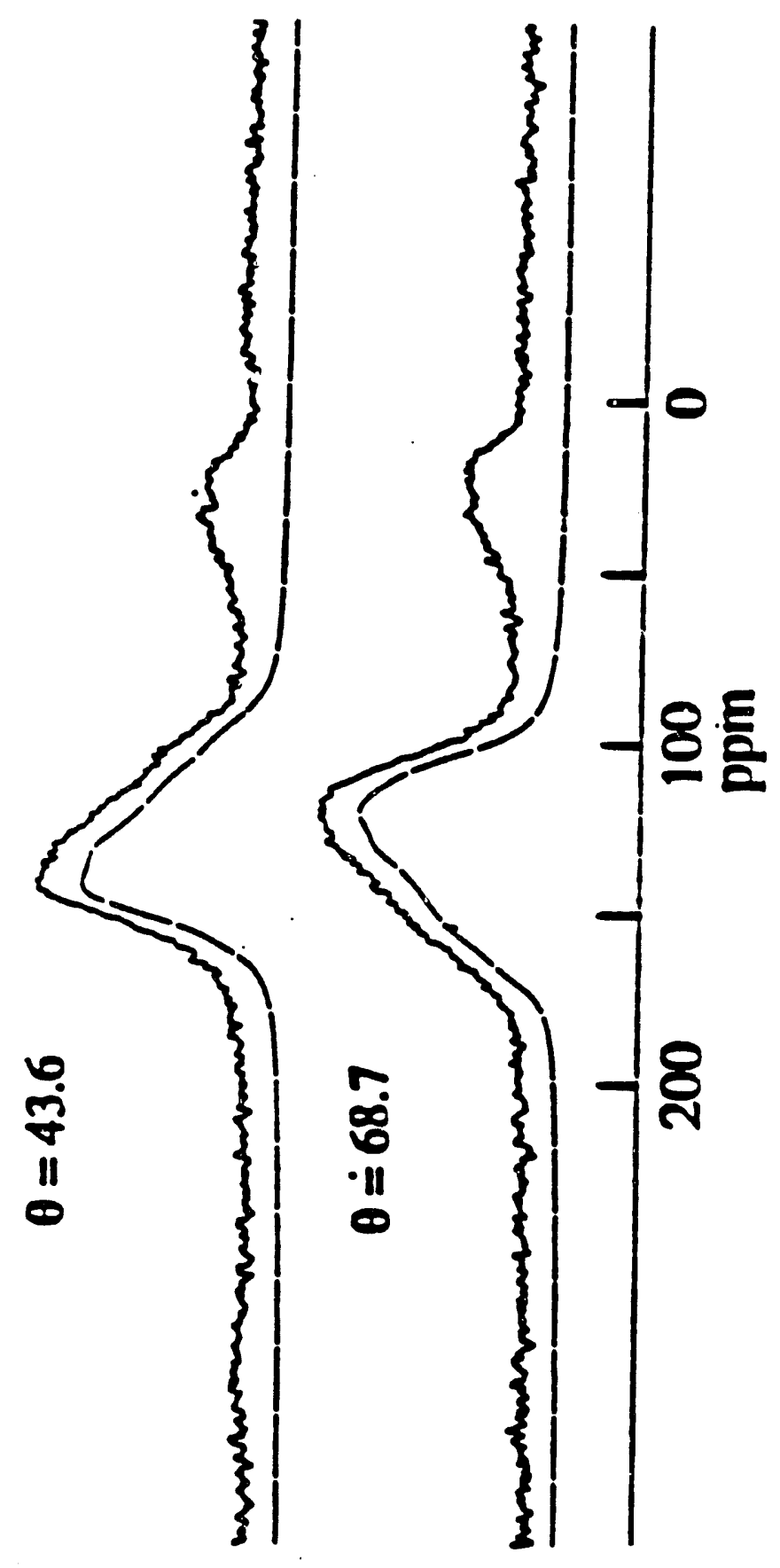

Figure 5. VASS spectra of Pocahontas coal along with best fits. 

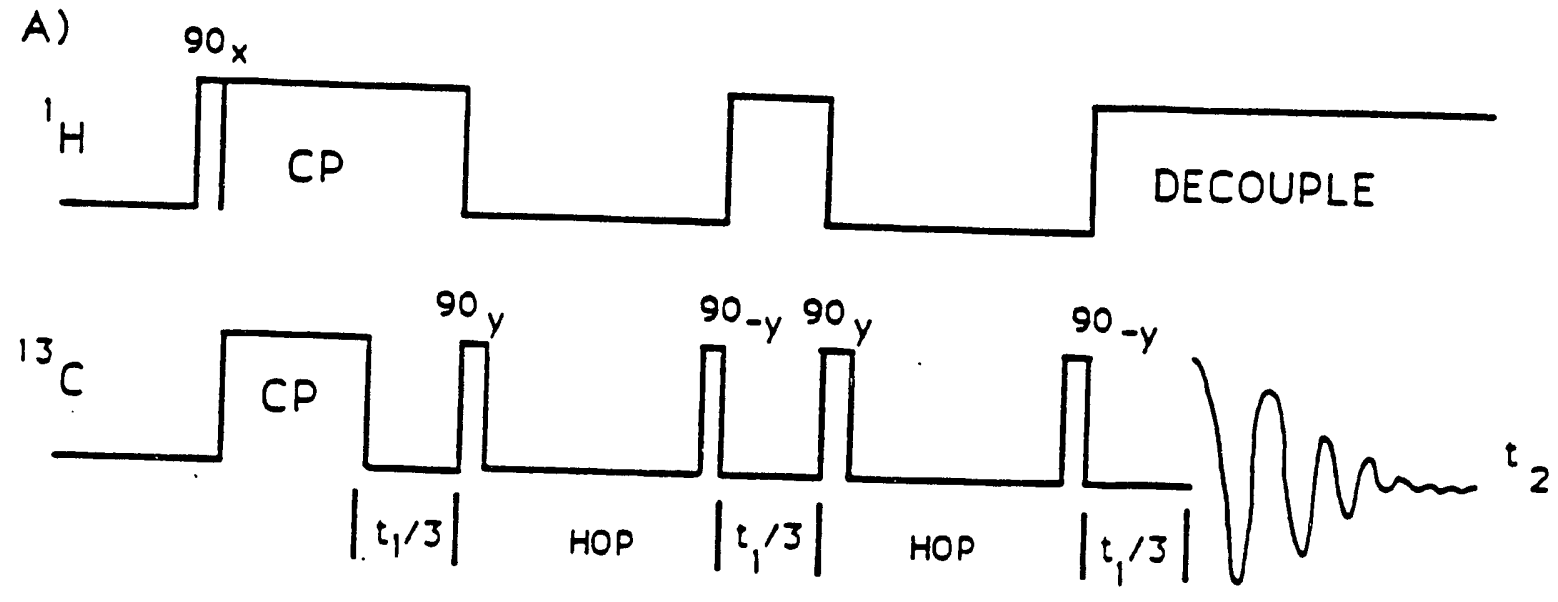

B) $90_{x}$
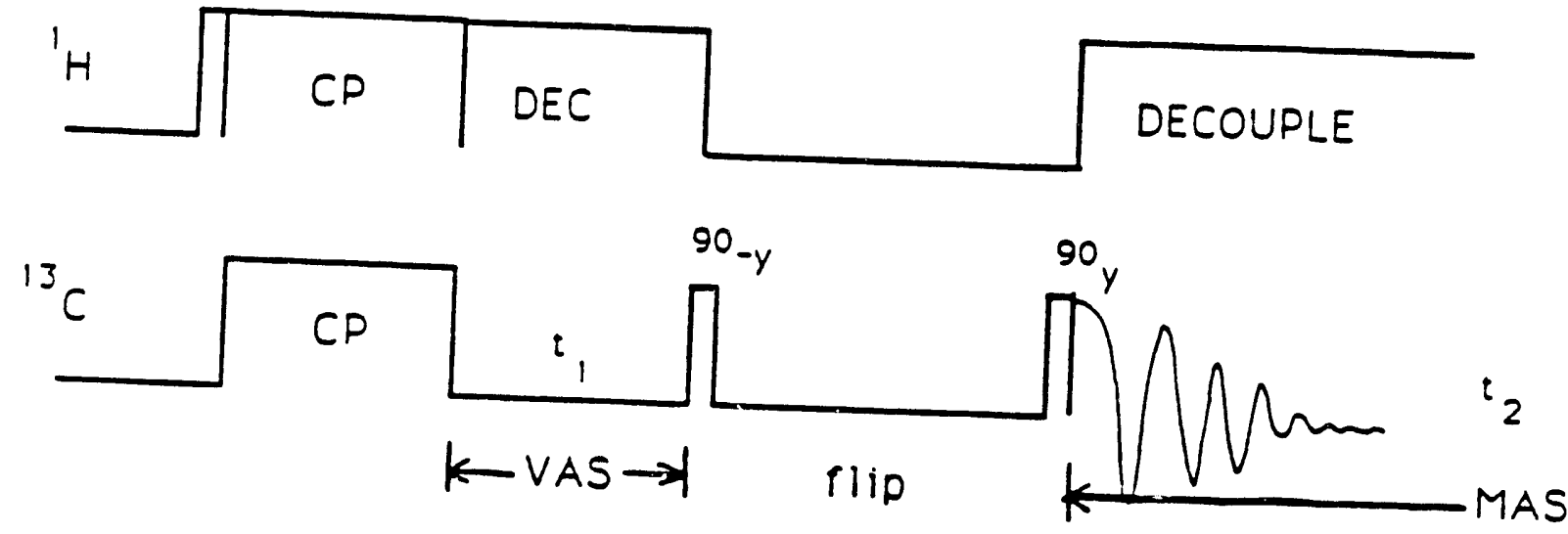

C) $90 x$
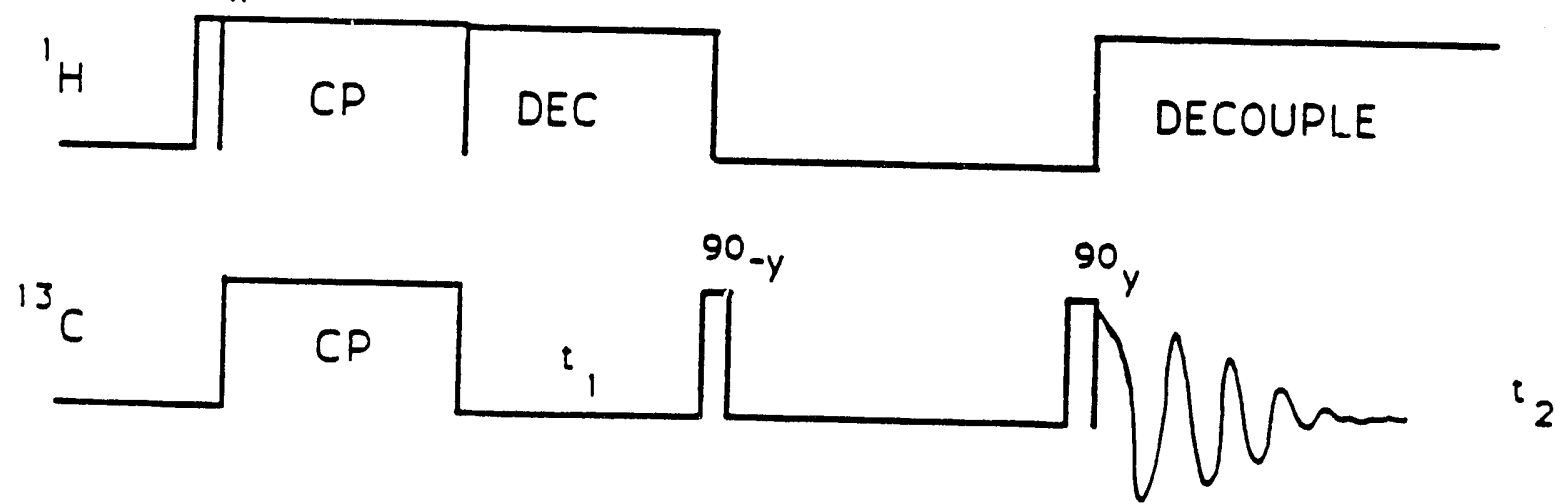

Spinner

Air OFF ON

Figure 6. Pulse sequences along with rotor orientation for (a) MAH, (b) DAS, and (c) 

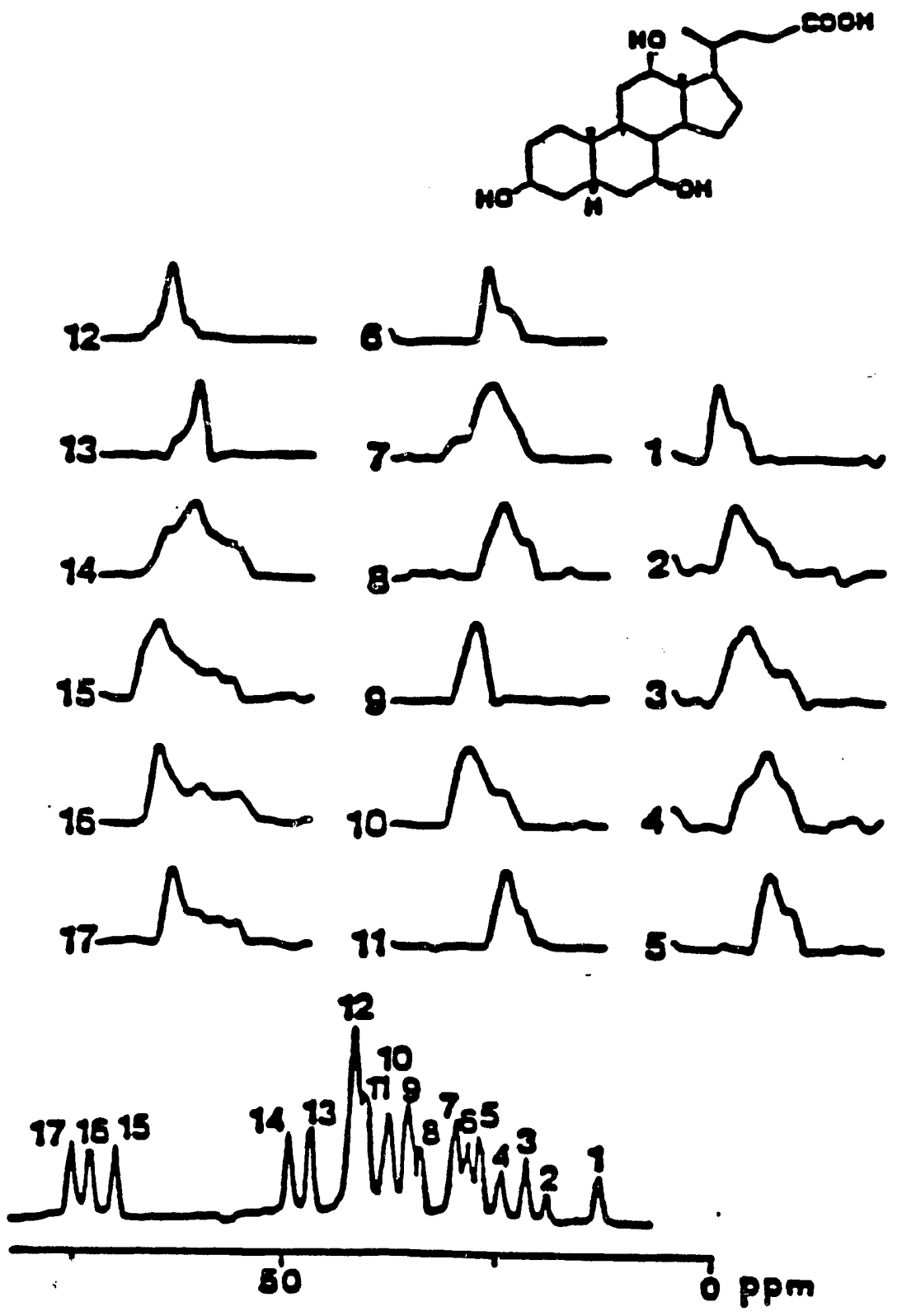

Figure

7. Results of a DAS experiment on cholic acid. Reproduced from Reference 8
with the permission of the authors. 


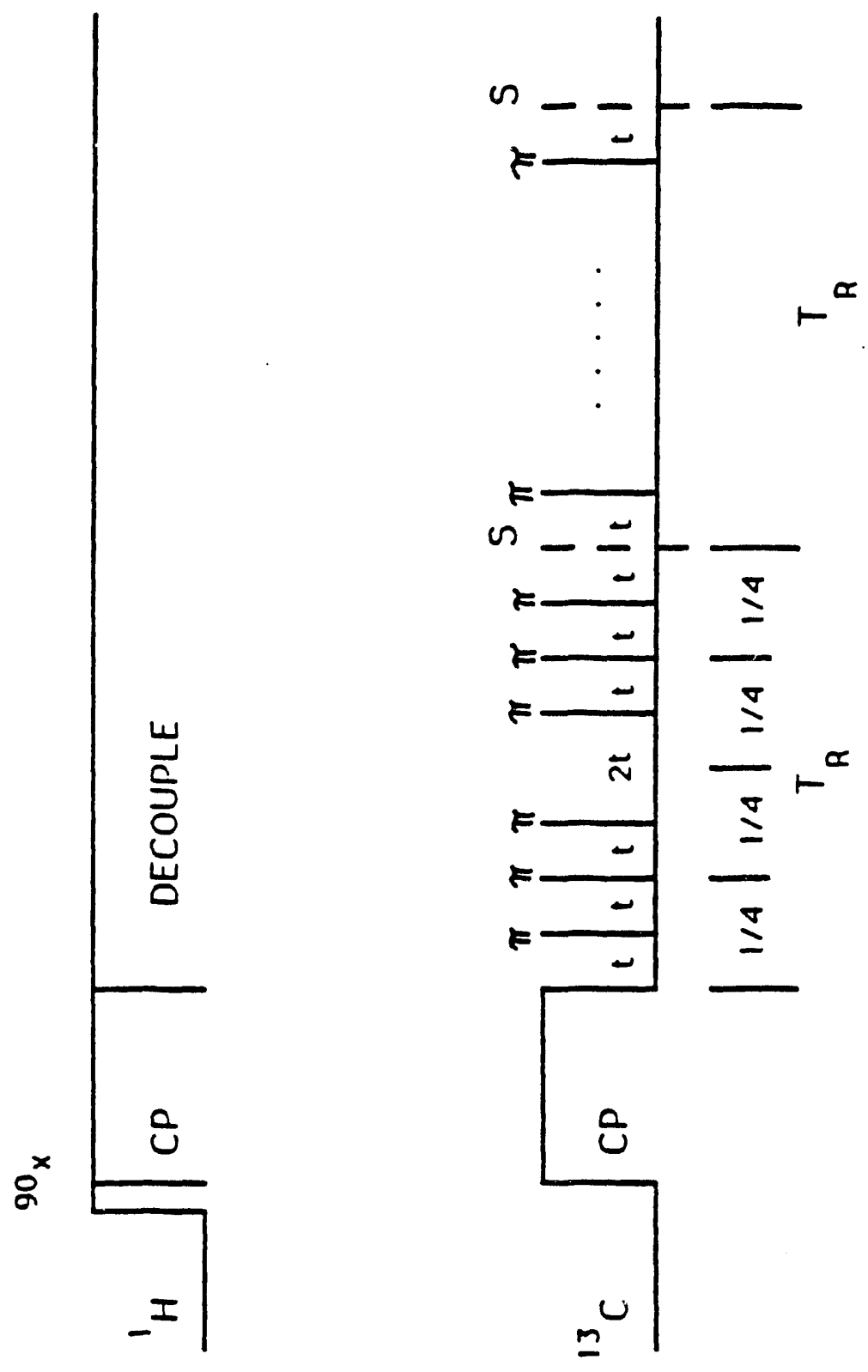

Figure 8. $6 \pi$-pulse sequence for MAS with synchronous application of $\pi$ pulses. $T_{R}$ is the rotor period and $S$ indicates the sampling time. 


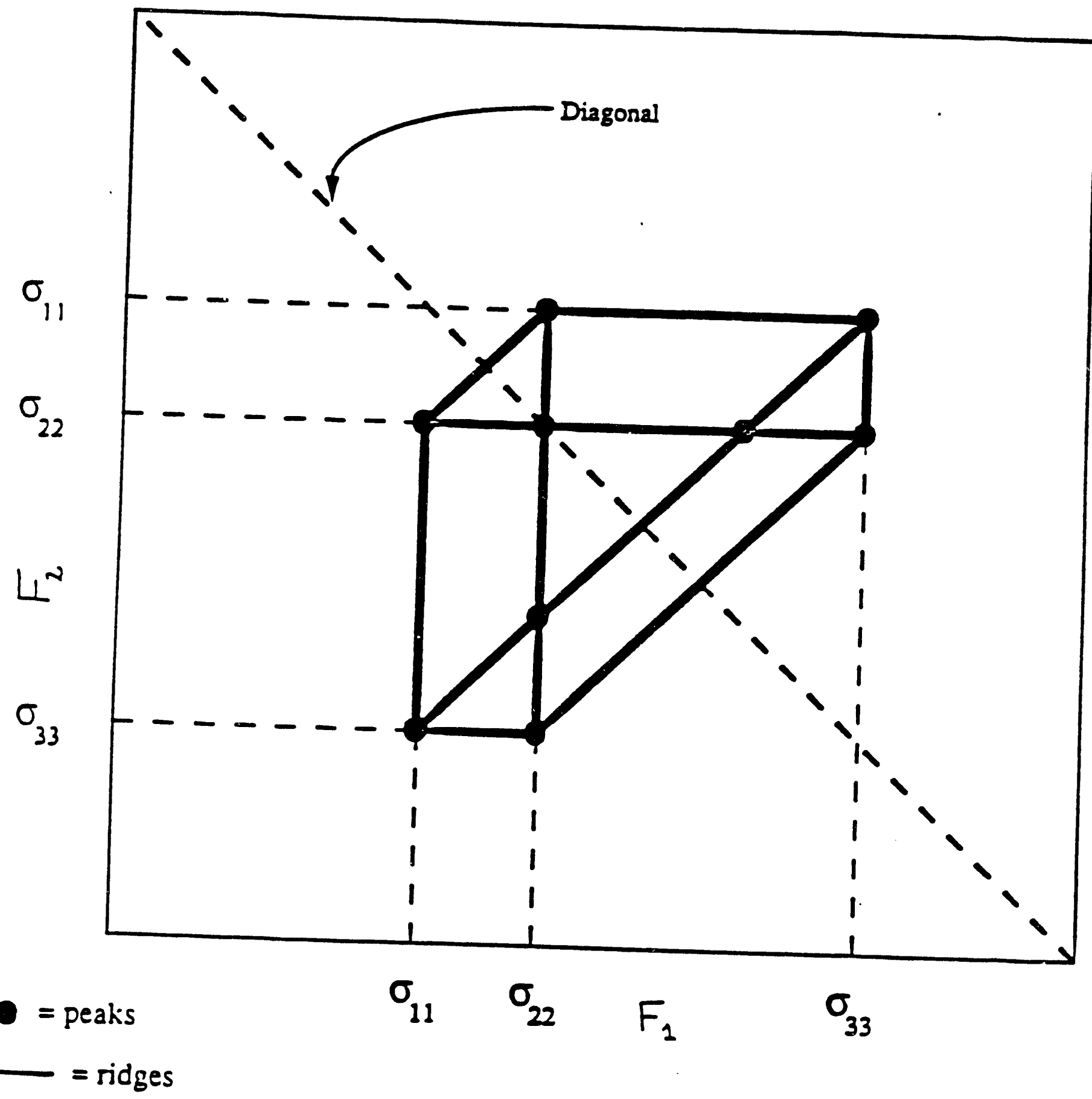

Figure 9.a: Ideal shielding patterns obtained in the 2-D CS-CS experiment for asymmetric tensor. The peaks (marked by ${ }^{\bullet}$ ) are the principal values of the chemical
shielding tensor. 


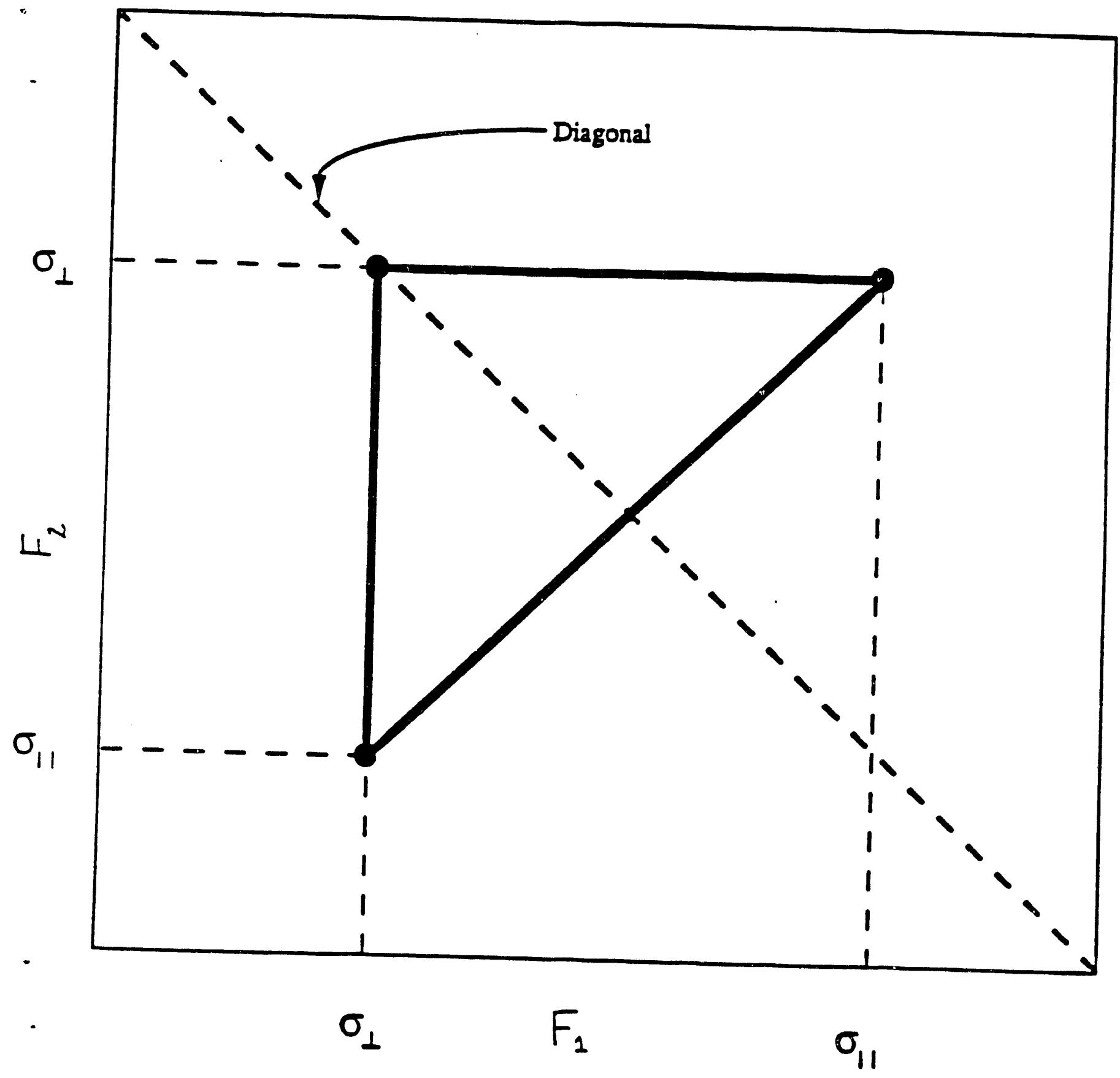

Figure 9. b: Ideal shielding parterns obtained in the 2-D CS-CS experiment for axially symmetric tensor. The peaks (marked by ${ }^{\bullet}$ ) are the principal values of the
chemical shielding tensor. 
Task V.2. MOLECULAR WEIGHT DETERMINATION OF COAL DERIVED LIQUIDS BY MASS SPECTROMETRIC TECHNIQUES (H.L.C. Meuzelaar and H. Huai)

\section{The Need for Improved MW Determination Methods}

One of the most challenging tasks for analytical chemists involved in characterizing the molecular properties of coal derived liquid (CDL) is to obtain a reliable estimate of the molecular weight distribution. Due to the relatively high MW, aromaticity and/or polarity of the major components conventional MW determination techniques, e.g. based on colligative properties tend to perform poorly with coal liquids. Among the MS techniques, Field Ionization MS (FIMS) has established a reputation for being especially well suited for MW determination of CDLs and other liquid fossil fuels. Unfortunately, FIMS instruments are only available for this purpose in a few laboratories worldwide. Moreover, although performing well in the medium to high MW range (e.g. MW 200-1000), all MS techniques may produce relatively large errors when applied to liquids containing relatively volatile components in the $C_{5}-C_{15}$ range, due to potentially large evaporation losses during sample introduction.

Consequently, we felt prompted to undertake the development of a novel $\mathrm{MW}$ determination method based on MS measurement of the relationship between vacuum distillation temperature and average molecular weight in combination with thermogravimetric (TG) measurement of the relationship between vacuum distillation temperature and relative mass loss. Combination of these two measurements then produces the relationship between molecular weight and mass fraction, in other words the molecular weight distribution of the sample. Obviously, this method can only be applied to vacuum distillable components. Within this limitation, however, the technique is relatively insensitive to mass losses during sample introduction and/or MW dependent differences in instrument response.

In order to test the applicability of the combined TG and MS approach to CDL tar oil fractions of four ANL coals (Beulah Zap, mlinois \#6, Blind Canyon and Pittsburgh \#8) produced

- by the Shabtai low temperature HT-BCD (Hydrotreatment-Base Catalyzed Depolymerization) process were analyzed.

\section{Vacuum Thermogravimetry}

Vacuum TG profiles of the 4 HT-BCD oil fractions obtained with a Perkin Elmer Model 7 TG/DTG system (Figure 1) show that $>92 \%$ of each oil fraction distills off below $350^{\circ} \mathrm{C}$ when heated at a rate of $25^{\circ} \mathrm{C} / \mathrm{min}$. This implies that the HT-BCD fraction might be suitable for the above described combined TG and MS approach to MW determination. 


\section{Chemical Ionization Mass Spectrometry}

A Finnigan MAT ITD 700 based GC/MS system with a short (4 m long) fused silica capillary column coated with $0.1 \mu \mathrm{m}$ BP-1 was used under isobutane chemical ionization (CI) conditions in order to facilitate the detection of relatively large and/or labile molecular components. As shown in Figure 2, this results in a low resolution gas chromatography (GC) profile. However, since we are only interested in determining the average molecular weight as a function of distillation temperature, i.e. in using the GC/MS data for a simulated distillation experiment, the lack of chromatographic resolution is of no consequence. Table 1 lists the average molecular weight of the four tar oil fractions, whereas Figure 3 shows the integrated (summed) mass spectrum over the entire elution period as well as partially summed mass spectra over successive temperature intervals, illustrating the gradual increase in average $\mathrm{MW}$ with increasing temperature.

Besides the use of isobutane $\mathrm{CI}$ which reduces fragmentation losses of molecular ion signals compared to electron ionization (EI), the simulated distillation GC approach used here differs from conventional methods in that the very short capillary column permits elution of much larger molecular components. This is illustrated by the spectra in Figure 4 representing (a) the use of a conventional $15 \mathrm{~m}$ long column in combination with EI and (b) the same column in combination with CI. Although CI does improve the detection of larger components, major improvement is obtained by means of a short capillary column, as can be verified by comparing Figures $3 \mathrm{a}$ and $4 \mathrm{~b}$.

In spite of the much improved detectability of large molecules, it would be imprudent to assume that all of the larger less volatile components are detected by the short column GC/CIMS technique. In fact, comparison with the FIMS profiles in Figure 5 and the average molecular weight (Table 1), obtained by $\sim 100 \mathrm{C} / \mathrm{min}$ programmed evaporation in front of the FI source, reveals the presence of much larger molecular components than observed by short column GC/CIMS (Figure 3a and Table 1). Before concluding, however, that the GC/CIMS profile in Figure 3a is completely wrong, we also need to remember that an unknown percentage of the lower MW components is likely to have been lost by early evaporation in the FIMS procedure.

In other words, we now have two, apparently conflicting, pieces of information: (1) the GC/CIMS curves which are likely to underestimate the contribution of high MW components, and (2) the FIMS profiles, which may severely underestimate the contribution of more volatile components. Fortunately, however, both techniques produce rather similar relationships between average MW and (simulated) distillation temperatures, as illustrated in Figure 6 . Apparently, the more or less selective losses of low and high end components in the FIMS and 
GC/CIMS data, respectively, have not caused a marked shift in the MW vs. temperature relationship, as can also be seen from the overlapping Hlinois \#6 curves in Figure 7 . In the center of the overlapping zone (e.g. MW 275 at $200 \mathrm{C}$ ) the two curves agree to within 10-15\%. Another interesting observation is the nearly linear relationship between temperature and average MW. Obviously, the shapes of the curves in Figure 5 may be expected to be dependent on the type of feed coal as well as conversion and fractionation processes used.

\section{Combination of TG and MS Data}

Figure 8 shows the calculated molecular weight distribution profile of nlinois \#6 HT-BCD oil obtained by multiplying the average molecular weights measured over each temperature interval of $25^{\circ} \mathrm{C}$ (GC/CIMS data) by the corresponding mass fractions involved, as measured from the vacuum TG curve of nlinois \#6 HT-BCD oil in Figure 1 and summing over all temperatures. Since the vacuum TG curve indicated that the largest mass fraction distilled between 200 and $250^{\circ} \mathrm{C}$ and too few reliable FIMS data points were available in that range only the GC/CIMS data could be used.

Obviously, the calculated MW distribution profile in Figure 8 and the average molecular weight in Table 1 differ markedly from the MW distribution suggested by the FIMS results (Figure 5 and Table 1). This suggests that the suspected evaporation loss during sample introduction must have been severe. Alternatively, some sample deterioration through spontaneous condensation reactions may have occurred during shipment of the samples to Professor H.-R. Schulten's laboratory at the Fresenius Institute in Wiesbaden (GFR).

Much more work, e.g. involving standard mixtures of known MW distribution, will need to be done before the practical usefulness and reliability of the combined TG and MS technique can be established. Although the first results are encouraging, especially in view of the fact that one of the least expensive, commercially available GC/MS instruments was used in these experiments, some of the underlying assumptions and approximations need to be analyzed critically. Obviously, this empirical approach will not be the method of choice when examining

- relatively simple, well behaved mixtures of molecules. With CDLs, however, the MW profiles calculated may be closer to the true MW distribution than has been achieved by other methods thus far. 


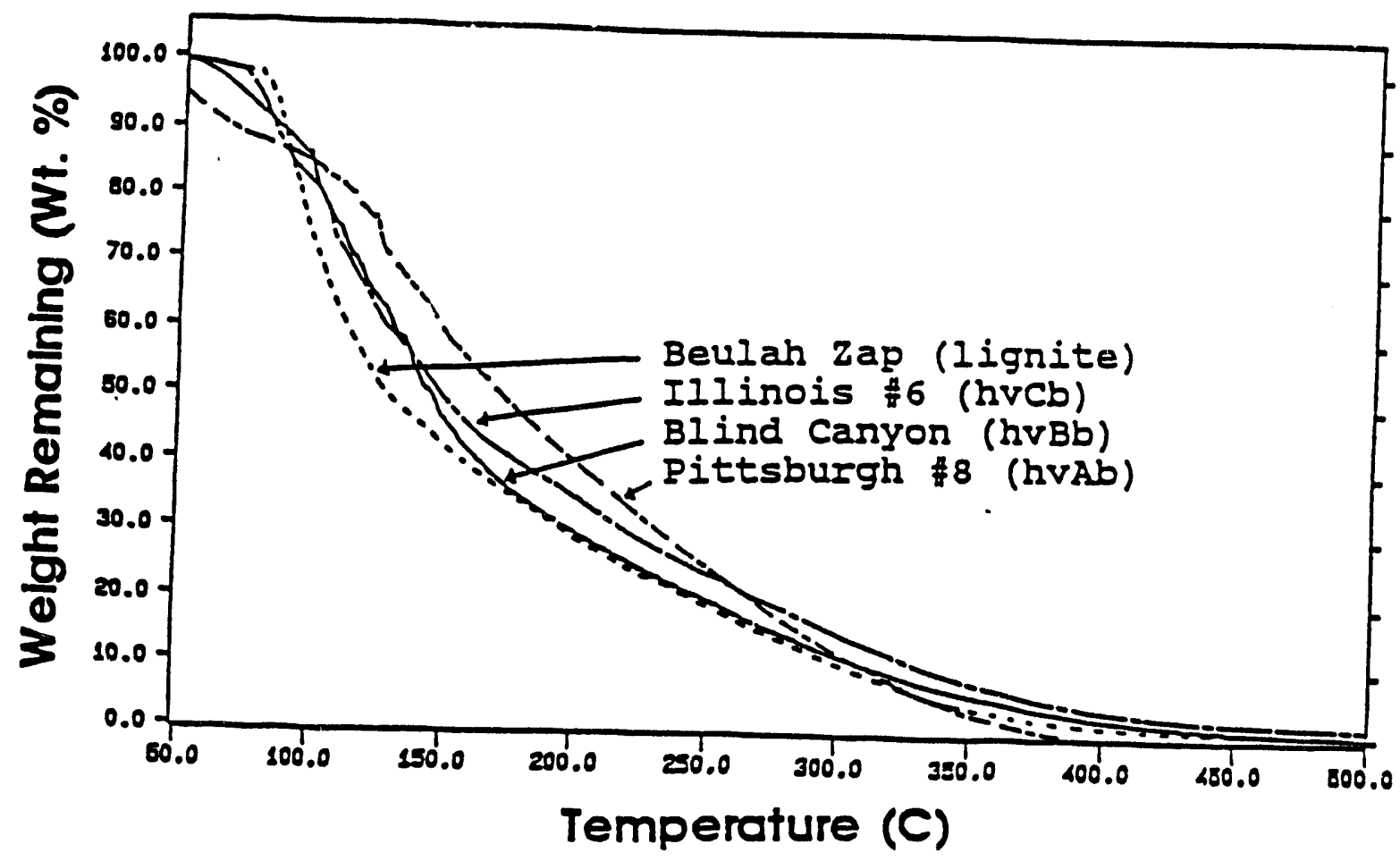
Figure 1. Thermogravimetric weight loss curves for HT-BCD oil fractions of four ANL-PC
coals obtained under vacuum conditions

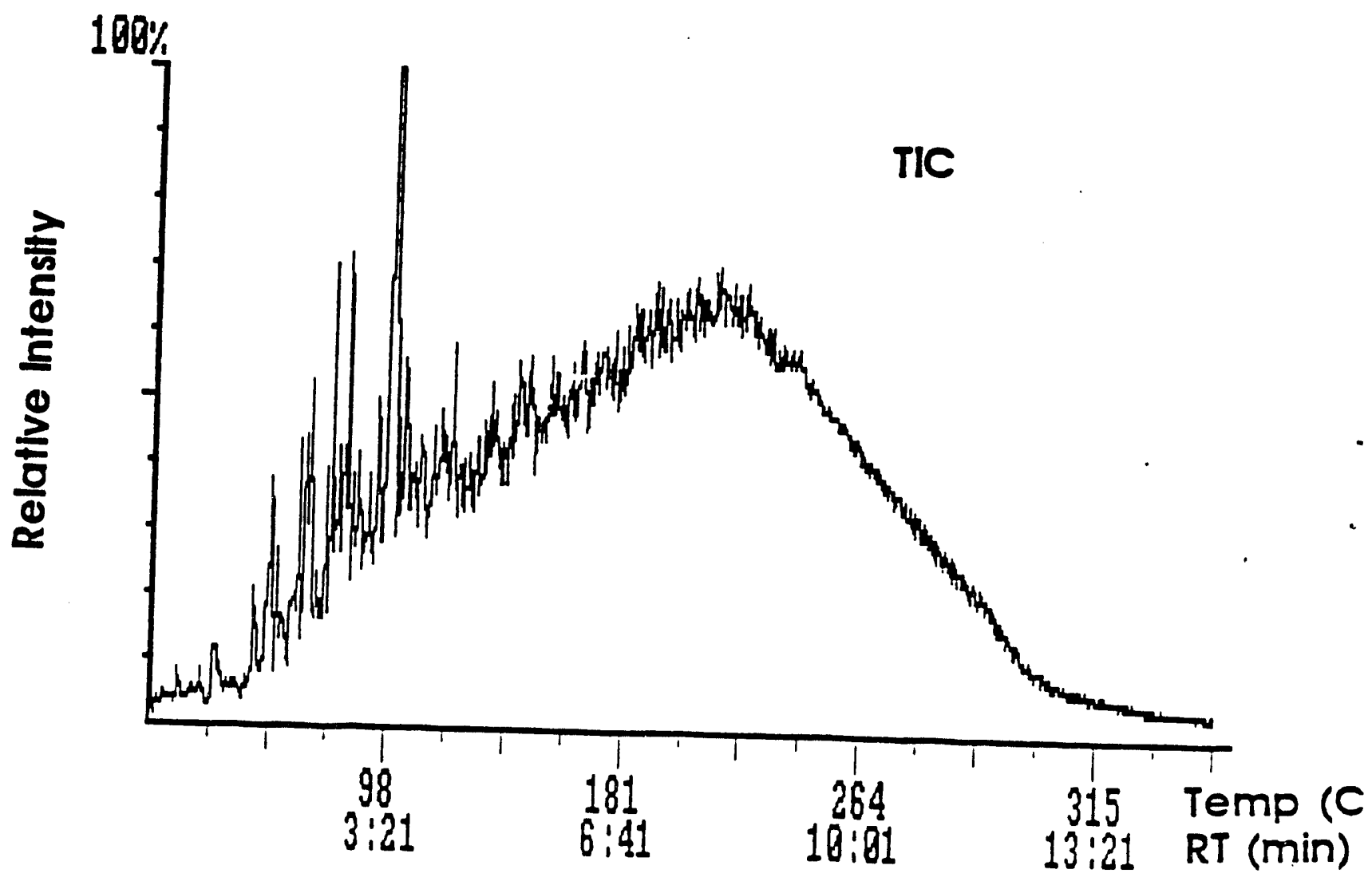

Figure 2. Short column GC/CIMS profile of Illinois \#6 HT-BCD oil 


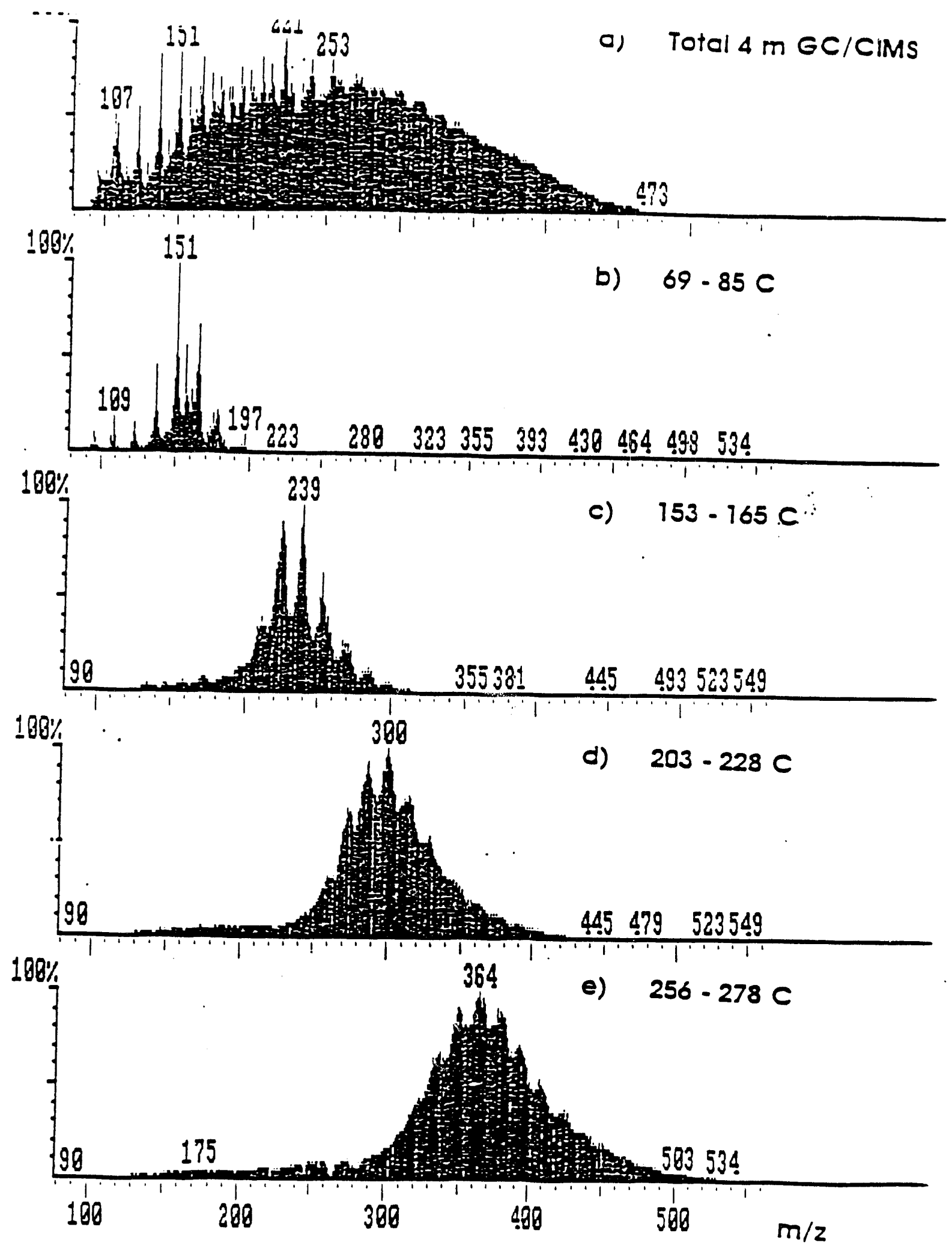

Figure .3. (a) Sumeed CIMS profile of Illinois $\# 6$ HT-BCD oil and (b-e) partial temperature ange CIMS profiles sbowing sucessive MW distrioutions. 


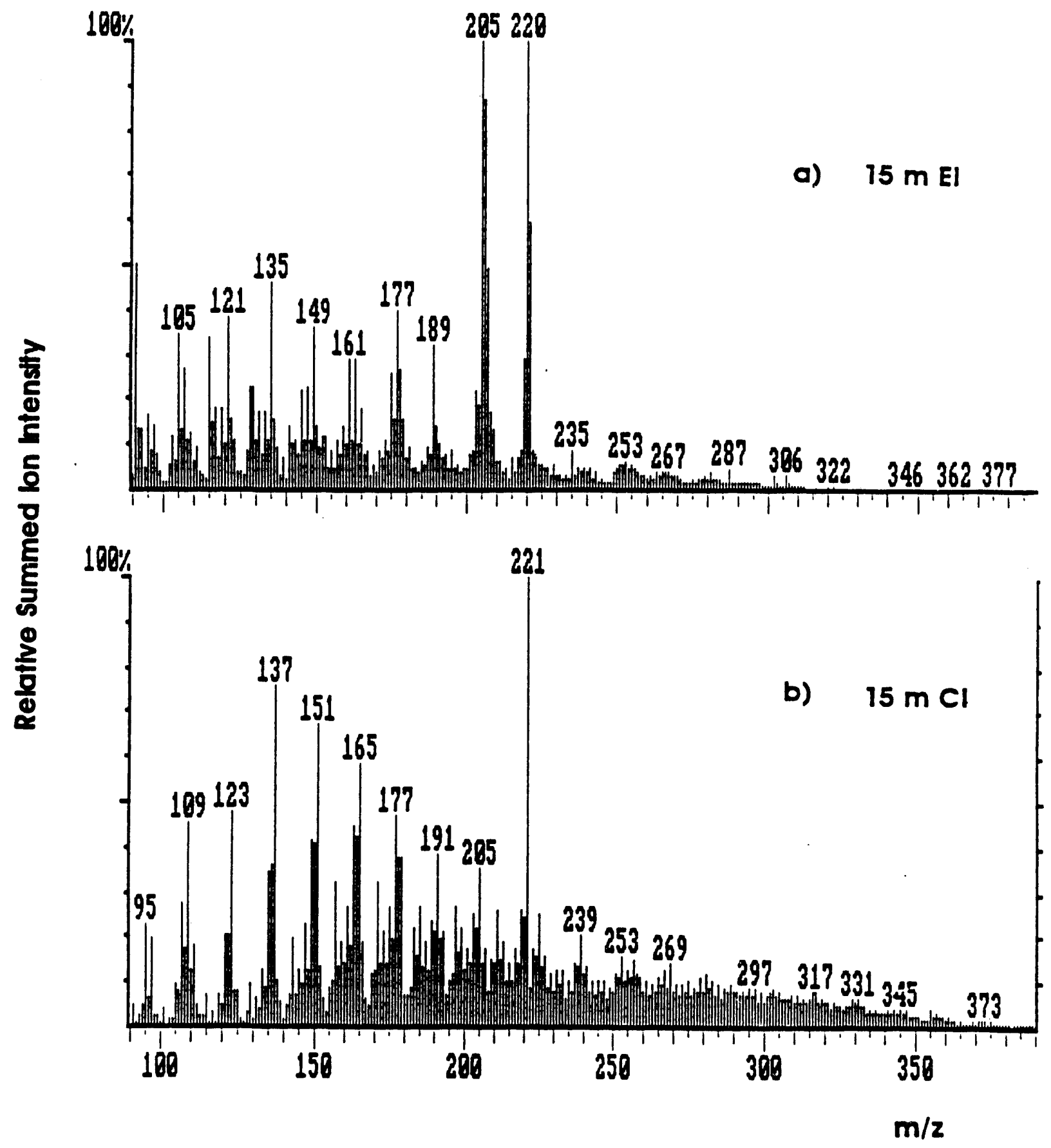

Figure 4. Summed MS profiles of llinois \#6 HT-BCD oil obtained $15 \mathrm{~m} \mathrm{GC}$ column in combination with (a) EI and (b) isobutane CI, respectively. 

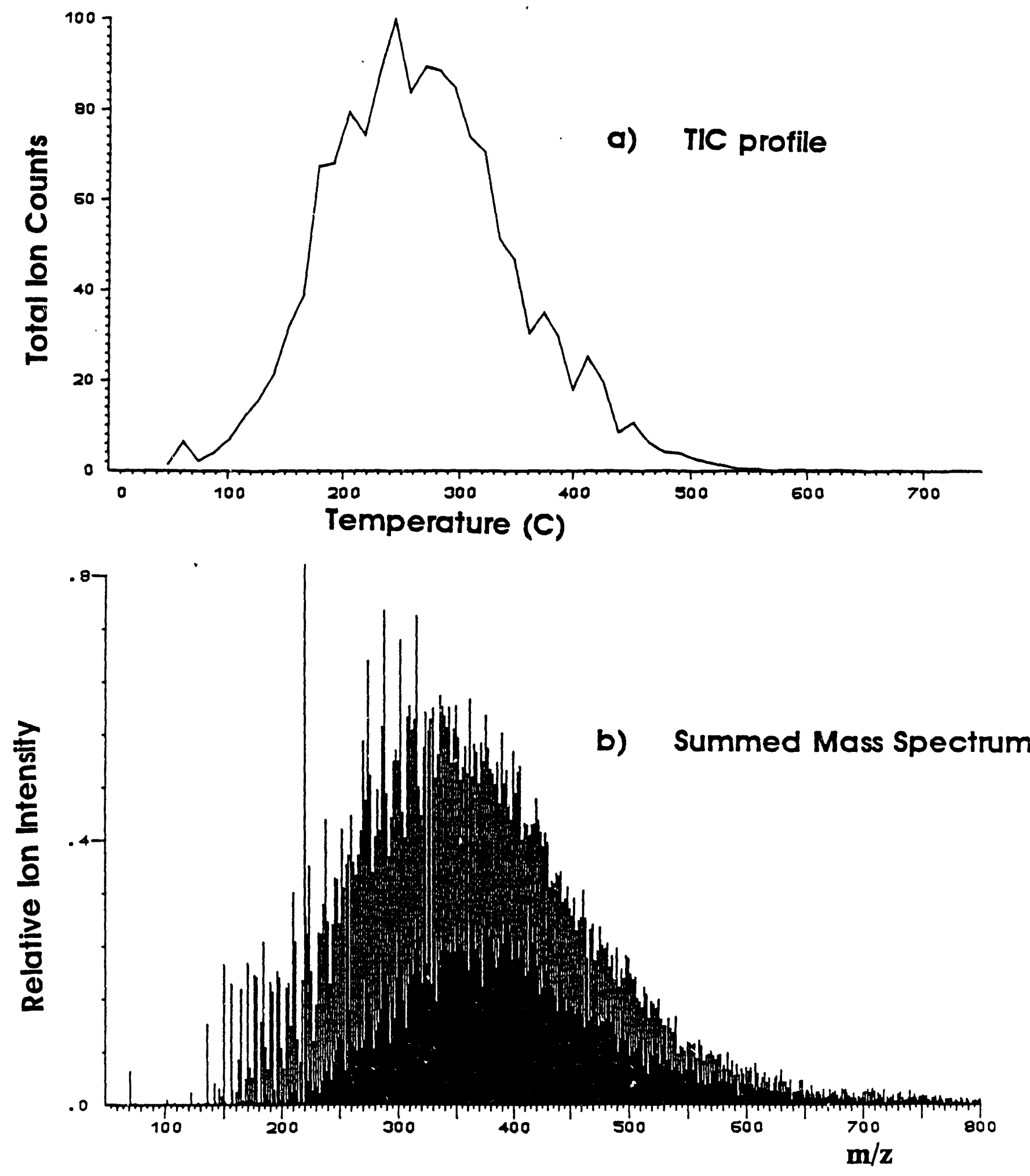

Figure 5. FIMS profiles of Illinois \#6 HT-BCD oil 
Table 1

Average Molecular Weights of HT-BCD-Oil Fractions Calculated by Different Methods

\begin{tabular}{lccc}
\hline Oil & FIMS & GC/CIMS & $\begin{array}{c}\text { Vacuum TG + } \\
\text { GC/CIMS }\end{array}$ \\
\hline Be!lah Zap & 372 & 277 & 235 \\
Illinois \#6 & 370 & 259 & 247 \\
Blind Canyon & 395 & 250 & 242 \\
Pittsburgh \#8 & 396 & n.a & n.a \\
\hline
\end{tabular}



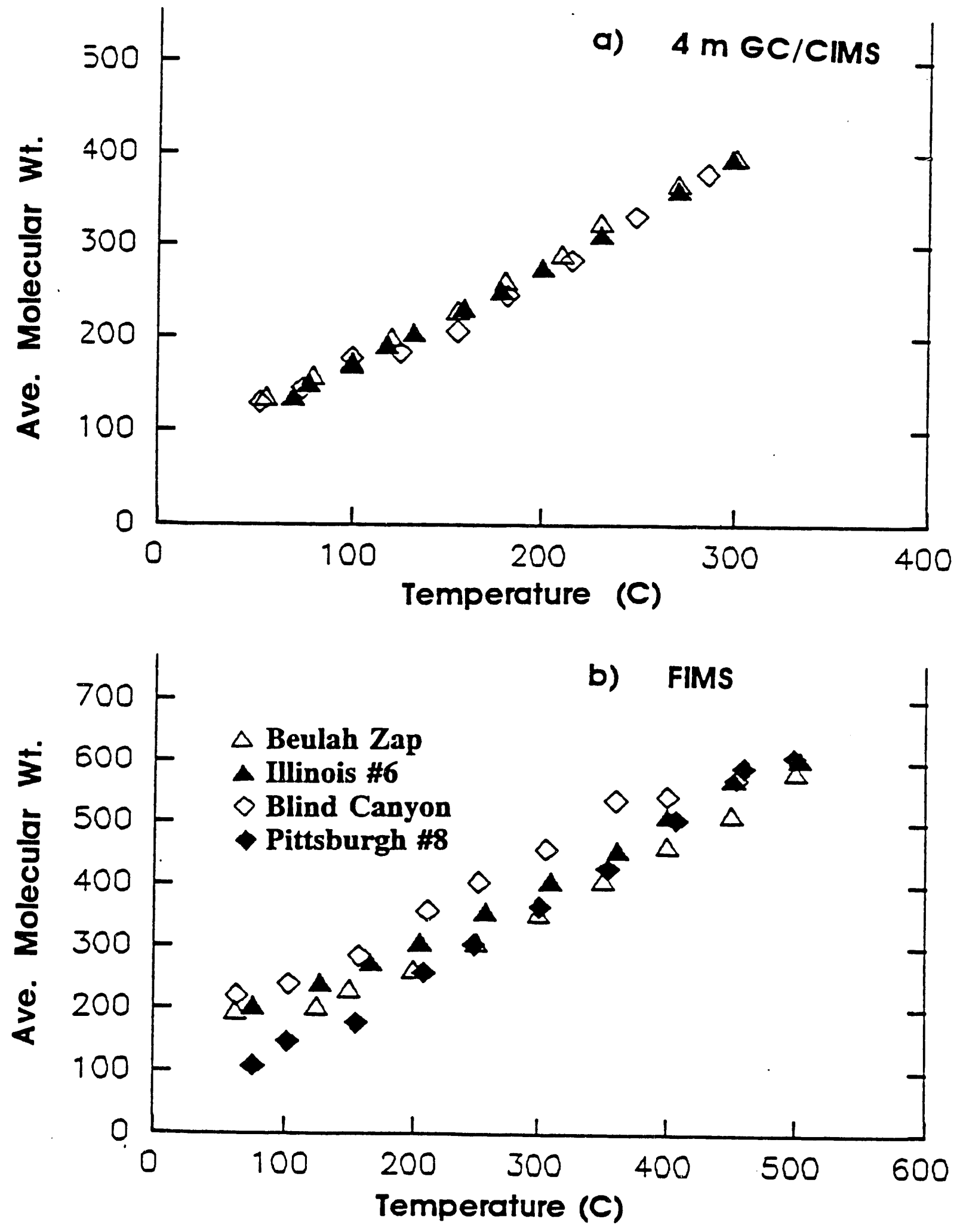

Figure 6. Relationship between (simulated) distillation temperature and average molecular weight of HT-BCD oils as observed by (a) GC/CIMS (4 m GC column) and (b) FIMS, respectively. 


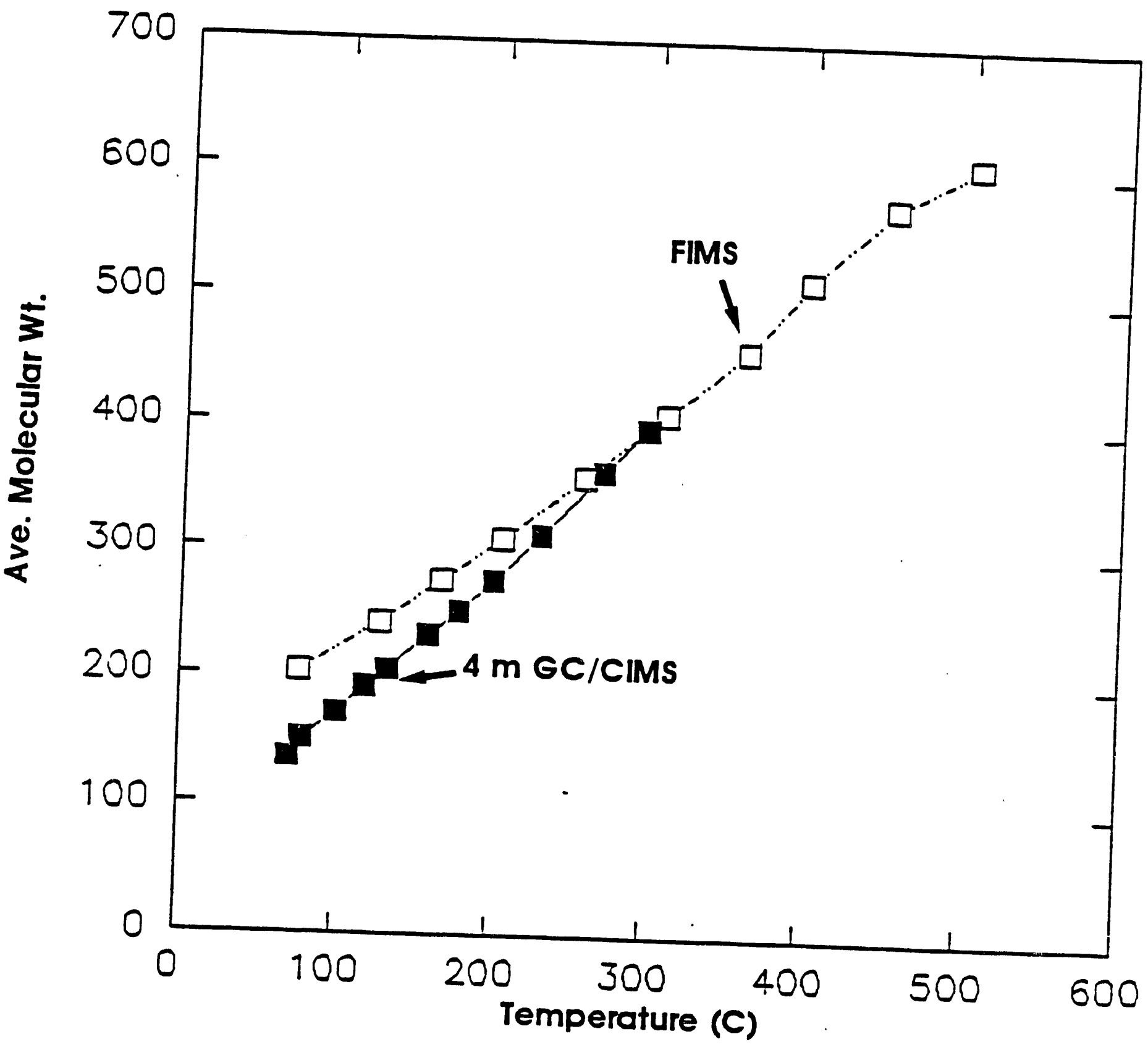

Figure 7. Curves of Illinois \#6 HT-BCD oil superposition of distillation temperature/molecular weight curves obtained by GC/CIMS $(\boldsymbol{\square})$ and FIMS (D), respectively. 


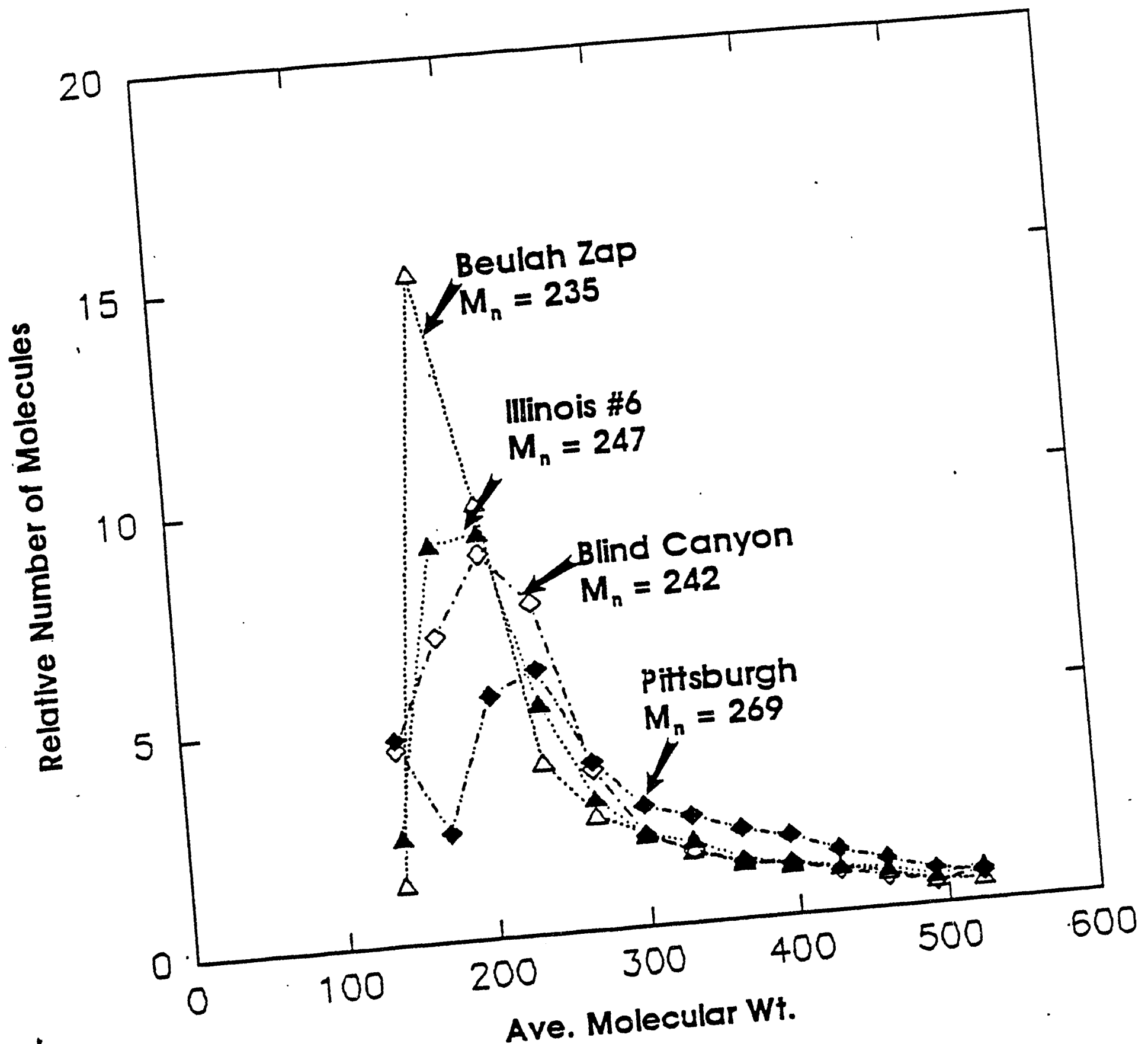

8. Molecular weight distribution profile of four HT-BCD
using vacuum IG information in combination with GC/CIMS data 


\section{Tracking a Soluble Iron Catalyst in a Coal Depolymerization Processes}

Acid catalysts are of interest for coal depolymerization since they offer the potential to liquefy coals at low temperatures with a high selectivity for liquid product. Many metal halides have been examined to evaluate their potential as coal depolymerization catalysts. However, the nature of the depolymerization catalysts is still not well understood. The objective of the present work is to track the soluble iron catalyst in a low temperature coal depolymerization process (that of Joseph S. Shabtai) [35] using electron microprobe analysis (EPM) and Mossbauer spectroscopy.

It is believed that the acid-catalyzed mechanism is strongly related to the distribution of the active species in the coal structure. We have studied the catalyst dispersion in the coal matrix using EPM. Iron chloride-impregnated coal samples (provided by Joseph S. Shabtai) were lightly sputter coated with carbon to reduce charging in the electron microscope (CAMECA, Model SX-50, Courbevoie Cedex, France). Electron photomicrographs and elemental distribution maps were obtained, and X-ray data acquisition was carried out using an energy dispersive spectrometer detector (EDS). X-ray data analysis was done using a Digimap program. The magnification used was x1600, which gave a Digimap scanned area of $50 \mu \mathrm{m} \times 50 \mu \mathrm{m}$.

The electron photomicrograph and the iron and chlorine distribution of a typical crosssection of an impregnated Blind Canyon coal sample are presented in Fig. 1. Two coal grains with the crystal size of $4.3 \mu \mathrm{m}$ and $50 \mu \mathrm{m}$ were selected for these studies. The elemental ( $\mathrm{Fe}$ ) distribution mapping shown in Fig. 1a is the result of computer data treatment (Equalizer and Median). The micrograph shows an even distribution of iron over the cross section of grains, suggesting that catalyst penetration into the coal particles from acetone solution has taken place at room temperature which is considerably lower than the melting point of $\mathrm{FeCl}_{3}\left(290{ }^{\circ} \mathrm{C}\right)$. However, the concentration of iron near the periphery of the large grain is relatively higher than that in the center of this grain. The small grain has an even and relatively high iron distribution compared with the larger grain of coal. This suggests that the impregnation of $\mathrm{FeCl}_{3}$ into the coal matrix may be, to some extent, a diffusion controlled process.

We also examined the chlorine distribution in these coal grains. If the chlorine is associated with iron, one would expect to observe similar distributions for these two elements in the coal grains. However, we find that the chlorine dispersion mapped in Fig. 1b, although quite even, is not identical to the iron distribution in Fig. 1a. A dissociation of the $\mathrm{FeCl}_{3}$ species during the impregnation process is therefore evident (see below). 
The elemental ( $\mathrm{Fe}$ and $\mathrm{Cl}$ ) distribution mapping technique is especially useful when combined with secondary electron imaging. The imaging of the coal grains (Fig. 1c) shows the pore dispersion in the cross-section of the typical coal grains. By examining the superposition of the iron distribution mapping on the secondary electron imaging, we find that the iron species have no preference for being around the pores of the coal sample.

Since EPM data give only the distribution of the catalyst in the coal matrix, it is important to understand the chemical state of the catalyst by other techniques. Mossbauer spectroscopy is a nuclear resonance technique which is particularly suited to the investigation of iron chemistry since ${ }^{57} \mathrm{Fe}$ is a commonly used Mössbauer active isotope. The isomer shift (IS) and the quadrupole splitting (QS), which describe the environment of the Fe species, usually suffice to fingerprint any particular Fe compound.

The room-temperature Mössbauer spectra of a $\mathrm{FeCl}_{3}$-impregnated Blind Canyon coal sample is shown in Fig. 2. The best fit of this spectrum consists of two quadrupole split doublets. Each doublet identified by comparison of the best-fit parameters with the Mossbauer parameters reported in the Mossbauer Effect Data Index is analyzed in Table 1. Also included in Table 1 is the area of each doublet expressed as a percentage of the total absorption area. The inner doublet (Fig. 2) centered about $0.33 \mathrm{~mm} / \mathrm{sec}$ velocity was readily identified as Fe(III). The outer doublet centered about $1.18 \mathrm{~mm} / \mathrm{sec}$ velocity was identified as $\mathrm{FeCl}_{2}$ or $\mathrm{FeS}$. The relative amount of $\mathrm{Fe}$ (II) species is about $74 \%$ in the $\mathrm{FeCl}_{3}$-impregnated Blind Canyon coal sample. Evidently, incorporation of the iron chloride $\left(\mathrm{FeCl}_{3}\right)$ in coal is conducive to the reduction of $\mathrm{Fe}$ (III) to $\mathrm{Fe}$ (II). It should be noted that $\mathrm{Fe}$ (II)-containing species such as iron sulfide (FeS) are included in a group of liquefaction catalysts which has been found to promote coal depolymerization and liquefaction [36].

In order to examine more thoroughly the functions of the $\mathrm{Fe}$ (II) species involved in the coal depolymerization reactions, we have measured the conversion of the iron chlorideimpregnated coal samples to oils (acetone soluble) at different Fe(II) contents (Table 2). It should be noted that the iron content was calculated from the Mössbauer data, and total iron amounts were measured by the inductively coupled plasma spectroscopic method. The data of Table 2 suggest that the Fe(II) species are more effective in coal depolymerization than the Fe(III) and can enhance coal conversion to oils.

In order to identify these iron species, room-temperature and cryogenic-temperature Mossbauer spectra of the coal samples were measured in Huffman's Mössbauer laboratory. When a sample is cooled to a sufficiently low temperature (the temperature below which crystalline domains behave as magnetically ordered crystals on the time scale of the observation), the splitting of a quadrupole doublet into a magnetic hyperfine six-line spectrum 
can be observed which can aid in the identification of the iron species. The Morssbauer data suggest that the iron species are $\mathrm{FeOCl}$ and $\mathrm{FeOOH}$.

In addition, X-ray powder pattern diffraction was used to help make the distinction between these species. Figure 3 shows the $\mathrm{X}$-ray diffractograms of $\mathrm{FeCl}_{3}$-impregnated $\mathrm{BC}$ coal and the best-fitted diffractograms of iron species through the computer search. Peaks corresponding to $\mathrm{Fe}_{2}(\mathrm{OH})_{3} \mathrm{Cl}$ and $\mathrm{FeCl}_{2} \cdot 2 \mathrm{H}_{2} \mathrm{O}$ are clearly present in the impregnated $\mathrm{BC}$ coal sample, but the major peaks of $\mathrm{FeOOH}$ species are not present in the impregnated coal sample.

\section{Benzene in Zeolite ZSM-5 Studied by DRIFTS}

The study of hydrocarbon adsorption on zeolite is important for what it reveals about selectivity in catalytic reaction and molecular-sieve effects. The benzene molecule has often been used as a probe of the behavior of hydrocarbons adsorbed on zeolites. The existence of at least two adsorbed benzene species has been observed in our laboratory using diffuse reflectance infrared spectroscopy. The distortion of the framework of ZSM-5 is indicated by the red-shift of the $\mathrm{Si}-\mathrm{O}$ asymmetric stretching vibrations when adsorbed benzene molecules are present. A manuscript describing this work has been in press for publication in a leading spectroscopic journal [37].

\section{Photoacoustic Enhancements of Surface IR Modes in Zeolite Channels}

Photoacoustic signal enhancement with adsorbed pyridine in the channels of zeolite ZSM5 and zeolite $H Y$ has been studied using photoacoustic, transmittance, and diffuse reflectance FT-IR spectroscopies. The experimental data indicate that the photoacoustic enhancements are 2.1 and 3.3 for HY and ZSM-5, respectively. Possible sources of the enhancement of the photoacoustic signal are discussed. The influence of coupling gases in the photoacoustic cell on the enhancement of the IR band is also reported [38]. 

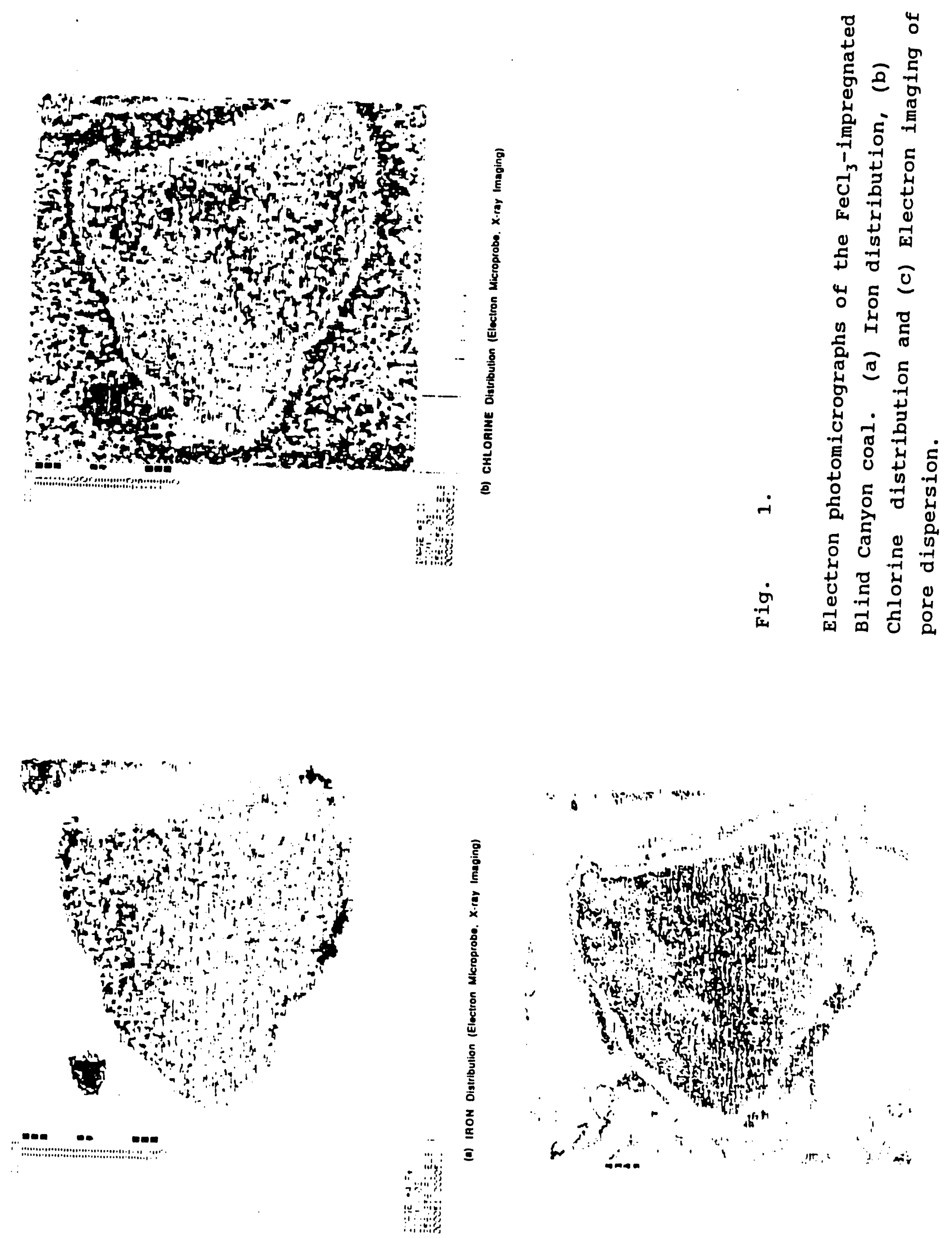
Table 1 .

Moessbauser parameters for the $\mathrm{FeCl}_{3}$-impregnated
Blind Canyon coal sample.

\begin{tabular}{llll}
\hline Species & IS $(\mathrm{mm} / \mathrm{s})$ & QS $(\mathrm{mm} / \mathrm{s})$ & $\%$ \\
\hline Fe(II) & 1.18 & 2.30 & 74 \\
$F e(I I I)$ & 0.33 & 0.73 & 26 \\
\hline
\end{tabular}

Table

2.

Effect of the iron content in the coal matrix on the conversion of the coal depolymerization

\begin{tabular}{ccc}
\hline Fe (III), \% & Fe(II), \% & Conversion, \% \\
\hline 1.3 & 3.6 & 14 \\
2.1 & 1.8 & 7 \\
4.7 & 0.9 & 6 \\
\hline
\end{tabular}

Note: Conversion: $\%$ conversion to oils (acetone soluble). Total Fe content was measured by the inductively coupled plasma spectroscopic method. Fe(III) and Fe(II) contents were calculated from the Moessbauer data. 


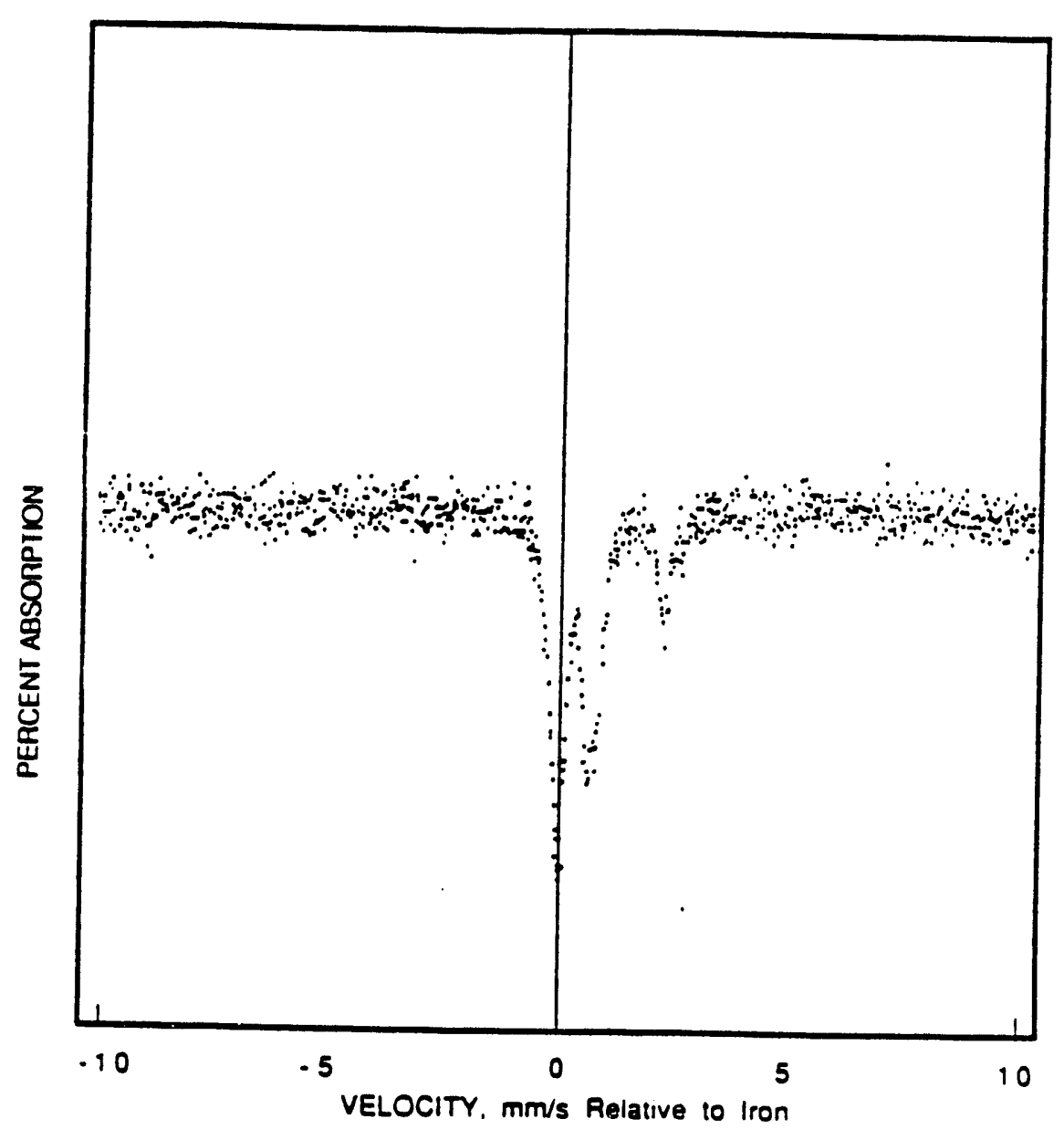

Fig. 2. Moessbauer spectrum of the FeCl $1_{3}$-impregnated Blind Canyon coal. 


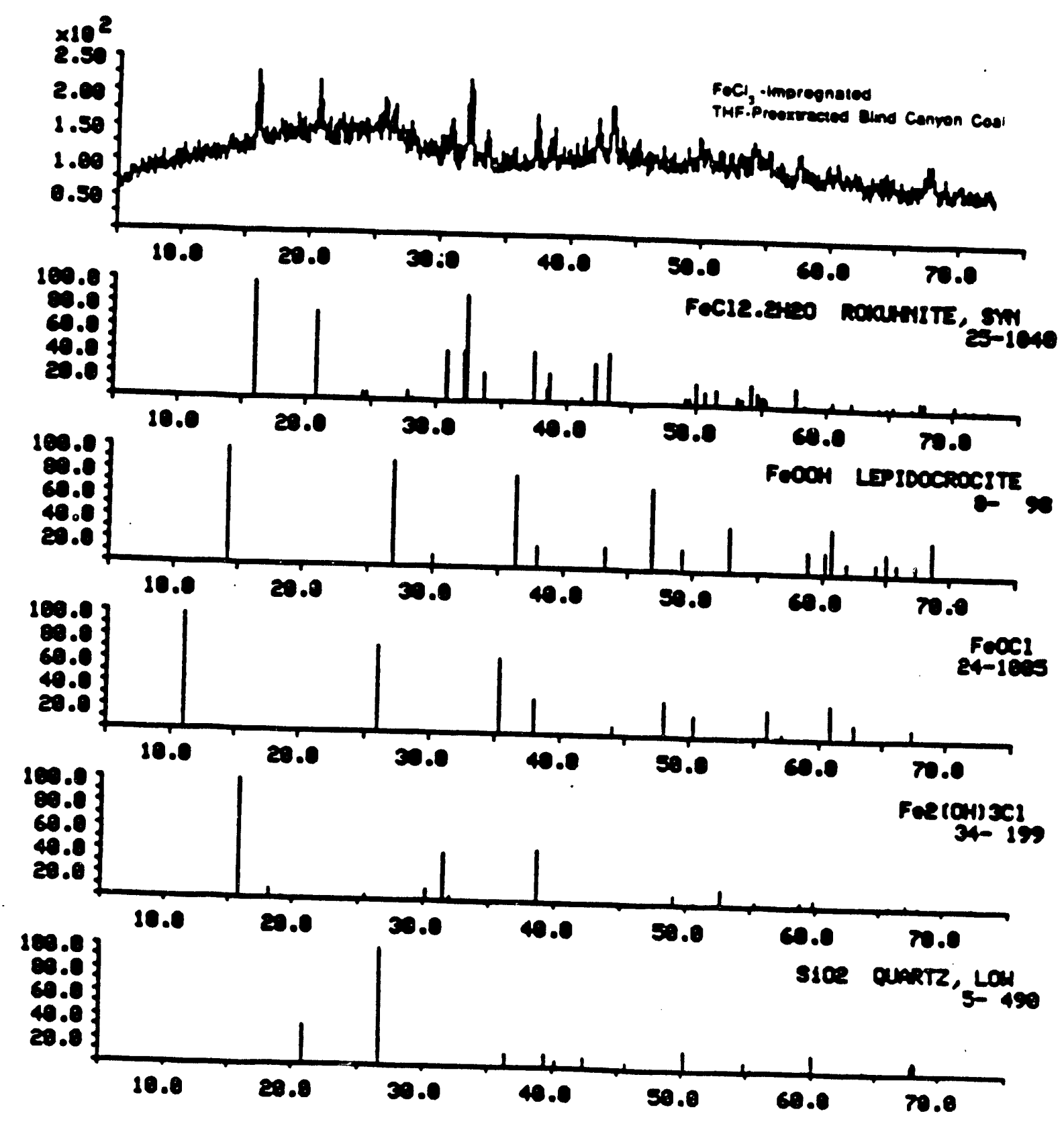

Figure V.3.1. X-ray diffractograms of $\mathrm{FeCl}_{3}$-impreguated $\mathrm{BC}$ coal and the best-fitted diffractograms of iron species through the computer search. The top diagram is the experimental $\mathrm{X}$-ray diffractogram. The computer generated spectrum shown immediately below the The $\mathrm{Fe}_{2}(\mathrm{OH})_{3} \mathrm{Cl}$ computer generated $\mathrm{H}_{2} \mathrm{O}$. It is clearly that the best fit of the experimental data. with the experimental data. 


.




\section{TASK V.4 SOLVENT EXIRACTION AND PYROLYSIS (L. L. Anderson)}

\section{Solvent Extraction and Pyrolysis}

Pyrolysis and solvent extraction of two additional Argonne Premium Coal Samples, namely Blind Canyon (HVB, Utah) and Lewiston-Stockton (HVB, West Virginia) have been studied. For pyrolysis runs, three samples from each coal were prepared: undried raw coal, dried raw coal and pre-extracted coal. The reaction conditions were $350^{\circ} \mathrm{C}, 1$ atmosphere of nitrogen (initial pressure) and a reaction time of 3 hours. Pyrolysis and solvent extraction yields of both coals and of three previously studied Argonne coals (see report for the period June 1988 May 1989) are shown in Table 1.

The results obtained with Blind Canyon and Lewiston-Stockton coals agreed with previous experiments on five other coals. When pyrolyzed, dried coals (at $110^{\circ} \mathrm{C}$, in vacuo) gave significantly lower liquid yields compared to undried coals. On the other hand, pre-extraction with tetrahydrofuran (THF) showed no apparent effect on subsequent pyrolysis yields, as compared with yields obtained without pre-extraction.

Elemental analyses of liquids from solvent extraction and pyrolysis of Blind Canyon and Lewiston-Stockton coals are shown in Table 2, along with the values of the two raw coals.

Carbon contents of the liquid products were slightly higher than those of the starting raw coals. Hydrogen contents of the liquids, however, were significantly higher than those of the raw coals. The liquid products of Blind Canyon coal contained as much as $7.7 \%$ hydrogen. The amount of nitrogen in the liquids were slightly lower than those of the raw coals. The atomic hydrogen-to-carbon ratio was higher (1.10) for Blind Canyon coal extract as compared to the starting coal (0.86). Likewise the $\mathrm{H} / \mathrm{C}$ ratio was higher $(0.91)$ for the Lewiston-Stockton coal extract as compared with that of the starting coal (0.76). The high hydrogen contents and hydrogen-to-carbon ratios of Blind Canyon coal and its liquids might be attributed to its high amount of resinite.

\section{Catalytic Hydropyrolysis}

Preliminary experiments on catalytic hydropyrolysis of Pittsburgh \#8 coal were conducted using "ferrophos" (a waste material produced in the electroreduction of phosphate ore to phosphorous) as a potential catalyst. The catalyst was ground to fine powder and physically mixed with the coal samples. Table 3 shows the yields of tetrahydrofuran (THF) soluble along with THF insoluble products.

Among these experiments, the highest total conversion was obtained at $450^{\circ} \mathrm{C}$ with ferrophos(FeP), however, the liquids to gases selectivity was poor. The THF soluble products 
were only $16 \%$ (maf coal). The highest THF soluble yield was produced at lower temperature, $350^{\circ} \mathrm{C}$, at which only a small portion of coal converted to gases. Ferrophos increased the conversion slightly (from $35 \%$ to $42 \%$ at $450^{\circ} \mathrm{C}$ ) for pittsburgh \#8 coal. The low activity of ferrophos was attributed to poor dispersion and the lack of shaking of the tubing bomb reactor used in these experiments. Carbon disulfide was added in one run (with the pre-estimated amount that should result in about $5 \%$ of $\mathrm{H}_{2} \mathrm{~S}$ in $\mathrm{H}_{2}$ ) in order to observe the effect of catalyst sulfiding. The result was quite surprising; the conversion was not only much lower than the one without carbon disulfide but also lower than the one without catalyst at all. This indicates that carbon disulfide, by some mechanism, strongly inhibited the coal liquefaction process in this system.

Future experiments are planned to further investigate this topic. A shaking mechanism for the tubing bomb reactor is being installed in order to facilitate better contact of catalyst, coal and hydrogen and better mass transfer. Improved dispersion techniques are also being implemented. The reaction condition parameters including temperature, reaction time, amount of catalyst are planned to be optimized. The effect of carbon disulfide will also be studied since for some catalysts traces of carbon disulfide are effective while higher concentrations completely deactivated the catalysts.

Literature surveys on iron as a catalyst for coal liquefaction are also being carried out. Specifically, soluble iron salts or complexes are selected for such studies because such catalyst can be better dispersed in coal. However, emphasis are on the soluble iron salts that are nonor less corrosive under coal liquefaction conditions. Different organic solvents will also be used as impregnation medium in order to swell the coal and heln in catalyst dispersion. The effectiveness of caralyst dispersion will be determined by reaction experiments and microsccpic analysis. 
Table 1. Product Yields from Extraction and Pyrolysis of Wyodak (WY), Pittsburgh \#8 (PT), IIlinois \#6 (II), BIind Canyon (BC) and Lewiston-stockton (WV) Coal's

\begin{tabular}{|c|c|c|c|c|c|}
\hline & & חथा & THF Insol. & $\begin{array}{l}\text { maf coal } \\
\text { THF soluble }\end{array}$ & Gases \\
\hline $\begin{array}{l}S X-W Y \\
P Y-W Y \\
P Y-W Y \\
P Y-W Y\end{array}$ & $\begin{array}{l}\# 1 \\
\# 1 \\
\# 2 \\
\# 3\end{array}$ & $\begin{array}{l}\text { (undried coal) } \\
\text { (pre-extracted coal) } \\
\text { (undried coal) } \\
\text { (dried coal) }\end{array}$ & $\begin{array}{l}97.1 \\
82.3 \\
76.6 \\
83.2\end{array}$ & $\begin{array}{r}19.9 \\
9.4 \\
19.6 \\
9.4\end{array}$ & $\begin{array}{l}-- \\
5.0 \\
5.6 \\
8.0\end{array}$ \\
\hline $\begin{array}{l}S X-P T \\
P Y-P T \\
P Y-P T \\
P Y-P T\end{array}$ & $\begin{array}{l}\# 2 \\
\# 2 \\
\# 1 \\
\# 3\end{array}$ & $\begin{array}{l}\text { (undried coal) } \\
\text { (pre-extracted coal) } \\
\text { (undried coal) } \\
\text { (aried coal) }\end{array}$ & $\begin{array}{l}86.2 \\
71.2 \\
61.8 \\
65.6\end{array}$ & $\begin{array}{l}16.6 \\
14.6 \\
39.6 \\
35.4\end{array}$ & $\begin{array}{l}-- \\
2 \cdot 7 \\
2 \cdot 3 \\
2 \cdot 2\end{array}$ \\
\hline $\begin{array}{l}S X-I I \\
P Y-I I \\
P Y-I I \\
P Y-I I\end{array}$ & $\begin{array}{l}\# 2 \\
\# 2 \\
\# 3 \\
\# 1\end{array}$ & $\begin{array}{l}\text { (undried coal) } \\
\text { (pre-extracted coal) } \\
\text { (undried coal) } \\
\text { (dried coal) }\end{array}$ & $\begin{array}{l}88.0 \\
73.2 \\
70.3 \\
72.5\end{array}$ & $\begin{array}{l}15.4 \\
12.3 \\
25.0 \\
23.3\end{array}$ & $\begin{array}{l}-- \\
4.7 \\
4.5 \\
3.1\end{array}$ \\
\hline $\begin{array}{l}S X-B C \\
P Y-B C \\
P Y-B C \\
P Y-B C\end{array}$ & $\begin{array}{l}\# 1 \\
\# 1 \\
\# 2 \\
\# 3\end{array}$ & $\begin{array}{l}\text { (undried coal) } \\
\text { (pre-extracted coal) } \\
\text { (undried coal) } \\
\text { (dried coal) }\end{array}$ & $\begin{array}{l}83.9 \\
71.5 \\
70.7 \\
81.5\end{array}$ & $\begin{array}{r}19.9 \\
9.9 \\
26.7 \\
21.7\end{array}$ & $\begin{array}{l}-- \\
1.1 \\
3.3 \\
1.6\end{array}$ \\
\hline $\begin{array}{l}S X-W V \\
P Y-W V \\
P Y-W V \\
P Y-W V\end{array}$ & $\begin{array}{l}\# 1 \\
\# 1 \\
\# 2 \\
\# 3\end{array}$ & $\begin{array}{l}\text { (undried coal) } \\
\text { (pre-extracted coal) } \\
\text { (undried coal) } \\
\text { (dried coal) }\end{array}$ & $\begin{array}{l}90.0 \\
80.4 \\
76.1 \\
78.7\end{array}$ & $\begin{array}{r}13.0 \\
8.2 \\
25.3 \\
21.0\end{array}$ & $\begin{array}{l}-- \\
0.7 \\
0.5 \\
0.5\end{array}$ \\
\hline
\end{tabular}


Table 2. -CFn Analyses of Blind Canyon (BC) and Iewistonstockton (WV) Coals and their Iiquid Products

Analyzed by the Fossil Fuel Characterization Laboratory, $U$. Of U.

\begin{tabular}{|c|c|c|c|c|c|}
\hline & & c & H & $\mathbf{N}$ & $\mathrm{H} / \mathrm{C}$ (atomic) \\
\hline $\begin{array}{l}B I \text { ind } \\
S X-B C \\
P Y-B C \\
P Y-B C \\
P Y-B C\end{array}$ & $\begin{array}{ll}\text { Canyon coal } \\
\# 1 & \text { (undried coal) } \\
\# 1 & \text { (pre-extracted) } \\
\# 2 & \text { (undried coal) } \\
\# 3 & \text { (dried coal) }\end{array}$ & $\begin{array}{l}80.7 \\
80.3 \\
81.2 \\
82.7 \\
81.5\end{array}$ & $\begin{array}{l}5.8 \\
7.4 \\
7.1 \\
7.7 \\
7.3\end{array}$ & $\begin{array}{l}1 \cdot 6 \\
1 \cdot 1 \\
1 \cdot 3 \\
1 \cdot 1 \\
1 \cdot 1\end{array}$ & $\begin{array}{l}0.86 \\
1.10 \\
1.05 \\
1.11 \\
1.08\end{array}$ \\
\hline $\begin{array}{l}\text { Lewist } \\
\text { SX-WV } \\
\text { PY-WV } \\
\text { PY-WV } \\
\text { PY-WV }\end{array}$ & $\begin{array}{ll}\text { :on-stockton coal } \\
\# 1 & \text { (undried coal) } \\
\# 1 & \text { (pre-extracted) } \\
\# 2 & \text { (undried coal) } \\
\# 3 & \text { (dried coal) }\end{array}$ & $\begin{array}{l}82.6 \\
83.7 \\
84.9 \\
84.1 \\
84.7\end{array}$ & $\begin{array}{l}5.2 \\
6.2 \\
6.5 \\
6.3 \\
6.1\end{array}$ & $\begin{array}{l}1 \cdot 6 \\
1 \cdot 3 \\
1.3 \\
1.3 \\
1.4\end{array}$ & $\begin{array}{l}0.76 \\
0.89 \\
0.91 \\
0.89 \\
0.86\end{array}$ \\
\hline
\end{tabular}


Table 3. Catalytic Eydropyrolysis (Ferrophos)

ơ maf coal

$\underline{\text { Run }}$

Char THF Sol.

FP-PT \#2 $350^{\circ} \mathrm{C}, 20$ wtz FeP

FP-PT \#3 $450^{\circ} \mathrm{C}, 20$ wt? FeP

FP-PT \#4 $450^{\circ} \mathrm{C}, 20$ wto $\mathrm{FeP}+\mathrm{CS}_{2}$

FP-PT \#5 $450^{\circ} \mathrm{C}$, no FeP

67.8

58.4

75.7

65.1

33.5

16.2

6.4

13.9

Reaction time $=1$ hour

Coal - Pittsburgh \#8 (Argonne Premium sample), undried

Hydrogen Pressure - FP-PT \#2 \#4 1040 psig (cold)

FP-PT \#3, \#4, \#5 890 psig (cold)

(all about 2000 psig at reaction temperature) 


\section{TASK V.5 LOW TEMPERATURE DEPOLYMERIZATION AND LIQUEFACTION OF PREMTUM U.S. COAL SAMPLES (J.S. Shabtai)}

During the above-indicated period, further studies were performed on the improvement and application of a recently-developed procedure for low-temperature coal depolymerizationliquefaction [35, 39-43]. The procedure includes an initial depolymerization stage comprising two sequential low-temperature reaction steps in which different types of intercluster linkages are subjected to preferential cleavage, i.e., (1) intercalation of the coal with catalytic amounts (3-15\%) of $\mathrm{FeCl}_{3}$ followed by mild hydrotreatment (HT) of the coal- $\mathrm{FeCl}_{3}$ intercalate; and (2) base-catalyzed depolymerization (BCD) of the product from step 1 . In the final stage of the procedure, the depolymerized coal product from the HT-BCD treatment (steps 1 and 2), consisting primarily of monocluster compounds, is subjected to hydroprocessing (HPR) with sulfided catalysts possessing augmented hydrogenolysis selectivity $[44,45]$ to obtain light hydrocarbon fuels as final products.

Research activities during the reporting period included; (1) systematic studies on the processing aspects of the low-temperature depolymerization-liquefaction of three selected U.S. coals provided by the Argonne National Laboratory, i.e., Blind Canyon (UT), Beulah Zap (ND), and Pittsburgh \#8 (PA) coals; (2) preparation of larger amounts of depolymerized coals for the purpose of in-depth characterization, including structural analysis by GC/MS (in collaboration with Task V.2; Professor H.L.C. Meuzelaar), ${ }^{13} \mathrm{C}$ NMR and PMR.(in collaboration with Task V.1; Professor R.J. Pugmire), and FTIR (in collaboration with Task V.3; Professor E.M. Eyring); (3) work on the simplification of the HT-BCD-HPR procedure and the development of alternative catalysts and solvents; and (4) elucidation of the chemistry and mode of catalytic action in each step of the procedure. Following is a summary of the main results obtained in the aboveindicated areas.

Low-Temperature Depolymerization-Liquefaction Studies of Blind Canyon (UT), Pittsburgh \#8 (PA), and Beulah Zap (ND) Coals.

Systematic studies were continued on the processing aspects of the low-temperature liquefaction of the above coals. Suitable conditions for high-yield conversion of the coals into low-boiling hydrocarbon oils, using the HT-BCD-HPR procedure, were determined. Although the optimal conditions for each step depend to some extent on the rank and source of the coal, some general ranges of processing conditions were determined and are summarized in Table 1. (In view of the current work under Section 3 [see below], the indicated conditions and catalyst systems could be considerably changed in the future.) Several mild HT experiments with Blind Canyon and Pittsburgh \#8 coals impregnated with catalytic amounts (3-15 wt\%) of $\mathrm{FeCl}_{3}$ were performed in a $150 \mathrm{~cm}^{3}$ autoclave at a temperature of $290^{\circ} \mathrm{C}$ for the purpose of determining 
whether under such conditions the metal halide has any corrosion effect upon the reactor. No corrosion could be detected, which indicates that the mild HT step at $\leq 290^{\circ} \mathrm{C}$ involves a solid phase coal depolymerization reaction in which the deeply impregnated (from $\mathrm{MeOH}$ or acetone) and uniformly dispersed $\mathrm{FeCl}_{3}$ catalyst essentially does not come in contact with the stainless steel reactor walls. This is completely different from previous high-temperature $\left(420-450^{\circ} \mathrm{C}\right)$ procedures in which copious excess amounts of metal halides, e.g., $\mathrm{ZnCl}_{2}$, were used (in the form of coal- $\mathrm{ZnCl}_{2}$ melts, or non-uniformly impregnated in the coal from aqueous solutions), resulting in reactor corrosion.

Table 1 shows that the depolymerizing stage of the procedure (steps HT and BCD) can be effectively performed under very mild processing conditions, i.e., temperature, $\leq 295^{\circ} \mathrm{C}$; pressure, $\leq 1500 \mathrm{psig}$; low catalyst concentration. Further, due to the simplified structure of the depolymerized coal products, consisting predominantly of monocluster compounds (see below, Section 2), the hydroprocessing stage (HPR step) of the procedure can be effectively performed under milder conditions (temperature, $350-370^{\circ} \mathrm{C}$ ) than those usually employed $\left(420-470^{\circ} \mathrm{C}\right.$ ) in direct catalytic hydroprocessing of non-depolymerized coals.

Table 2 summarizes the total conversion of Blind Canyon, Pittsburgh \#8, and Beulah Zap coals into depolymerized, THF-soluble and cyclohexane-soluble (oil fraction) products under the conditiors indicated in Table 1 . As seen, the total conversion of these coals into depolymerized (THF-soluble) products is in the approximate range of 88-92 wt\% (MAF basis), the difference to quantitative yield being apparently due to (a) presence of minor amounts of macerals resistant to breakdown reactions; (b) some occurrence of weight-losing reactions, e.g., decarboxylation and partial heteroatom removal (see below); and (c) some mechanical losses. Solubility fractionation of the THF-soluble product (Table 2) yielded about 68-71 wt\% of cyclohexane-solubles (oils) which predominantly consist of monocluster ccmpounds having molecular weights in the approximate region of 100-300. Most of the structural analysis work during the reporting period (se below, Section 2) was performed using this predominant oil fraction of the depolymerized HT-BCD product.

The incompletely depolymerized part of the HT-BCD product (asphaltenes and asphaltols), representing about $30 \mathrm{wt} \%$ of the total solubilized product was subjected to a preliminary study to determine if it is convertible into oils (cyclohexane-solubles) by recycling through the HT-BCD procedure. Initial recycling runs indicated that a significant part (at least

Monocluster compounds are defined as containing a single condensed aromatichydroaromatic ring system in their molecular structure. Bicluster or polycluster compounds are defined as containing two or more condensed ring systems interconnected by linking groups susceptible to hydrogenolytic or hydrolytic cleavage. 
40-50\%) of the small asphaltene plus asphaltol part of the product is converted into oils, bringing the total proportion of the latter to $>80 \mathrm{wt} \%$ of the depolymerized coals.

The hydroprocessing (HPR) of the depolymerized coals (both of the total product and of the predominant oil fractions) was also investigated. Table 3 summarizes the elemental compositions of the depolymerized Blind Canyon, Pittsburgh \#8, and Beulah Zap coals and of the final hydrocarbon oils derived from them by hydroprocessing (reaction conditions indicated in the footnotes). The depolymerized coals have a higher hydrogen content as compared with the starting coal feeds, due to (a) marked reduction of the heteroatom ( $S, N$, and $O$ ) contents during depolymerization; and (b) some extent of hydrogen transfer reactions from the methanol solvent during the BCD step. It should be noted that all of the inorganic sulfur is removed together with the ash at the end of the HT-BCD treatment, indicating the possible value of lowtemperature depolymerization as an approach to coal cleaning. As seen in Table 3, most ( $\geq 65 \%)$ of the organic sulfur is also removed during the HT-BCD treatment (organic $S$ content of the starting coals, wt\%: Blind Canyon, 0.37; Pittsburgh \#8, 0.89; Beulah Zap, 0.70). The O content of the Blind Canyon coal is reduced by -43 wt\%, whereas that of the Beulah Zap lignite by 38 wt\%, during depolymerization (the $O$ loss is lower for Pittsburgh \#8 coal, i.e., 20\%). The $\mathrm{N}$ content is reduced as a result of the depolymerization to an extent of $46 \mathrm{wt} \%$ for the Beulah Zap lignite, 38 wt\% for the Blind Canyon coal, and 36\% for the Pittsburgh \#8 coal. The hydroprocessing (HPR) step results in essentially complete removal of the heteroatoms ( $\leq 0.05$ $\% \mathrm{~S}, 0.08-0.21 \% \mathrm{~N}$, and 0.20-0.65\% O content in the final hydrocarbon oil product; Table 3). Only trace amounts of heteroatoms were observed after a slight increase in the severity of the HPR conditions.

One of the outstanding features of the HT-BCD procedure is the compositional simplicity and corresponding high volatility of the depolymerized coal products. Thermogravimetric analysis of the predominant (70-80 wt\%) oil fractions of the depolymerized coals showed (see Progress Report for the period May 4 - July 31, 1989, p. 99) that these products are fully distillable ( $\geq 98 \%$ ) under vacuum $\left(10^{-2}\right.$ torr), and distillable to an extent of $\sim 80 \%$ under $\mathrm{N}_{2}$ at - a pressure of 1 atm. It should be noted, however, that since the depolymerized coals still retain a major part ( $>50 \%$ ) of their original coal oxygen, mostly in the form of phenolic groups (and some hydrolysis-resistant dibenzofuranic groups) these depolymerized products distill only to the extent of about 25 wt\% in the gasoline, kerosene, and light gas oil boiling range (b.p. up to $325^{\circ} \mathrm{C} / 760$ torr), while the main portion distills in the heavy gas oil (40-45\%) and vacuum gas oil (20-25\%) ranges. In contrast, the final hydrocarbon oil products obtained by HPR, i.e., exhaustive $\mathrm{HDO}$, of the depolymerized coals, distill mostly at temperatures below $325^{\circ} \mathrm{C} / 760$ torr. 
Table 4 summarizes the boiling point distribution of the final hydrocarbon fuels obtained by hydroprocessing (HPR) of the depolymerized Blind Canyon, Pittsburgh \#8, and Beulah Zap coals. As seen, all of the final hydrocarbon fuels obtained contain a very high proportion (70.8$\mathbf{7 4 . 2 \% )}$ ) of low-boiling fractions (gasoline, kerosene, and gas oil; b.p. up to $325^{\circ} \mathrm{C} / 760$ torr). The very high values for total distillables $\left(<538^{\circ} \mathrm{C}\right)$ are fully consistent with the above-mentioned thermogravimetric analysis of the same products. Hydroprocessing (HPR) of the total depolymerized coals is being presently investigated and initial results indicate only slightly lower yields of low-boiling fractions up to $325^{\circ} \mathrm{C} / 760$ torr.

The highly promising results obtained during the reporting period confirm the efficiency of the HT-BCD-HPR procedure for high-yield conversion of premium U.S. coals of various ranks into light liquid fuels. Continuous flow reactors will be constructed and applied in a further investigation of the procedure during the May 1990 - April 1991 period.

\section{Characterization and Structural Analysis of Depolymerized Blind Canyon, Pittsburgh \#8, and Beulah Zap Coals}

In-depth structural analysis of the depolymerized coal products were performed in collaboration with Task V.1 (Professor Pugmire), Task V.2 (Professor Meuzelaar), and Task V.3 (Professor Eyring). For this purpose, sufficiently large samples were prepared in the framework of our Task V.5 and continuously provided to the other tasks.

Excellent progress was achieved under Task V.2 in the development of mass spectral techniques for molecular weight profiling of the depolymerized coals studied (see Progress and Final Reports for the May 1989 - May 1990 period; Task V.2). Results obtained showed that the predominant oil fractions of the products consist primarily of monocluster compounds of several types, i.e., phenols, alkylarenes, and alkanes.

Detailed ${ }^{13} \mathrm{C}$ NMR and PMR measurements of the depolymerized coal products were performed under Task V.1. Results obtained were fully consistent with the mass spectral data and showed that the depolymerized coals predominantly consist of mixtures of monocluster compounds showing well-resolved signals characteristic of phenols, alkylarenes and alkanes, together with small amounts of alcohols, alkenes, and carboxylic acids (especially in the case of the Beulah Zap lignite). Results obtained were summarized in previous Progress Reports (see for example Progress Report for the period November 1, 1989 - January 31, 1990, Task V.1, and R. White, M.S. Thesis, University of Utah, 1989). The PMR analyses were particularly valuable for determining the distribution of aliphatic hydrogens in alkyl substituents (present in alkylarenes or alkylphenols) as a function of their position relative to the aromatic rings. Further, the PMR data provided information on the distribution of aromatic hydrogens in 1-, 


\section{2-, and 3-ring systems.}

FTIR spectra of the depolymerized coal products were obtained through cooperation between Tasks V.3 and V.5. Examples of such spectra, together with full interpretations, were provided in the preceding Progress Reports. The data were valuable in corroborating the chemical functionalities of the depolymerized products as obtained by mass spectral and NMR techniques. The FTIR data were also particularly useful in determining the degree of aromatic

- ring substitution in product components, using for that purpose well-resolved C-H out-of-plane bending bands in the $600-900 \mathrm{~cm}^{-1}$ region. A quantitative method for determining the distribution of aromatic ring substitution types (mono-, di-, tri-, tetra-, and pentasubstituted rings) in depolymerized coal products is presently being developed.

Recently, a cooperation effort was initiated with Professor Milton Lee, Brigham Young University, on the compound class separation of depolymerized coal products, using selective elution chromatography. The products are being separated into four fractions, i.e., (1) saturates (alkanes and naphthenes); (2) arenes (mostly alkylarenes and some aromatic-hydroaromatic compounds); (3) N-containing compounds; and (4) O-containing compounds (mostly phenols). This preliminary separation allows for greatly improved simplicity and accuracy in subsequent mass spectral, NMR and FTIR analysis of the depolymerized coal products. This in turn leads to structural elucidation of the basic building units (monoclusters) of the coal polymer at a nearmolecular level. Further, the change in product composition, viz. the distribution of such building units, as a function of coal rank and source can be determined and used as a powerful coal characterization and differentiation method.

\section{Simplication of the HT-BCD-HPR Procedure}

Work was performed on the simplification of the HT-BCD-HPR coal liquefaction procedure and on the examination of alternative catalysts and solvents. The THF pre-extraction step was eliminated and $\mathrm{MeOH}$ is being gradually introduced as the only solvent in the process. Further, a search is being performed for (a) various soluble Fe-containing catalysts for possible use in the HT step; and (b) Ca-containing basic (or base-generating) compounds for replacement of $\mathrm{KOH}$ as catalyst in the BCD step. As mentioned, during the 1990-91 period continuous flow reactors will be constructed and applied in the studies. This will include an attempt to unify the performance of the depolymerization stage of the process (the HT and BCD steps), using a series reactor. 


\section{Elucidation of the Mode of Catalytic Action of Soluble Fe Catalysts in the HT Step}

Samples of $\mathrm{FeCl}_{3}$ - and $\mathrm{FeCl}_{2}$-impregnated coal samples were prepared and provided to Professor E.M. Eyring (see above, Task V.3) for the purpose of electron probe microanalysis (EPM), diffuse reflectance FTIR, and Mossbauer studies. Part of the impregnated samples were subjected to subsequent mild hydrotreatment (temperature, $275^{\circ} \mathrm{C} ; \mathrm{H}_{2}$ pressure, $1500 \mathrm{psig}$ ). This allows for tracking the action of the Fe catalyst during the HT coal depolymerization step. $\mathrm{FeCl}_{3}$-impregnated coal samples were provided also to Professor G.P. Huffman for his Mössbauer studies.

\section{References}

1. Mebring, M. Principles of High Resolution NMR in Solids; Springer-Verlag: New York, 1983.

2. Stejskal, E.O.; Schaefer, J.; McKay, R.A. J. Magn. Reson., 1977, 25, 569.

3. Sethi, N.K; Grant, D.M.; Pugmire, R.J. J. Magn. Reson., 1987, 71, 476.

4. Maricq, N.M.; Waugh, J.S. J. Chem. Phys., 1979, 70, 3300.

5. Herzfield, J.; Berger, A.E. J. Chem. Phys., 1980, 73, 6021.

6. Bax, A.; Szeverenyi, N.M.; Sardashti, M. J. Magn. Reson. 1983,52, 147.

7. Bax, A.; Szeverenyi, N.M.; Sardashti, M. J. Magn. Reson. 1983,55, 494.

8. Maciel, G.E.; Szeverenyi, N.M.; Sardashti, M. J. Magn. Res'ın. 1985,64, 365.

9. Zeigler, R.C.; Wind, R.A.; Maciel, G.E. J. Magn. Reson. 1988, 79, 299.

10. Lippmaa, E.; Alla, M.; Tuherm, T. Proceedings of the Nineteenth Congress Ampere, Heidelberg, 1976, p. 113.

11. Hughes, C.D.; Sherwood, M.H.; Alderman, D.W.; Grant, D.M. Manuscript in preparation.

12. (a) Veeman, W.S. Prog. NMR Spectrosc. 1984, 16, 193. (b) Facelli, J.C.; Grant, D.M.; Michl, J. Acc. Chem. Res. 1987, 20,152, and references therein.

13. Facelli, J.C.; Grant, D.M. Theor. Chim. Acta, 1987,71, 277.

14. Wemmer, D.E.; Pines, A.; Whitehurst, D.D. Phil. Trans. Roy. Soc. Lond. A,1981, 300, 15.

15. Duncan, T.M. J. Phys. Chem. Ref. Data, 1987, 16, 125.

16. Carter, C.M.; Alderman, D.W.; Facelli, J.C.; Grant, D.M. J. Am. Chem. Soc. 1987, 109, 2639.

17. Carter, C.M.; Facelli, J.C.; Alderman, D.W.; Grant, D.M.; Dalley, N.K; Wilson, B.E. J. Chem. Soc. Faraday Trans. 1, 1988, 84, 3673.

18. Facelli, J.C.; Grant, D.M. Molecular Structure and Carbon-13 Chemical Shielding Tensors Obtained from Nuclear Magnetic Resonance; Topics in Stereochemistry; John Wiley \& Sons: New York, 1989; Vol. 19, 1.

19. Morgan, K.R.; Newman, R.H. J. Am. Chem. Soc. 1990, 112, 4. 
20. Pines, A.; Gibby, M.G.; Waugh, J.S. J. Chem. Phys., 1973,59, 569.

21. Alderman, D.W.; Solum, M.S.; Grant, D.M. J. Chem. Phys., 1986, 84, 3717.

22. Sethi, N.K.: Pugmire, R.J.: Facelli, J.C.; Grant, D.M. Anal. Chem., 1988, 60,1574.

23. Solum, M.S.; Pugmire, R.J.; Grant, D.M. Energy and Fuels, 1989, 3, 187.

24. Soderquist, A.; Burton, D.J.; Pugmire, R. J.; Beeler, A.J., Grant, D.M.; Durand, B.; Huk, A.Y. Energy and Fuel, 1987, 1,50.

25. Aue, W.P.; Ruben, D.J.; Griffin, R.G. J. Magn. Reson., 1981, 47, 472.

26. Burgar, M.I. Fuel, 1984, 63, 1621.

27. Burgar, M.I.; Kalman, J.R.; Stephens, J.F. Proceedings of the 1985 International Conference on Coal Science, Sydney, Australia, 1985, p. 780.

28. Botto, R.E.; Axelson, D.E. ACS Fuel Division Preprints 1988, 33, 50.

29. Bax, A. Szeverenyi, N.M.; Maciel, G.E. J. Magn. Reson. 1983, 51,400.

30. Yarim-Agaev. Y.; Tutunjian, P.N.; Waugh, J.S. J. Magn. Reson. 1982, 47, 51.

31. Tycko, R; Dabbagh, G.; Mirau, P.A. J. Magn. Reson. 1989 85, 265.

32. (a) Jeener, J.; Meier, B.H.; Bachmann, P.; and Ernst, R.R. J. Chem. Phys., 1979, 71, 4546. (b) Carter, C.M.; Alderman, D.W.; Grant, D.M. J. Magn. Reson. 1985, 65, 183.

33. Henrichs, P.M. Macromolecules 1987, 20, 2099.

34. Unpublished results from this Laboratory.

35. Shabtai, J.S., Saito, I. US Pat., 4,728,418 (1988).

36. Derbyshire, F. Catalysis in Coal Liquefaction; IEA Coal Research: London, 1988; Energy \& Fuels, 3,273 (1989).

37. Wang, H.P.; Yu, T.; Garland, B.A.; Eyring, E.M. Appl. Spect., in press.

38. Wang, H.P.; Huai, H.; Eyring, E.M. 45th Northwest/10th Rocky Mountain Regional ACS Meeting, Salt Lake City, Utah, June 13, 1990.

39. Shabtai, J.S.; Zhang, Y. Proc. 1989 Internat. Confer. Coal Science, Tokyo, 1989, Vol. II, 807-810.

40. Shabtai, J.S.; Skulthai, T. Proc. 1987 Internat. Confer. Coal Science, Maastricht, The Netherlands, Elsevier, 761-764.

41. Shabtai, J.S.; Skulthai, T.; Saito, I. Amer. Chem. Soc. Div. Fuel Chem. Prepr., 1986, 31 (4), 15.

42. Skulthai, T. Ph.D. Dissertation, University of Utah, 1987.

43. White, R. M.S. Thesis, University of Utah, 1989.

44. Shabtai, J.S.; Guohe, Q.; Balusami, K.; Nag, N.K.; Massoth, K.E. J. Catal., 1988, 113, 206. 
45. Shabtai, J.S.; Nag, N.K; Massoth, F.E. J. Catal., 1987, 104, 413. 
Table 1 Ranges of Sultable Conditions for Low-Temperature and Beulah Zap Coals"

HT Step

$\mathrm{FeCl}_{3}$ catalystb concentration: 3-12 wts

$\mathrm{H}_{2}$ pressure: 1500 psig

Reaction temperature: $250-290^{\circ} \mathrm{C}$

Reaction time: $2 \mathrm{hr}^{\mathrm{a}}$

BCD Step

Depolymerizing agent: 5-108 $\mathrm{KOH}^{\mathrm{c}}$ in $\mathrm{MeOH}$

$\mathrm{N}_{2}$ pressure: $1,000 \mathrm{psig}$

Reaction temperature: $275-295^{\circ} \mathrm{C}$

Reaction time: $1 \mathrm{hr}^{\mathrm{a}}$

HPR SteP

Catalyst: sulfided 6Co8Mo or RuMod

$\mathrm{H}_{2}$ pressure: $2500-2700$ psig

Reaction temperature: $350-370^{\circ} \mathrm{C}^{\circ}$

Reaction time: $2-3 \mathrm{hr}^{2}$

- Processing conditions were determined in autoclave reactors. Flow reactor systems will be constructed during 1990-91, with the anticipated result of drastically decreased reaction time for each step of the procedure. HT experiments at $290^{\circ} \mathrm{C}$ with catalytic amounts of $\mathrm{FeCl}_{3}$ ( $3-15$ wto) impreg-
nated in the coals indicate the absence of any corrosion effect upon the
stainless steel reactor (see toxt). stainless steel reactor (see text). Experiments are currently underway to replace $\mathrm{KOH}$ with $\mathrm{Ca}(\mathrm{OH})_{2}$ as base
catalyst (see text).

d This catalyst has markedly higher $\mathrm{C}-\mathrm{O}$ and $\mathrm{C}-\mathrm{N}$ hydrogenolysis selectivity as compared with conventional CoMo and NiMu catalysts (see refs. 7,8 ). 


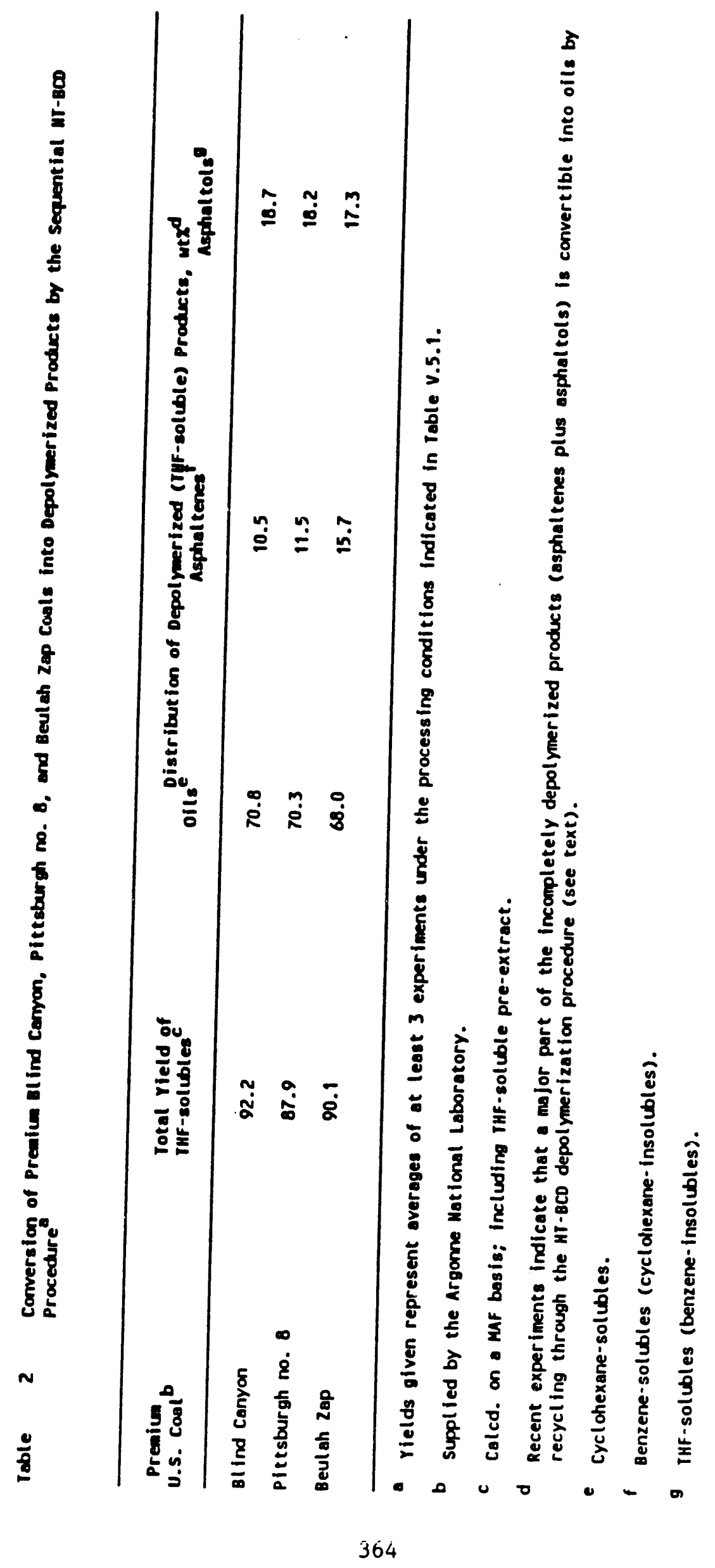




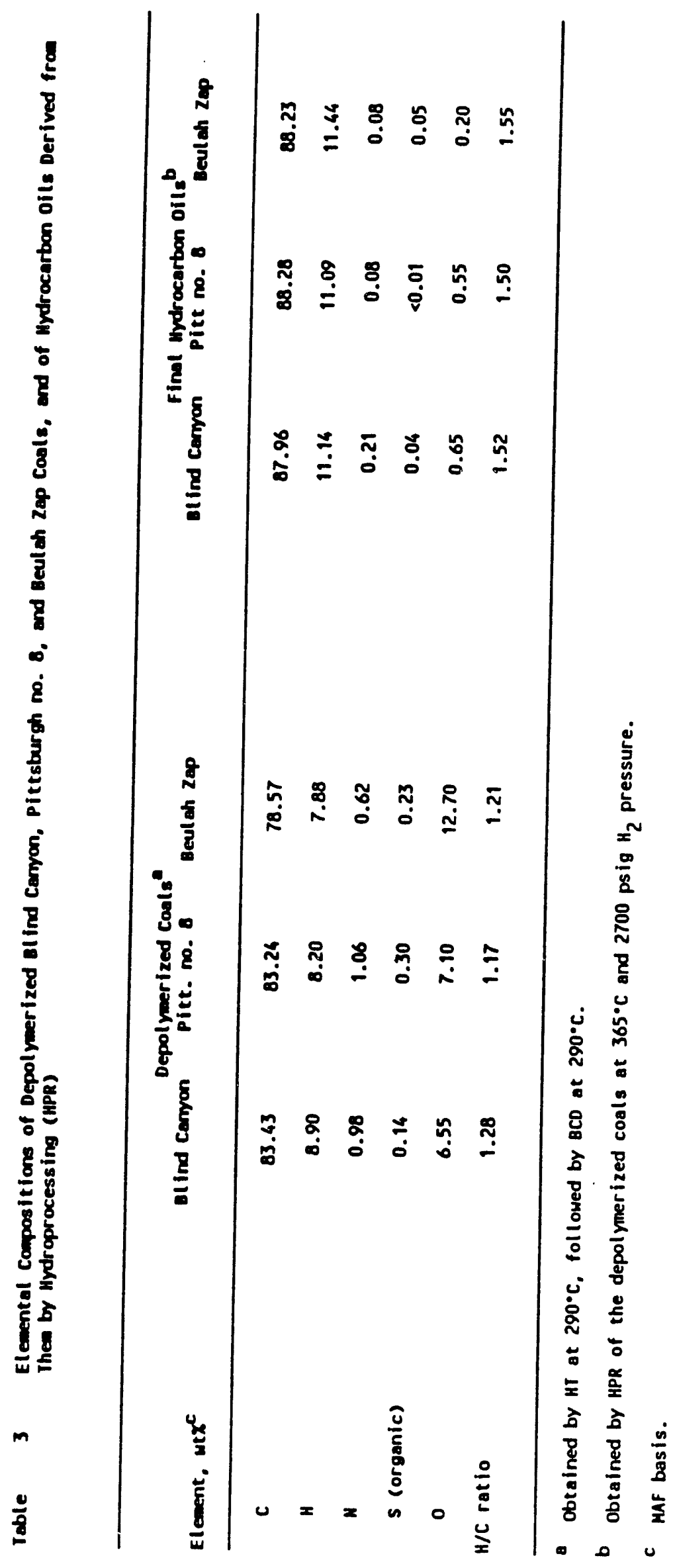




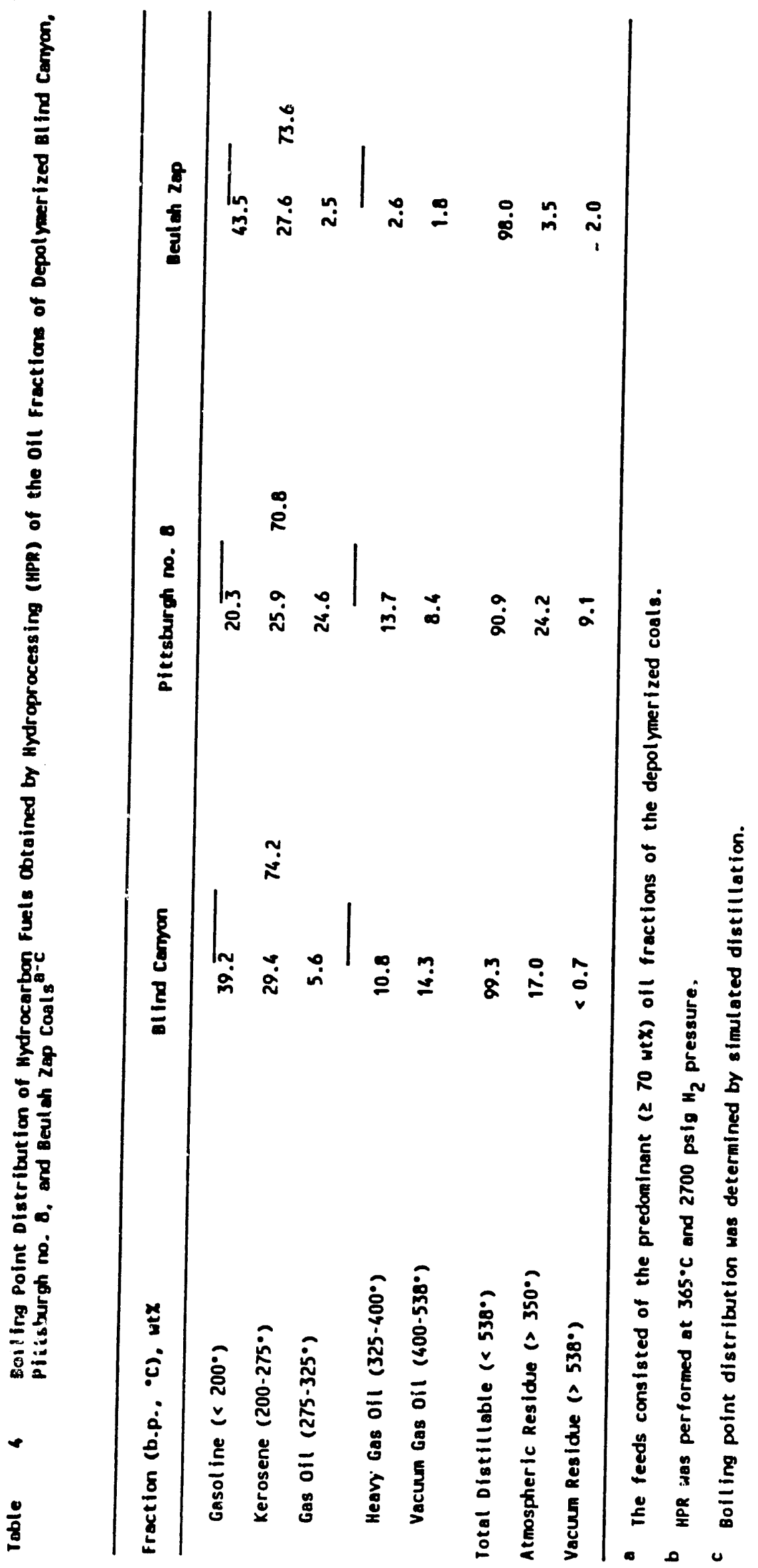




\section{Task VI}

\section{University of Kentucky Center for Applied Energy Research}




\section{Task VI}

\section{Basic Process/Resource Evaluation Task}

$\begin{array}{lll}\text { PI's: } & \text { Burt Davis } & \text { R. A. Keogh } \\ & \text { Diane Milburn } & \text { H. Dabbagh }\end{array}$

\section{Report}

Coordinator: Burt Davis

Phone Number: (606) 257-0251

Period: $\quad$ May 4, 1989 - May 3, 1990

\section{Task VI.1. Coal Maceral Characterization and Liquefaction}

\section{Objectives}

VI.1.1. Obtain, if possible, a coal with equal distribution of the three major maceral groups (vitrinites, inertinites, and liptinites) for separation by density gradient centrifugation.

VI.1.2. Characterize the maceral concentrates.

VI.1.3. Liquify the sample sets and characterize the products.

VI.1.4. Determine the thermal liquefaction pathway of the macerals.

\section{Introduction}

A classic experimental approach to the study of complex system, such as coal, is to reduce the system into its component parts. Recently, the use of density gradient centrifugation, DGC $(1,2)$ has been used to separate the different macerals of coal for structural and reactivity studies. The prerequisite fine grinding $(<3 \mu \mathrm{m})$ of the coal prior to separation using this technique has produced problems in identifying the majority of the material found in the isolated density fractions. In addition, only small quantities of the macerals were produced. However, DGC techniques developed in this laboratory $(3,4)$ have successfully separated coal ground to -100 mesh, (which allows identification of the majority of the material in each density fraction) in sufficient quantity and purity for analysis and liquefaction studies. 


\section{V1.1.1. Identification of the Coal}

A bench sample of the eastern Kentucky Stockton coal was identified as having approximately equal concentration of the three major maceral groups. The results of the extensive preliminary density gradient separations indicated that the maceral groups could not be separated in sufficient purities and quantities for the liquefaction studies. Therefore, no further separations on this coal was performed. Another coal which contained equal amounts of the maceral groups was not found during the course of the project. Therefore, two Ohio coals were selected for separation of the vitrinite macerals by density gradient centrifugation.

\section{VI.1.2. Maceral Characterization}

The ultimate, proximate and petrographic analyses of the parent, demineralized parent and vitrinite concentrates for the Ohio \#5 and Ohio \#6 coal are given in Table 1. The major difference in the characterization data of the two demineralized and parent coals is the total sulfur content. The Ohio \#5 coal set has approximately twice the sulfur content of the Ohio \#6 coal. The difference is not apparent in the two vitrinite concentrates. Both vitrinite concentrates are similar in their chemical and petrographic compositions.

\section{VI.1.3.}

The set of three sample types (parent, demineralized and vitrinite concentrate) were liquefied in the microautoclave reactors using three temperatures $\left(385^{\circ} \mathrm{C}, 427^{\circ} \mathrm{C}, 445^{\circ} \mathrm{C}\right)$, tetralin as the solvent, a hydrogen atmosphere ( $800 \mathrm{psig}$, ambient), and a solvent to coal ratio of 1:5. Two residence times (15 and $30 \mathrm{~min}$ ) were used at each of the reaction temperatures.

The distribution of the liquefaction products for the Ohio \#6 sample set is shown in Figure 1. All three types of sample follow the same thermal liquefaction pathway. In the initial dissolution stage of the pathway, the primary liquefaction products are the asphaltenes plus preasphaltenes. In this region, as the total conversion increases, the asphaltene plus preasphaltene $(A+P)$ yields also increase and the oil plus gas yields remain fairly constant (ca. $10 \mathrm{wt} . \%$ ). The maximum yield of $A+P$ corresponds to the maximum in total conversion and a change in the thermal pathway. In this region, the total conversion remains fairly constant. The primary reactions taking place are the conversion of the $A+P$ fraction to oil plus gas. The observation that all of the Ohio \#6 sample types have identical 
thermal liquefaction pathway indicates that the demineralization and DGC procedures has little, if any, effect on the liquefaction pathway of the Ohio \#6 samples.

The liquefaction product distribution for the Ohio \#5 demineralized parent and vitrinite concentrate are shown in Figure 2. It appears that the Ohio \#5 samples are significantly more reactive than the Ohio \#6 samples. It is also apparent from the data shown in Figure 2 that there is significant differences in product distributions as well as reactivity among the Ohio \#5 sample type. The Ohio \#5 demineralized coal reaches the maximum conversion rapidly (@ $385^{\circ} \mathrm{C}, 15 \mathrm{~min}$.) and appears to follow the same thermal pathway as the Ohio \#6 samples. However, the vitrinite concentrate appears to follow a different thermal pathway than was previously observed. As the total conversion of the vitrinite concentrate increases, there is a parallel increase in both the $A+P$ and $O+G$ yields. Although there was insufficient sample for further experiments, it is likely that the $A+P$ yield would still maximize at the highest conversion obtained. The major difference in the pathway of the Ohio \#5 vitrinite and the previous samples is the parallel increase in $O+G$ yields with conversion. The analysis of the Ohio \#5 and \#6 vitrinite are very similar and offer no explanation for the observed differences in their reactivities.

\section{References}

1. Dyrkacz, G. R., Bloomquist, C. A. A. and Howitz, E. P., Sep. Sci. Tech., 1981, 16, 1671.

2. Dyrkacz, G. R. and Howitz, E. P., Fuel, 1982, 61, 3.

3. Poe, S. H., Taulbee, D. N. and Keogh, R. A., "Density Gradient Separations of -100 Mesh Coal", accepted for publication in J. Org. Geochemistry.

4. Taulbee, D. N., Poe, S., Robl,T., and Keogh,R., "DGC Separation of the Major Maceral Groups from Mixed Maceral Bituminous Coal", submitted to Energy and Fuels. 


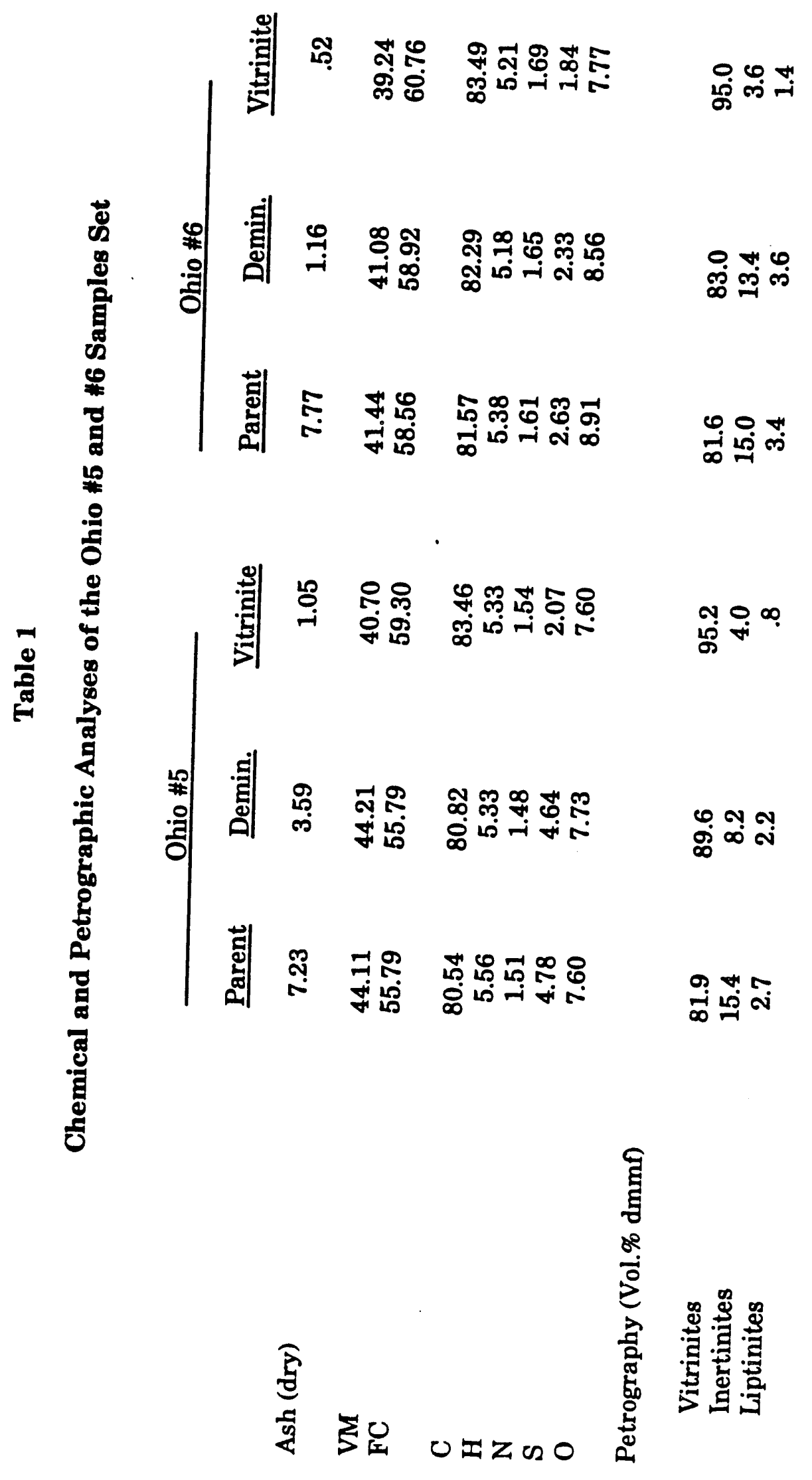




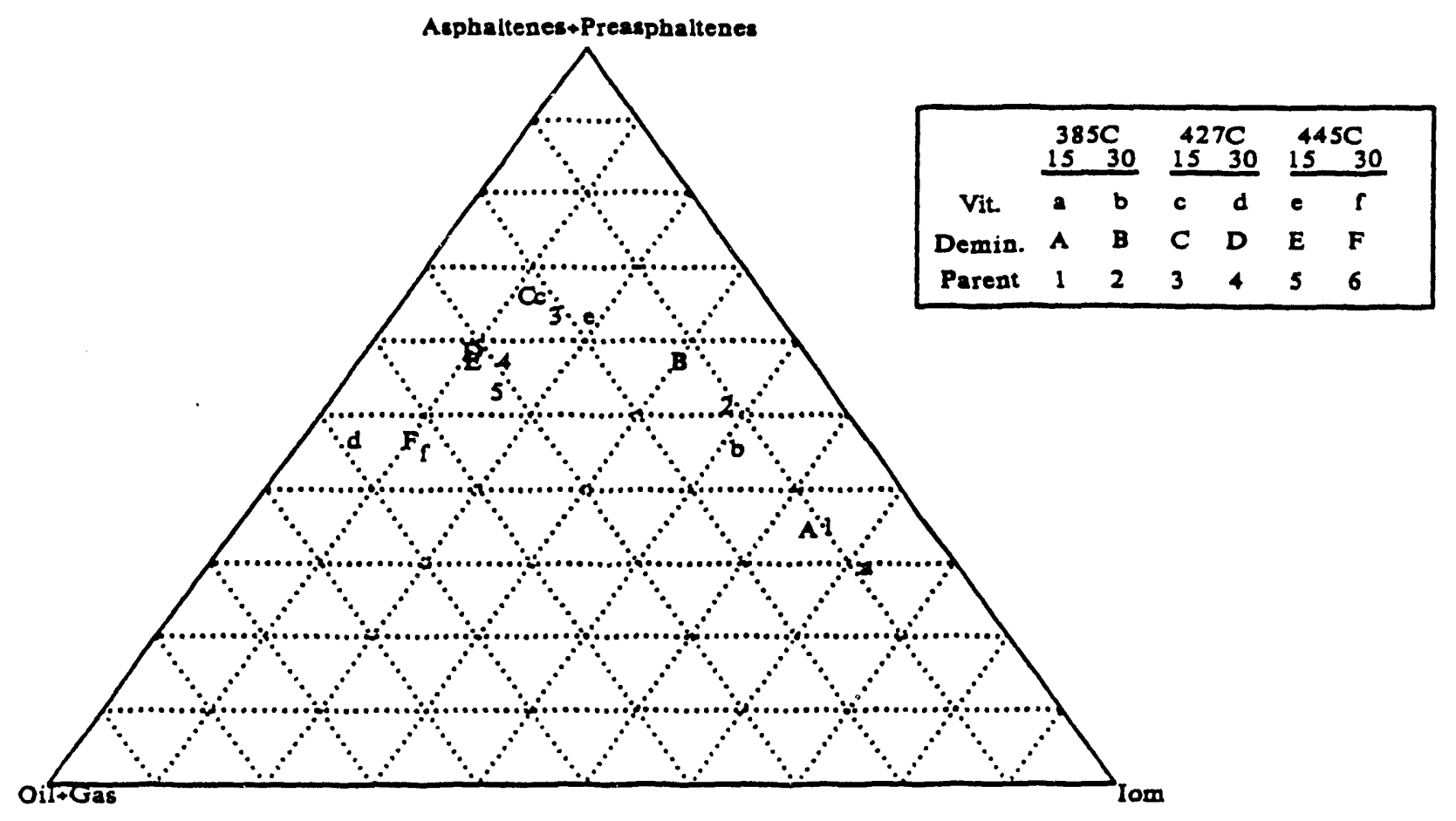

Figure 1. Product distribution of Ohio \#6 samples.

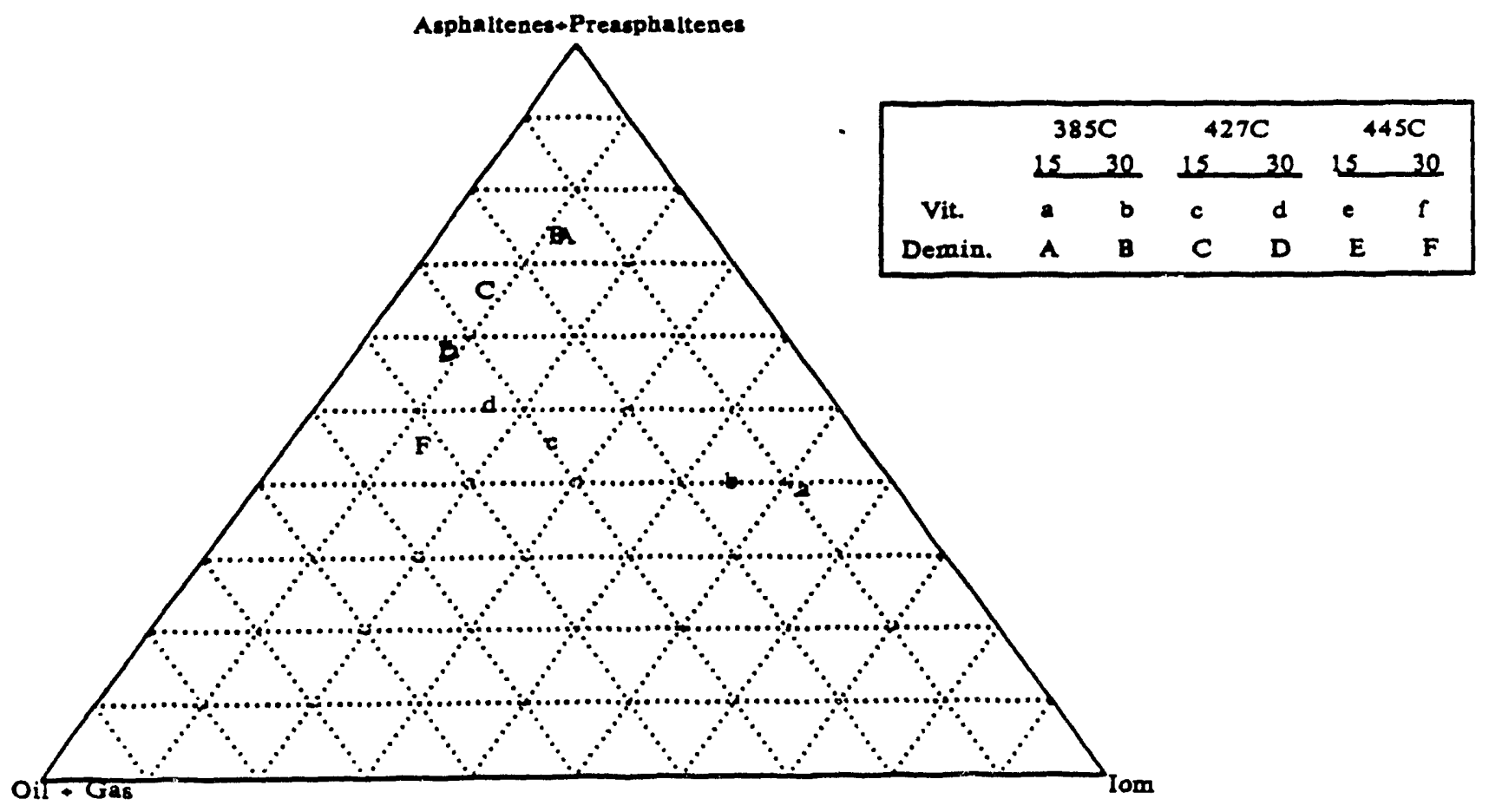

Figure 2. Product distributions of Ohio \#5 Samples. 


\section{Task VI.2. Coal Liquefaction Catalysis}

Catalyst samples for this aging study were obtained from Wilsonville's run 257.

Catalyst addition, as well as withdrawal, was started to the first reactor on the 18th day of operation; hence, reliable data for sulfur isotope exchange could not be obtained after day 18 . Catalyst addition was not started in the second stage reactor until day 31 . Wilsonville operators reported that the sulfur level in the as-received catalyst was less than their analytical limit ( 0.1 wt.\% or less). The sulfur isotope data for the presulfided material and for catalyst samples withdrawn at increasing operational time from each of the two reactors are shown in Figures 1 and 2. First, consider the data in Figure 1 for samples withdrawn from the second stage reactor since it contains more data points covering a longer time period. The data clearly show that there are two types of isotope exchange. First, there is a very rapid initial exchange of sulfur that was added during the presulfiding procedure with that of the coal. This rapid exchange corresponds to $33 \%$ of the sulfur that was initially present in the catalyst:

$$
\begin{aligned}
\% \text { S exchange } & =\left\{\left[(\delta){ }^{34} \mathrm{~S}_{\mathrm{t}=0}-(\delta){ }^{34} \mathrm{~S}_{\text {pretreat }} \|(\delta){ }^{34} \mathrm{~S}_{\text {coal olury }}-(\delta){ }^{34} \mathrm{~S}_{\text {pretreat }}\right]\right\} 100 \\
& =\{[0.6-(-0.4) \mathrm{y}[2.6-(-0.4)]\} 100=33
\end{aligned}
$$

Following the rapid exchange, there is a period of slow exchange during which the sulfur on the catalyst gradually approaches that of the added coal. The slope of the line in Figure 1 is $0.022 \delta^{34} \mathrm{~S} /$ day; it is calculated that it would take a minimum of 91 days for the sulfur isotope composition of the catalyst, calculated by extending the line defined by the measured $\delta^{34} \mathrm{~S}$ values toward the solid line representing the isotope composition of the coal, to attain that of the coal slurry. Thus, we obtain the surprising result that only about one-third of the sulfur initially present in the presulfided catalyst exchanges rapidly even when the catalyst is operated at commercial hydrotreating conditions (greater than 2000 psi and temperatures around $425^{\circ} \mathrm{C}$ ).

There are fewer data points shown in Figure 2 for samples withdrawn from the first reactor; however, the same pattern is exhibited in both figures 1 and 2 . Note that different batches of dimethyl disulfide were used to presulfide the two batches of catalysts; thus the material used in reactor 1 had an isotope composition that was shifted -1.15 from the standard rather while the batch added to the second reactor was shifted -0.4 . The line defining the slow exchange for the first reactor catalyst intercepts the zero time axis at 0.3 . However, only three of the four data points were used to determine the line that gave the value of 0.3 , and the data points could fit equally well the same line that was defined by the 
data in Figure 1. Using the data for Figure 2, we calculate that the rapid exchange corresponds to ca. $40 \%$ of the sulfur initially present in the catalyst. Thus we obtain similar data for catalysts exposed in either reactor 1 or 2 .

The data are intriguing but they do not provide sufficient information to define the structures that correspond to the rapid and slow exchange of sulfur. The first problem encountered in attempting to assign chemical species or structures to the exchange data is a definition of the compounds and their stoichiometry that are present in the catalyst. Massutin' reported in 1978 that in spite of the fact that these catalysts are either presulfided or become sulfided during use, surprisingly little work has been done on determining the stoichiometry of the sulfided catalyst state. When sulfur levels have been determined, the catalyst has usually been found to be incompletely sulfided to the respective sulfides, $\mathrm{MoS}_{2}$ and $\mathrm{Co}_{9} \mathrm{~S}_{8}{ }^{2,3}$ Massoth ${ }^{1}$ concluded that the sulfided catalyst consists of a mixed surface oxysulfide specie or some bulk $\mathrm{MoS}_{2}$ and another oxysulfide specie. The state of Co in the sulfided catalyst is uncertain. Knözinger summarized in 1988 the current results on the genesis and nature of molybdenum-based hydrodesulfurization catalysts, and did not significantly modify the picture presented by Massoth. Knözinger presents a more detailed description of the sulfided catalyst: highly dispersed $\mathrm{MoS}_{2}$-like slabs are the dominant Mo species in the sulfided catalysts. These slabs are stabilized by Mo-O-Al links after mild sulfidation at $\mathrm{T} \leq 670 \mathrm{~K}$ and in this state the slabs are preferentially oriented normal to the support surface. At higher sulfidation temperatures, the average size of the $\mathrm{MoS}_{2}$ slab is large; these large slabs are detached from the alumina and lie flat on the support surface. The presence of promoters $(\mathrm{Co}, \mathrm{Ni})$ may lead to somewhat higher dispersion but does not seem to significantly alter the local Mo environment. For typical catalyst compositions, the promoter atoms in or on the surface are sulfided but are not present in well ordered structures such as $\mathrm{Co}_{9} \mathrm{~S}_{8}$ or $\mathrm{Ni}_{3} \mathrm{~S}_{2}$.

There is considerable variation in the S/Mo ratio for catalysts from several runs at Wilsonville. Ratios reported by them and obtained by us are shown in Table 1. The ratio ranges from about 1.5 to 2.4. While these catalysts do contain other metals, it appears that a value for the atomic S/Mo greater than 2 is not that unusual. At the same time, it should be realized that there is considerable error associated with determining this ratio.

If $S$ is combined only with Mo the stoichiometry of the second stage catalyst is $\mathrm{MoS}_{2.78}$; assuming that all $\mathrm{Ni}$ is sulfided to form NiS reduces the stoichiometry to only $\mathrm{MoS}_{2.41}$. The first stage catalyst has a stoichiometry of $\mathrm{MoS}_{2.89}$ if all sulfur is combined with Mo; if NiS is 
formed then the stoichiometry becomes $\mathrm{MoS}_{2.5}$. Even if we assume that all of the $\mathrm{Ca}, \mathrm{Fe}$ and $\mathrm{Ni}$ are present as M(II)S, the stoichiometry for the second stage catalyst (for day 15) is $\mathrm{MoS}_{2.28}$. The data indicate that sulfidation at these severe conditions produces a molybdenum species with a stoichiometry considerably greater than $\mathrm{MoS}_{2}$ or that a large amount of sulfur is present in other metal sulfides, including $\mathrm{TiO}_{z} \mathrm{~S}_{y}$ and/or $\mathrm{AlO}_{z} \mathrm{~S}_{y}$, and/or elemental sulfur. Thus, the exchange data must be evaluated in the light of uncertain sulfide compounds and stoichiometry.

First we note that the assumption that organic sulfur compounds derived from the coal/solvent are adsorbed on the catalyst only makes the problem more perplexing. The sulfur compounds derived from the coal will have a $\delta^{34} \mathrm{~S}$ value of +2.5 ; correcting the $\delta^{34} \mathrm{~S}$ values of the catalyst for any coal derived from adsorbed organosulfur compounds decreases the amount of sulfur that rapidly exchanged.

If we assume that $\mathrm{Ni}$ and $\mathrm{Fe}$ are present as $\mathrm{NiS}$ and $\mathrm{FeS}$ and that these exchange rapidly, we can account for only $43 \%$ of the total rapid exchange; assuming only NiS is formed and rapidly exchanges can account for about 39\% of the total rapid exchange. If it is assumed that (1) $\mathrm{MoS}_{2}$ is formed and does not exchange rapidly and (2) all other sulfur is present in a form that rapidly exchanges, then the rapid exchange should be $27.8 \%$ of the total sulfur rather than the $34.5 \%$ that is observed. The data are suggestive that a significant fraction of the sulfur present in "molybdenum sulfide" does not exchange rapidly. To define the source of the rapidly exchanging sulfur will require further work with a series of sulfided preparations: alumina only, $\mathrm{Mo}-\mathrm{Al}_{2} \mathrm{O}_{3}, \mathrm{Ni}-\mathrm{Al}_{2} \mathrm{O}_{3}, \mathrm{Ni}-\mathrm{Mo}-\mathrm{Al}_{2} \mathrm{O}_{3}$, etc.

Isagulyants and coworkers ${ }^{5,6}$ utilized the ${ }^{36} \mathrm{~S}$ radioisotope to follow the exchange of sulfur isotope in Co- $\mathrm{Mo}-\mathrm{Al}_{2} \mathrm{O}_{3}$ catalysts using the pulse microreactor technique and lower temperature and pressure conditions. They found that about $60 \%$ of the sulfur in the catalyst is not involved in exchange during the conversion of thiophene. More recently Dobrovolszky et al. ${ }^{7}$ utilized a pulse reactor at low pressure conditions to follow the retention and exchange of radioactive sulfur with non-radioactive sulfur during the conversion of thiophene. These later workers found a release of about $20 \%$ of the sulfur added to the catalyst was removed during subsequent reactions.

The present results, obtained at high pressure in a large 6 ton per day plant, show remarkable agreement with data reported for small scale, low pressure laboratory reactors. It is apparent that stable sulfur isotopes can be utilized in appropriate situations even in large scale reactors and at industrial/process conditions. The efforts expended in catalyst 
preparation and pretreatment to produce highly dispersed sulfided molybdenum species appear to be merited since at least two-thirds of the sulfur initially present in Ni-Moalumina catalysts exchanges very slowly. This is taken to indicate that the small molybdenum sulfide crystals retain their original structure for long time periods (days or months); if rapid reorganization of these crystals were to occur it is expected that sulfur exchange would quickly occur between the sulfur present in the reactant and the sulfur pool initially presenc in the catalyst.

\section{References}

1. Massoth, F. E. Advan. Catal., 1978, $27,265$.

2. Massoth, F. E. J. Catal., 1975, 36, 164.

3. Massoth, F. E., Chung, K. S., and Ramachandran, R. Fuel Processing Technol., 1979, 2 , 57.

4. Knözinger, H. Catalysis. Theory to Practice [Proc. 9th Int. Congr. Catal.]" (M. J. Phillips and M. Ternan, Eds.), Chemical Inst. Canada, Ottawa, Ontario, Canada, 1988, p. 20.

5. Isagulyants, G. V., Greish, A. A., Kogan, V. M., Vyunova, G. M., and Antoshia, G. V. Kinetika i Kataliz, 1987, 28, 632,.

6. Isagulyants, G. V., Greish, A. A, and Kogan, V. M. in Catalysis. Theory to Practice (Proceedings 9th Int. Congr. Catalysis) (M. J. Phillips and M. Teman, Eds.) Vol. 1, 35 (1988).

7. Dobrovolszky, M., Tetenyi, P., and Paal, Z. Chem. Eng. Comm., 1989, $\underline{83}, 1$.

\section{Table 1}

Atomic Ratio, S/Mo, for Catalysts Withdrawn from the Wilsonville, Alabama Pilot Plant

$\begin{array}{llcc}\text { Run } & \underline{\text { Date }} & \begin{array}{c}\text { S/Mo, } \\ \text { Wilsonville }\end{array} & \begin{array}{c}\text { Atomic Ratio } \\ \text { CAER }\end{array} \\ 50 & 2 / 17 / 86 & 2.15 & 1.8 \\ 250 & 12 / 8 / 85 & 2.18 & 2.41 \\ 249 & 9 / 18 / 85 & 2.41 & 2.26 \\ 248 & 5 / 3 / 85 & 2.09^{*} & 2.11 \\ 247 & 1 / 6 / 85 & 1.99 & \cdots \\ \text { a Sample Date: } 5 / 5 / 85 & & \end{array}$




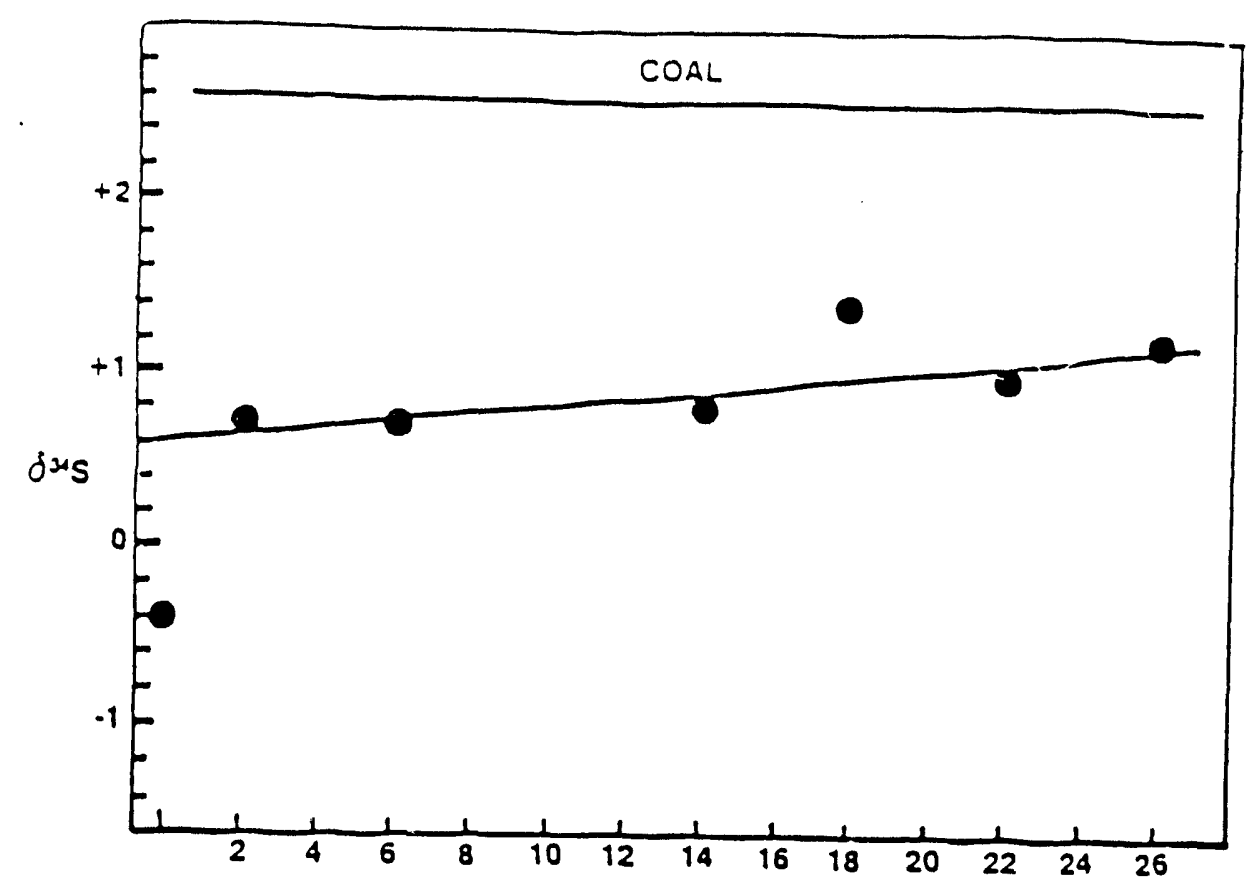

Time on Stream, Days

Figure 1. The $\delta{ }^{2} S$ isotope ratio $\left({ }^{2} S{ }^{2} S\right)$ for catalyst samples withdrawn from the second stage reactor atter presulfiding (day 0 ) and at increasing operating days. The upper solid line is the value of $\delta^{\text {hin }} \mathrm{S}$ in the feed coal.

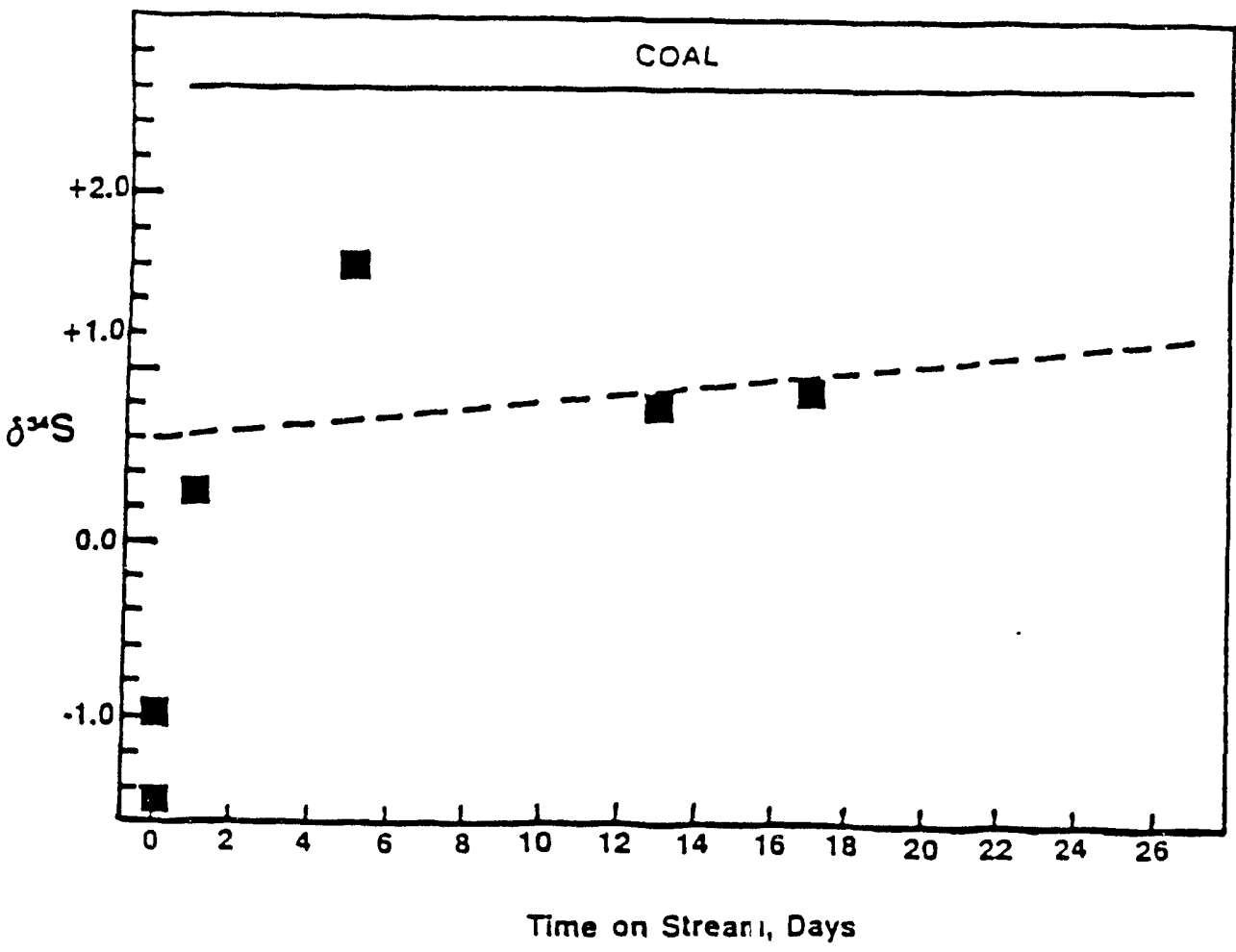

Figure 2. The $\delta^{24} \mathrm{~S}$ isotope ratio $\left({ }^{2} \mathrm{~S} / \mathrm{H}^{2} \mathrm{~S}\right)$ for catalyst samples withdrawn trom the first staçe rezctor ather presulfuding (day 0) and at increasing operating says. The solid line is the value of A $^{34} \mathrm{~S}$ in the tagd coal; the broken line is the one corresponding to the date fit in figure 1. 


\section{Task VI.3. The Role of Hydrogen During Liquefaction Using Donor and Non-Donor Solvents}

An objective of this work was to understand how gaseous hydrogen (deuterium) is utilized during the conversion of a coal that has as high a conversion in a non-donor solvent (1-methylnaphthalene) as a good donor (tetralin).

The effect of 1-methylnaphthalene-deuterium system on coal (Western Kentucky \#9 coal) conversion was studied at $385^{\circ} \mathrm{C}$ and $450^{\circ} \mathrm{C}$, ca. $2000 \mathrm{psi}$ and reaction times of 15,30 , and 60 minutes. The liquefaction experiment was conducted in a microautoclave reactor of $50 \mathrm{ml}$ capacity. After work-up, the oil fraction containing most of the 1-methylnaphthalene was subjected to GC-mass spectrometry. Small-scale, careful distillation of the oil fraction gave 1-methylnaphthalene (greater than $98 \%$ purity) for H-NMR analysis.

The deuterium content of methylnaphthalene increased with increase in reaction time and temperature (Table 1).

Table 1

Deuterogenation-Coal Liquefaction of a Western Kentucky \#9 Coal in the Presence of Methylnaphthalene

\begin{tabular}{|c|c|c|c|c|c|c|c|}
\hline \multirow[t]{2}{*}{$\begin{array}{l}\text { Run } \\
\text { Number } \\
\end{array}$} & \multirow[t]{2}{*}{$\begin{array}{l}\text { Time } \\
(\min ) \\
\end{array}$} & \multirow[t]{2}{*}{$\begin{array}{l}\text { Temperature } \\
\left({ }^{\circ} \mathrm{C}\right) \\
\end{array}$} & \multicolumn{3}{|c|}{$\begin{array}{l}\text { Deuterium Content of } \\
\text { Methylnaphthalene }\end{array}$} & \multirow[b]{2}{*}{$\underline{\mathrm{D}}_{3}$} & \multirow[b]{2}{*}{$\underline{D}_{A}$} \\
\hline & & & $\underline{\mathrm{D}}_{0}$ & $\underline{D_{1}}$ & $\underline{D}_{2}$ & & \\
\hline 1 & 15 & 385 & 84.3 & 14.5 & 1.2 & 0 & 0 \\
\hline 2 & 30 & 385 & 72.1 & 22.0 & 5.9 & 0 & 0 \\
\hline 3 & 60 & 385 & 62.7 & 30.0 & 7.3 & 0 & 0 \\
\hline 4 & 30 & 450 & 25.7 & 37.0 & 24.3 & 10.0 & 3. \\
\hline
\end{tabular}




\section{Table 2}

Deuterogenation - Coal Liquefaction of Western Kentucky \#9 Pyro in the Presence of 1-Methylnaphthalene in a 50cc Reactor at 800 psi $^{\AA}$ and Various Reaction Temperatures

\begin{tabular}{|c|c|c|c|c|c|}
\hline \multirow[t]{2}{*}{$\begin{array}{l}\text { Reaction } \\
\text { Time (min) }\end{array}$} & \multirow{2}{*}{$\begin{array}{l}\begin{array}{l}\text { Reaction } \\
\text { Temperature }\end{array} \\
{ }^{\circ} \mathrm{C}\end{array}$} & \multicolumn{4}{|c|}{$\begin{array}{l}\text { Deuterium Content (\%) } \\
\text { of } 1 \text {-Methylnaphthalene }\end{array}$} \\
\hline & & $\underline{D}_{0}$ & $\underline{D}_{1}$ & $\underline{D}_{2}$ & $\underline{D}_{3}$ \\
\hline $\begin{array}{l}50 \\
60 \\
30\end{array}$ & $\begin{array}{l}300 \\
385 \\
450\end{array}$ & $\begin{array}{l}97.5 \\
69 \\
30\end{array}$ & $\begin{array}{c}2 \\
26 \\
37\end{array}$ & $\begin{array}{l}0.5 \\
5 \\
24\end{array}$ & $\begin{array}{l}0 \\
0 \\
7\end{array}$ \\
\hline
\end{tabular}

a At room temperature.

\section{Table 3}

Blank Run: Effect of Reactor Wall on the Deuterogenation of 1-Methylnaphthalene in a $50 \mathrm{cc}$ Reactor at $385^{\circ} \mathrm{C}$, $2000 \mathrm{psi}$ and Reaction Time of $1 \mathrm{hr}$.

Reactor

Walls

Freshly-

Cleaned

Once Used

Once Used

Twice Used
Deuterium Content (\%)

of 1-Methylnaphthalene

a. A lubricant containing nickel-graphite flakes was used as a sealant to prevent loss of pressure.

b. No lubricant was used.

c. Reaction temperature $300^{\circ} \mathrm{C}$.

d. Two to three hundred coal liquefaction runs were made in the reactor prior to cleaning.

The deuterium content from a blank run with methylnaphthalene in the absence of coal at $385^{\circ} \mathrm{C}$ and 30 minutes was similar to that of run 2 (Table 1 ).

The preliminary results indicate that the isotope content of methylnaphthalene increases with temperature (Table 2) and reaction time. However, it appears that the walls 
of the reactor, and especially the iubricant (nickel-graphite flakes in petroleum oil) initially utilized, catalyzes deuterium exchange to a greater extent in the absence of coal then when coal is present during the conlersions. When the same reactor was reused without cleaning, the amount of deuterium excharge increases by about 13\% (Table 3). Elimination of the nickel containing lubricant lowers the deuterium exchange by about $10 \%$.

The deuterium NMR spectra of the 1-methylnaphthalene from the run at $450^{\circ} \mathrm{C}$ and one-half hour, with or without coal present, show that the ring contains $78 \%$ deuterium (nearly statistically distributed) and methyl contains $22 \%$ deuterium.

At $300^{\circ} \mathrm{C}$ and one hour, essentially all of the deuterium (equally distributed) is in the rings.

A reactor similar to the one used in the above experiments with a glass liner was utilized in order to decrease the reactor wall contact surface. A typical run with 1methylnaphthalene at $385^{\circ} \mathrm{C}, 1$ hour, and $2000 \mathrm{psi}$ of deuterium with the above reactor reduced the deuterium exchanged to about $3 \%$ (37\% without the glass liner).

The deuterium NMR of 1-methylnaphthalene from the run at $450^{\circ} \mathrm{C}$ and one-half hour, with or without coal present, show that the ring contains $78 \%$ deuterium and methyl contains $22 \%$ deuterium. A more careful deuterium NMR analysis with a larger sample indicated that deuterium distribution as follows: $38 \%$ at 4 position, 38\% equally distributed at the 5 and 8 position, $10 \%$ at the 2 position and 14\% equally distributed at 3,6 and 8 positions (Figure 1).

At $385^{\circ} \mathrm{C}$ and one hour, the deuterium distribution changed somewhat, that is within our experimental error of 5\%. The amount of deuterium exchange at 3, 4, 6 and 7 was the same as that of $450^{\circ} \mathrm{C}$ run, but there was $43.5 \%$ deuterium equally distributed at 2,5 and 8 positions (Figure 2).

A typical deuterium NMR (Figure 3) of pyridine soluble, pentane insolubles of coal fraction indicates that $42 \%$ of deuterium is in the aromatic NMR region, $45 \%$ in aliphatic region, and $13 \%$ active hydrogen (e.g., $-\mathrm{OH},-\mathrm{NH}, \mathrm{SH}$, etc.).

The selective deuteration of 1-methylnaphthalene at $2,4,5$ and 8 positions ( $85.5 \%)$ is a good indication of a catalytic mechanism rather than a free radical pathway. In a free radical mechanism one would expect near statistical distribution of deuterium in all positions, especially favoring the methyl group rather than the rir.s. At $385^{\circ} \mathrm{C}$ less than $2 \%$ exchange took place in the methyl group of the methylnaphthalene. At $45 n^{\circ} \mathrm{C}, 22 \%$ of the methyl group of the 1-methylnaphthalene was deuterated; however, the deuterium 
distribution in the ring (85.5\%) was similar to the $385^{\circ} \mathrm{C}$ experiment (see above) that favors $2,4,5$ and 8 positions of the ring.

The deuterium NMR of pyridine soluble, pentane insolubles fraction of the coal (at $450^{\circ} \mathrm{C}, 30 \mathrm{~min}$., $2000 \mathrm{psi}_{2}$ ) in the presence of 1-methylnaphthalene shows near equal distribution of deuterium in the aromatic and aliphatic region of NMR. This indicates that the aliphatic region of coal exchanges more than the aromatic region of the coal which is opposite to that of 1-methylnaphthalene. One explanation is that the coal molecule underwent more free radical exchange or that most of the aliphatic region of coal is not primary proton similar to 1-methylnaphthalene.

A $75 \mathrm{ml}$ stirred autoclave reactor with a glass liner was operated at $425^{\circ} \mathrm{C}$; deuterium exchange was $23 \%$ with more than half of the 1-methylnaphthaiene condensed outside of the glass liner. In this reactor, the deuterium exchange was about $62 \%$, similar to the microautoclave type reactor (MATR) without the glass liner (Table 4). A deuterium NMR spectra (Figure 4) of 1-methylnaphthalene shows $28 \%$ deuterium exchange at methyl group (only $2 \%$ for MATR) and increase exchange at 5 and 8 position with subsequent decrease at 4 position. The amount of exchange at 2, 3,6 and 7 was similar to MATR.

A number of model compounds were deuterated in similar experiments as reported for 1-methylnaphthalene. The GC mass spectra data indicate an increase in the amount deuterium exchange with an increase in the electron-donating ability of the group at 1 position (see Table 5). Deuterium NMR of ethyl naphthalene indicates nearly equal distribution of deuterium in the ring and no measurable amount of deuterium in the uniform ethyl group (Figure 5). This also indicates that a free radical reaction is not involved; however, it is not clear why ethylnaphthalene deuteration of the ring is not selective as was the case with 1-methylnaphthalene (see Figures 1 and 2).

Deuteration of phenathrene was somewhat more selective than ethylnaphthalene (see Figure 6). The relative $\% \mathrm{D}$ at the ring positions are: $1,8(21 \%) ; 4,5(15 \%) ; 9,10$ (35\%); and $2,3,6,7(28 \%)$.

When methoxynaphthalene was deuterated at $385^{\circ} \mathrm{C}, 1$ hour, 800 psi (cold) $\mathrm{D}_{2}$ more than $59 \%$ of the ring hyrogen and none of the methoxy hydrogen exchanged with deuterium. Deuterium NMR showed (Figure 7) showed 70\% deuterium at the 4 position and $30 \%$ at the 2 position. This is another good indication that an ortho-para directing effect is operating. Deuterium could not be detected at other ring positions or in the methyl group. 


\section{Coal Conversion with Carbon Monoxide and Water}

The reaction of carbon-14 carbon monoxide $-\mathrm{H}_{2} \mathrm{O}$ with Western Kentucky \#9 coal was carried out using a gas pressure of $800 \mathrm{psi}$ (cold). The preliminary results indicate that the conversion to pyridine solubles is in the range of $85-90 \%$ as was reported by Bianco et al. ${ }^{1}$ Analysis of the gas products shows carbon-14 labeled carbon dioxide was formed. Methane and ethane did not show any radioactivity. Three coal fractions (e.g., pyridine insolubles (PI), pyridine solubles/pentane insolubles (PSPI), and pentane solubles (PS)) were converted to carbon dioxide by means of Van Slyke-foch oxidation which was converted to barium carbonate. None of the coal fractions showed detectable carbon-14 activity. This is a good indication that carbon monoxide does not incorporate into any of the fractions during coal liquefaction by $\mathrm{CO}-\mathrm{H}_{2} \mathrm{O}$-base system.

Table 4

Deuteration of 1-Methylnaphthalene at $2000 \mathrm{psi} \mathrm{D}_{2}, 385^{\circ} \mathrm{C}, 1 \mathrm{Hr}$ Reaction Time

$\underline{\text { Reaction Type }}$

Used Reactor

Coal

Used Reactor

No Coal

Used Reactor

Ni Graphite

Flakes

New Reactor,

Glass Liner

Stirred
Reactor With
Glass Linear

at $425^{\circ} \mathrm{C}$

$\mathrm{CH}_{3} \mathrm{CO}_{2} \mathrm{D}$, - $100^{\circ} \mathrm{C}, 24 \mathrm{hr}$.
$\underline{\mathrm{D}_{0}}$

$\underline{\mathrm{D}_{1}}$

$\underline{\mathrm{D}_{2}}$

$\underline{D}_{3}$

$\underline{D}_{4} \quad \underline{D}_{5}$ 69 25

58

49

92.0

7.0

1

0

0

0

Condensed

Inside Glass

Condensed

Outside Glass

27.0

32

23

11

5

2

38.0

35

17

8.5

2.5

1

31

44

21

3.5

.5

0

1 A. Del Bianco, E. Girardi, F. Stroppa, Envideerche International Conference on Coal Sienle 1989 and references found thereafter. 


\section{Table 5}

Deuteration of Model Compounds at $385^{\circ} \mathrm{C}, \mathrm{D}_{2} 2000 \mathrm{psi}, 1 \mathrm{Hr}$.

Model

Compounds

Naphthalene*

1-Methyl-

naphthalene ${ }^{a}$

1-Ethyl-

naphthalene

1-Phenyl-

naphthalene ${ }^{*}$

1-Methoxy-

naphthalene

1-Hydroxy-

naphthalene ${ }^{a}$

Phenanthrene ${ }^{b}$

Pyrene

\section{Deuterium Content (\%)}

$\begin{array}{llllll}\underline{D}_{0} & \underline{D}_{1} & \underline{D}_{2} & \underline{D}_{3} & \underline{D}_{4} & \underline{D}_{5}\end{array}$

$\begin{array}{llllll}78 & 19.5 & 2.5 & 0 & 0 & 0\end{array}$

$\begin{array}{llllll}58 & 28 & 6 & 1 & 0 & 0\end{array}$

82

$\begin{array}{lllll}14.5 & 2.5 & 0.7 & 0.3 & 0\end{array}$

$\begin{array}{llllll}85.0 & 13.0 & 1.5 & 0.5 & 0 & 0\end{array}$

$\begin{array}{llllll}51 & 38 & 9.0 & 1.0 & 0 & 0\end{array}$

35.5

42

$\begin{array}{lll}17.5 & 5.0 & 0\end{array}$

0

$\begin{array}{llllll}3.4 & 13.2 & 26.4 & 26 & 17 & 9.3 \\ 22 & 40 & 22 & 11 & 3 & 2\end{array}$

Reaction time, a. 30 minutes, b. 2 hr. 


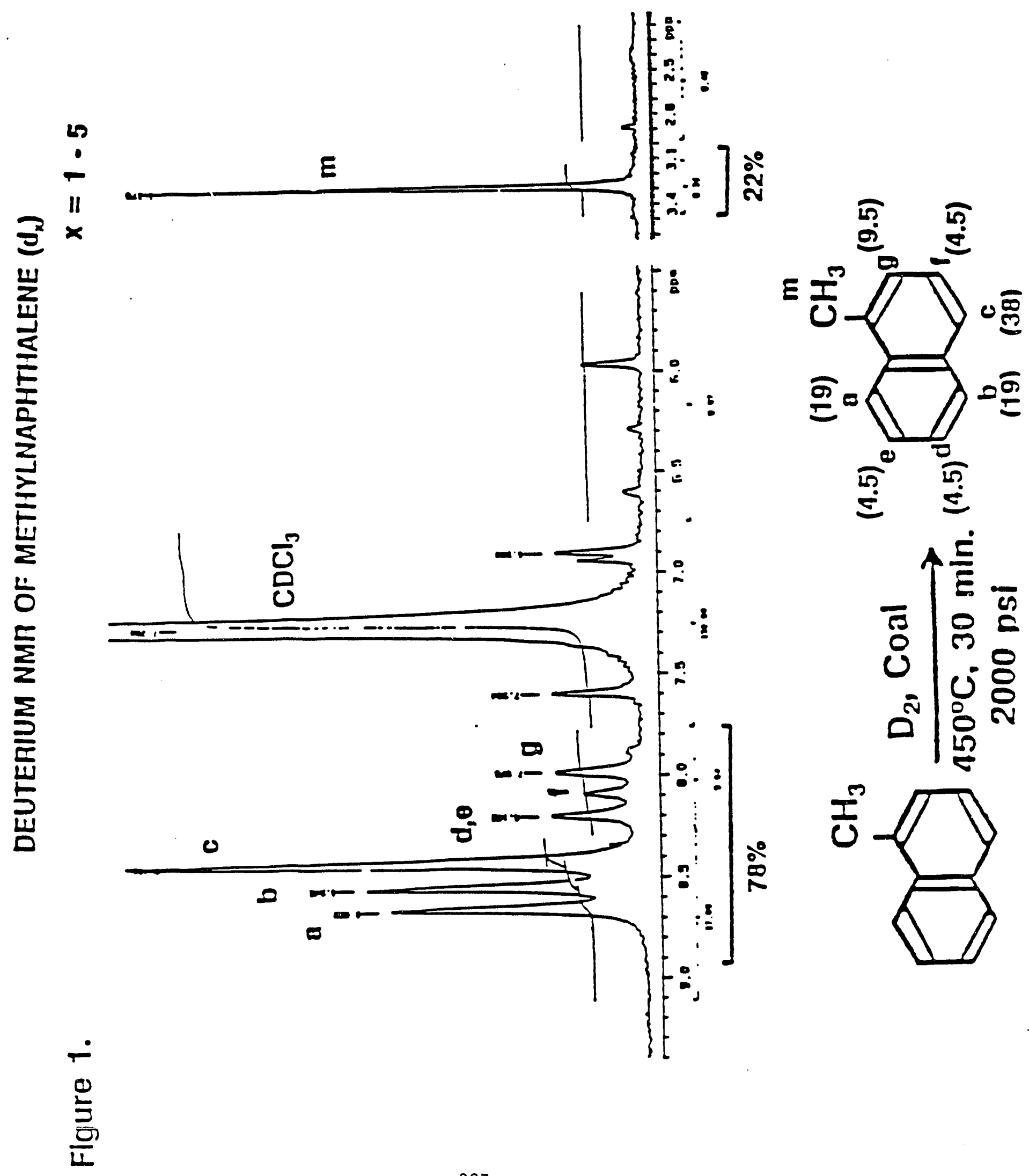



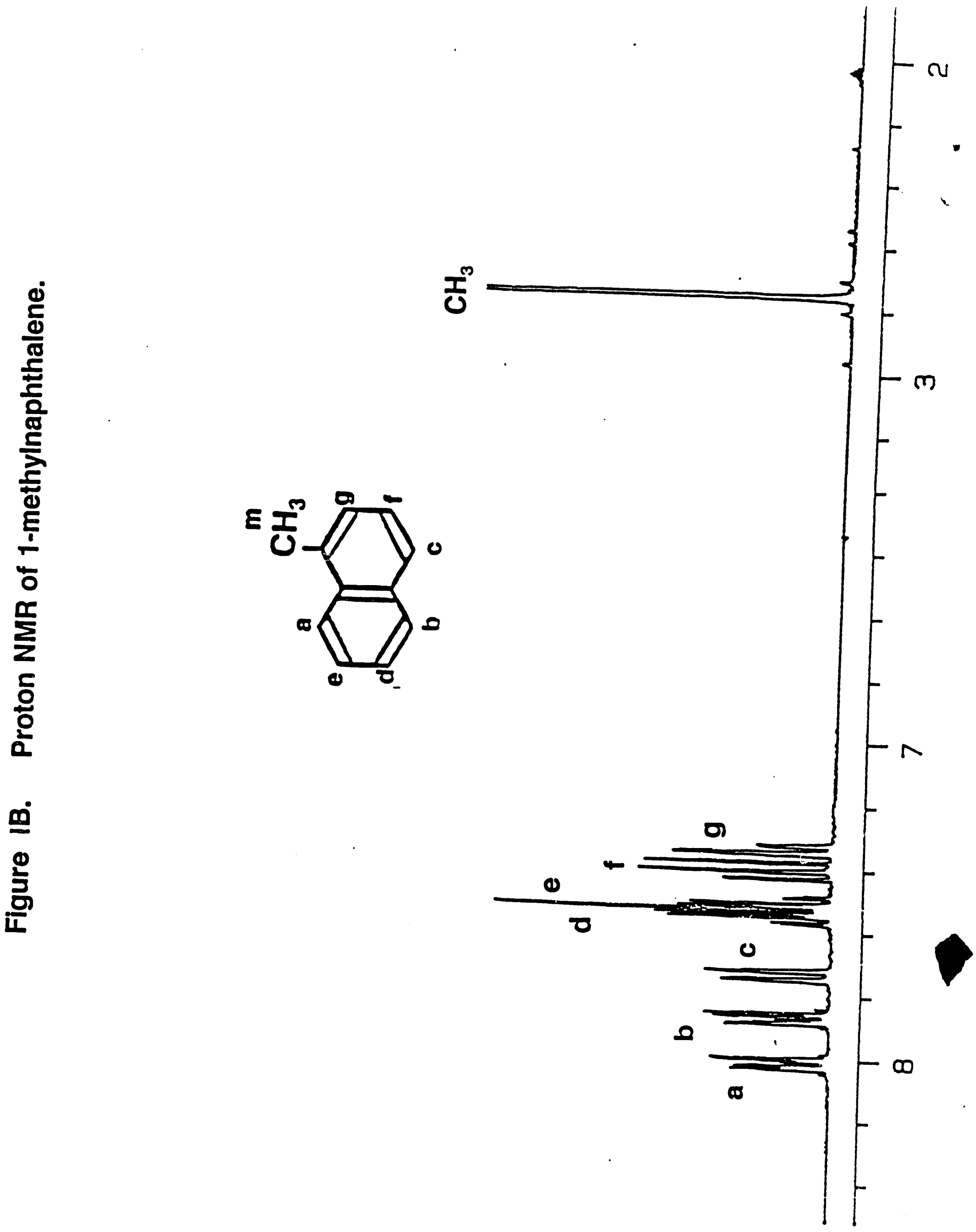

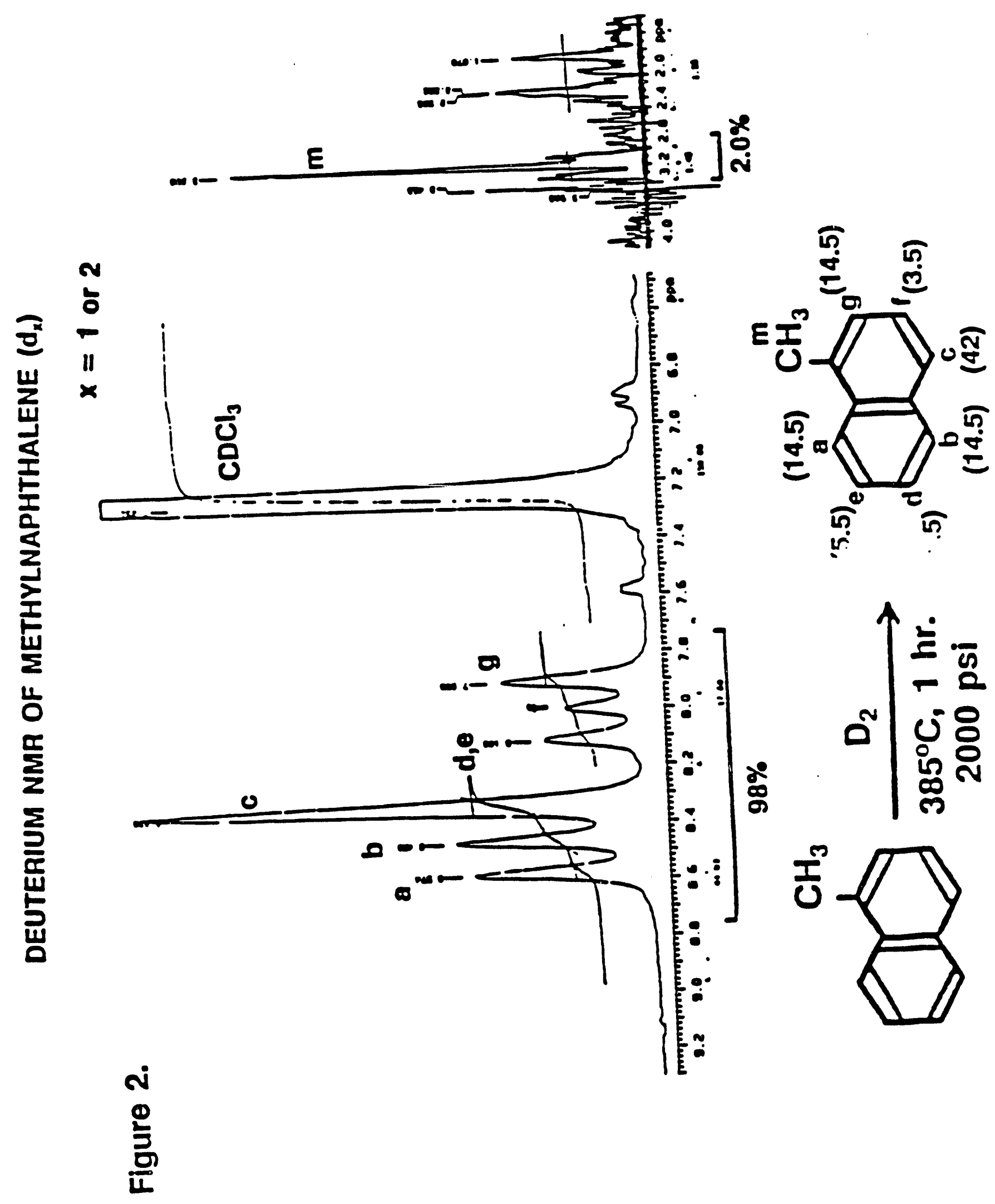


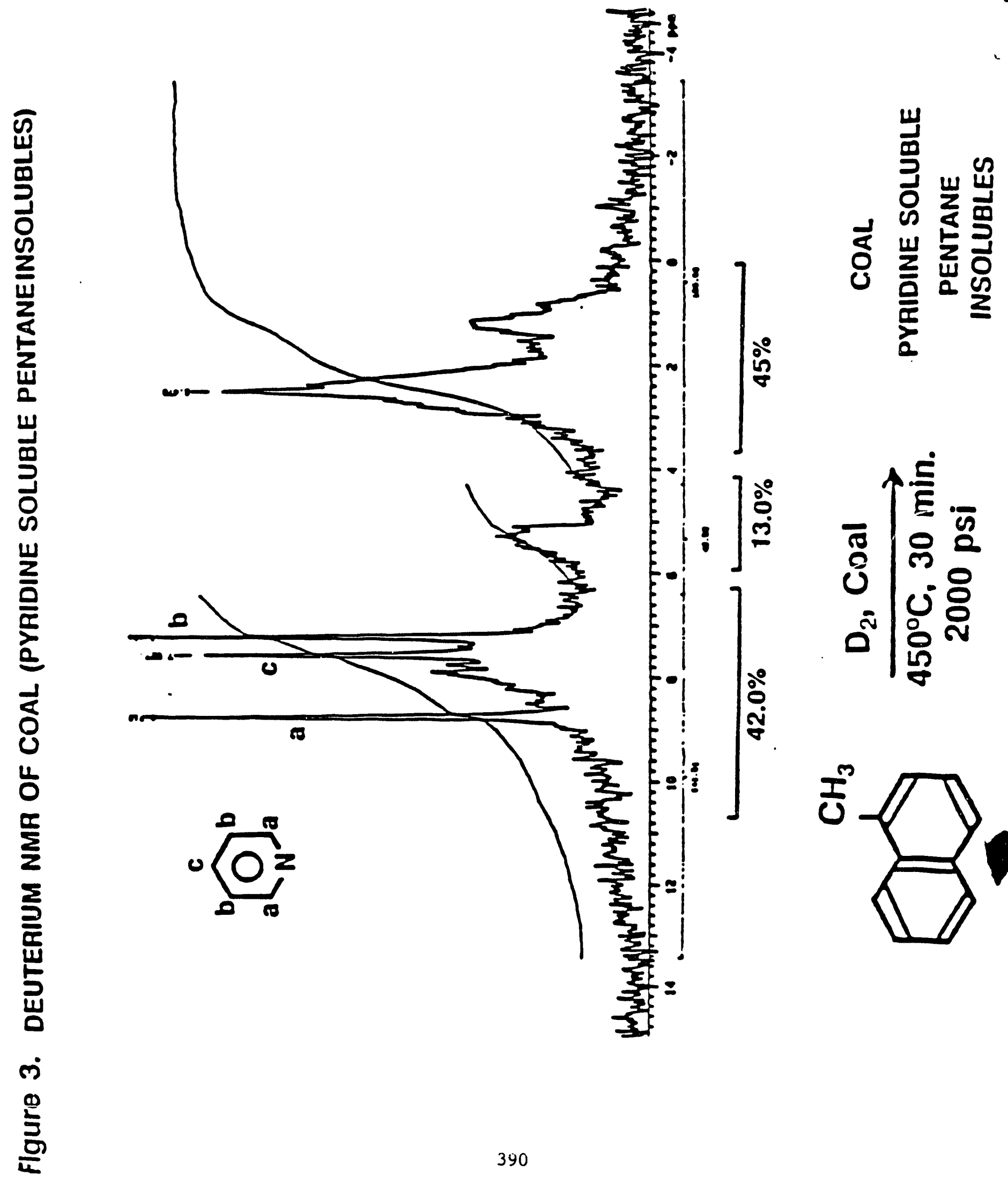




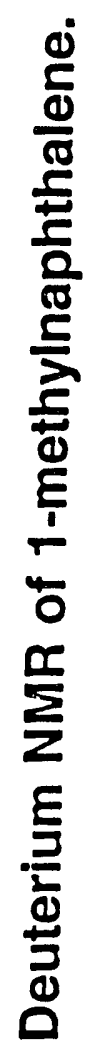

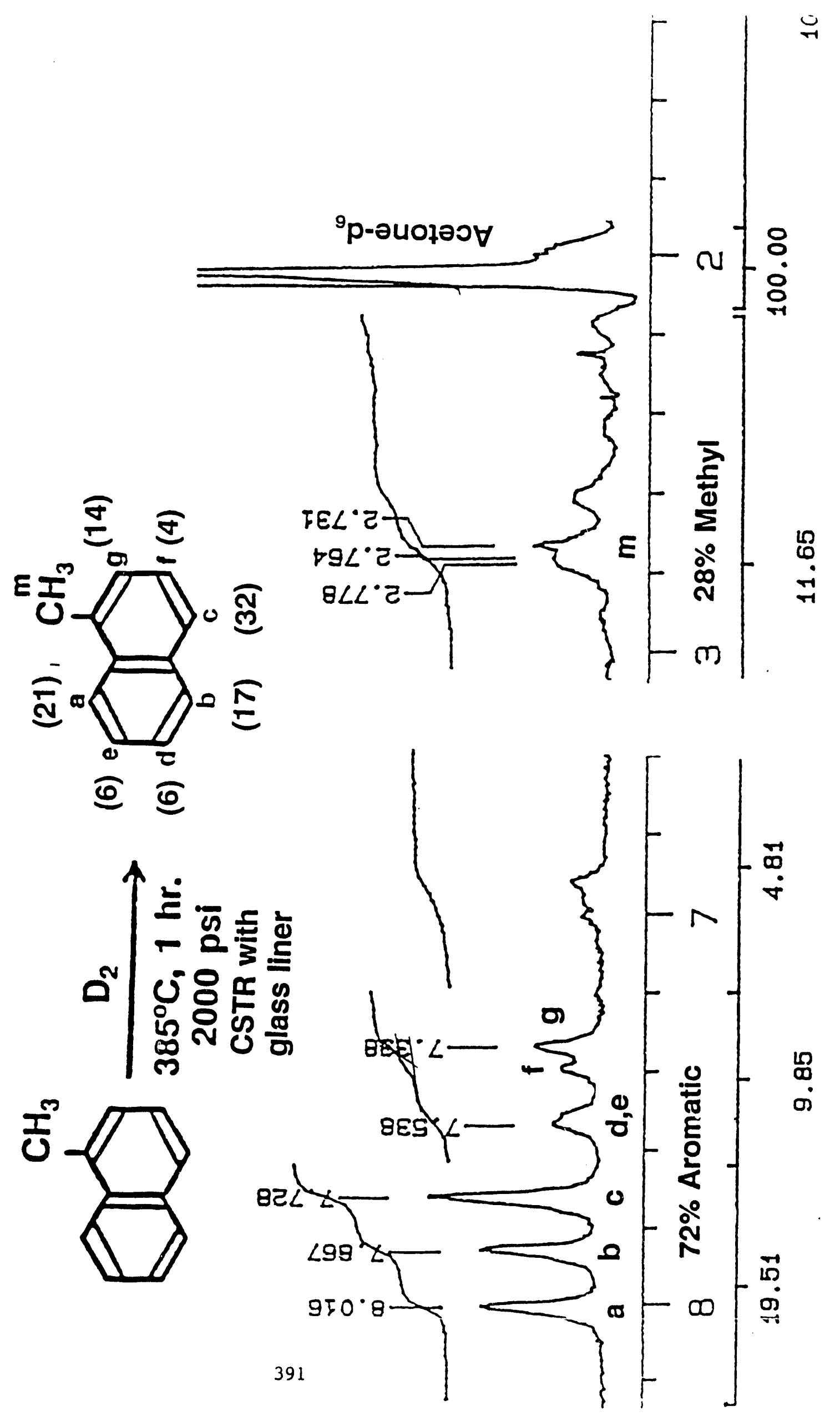




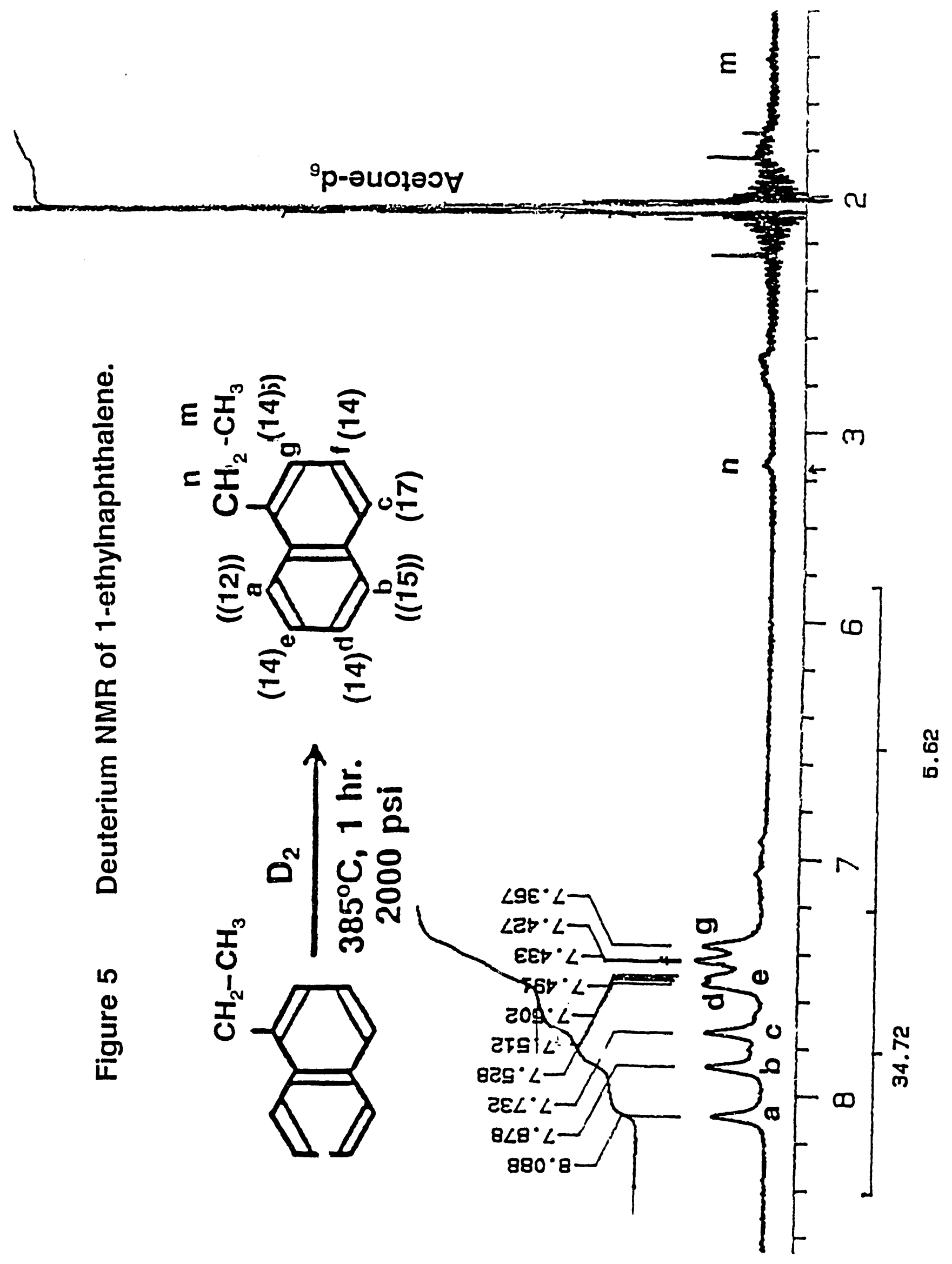



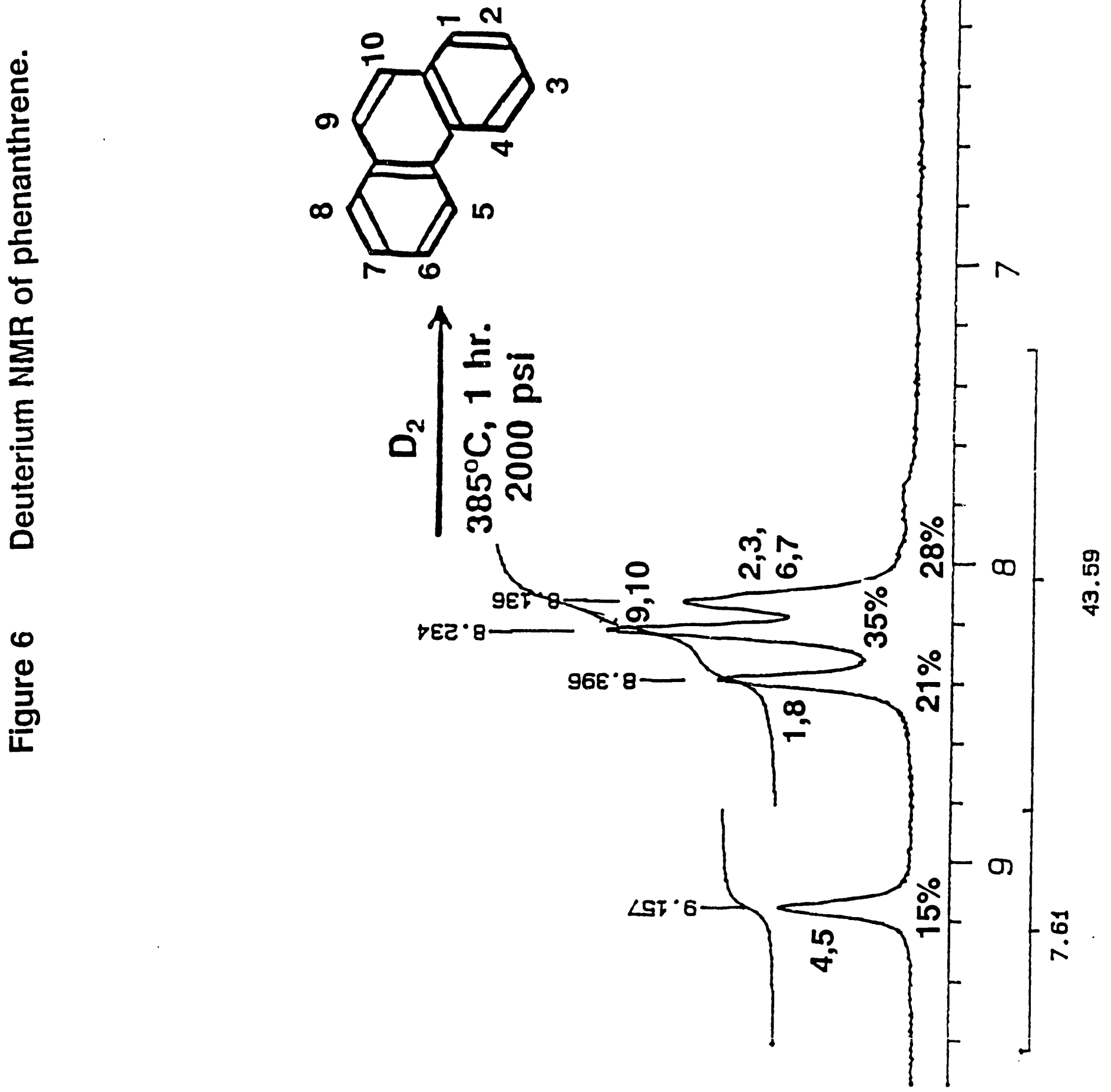

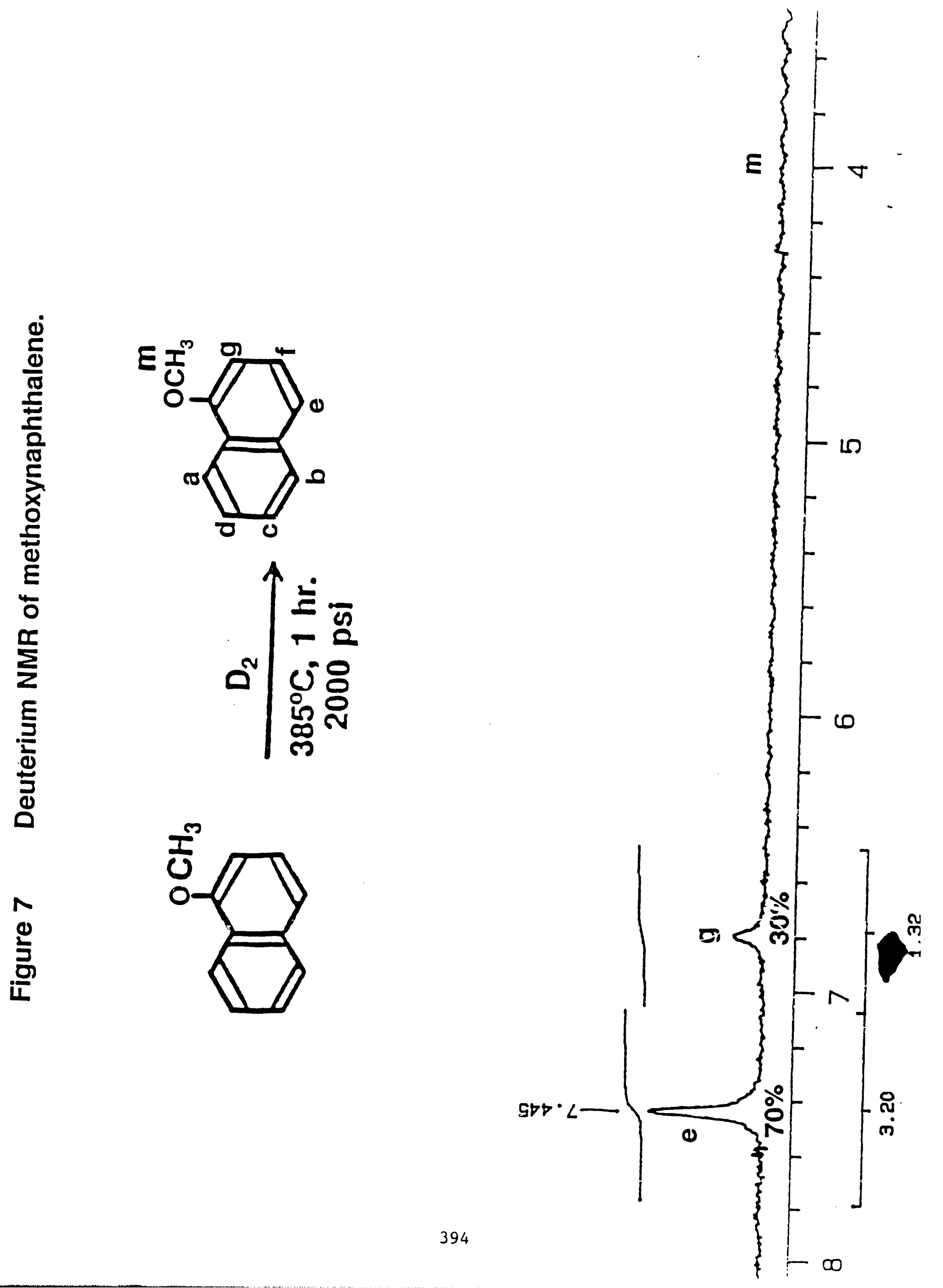


\section{Task VI.4. Liquefaction of U.S. Coals}

\section{Objectives}

VI.4.1. Collect and characterize approximately 60 bench, channel and/or run of mine coal samples from Illinois, Ohio, West Virginia and/or Indiana.

VI.4.2. Obtain liquefaction conversion data for the coals using the microautoclave reactors.

VI.4.3. Obtain a detailed characterization of the liquefaction products for selected samples.

VI.4.4. Continue establishing the relationship between coal properties and liquefaction responses.

VI.4.5. Construct and commission a CSTR liquefaction reactor.

\section{Introduction}

A systematic evaluation of the relationships between the basic characteristics of coals and their subsequent behavior during liquefaction using various process conditions have been carried out at the Center for Applied Energy Research. This year the program includes coals from Ohio, Mlinois, Indiana, W. Virginia and feedstock samples from the Wilsonville ITSL pilot plant facilities. Ultimately, this effort will provide an industrial client sufficient information to optimize feedstock selection for a number of processes.

\section{V1.4.1. Collection and Characterization of Project Coals}

During the contract year, 61 coal samples were obtained from Ohio, Dlinois, Indiana, W. Virginia and from the Wilsonville ITSL pilot plant for characterization. In addition, samples from the Argonne Premium Coal Data Bank were obtained from Professor Huffman for liquefaction studies. Samples, when possible, were collected at the working face of the mine. A channel sample was collected at each site and, when possible, the seam was benched based on visual differences. The samples were expedited in the preparation process to avoid oxidation. The samples were ground to the appropriaie mesh sizes for analysis and liquefaction studies. The initial splits $\left(1 / 4^{\prime \prime}\right)$ for liquefaction were stored under argon prior to final preparation ( -100 mesh) for liquefaction.

The chemical and petrographic analyses for all of the project coals are given in Appendix 1. A summary of selected coal property averages and ranges are giren in Table 1. 
The analyses indicate a large variation in the petrographic and chemical compositions of the project coals. Several unique coals were identified on the basis of the distribution of the forms of sulfur. One example of an unusualiy high sulfur coal was obtained in a bench sample of the Upper Freeport coal (Ohio). This coal contained $5.9 \mathrm{wt. \%}$ (daf) organic sulfur and $8.44 \mathrm{wt} . \%$ (daf) pyritic sulfur. The bench sample also contained 20 vol.\% (dmmf) liptinites which may account for the high organic sulfur content. Another example of a coal with unique distributions of the forms of sulfur was identified in several benches of the Indiana $\mathrm{V}$ seam. In these samples, the pyritic sulfur contents were relatively low (ca. . 5-1. wt.\%, maf) when compared to the organic sulfur content (ca. 2 to 4 wt.\%, maf). A number of these coals were provided to CFFLS members for their research activities.

\section{V1.4.2. and 4.3. Liquefaction anit Characterization}

All of the project and Argonne coals were liquetied in the microautoclave reactors using a temperature of $385^{\circ} \mathrm{C}$, a 15 minute residence time, a hydrogen atmosphere ( $800 \mathrm{psig}$, ambient), a solvent/coal ratio of 1.5 and tetralin as the solvent vehicle. In addition, the channels and other selected samples were studied using reaction temperatures of $427^{\circ} \mathrm{C}$ and $445^{\circ} \mathrm{C}$.

The solubility class distribution of the liquefaction products obtained using the $385^{\circ} \mathrm{C}$ reaction temperature are shown in Figure 1. The data are consistent with previously studied high volatile bituminous coals in that these coals have the same thermal pathway at this temperature. As the conversion increases there is a linear increase in the asphaltene plus preasphaltene yield. The oil plus gas yields remain fairly constant over the range of conversions obtained by these coals. The coals produce the largest range of conversion (30$95 \mathrm{wt} . \%$, maf) using this reaction temperature.

The distribution of liquefaction products of the channel samples obtained using the $427^{\circ} \mathrm{C}$ reaction temperature as shown in Figure 2. The data indicate that approximately one-half of the channel samples are still undergoing the primary liquefaction reactions. That is, conversion of the coal to produce asphaltenes plus preasphaltenes and a small increase in the oil plus gas yields. The remaining channels are undergoing secondary reactions at this temperature. The secondary reaction region of the liquefaction pathway defined by these coals is the region in which the asphaltenes plus preasphaltenes are converted to oll plus gas while the total coal conversion remains constant or slightly increases. 
At the reaction temperature of $445^{\circ} \mathrm{C}$, all of the channel samples, with two exceptions, are in the secondary reaction region of the pathway (Figure 3). An Upper Freeport coal sample remains in the primary liquefaction reaction region of the pathway using these conditions and is therefore shown to be very unreactive. The second exception is another sample of the Upper Freeport seam. This sample appears to readily undergo retrogressive reactions using the highest thermal severity $\left(445^{\circ} \mathrm{C}\right)$ in this study. The conversion of this coal sample decreases when the reaction temperature is increased from $427^{\circ} \mathrm{C}$ to $445^{\circ} \mathrm{C}$. Parallel to the decrease in conversion is a decrease in both the asphaltene plus preasphaltene and the oil plus gas yields.

The liquefaction data obtained for these coals have followed the same thermal conversion pathway defined by the Kentucky high volatile bituminous coals previously studied. In the initial section of the thermal pathway the primary liquefaction products are asphaltenes plus preasphaltenes $(A+P)$. As conversion increases in this region, there is a parallel, linear increase in A+P yields while the oil plus gas yield remains fairly constant. The switch from the primary liquefaction region to the secondary reaction region is characterized by a maximum in the $\mathrm{A}+\mathrm{P}$ yield and conversion. In the secondary reaction region of the thermal pathway, the major reactions taking place are the conversion of asphaltenes plus preasphaltenes to oils plus gases. In this region, there is little, if any, increase in the total conversion of the coals.

The Argonne Premium Coals have been liquefied using a $385^{\circ} \mathrm{C}$ reactor temperature, a 15 minute residence time, tetralin solvent and a hydrogen atmosphere ( $800 \mathrm{psig}$, ambient). Liquefaction experiments were run in duplicate when sufficient sample was available. The solubility class distributions of the liquefaction products of these coals are shown in Figure 4. As expected from the previous liquefaction data, the premium coals show a wide range of conversions ( $59 \%$ to $14 \%$, daf coal). The Illinois \#6 and the Blind Canyon high volatile bituminous coal seams exhibited the highest conversions and the low volatile bituminous Pocahontas \#3 coal showed the lowest conversion. The relationship between the solubility classes and conversions of the high, medium and low volatile premium coals are similar to those previously reported. As the conversion increases, there is a direct livear increase in the asphaltene plus preasphaltene yields and the oil plus gas yields remain relatively constant. The lignite (Beula-Zap) and subbituminous coals (Wyodak) appear to be outliers from the line determined by the bituminous coals and suggests that rank may be a factor in defining the thermal liquefaction pathway of coals. 


\section{VI.4.4. Data Correlation}

The liquefaction data obtained for the Argonne Premium coals were correlated with both the standard coal properties and the structure parameter data derived from NMR data reported by Professor Pugmire's research group (1).

The coal properties which appear to correlate with both coal conversion and the oil plus gas yields are the carbon coutents and $\mathrm{O} / \mathrm{C}$ ratios. The relationship between conversion and the carbon content is shown in Figure 5. As can be seen the relationship for the premium coals appears to be nonlinear. The conversion curve shows a maximum at a carbon content of approximately $77 \mathrm{wt} . \%$. The curve is similar to that reported by Whitehurst (2) for short residence times. The only outlier of this curve is the Stockton coal. The lower than expected conversion obtained is most likely due to the higher inertinite content of this sample.

The oil plus gas yields are a relative measure of the distillate yields during the conversion of coais. This liquefaction parameter also shows a relationship with the carbon content of the coal (Figure 6). As can be seen in Figure 6, the distillate yields decrease with increasing carbon content of the coals. The Stockton and Blind Canyon appear to be outliers from the curve defined by the other premium coals. Again, the reason for this appears to be related to the petrographic composition of the coals. The high inertinite content of the Stockton coal may be responsible for the lower than expected oil plus gas yield and the high liptinite content of the Blind-Canyon coal may be responsible for the higher than expected oil plus gas yields. The distillate yields also appear to correlate directly with the $\mathrm{O} / \mathrm{C}$ atomic ratio of the coal as shown in Figure 7. The higher the O/C ratio, the greater the oil plus gas yields. Again, the Stockholm coal is an outlier and the reason for this is most likely due to the inertinite concentration of the coal. There appears to be a number of correlations between the liquefaction data and the coal structure parameters derived from NMR. The oil plus gas yields show correlations between the fraction of aliphatic carbon as $\mathrm{CH}$ or $\mathrm{CH}_{2}$ (Figure 8), coal aromaticity, $\mathrm{Fa}$ (Figure 9), and the fraction of aliphatic carbon bonded to oxygen (Figure 10). The structural implications of these correlations suggest that both the $\mathrm{CH}_{2}$ and ether crosslinking groups between aromatic clusters are important parameters in defining the reactivity of coals. 


\section{VI.4.5. Construction of a CSTR Liquefaction Reactor}

A one liter CSTR autoclave reactor was successfully constructed and commissioned.

The reactor system utilizes the slurry preparation, gas feed, pumping and separator sections of the 1/8 tpd pilot plant. The new CSTR reactor has successfully been operated in thermal and catalytic coprocessing runs to date.

\section{References}

1. Solumin, M. S., Pugmire, R. J. and Grant, D. M., Energy and Fuels, $\underline{3}$ (2), 1989.

2. Whitehurst, D. D., Mitchell, T. O., and Fircasiu, M., Coal Liquefaction - The Chemistry and Technology of Thermal Processes, New York, NY, Academic Press, 1980. 


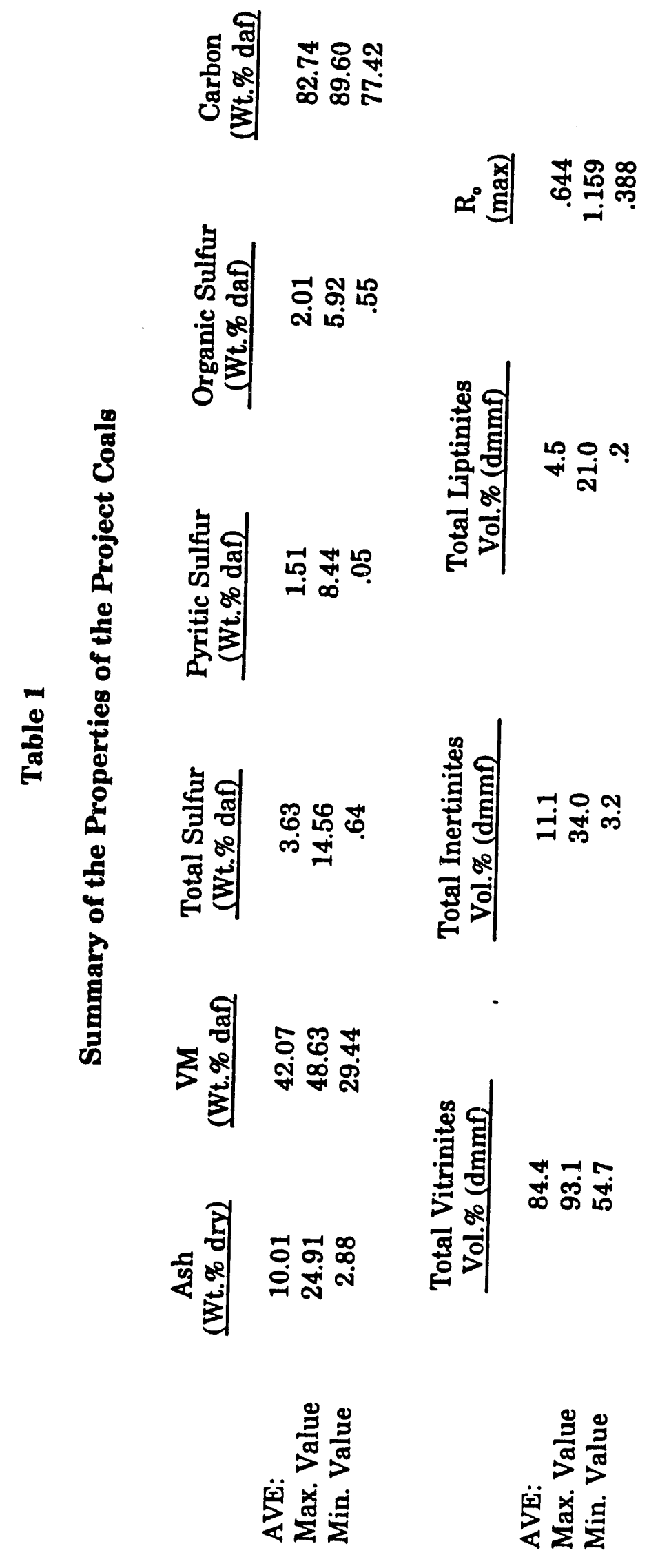




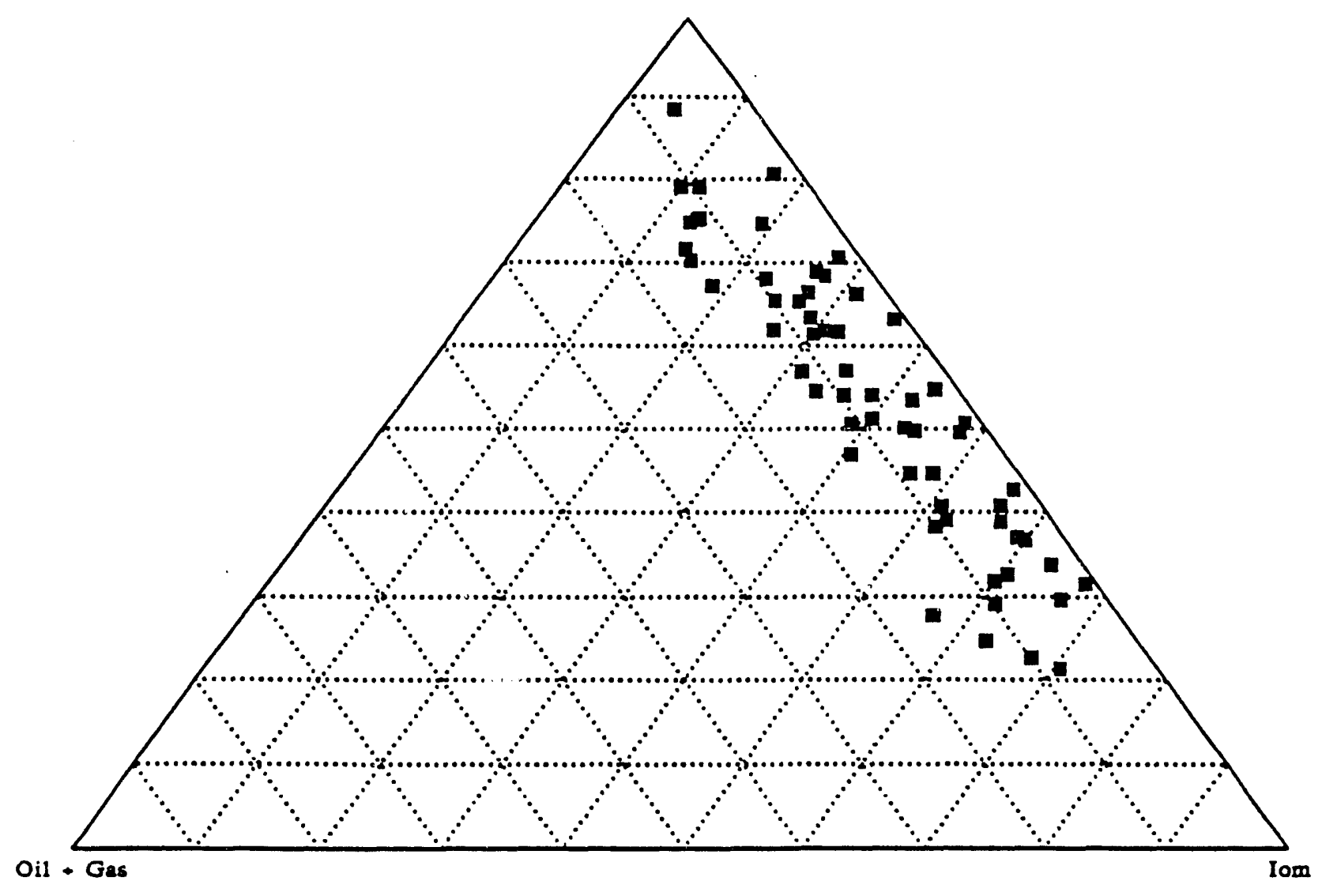

Figure 1. Liquefaction product distributions @ $385 \mathrm{C}$.

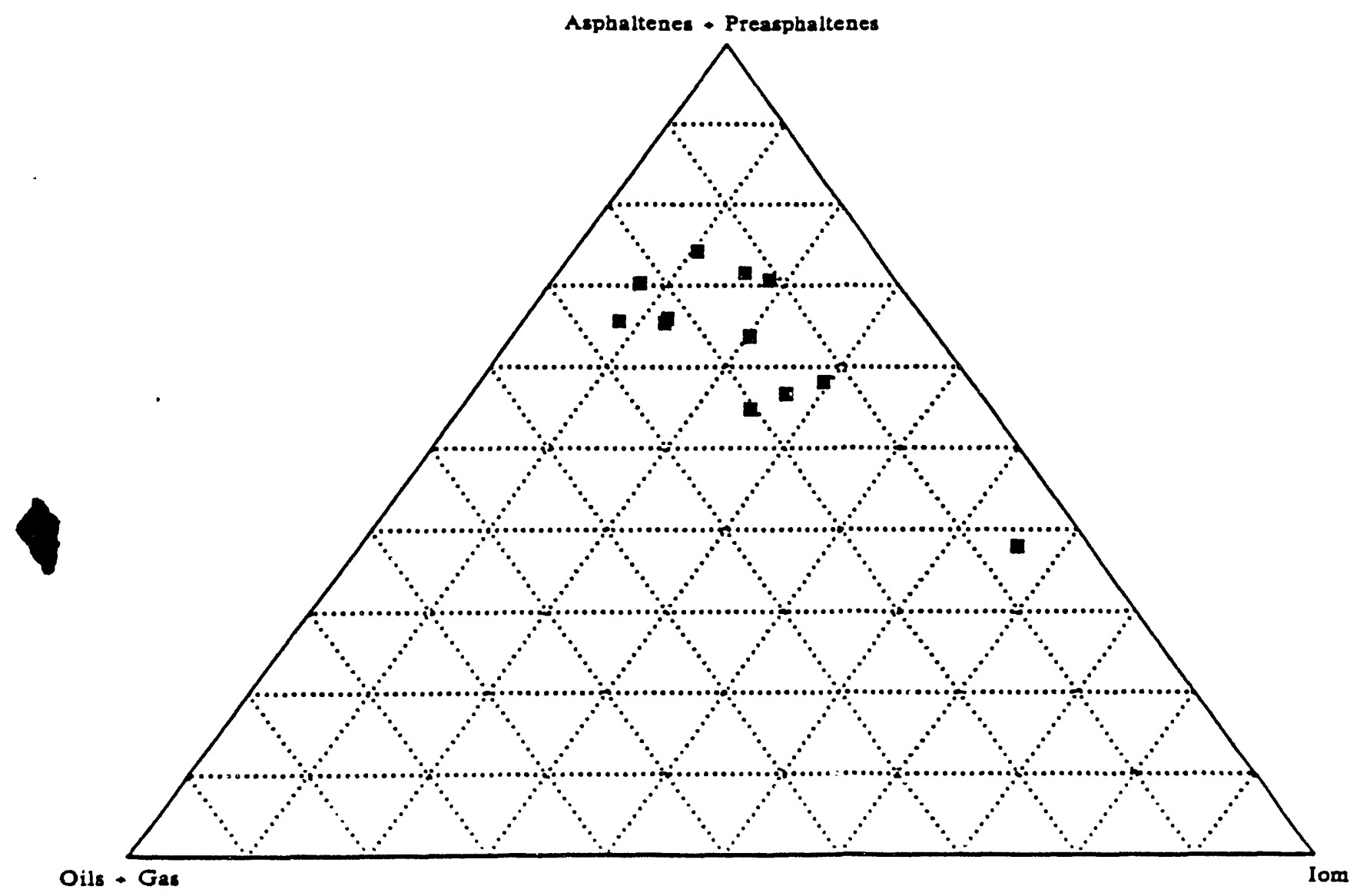

Figure 2. Liquefaction product yields @ $427 \mathrm{C}$. 


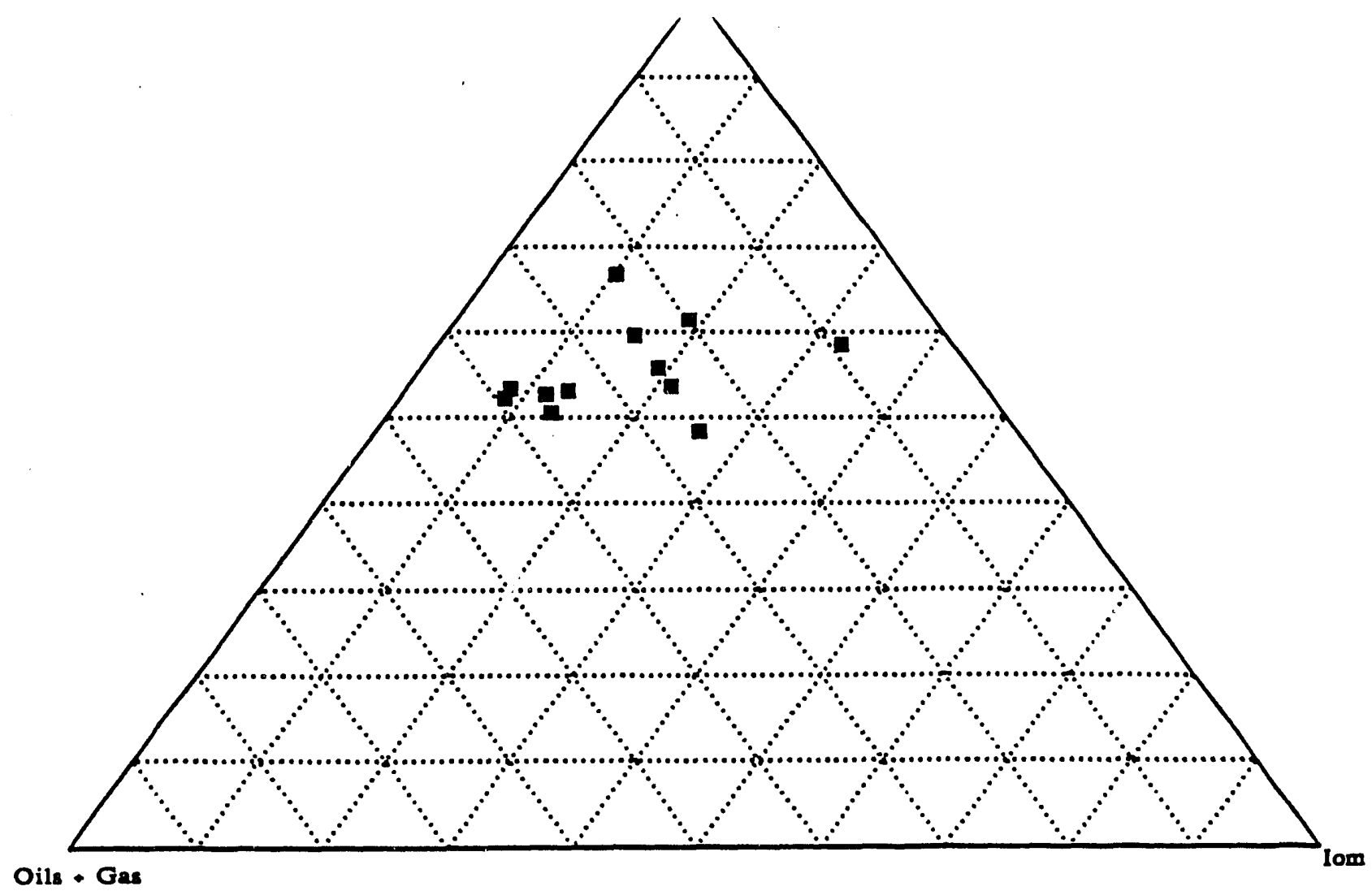

Figure 3. Liquefaction product distributions @ 445 C.

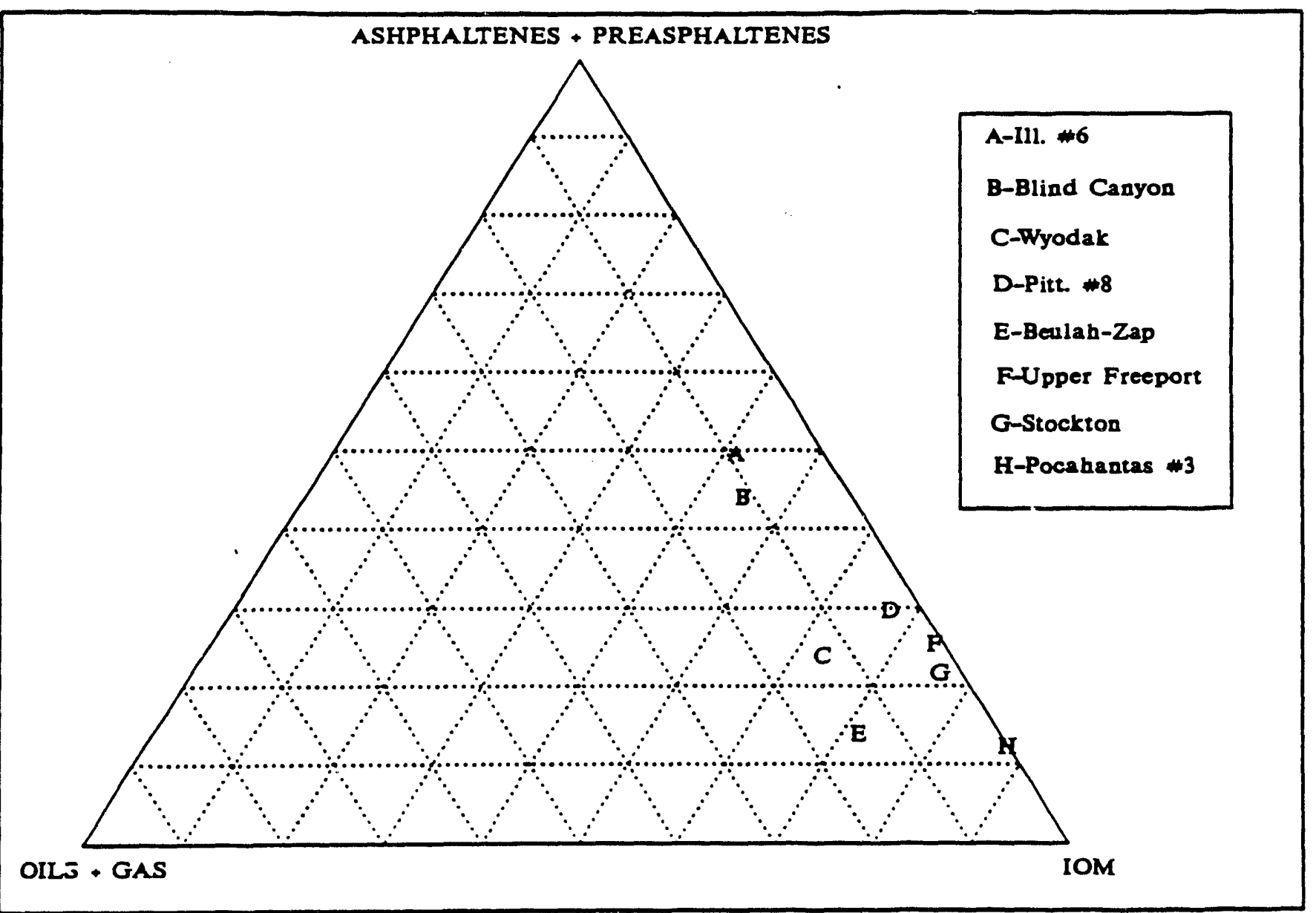

Figure 4. Liquefaction data of the Argonne Premium Coals 


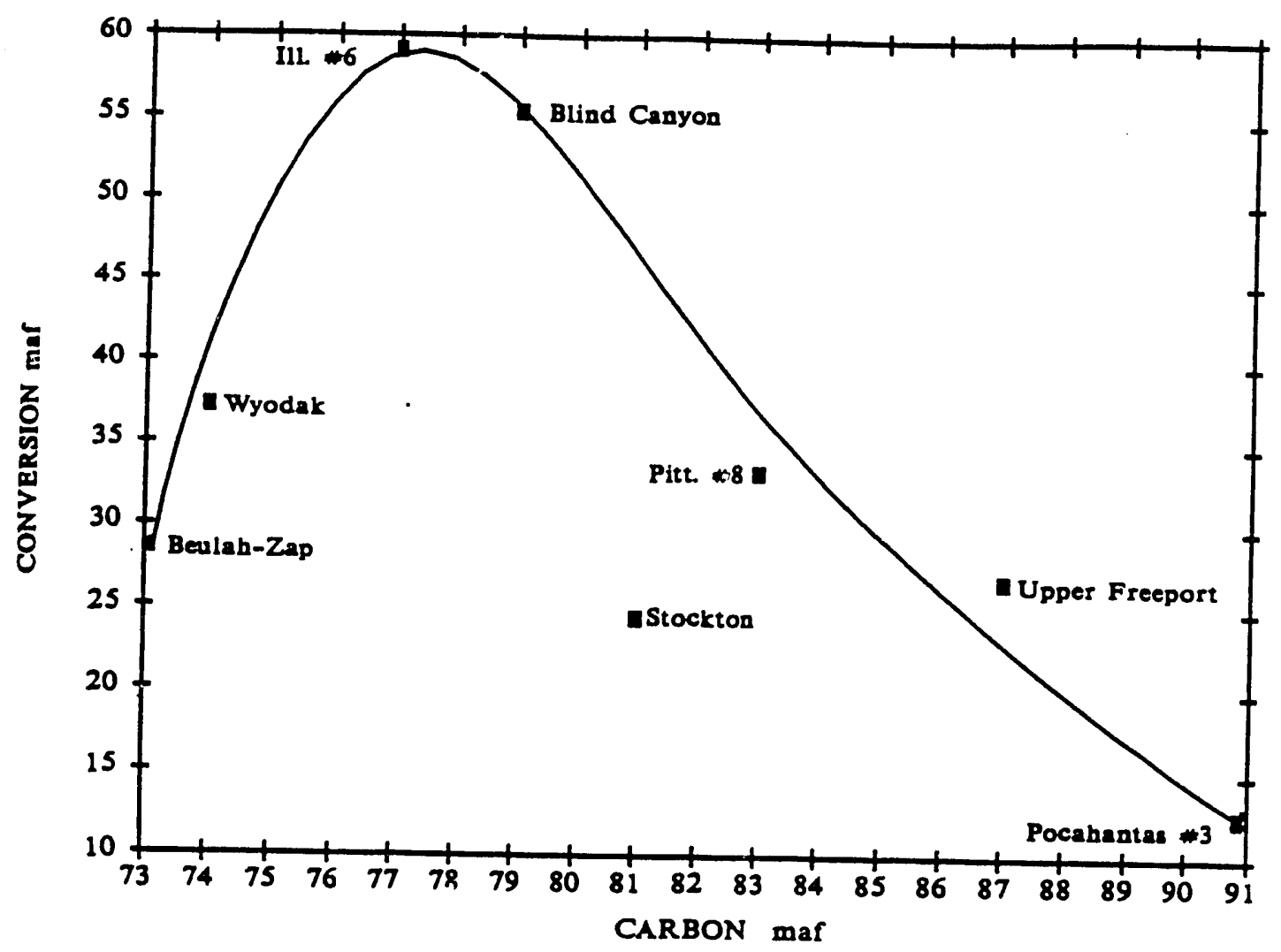

Figure 5. Variation of conversion with the carbon contents of the Argonne Premium Coals.

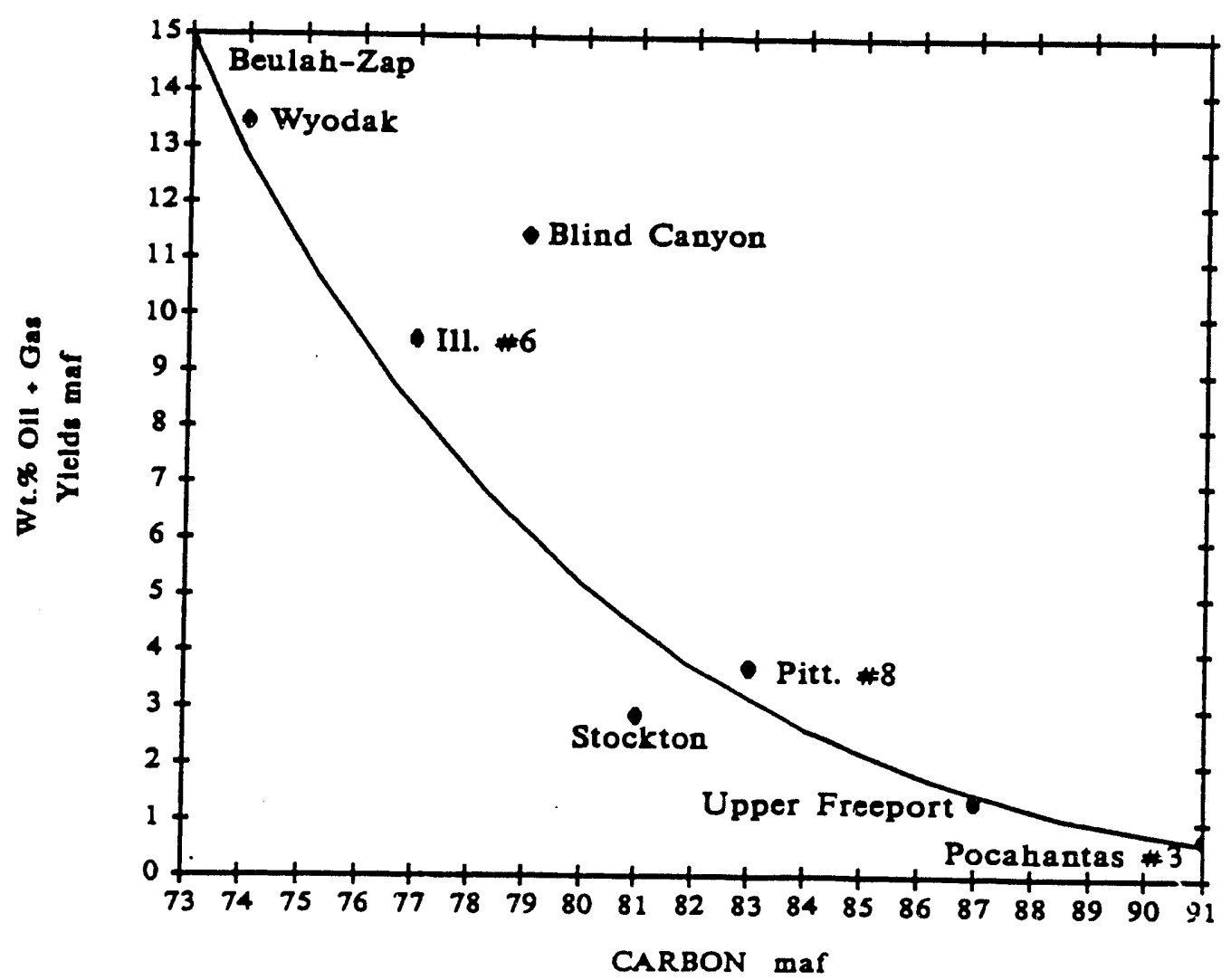

Figure 6. Variation of the oil + gas yields with the carbon contents 


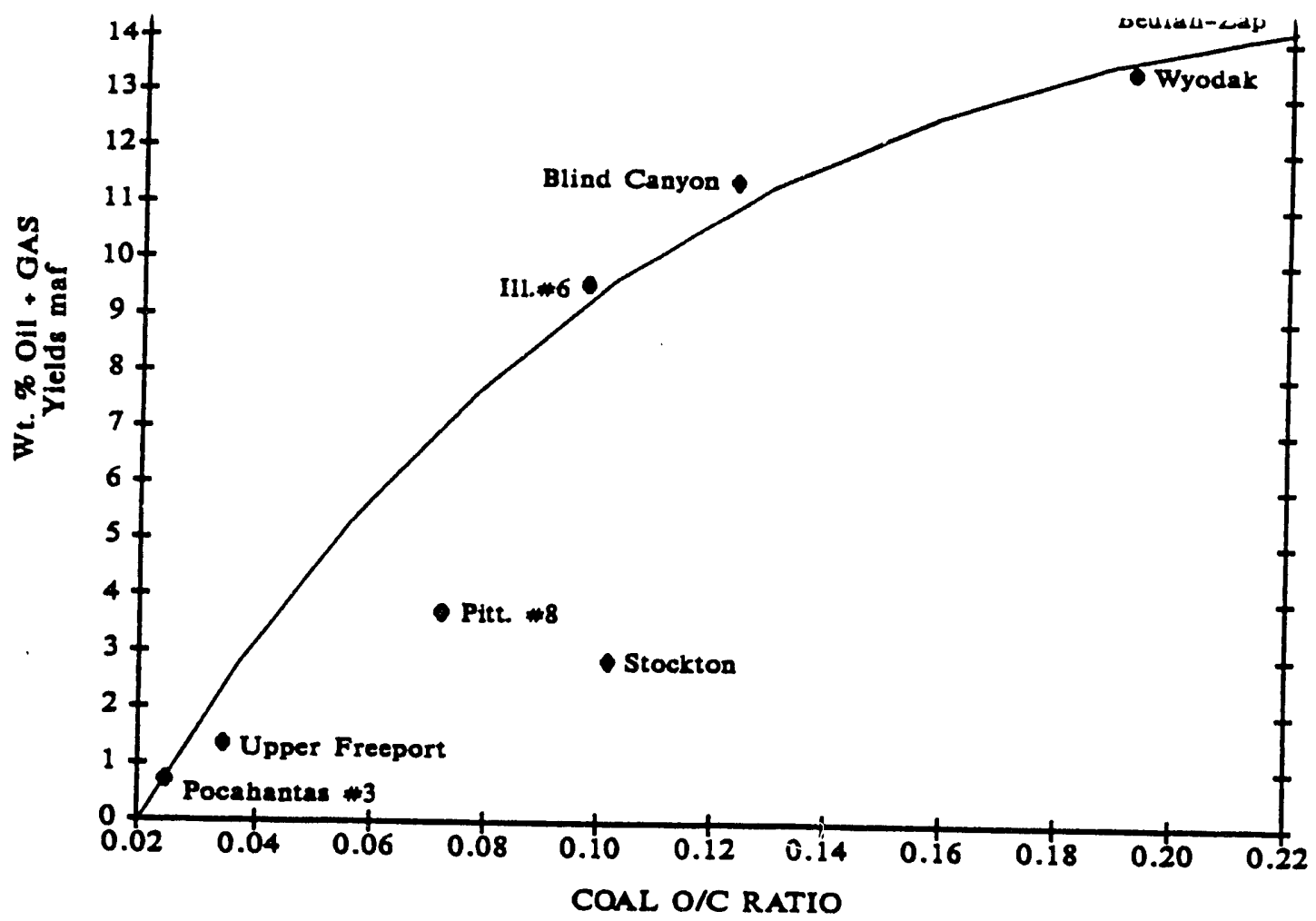

Figure 7. Variation of the oil + gas yields with the o/c ratios.

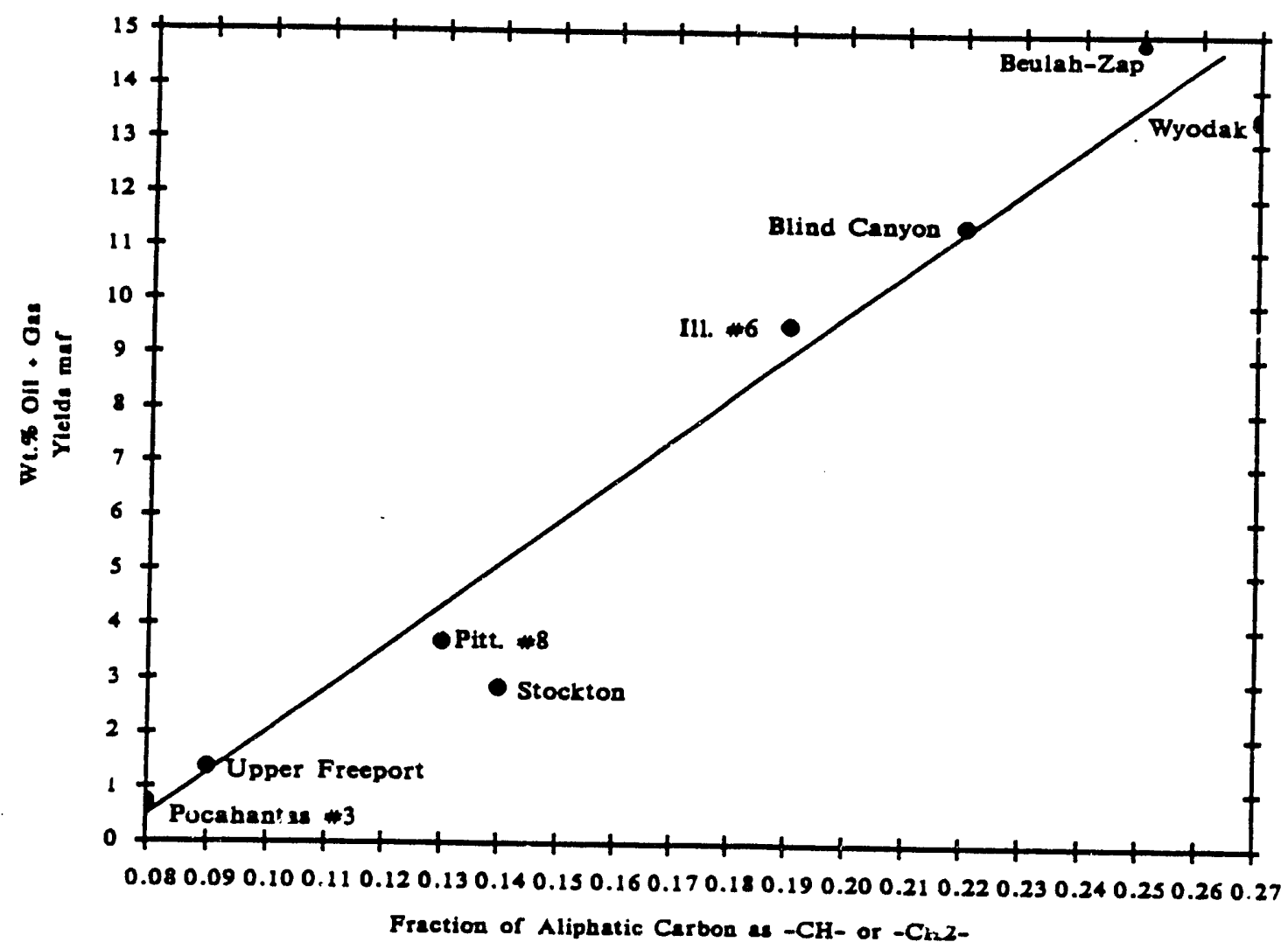

Figure 8. Variation of oil + gas yields with the fraction of aliphatic carbon as - $\mathrm{CH}-$ or - $\mathrm{CH} 2-$. 


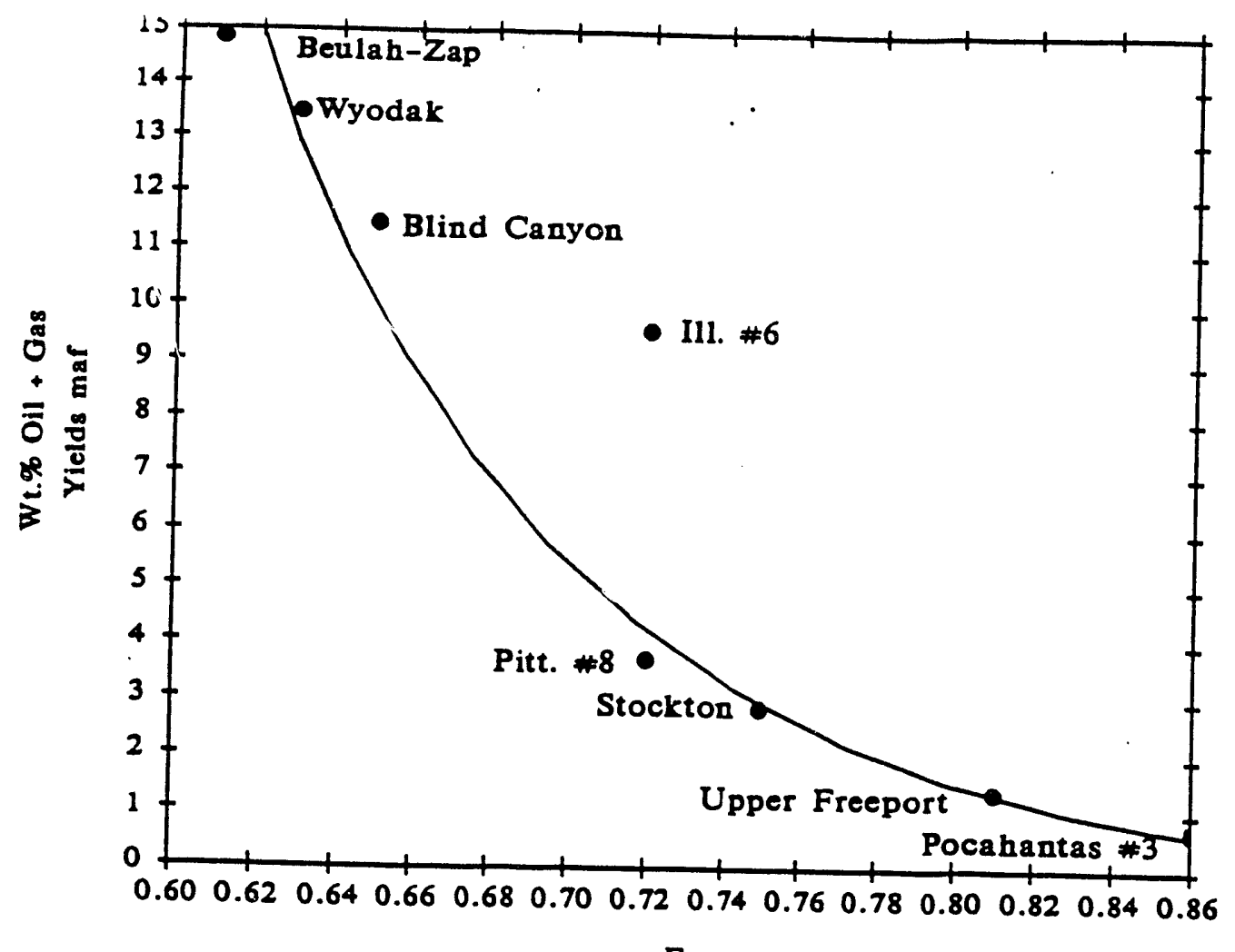

Fa

Figure 9. Variation of oil + gas yields vs the aromaticity of the Argonne Premium Coals.

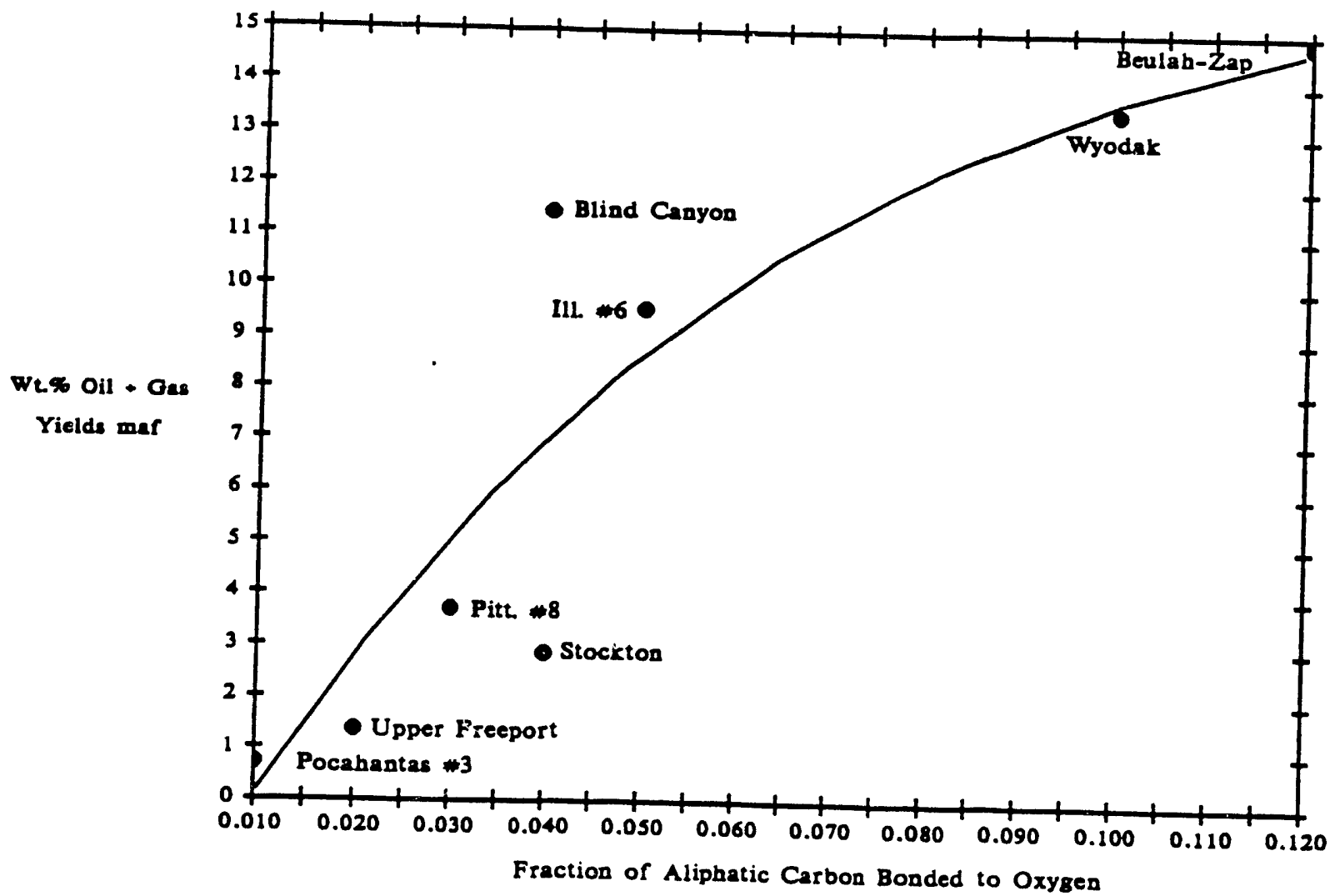

Figare 10.Variation of oil + gas yields with the fraction of aliphatic carbon bonded to oxygen 


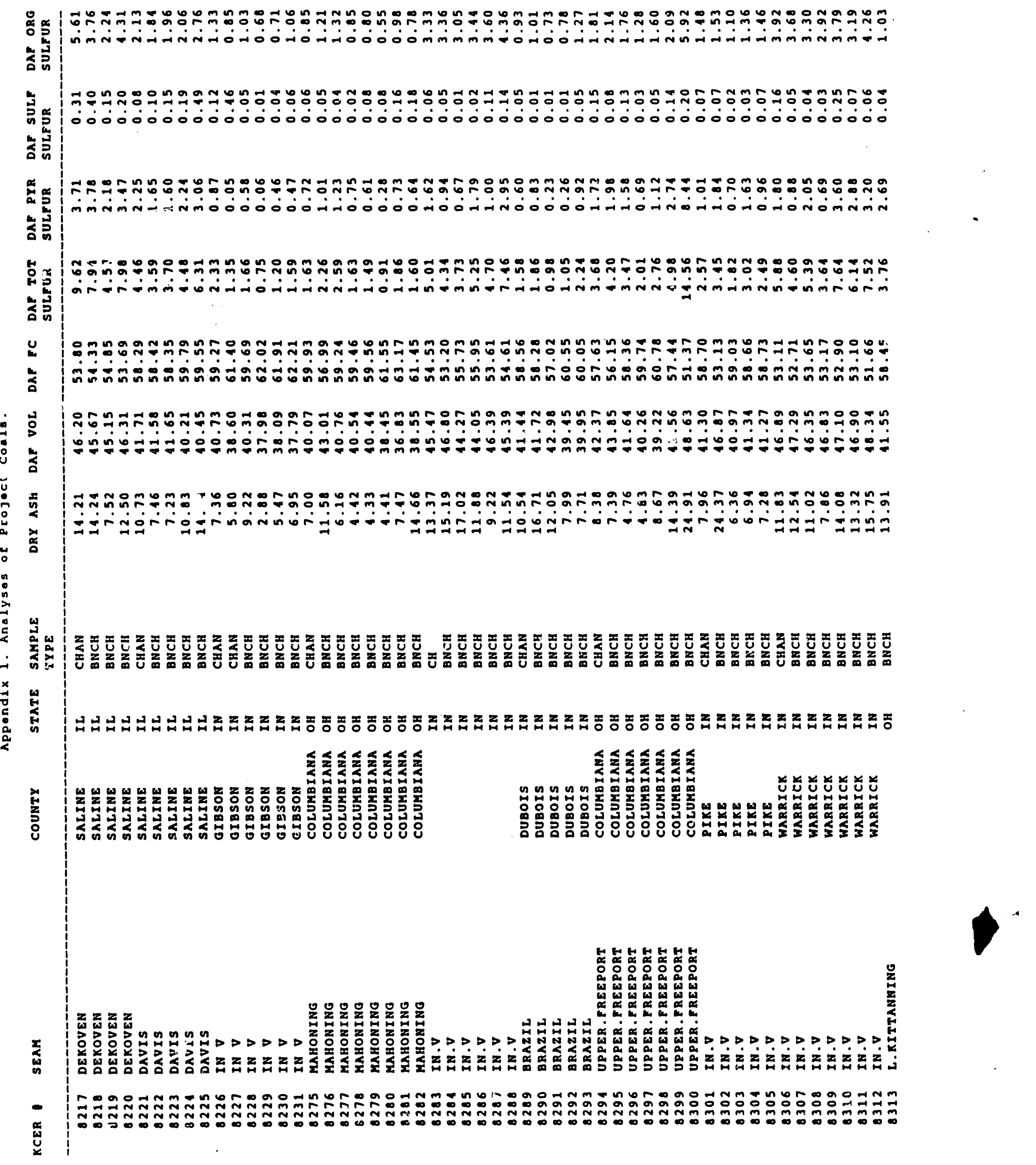




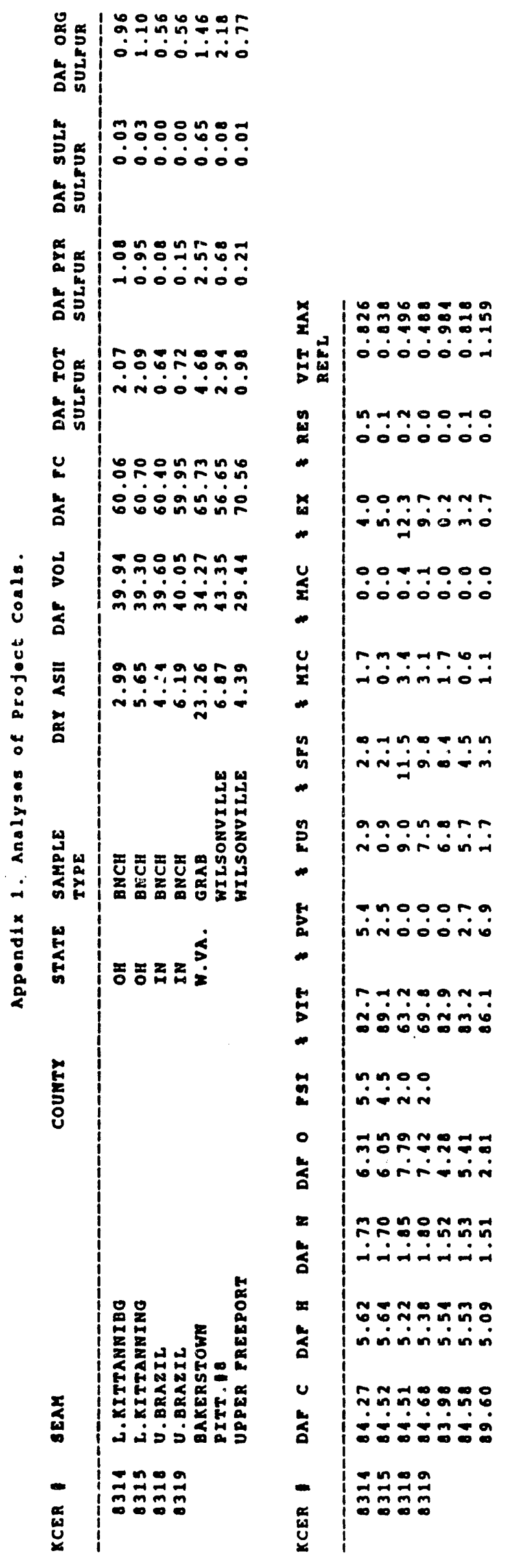




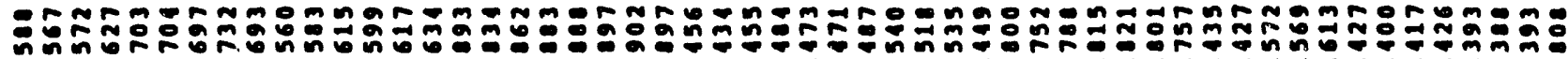

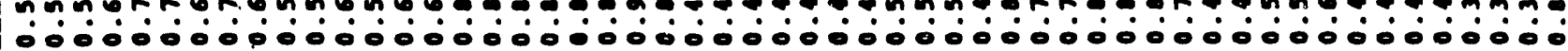

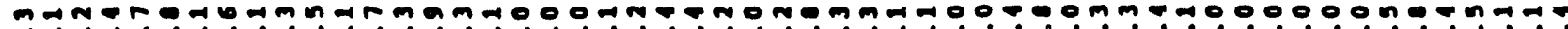

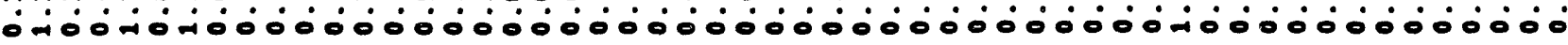

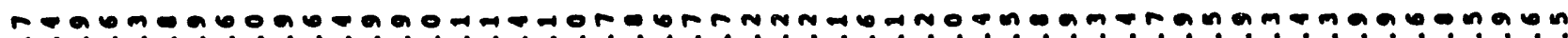

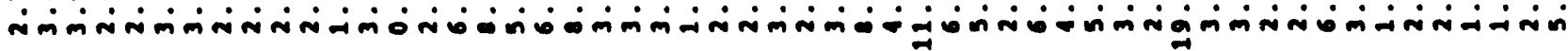

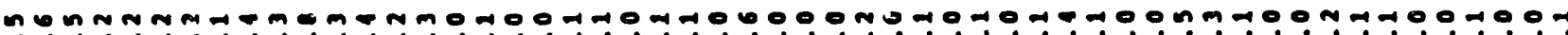
O000000000000000000000000000000000000000000000000 10

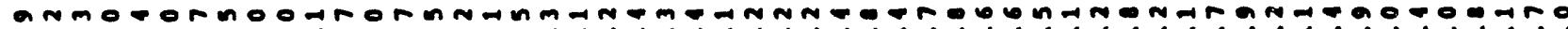
耳ं

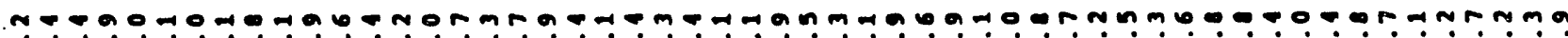

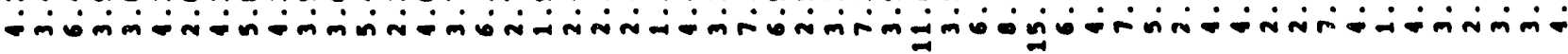

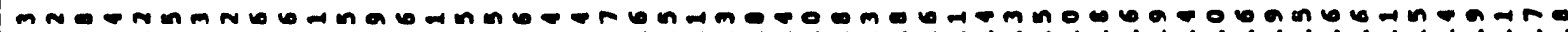

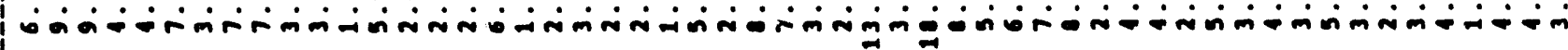

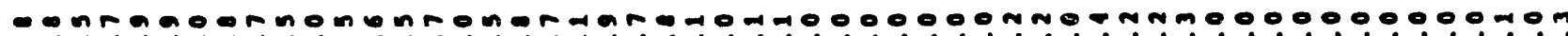
ம

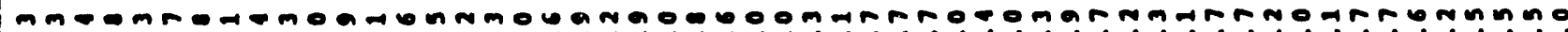

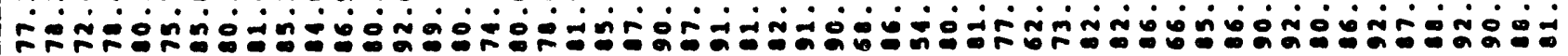

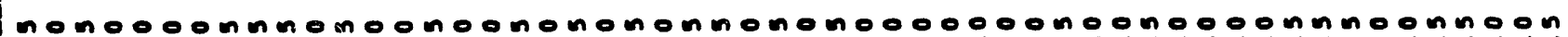

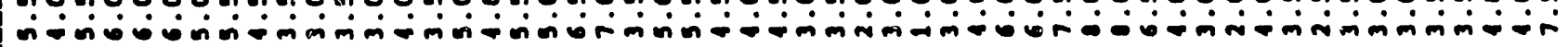

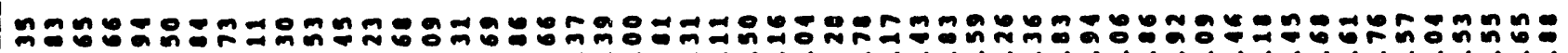

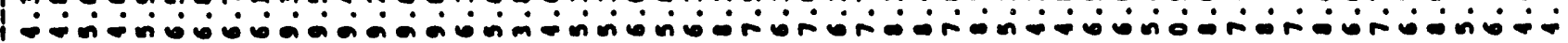

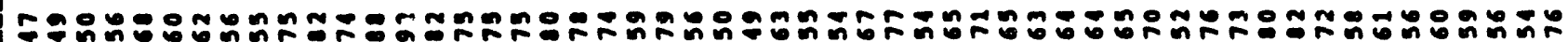

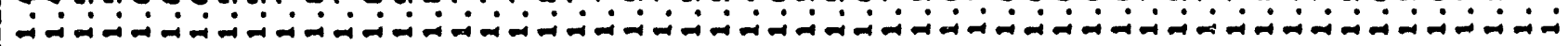

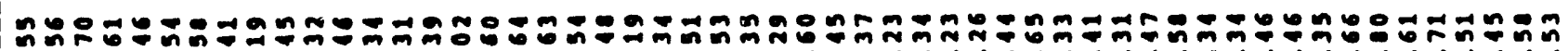

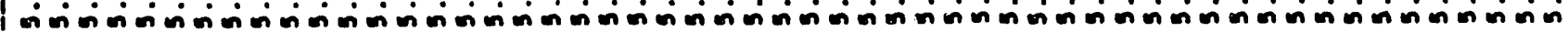

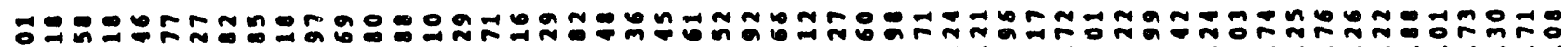

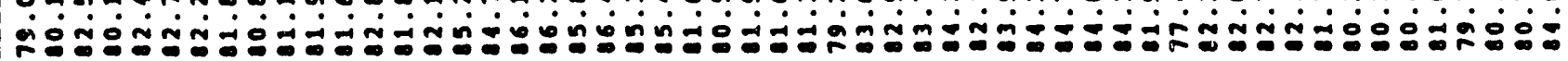

Haㅜㄴ

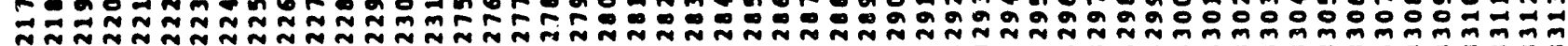

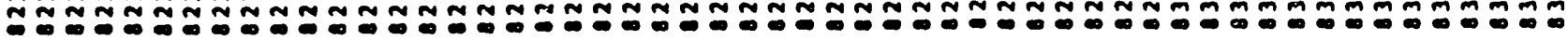



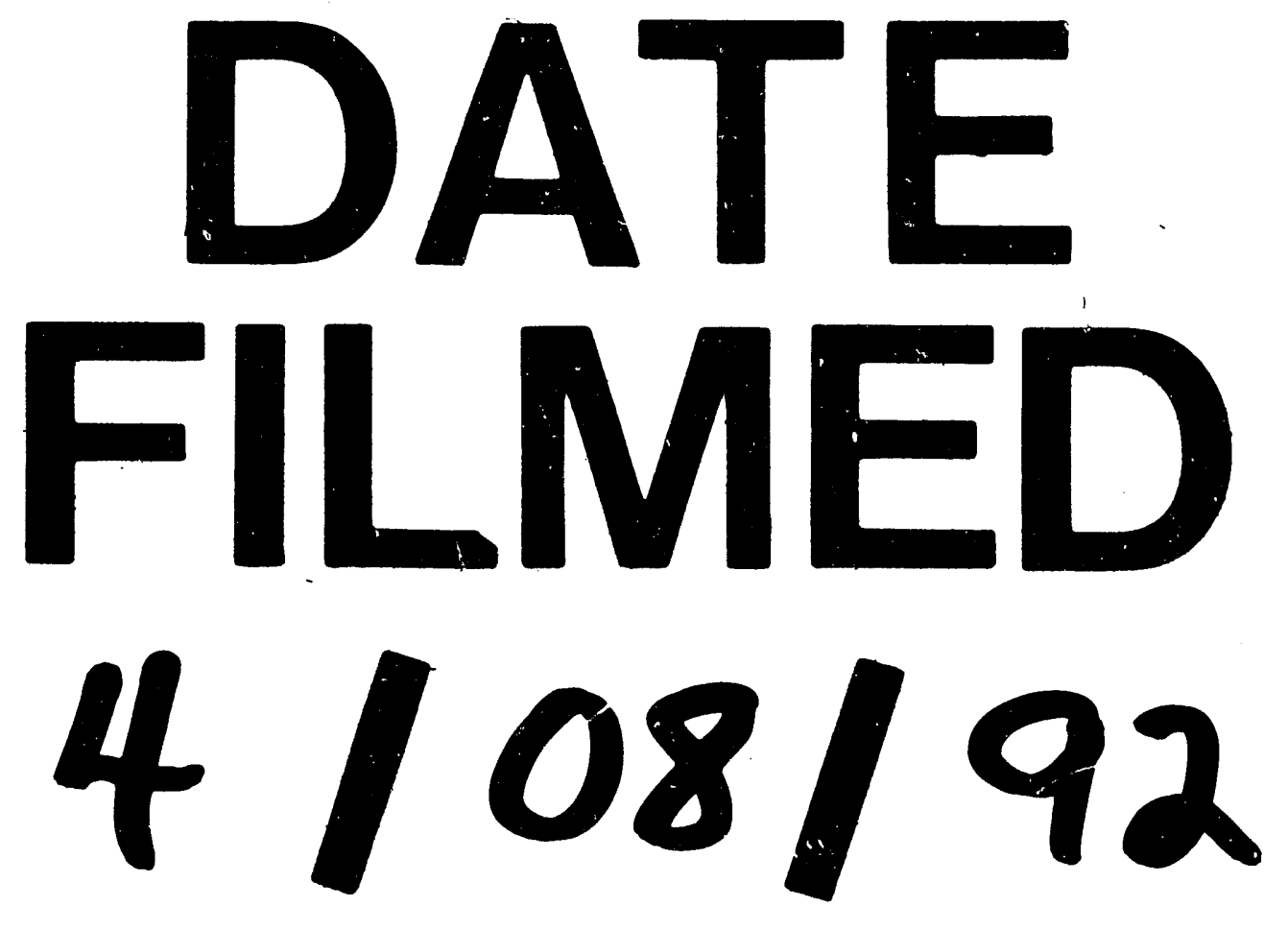

$4 / 08 / 92$ 
